WSRC-STI-2007-00150, Rev 0

KEYWORDS:

Groundwater Modeling Flow and Contaminant Transport Richard's Equation Porous Media Variably Saturated Code Verification

RETENTION - Permanent

\title{
PORFLOW Testing and Verification Document
}

Sebastian E. Aleman

Savannah River National Laboratory

Publication Date: June 2007

Washington Savannah River Company

Savannah River National Laboratory

Aiken, SC 29808

Prepared for the U.S. Department of Energy

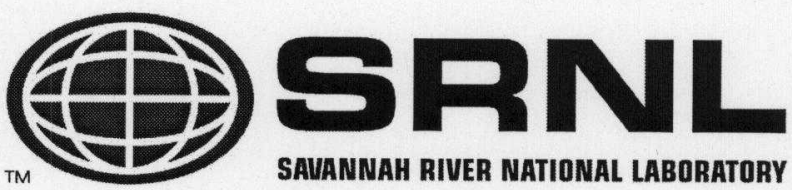

Under Contract No. DE-AC09-96SR18500 
DOCUMENT: WSRC-STI-2007-00150, Rev 0

TITLE: PORFLOW Testing and Verification Document

\section{APPROVALS}

Date:

Sebastian E. Aleman, Author

Engineering, Modeling and Simulation Group, SRNL

Date:

Thong Hang, Technical Reviewer

Engineering, Modeling and Simulation Group, SRNL

Date:

Jeff Jordan, Technical Reviewer

Engineering, Modeling and Simulation Group, SRNL

Date:

Steven J. Hensel, Manager

Engineering, Modeling and Simulation Group, SRNL

Date:

B. Tom Butcher, Manager

Environmental Assessment \& Performance Modeling Group, SRNL 


\section{PORFLOW Testing and Verification Document}

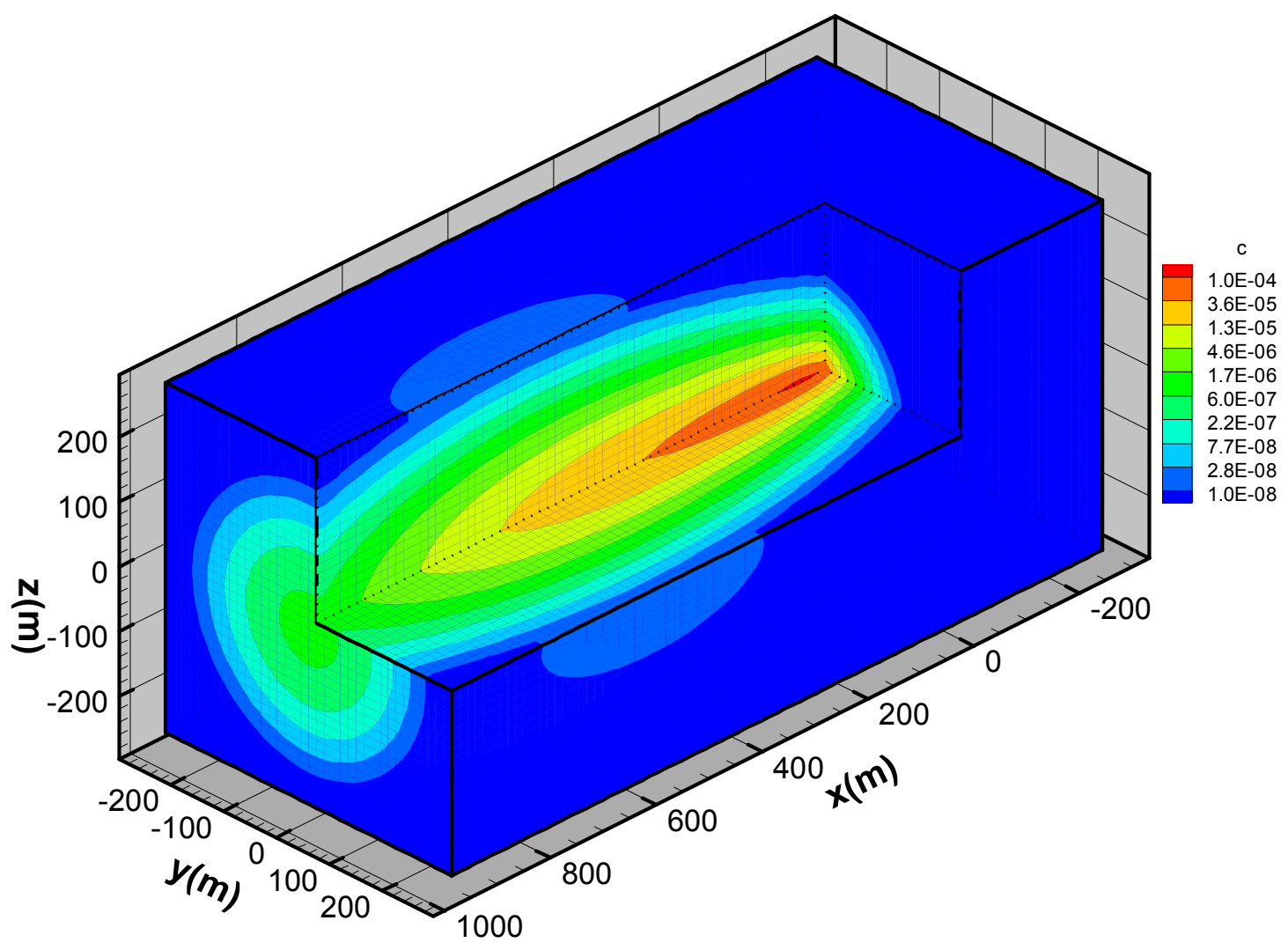

Washington Savannah River Company

Savannah River National Laboratory

Aiken, SC 29808

Prepared for the U.S. Department of Energy

Under Contract No. DE-AC09-96SR18500

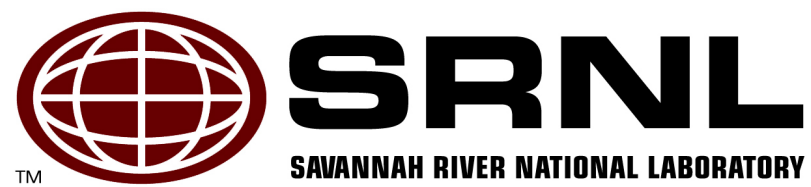




\section{DISCLAIMER}

This report was prepared by Washington Savannah River Company (WSRC) for the United States Department of Energy under Contract No. DE-AC09-96SR18500 and is an account of work performed under that contract. Neither the United States Department of Energy, nor WSRC, nor any of their employees makes any warranty, expressed or implied, or assumes any legal liability or responsibility for the accuracy, completeness, or usefulness, of any information, apparatus, or product or process disclosed herein or represents that its use will not infringe privately owned rights. Reference herein to any specific commercial product, process, or service by trademark, name, manufacturer or otherwise does not necessarily constitute or imply endorsement, recommendation, or favoring of same by WSRC or by the United States Government or any agency thereof. The views and opinions of the authors expressed herein do not necessarily state or reflect those of the United States Government or any agency thereof.

\section{Printed in the United States of America}

Prepared For

U.S. Department of Energy 
WSRC-STI-2007-00150, Rev 0

\author{
KEYWORDS: \\ Groundwater Modeling \\ Flow and Contaminant Transport \\ Richard's Equation \\ Porous Media \\ Variably Saturated \\ Code Verification \\ RETENTION - Permanent
}

\title{
PORFLOW Testing and Verification Document
}

Sebastian E. Aleman

Savannah River National Laboratory

Publication Date: June 2007

Washington Savannah River Company Savannah River National Laboratory Aiken, SC 29808

Prepared for the U.S. Department of Energy

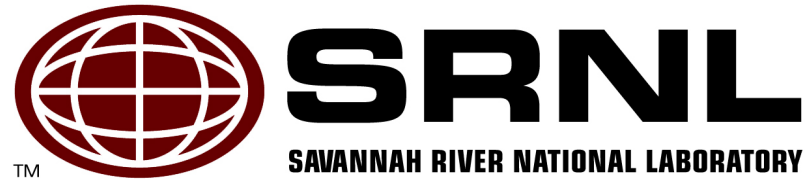


WSRC-STI-2007-00150

Under Contract No. DE-AC09-96SR18500 


\section{TABLE OF CONTENTS}

ABSTRACT . .1

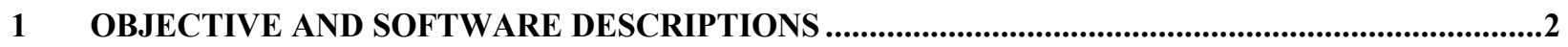

2 SOFTWARE INSTALLATION AND COMPUTING PLATFORM......................................................2

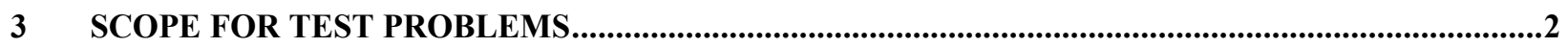

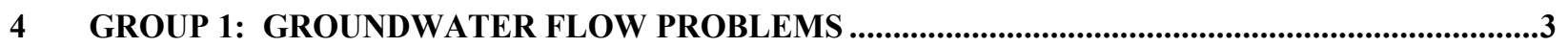

4.1 StEAdY-STATE, ONE-DimENSIONAL FLOW IN A CONFINED AQUIFER ..................................................

4.2 STEADY-STATE, ONE-DiMENSIONAL FLOW IN AN UNCONFINED AQUIFER ..............................................

4.3 Steady-STAte, Two-Dimensional Flow through a Heterogeneous AQuifer SYSTEM...................15

4.4 UnConfined AQUiFER SubJeCt To Combined ReCharge/Drain BC .............................................20

4.5 Transient, ONE-Dimensional Flow to A Well In A Confined AquifER (Theis, 1935) ...................27

4.6 Transient, Two-Dimensional Flow to a Well in an Anisotropic Confined Aquifer (Hantush

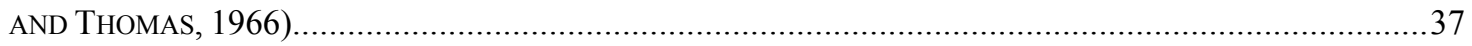

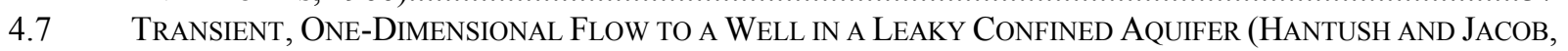

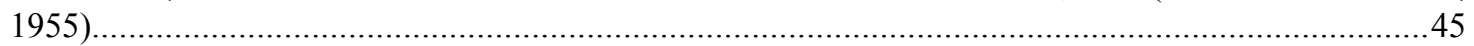

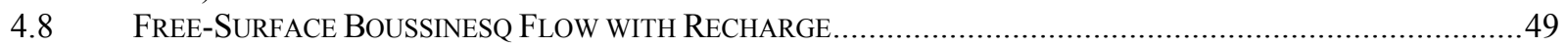

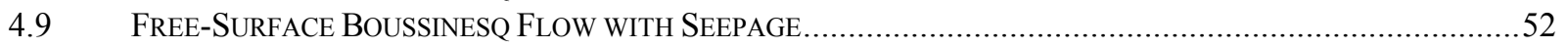

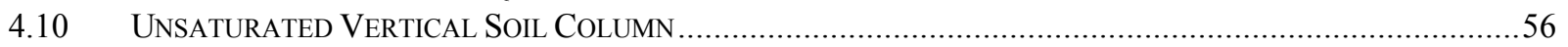

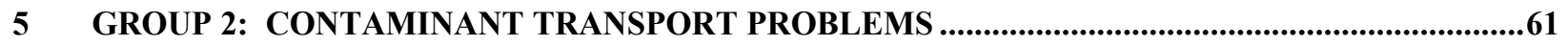

5.1 One-Dimensional Saturated Solute Transport in A Uniform Flow Field .................................61

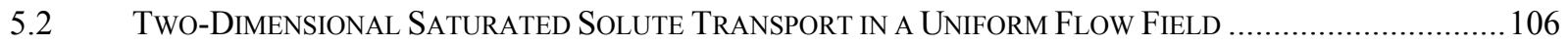

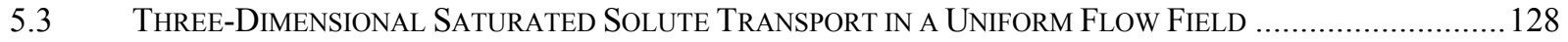

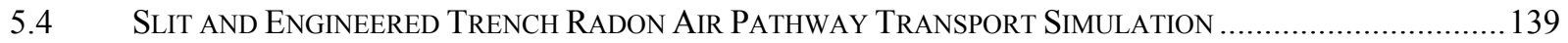

5.5 IMPACT OF PORFLOW RETARDATION MOdEL ON VARIABLy SATURATED SOlUTE TRANSPORT............148

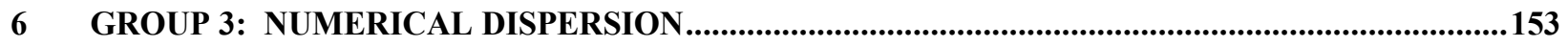

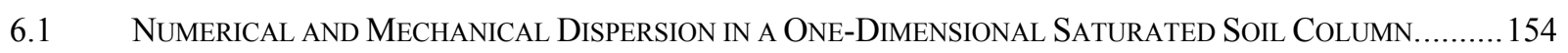

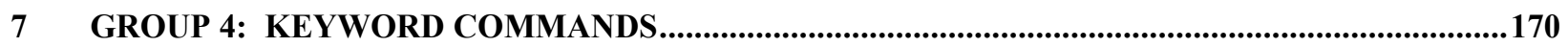

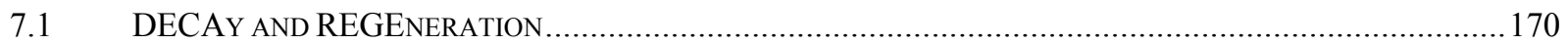

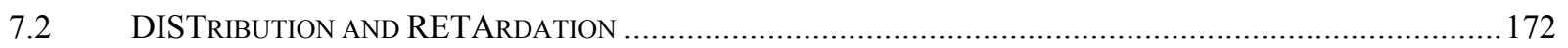

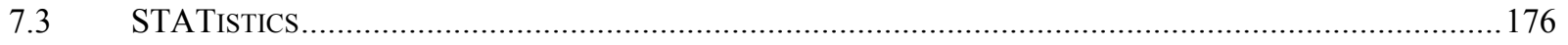

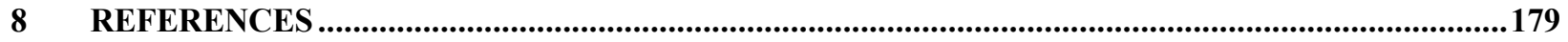

A HANTUSH AND JACOB NUMERICAL CODE .................................................................................180

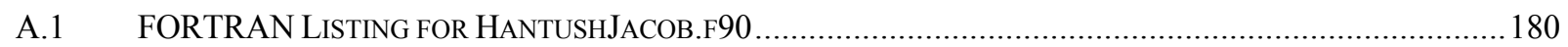

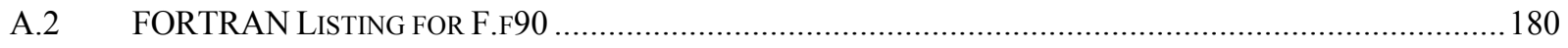

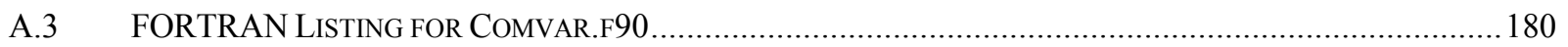

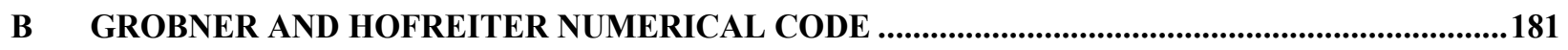

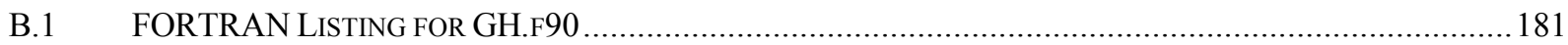

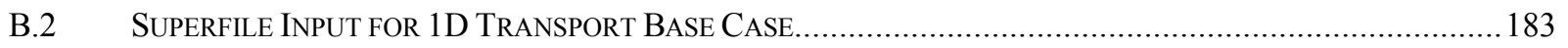

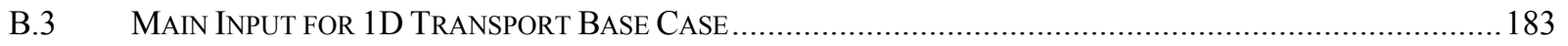

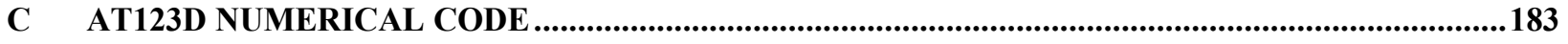


WSRC-STI-2007-00150, Rev 0

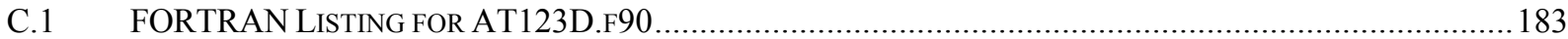

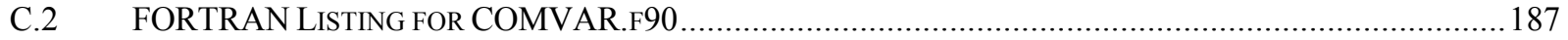

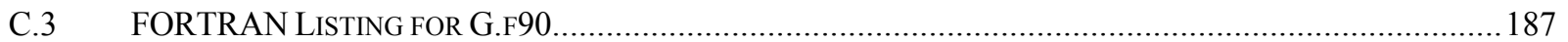

C.4 FORTRAN LISTING FOR GX.F90

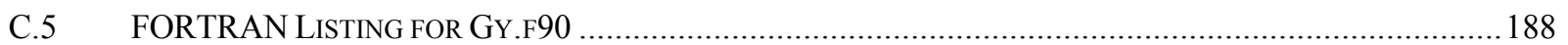

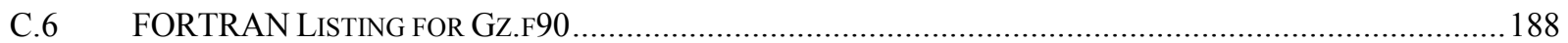

C.7 SUPERFILE INPUT FOR 2D TRANSPORT BASE CASE (CASE A) ........................................................... 189

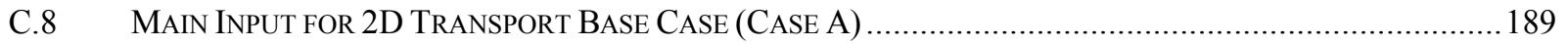

D BATEMAN NUMERICAL CODE …..................................................................................................189

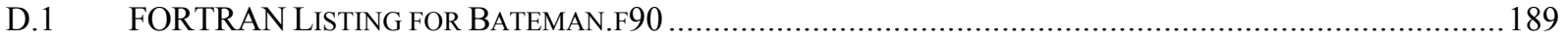

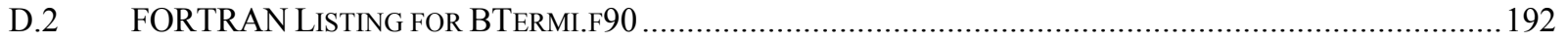

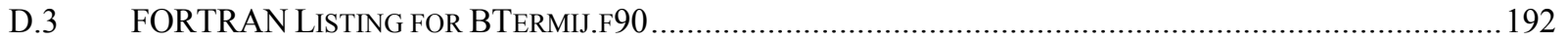

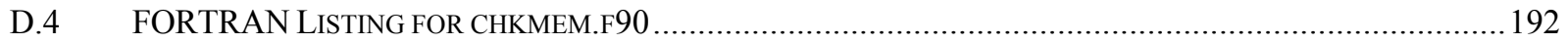

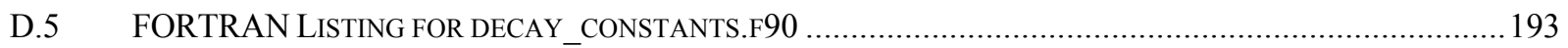

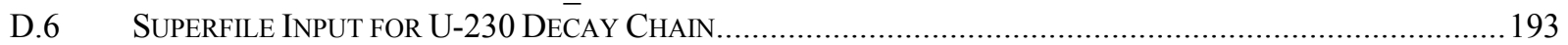

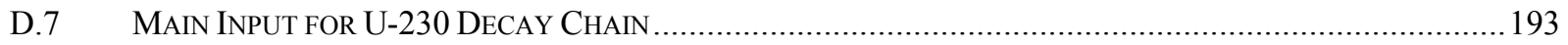




\section{WSRC-STI-2007-00150, Rev 0}

\section{LIST OF TABLES}

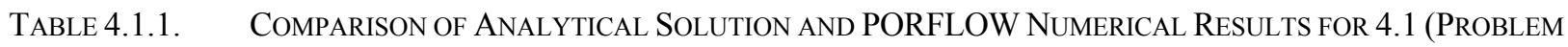

TABLE 4.1.2.

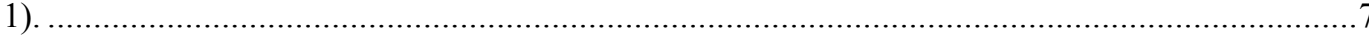

TABLE 4.1.3. PORFLOW DARCY VELOCITY AT NODE 201 FOR 4.1 (PROBLEM 2) ........................................... 7

TABLE 4.1.4.

TABLE 4.2.1.

TABLE 4.2.2.

TABLE 4.2.3.

TABLE 4.3.1.

TABLE 4.3.2.

TABLE 4.4.1.

TABLE 4.4.2.

TABLE 4.4.3.

TABLE 4.5.1.

TABLE 4.5.2.

TABLE 4.5.3.

TABLE 4.5.4.

TABLE 4.6.1.

INPUT COMMANDS FOR 4.1 (PROBLEM 1)

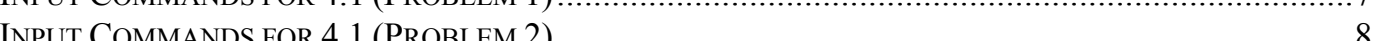

COMPARISON OF ANALYTICAL SOLUTION AND PORFLOW NU..............................................

INPUT COMMANDS FOR 4.2 (PROBLEM 1) ...................................................................... 13

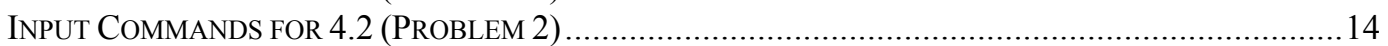

APPROXIMATE ANALYTICAL AND PORFLOW HydRAULIC HEAd SOLUTION FOR THE UNCONFINED

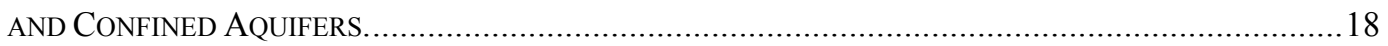

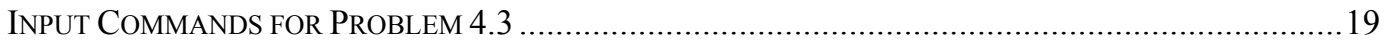

ANALYTICAL, COMSOL AND PORFLOW WATER TABLE COMPARISON....................................22

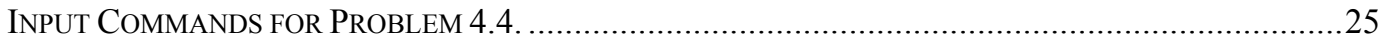

NODAL GRID FOR PROBLEM 4.4 ............................................................................26

COMPARISON OF THEIS, COMSOL AND PORFLOW TRANSIENT DRAWDOWN (0 TO 1 HOUR) .........33

COMPARISON OF THEIS, COMSOL AND PORFLOW TRANSIENT DRAWNDOWN (0 TO 1 DAY) .........33

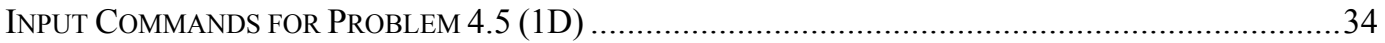

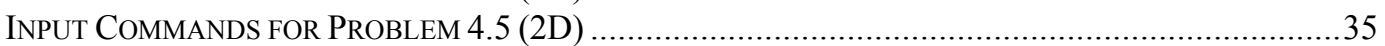

COMPARISON OF HANTUSH AND THOMAS, COMSOL AND PORFLOW NUMERICAL RESULTS AT X =

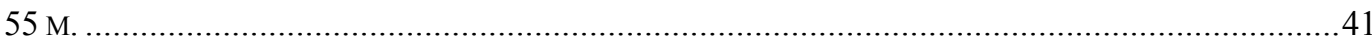

TABLE 4.6.2. COMPARISON OF HANTUSH AND THOMAS, COMSOL AND PORFLOW NUMERICAL RESULTS AT Y =

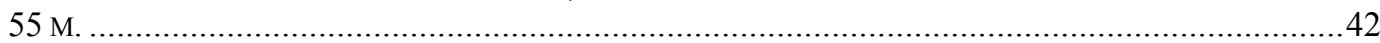

TABLE 4.6.3. COMPARISON OF HANTUSH AND THOMAS, COMSOL AND PORFLOW NUMERICAL RESULTS AT X $=$

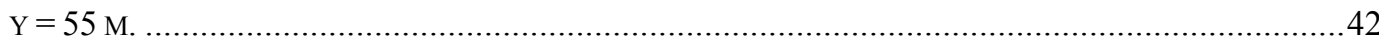

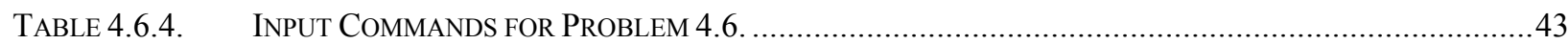

TABLE 4.7.1. COMPARISON OF HANTUSH AND JACOB, COMSOL AND PORFLOW TRANSIENT DRAWDOWN AT R $=$

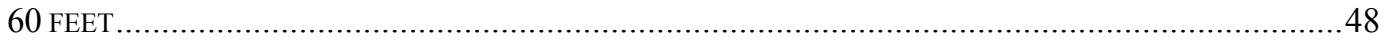

TABLE 4.7.2. INPUT COMMANDS FOR PROBLEM 4.7 ..........................................................................48

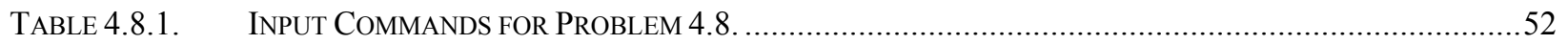

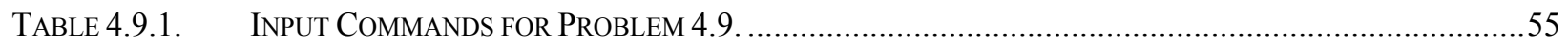

TABle 4.10.1. InPUt COMMANDS For Problem 4.10 (WATER RETENTION Profile)......................................60

TABlE 4.10.2. InPUT COMMANDS FOR PROBLEM 4.10 (CONSTANT SATURATION)...........................................60

Table 5.1.1. Values of the Physical Parameters, Mesh Spacing, Time Steps, and Key Computed

PARAMETERS USED IN THE ONE-DiMENSIONAL TRANSPORT SiMULATIONS..................................65

TABle 5.1.2. SumMARY OF Simulations PERFormed (BASE CASE AND VARIATIONS) ON THE ONE-DiMENSIONAL

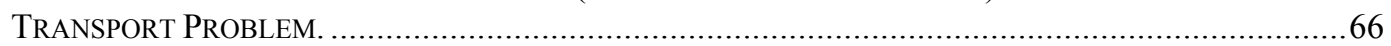

TABLE 5.1.3. COMPARISON OF ANALYTICAL AND PORFLOW NuMERICAL RESUltS FOR THE TRANSIENT 1D TRANSPORT PROBLEM (BASE CASE) ..................................................................... 75

TABLE 5.1.4. COMPARISON OF ANALYTICAL AND PORFLOW NUMERICAL RESUltS FOR THE TRANSIENT 1D TRANSPORT PROBLEM (CASE A) ........................................................................ 76

TABLE 5.1.5. COMPARISON OF ANALYTICAL AND PORFLOW NuMERICAL RESUltS FOR THE TRANSIENT 1D TRANSPORT PROBLEM (CASE B) ............................................................................. 77

TABLE 5.1.6. COMPARISON OF ANALYTICAL AND PORFLOW NUMERICAL RESUltS FOR THE TRANSIENT 1D TRANSPORT PROBLEM (CASE C) ...............................................................................78

TABLE 5.1.7. COMPARISON OF ANALYTICAL AND PORFLOW NUMERICAL RESUltS FOR THE TRANSIENT 1D

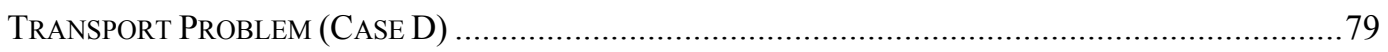

TABLE 5.1.8. COMPARISON OF ANALYTICAL AND PORFLOW NUMERICAL RESULTS FOR THE TRANSIENT 1D

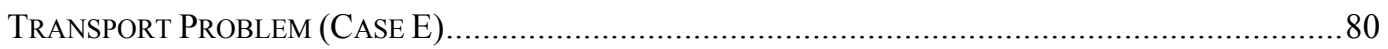

TABLE 5.1.9. COMPARISON OF ANALYTICAL AND PORFLOW NuMERICAL RESUltS FOR THE TRANSIENT 1D

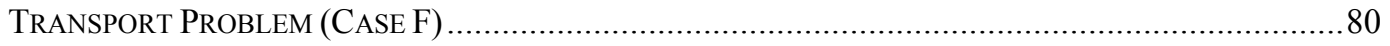

TABLE 5.1.10. COMPARISON OF ANALYTICAL AND PORFLOW NuMERICAL RESUltS FOR THE TRANSIENT 1D TRANSPORT PROBLEM (CASE G) .............................................................................. 81 
WSRC-STI-2007-00150, Rev 0

TABLE 5.1.11. COMPARISON OF ANALYTICAL AND PORFLOW NUMERICAL RESUlts FOR THE TRANSIENT 1D

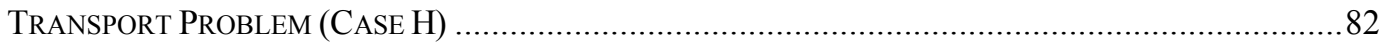

TABLE 5.1.12. COMPARISON OF ANALYTICAL AND PORFLOW NUMERICAL RESUlTS FOR THE TRANSIENT 1D

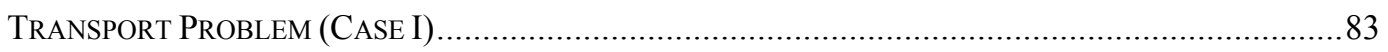

TABLE 5.1.13. COMPARISON OF ANALYTICAL AND PORFLOW NUMERICAL RESULTS FOR THE TRANSIENT 1D

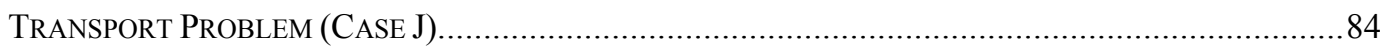

TABLE 5.1.14. COMPARISON OF ANALYTICAL AND PORFLOW NUMERICAL RESUlTS FOR THE TRANSIENT 1D

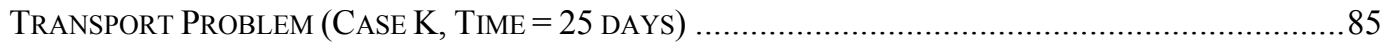

TABLE 5.1.15. COMPARISON OF ANALYTICAL AND PORFLOW NUMERICAL RESUlTS FOR THE TRANSIENT 1D

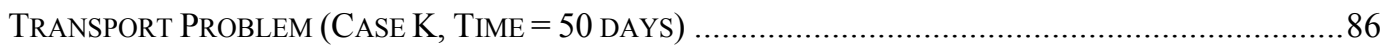

TABLE 5.1.16. COMPARISON OF ANALYTICAL AND PORFLOW NUMERICAL RESUltS FOR THE TRANSIENT 1D

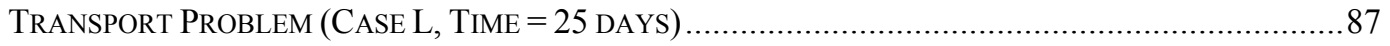

TABLE 5.1.17. COMPARISON OF ANALYTICAL AND PORFLOW NUMERICAL RESUltS FOR THE TRANSIENT 1D

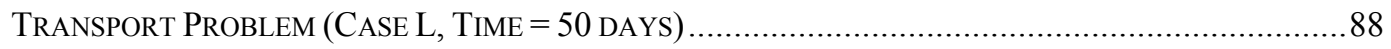

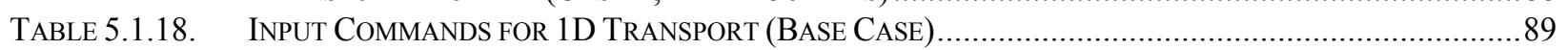

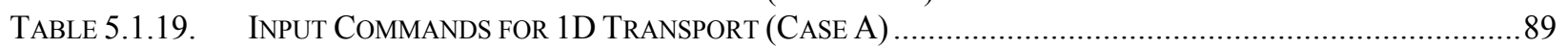

TABLE 5.1.20. INPUT COMMANDS FOR 1D TRANSPORT (CASE B) ............................................................. 90

TABLE 5.1.21. INPUT COMMANDS FOR 1D TRANSPORT (CASE C) …............................................................. 91

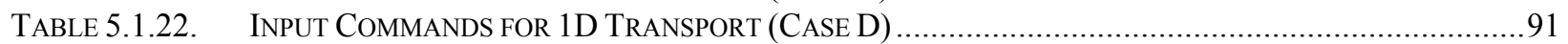

TABLE 5.1.23. INPUT COMMANDS FOR 1D TRANSPORT (CASE E) ..............................................................92

TABLE 5.1.24. INPUT COMMANDS FOR 1D TRANSPORT (CASE F) .................................................................93

TABLE 5.1.25. INPUT COMMANDS FOR 1D TRANSPORT (CASE G) ...................................................................99

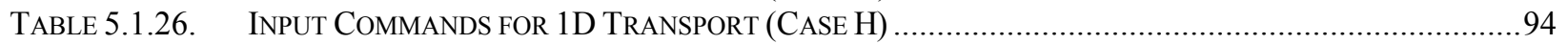

TABLE 5.1.27. INPUT COMMANDS FOR 1D TRANSPORT (CASE I) ……............................................................95

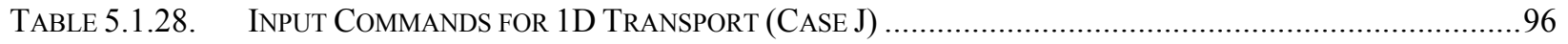

TABLE 5.1.29A. INPUT COMMANDS FOR 1D TRANSPORT (CASE K, T = 25 DAYS) …….......................................96

TABLE 5.1.29B. INPUT COMMANDS FOR 1D TRANSPORT (CASE K, T $=50$ DAYS) …….......................................99

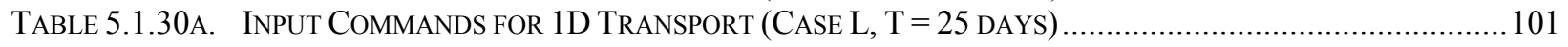

TABLE 5.1.30B. INPUT COMMANDS FOR 1D TRANSPORT (CASE L, T = 50 DAYS) ……....................................... 103

Table 5.2.1. Values of the Physical Parameters, Mesh Spacing, Time Steps, and Key Computed PARAMETERS USED IN THE TWO-DiMENSIONAL TRANSPORT SiMULATIONS................................110

TAble 5.2.2. Summary of Simulations Performed (BASE CASE AND VARiations) ON THE Two-Dimensional

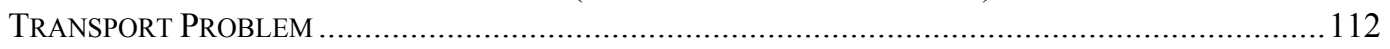

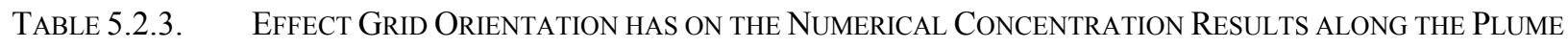
Centerline for the Transient 2D Transport Simulation (CASEs A and B, T = 1400 Days).

TABLE 5.2.4. EFFECT GRID ORIENTATION HAS ON THE NUMERICAL CONCENTRATION RESUltS TRANSVERSE TO the Plume Centerline (424 M Downstream of the sourCe) FOR the Transient 2D

TRANSPORT SiMUlation (CASES A AND B, T = 1400 DAYS)..................................................... 123

TABLE 5.2.5. EFFECT GRID ORIENTATION HAS ON THE NUMERICAL CONCENTRATION RESUltS TRANSVERSE TO the Plume Centerline (0 M Downstream of the source) For the Transient 2D Transport

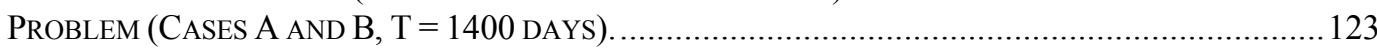

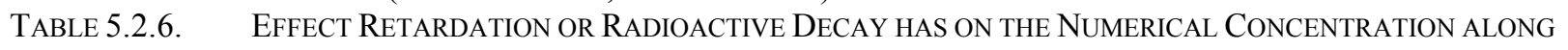
the Plume Centerline for the Transient 2D Transport Problem (Cases C and D, T = 1400

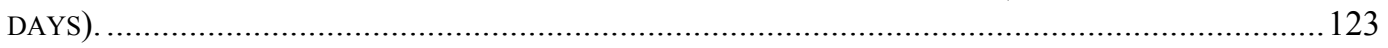

TABLE 5.2.7. EFFECT RETARDATION OR RADIOACTIVE DECAY HAS ON THE NUMERICAL CONCENTRATION Transverse to the Plume Centerline for the Transient 2D Transport Problem at X $=420$

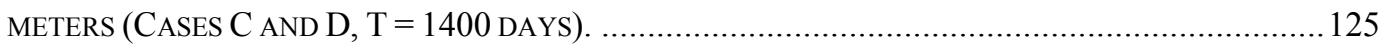

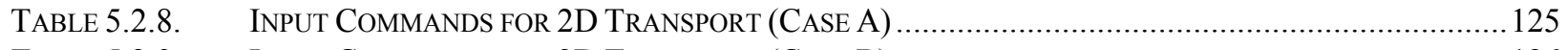

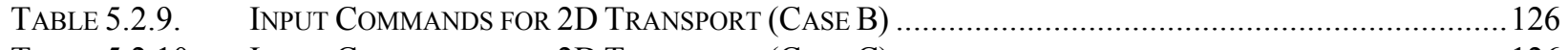

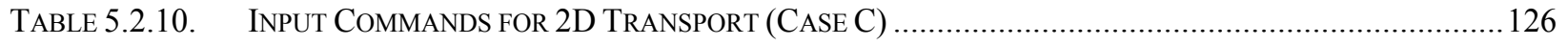

TABLE 5.2.11. INPUT COMMANDS FOR 2D TRANSPORT (CASE D) ................................................................. 127

Table 5.3.1. Values of the Physical Parameters, Mesh Spacing, Time Steps, and Key Computed

PARAMETERS USED IN THE THREe-DimENSIONAL TRANSPORT SimULATIONS ...............................133 
WSRC-STI-2007-00150, Rev 0

Table 5.3.2. Concentration Profile for 3D Transport of the Base Case along the Plume Centerline

AT 1400 DAYS. 137

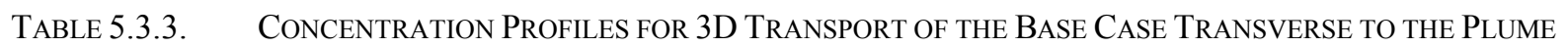
CENTERLINE LOCATED AT X $=120$ METERS (1400 DAYS). ..................................................... 138

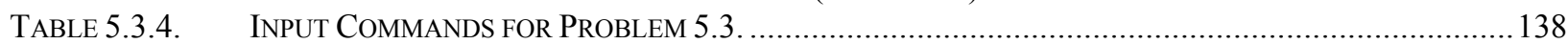

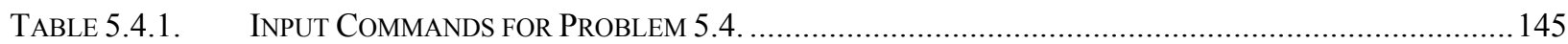

TABLE 5.4.2. GEOMETRY SPECIFICATION FOR PROBLEM 5.4 ..................................................................... 146

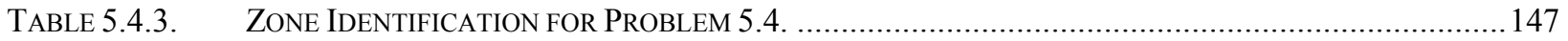

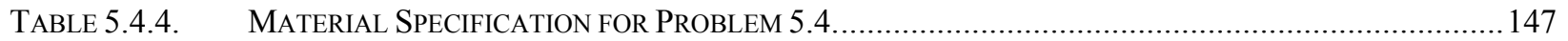

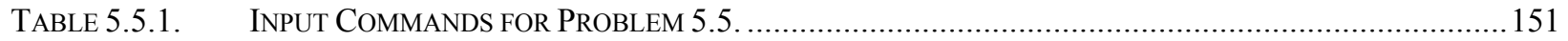

TABLE 6.1.1. INPUT COMMANDS FOR PROBLEM 6.1 (NuMERICAL DiSPERSION). ............................................ 156

TABLE 6.1.2. INPUT COMMANDS FOR PROBLEM 6.1 (MECHANICAL DiSPERSION)............................................ 163

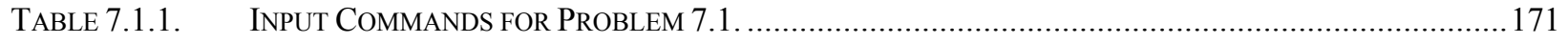

TABLE 7.2.1. INPUT COMMANDS FOR PROBLEM 7.2 (DIST COMMAND) ...................................................... 174

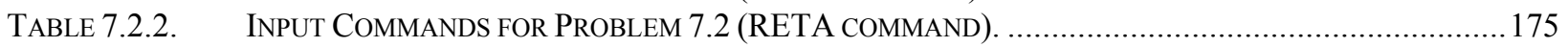

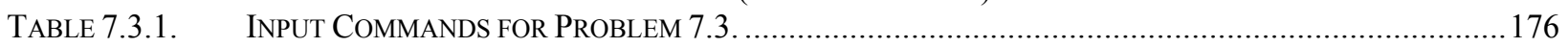

TABLE 7.3.2. LOCATION OF POINT SOURCE FOR PROBLEM 7.3 …........................................................... 177

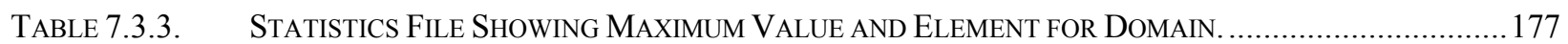

TABLE 7.3.4. LOCATION OF PluME CENTERLINE NODES BEYOND X $=700$ METERS. ........................................ 178

Table 7.3.5. Statistics File Showing MaXimum Value and Element Beyond X $=700$ M...................... 178 


\section{WSRC-STI-2007-00150, Rev 0}

\section{LIST OF FIGURES}

Figure 4.1.1. Schematic Diagram of a Confined Aquifer with a Constant Head Boundary Condition AT $X=0$ AND EITHER A GENERAL HEAD OR RIVER BOUNDARY CONDITION AT X $=200 \ldots \ldots \ldots \ldots \ldots \ldots . . . . . .3$

FIGURE 4.1.2. PORFLOW GRID FOR PROBLEM 4.1 ................................................................................5

Figure 4.1.3. COMPARISON OF ANALytical Solution to PORFLOW Numerical Results for 4.1 (Problem

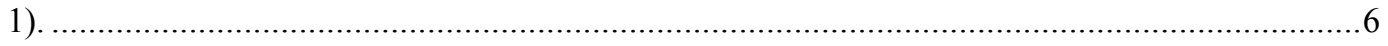

FIGURE 4.1.4. COMPARISON OF ANALYTICAL SOLUTION TO PORFLOW DARCY VELOCITY FOR 4.1 (PROBLEM 2)..6

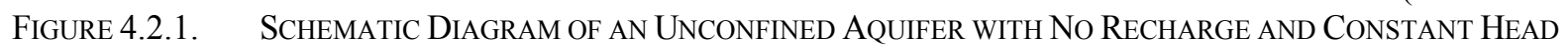

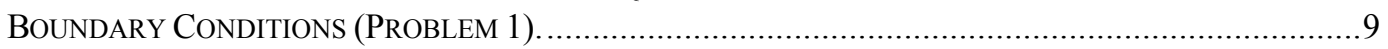

FIGURE 4.2.2. SCHEMATIC DIAGRAM OF AN UNCONFINED AQUIFER WITH RECHARGE AND MIXED BOUNDARY

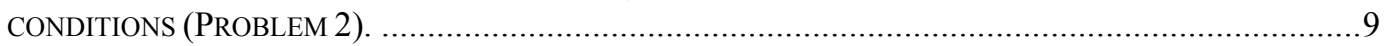

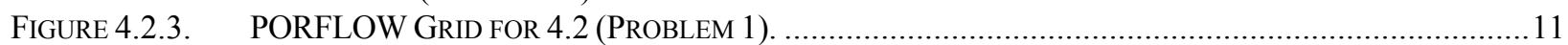

FIGURE 4.2.4. PORFLOW GRID FOR 4.2 (PROBLEM 2). ............................................................................. 11

Figure 4.2.5. COMPARISON OF ANALYTICAL SOLUTION AND PORFLOW RESUltS For 4.2 (PROBlem 1).............12

Figure 4.2.6. COMPARISON OF ANALYTICAL SOLUTION AND PORFLOW RESUltS FOR 4.2 (PROBlem 2).............12

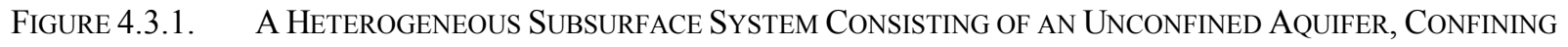

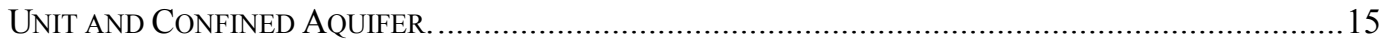

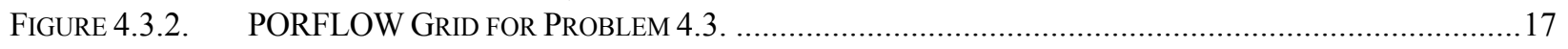

Figure 4.3.3. APPROXIMATE ANALYTICAL AND PORFLOW HydRaUlic HEAD ProfiLES FOR THE UNCONFINED

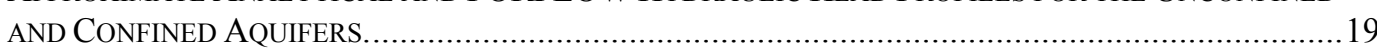

FigURE 4.4.1. SCHEMATIC ILLUSTRATION OF AN UNCONFINED AQUIFER EXPERIENCING BOTH RECHARGE AND DRAINAGE AT THE GROUND SURFACE; SEEPLINE UNKNOWN A PRIORI............................................20

Figure 4.4.2. CAUCHY BOUNDARY CONDITION FOR THE SiMULTANEOUS TREATMENT OF GROUNDWATER

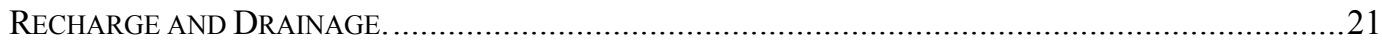

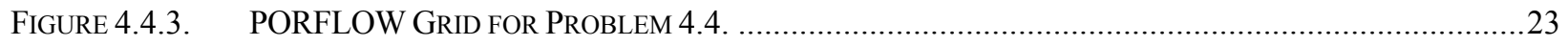

FigURE 4.4.4. COMSOL FINITE-ELEMENT MESH FOR PROBLEM 4.4 ...........................................................24

Figure 4.4.5. COMPARISON OF PORFLOW HYdRAULIC HEAD DisTRIBUTION TO ANALYTICAL SOLUTION AND

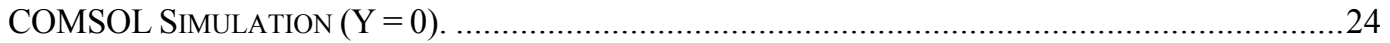

Figure 4.5.1. RAdial Flow TO A PUMPING WELL IN A CONFINED AQUIFER..................................................28

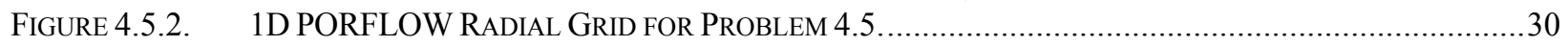

FIGURE 4.5.3. 2D PORFLOW CARTESIAN GRID FOR PROBLEM 4.5 ........................................................... 31

FIGURE 4.5.4. THEIS, COMSOL AND PORFLOW TRANSIENT DRAWDOWN AT R $=55$ METERS..........................31

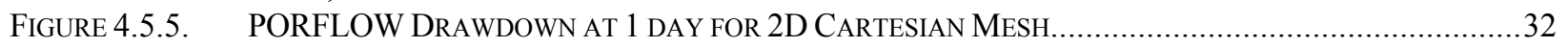

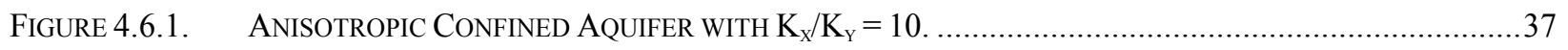

FigURE 4.6.2. COMSOL FINITE-ELEMENT MESH FOR PROBLEM 4.6 ............................................................. 38

Figure 4.6.3. HANTUSH AND THOMAS, COMSOL AND PORFLOW TRANSIENT DRAWDOWN AT X $=55$ METERS.....

FIGURE 4.6.4. HANTUSH AND THOMAS, COMSOL AND PORFLOW TRANSIENT DRAWDOWN AT Y $=55$ METERS.....

FIGURE 4.6.5. HANTUSH AND THOMAS, COMSOL AND PORFLOW TRANSIENT DRAWDOWN AT X $=\mathrm{Y}=55$

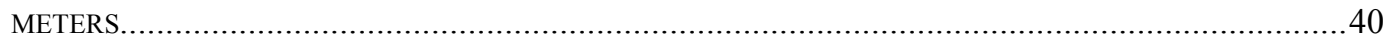

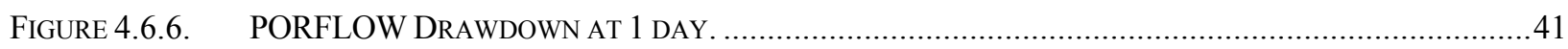

Figure 4.7.1. SCHEMATIC Diagram OF A LEAKY CONFINED AQUiFER WiTH CONSTANT Discharge From A

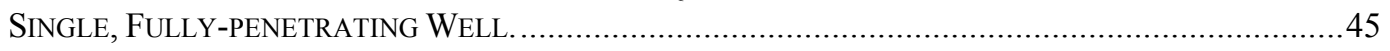

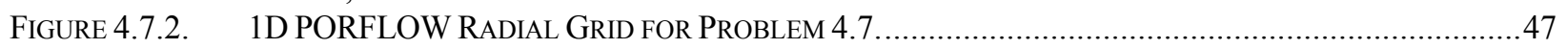

FigURE 4.7.3. HANTUSH AND JACOB, COMSOL AND PORFLOW TRANSIENT DRAWDOWN AT R $=60$ FEET $\ldots \ldots . . .47$

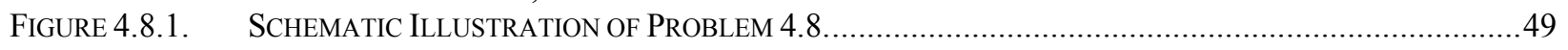

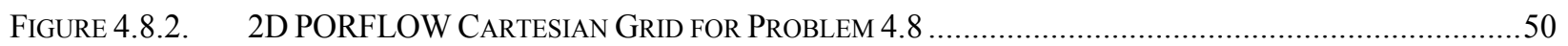

FigURE 4.8.3. COMSOL FINITE-ELEMENT MESH FOR PROBLEM 4.8 .......................................................... 51

Figure 4.8.4. Time History of Phreatic Surface Due to ReCharge. .....................................................51

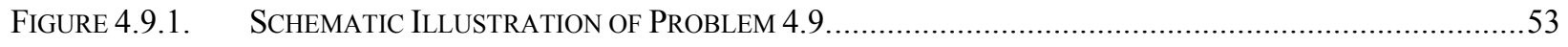

FIGURE 4.9.2. 2D PORFLOW CARTESIAN GRID FOR PROBLEM 4.9 ..........................................................5

FiguRE 4.9.3. COMSOL FINITE-ElEMENT MESH For ProBlem 4.9 ..............................................................5 
WSRC-STI-2007-00150, Rev 0

Figure 4.9.4. Time History of Phreatic Surface Due to SeEPage. ............................................................55

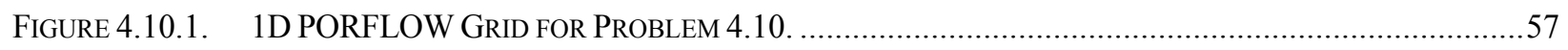

FigURE 4.10.2. COMSOL FINITE-ELEMENT MESH FOR PROBLEM 4.10 .............................................................58

Figure 4.10.3. PREDicted COLUMN SATURATION PRofile FOR COMSOL AND PORFLOW Simulations. ..........59

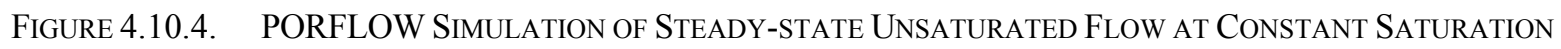
$(75 \%)$

Figure 5.1.1. SCHEMATIC DiAGRAM FOR 1D SOLUTE TRANSPORT IN A CONFINED AQUIFER...............................61

FIGURE 5.1.2. PORFLOW GRID FOR 1-D BASE CASE TRANSPORT SIMULATION...............................................6

Figure 5.1.3. CONCENTRATION PROFILES For THE 1D TRANSPORT OF THE BASE CASE. ....................................69

Figure 5.1.4 Concentration Profiles for the 1D Transport Showing EfFect of Retardation (CASe A)..

Figure 5.1.5 CONCENTRATION PROFILES FOR THE 1D TRANSPORT ShOWING EFFECT OF RADiOACTIVE DeCAY

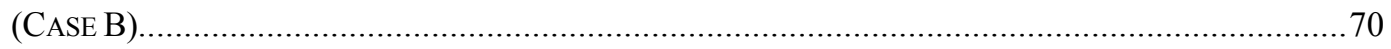

Figure 5.1.6 CONCENTRATION PROFILES FOR THE 1D TRANSPORT Showing COMBINED EFFECT OF

RETARDATION AND RADIOACTIVE DECAY (CASE C) ............................................................... 70

Figure 5.1.7 Concentration Profiles for the 1D Transport Showing EfFect of Grid Size, $\Delta \mathrm{X}=20 \mathrm{M}$

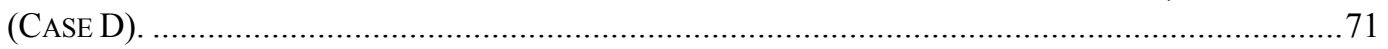

Figure 5.1.8 Concentration Profiles For the 1D TRANSPort Showing EFFECT OF Grid Size, $\Delta \mathrm{X}=40 \mathrm{M}$

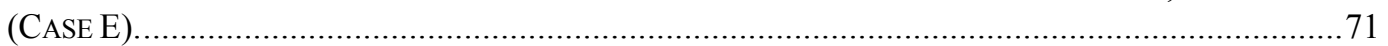

Figure 5.1.9 CONCENTRATION PROFILES FOR THE 1D TRANSPORT SHOWING EFFECT OF GRID Size, $\Delta \mathrm{X}=80 \mathrm{M}$

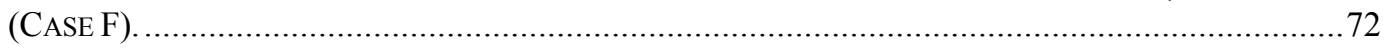

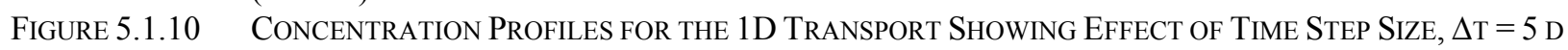

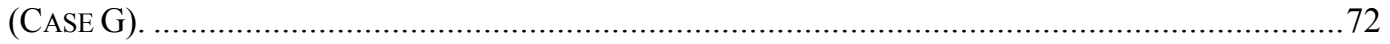

Figure 5.1.11 Concentration Profiles for the 1D Transport Showing EFfect of Time Step Size, $\Delta \mathrm{T}=10$

D (CASE H). .........................................................................................................

Figure 5.1.12 Concentration Profiles for the 1D TRANSPort Showing EFFeCt Of Time Step Size, $\Delta \mathrm{T}=25$

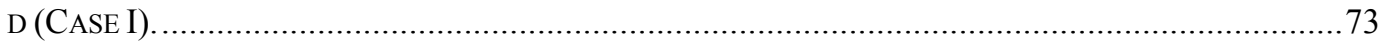

Figure 5.1.13 Concentration Profiles for the 1D Transport at High Peclet Number Showing Effect of DISPERSION (CASE J) .................................................................................................... 74

Figure 5.1.14 Concentration Profiles for the 1D Transport at High Peclet Number Showing Effect of NODAL INTEGRATION SCHEMES (CASE K). .........................................................................

Figure 5.1.15 Concentration Profiles for the 1D Transport Showing EFFect of Property Averaging

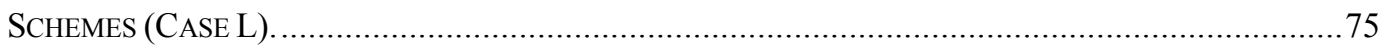

Figure 5.2.1. SCHEMATIC DiAGRAM FOR 2D SOLUTE TRANSPORT IN A CONFINED AQUIFER............................... 106

FIGURE 5.2.2. PORFLOW PARALLEL GRID FOR 2D TRANSPORT SiMULATIONS................................................114

FigURE 5.2.3. PORFLOW DiAgONAL GRID FOR 2D TRANSPORT SiMULATION. .............................................. 114

Figure 5.2.4. PORFLOW CONCENTRATION Plume FOR 2D TRANSPORT OF THE BASE CASE ON THE PARAllel

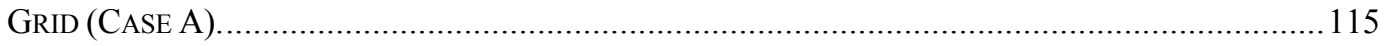

Figure 5.2.5. Concentration Profiles for 2D Transport of the Base Case along the Plume Centerline

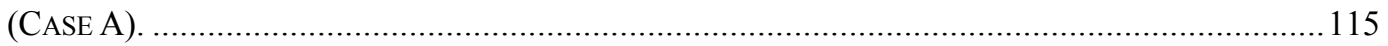

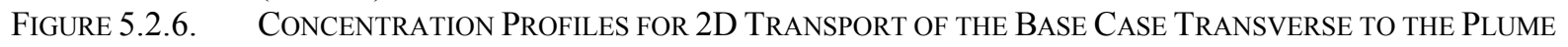
CENTERLINE AT X = 420 METERS (CASE A)......................................................................... 116

Figure 5.2.7. PORFLOW CONCENTRATION Plume FOR 2D TRANSPORT OF THE BASE CASE ON THE Diagonal

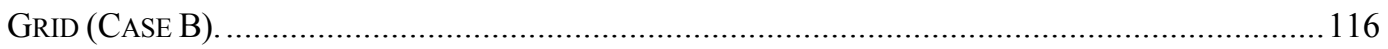

Figure 5.2.8. Concentration Profiles for 2D Transport of the Base CaSe along the Plume Centerline

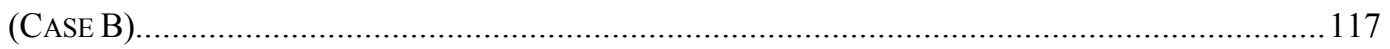

Figure 5.2.9. Concentration Profiles for 2D Transport of the Base Case Transverse to the Plume CENTERLINE AT X = 424 METERS (CASE B) . ...................................................................... 117

Figure 5.2.10 PORFLOW CONCENTRATION PlUME FOR 2D TRANSPORT SHOWING THE EFFECT OF RETARDATION

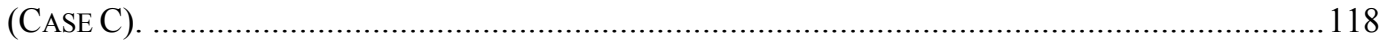

Figure 5.2.11 COncentration Profiles for 2D Transport Showing the EFFeCt OF Retardation along

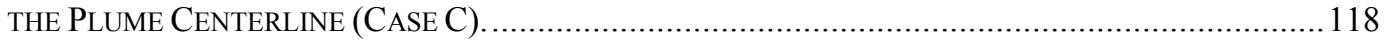

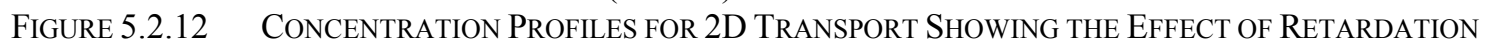
TRANSVERSE to THE Plume Centerline AT X = 420 Meters (CASE C). ...................................119 


\section{WSRC-STI-2007-00150, Rev 0}

Figure 5.2.13 PORFLOW Concentration Plume for 2D Transport Showing the Effect of Radioactive

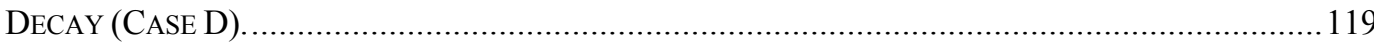

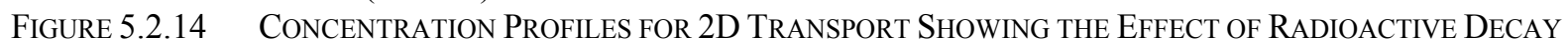
ALONG THE Plume CENTERLINE (CASE D). ........................................................................ 120

Figure 5.2.15 Concentration Profiles for 2D Transport Showing the EFfect of Radioactive Decay TRANSVERSE TO THE Plume CENTERLINE AT X $=420$ METERS (CASE D) ...................................... 120

FIGURE 5.3.1. PORFLOW GRID FOR 3D TRANSPORT SIMULATION. ............................................................... 135

Figure 5.3.2 PORFlOW CONCENTRATION Plume For 3D TRANSPORT OF THE BASE CASE ON A PARAllel Grid.

Figure 5.3.3 Concentration Profile for 3D Transport of the Base Case along the Plume Centerline.

Figure 5.3.4 Concentration Profiles for 3D Transport of the Base CaSe Transverse to the Plume CENTERLINE (IN BOTH Y AND Z DIRECTIONS) LOCATED AT X = 120 METERS. ................................136

FiguRE 5.4.1. RADIOACTIVE DECAY ChaINS LEADING TO RN-222 ........................................................... 142

Figure 5.4.2. PORFLOW 1D CONCEPTUAL MODEL AND MESH FOR RADON TRANSPORT IN THE SLIT AND

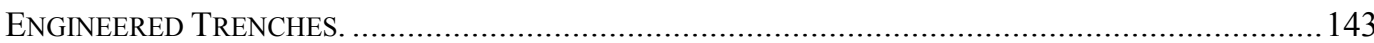

Figure 5.4.3. COMPARISON OF COMSOL AND PORFLOW Th-230 CONCENTRATION PROFILES AT 1000 YeARS.....

FIGURE 5.4.4. COMPARISON OF COMSOL AND PORFLOW RA-226 CONCENTRATION PROFILES. ...................... 144

FigURE 5.4.5. COMPARISON OF COMSOL AND PORFLOW RN-222 CONCENTRATION PROFILES AT 1000 YeARS.....

Figure 5.4.6. COMPARISON OF COMSOL AND PORFLOW RN-222 DiSPERSIVE FlUX AT THE LAND SURFACE. . 145

FIGURE 5.5.1. PORFLOW GRID FOR 1D VARIABLY SATURATED TRANSPORT............................................... 150

Figure 5.5.2. COMPARISON OF COMSOL AND PORFLOW PARENT AND DAUGHTER SOLUTE CONCENTRATION

PROFILES AT 25 AND 50 YEARS (PORFLOW USING EQ. 5.5.2) ................................................. 150

Figure 5.5.3. COMPARISON OF COMSOL AND PORFLOW PARENT AND DAUGHTER SOLUTE CONCENTRATION PROFILES AT 25 AND 50 YEARS (PORFLOW USING EQ. 5.5.1) ................................................ 151

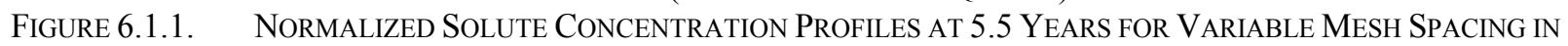
PORFLOW WITH NUMERICAL DISPERSION. ...................................................................... 155

Figure 6.1.2. Peak Normalized Solute Concentration as a Function of MeSh SPaCing in PORFLOW at 5.5 YEARS WITH NUMERICAL DISPERSION. .............................................................................. 155

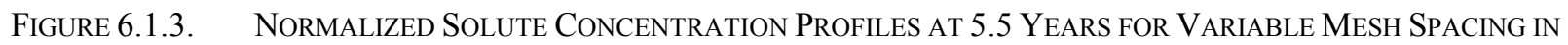

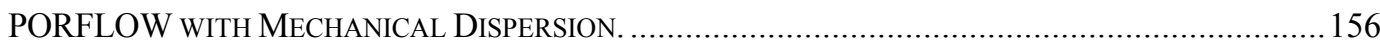

Figure 7.1.1. COMPARISON OF RADIONUClide CONCENTRATIONS IN THE U-230 DeCAy Chain to THE BATEMAN

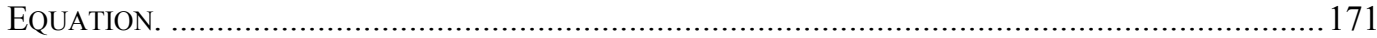

FiguRE 7.2.1. IMPLEMENTATION OF SOLUBILITY Limited SOURCE BASED ON THE DIST AND RETA COMMAND...... 174 


\begin{abstract}
The PORFLOW software package is a comprehensive mathematical model for simulation of multi-phase fluid flow, heat transfer and mass transport in variably saturated porous and fractured media. PORFLOW can simulate transient or steady-state problems in Cartesian or cylindrical geometry. The porous medium may be anisotropic and heterogeneous and may contain discrete fractures or boreholes with the porous matrix. The theoretical models within the code provide a unified treatment of concepts relevant to fluid flow and transport.

The main features of PORFLOW that are relevant to Performance Assessment modeling at the Savannah River National Laboratory (SRNL) include variably saturated flow and transport of parent and progeny radionuclides. This document involves testing a relevant sample of problems in PORFLOW and comparing the outcome of the simulations to analytical solutions or other commercial codes. The testing consists of the following four groups.
\end{abstract}

- Group 1: Groundwater Flow

- Group 2: Contaminant Transport

- Group 3: Numerical Dispersion

- Group 4: Keyword Commands 


\section{Objective and Software Descriptions}

PORFLOW is a commercially developed computer code for use in simulating groundwater flow and contaminant transport in the vadose zone and underlying aquifers. Since the results from calculations performed using PORFLOW are used to comply with regulatory laws, environmental permits and regulations, its software classification is upgraded from the current Level D to Level $\mathrm{C}$

PORFLOW is used in many modeling applications including work for Solid Waste (e.g., waste disposal in Saltstone, Components in Grout, Low Activity Waste Vaults etc.) and High-Level Waste Tank Closures. The results from PORFLOW calculations will form a basis to support many types of analyses such as the Composite Analysis or the Performance Assessment to comply with DOE Orders and environmental regulations. Therefore, PORFLOW is classified as Level C software.

\section{Software Installation and Computing Platform}

PORFLOW version 5.97.0 has been installed on the IBM PC workstation tegu4. The PORFLOW executable, porflow.exe, is a Windows 2000/XP console program and is located in the network directory \} \backslash \text { tegu4 } 4 \text { porflow. The testing of PORFLOW was performed on an IBM IntelliStation } \mathrm { Z } Pro with dual 3.4GHz Xeon processors running Windows 2000.

\section{Scope for Test Problems}

The test problems were selected based on the fact that analytical solutions (or code-to-code comparisons) exist that definitively establish the code accuracy capability and the resulting impact that mesh and control parameter settings have on accuracy. Four groups of test problems are described to verify the capability of the software to represent the physical phenomena characteristic of groundwater flow and transport applications at the Savannah River Site.

They are:

1. Group 1: Saturated and variably saturated groundwater flow in one and two dimensions (steady-state and transient conditions)

2. Group 2: Contaminant transport in one, two and three dimensions (transient).

3. Group 3: Numerical dispersion.

4. Group 4: Keyword Commands (e.g. STATistics) 


\section{Group 1: Groundwater Flow Problems}

\subsection{Steady-state, One-Dimensional Flow in a Confined Aquifer}

Figure 4.1.1 illustrates two confined aquifers experiencing steady, one-dimensional flow. The test cases are designed to confirm correct implementation of the general head (mixed) and river bed boundary conditions. The problems can be easily solved analytically.

PROBLEM 1

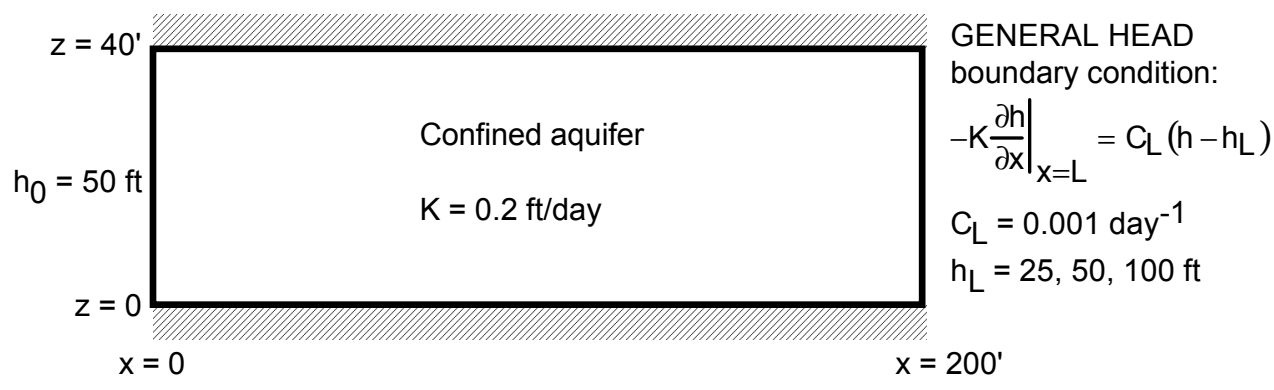

PROBLEM 2

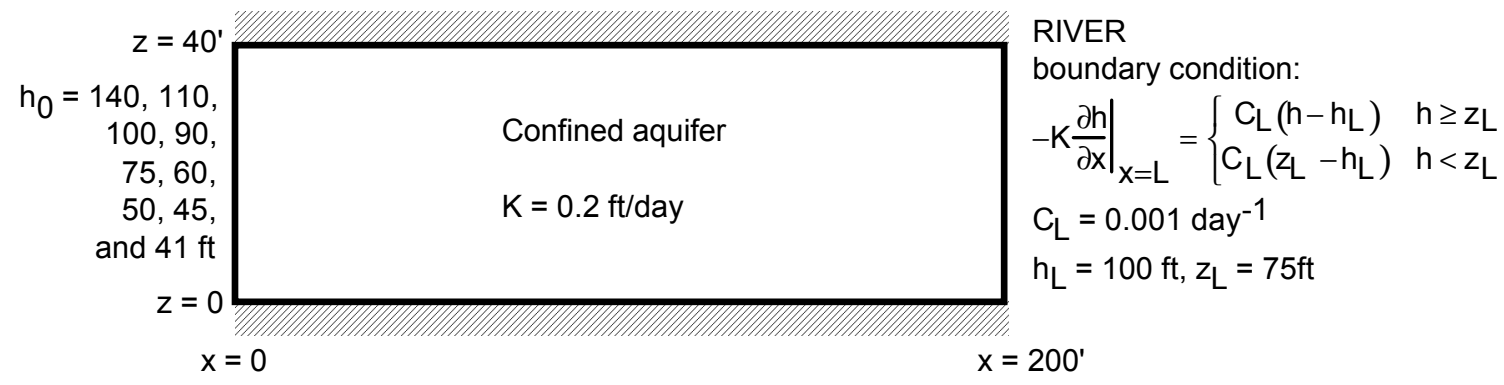

Figure 4.1.1. Schematic Diagram of a Confined Aquifer with a Constant Head Boundary Condition at $x=0$ and Either a General Head or River Boundary Condition at $x=200$.

Analytical solution: Invoking the Dupuit assumption, the following governing equation can be developed for a confined aquifer (de Marsily, 1986, Eq 5.3.11)

$$
\nabla^{2} h=\frac{\partial^{2} h}{\partial x^{2}}+\frac{\partial^{2} h}{\partial y^{2}}=\frac{S}{T} \frac{\partial h}{\partial t}+\frac{Q}{T}
$$

where

$\mathrm{h}$ hydraulic head, L

$\mathrm{S}$................. storage coefficient of a confined aquifer

$\mathrm{T}$................. transmissivity of the aquifer, $\mathrm{L}^{2} / \mathrm{T}$

Q ................volumetric flow rate per unit surface area withdrawn from the aquifer, $\mathrm{L} / \mathrm{T}$

For constant aquifer thickness, constant properties, one-dimensional steady flow and no recharge, Eq. 4.1.1 becomes 


$$
\frac{d^{2} h}{d x^{2}}=0
$$

For the boundary conditions of Problem 1

$$
\begin{aligned}
& \mathrm{h}=\mathrm{h}_{0} @ \mathrm{x}=0 \\
&-\left.\mathrm{K} \frac{\mathrm{dh}}{\mathrm{dx}}\right|_{\mathrm{x}=\mathrm{L}}=\mathrm{C}_{\mathrm{L}}\left(\mathrm{h}-\mathrm{h}_{\mathrm{L}}\right) @ \mathrm{x}=\mathrm{L}
\end{aligned}
$$

the solution can be derived using direct integration as

$$
\mathrm{h}=\mathrm{h}_{0}+\frac{\mathrm{h}_{\mathrm{L}}-\mathrm{h}_{0}}{1+\frac{\mathrm{K}}{\mathrm{C}_{\mathrm{L}} \mathrm{L}}} \frac{\mathrm{x}}{\mathrm{L}}
$$

For the boundary conditions of Problem 2

$$
\begin{gathered}
\mathrm{h}=\mathrm{h}_{0} \quad @ \quad \mathrm{x}=0 \\
\mathrm{U}_{\mathrm{x}}=-\left.\mathrm{K} \frac{\mathrm{dh}}{\mathrm{dx}}\right|_{\mathrm{x}=\mathrm{L}}=\left\{\begin{array}{cc}
\mathrm{C}_{\mathrm{L}}\left(\mathrm{h}-\mathrm{h}_{\mathrm{L}}\right) & \mathrm{h} \mathrm{z}_{\mathrm{L}} \\
\mathrm{C}_{\mathrm{L}}\left(\mathrm{z}_{\mathrm{L}}-\mathrm{h}_{\mathrm{L}}\right) & \mathrm{h}<\mathrm{z}_{\mathrm{L}}
\end{array} \quad \mathrm{x}=\mathrm{L}\right.
\end{gathered}
$$

the solution is

$$
h=\left\{\begin{array}{cc}
\mathrm{h}_{0}+\frac{\mathrm{h}_{\mathrm{L}}-\mathrm{h}_{0}}{1+\frac{\mathrm{K}}{\mathrm{C}_{\mathrm{L}} \mathrm{L}} \frac{\mathrm{x}}{\mathrm{L}}} & \mathrm{h} \mathrm{z}_{\mathrm{L}} \\
\mathrm{h}_{0}+\mathrm{C}_{\mathrm{L}}\left(\mathrm{h}_{\mathrm{L}}-\mathrm{z}_{\mathrm{L}}\right) \frac{\mathrm{x}}{\mathrm{L}} & \mathrm{h}<\mathrm{z}_{\mathrm{L}}
\end{array}\right.
$$

PORFLOW simulation and comparison: Figure 4.1.2 illustrates the PORFLOW mesh chosen for both problems. The mesh includes 201 nodes in the $\mathrm{x}$ direction and 3 nodes in the y direction. Even though the problem is one-dimensional, PORFLOW requires a minimum of 3 nodes in the $y$ direction. Table 4.1.1 and Figure 4.1.3 present the PORFLOW results for Problem 1 in comparison with the analytical solution. Inspection of Table 4.1.1 shows that PORFLOW sets the pressure at the right boundary node equal to the mixed or Cauchy boundary condition pressure value. In order to test the river boundary condition the Darcy velocity is computed at node 201 $(\mathrm{x}=201, \mathrm{y}=0)$ using the volumetric flow and the river boundary condition. The Darcy velocity is given by

$$
\mathrm{U}_{\mathrm{x}}=\frac{\mathrm{Q}}{\mathrm{A}}
$$


where the volumetric flow Q at node 201 can be found in the FC array of the PORFLOW archive file and the effective flow area $\mathrm{A}$ at node 201 is $40 \mathrm{ft}^{2}$. The Darcy velocity is also given by

$$
U_{x}=\left\{\begin{array}{cc}
C_{L}\left(h-h_{L}\right) & h \geq z_{L} \\
C_{L}\left(z_{L}-h_{L}\right) & h<z_{L}
\end{array}\right.
$$

where $\mathrm{h}$ is hydraulic head at node 201 and $\mathrm{h}_{\mathrm{L}}=100 \mathrm{ft}, \mathrm{z}_{\mathrm{L}}=75 \mathrm{ft}$.

Table 4.1.2 and Figure 4.1.4 present the PORFLOW results for Problem 2 that compares the two calculations of Darcy velocity at node 201. PORFLOW has excellent agreement with the analytic results for both problems.

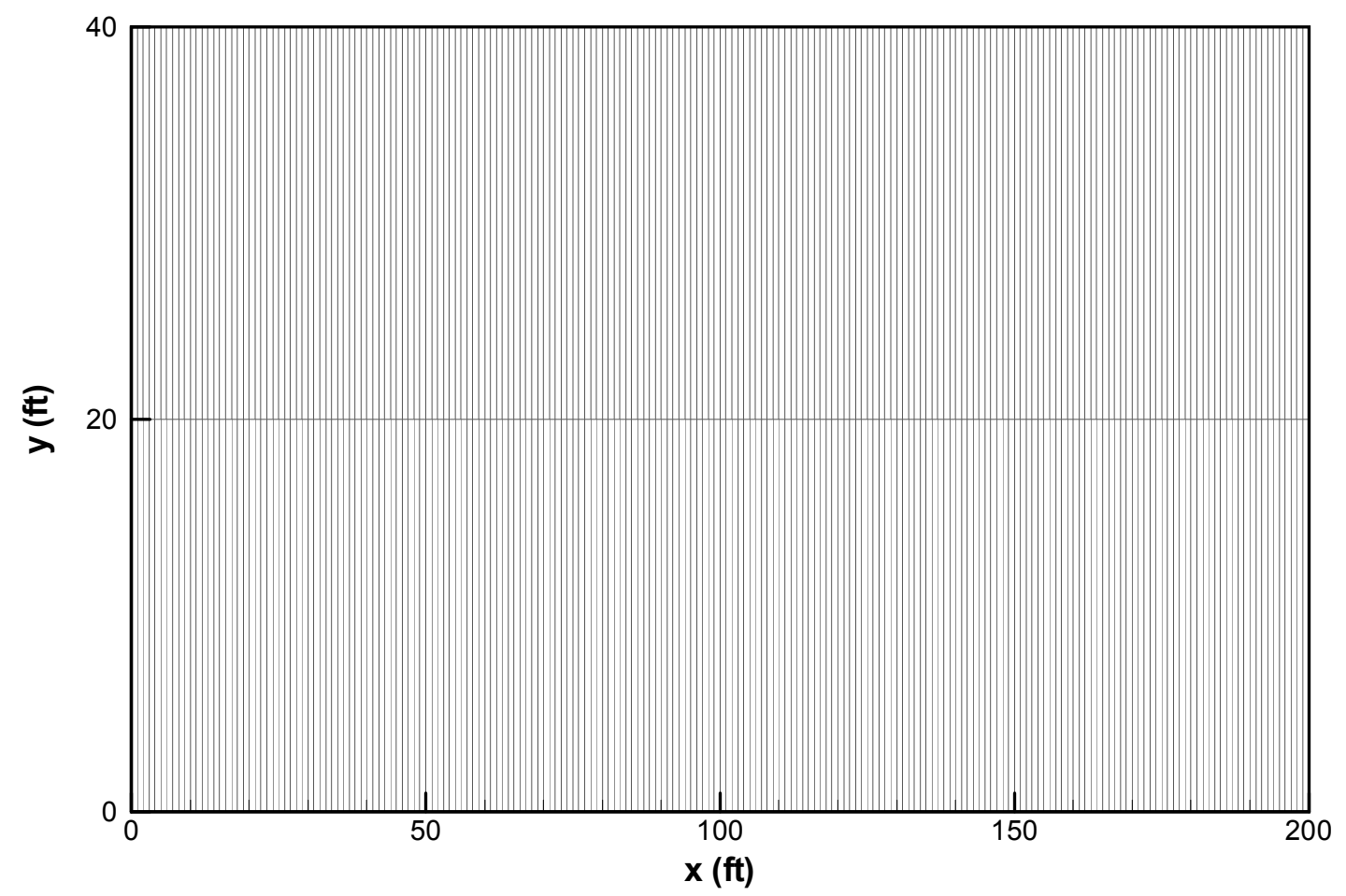

Figure 4.1.2. PORFLOW Grid for Problem 4.1. 


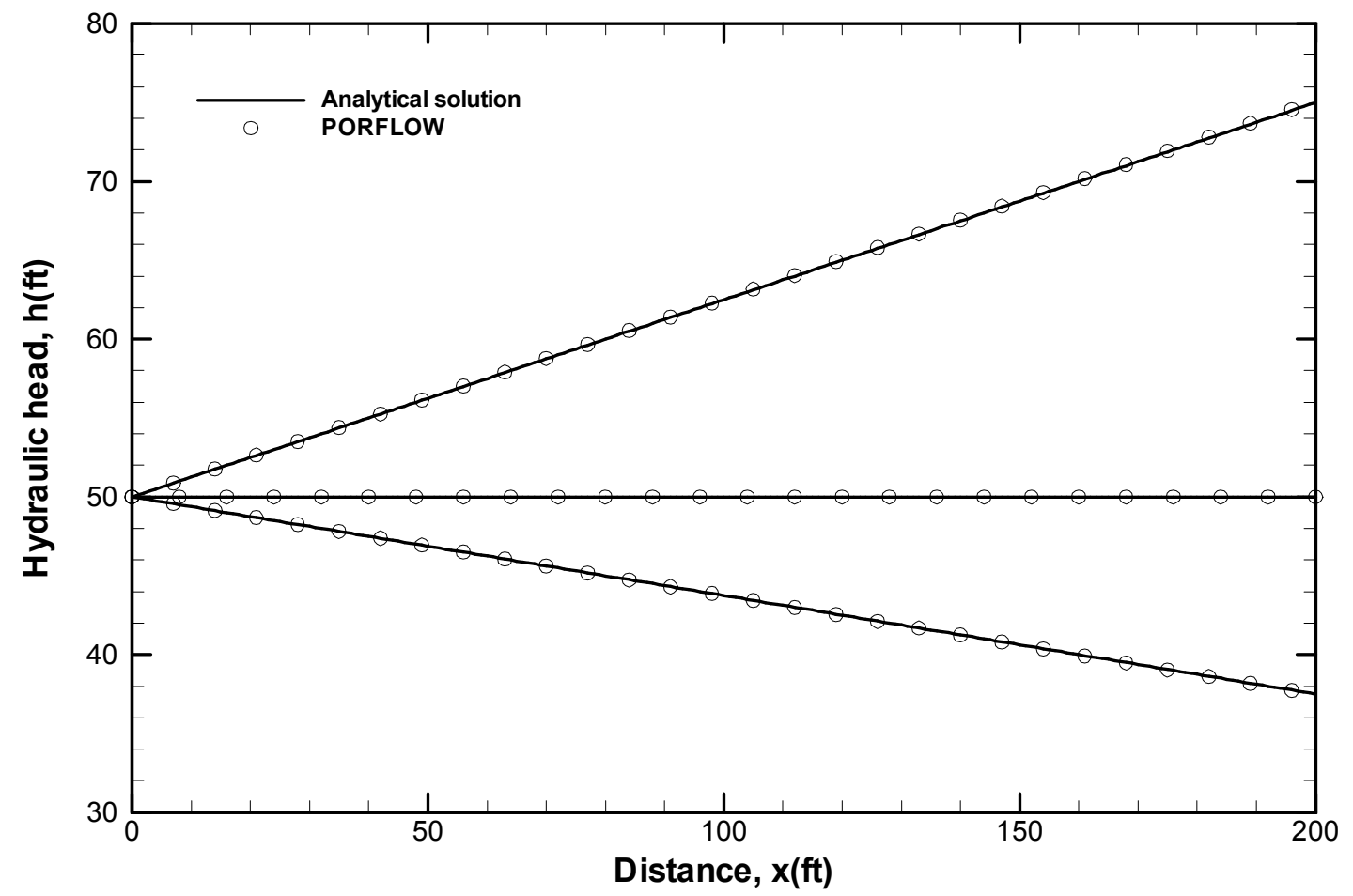

Figure 4.1.3. Comparison of Analytical Solution to PORFLOW Numerical Results for 4.1 (Problem 1).

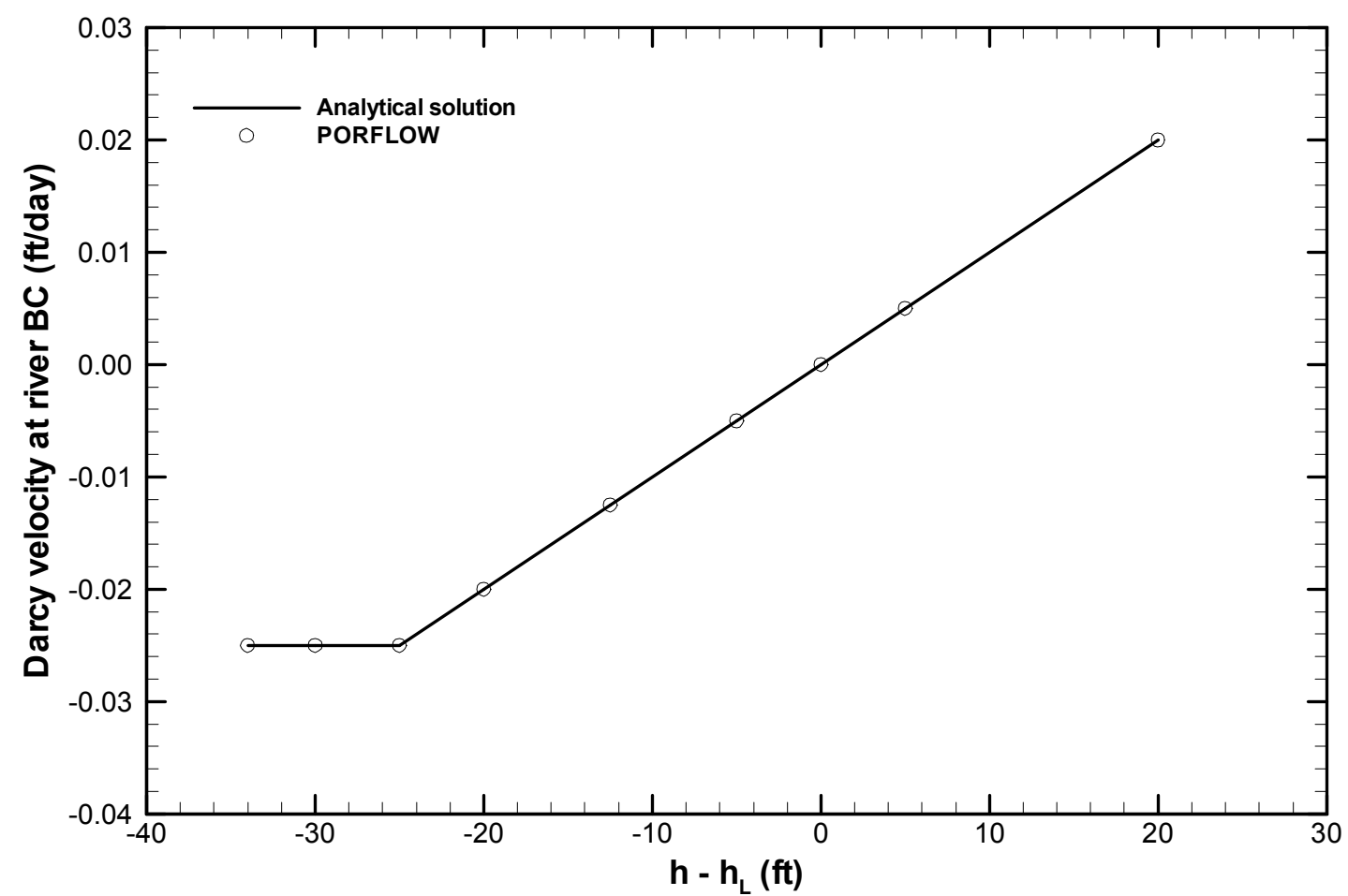

Figure 4.1.4. Comparison of Analytical Solution to PORFLOW Darcy Velocity for 4.1 (Problem 2). 
Table 4.1.1. Comparison of Analytical Solution and PORFLOW Numerical Results for 4.1 (Problem 1).

\begin{tabular}{|c|cc|cc|cc|}
\hline \multirow{2}{*}{$\begin{array}{c}\mathrm{x} \\
(\mathrm{ft})\end{array}$} & \multicolumn{2}{|c|}{$\mathrm{h}_{\mathrm{L}}=25 \mathrm{ft}$} & \multicolumn{2}{c|}{$\mathrm{h}_{\mathrm{L}}=50 \mathrm{ft}$} & \multicolumn{2}{c|}{$\mathrm{h}_{\mathrm{L}}=100 \mathrm{ft}$} \\
\cline { 2 - 7 }$(\mathrm{ft})$ & $\begin{array}{c}\text { Analytic } \\
(\mathrm{ft})\end{array}$ & $\begin{array}{c}\text { PORFOW } \\
(\mathrm{ft})\end{array}$ & $\begin{array}{c}\text { PORFLOW } \\
(\mathrm{ft})\end{array}$ & $\begin{array}{c}\text { Analytic } \\
(\mathrm{ft})\end{array}$ & $\begin{array}{c}\text { PORFLOW } \\
(\mathrm{ft})\end{array}$ \\
\hline 0 & 50.00 & 50.00 & 50.0 & 50.0 & 50.0 & 50.0 \\
\hline 20 & 48.75 & 48.75 & 50.0 & 50.0 & 52.5 & 52.5 \\
\hline 40 & 47.50 & 47.49 & 50.0 & 50.0 & 55.0 & 55.0 \\
\hline 60 & 46.25 & 46.24 & 50.0 & 50.0 & 57.5 & 57.5 \\
\hline 80 & 45.00 & 44.99 & 50.0 & 50.0 & 60.0 & 60.0 \\
\hline 100 & 43.75 & 43.73 & 50.0 & 50.0 & 62.5 & 62.5 \\
\hline 120 & 42.50 & 42.48 & 50.0 & 50.0 & 65.0 & 65.0 \\
\hline 140 & 41.25 & 41.23 & 50.0 & 50.0 & 67.5 & 67.6 \\
\hline 160 & 40.00 & 39.98 & 50.0 & 50.0 & 70.0 & 70.1 \\
\hline 180 & 38.75 & 38.72 & 50.0 & 50.0 & 72.5 & 72.6 \\
\hline 199 & 37.56 & 37.53 & 50.0 & 50.0 & 74.9 & 74.9 \\
\hline 200 & 37.50 & 25.00 & 50.0 & 50.0 & 75.0 & 100.0 \\
\hline
\end{tabular}

Table 4.1.2. PORFLOW Darcy Velocity at Node 201 for 4.1 (Problem 2)

\begin{tabular}{|ccc|cc|cc|}
\hline & & & \multicolumn{2}{|c|}{ PORFLOW } & \multicolumn{2}{|c|}{ Analytic } \\
\cline { 4 - 7 } $\mathrm{h}_{0}$ & $\mathrm{~h}_{\mathrm{L}}$ & $\mathrm{zL}_{\mathrm{L}}$ & $\mathrm{h}_{\mathrm{X}=\mathrm{L}}$ & $\begin{array}{c}\mathrm{U} \\
(\mathrm{ft} / \mathrm{day})\end{array}$ & $\begin{array}{c}\mathrm{h}_{\mathrm{X}=\mathrm{L}} \\
(\mathrm{ft})\end{array}$ & $\begin{array}{c}\mathrm{U} \\
(\mathrm{ft} / \mathrm{day})\end{array}$ \\
\hline 140 & 100 & 75 & 120 & 0.0200 & 120.0 & 0.0200 \\
\hline 110 & 100 & 75 & 105 & 0.0050 & 105.0 & 0.0050 \\
\hline 100 & 100 & 75 & 100 & 0.0000 & 100.0 & 0.0000 \\
\hline 90 & 100 & 75 & 95 & -0.0050 & 95.0 & -0.0050 \\
\hline 75 & 100 & 75 & 87.5 & -0.0125 & 87.5 & -0.0125 \\
\hline 60 & 100 & 75 & 80 & -0.0200 & 80.0 & -0.0200 \\
\hline 50 & 100 & 75 & 75 & -0.0250 & 75.0 & -0.0250 \\
\hline 45 & 100 & 75 & 70 & -0.0250 & 70.0 & -0.0250 \\
\hline 41 & 100 & 75 & 66 & -0.0250 & 66.0 & -0.0250 \\
\hline
\end{tabular}

Table 4.1.3. Input Commands for 4.1 (Problem 1)

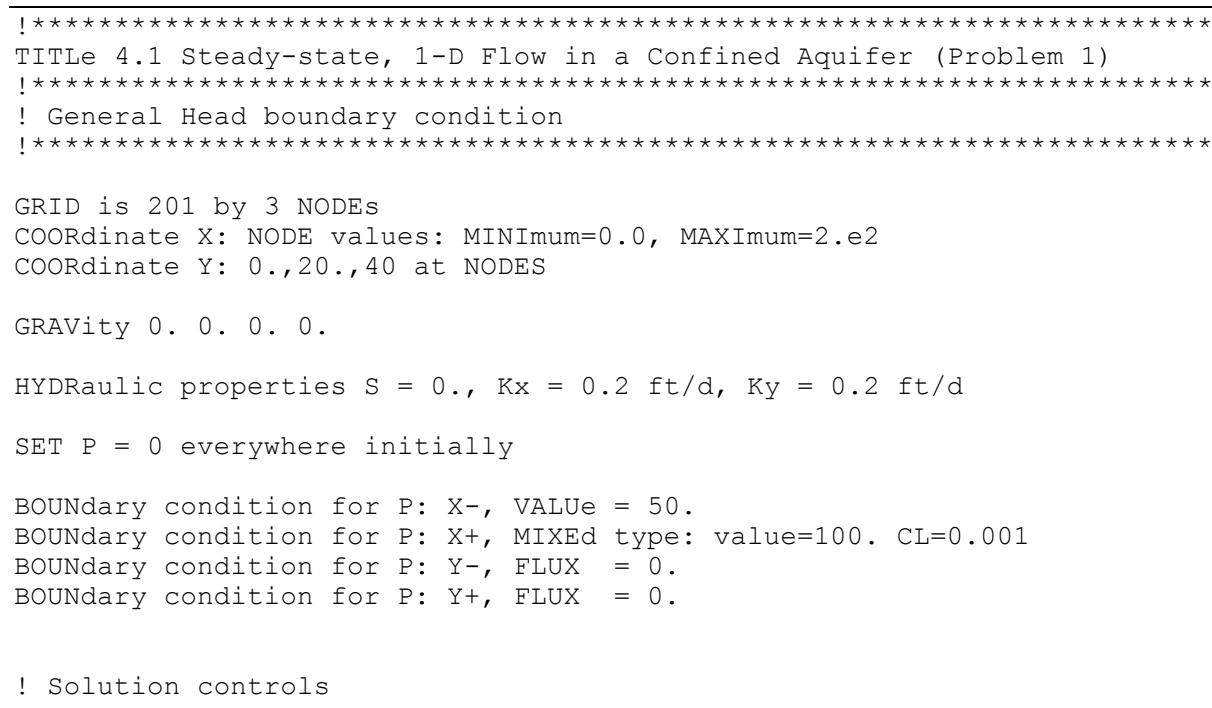


!MATRix NSPC for $\mathrm{P}$ precon=CHOL, accel=CONJ, MODI

\section{Table 4.1.4. Input Commands for 4.1 (Problem 2)}

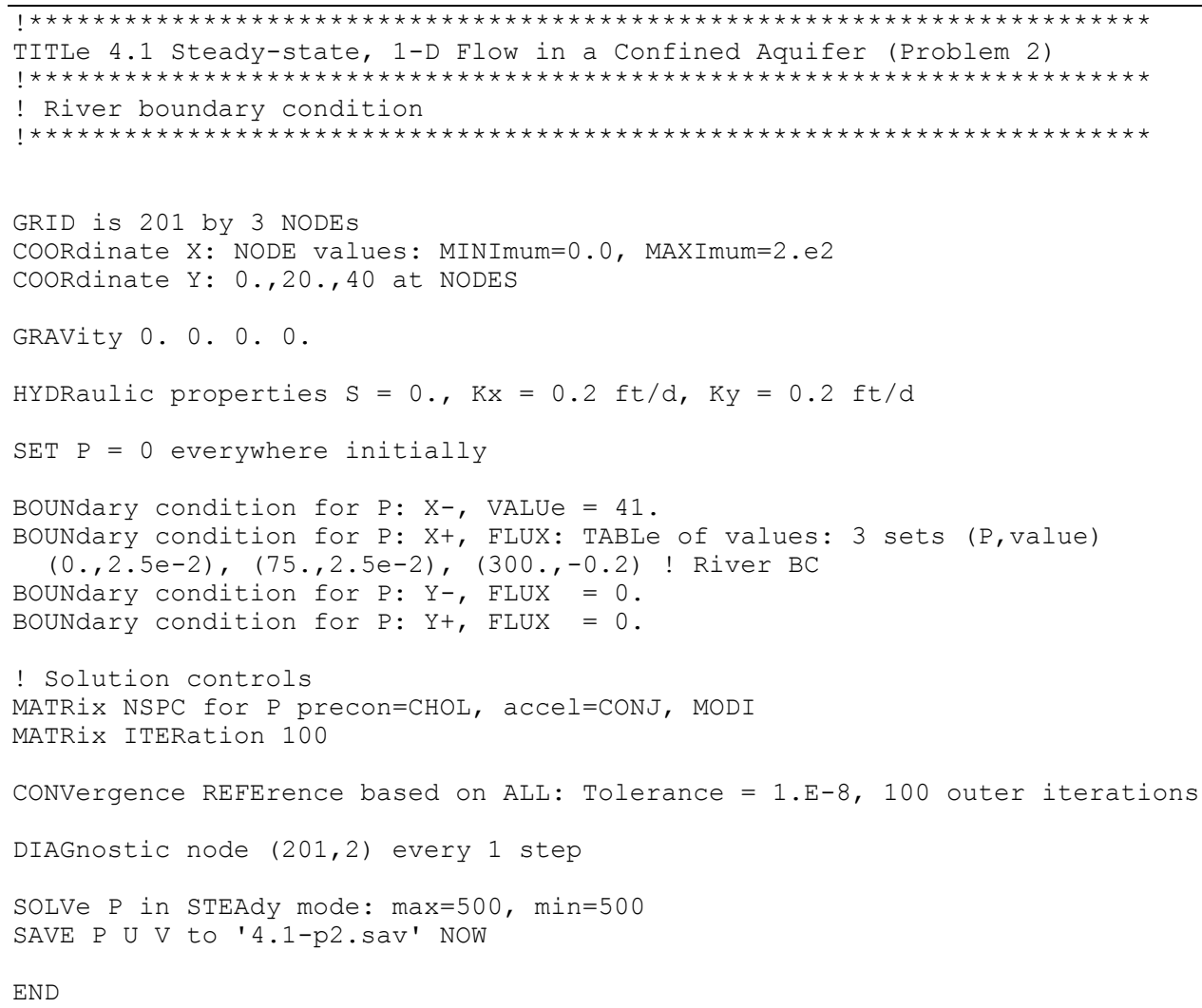

\subsection{Steady-state, One-Dimensional Flow in an Unconfined Aquifer}

Figures 4.2.1 and 4.2.2 illustrate a pair of unconfined aquifers experiencing steady, onedimensional flow without and with recharge, respectively. The second problem is essentially FTWORK Problem 4.1.1 (GeoTrans, 1993). Both problems can be easily solved analytically. 


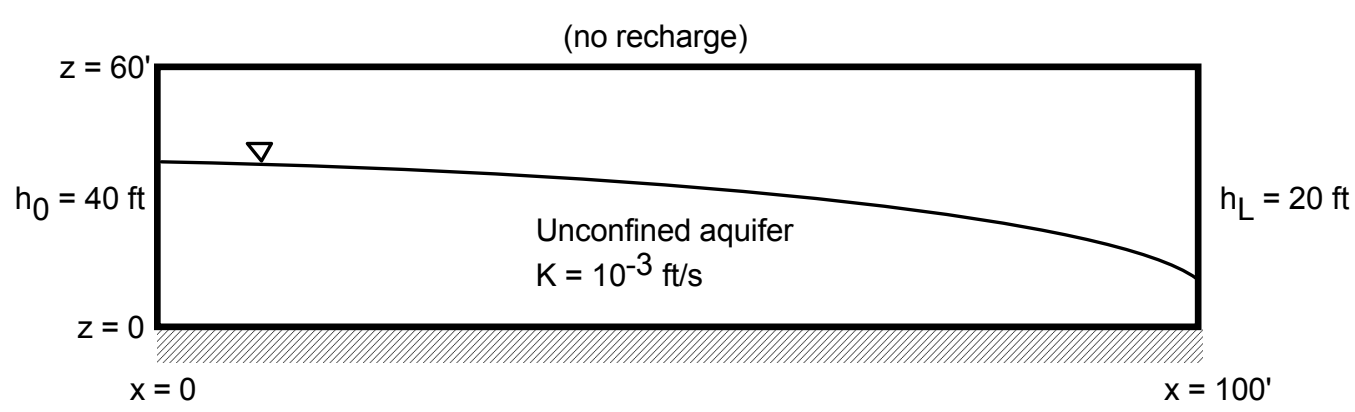

Figure 4.2.1. Schematic Diagram of an Unconfined Aquifer with No Recharge and Constant Head Boundary Conditions (Problem 1).

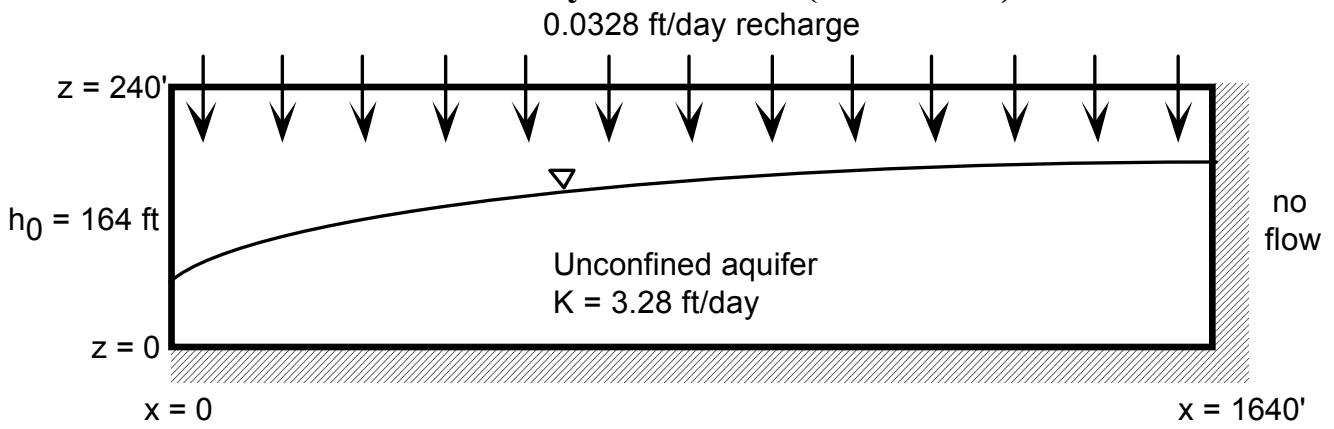

Figure 4.2.2. Schematic Diagram of an unconfined aquifer with recharge and mixed boundary conditions (Problem 2).

Analytical solutions: Invoking the Dupuit assumption (de Marsily, 1986, Eq. (5.1.1)) gives the following general expression for flow in an unconfined aquifer

$$
\frac{\partial}{\partial x}\left[\int_{\sigma}^{h} K_{x x} d z \frac{\partial h}{\partial x}\right]+\frac{\partial}{\partial y}\left[\int_{\sigma}^{h} K_{y y} d z \frac{\partial h}{\partial y}\right]=\omega_{d} \frac{\partial h}{\partial t}+Q
$$

where

$$
\begin{aligned}
& \mathrm{K} \text {................ saturated hydraulic conductivity tensor, L/T } \\
& \sigma \text {................. elevation of the aquifer base, } L \\
& \omega_{\mathrm{d}} \ldots \ldots \ldots \ldots . . . . . . . \text { specific yield or drainage porosity }
\end{aligned}
$$

If $\mathrm{K}_{\mathrm{xx}}$ and $\mathrm{K}_{\mathrm{yy}}$ are constant along the $\mathrm{z}$-axis, we can evaluate the integral to arrive at

$$
\frac{\partial}{\partial x}\left[K_{x x}(h-\sigma) \frac{\partial h}{\partial x}\right]+\frac{\partial}{\partial y}\left[K_{y y}(h-\sigma) \frac{\partial h}{\partial y}\right]=\omega_{d} \frac{\partial h}{\partial t}+Q
$$

For the special case of a horizontal aquifer $(\sigma=0)$, isotropic and uniform medium $\left(\mathrm{K}_{\mathrm{xx}}=\mathrm{K}_{\mathrm{yy}}=\mathrm{K}\right)$ and one-dimensional steady-state flow, Eq. 4.2.2 becomes

$$
\frac{\mathrm{d}^{2} \mathrm{~h}^{2}}{\mathrm{dx}}=\frac{2 \mathrm{Q}}{\mathrm{K}}
$$


For the prescribed head boundary conditions for Problem 1

$$
\begin{aligned}
& \mathrm{h}=\mathrm{h}_{0} @ \quad \mathrm{x}=0 \\
& \mathrm{~h}=\mathrm{h}_{\mathrm{L}} @ \mathrm{x}=\mathrm{L}
\end{aligned}
$$

the solution can be derived using direct integration as

$$
\mathrm{h}^{2}=\mathrm{h}_{0}^{2}+\left(\mathrm{h}_{\mathrm{L}}^{2}-\mathrm{h}_{0}^{2}\right) \frac{\mathrm{x}}{\mathrm{L}}+\frac{\mathrm{Q}_{\mathrm{src}} \mathrm{L}^{2}}{\mathrm{~K}}\left(\frac{\mathrm{x}}{\mathrm{L}}\right)\left(1-\frac{\mathrm{x}}{\mathrm{L}}\right)
$$

where $\mathrm{Q}_{\mathrm{src}}=-\mathrm{Q}$. For the boundary conditions for Problem 2

$$
\begin{aligned}
& \mathrm{h}=\mathrm{h}_{0} @ \quad \mathrm{x}=0 \\
& \mathrm{~h}^{\prime}=0 @ \mathrm{x}=\mathrm{L}
\end{aligned}
$$

the solution is

$$
\mathrm{h}^{2}=\mathrm{h}_{0}^{2}-\frac{\mathrm{Q}_{\mathrm{src}} \mathrm{L}^{2}}{\mathrm{~K}}\left(\frac{\mathrm{x}}{\mathrm{L}}\right)\left(\frac{\mathrm{x}}{\mathrm{L}}-2\right)
$$

PORFLOW simulation and comparison: Figures 4.2.3 and 4.2.4 present the two grids chosen for the PORFLOW simulations. The mesh for Problem 1 contains 101 nodes in the $\mathrm{x}$-direction and 61 nodes in y-direction. The mesh for Problem 2 contains 165 nodes in the x-direction and 25 nodes in the y-direction. Table 4.2.1 and Figures 4.2.5 and 4.2.6 present the analytical solution and PORFLOW results. The agreement is excellent for both problems. The slight differences between the Dupuit assumption and PORFLOW are due to curvature of the phreatic surface (watertable). In reality, the flow is not purely horizontal and the flow near the phreatic surface has small components of velocity in the vertical direction. The PORFLOW input commands for Problem 1 and 2 are shown in Tables 4.2.2 and 4.2.3, respectively. 
WASHINGTON SAVANNAH RIVER COMPANY

PORFLOW Testing and Verification Document

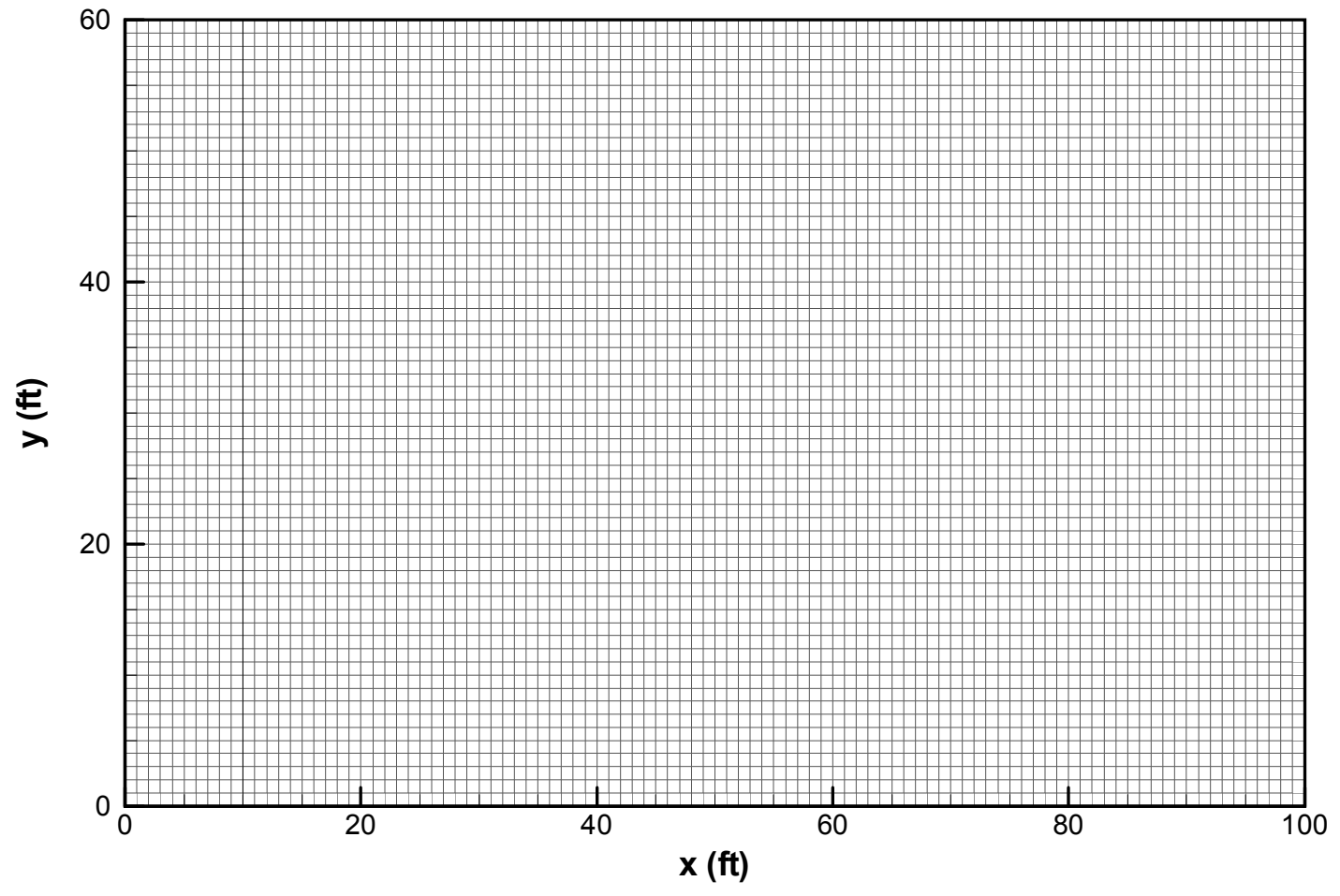

Figure 4.2.3. PORFLOW Grid for 4.2 (Problem 1).

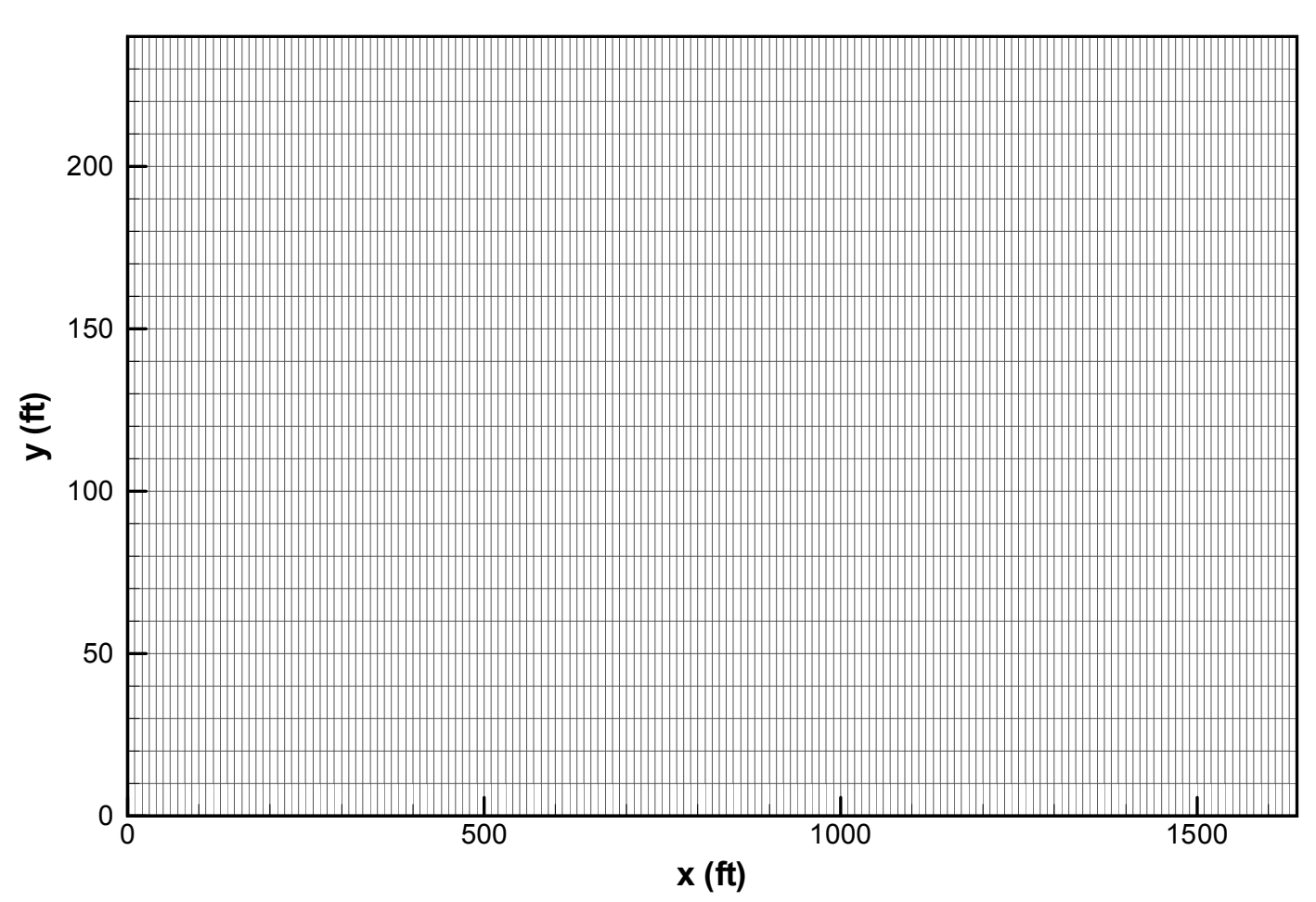

Figure 4.2.4. PORFLOW Grid for 4.2 (Problem 2).
Report: WSRC-STI-2007-00150

Revision (Date): $\quad \operatorname{Rev} 0$ (6/15/2007)

Page:

11 of 193 


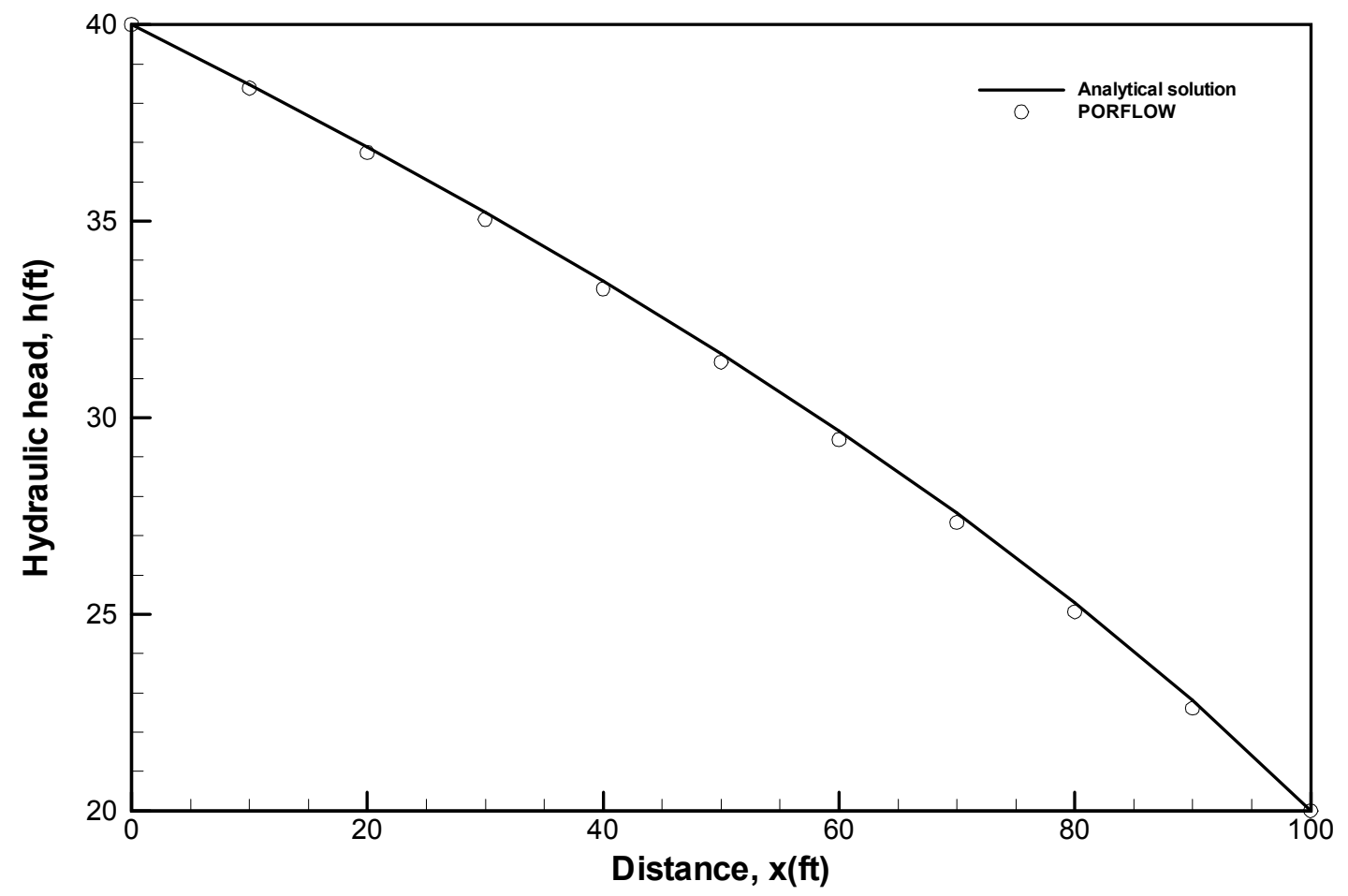

Figure 4.2.5. Comparison of Analytical Solution and PORFLOW Results for 4.2 (Problem 1).

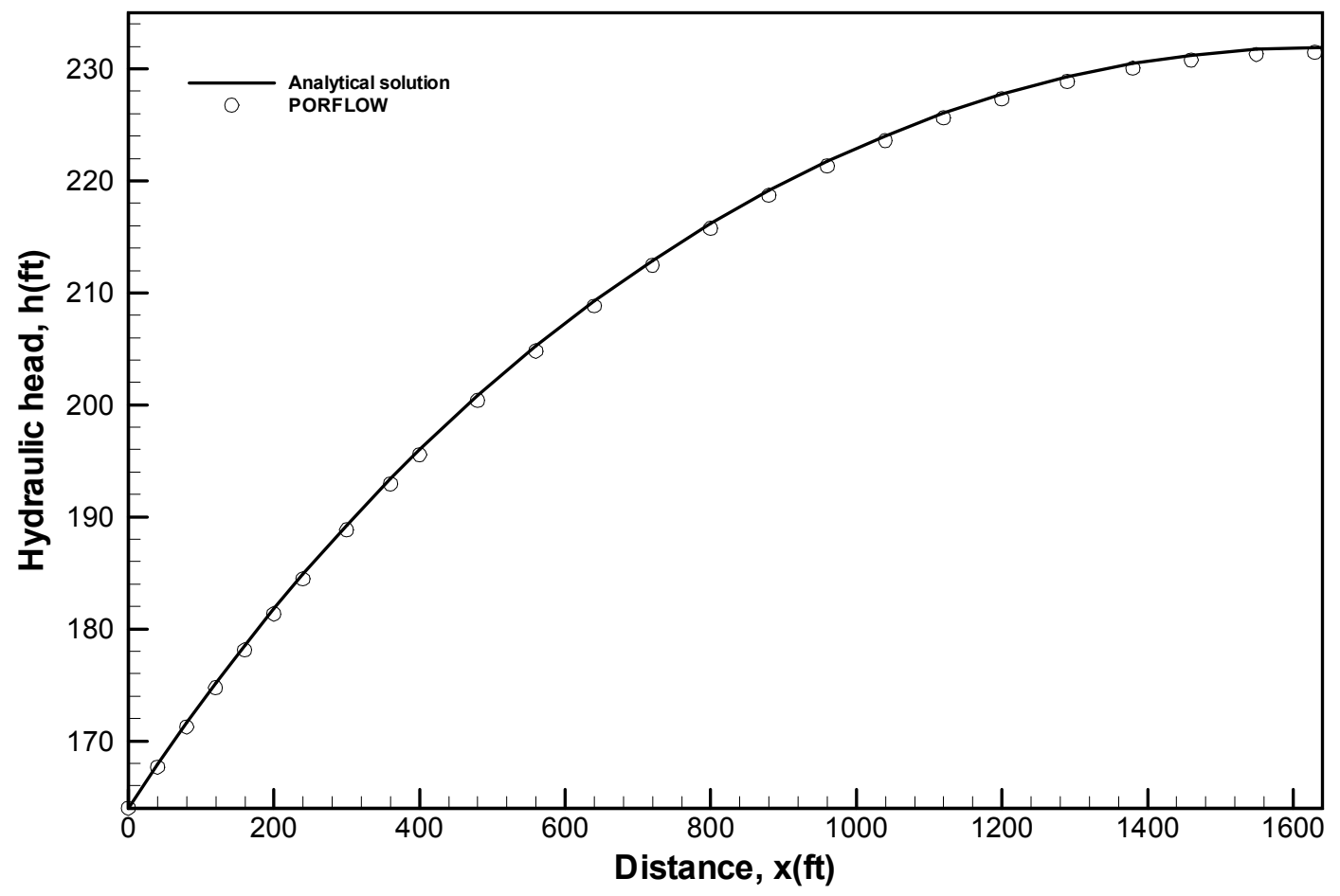

Figure 4.2.6. Comparison of Analytical Solution and PORFLOW Results for 4.2 (Problem 2). 
Table 4.2.1. Comparison of Analytical Solution and PORFLOW Numerical Results.

\begin{tabular}{|c|c|c|c|c|c|}
\hline \multicolumn{3}{|c|}{ Problem 1} & \multicolumn{3}{|c|}{ Problem 2} \\
\hline $\begin{array}{c}\mathrm{X} \\
\text { (ft) }\end{array}$ & $\begin{array}{c}\text { Analytic } \\
\text { (ft) }\end{array}$ & $\begin{array}{c}\text { PORFLOW } \\
(\mathrm{ft})\end{array}$ & $\begin{array}{c}\mathrm{X} \\
(\mathrm{ft})\end{array}$ & $\begin{array}{c}\text { Analytic } \\
\text { (ft) }\end{array}$ & $\begin{array}{c}\text { PORFLOW } \\
(\mathrm{ft})\end{array}$ \\
\hline 0 . & 40.000 & 40.000 & 0 & $\begin{array}{l}164.000 \\
\end{array}$ & 164.000 \\
\hline 10 & 38.471 & 38.383 & 40 & 167.905 & 167.660 \\
\hline 20 & 36.878 & 36.878 & 80 & 171.628 & 171.250 \\
\hline 30 & 35.214 & 35.041 & 120 & 175.180 & 174.750 \\
\hline 40 & 33.466 & 33.271 & 160 & 178.572 & 178.110 \\
\hline 50 & 31.623 & 31.410 & 200 & 181.813 & 181.340 \\
\hline 60 & 29.665 & 29.438 & 240 & 184.911 & 184.440 \\
\hline 70 & 27.568 & 27.331 & 300 & 189.304 & 188.830 \\
\hline 80 & 25.298 & 25.060 & 360 & 193.411 & 192.940 \\
\hline 90 & 22.804 & 22.605 & 400 & 196.000 & 195.540 \\
\hline 100 & 20.000 & 20.000 & 480 & 200.838 & 200.380 \\
\hline & & & 560 & 205.251 & 204.800 \\
\hline & & & 640 & 209.265 & 208.820 \\
\hline & & & 720 & 212.904 & 212.460 \\
\hline & & & 800 & 216.185 & 215.750 \\
\hline & & & 880 & 219.126 & 218.690 \\
\hline & & & 960 & 221.739 & 221.310 \\
\hline & & & 1040 & 224.036 & 223.600 \\
\hline & & & 1120 & 226.027 & 225.600 \\
\hline & & & 1200 & 227.719 & 227.300 \\
\hline & & & 1290 & 229.275 & 228.850 \\
\hline & & & 1380 & 230.469 & 230.030 \\
\hline & & & 1460 & 231.231 & 230.780 \\
\hline & & & 1550 & 231.756 & 231.290 \\
\hline & & & 1630 & 231.929 & 231.460 \\
\hline
\end{tabular}

Table 4.2.2. Input Commands for 4.2 (Problem 1)

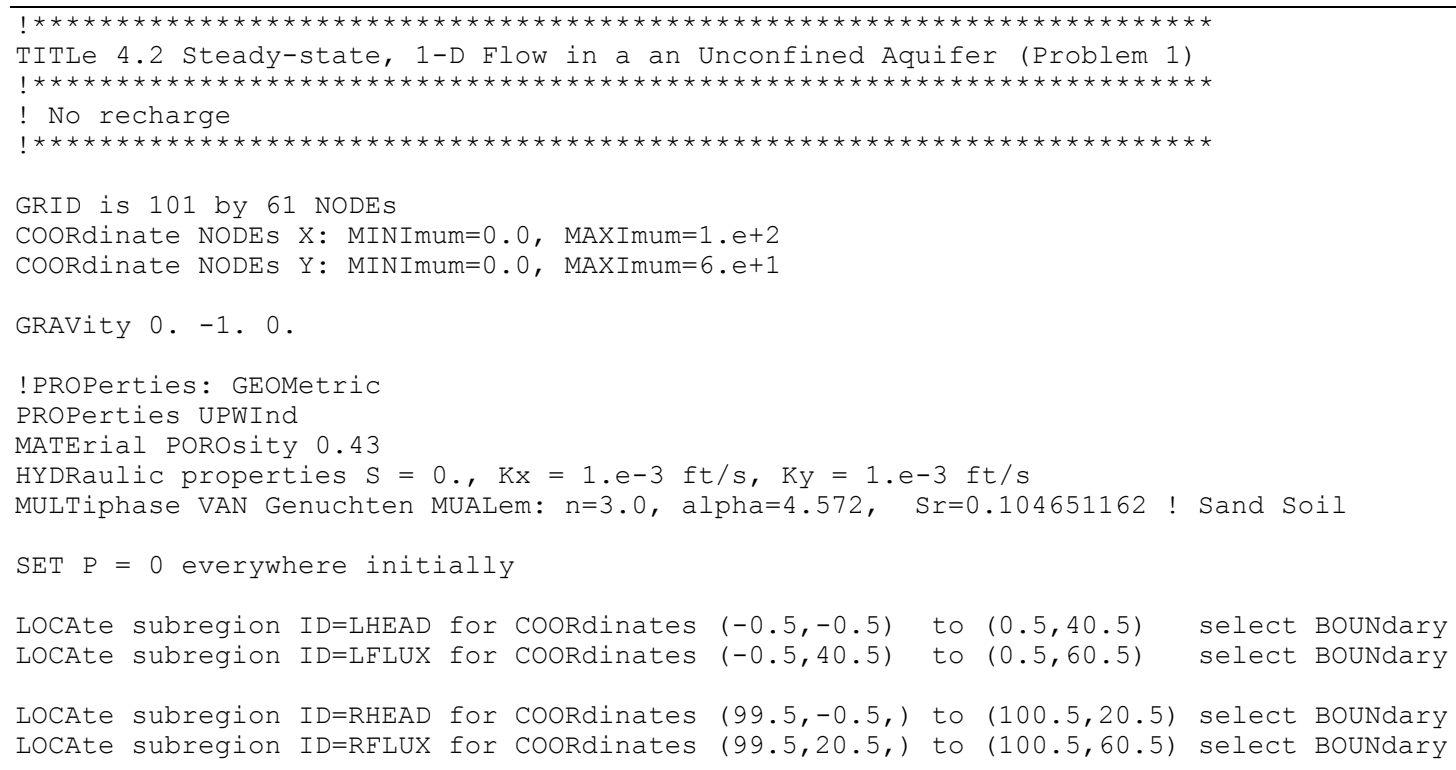


BOUNdary condition for P: VALUe is LINEar function (40.) (-1) (Y) at ID=LHEAD BOUNdary condition for $\mathrm{P}$ : FLUX $=0$. at ID=LFLUX

BOUNdary condition for P: VALUe is LINEar function (20.) (-1) (Y) at ID=RHEAD BOUNdary condition for $\mathrm{P}$ : FLUX $=0$. at ID=RFLUX

BOUNdary condition for $\mathrm{P}: \mathrm{Y}-, \mathrm{FLUX}=0$.

BOUNdary condition for $\mathrm{P}: \mathrm{Y}+, \mathrm{FLUX}=0$.

! Solution controls

MATRix NSPC for P precon=CHOL, accel=CONJ, MODI

MATRix ITERation 100

CONVergence REFErence based on ALL: Tolerance $=1 . E-6,100$ outer iterations

DIAGnostic P node $(100,60)$ every 1 step

RELAX S 0.01

SOLVe $P$ in STEAdy mode: $\max =200, \min =200$

SAVE P H to '4.2-p1.sav' NOW

END

\section{Table 4.2.3. Input Commands for 4.2 (Problem 2)}

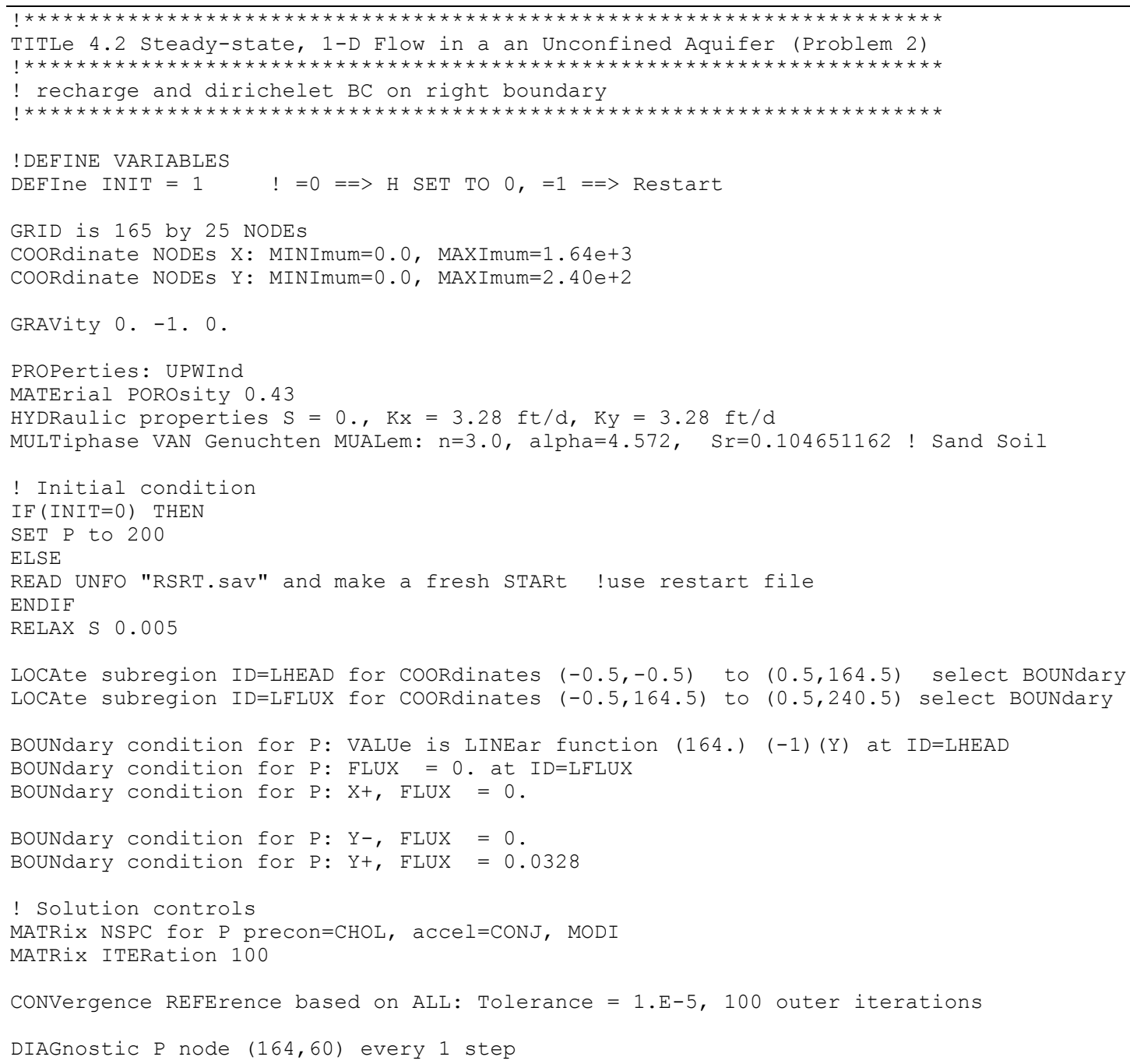


SoLVe $\mathrm{P}$ in STEAdy mode: $\max =100$, $\min =100$

SAVE $P$ S MOIS to "INIT.SaV" UNFOrmatted using COMPact NOW

SAVE P H to '4.2-p2.sav' NOW

END

\subsection{Steady-state, Two-Dimensional Flow through a Heterogeneous Aquifer System.}

Figure 4.3.1 schematically illustrates a particular problem involving steady-state groundwater flow through a heterogeneous subsurface system. The problem shown in Figure 4.3.1 was chosen as a test case to verify that PORFLOW can correctly solve a groundwater flow problem involving a non-uniform hydraulic conductivity field. Problem parameters were carefully chosen to enable analytic solution. Specifically, the boundary conditions and conductivity field were chosen to create two aquifers with a constant head difference. A constant head difference coupled with a uniform conductivity in the confining unit yields a uniform leakance between the two aquifers. Assuming flow in the aquifers is essentially one-dimensional (Dupuit assumption; typically an excellent assumption), analytical solutions can be derived for both the unconfined and confined aquifers for a constant source/sink term. These analytical solutions are presented below followed by PORFLOW simulation and comparison results.

(no recharge)

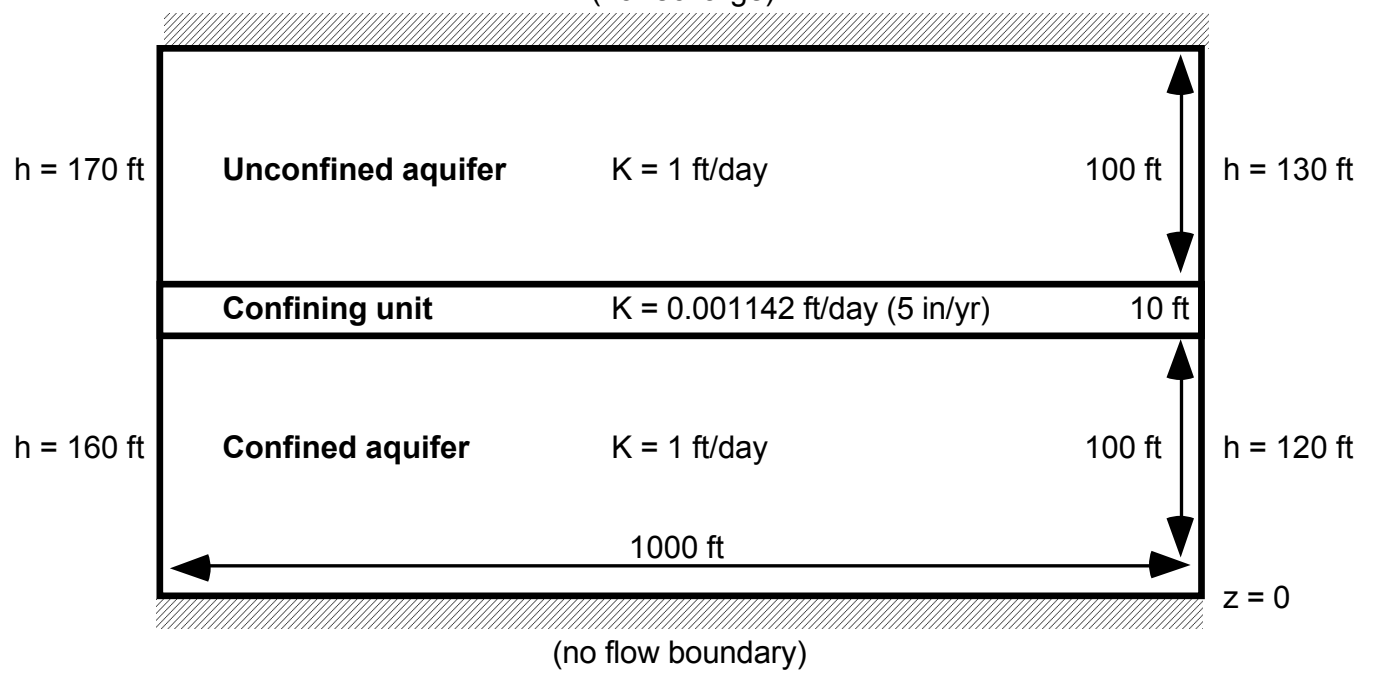

Figure 4.3.1. A Heterogeneous Subsurface System Consisting of an Unconfined Aquifer, Confining Unit and Confined Aquifer.

Unconfined aquifer analytical solution: Invoking the Dupuit assumption (de Marsily, 1986, Eq. (5.1.1)) gives the following general expression for flow in an unconfined aquifer

$$
\frac{\partial}{\partial x}\left[\int_{\sigma}^{h} K_{x x} d z \frac{\partial h}{\partial x}\right]+\frac{\partial}{\partial y}\left[\int_{\sigma}^{h} K_{y y} d z \frac{\partial h}{\partial y}\right]=\omega_{d} \frac{\partial h}{\partial t}+Q
$$

If $\mathrm{K}_{\mathrm{xx}}$ and $\mathrm{K}_{\mathrm{yy}}$ are constant along the $\mathrm{z}$-axis, we can evaluate the integral to arrive at 


$$
\frac{\partial}{\partial x}\left[K_{x x}(h-\sigma) \frac{\partial h}{\partial x}\right]+\frac{\partial}{\partial y}\left[K_{y y}(h-\sigma) \frac{\partial h}{\partial y}\right]=\omega_{d} \frac{\partial h}{\partial t}+Q
$$

For the special case of a horizontal aquifer $(\sigma=0)$, isotropic and uniform medium $\left(K_{x x}=K_{y y}=K\right)$ and one-dimensional steady-state flow, Eq. 4.3.2 becomes

$$
\frac{\mathrm{d}^{2} \mathrm{~h}^{2}}{\mathrm{dx} \mathrm{x}^{2}}=\frac{2 \mathrm{Q}}{\mathrm{K}}
$$

For the prescribed head boundary conditions

$$
\begin{aligned}
& \mathrm{h}=\mathrm{h}_{0} @ \quad \mathrm{x}=0 \\
& \mathrm{~h}=\mathrm{h}_{\mathrm{L}} @ \quad \mathrm{x}=\mathrm{L}
\end{aligned}
$$

the solution can be derived using direct integration as

$$
\mathrm{h}^{2}=\mathrm{h}_{0}^{2}+\left(\mathrm{h}_{\mathrm{L}}^{2}-\mathrm{h}_{0}^{2}\right) \frac{\mathrm{x}}{\mathrm{L}}+\frac{\mathrm{Q}_{\mathrm{src}} \mathrm{L}^{2}}{\mathrm{~K}}\left(\frac{\mathrm{x}}{\mathrm{L}}\right)\left(1-\frac{\mathrm{x}}{\mathrm{L}}\right)
$$

where $\mathrm{Q}_{\text {src }}=-\mathrm{Q}$.

Confined aquifer analytical solution: Invoking the Dupuit assumption (de Marsily, 1986, Eq. (5.3.11)) gives the following general expression for flow in an confined aquifer

$$
\nabla^{2} \mathrm{~h}=\frac{\partial^{2} \mathrm{~h}}{\partial \mathrm{x}^{2}}+\frac{\partial^{2} \mathrm{~h}}{\partial \mathrm{y}^{2}}=\frac{\mathrm{S}}{\mathrm{T}} \frac{\partial \mathrm{h}}{\partial \mathrm{t}}+\frac{\mathrm{Q}}{\mathrm{T}}
$$

where the ratio $\mathrm{T} / \mathrm{S}$ is the aquifer diffusivity. For constant aquifer thickness, constant properties and one-dimensional steady flow, Eq. 3.3.6 becomes

$$
\frac{\mathrm{d}^{2} \mathrm{~h}}{\mathrm{dx}^{2}}=\frac{\mathrm{Q}}{\mathrm{Ke}}
$$

where e is the thickness of the aquifer. For the prescribed head boundary conditions

$$
\begin{aligned}
& \mathrm{h}=\mathrm{h}_{0} @ \quad \mathrm{x}=0 \\
& \mathrm{~h}=\mathrm{h}_{\mathrm{L}} @ \quad \mathrm{x}=\mathrm{L}
\end{aligned}
$$

the solution can be derived using direct integration as 


$$
\mathrm{h}=\mathrm{h}_{0}\left(1-\frac{\mathrm{x}}{\mathrm{L}}\right)+\mathrm{h}_{\mathrm{L}}\left(\frac{\mathrm{x}}{\mathrm{L}}\right)+\frac{\mathrm{Q}_{\mathrm{src}} \mathrm{L}^{2}}{2 \mathrm{Ke}}\left(\frac{\mathrm{x}}{\mathrm{L}}\right)\left(1-\frac{\mathrm{x}}{\mathrm{L}}\right)
$$

where $\mathrm{Q}_{\mathrm{src}}=-\mathrm{Q}$.

PORFLOW numerical simulation and comparison: Figure 4.3.2 illustrates the grid chosen for the PORFLOW simulation. The mesh contains 51 nodes in the x-direction for a mesh spacing of 20 feet and 106 nodes in the y-direction for a mesh spacing of 2 feet. Table 4.3.1 and Figure 4.3.3 present the PORFLOW results alongside the approximate analytical results. As the water flows from the left boundary of the unconfined aquifer, vertical velocity components become evident due to the curvature of the watertable. This impact can be shown by the hydraulic head in the unconfined aquifer falling below the analytical solution and consequently raising the hydraulic head in the confined aquifer. The agreement between the code and analytical solution is excellent. The PORFLOW input commands are shown in Table 4.3.2.

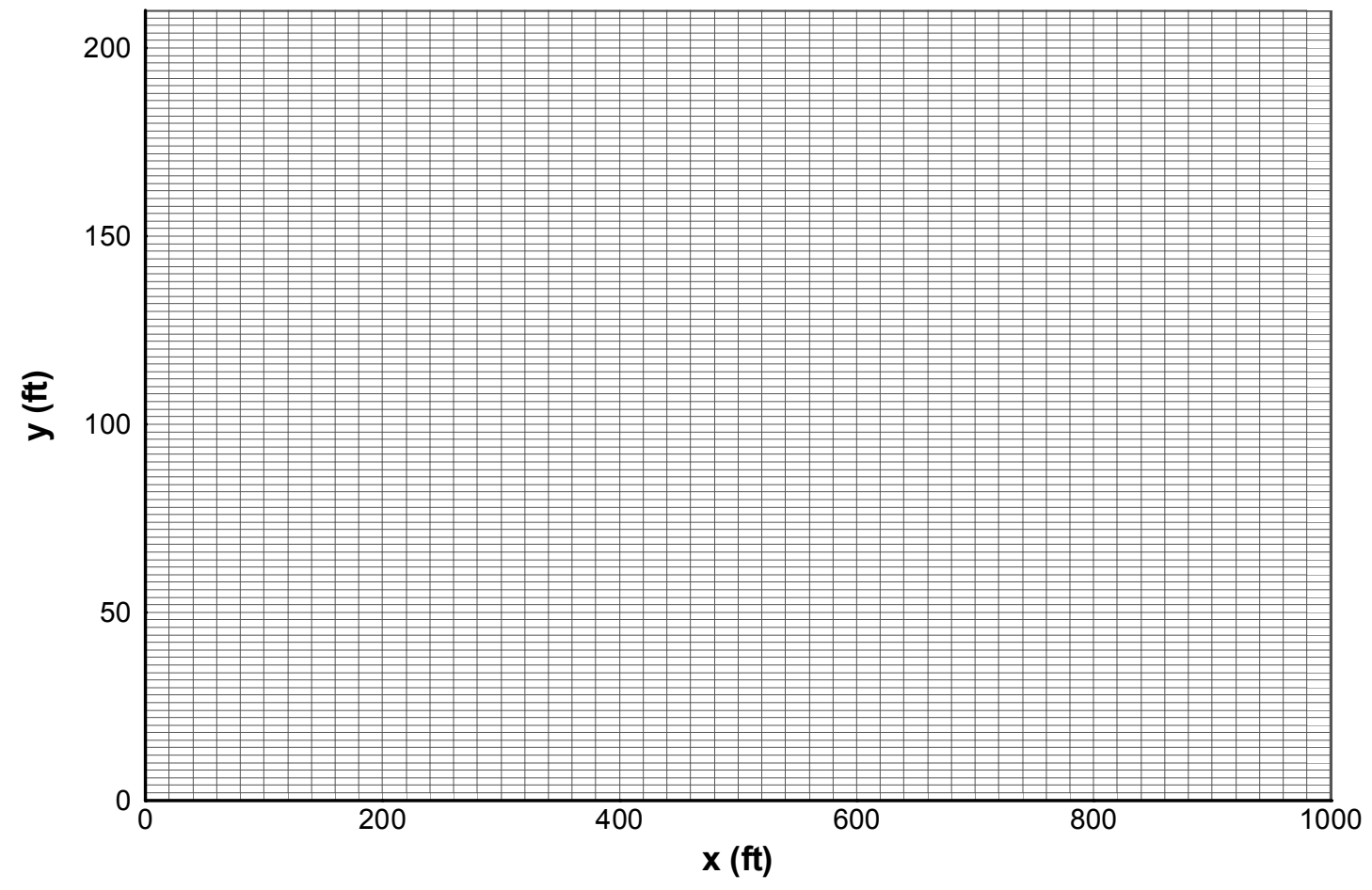

Figure 4.3.2. $\quad$ PORFLOW Grid for Problem 4.3. 
Table 4.3.1. Approximate Analytical and PORFLOW Hydraulic Head Solution for the Unconfined and Confined Aquifers.

\begin{tabular}{|c|c|c|c|c|c|}
\hline \multirow{2}{*}{$\mathrm{x} / \mathrm{L}$} & $\mathrm{x}$ & \multicolumn{2}{|c|}{ Unconfined aquifer head } & \multicolumn{2}{c|}{ Confined aquifer head } \\
\cline { 3 - 6 } & $(\mathrm{ft})$ & $\begin{array}{c}\text { Analytical } \\
(\mathrm{ft})\end{array}$ & $\begin{array}{c}\text { PORFLOW } \\
\mathrm{y}=160^{\prime} \\
(\mathrm{ft})\end{array}$ & $\begin{array}{c}\text { Analytical } \\
(\mathrm{ft})\end{array}$ & $\begin{array}{c}\text { PORFLOW } \\
\mathrm{y}=50^{\prime} \\
(\mathrm{ft})\end{array}$ \\
\hline 0.00 & 0 & 170.000 & 170.000 & 160.000 & 160.000 \\
\hline 0.05 & 50 & 168.187 & 168.093 & 158.271 & 158.260 \\
\hline 0.10 & 100 & 166.367 & 166.224 & 156.514 & 156.498 \\
\hline 0.15 & 150 & 164.539 & 164.405 & 154.728 & 154.707 \\
\hline 0.20 & 200 & 162.701 & 162.631 & 152.913 & 152.889 \\
\hline 0.25 & 250 & 160.852 & 160.955 & 151.070 & 151.044 \\
\hline 0.30 & 300 & 158.993 & 159.145 & 149.199 & 149.170 \\
\hline 0.35 & 350 & 157.120 & 157.011 & 147.299 & 147.269 \\
\hline 0.40 & 400 & 155.233 & 154.990 & 145.370 & 145.340 \\
\hline 0.45 & 450 & 153.330 & 153.037 & 143.413 & 143.384 \\
\hline 0.50 & 500 & 151.408 & 151.146 & 141.427 & 141.401 \\
\hline 0.55 & 550 & 149.465 & 149.310 & 139.413 & 139.389 \\
\hline 0.60 & 600 & 147.497 & 147.606 & 137.370 & 137.350 \\
\hline 0.65 & 650 & 145.501 & 145.779 & 135.299 & 135.281 \\
\hline 0.70 & 700 & 143.470 & 143.273 & 133.199 & 133.184 \\
\hline 0.75 & 750 & 141.400 & 141.128 & 131.070 & 131.059 \\
\hline 0.80 & 800 & 139.281 & 139.028 & 128.913 & 128.904 \\
\hline 0.85 & 850 & 137.101 & 137.017 & 126.728 & 126.721 \\
\hline 0.90 & 900 & 134.845 & 135.072 & 124.514 & 124.509 \\
\hline 0.95 & 950 & 132.489 & 132.870 & 122.271 & 122.266 \\
\hline 1.00 & 1000 & 130.000 & 130.000 & 120.000 & 120.000 \\
\hline & & & & & \\
\hline
\end{tabular}

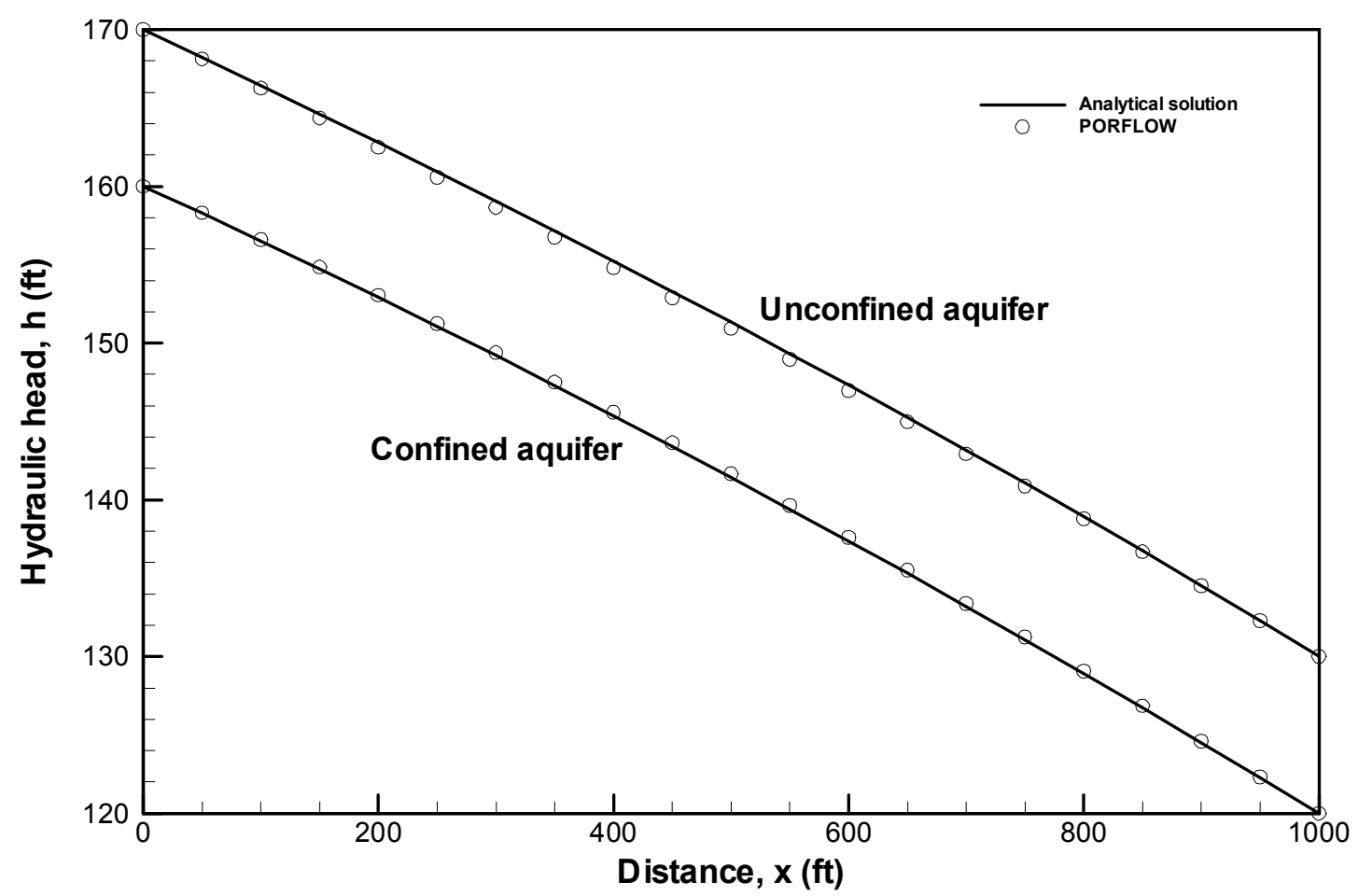




\section{Figure 4.3.3. Approximate Analytical and PORFLOW Hydraulic Head Profiles for the Unconfined and Confined Aquifers.}

Table 4.3.2. Input Commands for Problem 4.3

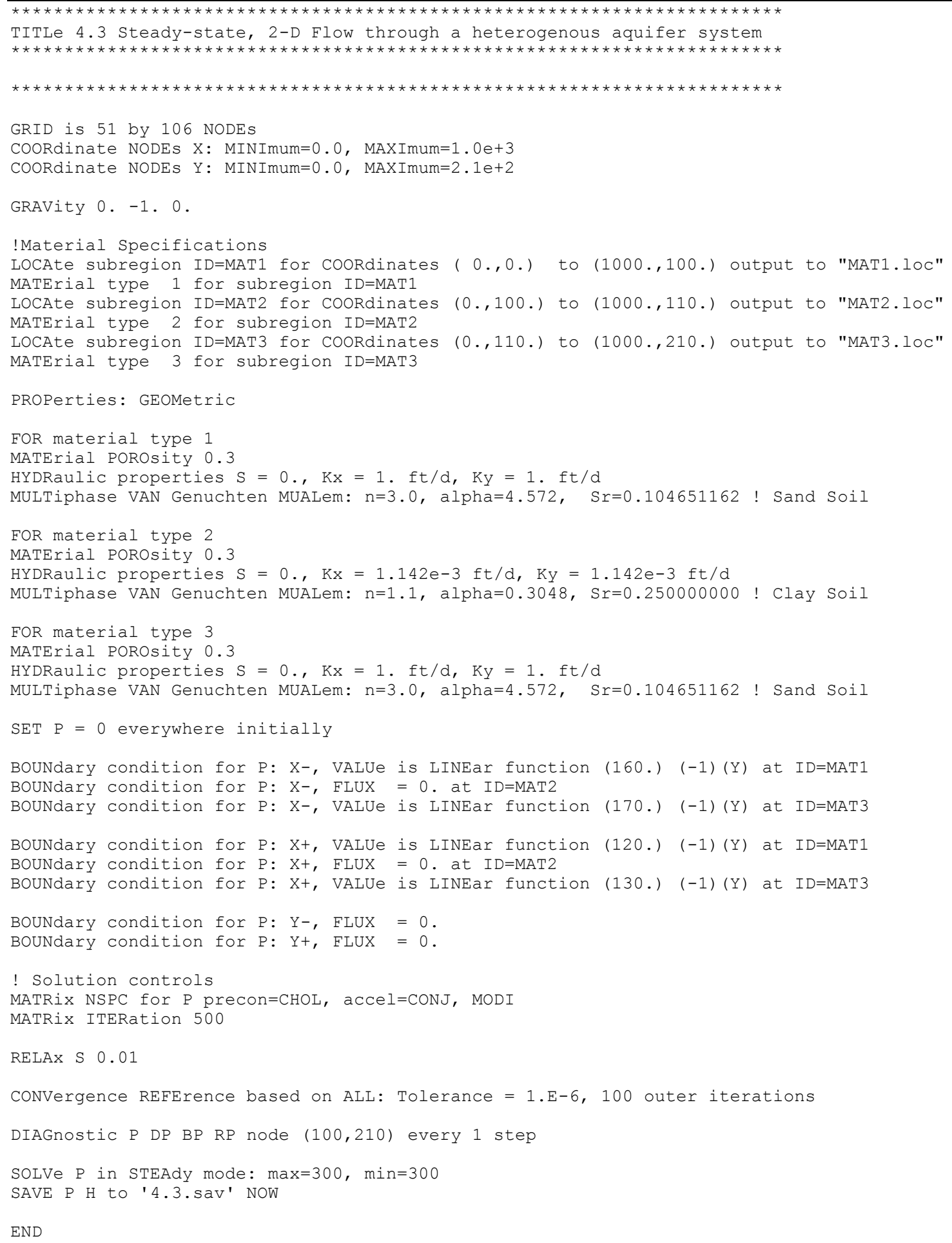




\subsection{Unconfined Aquifer Subject to Combined Recharge/Drain BC}

Figure 4.4.1 schematically illustrates an unconfined aquifer experiencing both recharge and drainage at the ground surface. The position of the seepline is unknown a priori. This test case is designed to test the implementation of a combined recharge/drain Cauchy boundary condition. The results of the PORFLOW simulation will be compared to an approximate analytical solution and to a finite element simulation of Richard's equation using the Earth Science Module in COMSOL Multiphysics (COMSOL AB, 2006).

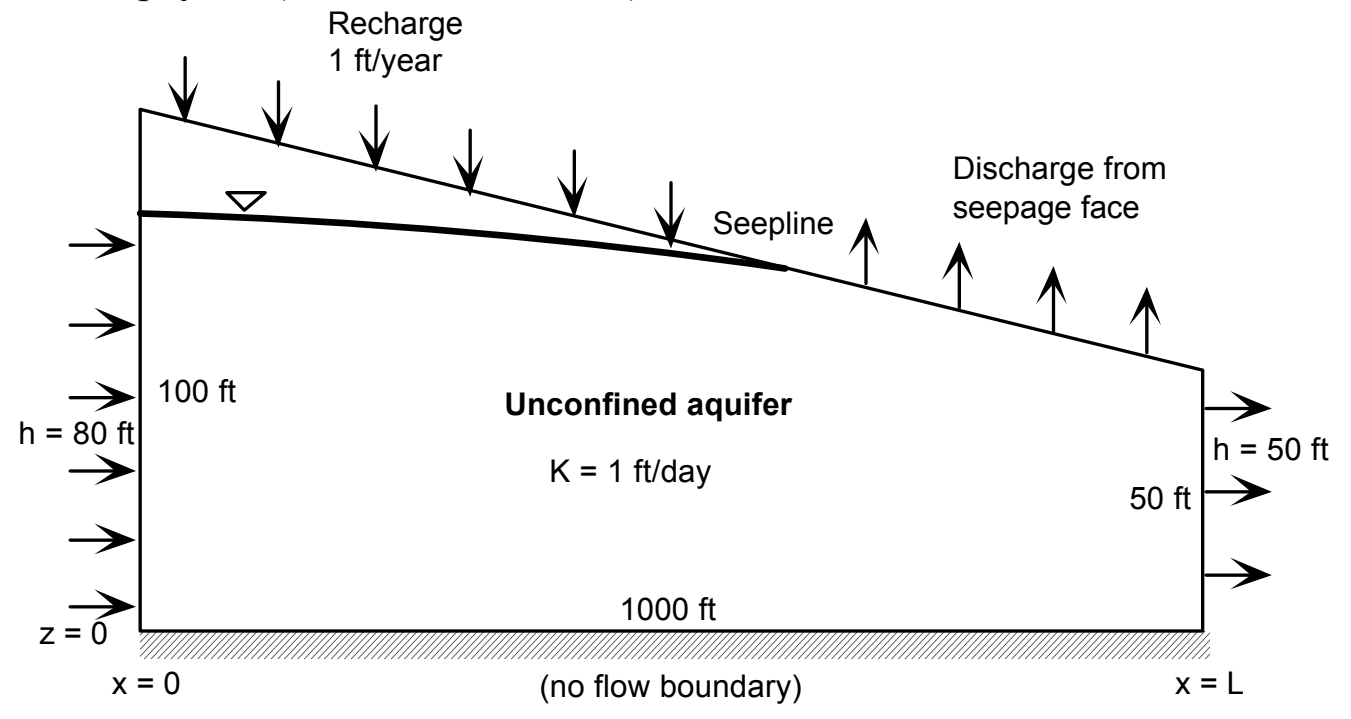

Figure 4.4.1. Schematic Illustration of an Unconfined Aquifer Experiencing both Recharge and Drainage at the Ground Surface; Seepline unknown a priori.

Analytical solution: With the Dupuit assumption, an analytical solution can be derived for the problem. Between the left boundary and the seepline, there is an unconfined aquifer subject to a recharge rate of $1 \mathrm{ft} / \mathrm{yr}$. From inspection of Eq. 4.3.5, the analytical solution for hydraulic head is

$$
\mathrm{h}^{2}=\mathrm{h}_{0}^{2}+\left(\mathrm{h}_{\mathrm{s}}^{2}-\mathrm{h}_{0}^{2}\right) \frac{\mathrm{x}}{\mathrm{L}_{\mathrm{s}}}+\frac{\mathrm{Q}_{\mathrm{src}} \mathrm{L}^{2}}{\mathrm{~K}}\left(\frac{\mathrm{x}}{\mathrm{L}_{\mathrm{s}}}\right)\left(1-\frac{\mathrm{x}}{\mathrm{L}_{\mathrm{s}}}\right) \quad 0 \leq \mathrm{x} \leq \mathrm{L}_{\mathrm{s}}
$$

where the seepline is positioned at $\mathrm{x}=\mathrm{L}_{\mathrm{s}}$, the hydraulic head at the seepline is $\mathrm{h}_{\mathrm{s}}$. The other variables are defined as before. Along the seepage face, the drain coefficient is assumed sufficiently large that the hydraulic head is the same as the ground elevation. That is,

$$
\mathrm{h}=50\left(2-\frac{\mathrm{x}}{\mathrm{L}}\right) \quad \mathrm{L}_{\mathrm{s}} \leq \mathrm{x} \leq \mathrm{L}
$$

The location of the seepline is obtained by simultaneous solution of the following nonlinear equation set: 


$$
\begin{gathered}
\mathrm{h}=50\left(2-\frac{\mathrm{L}_{\mathrm{s}}}{\mathrm{L}}\right) \\
\left.\frac{\mathrm{dh}}{\mathrm{dx}}\right|_{\mathrm{x}=\mathrm{L}_{\mathrm{s}}^{-}}=\frac{1}{\mathrm{~h}_{\mathrm{s}}}\left[\frac{\mathrm{h}_{\mathrm{s}}^{2}-\mathrm{h}_{0}^{2}}{2 \mathrm{~L}_{\mathrm{s}}}-\frac{\mathrm{Q}_{\mathrm{src}} \mathrm{L}_{\mathrm{s}}}{2 \mathrm{~K}}\right]=\frac{\mathrm{h}_{\mathrm{L}}-\mathrm{h}_{\mathrm{s}}}{\mathrm{L}-\mathrm{L}_{\mathrm{s}}}=\left.\frac{\mathrm{dh}}{\mathrm{dx}}\right|_{\mathrm{x}=\mathrm{L}_{\mathrm{s}}^{+}}
\end{gathered}
$$

For the parameter values indicated in Figure 4.4.1, the location of the seepline is $\mathrm{L}_{\mathrm{s}}=829 \mathrm{ft}$.

Implementation of Recharge/Drain Boundary Condition in PORFLOW and COMSOL: A full discussion the head-dependent recharge and drain boundary condition can be found in Section 3.1.7.1 of the FACT Version 2.0 manual (Hamm and Aleman, 2000). In summary, the recharge/drain boundary condition is implemented as shown in Figure 4.4.2.

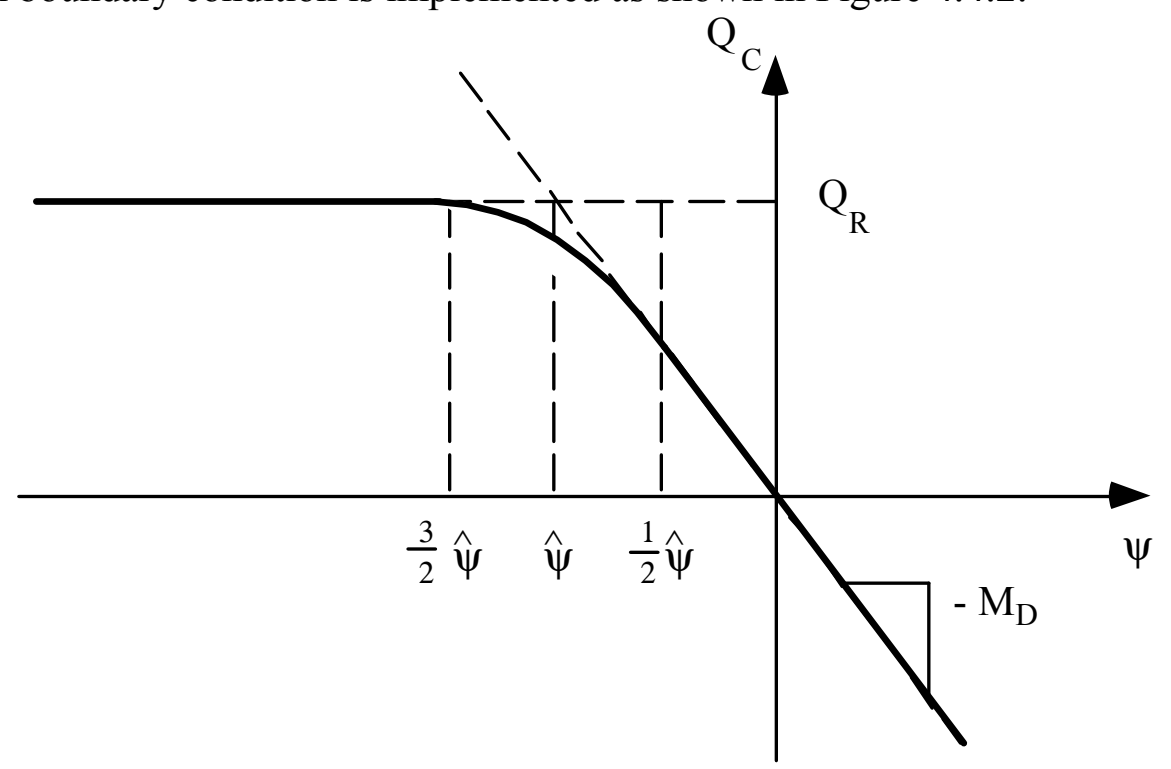

Figure 4.4.2. Cauchy Boundary Condition for the Simultaneous Treatment of Groundwater Recharge and Drainage.

The mathematical formulation chosen for this function, as shown in Figure 4.4.2 is

$$
Q_{c}=\left\{\begin{array}{ccc}
Q_{R} & \text { for } & \psi \leq \frac{3}{2} \hat{\psi} \\
\frac{Q_{R}}{8}\left[7-2 x-x^{2}\right] & \text { for } & \frac{3}{2} \hat{\psi}<\psi<\frac{1}{2} \hat{\psi} \\
-M_{D} \psi & \text { for } & \frac{1}{2} \hat{\psi} \leq \psi
\end{array}\right.
$$

where

$$
\begin{gathered}
\psi=\mathrm{h}-\mathrm{y}_{\mathrm{c}} \\
\mathrm{Q}_{\mathrm{R}}=\mathrm{A}_{\mathrm{D}} \mathrm{R}_{\max }
\end{gathered}
$$




$$
\begin{gathered}
M_{D}=A_{D}\left(\frac{K}{b}\right)_{D} \\
x=2 \frac{\hat{\psi}-\psi}{\hat{\psi}} \\
\hat{\psi}=-\frac{Q_{R}}{M_{D}}
\end{gathered}
$$

and

$$
\begin{aligned}
& \mathrm{y}_{\mathrm{c}} \ldots \ldots \ldots \ldots \ldots . . . . . . \text { nodal surface elevation, } \mathrm{ft} \\
& \psi \text {.................nodal surface pressure head, } \mathrm{ft} \\
& \mathrm{Q}_{\mathrm{c}} \text {.................nodal volumetric source or sink flow at surface, } \mathrm{ft}^{3} / \text { day } \\
& \mathrm{R}_{\text {max }} \text { …......... maximum recharge, } \mathrm{ft} / \text { day } \\
& A_{D} \text { ….............nodal area available for recharge and drainage (computed), } \mathrm{ft}^{2} \\
& (\mathrm{~K} / \mathrm{b})_{\mathrm{D}} \ldots \ldots \ldots . . . . \text { surface leakance coefficient, } 1 / \text { day }
\end{aligned}
$$

Equation 4.4.5 is divided by the nodal area, $A_{D}$, to get the expression back to a flux boundary condition, that is required by both PORFLOW and COMSOL as

$$
\mathrm{R}_{\mathrm{c}}=\left\{\begin{array}{ccc}
\mathrm{R}_{\max } & \text { for } & \psi \leq \frac{3}{2} \hat{\psi} \\
\frac{\mathrm{R}_{\max }\left[7-2 \mathrm{x}-\mathrm{x}^{2}\right]}{8}[ & \text { for } & \frac{3}{2} \hat{\psi}<\psi<\frac{1}{2} \hat{\psi} \\
-(\mathrm{K} / \mathrm{b})_{\mathrm{D}} \psi & \text { for } & \frac{1}{2} \hat{\psi} \leq \psi
\end{array}\right.
$$

The maximum recharge, $\mathrm{R}_{\max }$, is $1 \mathrm{ft} / \mathrm{day}$ as shown in Figure 4.4.1. The surface leakance coefficient was set to 1 day $^{-1}$.

PORFLOW numerical simulation and comparison: The PORFLOW mesh is show in Figure 4.4.3. The mesh contains 21 nodes in the $\mathrm{x}$-direction for a mesh spacing of 50 feet and 21 nodes in the y-direction. The mesh is non-orthogonal in y and has a mesh spacing of 5 feet at the left boundary and a mesh spacing of 2.5 feet at the right boundary. The COMSOL finite-element mesh is shown in Figure 4.4.4 and consists of 9648 triangular elements and 5112 mesh points.

The analytical solution assumes that the recharge is constant at $1 \mathrm{ft} /$ day from the left boundary to the seepline. Figure 4.4.5 and Table 4.4.1 illustrate that the analytical solution (1) predicts higher hydraulic heads than PORFLOW or COMSOL. The implementation of Eq. 4.4.7 in PORFLOW and COMSOL results in the recharge rate varying from a maximum of $1 \mathrm{ft} /$ day to $0 \mathrm{ft} /$ day at the seepline. The effective recharge into the PORFLOW and COMSOL mesh is less than $1 \mathrm{ft} /$ day. An effective recharge rate of $0.67 \mathrm{ft} /$ day in Eq. 4.4 .1 provides excellent agreement between the analytical solution (2) and the numerical simulations (Figure 4.4.5). The PORFLOW input commands and grid file are given in Tables 4.4.2 and 4.4.3, respectively. 
The drain portion of the recharge/drain boundary condition could not be implemented in PORFLOW due to convergence issues. The Earth Science Module in COMSOL with a fine mesh converged quite rapidly for this problem.

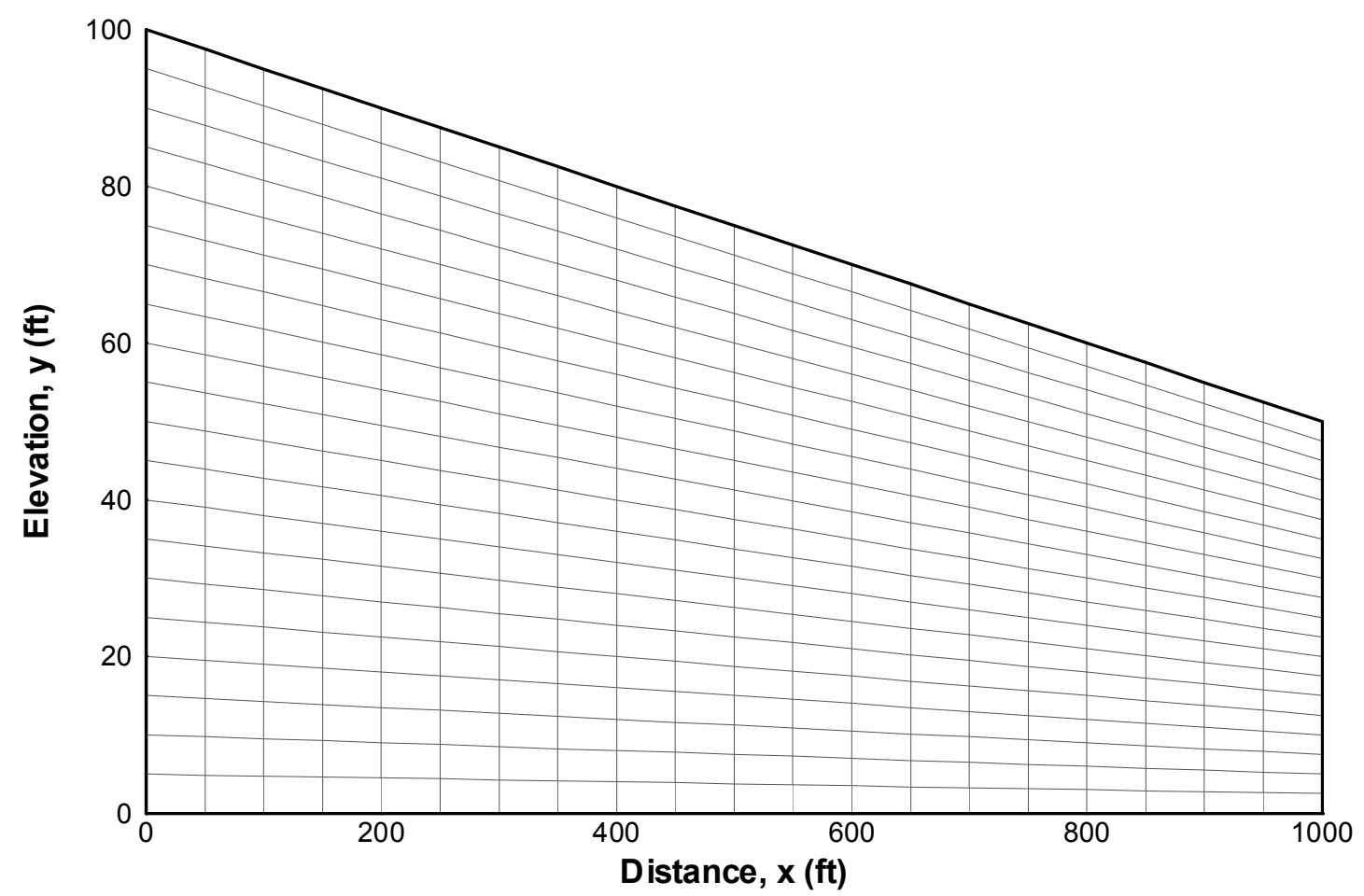

Figure 4.4.3. PORFLOW Grid for Problem 4.4. 


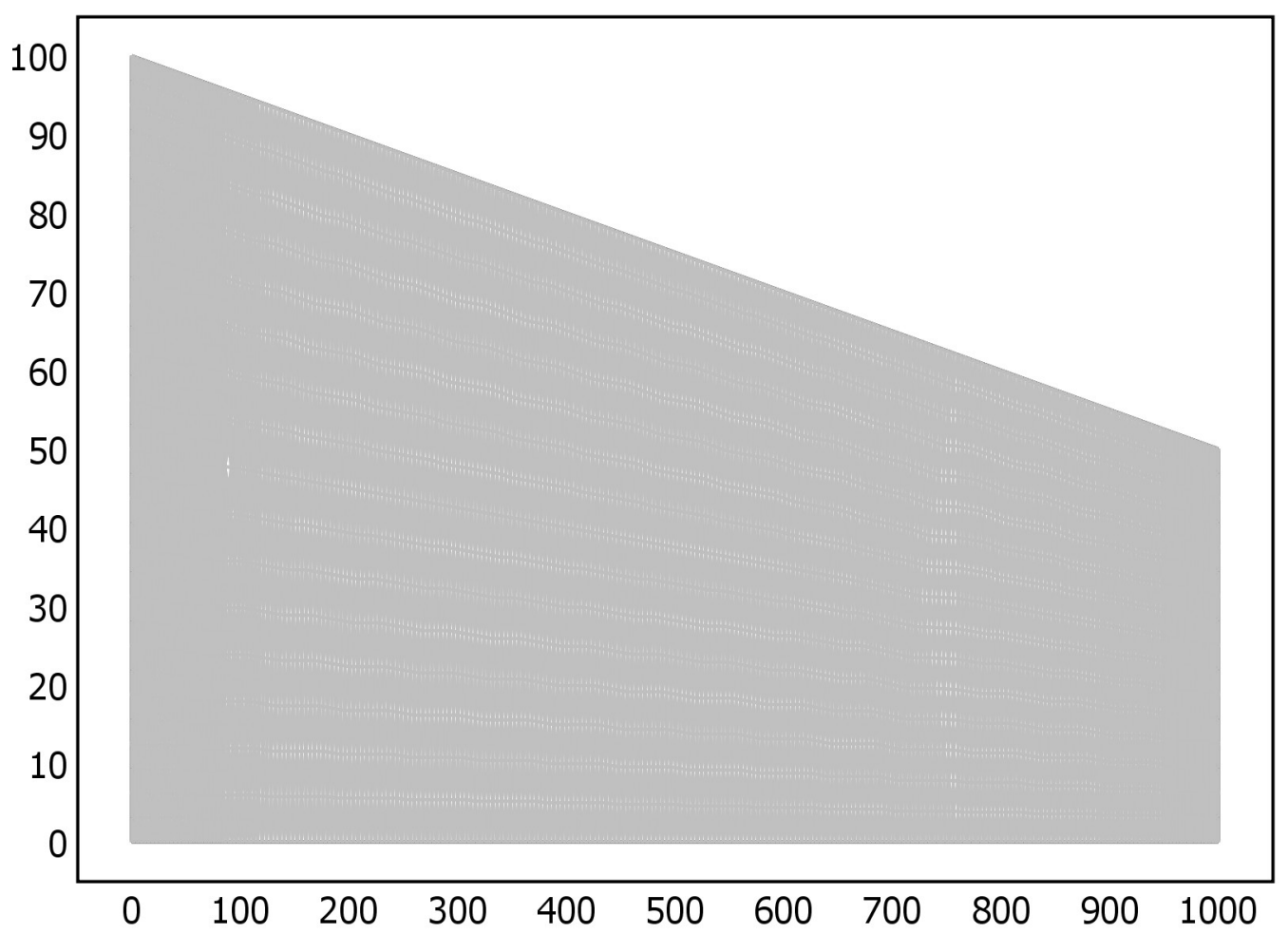

Figure 4.4.4. COMSOL Finite-Element Mesh for Problem 4.4.

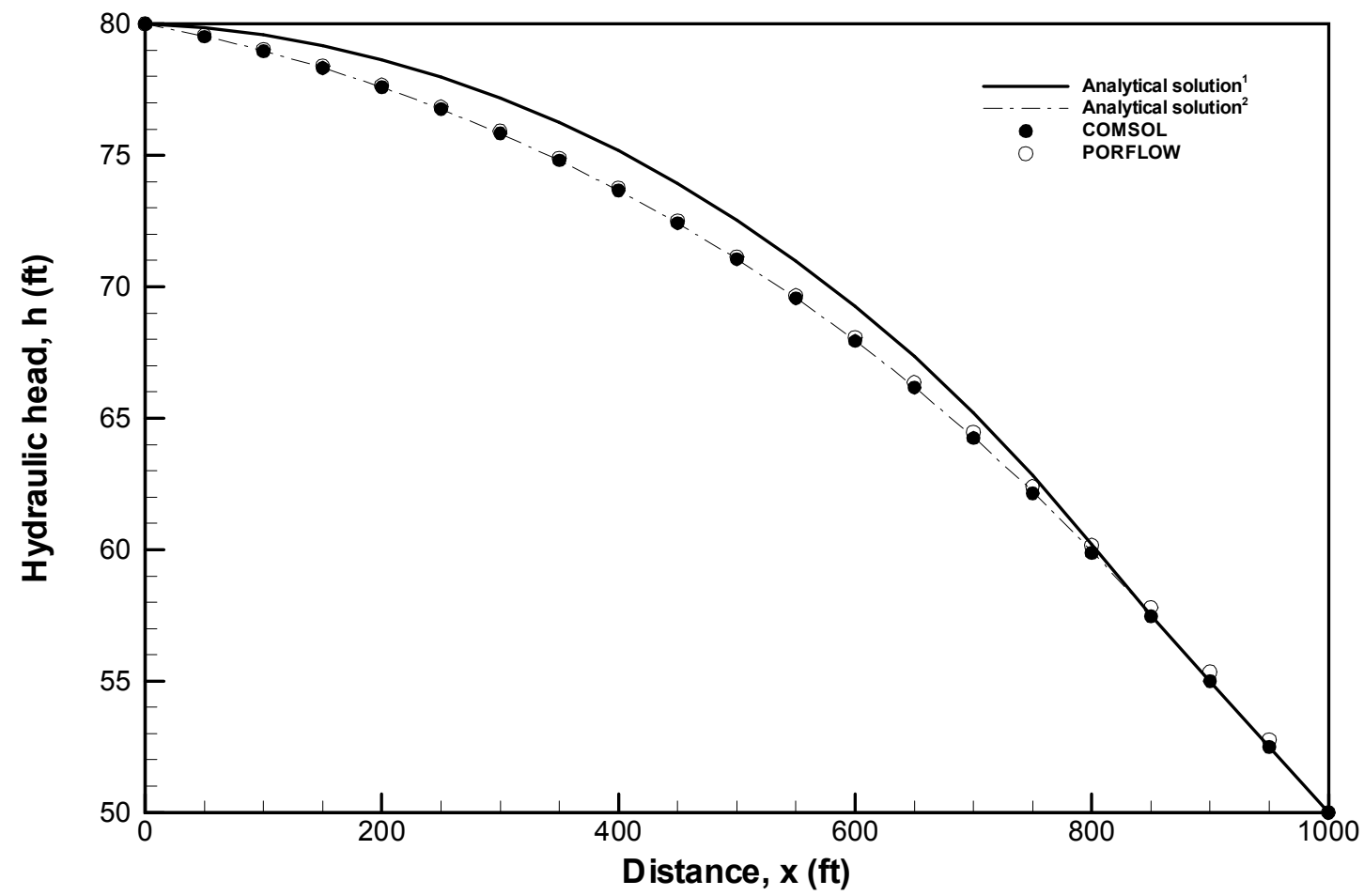

Figure 4.4.5. Comparison of PORFLOW Hydraulic Head Distribution to Analytical Solution and COMSOL Simulation $(\mathrm{Y}=\mathbf{0})$. 
Table 4.4.1. Analytical, COMSOL and PORFLOW Water Table Comparison

\begin{tabular}{|c|c|c|c|c|}
\hline $\begin{array}{c}\text { Horizontal } \\
\text { distance, } x \\
(\mathrm{ft})\end{array}$ & $\begin{array}{c}\text { Analytical }^{1} \\
\text { head } \\
(\mathrm{ft})\end{array}$ & $\begin{array}{c}\text { Analytical }^{2} \\
\text { head } \\
(\mathrm{ft})\end{array}$ & $\begin{array}{c}\text { COMSOL } \\
\text { head } \\
(\mathrm{ft})\end{array}$ & $\begin{array}{c}\text { PORFLOW } \\
\text { head } \\
(\mathrm{ft})\end{array}$ \\
\hline 0 & 80.000 & 80.000 & 80.000 & 80.000 \\
\hline 50 & 79.850 & 79.532 & 79.512 & 79.574 \\
\hline 100 & 79.575 & 78.976 & 78.960 & 79.031 \\
\hline 150 & 79.173 & 78.331 & 78.322 & 78.394 \\
\hline 200 & 78.642 & 77.594 & 77.591 & 77.661 \\
\hline 250 & 77.980 & 76.762 & 76.764 & 76.839 \\
\hline 300 & 77.184 & 75.833 & 75.838 & 75.912 \\
\hline 350 & 76.248 & 74.803 & 74.808 & 74.880 \\
\hline 400 & 75.168 & 73.667 & 73.672 & 73.753 \\
\hline 450 & 73.938 & 72.421 & 72.424 & 72.507 \\
\hline 500 & 72.550 & 71.058 & 71.058 & 71.136 \\
\hline 550 & 70.994 & 69.572 & 69.564 & 69.654 \\
\hline 600 & 69.259 & 67.956 & 67.937 & 68.075 \\
\hline 650 & 67.333 & 66.198 & 66.170 & 66.352 \\
\hline 700 & 65.196 & 64.289 & 64.243 & 64.463 \\
\hline 750 & 62.829 & 62.213 & 62.149 & 62.397 \\
\hline 800 & 60.204 & 59.953 & 59.880 & 60.157 \\
\hline 850 & 57.500 & 57.500 & 57.468 & 57.804 \\
\hline 900 & 55.000 & 55.000 & 54.994 & 55.344 \\
\hline 950 & 52.500 & 52.500 & 52.500 & 52.768 \\
\hline 1000 & 50.000 & 50.000 & 50.000 & 50.000 \\
\hline
\end{tabular}

Table 4.4.2. Input Commands for Problem 4.4.

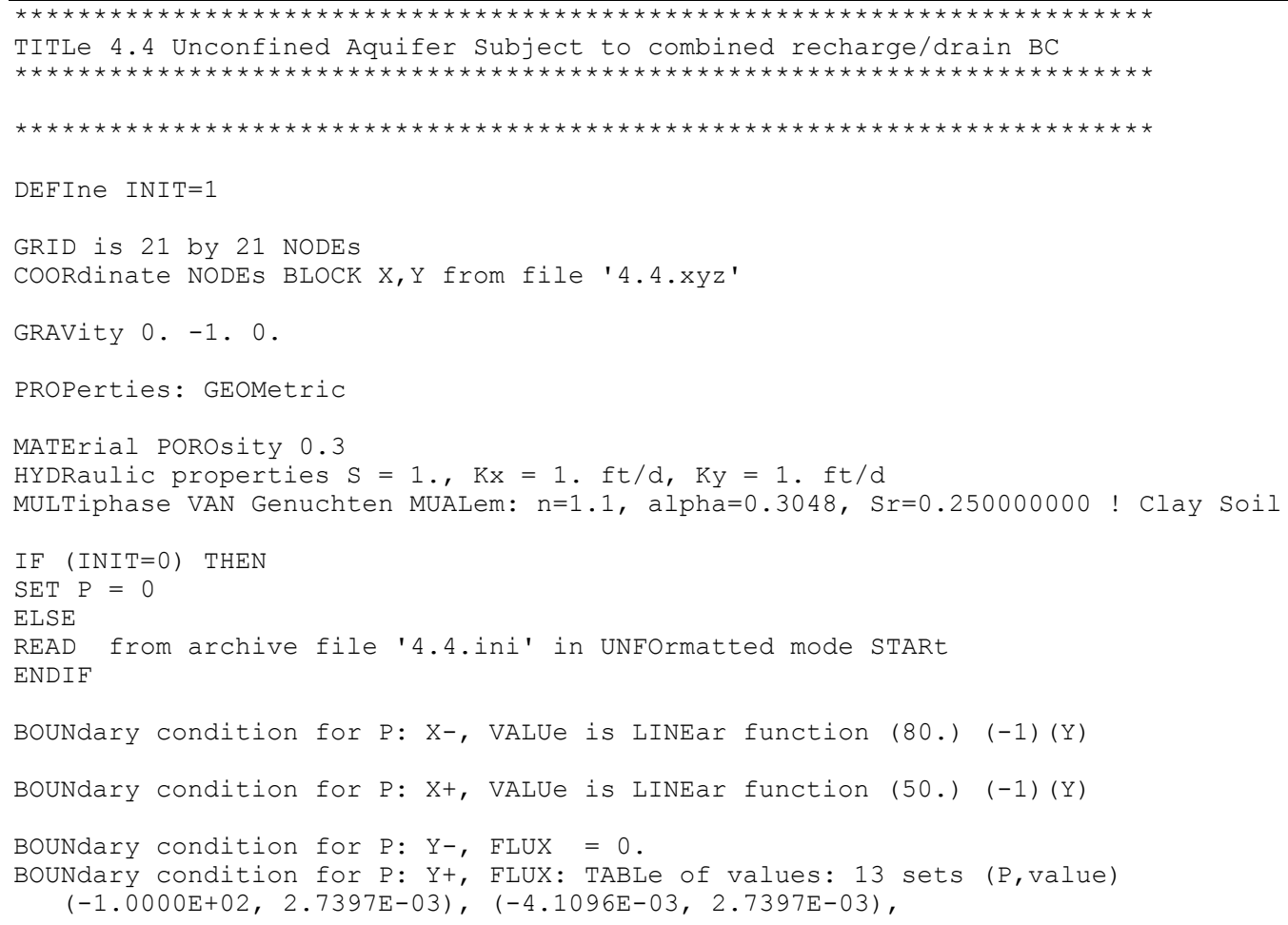


$(-3.8356 \mathrm{E}-03,2.7260 \mathrm{E}-03),(-3.5616 \mathrm{E}-03,2.6849 \mathrm{E}-03)$, $(-3.2877 \mathrm{E}-03,2.6164 \mathrm{E}-03),(-3.0137 \mathrm{E}-03,2.5205 \mathrm{E}-03)$, $(-2.7397 \mathrm{E}-03,2.3973 \mathrm{E}-03),(-2.4658 \mathrm{E}-03,2.2466 \mathrm{E}-03)$, $(-2.1918 \mathrm{E}-03,2.0685 \mathrm{E}-03),(-1.9178 \mathrm{E}-03,1.8630 \mathrm{E}-03)$, $(-1.6438 \mathrm{E}-03,1.6301 \mathrm{E}-03),(-1.3699 \mathrm{E}-03,1.3699 \mathrm{E}-03)$, $(0.0000 \mathrm{E}+00,0.0000 \mathrm{E}+00)$ !Recharge/Drain

! Solution controls

MATRix NSPC for $P$ precon=CHOL, accel=CONJ, MODI

MATRix ITERation 100

RELAX P 0.5

CONVergence REFErence for ALL: Tolerance = 1.E-4, 200 outer iterations

DIAGnostic H DP BP RP node $(20,21)$ every 1 step

TIME $=0$

SOLVe $\mathrm{P}$ in STEAdy mode: $\max =5000$, $\mathrm{min}=5000$

SAVE $P$ H U V to '4.4.sav' NOW

SAVE file to '4.4.rst' in UNFOrmatted mode

END

Table 4.4.3. Nodal Grid for Problem 4.4.

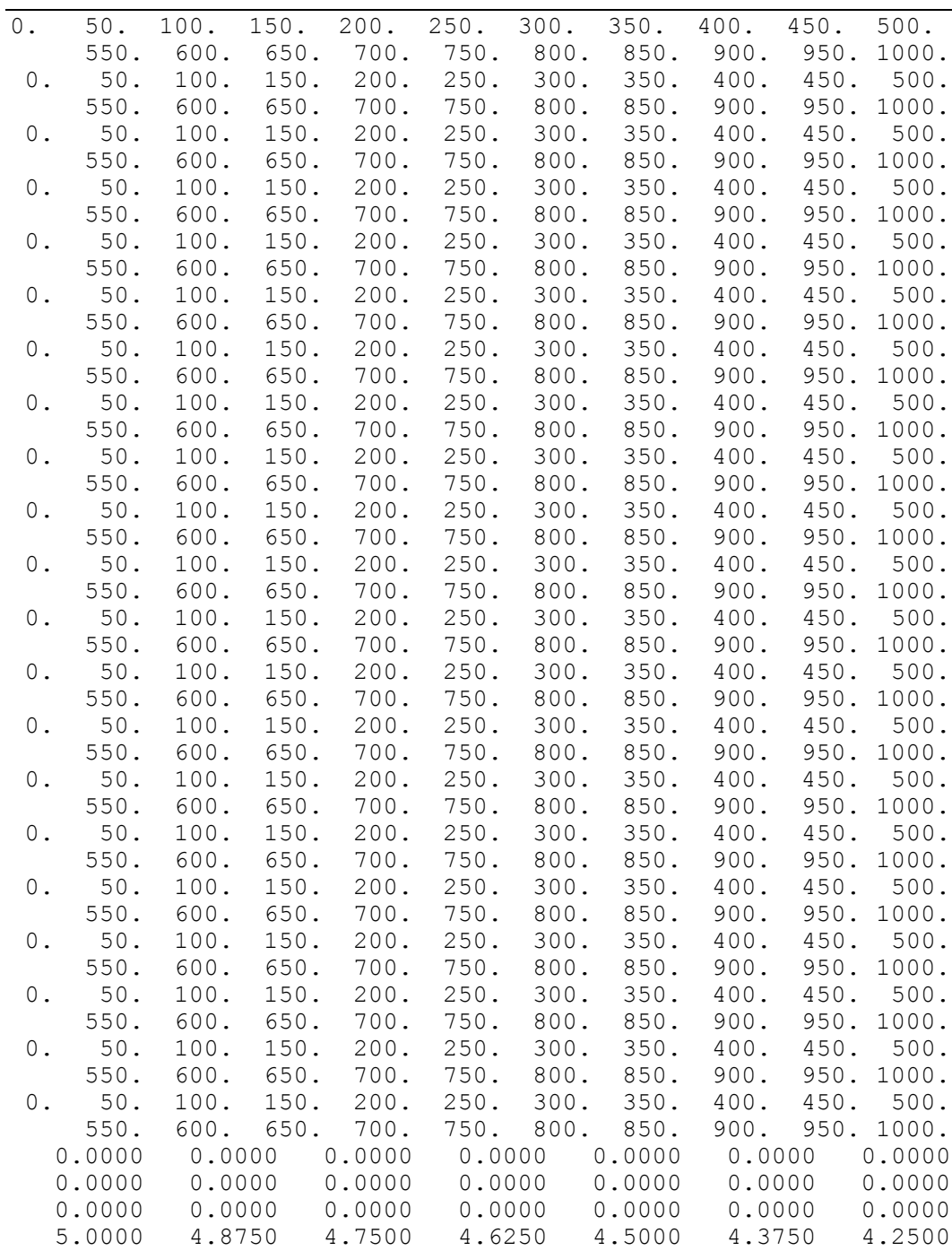




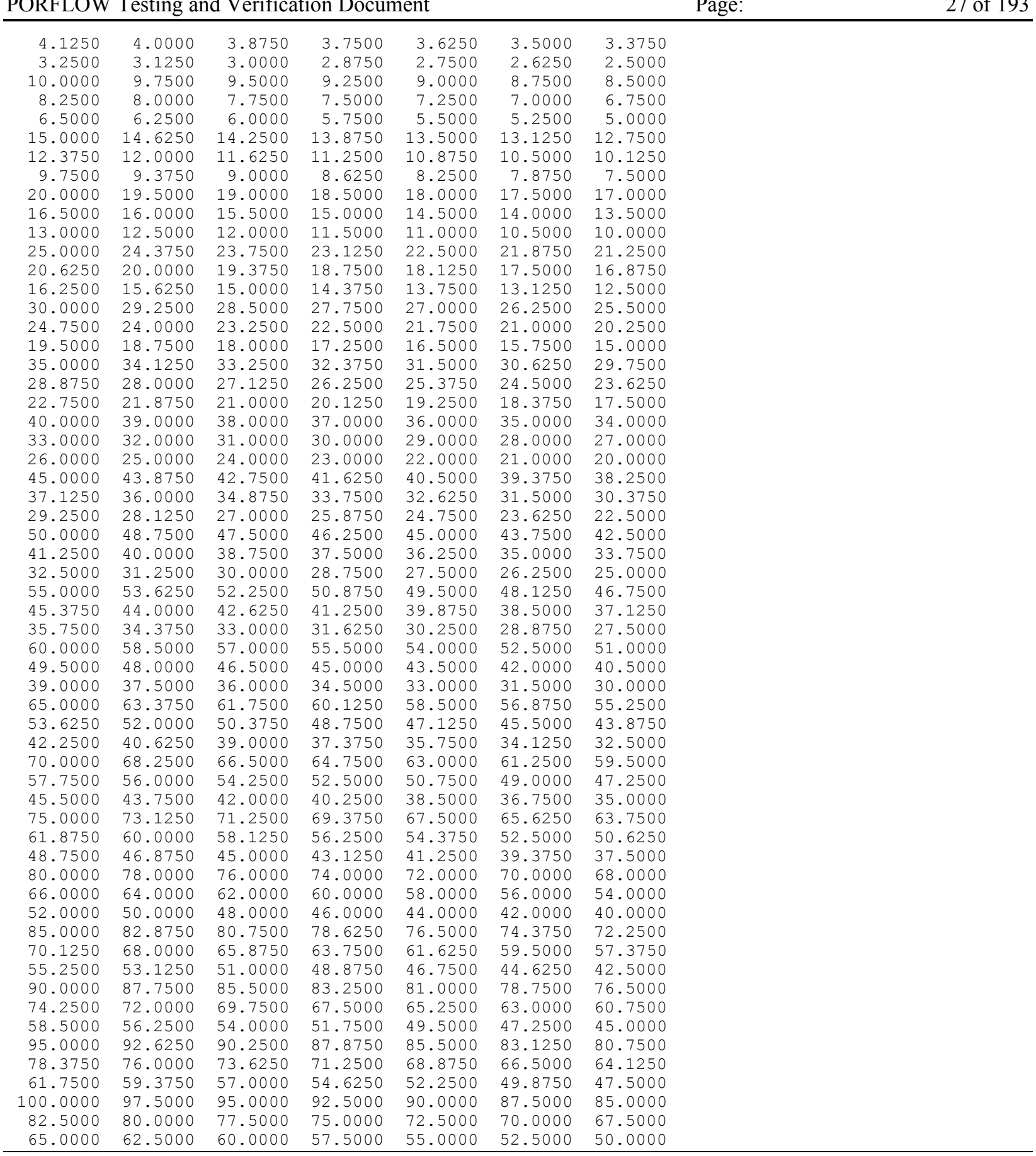

\subsection{Transient, One-Dimensional Flow to a Well in a Confined Aquifer (Theis, 1935)}

The problem involves radial flow to a well in a confined aquifer whose classic solution is given by Theis (1935). The problem is illustrated in Figure 4.5.1. Specifically we consider an aquifer with the following attributes:

- homogeneous, isotropic, and of uniform thickness 
- fully confined above and below by impervious layers

- infinite in horizontal extent

- water is released instantaneously from storage when hydraulic head decreases

- homogeneous and isotropic properties

- the screen of the pumped well fully penetrates the aquifer

- well diameter is very small, so that storage in the well can be neglected

- water is pumped at a constant rate from the well, and enters uniformly along the well screen

- prior to pumping, the aquifer is in a state of equilibrium

The parameter values chosen for PORFLOW are given in Figure 4.5.1 and are equivalent to those chosen by Anderson (1993, Problem 1) for MODFLOW simulations.

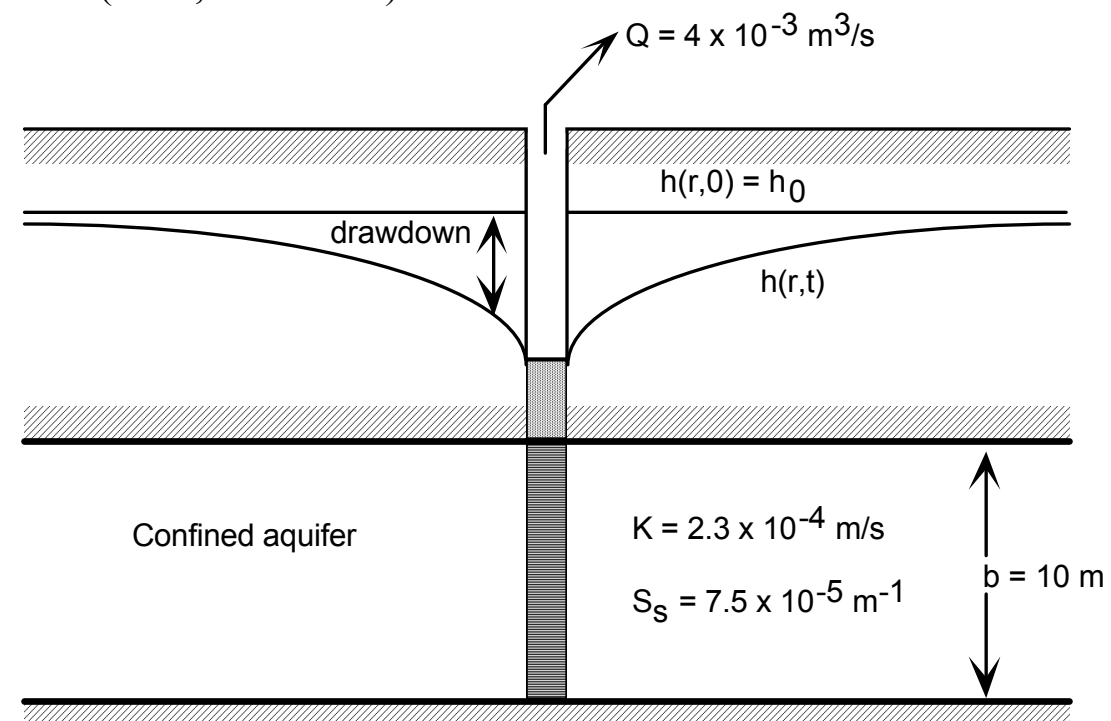

Figure 4.5.1. Radial Flow to a Pumping Well in a Confined Aquifer.

Theis analytical solution: The governing equation for the flow problem described above is (Freeze and Cherry, 1979, Section 8.3)

$$
\frac{1}{\mathrm{r}} \frac{\partial}{\partial \mathrm{r}}\left(\mathrm{r} \frac{\partial \mathrm{h}}{\partial \mathrm{r}}\right)=\frac{\mathrm{S}}{\mathrm{T}} \frac{\partial \mathrm{h}}{\partial \mathrm{t}}
$$

The initial condition is

$$
\mathrm{h}(\mathrm{r}, 0)=\mathrm{h}_{0}
$$


where $\mathrm{h} 0$ is the constant initial head.

The boundary conditions assume no drawdown in hydraulic head at the infinite boundary

$$
\mathrm{h}(\infty, \mathrm{t})=\mathrm{h}_{0}
$$

and a constant pumping rate $\mathrm{Q}$ at the well:

$$
\lim _{r \rightarrow 0}\left(r \frac{\partial h}{\partial r}\right)=\frac{Q}{2 \pi T} \quad \text { for } t>0
$$

Because the aquifer properties are homogeneous

$$
\begin{aligned}
& \mathrm{S}=\mathrm{S}_{\mathrm{S}} \mathrm{b} \\
& \mathrm{T}=\mathrm{Kb}
\end{aligned}
$$

An analytical solution to Equation 4.5.1 subject to the initial and boundary conditions of Eqs. 4.5.2 through 4.5.5 is given by Theis (1935) in terms of drawdown as

$$
\mathrm{s}=\mathrm{h}_{0}-\mathrm{h}(\mathrm{r}, \mathrm{t})=\frac{\mathrm{Q}}{4 \pi \mathrm{T}} \int_{\mathrm{u}}^{\infty} \frac{\mathrm{e}^{-\tau}}{\tau} \mathrm{d} \tau=\frac{\mathrm{Q}}{4 \pi \mathrm{T}} \mathrm{W}(\mathrm{u})
$$

where

$$
\mathrm{u}=\frac{\mathrm{r}^{2} \mathrm{~S}}{4 \mathrm{Tt}}
$$

and $\mathrm{W}(\mathrm{u})$ is known as the Theis well function. The well function (or the exponential integral) can be evaluated by polynomial and rational approximations given by Abramowitz and Stegun (1970, p. 231).

$$
W(u)=\left\{\begin{array}{cc}
-0.5772+u-0.25 u^{2}+0.055 u^{3}-0.01 u^{4}+0.001 u^{5}-\ln (u) & u<1 \\
\frac{\exp (-u)}{u} \frac{u^{4}+8.58 u^{3}+18.06 u^{2}+8.64 u+0.27}{u^{4}+9.57 u^{3}+25.63 u^{2}+21.1 u+3.96} & u \geq 1
\end{array}\right.
$$

Evaluations of the Theis solution for the parameter values specified in Figure 4.5.1 at a radial position of 55 meters from the well are presented in Tables 4.5.1 and 4.5.2 for various times.

PORFLOW simulation and comparison: This problem was solved using PORFLOW in one and two dimensions. Figure 4.5.2 shows the PORFLOW mesh in the radial coordinate system. The mesh consists of 1000 nodes in the radial direction with a finer mesh as you approach the pumping well at the left boundary. The mesh extends from 0.1 meters to 10000 meters in the radial direction. The three nodes in the $\mathrm{x}$-direction are automatically added by PORFLOW for the 
1D simulation. Refer to Table 4.5.3 for details of the simulation (material properties, initial and boundary conditions).

The COMSOL mesh, which is not shown, includes 916 finite elements and 917 nodes in the radial direction. The mesh extends from 0 meters to 10000 meters in the radial direction. COMSOL was also used to compute the Theis drawdown using Eqs. 4.5.6 to 4.5.8.

Figure 4.5.3 shows the PORFLOW mesh in the 2D Cartesian coordinate system. The mesh extends from -1200 to +1200 meters in each direction. There are 241 nodes in each direction for a total nodal count of 58081. The mesh is refined locally near the pumping well at the center of the mesh $(\mathrm{x}=\mathrm{y}=0)$ because steep head gradients are expected there. Refer to Table 4.5.4 for details of the simulation.

Figure 4.5.4 illustrates the transient drawdown at a radial distance of 55 meters from the well for the Theis, COMSOL and PORFLOW numerical simulations. There is excellent agreement among the three numerical simulations. Tables 4.5.1 and 4.5.2 show the transient drawdown at short and longer times, respectively.

Figure 4.5.5 shows the PORFLOW drawdown at 1 day for the 2D Cartesian simulation. The near field results show the concentric circles of drawdown as expected while near the boundaries there is distortion of the drawdown contours due to boundary effects.

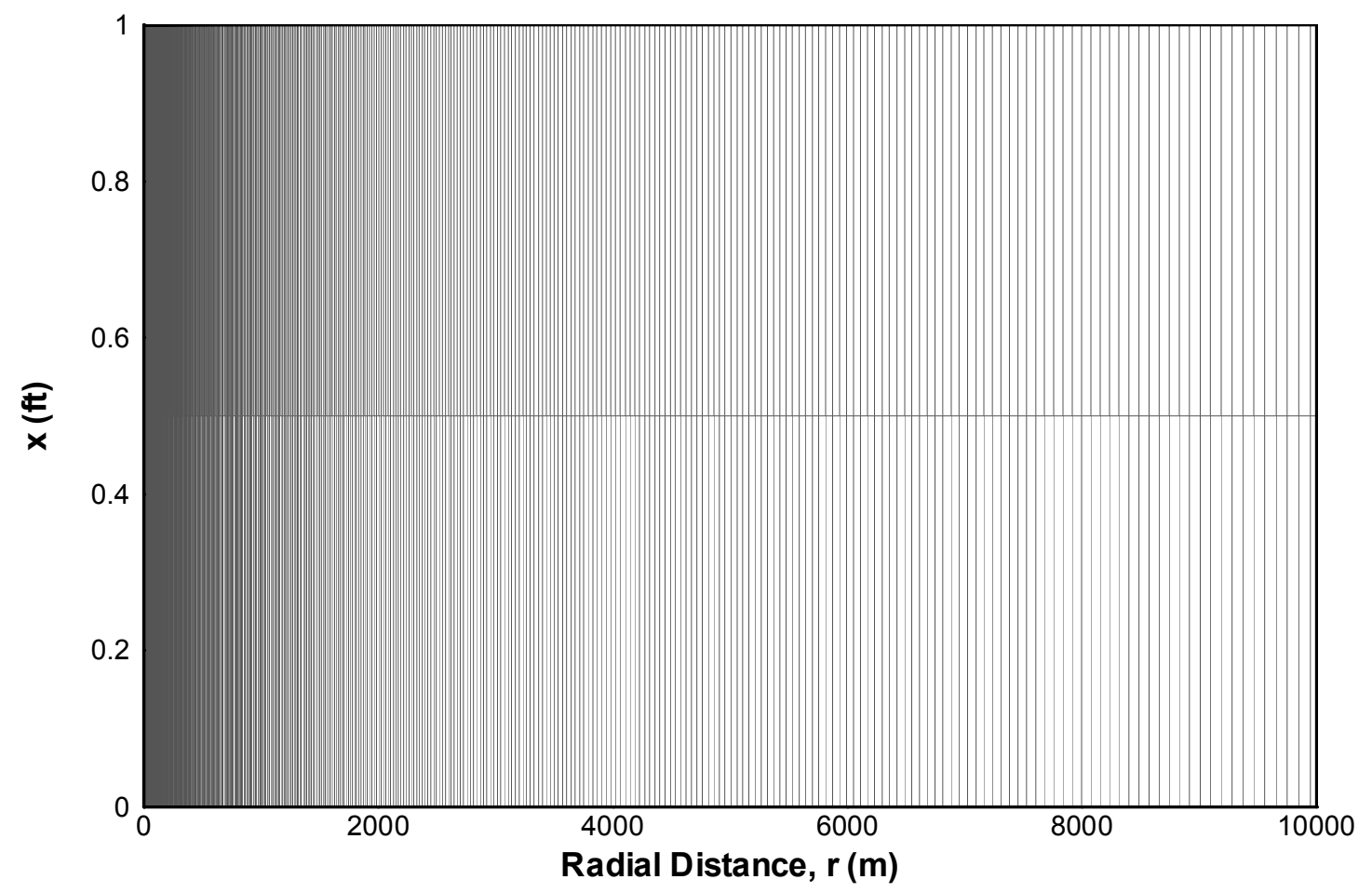

Figure 4.5.2. 1D PORFLOW Radial Grid for Problem 4.5. 


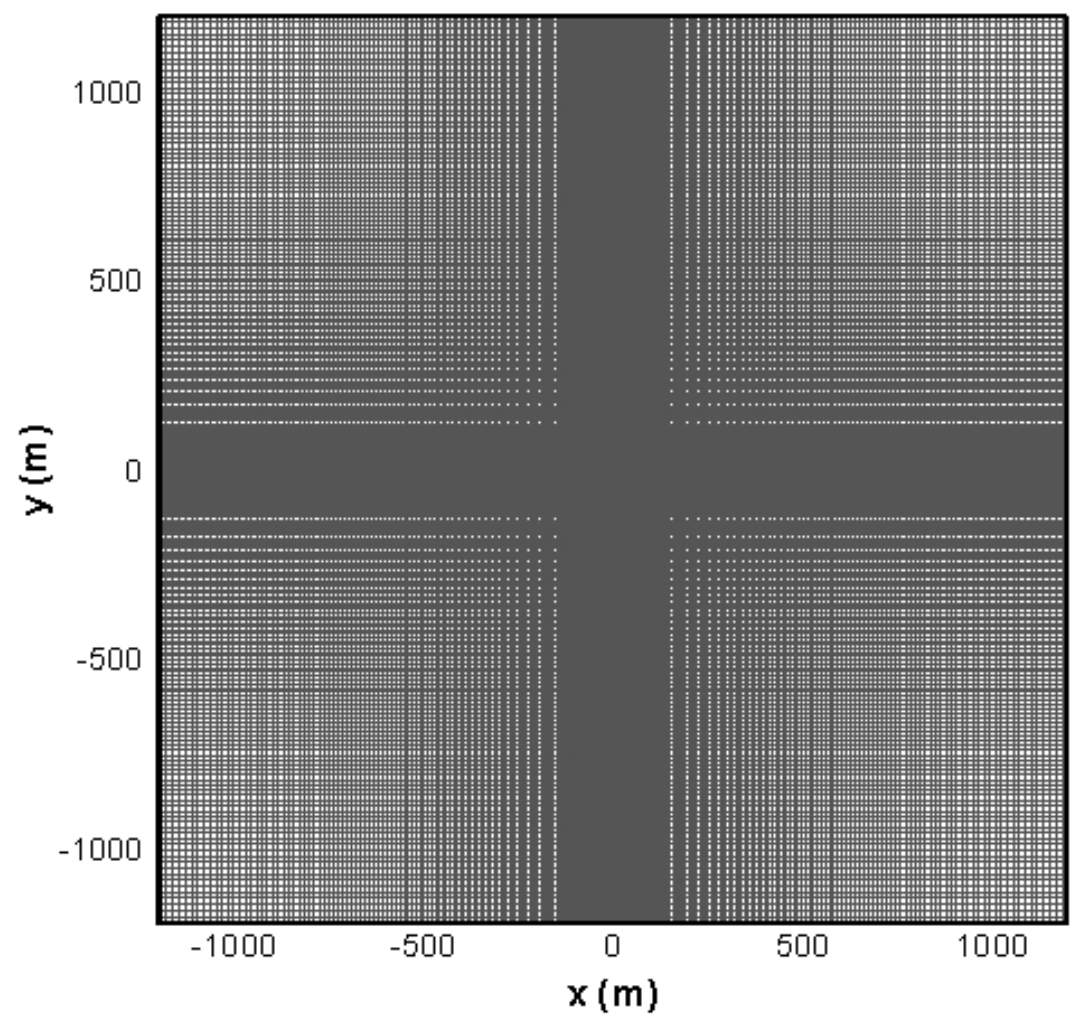

Figure 4.5.3. 2D PORFLOW Cartesian Grid for Problem 4.5.

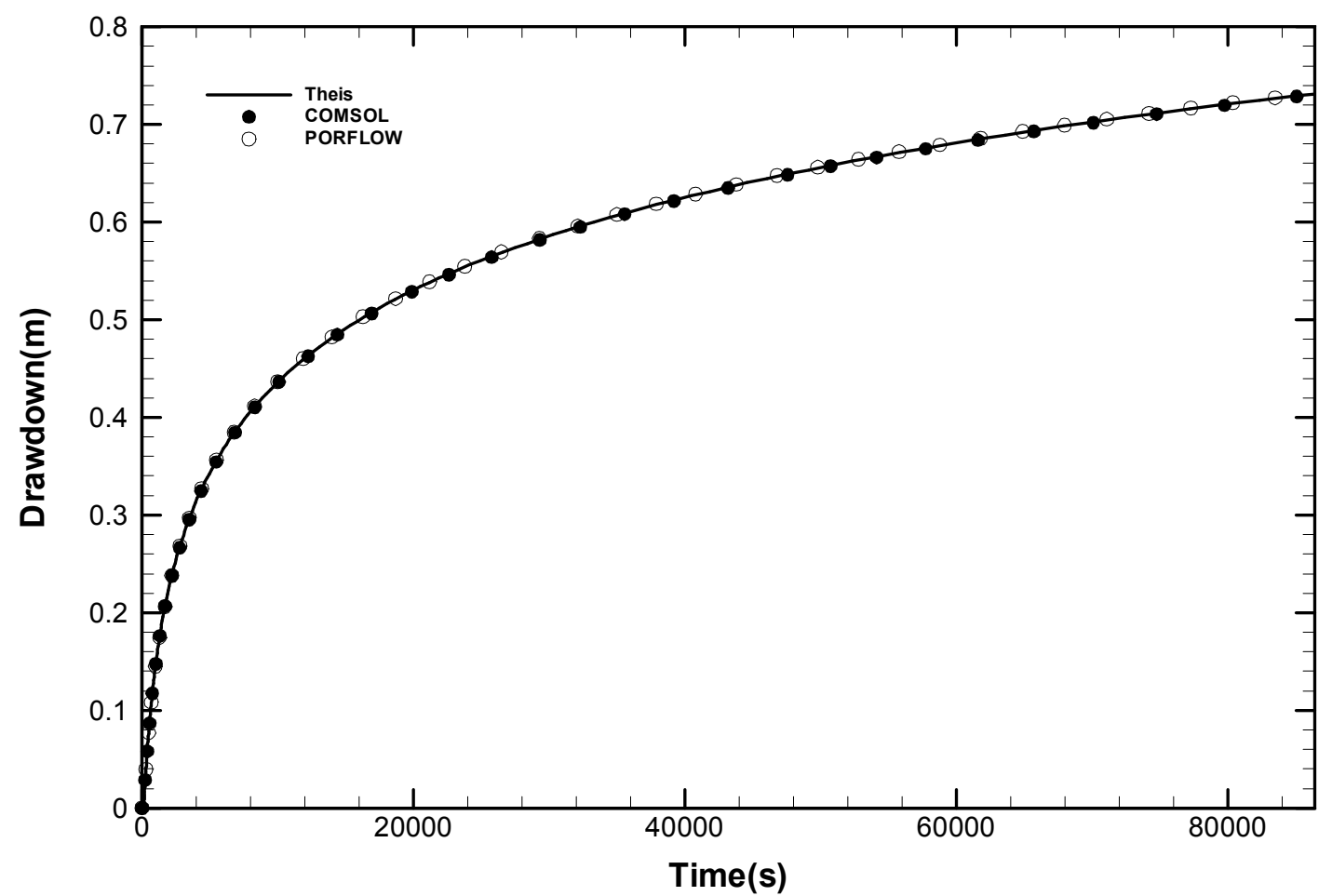

Figure 4.5.4. Theis, COMSOL and PORFLOW Transient Drawdown at $r=55$ meters. 


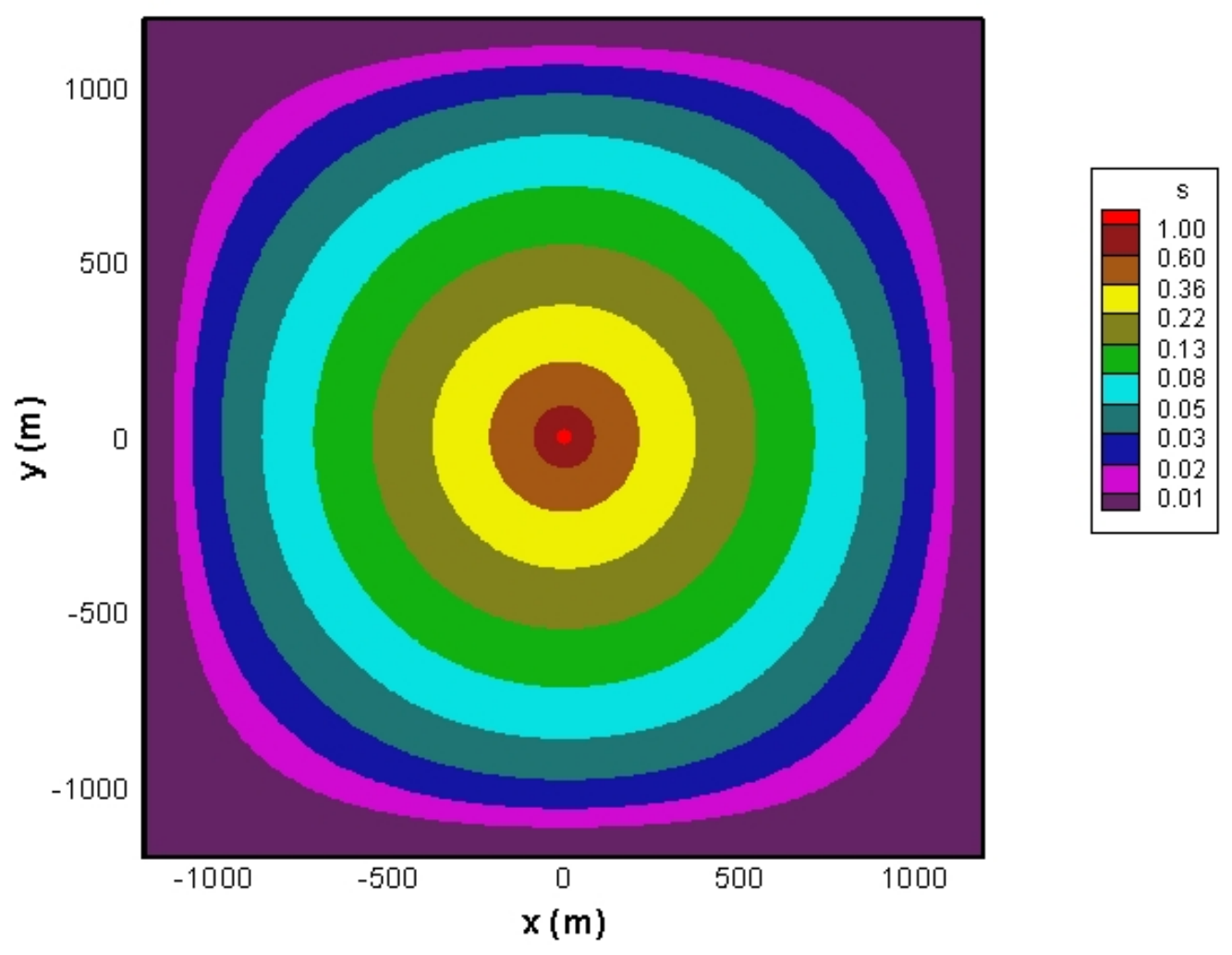

Figure 4.5.5. PORFLOW Drawdown at 1 day for 2D Cartesian Mesh. 
Table 4.5.1. Comparison of Theis, COMSOL and PORFLOW Transient Drawdown (0 to 1 hour)

\begin{tabular}{|c|c|c|c|}
\hline $\begin{array}{l}\text { Time } \\
(\mathrm{sec})\end{array}$ & $\begin{array}{l}\text { Theis } \\
(\mathrm{m})\end{array}$ & $\begin{array}{l}\text { COMSOL } \\
(\mathrm{m})\end{array}$ & $\begin{array}{c}\text { PORFLOW } \\
(\mathrm{m})\end{array}$ \\
\hline $0.00 \mathrm{E}+00$ & $0.0000 \mathrm{E}+00$ & $1.1855 \mathrm{E}-14$ & $0.0000 \mathrm{E}+00$ \\
\hline $1.20 \mathrm{E}+02$ & $6.2764 \mathrm{E}-03$ & $8.1695 \mathrm{E}-03$ & $5.6947 \mathrm{E}-03$ \\
\hline $2.40 \mathrm{E}+02$ & $2.9013 \mathrm{E}-02$ & $2.9221 \mathrm{E}-02$ & $2.7228 \mathrm{E}-02$ \\
\hline $3.60 \mathrm{E}+02$ & $5.3206 \mathrm{E}-02$ & $5.1830 \mathrm{E}-02$ & $5.1300 \mathrm{E}-02$ \\
\hline $4.80 \mathrm{E}+02$ & $7.5200 \mathrm{E}-02$ & 7.3004E-02 & 7.3423E-02 \\
\hline $6.00 \mathrm{E}+02$ & $9.4700 \mathrm{E}-02$ & $9.2141 \mathrm{E}-02$ & $9.3116 \mathrm{E}-02$ \\
\hline $7.20 \mathrm{E}+02$ & $1.1203 \mathrm{E}-01$ & $1.0941 \mathrm{E}-01$ & 1.1064E-01 \\
\hline $8.40 \mathrm{E}+02$ & $1.2757 \mathrm{E}-01$ & $1.2501 \mathrm{E}-01$ & $1.2635 \mathrm{E}-01$ \\
\hline $9.60 \mathrm{E}+02$ & $1.4161 \mathrm{E}-01$ & $1.3886 \mathrm{E}-01$ & $1.4054 \mathrm{E}-01$ \\
\hline $1.08 \mathrm{E}+03$ & $1.5440 \mathrm{E}-01$ & $1.5157 \mathrm{E}-01$ & $1.5346 \mathrm{E}-01$ \\
\hline $1.20 \mathrm{E}+03$ & $1.6614 \mathrm{E}-01$ & $1.6328 \mathrm{E}-01$ & $1.6532 \mathrm{E}-01$ \\
\hline $1.32 \mathrm{E}+03$ & $1.7699 \mathrm{E}-01$ & $1.7445 \mathrm{E}-01$ & $1.7626 \mathrm{E}-01$ \\
\hline $1.44 \mathrm{E}+03$ & $1.8706 \mathrm{E}-01$ & $1.8481 \mathrm{E}-01$ & $1.8641 \mathrm{E}-01$ \\
\hline $1.56 \mathrm{E}+03$ & $1.9645 \mathrm{E}-01$ & $1.9451 \mathrm{E}-01$ & 1.9589E-01 \\
\hline $1.68 \mathrm{E}+03$ & $2.0526 \mathrm{E}-01$ & $2.0357 \mathrm{E}-01$ & $2.0476 \mathrm{E}-01$ \\
\hline $1.80 \mathrm{E}+03$ & $2.1355 \mathrm{E}-01$ & $2.1207 \mathrm{E}-01$ & $2.1311 \mathrm{E}-01$ \\
\hline $1.92 \mathrm{E}+03$ & $2.2137 \mathrm{E}-01$ & $2.2005 \mathrm{E}-01$ & $2.2098 \mathrm{E}-01$ \\
\hline $2.04 \mathrm{E}+03$ & $2.2877 \mathrm{E}-01$ & $2.2759 \mathrm{E}-01$ & 2.2844E-01 \\
\hline $2.16 \mathrm{E}+03$ & $2.3581 \mathrm{E}-01$ & $2.3473 \mathrm{E}-01$ & $2.3552 \mathrm{E}-01$ \\
\hline $2.28 \mathrm{E}+03$ & $2.4250 \mathrm{E}-01$ & $2.4151 \mathrm{E}-01$ & $2.4225 \mathrm{E}-01$ \\
\hline $2.40 \mathrm{E}+03$ & $2.4889 \mathrm{E}-01$ & $2.4798 \mathrm{E}-01$ & $2.4868 \mathrm{E}-01$ \\
\hline $2.52 \mathrm{E}+03$ & $2.5500 \mathrm{E}-01$ & $2.5416 \mathrm{E}-01$ & $2.5482 \mathrm{E}-01$ \\
\hline $2.64 \mathrm{E}+03$ & $2.6085 \mathrm{E}-01$ & $2.6008 \mathrm{E}-01$ & $2.6070 \mathrm{E}-01$ \\
\hline $2.76 \mathrm{E}+03$ & $2.6647 \mathrm{E}-01$ & $2.6575 \mathrm{E}-01$ & $2.6635 \mathrm{E}-01$ \\
\hline $2.88 \mathrm{E}+03$ & $2.7186 \mathrm{E}-01$ & $2.7120 \mathrm{E}-01$ & $2.7177 \mathrm{E}-01$ \\
\hline $3.00 \mathrm{E}+03$ & $2.7706 \mathrm{E}-01$ & $2.7644 \mathrm{E}-01$ & 2.7699E-01 \\
\hline $3.12 \mathrm{E}+03$ & $2.8207 \mathrm{E}-01$ & $2.8149 \mathrm{E}-01$ & $2.8202 \mathrm{E}-01$ \\
\hline $3.24 \mathrm{E}+03$ & $2.8690 \mathrm{E}-01$ & $2.8636 \mathrm{E}-01$ & $2.8688 \mathrm{E}-01$ \\
\hline $3.36 \mathrm{E}+03$ & $2.9157 \mathrm{E}-01$ & $2.9107 \mathrm{E}-01$ & $2.9157 \mathrm{E}-01$ \\
\hline $3.48 \mathrm{E}+03$ & $2.9609 \mathrm{E}-01$ & $2.9562 \mathrm{E}-01$ & $2.9611 \mathrm{E}-01$ \\
\hline $3.60 \mathrm{E}+03$ & $3.0046 \mathrm{E}-01$ & $3.0002 \mathrm{E}-01$ & $3.0050 \mathrm{E}-01$ \\
\hline
\end{tabular}

Table 4.5.2. Comparison of Theis, COMSOL and PORFLOW Transient Drawndown (0 to 1 day)

\begin{tabular}{|c|c|c|c|}
\hline $\begin{array}{c}\text { Time } \\
(\mathrm{sec})\end{array}$ & $\begin{array}{c}\text { Theis } \\
(\mathrm{m})\end{array}$ & $\begin{array}{c}\text { COMSOL } \\
(\mathrm{m})\end{array}$ & $\begin{array}{c}\text { PORFLOW } \\
(\mathrm{m})\end{array}$ \\
\hline $0.00 \mathrm{E}+00$ & $0.0000 \mathrm{E}+00$ & $1.1855 \mathrm{E}-14$ & $0.0000 \mathrm{E}+00$ \\
\hline $3.60 \mathrm{E}+03$ & $3.0046 \mathrm{E}-01$ & $2.8601 \mathrm{E}-01$ & $3.0050 \mathrm{E}-01$ \\
\hline $7.20 \mathrm{E}+03$ & $3.9177 \mathrm{E}-01$ & $3.7997 \mathrm{E}-01$ & $3.9208 \mathrm{E}-01$ \\
\hline $1.08 \mathrm{E}+04$ & $4.4633 \mathrm{E}-01$ & $4.3591 \mathrm{E}-01$ & $4.4673 \mathrm{E}-01$ \\
\hline $1.44 \mathrm{E}+04$ & $4.8536 \mathrm{E}-01$ & $4.7708 \mathrm{E}-01$ & $4.8581 \mathrm{E}-01$ \\
\hline $1.80 \mathrm{E}+04$ & $5.1577 \mathrm{E}-01$ & $5.0790 \mathrm{E}-01$ & $5.1626 \mathrm{E}-01$ \\
\hline $2.16 \mathrm{E}+04$ & $5.4069 \mathrm{E}-01$ & $5.3396 \mathrm{E}-01$ & $5.4119 \mathrm{E}-01$ \\
\hline $2.52 \mathrm{E}+04$ & $5.6180 \mathrm{E}-01$ & $5.5606 \mathrm{E}-01$ & $5.6232 \mathrm{E}-01$ \\
\hline $2.88 \mathrm{E}+04$ & $5.8011 \mathrm{E}-01$ & $5.7564 \mathrm{E}-01$ & $5.8064 \mathrm{E}-01$ \\
\hline
\end{tabular}




\begin{tabular}{|c|c|c|c|}
\hline $\begin{array}{c}\text { Time } \\
(\mathrm{sec})\end{array}$ & $\begin{array}{c}\text { Theis } \\
(\mathrm{m})\end{array}$ & $\begin{array}{c}\text { COMSOL } \\
(\mathrm{m})\end{array}$ & $\begin{array}{c}\text { PORFLOW } \\
(\mathrm{m})\end{array}$ \\
\hline $3.24 \mathrm{E}+04$ & $5.9628 \mathrm{E}-01$ & $5.9285 \mathrm{E}-01$ & $5.9682 \mathrm{E}-01$ \\
\hline $3.60 \mathrm{E}+04$ & $6.1076 \mathrm{E}-01$ & $6.0809 \mathrm{E}-01$ & $6.1130 \mathrm{E}-01$ \\
\hline $3.96 \mathrm{E}+04$ & $6.2386 \mathrm{E}-01$ & $6.2174 \mathrm{E}-01$ & $6.2441 \mathrm{E}-01$ \\
\hline $4.32 \mathrm{E}+04$ & $6.3583 \mathrm{E}-01$ & $6.3405 \mathrm{E}-01$ & $6.3639 \mathrm{E}-01$ \\
\hline $4.68 \mathrm{E}+04$ & $6.4685 \mathrm{E}-01$ & $6.4530 \mathrm{E}-01$ & $6.4741 \mathrm{E}-01$ \\
\hline $5.04 \mathrm{E}+04$ & $6.5705 \mathrm{E}-01$ & $6.5569 \mathrm{E}-01$ & $6.5762 \mathrm{E}-01$ \\
\hline $5.40 \mathrm{E}+04$ & $6.6656 \mathrm{E}-01$ & $6.6535 \mathrm{E}-01$ & $6.6712 \mathrm{E}-01$ \\
\hline $5.76 \mathrm{E}+04$ & $6.7545 \mathrm{E}-01$ & $6.7437 \mathrm{E}-01$ & $6.7602 \mathrm{E}-01$ \\
\hline $6.12 \mathrm{E}+04$ & $6.8381 \mathrm{E}-01$ & $6.8285 \mathrm{E}-01$ & $6.8438 \mathrm{E}-01$ \\
\hline $6.48 \mathrm{E}+04$ & $6.9168 \mathrm{E}-01$ & $6.9083 \mathrm{E}-01$ & $6.9226 \mathrm{E}-01$ \\
\hline $6.84 \mathrm{E}+04$ & $6.9914 \mathrm{E}-01$ & $6.9838 \mathrm{E}-01$ & $6.9971 \mathrm{E}-01$ \\
\hline $7.20 \mathrm{E}+04$ & $7.0621 \mathrm{E}-01$ & $7.0553 \mathrm{E}-01$ & $7.0679 \mathrm{E}-01$ \\
\hline $7.56 \mathrm{E}+04$ & $7.1294 \mathrm{E}-01$ & $7.1232 \mathrm{E}-01$ & $7.1352 \mathrm{E}-01$ \\
\hline $7.92 \mathrm{E}+04$ & $7.1936 \mathrm{E}-01$ & $7.1879 \mathrm{E}-01$ & $7.1994 \mathrm{E}-01$ \\
\hline $8.28 \mathrm{E}+04$ & $7.2549 \mathrm{E}-01$ & $7.2497 \mathrm{E}-01$ & $7.2608 \mathrm{E}-01$ \\
\hline $8.64 \mathrm{E}+04$ & $7.3137 \mathrm{E}-01$ & $7.3088 \mathrm{E}-01$ & $7.3195 \mathrm{E}-01$ \\
\hline
\end{tabular}

Table 4.5.3. Input Commands for Problem 4.5 (1D)

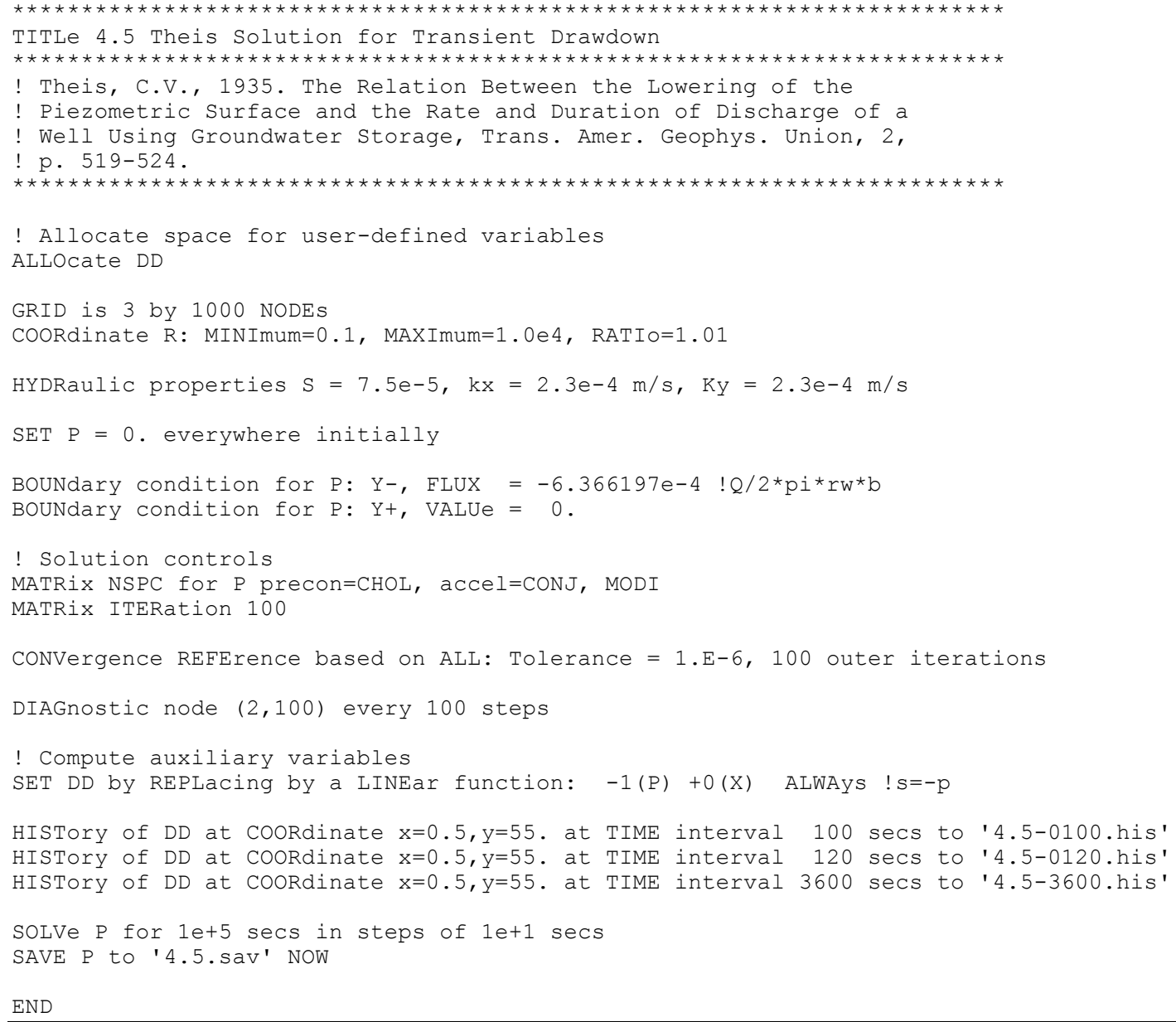


Table 4.5.4. Input Commands for Problem 4.5 (2D)

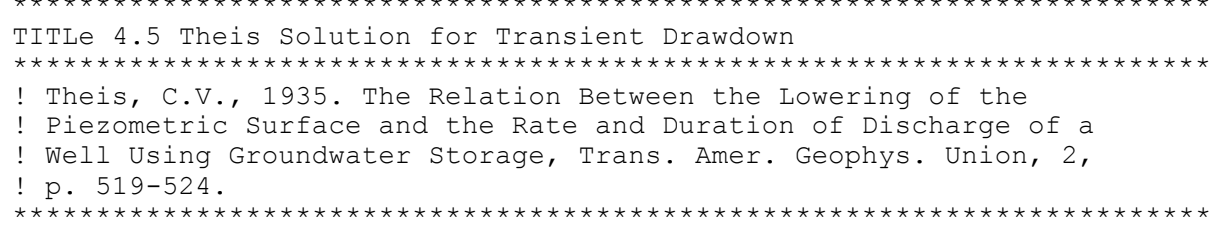

$-1.20000000 \mathrm{E}+003-1.18304190 \mathrm{E}+003-1.16608380 \mathrm{E}+003-1.14929528 \mathrm{E}+003-1.13267464 \mathrm{E}+003$ $-1.11622022 \mathrm{E}+003-1.09993033 \mathrm{E}+003-1.08380335 \mathrm{E}+003-1.06783763 \mathrm{E}+003-1.05203157 \mathrm{E}+003$ $-1.03638358 \mathrm{E}+003-1.02089206 \mathrm{E}+003-1.00555546 \mathrm{E}+003-9.90372222 \mathrm{E}+002-9.75340818 \mathrm{E}+002$ $-9.60459728 \mathrm{E}+002-9.45727449 \mathrm{E}+002-9.31142493 \mathrm{E}+002-9.16703387 \mathrm{E}+002-9.02408671 \mathrm{E}+002$ $-8.88256903 \mathrm{E}+002-8.74246652 \mathrm{E}+002-8.60376504 \mathrm{E}+002-8.46645057 \mathrm{E}+002-8.33050925 \mathrm{E}+002$ $-8.19592734 \mathrm{E}+002-8.06269125 \mathrm{E}+002-7.93078753 \mathrm{E}+002-7.80020283 \mathrm{E}+002-7.67092399 \mathrm{E}+002$ $-7.54293793 \mathrm{E}+002-7.41623174 \mathrm{E}+002-7.29079261 \mathrm{E}+002-7.16660786 \mathrm{E}+002-7.04366497 \mathrm{E}+002$ 


\begin{tabular}{|c|c|c|c|c|}
\hline$-6.92195150 E+002$ & $-6.80145517 E+002$ & $-6.68216380 \mathrm{E}+002$ & $-6.56406535 E+002$ & $-6.44714788 E+002$ \\
\hline 002 & & 002 & $9105341 E+002$ & $-5.87986606 \mathrm{E}+002$ \\
\hline 002 & $7 E+002$ & $89 E+002$ & $-5.44612477 \mathrm{E}+002$ & $-5.34038670 \mathrm{E}+002$ \\
\hline ?ח? & $214 E+002$ & 002 & $-4.92790304 \mathrm{E}+002$ & $-4.82734720 \mathrm{E}+0$ \\
\hline & (1) & 002 & $-4.43507934 \mathrm{E}+002$ & $-4.33945173 E+0$ \\
\hline 02 & 002 & 002 & $-3.96640890 E+002$ & -3.875468001 \\
\hline & 0 & 002 & -3.52070798 & -3.4 \\
\hline & & 002 & -3.0968508 & -3.0146 \\
\hline & - & 002 & -2.6937669 & -2.6155 \\
\hline $0 ?$ & - & 002 & -2.3104381 & \\
\hline & & 002 & -1 & -1 \\
\hline & & 002 & -1.59 & -1.5 \\
\hline 0 & & 002 & -1.269 & -1 \\
\hline & & 02 & -9 & - \\
\hline & & & -6 & -5 \\
\hline & & & -3 & \\
\hline & 001 & -1.5749 & -1.046 & -5 \\
\hline$c$ & 5.2691 & $1.05383819 \mathrm{E}+001$ & $1.58602648 \mathrm{E}+001$ & 2.1 \\
\hline 2 & 3.21 & $53332 \mathrm{E}$ & 001 & 4 \\
\hline 5 & 6. & 001 & 001 & \\
\hline 001 & $76 E+0$ & $47 E+001$ & $1.02382716 \mathrm{E}+002$ & 1.0862 \\
\hline 02 & $28 E+0$ & $20942 \mathrm{E}$ & 1.34 & 1 \\
\hline 02 & 1 & 02 & 1.6 & 1 \\
\hline 002 & $27 E$ & $95659501 \mathrm{E}+002$ & $2595 E+002$ & 2.1 \\
\hline+002 & $39 E+002$ & $.32249489 E+002$ & $83 E+002$ & 2.4 \\
\hline 02 & 2 & 02 & 02 & 2 \\
\hline 02 & $60 \mathrm{E}$ & $4047 E+002$ & $786 \mathrm{E}+002$ & 3.2 \\
\hline 02 & $08 E+00$ & $503888 E+002$ & $425 E+002$ & 3 . \\
\hline 3 & 3. & $7 \mathrm{E}$ & 02 & 4. \\
\hline 02 & $78 \mathrm{E}+$ & $4.45174800 \mathrm{E}+002$ & $3047 E+002$ & $76 \mathrm{E}+0$ \\
\hline+002 & $760 E+002$ & $92576 \mathrm{E}+002$ & $4001 E+002$ & 5.1 \\
\hline 5 & 5. & 02 & 02 & 5. \\
\hline 5 & $62 \mathrm{E}+$ & $3563 E+002$ & $28097 E+002$ & 6.2 \\
\hline 5 & $6705+0$ & $970 \mathrm{E}+002$ & $69 E+002$ & 6.8 \\
\hline 6 & 7 . & 2 & $E+002$ & 7. \\
\hline 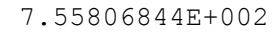 & $412 \mathrm{E}+0$ & $7.81483725 \mathrm{E}+002$ & $7.94515061 \mathrm{E}+002$ & 8.0767 \\
\hline $\mathrm{E}+\mathrm{C}$ & $176 \mathrm{E}+0$ & $8.47956637 \mathrm{E}+002$ & $8.61652702 \mathrm{E}+002$ & $8 \mathrm{E}+0$ \\
\hline $\mathrm{E}+0 \mathrm{C}$ & $4 E+0$ & $35 E+002$ & $8 E+002$ & $7 \mathrm{E}+\mathrm{C}-2 \cdot 2 \cdot(-1)$ \\
\hline $3 F+00 ?$ & $68599 \mathrm{E}+0$ & $9.91247784 \mathrm{E}+002$ & $1.00637676 \mathrm{E}+003$ & $703 E+0$ \\
\hline ut & $50 \mathrm{E}+\mathrm{C}$ & $77 E+003$ & $8 E+C$ & $9 \mathrm{E}+$ \\
\hline$E+C$ & $402 \mathrm{E}+0$ & $36 E+003$ & $16 E+0$ & $8 E+C$ \\
\hline
\end{tabular}

\footnotetext{
HYDRaulic properties $\mathrm{S}=7.5 e-5, \mathrm{kx}=2.3 e-4 \mathrm{~m} / \mathrm{s}, \mathrm{Ky}=2.3 e-4 \mathrm{~m} / \mathrm{s}$
}

SET $\mathrm{P}=0$. everywhere initially

BOUNdary condition for $\mathrm{P}: \mathrm{X}-$, $\mathrm{VALUe}=0$. Boundary condition for $\mathrm{P}: \mathrm{X}+$, VALUe $=0$. Boundary condition for $\mathrm{P}$ : $\mathrm{Y}-, \mathrm{VALUe}=0$. BoUndary condition for $\mathrm{P}$ : $\mathrm{Y}+$, $\mathrm{VALUe}=0$.

! Well

LocAte $(121,121)$ source for pumping well

SOURce for $\mathrm{P}=-0.0004$ in SELEcted zone

! Solution controls

MATRix NSPC for $P$ precon=CHOL, accel=CONJ, MODI

MATRix ITERation 100

CONVergence REFErence based on ALL: Tolerance $=1 . \mathrm{E}-6,100$ outer iterations

DIAGnostic node $(2,18)$ every 100 steps

SAVE P to' '4.5-XY.Sav' at TIME interval of 86400 secs

HISTory of $P$ at CooRdinate $x=55 ., y=55$. at TIME interval 100 secs to '4.5-XY.his'

Solve $\mathrm{P}$ for $1 \mathrm{e}+5$ secs in steps of 86.4 secs

END 


\subsection{Transient, Two-Dimensional Flow to a Well in an Anisotropic Confined Aquifer (Hantush and Thomas, 1966)}

We next consider a confined aquifer identical to Problem 4.5 except that the hydraulic conductivity is anisotropic in the horizontal plane as shown in Figure 4.6.1. The principal axes of the conductivity tensor are assumed to be aligned with the coordinate axes.

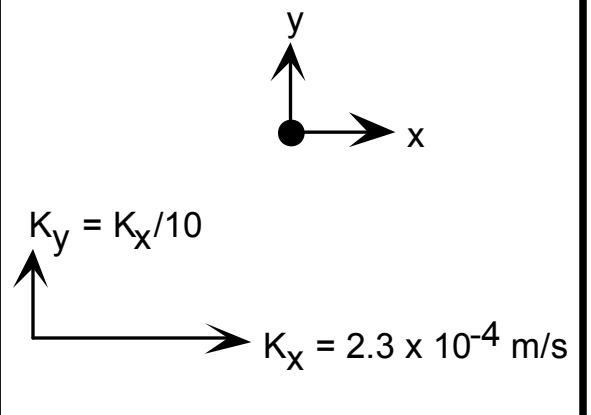

Figure 4.6.1. Anisotropic Confined Aquifer with $K_{x} / K_{y}=10$.

Analytic solution: The governing equation for the flow problem described above can be written as

$$
\frac{\partial}{\partial x}\left(\mathrm{~T}_{\mathrm{x}} \frac{\partial \mathrm{h}}{\partial \mathrm{x}}\right)+\frac{\partial}{\partial \mathrm{y}}\left(\mathrm{T}_{\mathrm{y}} \frac{\partial \mathrm{h}}{\partial \mathrm{y}}\right)=\mathrm{S} \frac{\partial \mathrm{h}}{\partial \mathrm{t}}+\mathrm{Q} \delta(\mathrm{x}) \delta(\mathrm{y}) \quad-\infty<\mathrm{x}<\infty,-\infty<\mathrm{y}<\infty
$$

The initial condition is

$$
\mathrm{h}(\mathrm{x}, \mathrm{y}, 0)=\mathrm{h}_{0}
$$

Because the aquifer hydraulic conductivity is anisotropic, Eq. $4.5 .5 \mathrm{~b}$ becomes

$$
\mathrm{T}_{\mathrm{x}}=\mathrm{K}_{\mathrm{x}} \mathrm{b} \text { and } \mathrm{T}_{\mathrm{y}}=\mathrm{K}_{\mathrm{y}} \mathrm{b}
$$

The solution to the governing equation is given by Hantush and Thomas (1966) as

$$
\mathrm{s}=\mathrm{h}_{0}-\mathrm{h}(\mathrm{r}, \mathrm{t})=\frac{\mathrm{Q}}{4 \pi \sqrt{\mathrm{T}_{\mathrm{x}} \mathrm{T}_{\mathrm{y}}}} \int_{\mathrm{u}^{\prime}}^{\infty} \frac{\mathrm{e}^{-\tau}}{\tau} \mathrm{d} \tau=\frac{\mathrm{Q}}{4 \pi \sqrt{\mathrm{T}_{\mathrm{x}} \mathrm{T}_{\mathrm{y}}}} \mathrm{W}\left(\mathrm{u}^{\prime}\right)
$$

where

$$
u^{\prime}=\frac{\left(x^{2} T_{y}+y^{2} T_{x}\right) S}{4 T_{x} T_{y} t}
$$


and $\mathrm{W}\left(\mathrm{u}^{\prime}\right)$ is the well function. Note that for $\mathrm{T}_{\mathrm{x}}=\mathrm{T}_{\mathrm{y}}$, the above solution reduces to the Theis solution presented in Section 4.5.

Evaluation of the Hantush and Thomas solution for the parameter values specified in Figures 4.5.1 and 4.6.1 are presented in Tables 4.6.1 through 4.6.3 for various spatial locations and times.

PORFLOW simulation and comparison: The same 2D grid considered in Problem 4.5 was used for the PORFLOW simulation in this problem. The PORFLOW mesh and model settings are given in Table 4.6.4. Figure 4.6.2 shows the COMSOL finite-element mesh which consists of 12440 triangular elements and 6393 nodes.

Due to the anisotropy of the hydraulic conductivity, the spatial contours of drawdown will be elliptical at any point in time. Therefore, we will look at the transient drawdown of a virtual piezometer along the x-axis, y-axis and at a 45 degree angle. Figures 4.6.3, 4.6.4 and 4.6.5 illustrates the Hantush and Thomas, COMSOL and PORFLOW transient drawdown at $\mathrm{x}=55 \mathrm{~m}$, $\mathrm{y}=55 \mathrm{~m}$ and $\mathrm{x}=\mathrm{y}=55 \mathrm{~m}$, respectively. The agreement between the Hantush and Thomas solution and the numerical simulators is excellent. Figure 4.6.6 shows the PORFLOW drawdown at 1 day. The drawdown contours are elliptical near the well and become distorted near the boundaries. Tables 4.6.1 through 4.6.3 summarize the data shown in Figures 4.6.3 through 4.6.5, respectively.

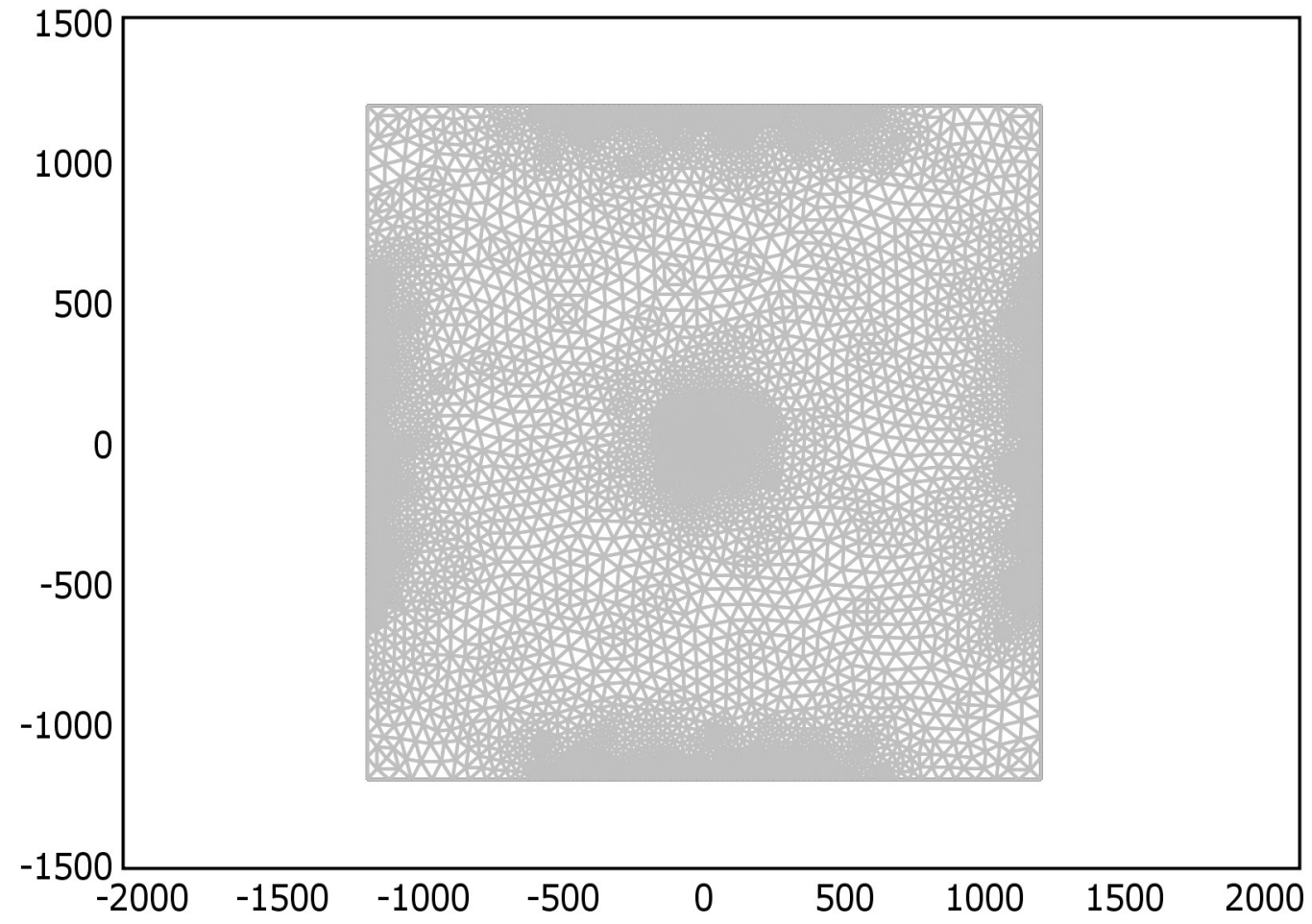

Figure 4.6.2. COMSOL Finite-Element Mesh for Problem 4.6. 


\section{PORFLOW Testing and Verification Document}

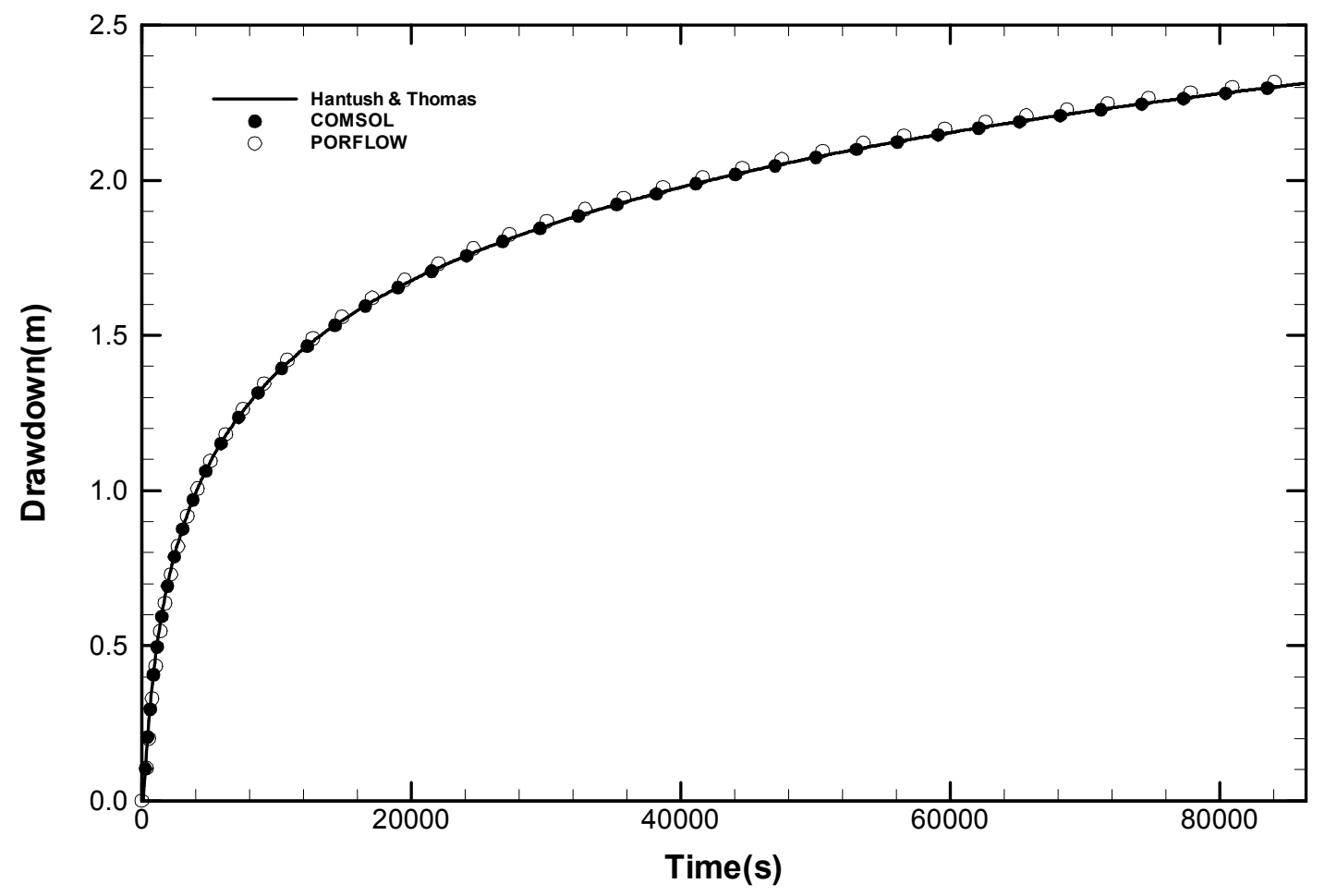

Figure 4.6.3. Hantush and Thomas, COMSOL and PORFLOW Transient Drawdown at $\mathbf{x}=55$ meters.

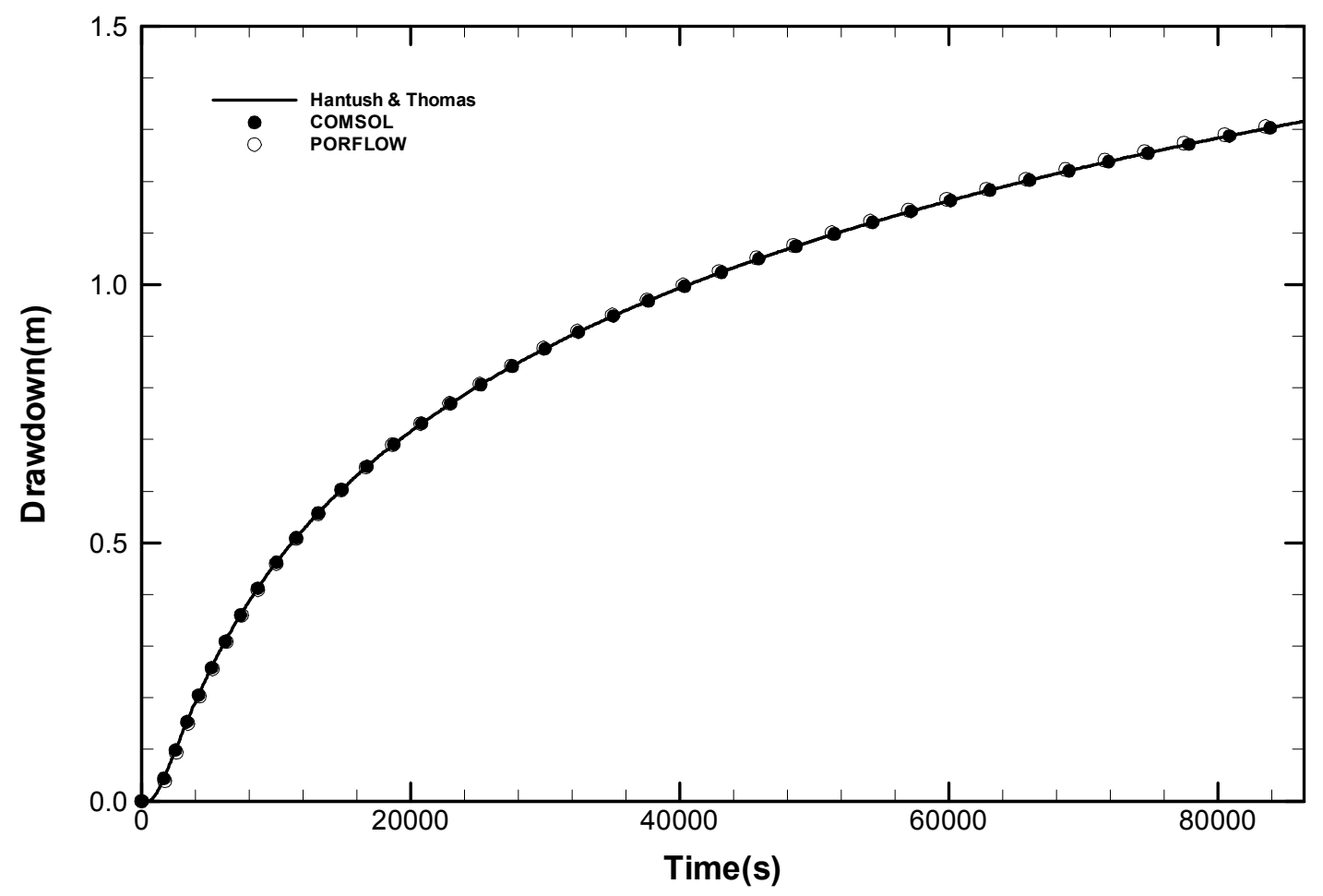

Figure 4.6.4. Hantush and Thomas, COMSOL and PORFLOW Transient Drawdown at $\mathbf{y}=\mathbf{5 5}$ meters. 


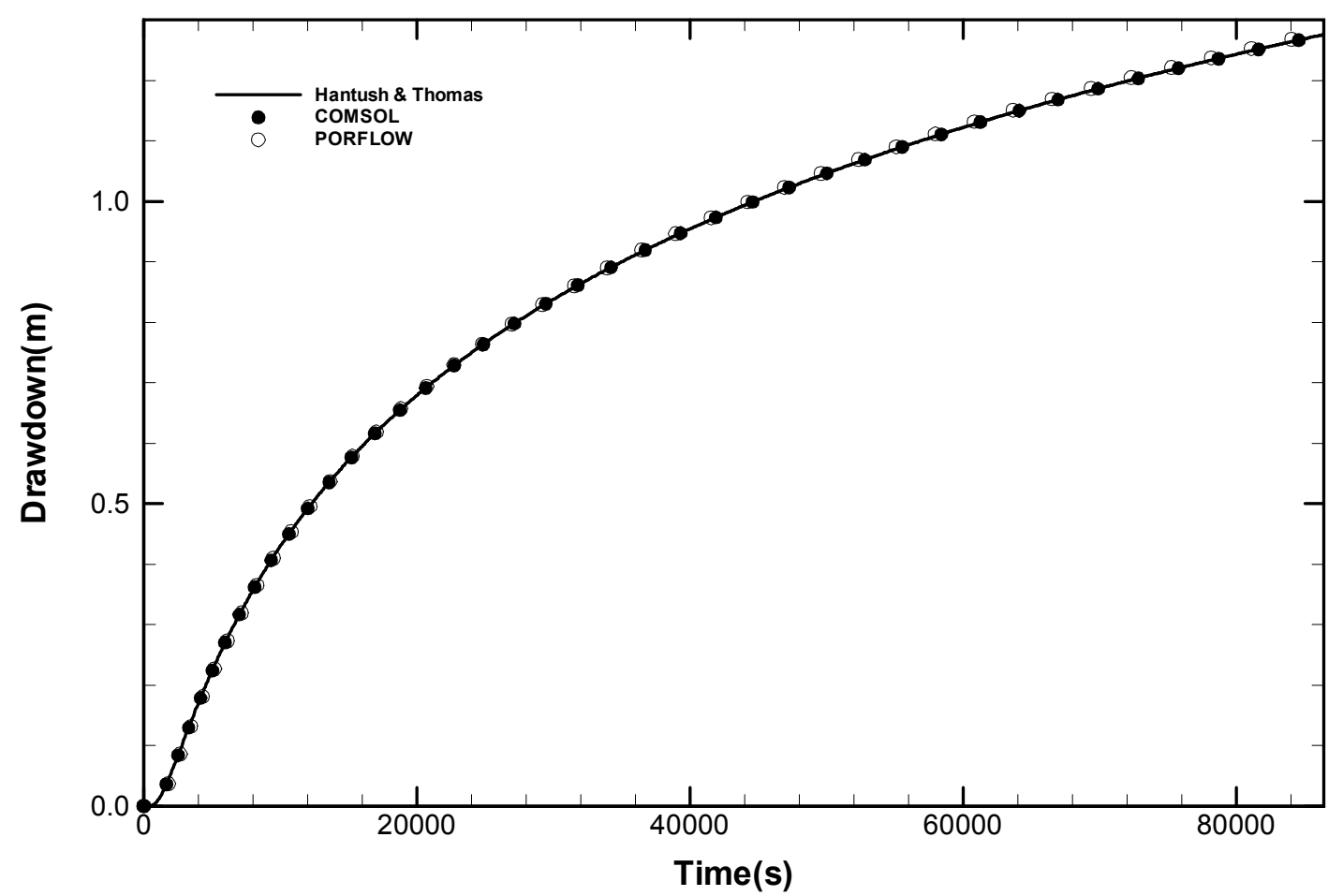

Figure 4.6.5. Hantush and Thomas, COMSOL and PORFLOW Transient Drawdown at $\mathbf{x}=\mathbf{y}=\mathbf{5 5}$ meters. 


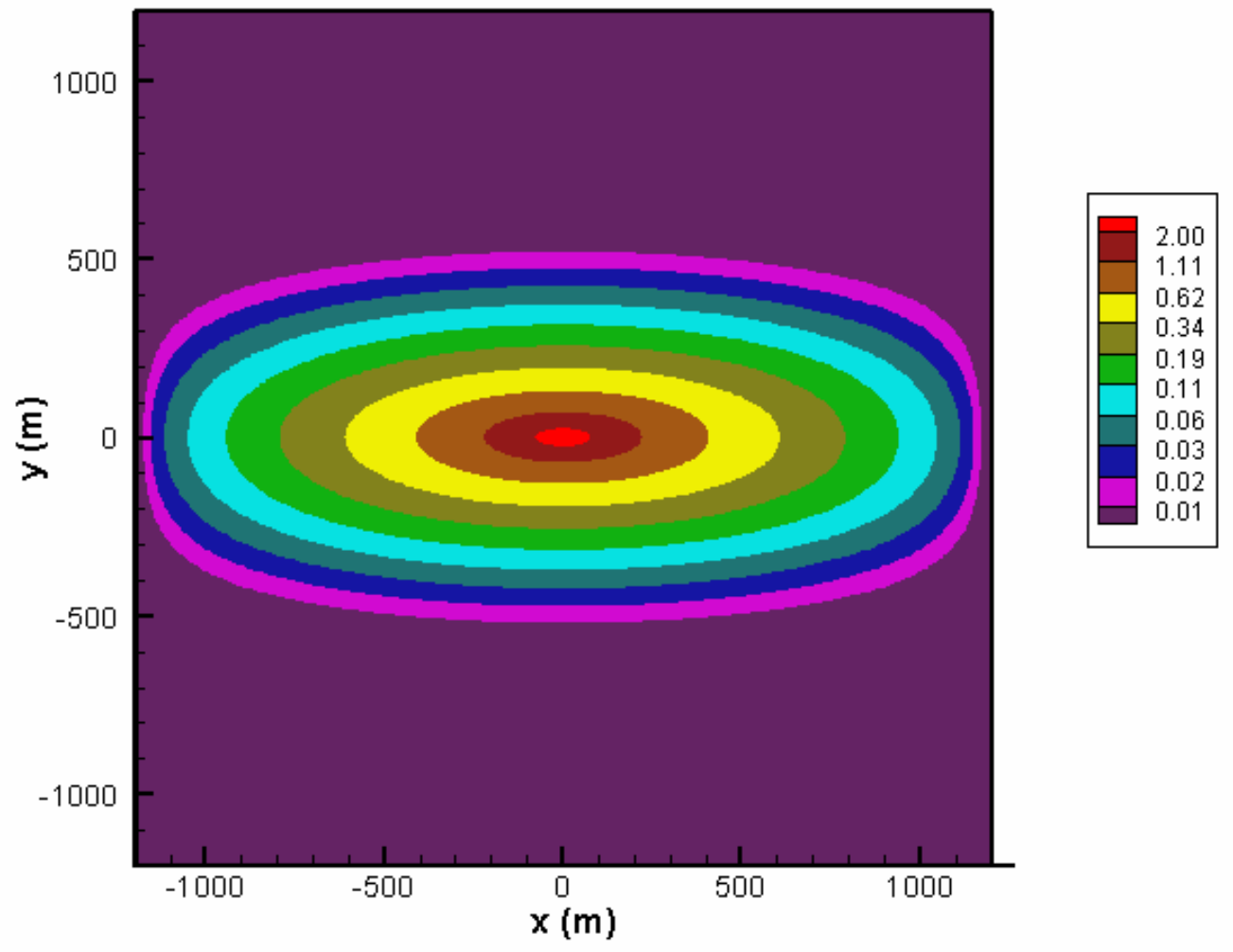

Figure 4.6.6. PORFLOW Drawdown at 1 day.

Table 4.6.1. Comparison of Hantush and Thomas, COMSOL and PORFLOW Numerical Results at $x=55 \mathrm{~m}$.

\begin{tabular}{|c|c|c|c|}
\hline $\begin{array}{c}\text { Time } \\
(\mathrm{sec})\end{array}$ & $\begin{array}{c}\text { Hantush \& } \\
\text { Thomas } \\
(m)\end{array}$ & $\begin{array}{c}\text { COMSOL } \\
(\mathrm{m})\end{array}$ & $\begin{array}{c}\text { PORFLOW } \\
(\mathrm{m})\end{array}$ \\
\hline $1.7280 \mathrm{E}+02$ & $4.8846 \mathrm{E}-02$ & $5.4038 \mathrm{E}-02$ & $2.5966 \mathrm{E}-02$ \\
\hline $3.4560 \mathrm{E}+02$ & $1.5936 \mathrm{E}-01$ & $1.5551 \mathrm{E}-01$ & $1.0499 \mathrm{E}-01$ \\
\hline $5.1840 \mathrm{E}+02$ & $2.5835 \mathrm{E}-01$ & $2.5186 \mathrm{E}-01$ & $2.0017 \mathrm{E}-01$ \\
\hline $7.7760 \mathrm{E}+02$ & $3.7851 \mathrm{E}-01$ & $3.7191 \mathrm{E}-01$ & $3.2893 \mathrm{E}-01$ \\
\hline $1.2096 \mathrm{E}+03$ & $5.2824 \mathrm{E}-01$ & $5.2259 \mathrm{E}-01$ & $4.9368 \mathrm{E}-01$ \\
\hline $1.7280 \mathrm{E}+03$ & $6.5976 \mathrm{E}-01$ & $6.5517 \mathrm{E}-01$ & $6.3681 \mathrm{E}-01$ \\
\hline $2.4192 \mathrm{E}+03$ & $7.9022 \mathrm{E}-01$ & $7.8654 \mathrm{E}-01$ & $7.7654 \mathrm{E}-01$ \\
\hline $3.1968 \mathrm{E}+03$ & $9.0181 \mathrm{E}-01$ & $8.9865 \mathrm{E}-01$ & $8.9443 \mathrm{E}-01$ \\
\hline $4.3200 \mathrm{E}+03$ & $1.0251 \mathrm{E}+00$ & $1.0229 \mathrm{E}+00$ & $1.0232 \mathrm{E}+00$ \\
\hline $5.7888 \mathrm{E}+03$ & $1.1470 \mathrm{E}+00$ & $1.1455 \mathrm{E}+00$ & $1.1493 \mathrm{E}+00$ \\
\hline $7.6896 \mathrm{E}+03$ & $1.2667 \mathrm{E}+00$ & $1.2656 \mathrm{E}+00$ & $1.2723 \mathrm{E}+00$ \\
\hline $1.0022 \mathrm{E}+04$ & $1.3795 \mathrm{E}+00$ & $1.3785 \mathrm{E}+00$ & $1.3874 \mathrm{E}+00$ \\
\hline $1.3306 \mathrm{E}+04$ & $1.5009 \mathrm{E}+00$ & $1.5000 \mathrm{E}+00$ & $1.5108 \mathrm{E}+00$ \\
\hline $1.7539 \mathrm{E}+04$ & $1.6198 \mathrm{E}+00$ & $1.6190 \mathrm{E}+00$ & $1.6313 \mathrm{E}+00$ \\
\hline $2.2896 \mathrm{E}+04$ & $1.7350 \mathrm{E}+00$ & $1.7343 \mathrm{E}+00$ & $1.7477 \mathrm{E}+00$ \\
\hline $3.0067 \mathrm{E}+04$ & $1.8532 \mathrm{E}+00$ & $1.8524 \mathrm{E}+00$ & $1.8667 \mathrm{E}+00$ \\
\hline $3.9053 \mathrm{E}+04$ & $1.9668 \mathrm{E}+00$ & $1.9660 \mathrm{E}+00$ & $1.9810 \mathrm{E}+00$ \\
\hline $5.0026 \mathrm{E}+04$ & $2.0745 \mathrm{E}+00$ & $2.0738 \mathrm{E}+00$ & $2.0893 \mathrm{E}+00$ \\
\hline & & \multicolumn{2}{|l}{} \\
\hline
\end{tabular}




\begin{tabular}{|c|c|c|c|}
\hline $\begin{array}{c}\text { Time } \\
(\mathrm{sec})\end{array}$ & $\begin{array}{c}\text { Hantush \& } \\
\text { Thomas } \\
(\mathrm{m})\end{array}$ & $\begin{array}{c}\text { COMSOL } \\
(\mathrm{m})\end{array}$ & $\begin{array}{c}\text { PORFLOW } \\
(\mathrm{m})\end{array}$ \\
\hline $6.6010 \mathrm{E}+04$ & $2.1954 \mathrm{E}+00$ & $2.1945 \mathrm{E}+00$ & $2.2105 \mathrm{E}+00$ \\
\hline $8.6400 \mathrm{E}+04$ & $2.3128 \mathrm{E}+00$ & $2.3114 \mathrm{E}+00$ & $2.3277 \mathrm{E}+00$ \\
\hline
\end{tabular}

Table 4.6.2. Comparison of Hantush and Thomas, COMSOL and PORFLOW Numerical Results at $\mathbf{y}=55 \mathrm{~m}$.

\begin{tabular}{|c|c|c|c|}
\hline $\begin{array}{c}\text { Time } \\
(\mathrm{sec})\end{array}$ & $\begin{array}{c}\text { Hantush \& } \\
\text { Thomas } \\
(\mathrm{m})\end{array}$ & $\begin{array}{c}\text { COMSOL } \\
(\mathrm{m})\end{array}$ & $\begin{array}{c}\text { PORFLOW } \\
(\mathrm{m})\end{array}$ \\
\hline $1.7280 \mathrm{E}+02$ & $1.8251 \mathrm{E}-08$ & $2.3841 \mathrm{E}-05$ & $8.3667 \mathrm{E}-10$ \\
\hline $3.4560 \mathrm{E}+02$ & $4.3407 \mathrm{E}-05$ & $2.4897 \mathrm{E}-04$ & $1.2249 \mathrm{E}-06$ \\
\hline $5.1840 \mathrm{E}+02$ & $6.6941 \mathrm{E}-04$ & $1.3030 \mathrm{E}-03$ & $6.8076 \mathrm{E}-05$ \\
\hline $7.7760 \mathrm{E}+02$ & $4.6002 \mathrm{E}-03$ & $5.6579 \mathrm{E}-03$ & $1.5563 \mathrm{E}-03$ \\
\hline $1.2096 \mathrm{E}+03$ & $2.0298 \mathrm{E}-02$ & $2.1288 \mathrm{E}-02$ & $1.2984 \mathrm{E}-02$ \\
\hline $1.7280 \mathrm{E}+03$ & $4.8846 \mathrm{E}-02$ & $4.9342 \mathrm{E}-02$ & $3.9031 \mathrm{E}-02$ \\
\hline $2.4192 \mathrm{E}+03$ & $9.3000 \mathrm{E}-02$ & $9.2968 \mathrm{E}-02$ & $8.2556 \mathrm{E}-02$ \\
\hline $3.1968 \mathrm{E}+03$ & $1.4309 \mathrm{E}-01$ & $1.4287 \mathrm{E}-01$ & $1.3346 \mathrm{E}-01$ \\
\hline $4.3200 \mathrm{E}+03$ & $2.1097 \mathrm{E}-01$ & $2.1055 \mathrm{E}-01$ & $2.0278 \mathrm{E}-01$ \\
\hline $5.7888 \mathrm{E}+03$ & $2.8914 \mathrm{E}-01$ & $2.8878 \mathrm{E}-01$ & $2.8279 \mathrm{E}-01$ \\
\hline $7.6896 \mathrm{E}+03$ & $3.7495 \mathrm{E}-01$ & $3.7468 \mathrm{E}-01$ & $3.7047 \mathrm{E}-01$ \\
\hline $1.0022 \mathrm{E}+04$ & $4.6247 \mathrm{E}-01$ & $4.6227 \mathrm{E}-01$ & $4.5963 \mathrm{E}-01$ \\
\hline $1.3306 \mathrm{E}+04$ & $5.6258 \mathrm{E}-01$ & $5.6245 \mathrm{E}-01$ & $5.6132 \mathrm{E}-01$ \\
\hline $1.7539 \mathrm{E}+04$ & $6.6541 \mathrm{E}-01$ & $6.6532 \mathrm{E}-01$ & $6.6548 \mathrm{E}-01$ \\
\hline $2.2896 \mathrm{E}+04$ & $7.6852 \mathrm{E}-01$ & $7.6845 \mathrm{E}-01$ & $7.6965 \mathrm{E}-01$ \\
\hline $3.0067 \mathrm{E}+04$ & $8.7704 \mathrm{E}-01$ & $8.7699 \mathrm{E}-01$ & $8.7907 \mathrm{E}-01$ \\
\hline $3.9053 \mathrm{E}+04$ & $9.8351 \mathrm{E}-01$ & $9.8347 \mathrm{E}-01$ & $9.8624 \mathrm{E}-01$ \\
\hline $5.0026 \mathrm{E}+04$ & $1.0860 \mathrm{E}+00$ & $1.0859 \mathrm{E}+00$ & $1.0892 \mathrm{E}+00$ \\
\hline $6.6010 \mathrm{E}+04$ & $1.2022 \mathrm{E}+00$ & $1.2021 \mathrm{E}+00$ & $1.2059 \mathrm{E}+00$ \\
\hline $8.6400 \mathrm{E}+04$ & $1.3162 \mathrm{E}+00$ & $1.3156 \mathrm{E}+00$ & $1.3198 \mathrm{E}+00$ \\
\hline
\end{tabular}

Table 4.6.3. Comparison of Hantush and Thomas, COMSOL and PORFLOW Numerical Results at $x=y=55 \mathrm{~m}$.

\begin{tabular}{|c|c|c|c|}
\hline $\begin{array}{c}\text { Time } \\
(\mathrm{sec})\end{array}$ & $\begin{array}{c}\text { Hantush \& } \\
\text { Thomas } \\
(\mathrm{m})\end{array}$ & $\begin{array}{c}\text { COMSOL } \\
(\mathrm{m})\end{array}$ & $\begin{array}{c}\text { PORFLOW } \\
(\mathrm{m})\end{array}$ \\
\hline $1.7280 \mathrm{E}+02$ & $4.0034 \mathrm{E}-09$ & $1.4498 \mathrm{E}-05$ & $2.0199 \mathrm{E}-08$ \\
\hline $3.4560 \mathrm{E}+02$ & $1.9514 \mathrm{E}-05$ & $1.5601 \mathrm{E}-04$ & $4.9338 \mathrm{E}-06$ \\
\hline $5.1840 \mathrm{E}+02$ & $3.8299 \mathrm{E}-04$ & $8.6546 \mathrm{E}-04$ & $1.1264 \mathrm{E}-04$ \\
\hline $7.7760 \mathrm{E}+02$ & $3.0962 \mathrm{E}-03$ & $4.0324 \mathrm{E}-03$ & $1.4715 \mathrm{E}-03$ \\
\hline $1.2096 \mathrm{E}+03$ & $1.5375 \mathrm{E}-02$ & $1.6422 \mathrm{E}-02$ & $1.0586 \mathrm{E}-02$ \\
\hline $1.7280 \mathrm{E}+03$ & $3.9502 \mathrm{E}-02$ & $4.0179 \mathrm{E}-02$ & $3.2179 \mathrm{E}-02$ \\
\hline $2.4192 \mathrm{E}+03$ & $7.8673 \mathrm{E}-02$ & $7.8863 \mathrm{E}-02$ & $7.0160 \mathrm{E}-02$ \\
\hline $3.1968 \mathrm{E}+03$ & $1.2448 \mathrm{E}-01$ & $1.2451 \mathrm{E}-01$ & $1.1626 \mathrm{E}-01$ \\
\hline $4.3200 \mathrm{E}+03$ & $1.8803 \mathrm{E}-01$ & $1.8779 \mathrm{E}-01$ & $1.8071 \mathrm{E}-01$ \\
\hline $5.7888 \mathrm{E}+03$ & $2.6246 \mathrm{E}-01$ & $2.6221 \mathrm{E}-01$ & $2.5659 \mathrm{E}-01$ \\
\hline $7.6896 \mathrm{E}+03$ & $3.4516 \mathrm{E}-01$ & $3.4498 \mathrm{E}-01$ & $3.4091 \mathrm{E}-01$ \\
\hline $1.0022 \mathrm{E}+04$ & $4.3024 \mathrm{E}-01$ & $4.3013 \mathrm{E}-01$ & $4.2750 \mathrm{E}-01$ \\
\hline $1.3306 \mathrm{E}+04$ & $5.2824 \mathrm{E}-01$ & $5.2818 \mathrm{E}-01$ & $5.2697 \mathrm{E}-01$ \\
\hline
\end{tabular}




\begin{tabular}{|c|c|c|c|}
\hline $\begin{array}{c}\text { Time } \\
(\mathrm{sec})\end{array}$ & $\begin{array}{c}\text { Hantush \& } \\
\text { Thomas } \\
(\mathrm{m})\end{array}$ & $\begin{array}{c}\text { COMSOL } \\
(\mathrm{m})\end{array}$ & $\begin{array}{c}\text { PORFLOW } \\
(\mathrm{m})\end{array}$ \\
\hline $1.7539 \mathrm{E}+04$ & $6.2942 \mathrm{E}-01$ & $6.2940 \mathrm{E}-01$ & $6.2943 \mathrm{E}-01$ \\
\hline $2.2810 \mathrm{E}+04$ & $7.2979 \mathrm{E}-01$ & $7.2980 \mathrm{E}-01$ & $7.3082 \mathrm{E}-01$ \\
\hline $3.0067 \mathrm{E}+04$ & $8.3876 \mathrm{E}-01$ & $8.3878 \mathrm{E}-01$ & $8.4069 \mathrm{E}-01$ \\
\hline $3.9053 \mathrm{E}+04$ & $9.4447 \mathrm{E}-01$ & $9.4450 \mathrm{E}-01$ & $9.4709 \mathrm{E}-01$ \\
\hline $5.0026 \mathrm{E}+04$ & $1.0464 \mathrm{E}+00$ & $1.0464 \mathrm{E}+00$ & $1.0495 \mathrm{E}+00$ \\
\hline $6.6010 \mathrm{E}+04$ & $1.1621 \mathrm{E}+00$ & $1.1620 \mathrm{E}+00$ & $1.1657 \mathrm{E}+00$ \\
\hline $8.6400 \mathrm{E}+04$ & $1.2757 \mathrm{E}+00$ & $1.2752 \mathrm{E}+00$ & $1.2791 \mathrm{E}+00$ \\
\hline
\end{tabular}

Table 4.6.4. Input Commands for Problem 4.6.

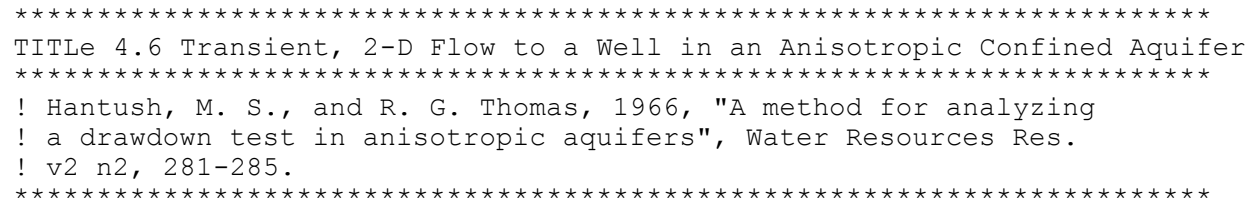

! Allocate space for user-defined variables

ALLOcate DD

GRID is 241 by 241 NODES

COORdinate NODEs X:

$-1.20000000 \mathrm{E}+003-1.18304190 \mathrm{E}+003-1.16608380 \mathrm{E}+003-1.14929528 \mathrm{E}+003-1.13267464 \mathrm{E}+003$ $-1.11622022 \mathrm{E}+003-1.09993033 \mathrm{E}+003-1.08380335 \mathrm{E}+003-1.06783763 \mathrm{E}+003-1.05203157 \mathrm{E}+003$ $-1.03638358 \mathrm{E}+003-1.02089206 \mathrm{E}+003-1.00555546 \mathrm{E}+003-9.90372222 \mathrm{E}+002-9.75340818 \mathrm{E}+002$ $-9.60459728 \mathrm{E}+002-9.45727449 \mathrm{E}+002-9.31142493 \mathrm{E}+002-9.16703387 \mathrm{E}+002-9.02408671 \mathrm{E}+002$ $-8.88256903 \mathrm{E}+002-8.74246652 \mathrm{E}+002-8.60376504 \mathrm{E}+002-8.46645057 \mathrm{E}+002-8.33050925 \mathrm{E}+002$ $-8.19592734 \mathrm{E}+002-8.06269125 \mathrm{E}+002-7.93078753 \mathrm{E}+002-7.80020283 \mathrm{E}+002-7.67092399 \mathrm{E}+002$ $-7.54293793 \mathrm{E}+002-7.41623174 \mathrm{E}+002-7.29079261 \mathrm{E}+002-7.16660786 \mathrm{E}+002-7.04366497 \mathrm{E}+002$ $-6.92195150 \mathrm{E}+002-6.80145517 \mathrm{E}+002-6.68216380 \mathrm{E}+002-6.56406535 \mathrm{E}+002-6.44714788 \mathrm{E}+002$ $-6.33139958 \mathrm{E}+002-6.21680877 \mathrm{E}+002-6.10336387 \mathrm{E}+002-5.99105341 \mathrm{E}+002-5.87986606 \mathrm{E}+002$ $-5.76979059 \mathrm{E}+002-5.66081587 \mathrm{E}+002-5.55293089 \mathrm{E}+002-5.44612477 \mathrm{E}+002-5.34038670 \mathrm{E}+002$ $-5.23570602 \mathrm{E}+002-5.13207214 \mathrm{E}+002-5.02947460 \mathrm{E}+002-4.92790304 \mathrm{E}+002-4.82734720 \mathrm{E}+002$ $-4.72779691 \mathrm{E}+002-4.62924212 \mathrm{E}+002-4.53167289 \mathrm{E}+002-4.43507934 \mathrm{E}+002-4.33945173 \mathrm{E}+002$ $-4.24478040 \mathrm{E}+002-4.15105578 \mathrm{E}+002-4.05826840 \mathrm{E}+002-3.96640890 \mathrm{E}+002-3.87546800 \mathrm{E}+002$ $-3.78543650 \mathrm{E}+002-3.69630532 \mathrm{E}+002-3.60806545 \mathrm{E}+002-3.52070798 \mathrm{E}+002-3.43422409 \mathrm{E}+002$ $-3.34860503 \mathrm{E}+002-3.26384216 \mathrm{E}+002-3.17992692 \mathrm{E}+002-3.09685084 \mathrm{E}+002-3.01460551 \mathrm{E}+002$ $-2.93318264 \mathrm{E}+002-2.85257400 \mathrm{E}+002-2.77277144 \mathrm{E}+002-2.69376691 \mathrm{E}+002-2.61555243 \mathrm{E}+002$ $-2.53812009 \mathrm{E}+002-2.46146207 \mathrm{E}+002-2.38557063 \mathrm{E}+002-2.31043811 \mathrm{E}+002-2.23605691 \mathrm{E}+002$ $-2.16241953 \mathrm{E}+002-2.08951852 \mathrm{E}+002-2.01734652 \mathrm{E}+002-1.94589624 \mathrm{E}+002-1.87516046 \mathrm{E}+002$ $-1.80513204 \mathrm{E}+002-1.73580390 \mathrm{E}+002-1.66716904 \mathrm{E}+002$ $-1.46535519 \mathrm{E}+002-1.39942482 \mathrm{E}+002-1.33415375 \mathrm{E}+002$ $-1.14223078 \mathrm{E}+002-1.07953166 \mathrm{E}+002-1.01745952 \mathrm{E}+002$ $-8.34942688 \mathrm{E}+001-7.75316445 \mathrm{E}+001-7.16286464 \mathrm{E}+001$ $-5.42714768 \mathrm{E}+001-4.86010805 \mathrm{E}+001-4.29873881 \mathrm{E}+001$

$-2.64808925 \mathrm{E}+001-2.10884020 \mathrm{E}+001$ $0.00000000 \mathrm{E}+000 \quad 5.26919097 \mathrm{E}+000$ $2.66642193 \mathrm{E}+001 \quad 3.21473605 \mathrm{E}+001$ $5.46337503 \mathrm{E}+001 \quad 6.03965868 \mathrm{E}+001$ $8.40300084 \mathrm{E}+001 \quad 9.00868076 \mathrm{E}+001$ $1.14925771 \mathrm{E}+002 \quad 1.21291528 \mathrm{E}+002$ $1.47397528 \mathrm{E}+002 \quad 1.54088003 \mathrm{E}+002$ $1.81525672 \mathrm{E}+002 \quad 1.88557427 \mathrm{E}+002$ $2.17394693 \mathrm{E}+002 \quad 2.24785139 \mathrm{E}+002$ $2.55093395 \mathrm{E}+002 \quad 2.62860828 \mathrm{E}+002$ $2.94715110 \mathrm{E}+002 \quad 3.02878760 \mathrm{E}+002$ $3.36357930 \mathrm{E}+002 \quad 3.44938008 \mathrm{E}+002$ $3.80124953 \mathrm{E}+002 \quad 3.89142701 \mathrm{E}+002$ $4.26124533 \mathrm{E}+002 \quad 4.35602278 \mathrm{E}+002$ $4.74470555 \mathrm{E}+002 \quad 4.84431760 \mathrm{E}+002$ $5.25282710 \mathrm{E}+002 \quad 5.35752036 \mathrm{E}+002$ $5.78686795 \mathrm{E}+002 \quad 5.89690162 \mathrm{E}+002$ $6.34815025 \mathrm{E}+002 \quad 6.46379674 \mathrm{E}+002$ $6.93806359 \mathrm{E}+002 \quad 7.05960922 \mathrm{E}+002$

$-1.57498363 \mathrm{E}+001$ $1.05383819 \mathrm{E}+001$ $3.76853332 \mathrm{E}+001$ $6.62170517 \mathrm{E}+001$ $9.62041747 \mathrm{E}+001$ $1.27720942 \mathrm{E}+002$ $1.60845382 \mathrm{E}+002$ $1.95659501 \mathrm{E}+002$ $2.32249489 \mathrm{E}+002$ $2.70705935 \mathrm{E}+002$ $3.11124047 \mathrm{E}+002$ $3.53603888 \mathrm{E}+002$ $3.98250627 \mathrm{E}+002$ $4.45174800 \mathrm{E}+002$ $4.94492576 \mathrm{E}+002$ $5.46326055 \mathrm{E}+002$ $6.00803563 \mathrm{E}+002$ $6.58059970 \mathrm{E}+002$ $7.18237030 \mathrm{E}+002$
$-1.59922054 \mathrm{E}+002-1.53195152 \mathrm{E}+002$ $-1.26953540 \mathrm{E}+002-1.20556323 \mathrm{E}+002$ $-9.56008113 \mathrm{E}+001-8.95171216 \mathrm{E}+001$ $-6.57846783 \mathrm{E}+001-5.99991500 \mathrm{E}+001$ $-3.74298326 \mathrm{E}+001-3.19278526 \mathrm{E}+001$ $-1.04646563 \mathrm{E}+001-5.23232817 \mathrm{E}+000$ $1.58602648 \mathrm{E}+001 \quad 2.12353665 \mathrm{E}+001$ $4.32786856 \mathrm{E}+001 \quad 4.89279715 \mathrm{E}+001$ $7.20957213 \mathrm{E}+001 \quad 7.80331776 \mathrm{E}+001$ $1.02382716 \mathrm{E}+002 \quad 1.08623042 \mathrm{E}+002$ $1.34214651 \mathrm{E}+002 \quad 1.40773296 \mathrm{E}+002$ $1.67670335 \mathrm{E}+002 \quad 1.74563537 \mathrm{E}+002$ $2.02832595 \mathrm{E}+002 \quad 2.10077420 \mathrm{E}+002$ $2.39788483 \mathrm{E}+002 \quad 2.47402867 \mathrm{E}+002$ $2.78629494 \mathrm{E}+002 \quad 2.86632288 \mathrm{E}+002$ $3.19451786 \mathrm{E}+002 \quad 3.27862803 \mathrm{E}+002$ $3.62356425 \mathrm{E}+002 \quad 3.71196489 \mathrm{E}+002$ $4.07449633 \mathrm{E}+002 \quad 4.16740628 \mathrm{E}+002$ $4.54843047 \mathrm{E}+002 \quad 4.64607976 \mathrm{E}+002$ $5.04654001 \mathrm{E}+002 \quad 5.14917040 \mathrm{E}+002$ $5.57005815 \mathrm{E}+002 \quad 5.67792372 \mathrm{E}+002$ $6.12028097 \mathrm{E}+002 \quad 6.23364877 \mathrm{E}+002$ $6.69857069 \mathrm{E}+002 \quad 6.81772139 \mathrm{E}+002$ 7.30635900E+002 7.43158758E+002 
$7.55806844 \mathrm{E}+002$

$8.20969977 \mathrm{E}+002$

$8.89457085 \mathrm{E}+002$

$9.61437723 \mathrm{E}+002$

$1.03709010 \mathrm{E}+003$

$1.11660150 \mathrm{E}+003$

$1.20000000 \mathrm{E}+003$

COORdinate NODES Y:

$-1.20000000 \mathrm{E}+003-1.18304190 \mathrm{E}+003-1.16608380 \mathrm{E}+003-1.14929528 \mathrm{E}+003-1.13267464 \mathrm{E}+003$

$-1.11622022 \mathrm{E}+003-1.09993033 \mathrm{E}+003-1.08380335 \mathrm{E}+003-1.06783763 \mathrm{E}+003-1.05203157 \mathrm{E}+003$

$-1.03638358 \mathrm{E}+003-1.02089206 \mathrm{E}+003-1.00555546 \mathrm{E}+003-9.90372222 \mathrm{E}+002-9.75340818 \mathrm{E}+002$

$-9.60459728 \mathrm{E}+002-9.45727449 \mathrm{E}+002-9.31142493 \mathrm{E}+002-9.16703387 \mathrm{E}+002-9.02408671 \mathrm{E}+002$

$-8.88256903 \mathrm{E}+002-8.74246652 \mathrm{E}+002-8.60376504 \mathrm{E}+002-8.46645057 \mathrm{E}+002-8.33050925 \mathrm{E}+002$

$-8.19592734 \mathrm{E}+002-8.06269125 \mathrm{E}+002-7.93078753 \mathrm{E}+002-7.80020283 \mathrm{E}+002-7.67092399 \mathrm{E}+002$

$-7.54293793 \mathrm{E}+002-7.41623174 \mathrm{E}+002-7.29079261 \mathrm{E}+002-7.16660786 \mathrm{E}+002-7.04366497 \mathrm{E}+002$

$-6.92195150 \mathrm{E}+002-6.80145517 \mathrm{E}+002-6.68216380 \mathrm{E}+002-6.56406535 \mathrm{E}+002-7.0436649$

$-6.33139958 \mathrm{E}+002-6.21680877 \mathrm{E}+002-6.10336387 \mathrm{E}+002-5.99105341 \mathrm{E}+002-5.87986606 \mathrm{E}+002$

$-5.76979059 \mathrm{E}+002-5.66081587 \mathrm{E}+002-5.55293089 \mathrm{E}+002-5.44612477 \mathrm{E}+002-5.34038670 \mathrm{E}+002$

$-5.23570602 \mathrm{E}+002-5.13207214 \mathrm{E}+002-5.02947460 \mathrm{E}+002-4.92790304 \mathrm{E}+002-4.82734720 \mathrm{E}+002$

$-4.72779691 \mathrm{E}+002-4.62924212 \mathrm{E}+002-4.53167289 \mathrm{E}+002-4.43507934 \mathrm{E}+002-4.33945173 \mathrm{E}+002$

$-4.24478040 \mathrm{E}+002-4.15105578 \mathrm{E}+002-4.05826840 \mathrm{E}+002-3.96640890 \mathrm{E}+002-3.87546800 \mathrm{E}+002$

$-3.78543650 \mathrm{E}+002-3.69630532 \mathrm{E}+002-3.60806545 \mathrm{E}+002-3.52070798 \mathrm{E}+002-3.43422409 \mathrm{E}+002$

$-3.34860503 \mathrm{E}+002-3.26384216 \mathrm{E}+002-3.17992692 \mathrm{E}+002-3.09685084 \mathrm{E}+002-3.01460551 \mathrm{E}+002$

$-2.93318264 \mathrm{E}+002-2.85257400 \mathrm{E}+002-2.77277144 \mathrm{E}+002-2.69376691 \mathrm{E}+002-2.61555243 \mathrm{E}+002$

$-2.53812009 \mathrm{E}+002-2.46146207 \mathrm{E}+002-2.38557063 \mathrm{E}+002-2.31043811 \mathrm{E}+002-2.23605691 \mathrm{E}+002$

$-2.16241953 \mathrm{E}+002-2.08951852 \mathrm{E}+002-2.01734652 \mathrm{E}+002-1.94589624 \mathrm{E}+002-1.87516046 \mathrm{E}+002$

$-1.80513204 \mathrm{E}+002-1.73580390 \mathrm{E}+002-1.66716904 \mathrm{E}+002-1.59922054 \mathrm{E}+002-1.53195152 \mathrm{E}+002$

$-1.46535519 \mathrm{E}+002-1.39942482 \mathrm{E}+002-1.33415375 \mathrm{E}+002-1.26953540 \mathrm{E}+002-1.20556323 \mathrm{E}+002$

$-1.14223078 \mathrm{E}+002-1.07953166 \mathrm{E}+002-1.01745952 \mathrm{E}+002-9.56008113 \mathrm{E}+001-8.9517121$

$-8.34942688 \mathrm{E}+001-7.75316445 \mathrm{E}+001-7.16286464 \mathrm{E}+001-6.57846783 \mathrm{E}+001-5.99991500 \mathrm{E}+001$

$-5.42714768 \mathrm{E}+001-4.86010805 \mathrm{E}+001-4.29873881 \mathrm{E}+001-3.74298326 \mathrm{E}+001-3.19278526 \mathrm{E}+001$

$-2.64808925 \mathrm{E}+001-2.10884020 \mathrm{E}+001-12$

$0.00000000 \mathrm{E}+000 \quad 5.26919097 \mathrm{E}+000 \quad 1.05383819 \mathrm{E}+001$

$\begin{array}{llll}2.66642193 \mathrm{E}+001 & 3.21473605 \mathrm{E}+001 & 3.76853332 \mathrm{E}+001\end{array}$

$\begin{array}{lll}5.46337503 \mathrm{E}+001 & 6.03965868 \mathrm{E}+001 & 6.62170517 \mathrm{E}+001\end{array}$

$8.40300084 \mathrm{E}+001 \quad 9.00868076 \mathrm{E}+001 \quad 9.62041747 \mathrm{E}+001$

$\begin{array}{lll}1.14925771 \mathrm{E}+002 & 1.21291528 \mathrm{E}+002 & 1.27720942 \mathrm{E}+002\end{array}$

$\begin{array}{lll}1.47397528 \mathrm{E}+002 & 1.54088003 \mathrm{E}+002 & 1.60845382 \mathrm{E}+002\end{array}$

$\begin{array}{lll}1.81525672 \mathrm{E}+002 & 1.88557427 \mathrm{E}+002 & 1.95659501 \mathrm{E}+002\end{array}$

$\begin{array}{lll}2.17394693 \mathrm{E}+002 & 2.24785139 \mathrm{E}+002 & 2.32249489 \mathrm{E}+002\end{array}$

$\begin{array}{lll}2.55093395 \mathrm{E}+002 & 2.62860828 \mathrm{E}+002 & 2.70705935 \mathrm{E}+002\end{array}$

$2.94715110 \mathrm{E}+002$

$3.36357930 \mathrm{E}+002$

$3.80124953 \mathrm{E}+002$

$4.26124533 \mathrm{E}+002$

$4.74470555 \mathrm{E}+002$

$5.25282710 \mathrm{E}+002$

$5.78686795 \mathrm{E}+002$

$6.34815025 \mathrm{E}+002$

$6.93806359 \mathrm{E}+002$

$7.55806844 \mathrm{E}+002$

$8.20969977 \mathrm{E}+002$

$8.89457085 \mathrm{E}+002$

$9.61437723 \mathrm{E}+002$

$1.03709010 \mathrm{E}+003$

3. $02878760 \mathrm{E}+002$

$3.44938008 \mathrm{E}+002$

$3.89142701 \mathrm{E}+002$

$4.35602278 \mathrm{E}+002$

$4.84431760 \mathrm{E}+002$

$5.35752036 \mathrm{E}+002$

$5.89690162 \mathrm{E}+002$

$6.46379674 \mathrm{E}+002$

$7.05960922 \mathrm{E}+002$

$7.68581412 \mathrm{E}+002$

$8.34396176 \mathrm{E}+002$

$9.03568154 \mathrm{E}+002$

$9.76268599 \mathrm{E}+002$

$1.05267750 \mathrm{E}+003$

$3.11124047 \mathrm{E}+002$

$3.53603888 \mathrm{E}+002$

3. $98250627 \mathrm{E}+002$

4. $45174800 \mathrm{E}+002$

$4.94492576 \mathrm{E}+002$

$5.46326055 \mathrm{E}+002$

$6.00803563 \mathrm{E}+002$

$6.58059970 \mathrm{E}+002$

$7.18237030 \mathrm{E}+002$

$7.81483725 \mathrm{E}+002$

$8.47956637 \mathrm{E}+002$

$9.17820335 \mathrm{E}+002$

$9.91247784 \mathrm{E}+002$

$1.06842077 \mathrm{E}+003$

$1.13298402 \mathrm{E}+003$

1. $14953036 \mathrm{E}+003$

$-1.04646563 \mathrm{E}+001-5.23232817 \mathrm{E}+000$

$1.58602648 \mathrm{E}+001 \quad 2.12353665 \mathrm{E}+001$

$4.32786856 \mathrm{E}+001 \quad 4.89279715 \mathrm{E}+001$

$\begin{array}{ll}7.20957213 \mathrm{E}+001 & 7.80331776 \mathrm{E}+001\end{array}$

$1.02382716 \mathrm{E}+002 \quad 1.08623042 \mathrm{E}+002$

$1.34214651 \mathrm{E}+002 \quad 1.40773296 \mathrm{E}+002$

$1.67670335 \mathrm{E}+002 \quad 1.74563537 \mathrm{E}+002$

$2.02832595 \mathrm{E}+002 \quad 2.10077420 \mathrm{E}+002$

$2.39788483 \mathrm{E}+002 \quad 2.47402867 \mathrm{E}+002$

$2.78629494 \mathrm{E}+002 \quad 2.86632288 \mathrm{E}+002$

$3.19451786 \mathrm{E}+002 \quad 3.27862803 \mathrm{E}+002$

$3.62356425 \mathrm{E}+002 \quad 3.71196489 \mathrm{E}+002$

$4.07449633 \mathrm{E}+002 \quad 4.16740628 \mathrm{E}+002$

$4.54843047 \mathrm{E}+002 \quad 4.64607976 \mathrm{E}+002$

$5.04654001 \mathrm{E}+002 \quad 5.14917040 \mathrm{E}+002$

$5.57005815 \mathrm{E}+002 \quad 5.67792372 \mathrm{E}+002$

$6.12028097 \mathrm{E}+002 \quad 6.23364877 \mathrm{E}+002$

$6.69857069 \mathrm{E}+002 \quad 6.81772139 \mathrm{E}+002$

$7.30635900 \mathrm{E}+002 \quad 7.43158758 \mathrm{E}+002$

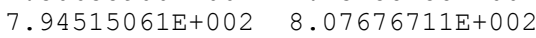

$8.61652702 \mathrm{E}+002 \quad 8.75485728 \mathrm{E}+002$

$9.32215038 \mathrm{E}+002 \quad 9.46753687 \mathrm{E}+002$

$1.00637676 \mathrm{E}+003 \quad 1.02165703 \mathrm{E}+003$

$1.08432148 \mathrm{E}+003 \quad 1.10038119 \mathrm{E}+003$

$1.16624216 \mathrm{E}+003$

$1.10038119 \mathrm{E}+003$
$1.18312108 \mathrm{E}+003$

$1.20000000 \mathrm{E}+003$

HYDRaulic properties $S=7.5 e-5, \mathrm{kx}=2.3 e-4 \mathrm{~m} / \mathrm{s}, \mathrm{Ky}=2.3 e-5 \mathrm{~m} / \mathrm{s}$

SET $\mathrm{P}=0$. everywhere initially

Boundary condition for $\mathrm{P}: \mathrm{X}-$, VALUe $=0$.

Boundary condition for $\mathrm{P}: \mathrm{X}+$, VALUe $=0$.

BOUNdary condition for $\mathrm{P}: \mathrm{Y}-$, VALUe $=0$.

Boundary condition for $\mathrm{P}$ : $\mathrm{Y}+$, $\mathrm{VALUe}=0$.

!Well

LocAte $(121,121)$ source for pumping well

SOURce for $\mathrm{P}=-0.0004$ in SELEcted zone

! Solution controls

MATRix NSPC for $P$ precon=CHOL, accel=CONJ, MODI 
MATRix ITERation 100

CONVergence REFErence based on ALL: Tolerance $=1 . \mathrm{E}-6,100$ outer iterations

DIAGnostic node $(121,121)$ every 10 steps

! Compute auxiliary variables

SET DD by REPLacing by a LINEar function: - $1(\mathrm{P})$ +0 (X) ALWAys ! $\mathrm{s}=-\mathrm{p}$

LOCATE STATion at coordinates $(x=55 ., y=0$.

HISTory of DD at TIME interval 86.4 secs to '4.6-X55-Y00.his' for SELEcted zone

LOCATE STATion at coordinates ( $\mathrm{x}=0 ., \mathrm{y}=55$. )

HISTory of DD at TIME interval 86.4 secs to '4.6-X00-Y55.his' for SELEcted zone

LOCATE STATion at coordinates $(x=55 ., y=55$.

HISTOry of DD at TIME interval 86.4 secs to '4.6-X55-Y55.his' for SELEcted zone

SOLVe $P$ for 86400 secs in steps of 86.4 secs

SAVE P H DD to '4.6.sav' NOW

END

\subsection{Transient, One-Dimensional Flow to a Well in a Leaky Confined Aquifer (Hantush and Jacob, 1955)}

We next consider a confined aquifer identical to Problem 4.5 except that the aquifer is recharged from an overlying constant head aquifer through an aquitard separating them, as shown in Figure 4.7.1. The aquitard is assumed to have uniform thickness and vertical conductivity. Flow through the aquitard is assumed to be vertical and proportional to the head difference between the adjoining aquifers (storage capacity assume to be zero). Parameter values are taken from a GeoTrans (1993) problem.

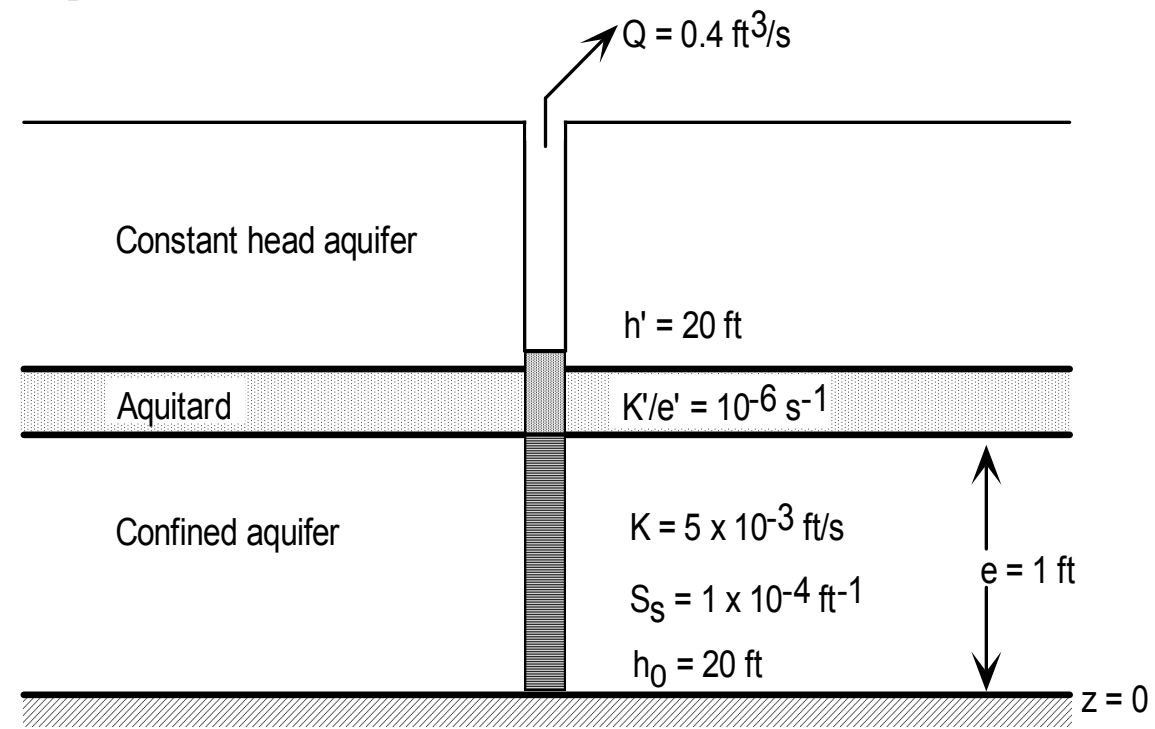

Figure 4.7.1. Schematic Diagram of a Leaky Confined Aquifer with Constant Discharge from a Single, Fully-penetrating Well.

Analytical solution: The governing equation for the flow problem described above is 


$$
\frac{1}{\mathrm{r}} \frac{\partial}{\partial \mathrm{r}}\left(\mathrm{r} \frac{\partial \mathrm{h}}{\partial \mathrm{r}}\right)=\frac{\mathrm{S}}{\mathrm{T}} \frac{\partial \mathrm{h}}{\partial \mathrm{t}}+\mathrm{F}_{\mathrm{t}}
$$

The initial condition is

$$
\mathrm{h}(\mathrm{r}, 0)=\mathrm{h}_{0}
$$

The boundary conditions assume no drawdown in hydraulic head at the infinite boundary:

$$
\mathrm{h}(\infty, \mathrm{t})=\mathrm{h}_{0}
$$

and a constant pumping rate $\mathrm{Q}$ at the well:

$$
\lim _{r \rightarrow 0}\left(r \frac{\partial h}{\partial r}\right)=\frac{Q}{2 \pi T} \quad \text { for } t>0
$$

where $F_{t}$ represents leakance from the overlying aquifer. The leakage flux is given by

$$
F_{t}=-\frac{K^{\prime}}{e^{\prime}}\left(h^{\prime}-h\right)
$$

where $\mathrm{K}^{\prime} / \mathrm{e}^{\prime}$ is the aquitard leakance coefficient. The solution to Eq. 4.7.1 is given by Hantush and Jacob (1955) as

$$
\mathrm{s}=\mathrm{h}_{0}-\mathrm{h}(\mathrm{r}, \mathrm{t})=\frac{\mathrm{Q}}{4 \pi \mathrm{T}} \int_{\mathrm{u}}^{\infty} \frac{\mathrm{e}^{-\tau-\mathrm{r}^{2} / 4 \mathrm{~B}^{2} \tau}}{\tau} \mathrm{d} \tau=\frac{\mathrm{Q}}{4 \pi \mathrm{T}} \mathrm{W}^{\prime}\left(\mathrm{u}, \frac{\mathrm{r}}{\mathrm{B}}\right)
$$

where the Hantush leakage factor $\mathrm{B}=\sqrt{\mathrm{T \textrm {e } ^ { \prime }} / \mathrm{K}^{\prime}}$.

Note that for $\mathrm{B}=\infty$ (no leakance) the above solution reduces to the Theis solution presented in Section 4.5 .

The Hantush and Jacob drawdown shown in Equation 4.7.6 is computed using the HantushJacob numerical code in Section A.

PORFLOW simulation and comparison: Figure 4.7.2 shows the radial mesh used in the PORFLOW simulation. The mesh extends to a radial distance of 10000 feet using 1000 nodes with mesh refinement near the pumping well at $r=0.1$ feet. The PORFLOW model details are given in Table 4.7.2. A similar COMSOL model was generated with 916 linear radial elements.

Figure 4.7.2 and Table 4.7.1 show a comparison of the Hantush \& Jacob solution, COMSOL and PORFLOW transient drawdown at a radial distance of 60 feet. The agreement among the three models is excellent. 


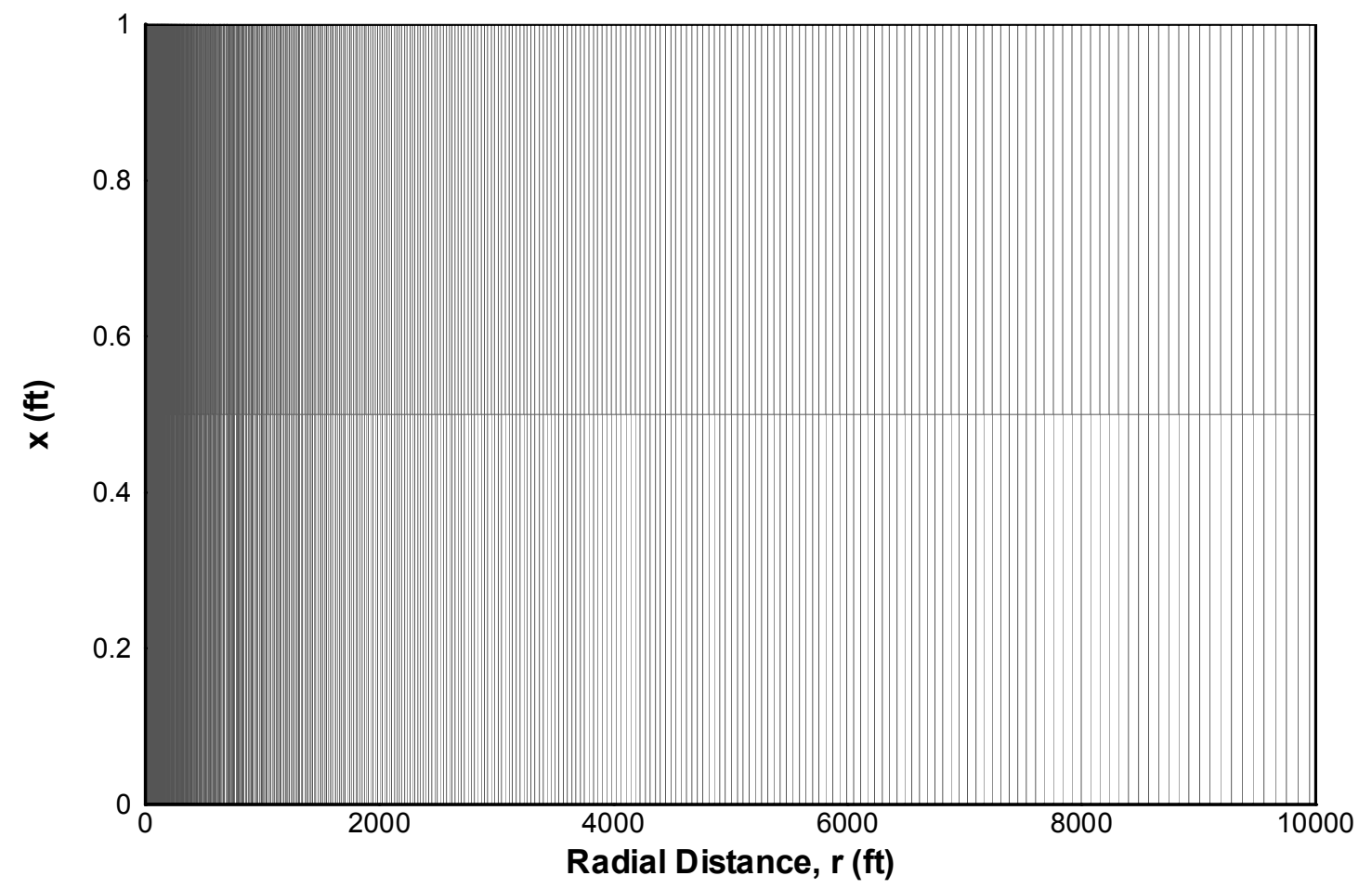

Figure 4.7.2. 1D PORFLOW Radial Grid for Problem 4.7.

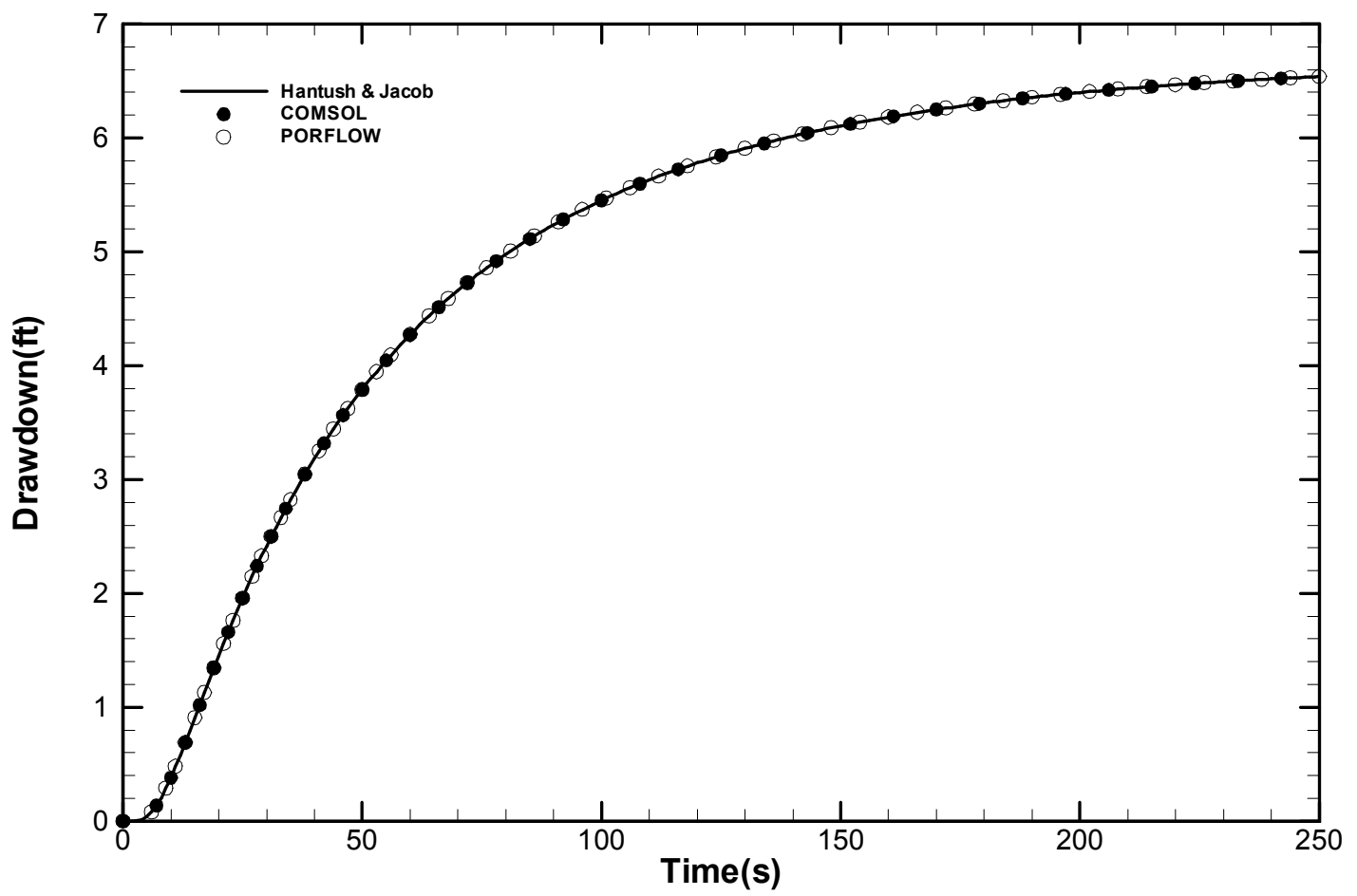

Figure 4.7.3. Hantush and Jacob, COMSOL and PORFLOW Transient Drawdown at $r$ $=60$ feet. 
Table 4.7.1. Comparison of Hantush and Jacob, COMSOL and PORFLOW Transient Drawdown at $r=60$ feet

\begin{tabular}{|c|c|c|c|}
\hline $\begin{array}{c}\text { Time } \\
(\text { sec})\end{array}$ & $\begin{array}{c}\text { Hantush \& Jacob } \\
(\mathrm{ft})\end{array}$ & $\begin{array}{c}\text { COMSOL } \\
(\mathrm{ft})\end{array}$ & $\begin{array}{c}\text { PORFLOW } \\
(\mathrm{ft})\end{array}$ \\
\hline $1.00 \mathrm{E}+00$ & $5.0675 \mathrm{E}-09$ & $1.4877 \mathrm{E}-08$ & $1.7054 \mathrm{E}-08$ \\
\hline $2.00 \mathrm{E}+00$ & $7.7806 \mathrm{E}-05$ & $8.0141 \mathrm{E}-05$ & $9.0138 \mathrm{E}-05$ \\
\hline $3.00 \mathrm{E}+00$ & $2.2324 \mathrm{E}-03$ & $2.2312 \mathrm{E}-03$ & $2.3117 \mathrm{E}-03$ \\
\hline $4.00 \mathrm{E}+00$ & $1.2755 \mathrm{E}-02$ & $1.2719 \mathrm{E}-02$ & $1.2884 \mathrm{E}-02$ \\
\hline $5.00 \mathrm{E}+00$ & $3.7615 \mathrm{E}-02$ & $3.7498 \mathrm{E}-02$ & $3.7708 \mathrm{E}-02$ \\
\hline $6.00 \mathrm{E}+00$ & $7.9104 \mathrm{E}-02$ & $7.9019 \mathrm{E}-02$ & $7.9081 \mathrm{E}-02$ \\
\hline $8.00 \mathrm{E}+00$ & $2.0790 \mathrm{E}-01$ & $2.0767 \mathrm{E}-01$ & $2.0753 \mathrm{E}-01$ \\
\hline $1.00 \mathrm{E}+01$ & $3.8210 \mathrm{E}-01$ & $3.8144 \mathrm{E}-01$ & $3.8139 \mathrm{E}-01$ \\
\hline $1.50 \mathrm{E}+01$ & $9.0814 \mathrm{E}-01$ & $9.0757 \mathrm{E}-01$ & $9.0688 \mathrm{E}-01$ \\
\hline $1.60 \mathrm{E}+01$ & $1.0182 \mathrm{E}+00$ & $1.0178 \mathrm{E}+00$ & $1.0169 \mathrm{E}+00$ \\
\hline $2.00 \mathrm{E}+01$ & $1.4521 \mathrm{E}+00$ & $1.4519 \mathrm{E}+00$ & $1.4506 \mathrm{E}+00$ \\
\hline $2.60 \mathrm{E}+01$ & $2.0547 \mathrm{E}+00$ & $2.0548 \mathrm{E}+00$ & $2.0532 \mathrm{E}+00$ \\
\hline $3.50 \mathrm{E}+01$ & $2.8231 \mathrm{E}+00$ & $2.8233 \mathrm{E}+00$ & $2.8216 \mathrm{E}+00$ \\
\hline $4.30 \mathrm{E}+01$ & $3.3813 \mathrm{E}+00$ & $3.3815 \mathrm{E}+00$ & $3.3799 \mathrm{E}+00$ \\
\hline $5.50 \mathrm{E}+01$ & $4.0453 \mathrm{E}+00$ & $4.0455 \mathrm{E}+00$ & $4.0441 \mathrm{E}+00$ \\
\hline $6.70 \mathrm{E}+01$ & $4.5536 \mathrm{E}+00$ & $4.5538 \mathrm{E}+00$ & $4.5525 \mathrm{E}+00$ \\
\hline $8.00 \mathrm{E}+01$ & $4.9778 \mathrm{E}+00$ & $4.9779 \mathrm{E}+00$ & $4.9768 \mathrm{E}+00$ \\
\hline $1.01 \mathrm{E}+02$ & $5.4715 \mathrm{E}+00$ & $5.4716 \mathrm{E}+00$ & $5.4706 \mathrm{E}+00$ \\
\hline $1.20 \mathrm{E}+02$ & $5.7812 \mathrm{E}+00$ & $5.7813 \mathrm{E}+00$ & $5.7805 \mathrm{E}+00$ \\
\hline $1.49 \mathrm{E}+02$ & $6.0975 \mathrm{E}+00$ & $6.0976 \mathrm{E}+00$ & $6.0968 \mathrm{E}+00$ \\
\hline $1.70 \mathrm{E}+02$ & $6.2502 \mathrm{E}+00$ & $6.2502 \mathrm{E}+00$ & $6.2495 \mathrm{E}+00$ \\
\hline $2.00 \mathrm{E}+02$ & $6.3988 \mathrm{E}+00$ & $6.3989 \mathrm{E}+00$ & $6.3982 \mathrm{E}+00$ \\
\hline $2.17 \mathrm{E}+02$ & $6.4582 \mathrm{E}+00$ & $6.4582 \mathrm{E}+00$ & $6.4576 \mathrm{E}+00$ \\
\hline $2.50 \mathrm{E}+02$ & $6.5396 \mathrm{E}+00$ & $6.5396 \mathrm{E}+00$ & $6.5390 \mathrm{E}+00$ \\
\hline
\end{tabular}

Table 4.7.2. Input Commands for Problem 4.7.

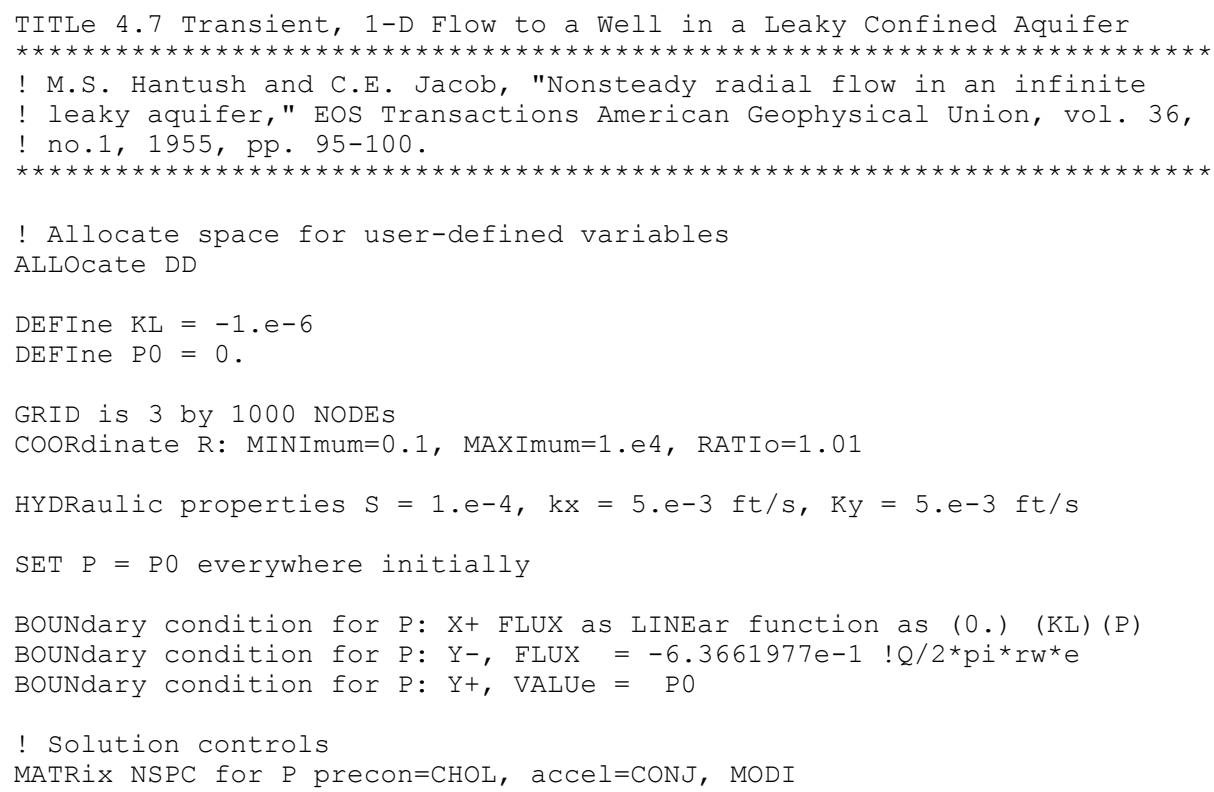


MATRix ITERation 100

CONVergence REFErence based on ALL: Tolerance $=1 . E-6,30$ outer iterations

DIAGnostic node $(2,18)$ every 100 steps

! Compute auxiliary variables

SET DD by REPLacing by a LINEar function: -1(P) +0(X) ALWAys ! $s=-p$

HISTory of DD at COORdinate $\mathrm{x}=0.5, \mathrm{y}=60$. at TIME interval 1 secs to '4.7.his'

SOLVe $P$ for 250. secs in steps of $1 e-2$ secs

END

\subsection{Free-Surface Boussinesq Flow with Recharge}

This test case concerns a semi-infinite, unconfined aquifer. Initially the phreatic surface is at 10 meters everywhere. At time zero, the water level at the left boundary is suddenly raised to 11 meters. The schematic is shown in Figure 4.8.1. The horizontal extent of the computational domain is set at 200 meters and the vertical extent at 11 meters. The objective is to determine the phreatic surface at specified times. This problem is often referred to as the Boussinesq problem. It is described in detail by Polubarinova-Kochina (1962). This problem and description was taken from Problem V10 in the ACRI PORFLOW validation report version 2.50.

No Flow

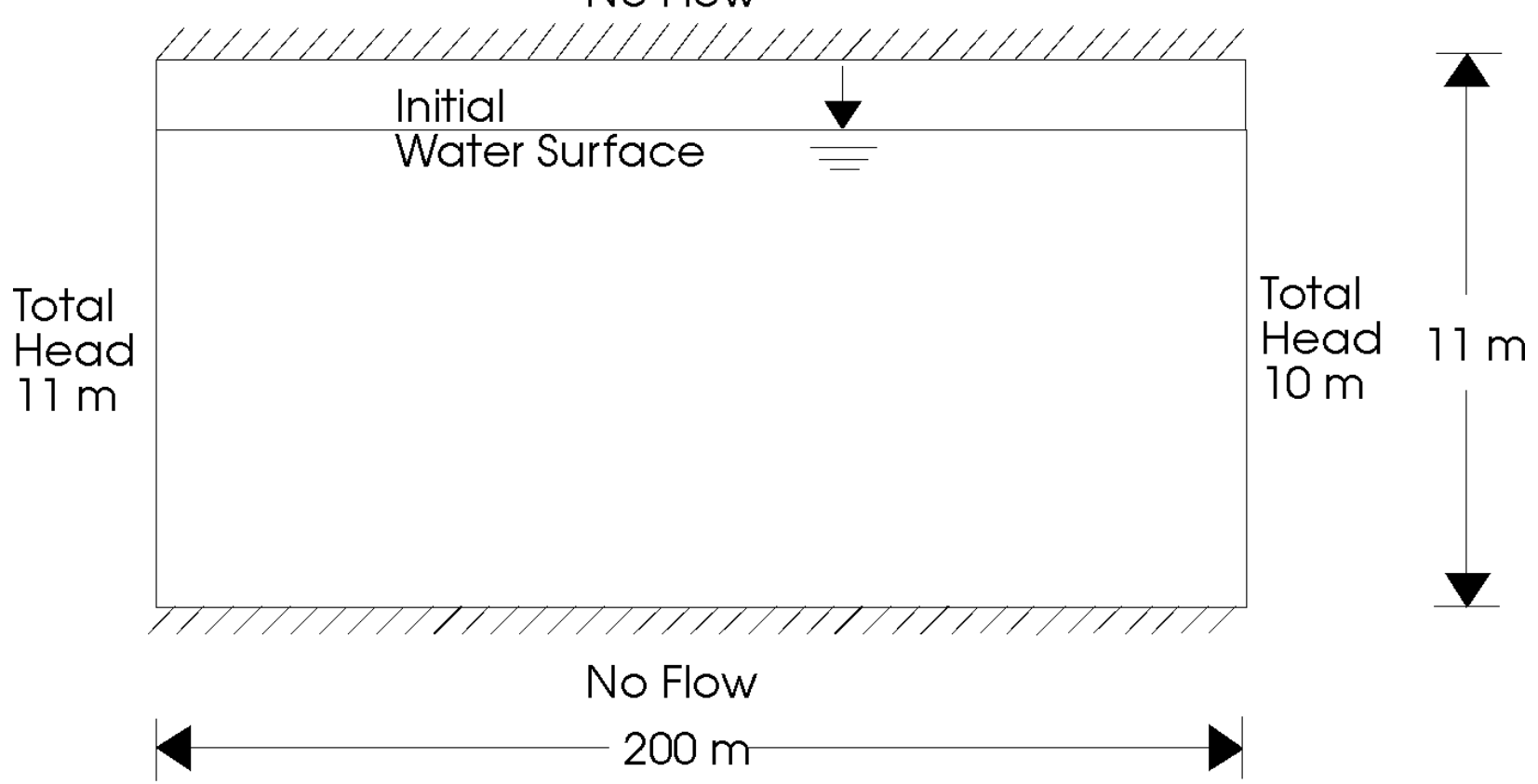

Figure 4.8.1. Schematic Illustration of Problem 4.8.

PORFLOW simulation and comparison: The problem is simulated with a grid of 44 nodes in the horizontal and 23 in the vertical direction. The PORFLOW mesh is shown in Figure 4.8.2. The grid spacing in the horizontal direction increases in a geometric ratio of 1.1. The minimum grid spacing is $0.19 \mathrm{~m}$ and the maximum is $17.68 \mathrm{~m}$. The grid spacing in the vertical varies from $0.1 \mathrm{~m}$ at the top to $2 \mathrm{~m}$ at the bottom. The grid spacing near the top is smaller to allow better resolution near the phreatic surface. The total simulation time is 324 days. In the first 9 days of the simulation, the time step is increased from an initial value of 0.01 days to 1 day in a geometric 
ratio of 1.01; thereafter the time step is kept constant at 1 day. The convergence criterion is specified at $10^{-6}$ and the maximum number of iterations is set to 200 to assure that the transient solution is accurate. The PORFLOW input commands for this problem are shown in Table 4.8.1.

Figure 4.8.3 shows the COMSOL finite-element mesh which consists of 12905 triangular elements and 6858 nodes.

The time history of the phreatic surface due to recharge is shown in Figure 4.8 .4 for the PORFLOW and COMSOL numerical simulations for times of 9, 36, 81, 144,225 and 324 days. Qualitatively the two sets of results are in good agreement. The Polubarinova-Kochina analytical solution was not available for comparison to the numerical results.

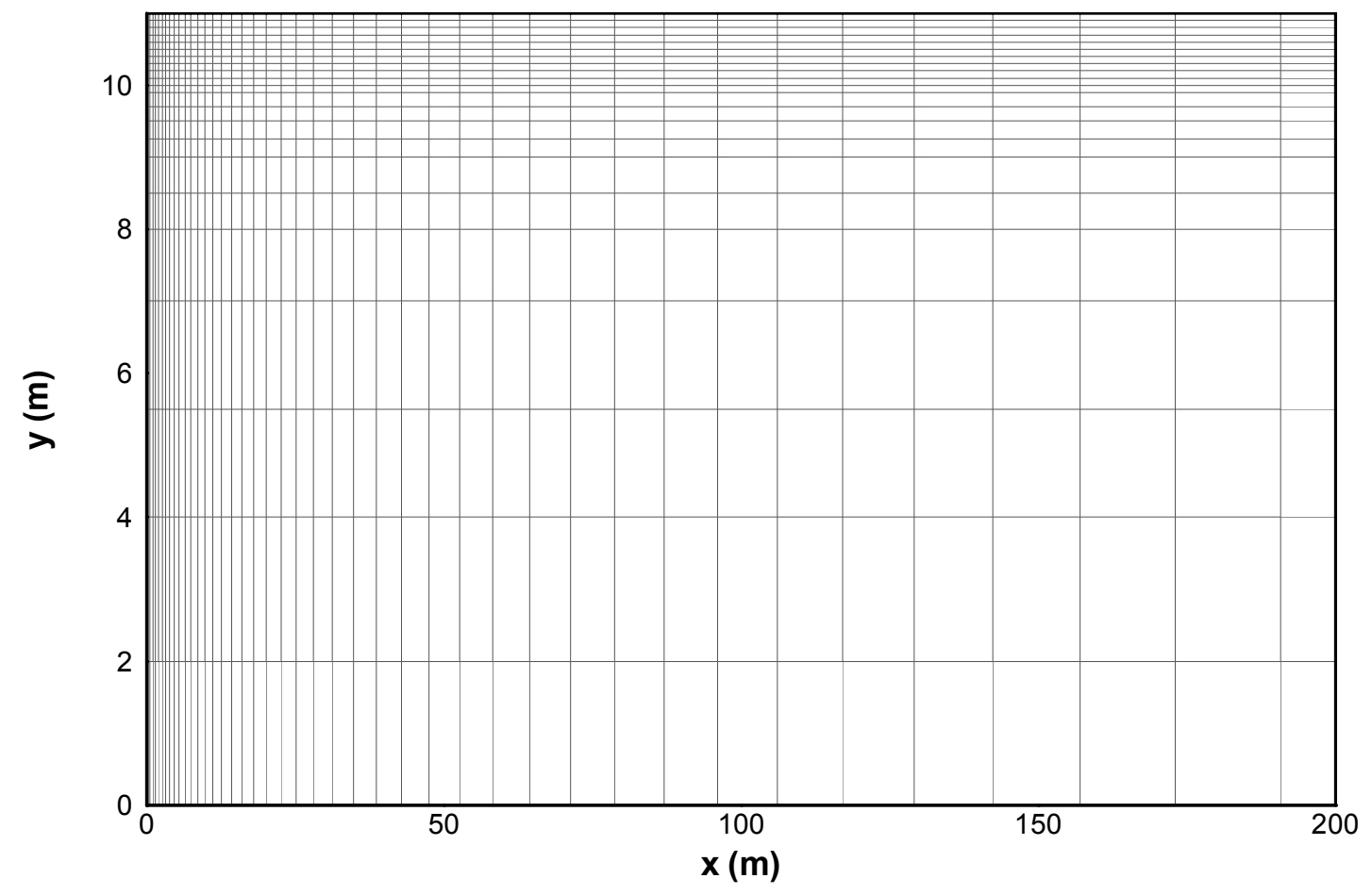

Figure 4.8.2. 2D PORFLOW Cartesian Grid for Problem 4.8 


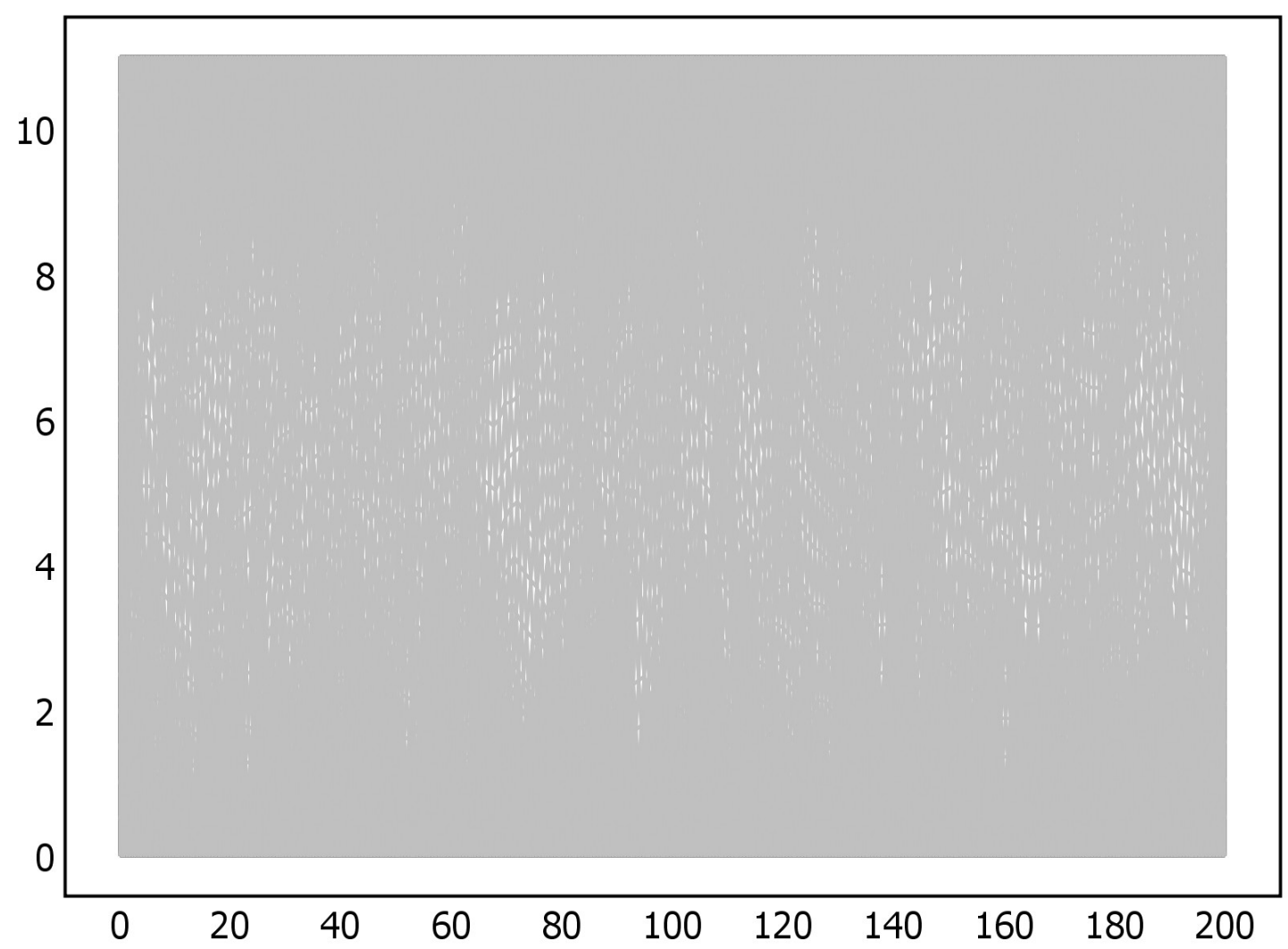

Figure 4.8.3. COMSOL Finite-Element Mesh for Problem 4.8.

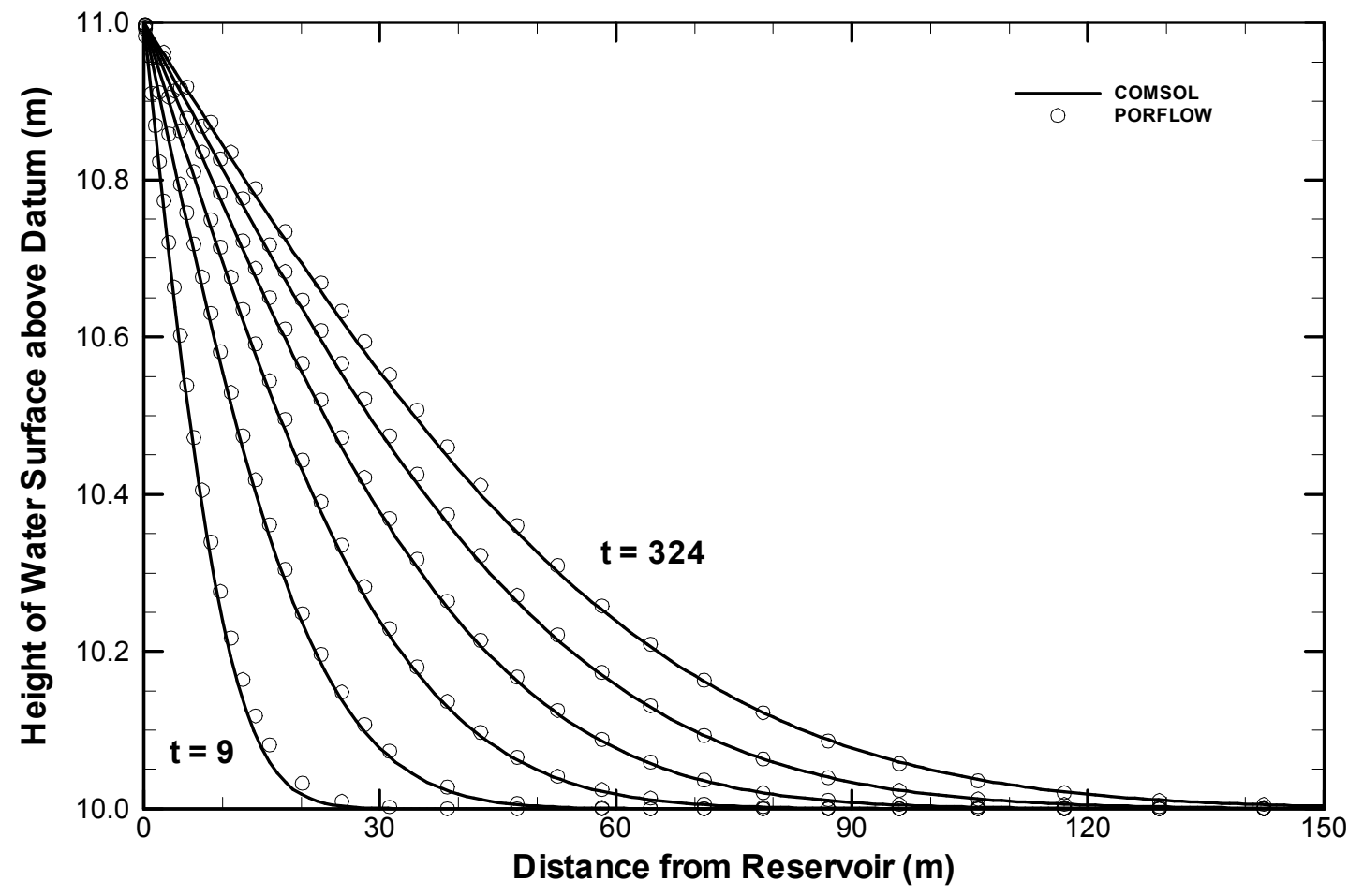

Figure 4.8.4. Time History of Phreatic Surface Due to Recharge. 
Table 4.8.1. Input Commands for Problem 4.8.

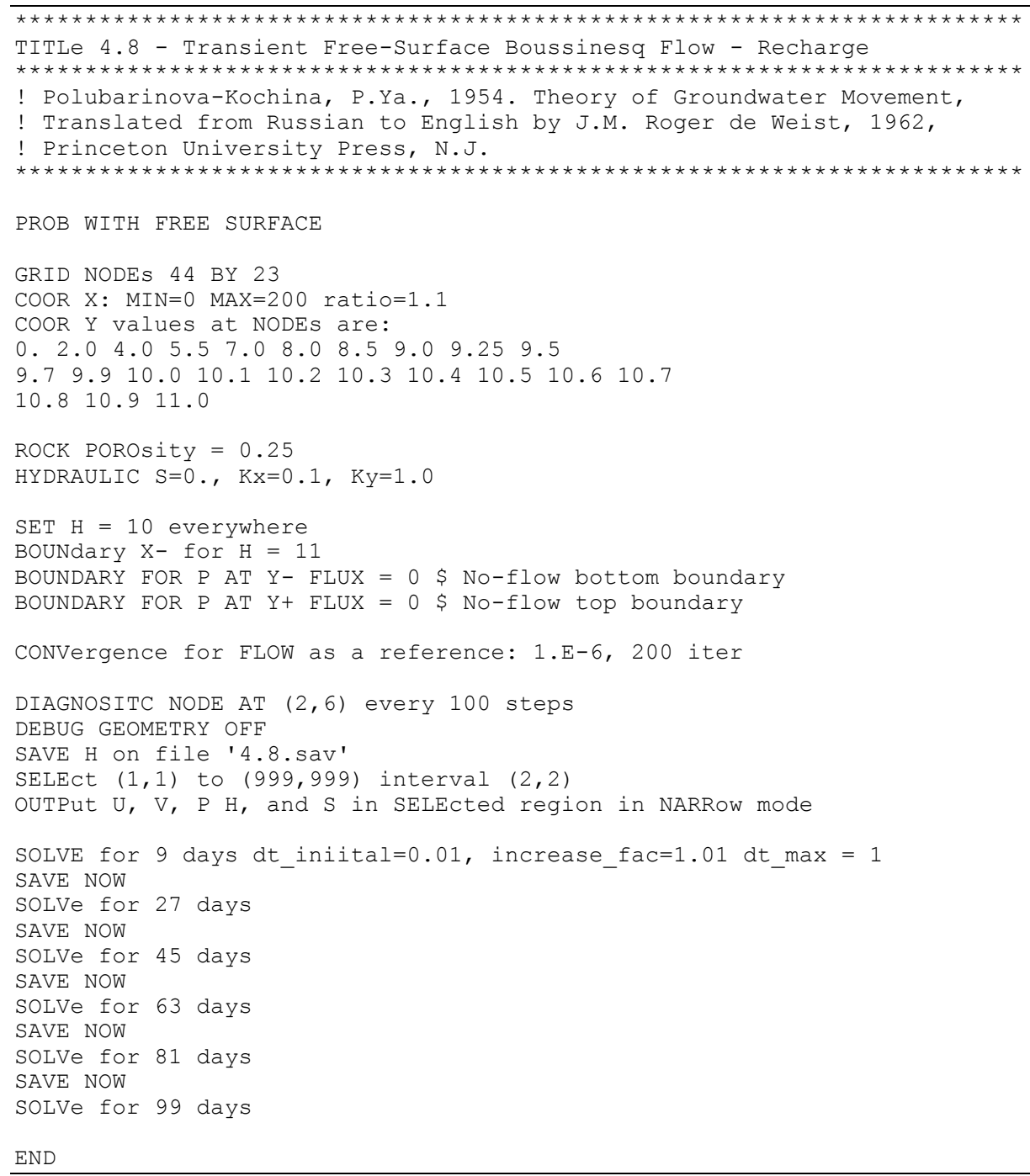

\subsection{Free-Surface Boussinesq Flow with Seepage}

This test problem is a variation on the previous Boussinesq problem. In this case, the initial phreatic surface is at 10 meters. At time zero, the water level at the left boundary is suddenly lowered to 9 meters. The schematic is shown in Figure 4.9.1. The horizontal extent of the mesh is 200 meters and the vertical extent is 10 meters. The objective is to determine the phreatic surface at selected times. It is described in detail by Polubarinova-Kochina (1962). This problem and description was taken from Problem V11 in the ACRI PORFLOW validation report version 2.50 . 


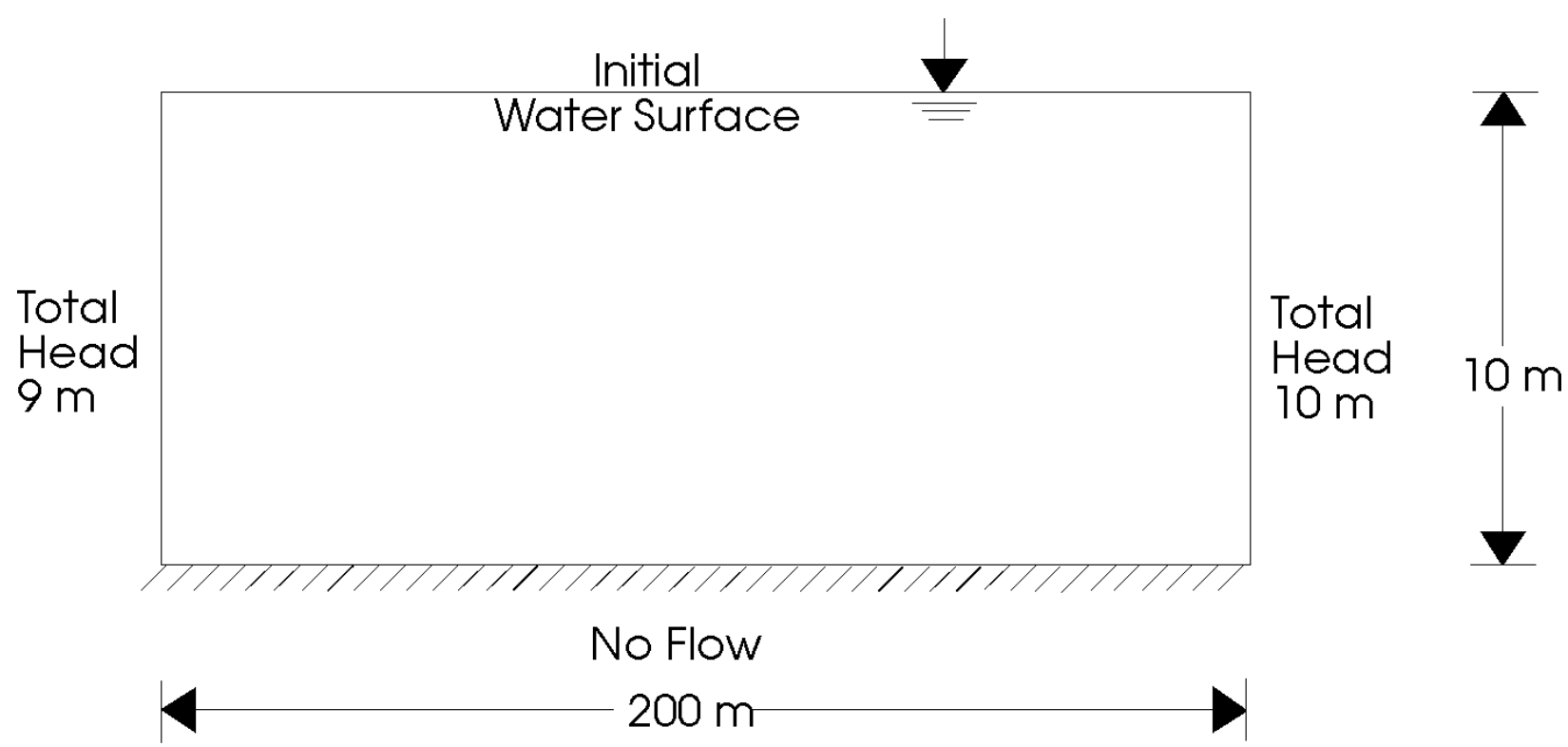

Figure 4.9.1. Schematic Illustration of Problem 4.9.

PORFLOW simulation and comparison: The problem is simulated with a grid of 44 nodes in the horizontal and 23 in the vertical direction. The PORFLOW mesh is shown in Figure 4.9.2. The grid spacing in the horizontal direction increases in a geometric ratio of 1.1. The minimum grid spacing is $0.19 \mathrm{~m}$ and the maximum is $17.68 \mathrm{~m}$. The grid spacing in the vertical varies from $0.1 \mathrm{~m}$ at the top to $1.5 \mathrm{~m}$ at the bottom. The grid spacing near the top is smaller to allow better resolution near the phreatic surface. The total simulation time is 324 days. In the first 9 days of the simulation, the time step is increased from an initial value of 0.01 days to 1 day in a geometric ratio of 1.01; thereafter the time step is kept constant at 1 day. The convergence criterion is specified at $10^{-6}$ and the maximum number of iterations is set to 200 to assure that the transient solution is accurate. The PORFLOW input commands for this problem are shown in Table 4.9.1.

Figure 4.9.3 shows the COMSOL finite-element mesh which consists of 12616 triangular elements and 6711 nodes.

The time history of the phreatic surface due to seepage is shown in Figure 4.9.4 for the PORFLOW and COMSOL numerical simulations for times of 9, 36, 81, 144,225 and 324 days. Qualitatively the two sets of results are in good agreement. The Polubarinova-Kochina analytical solution was not available for comparison to the numerical results. 


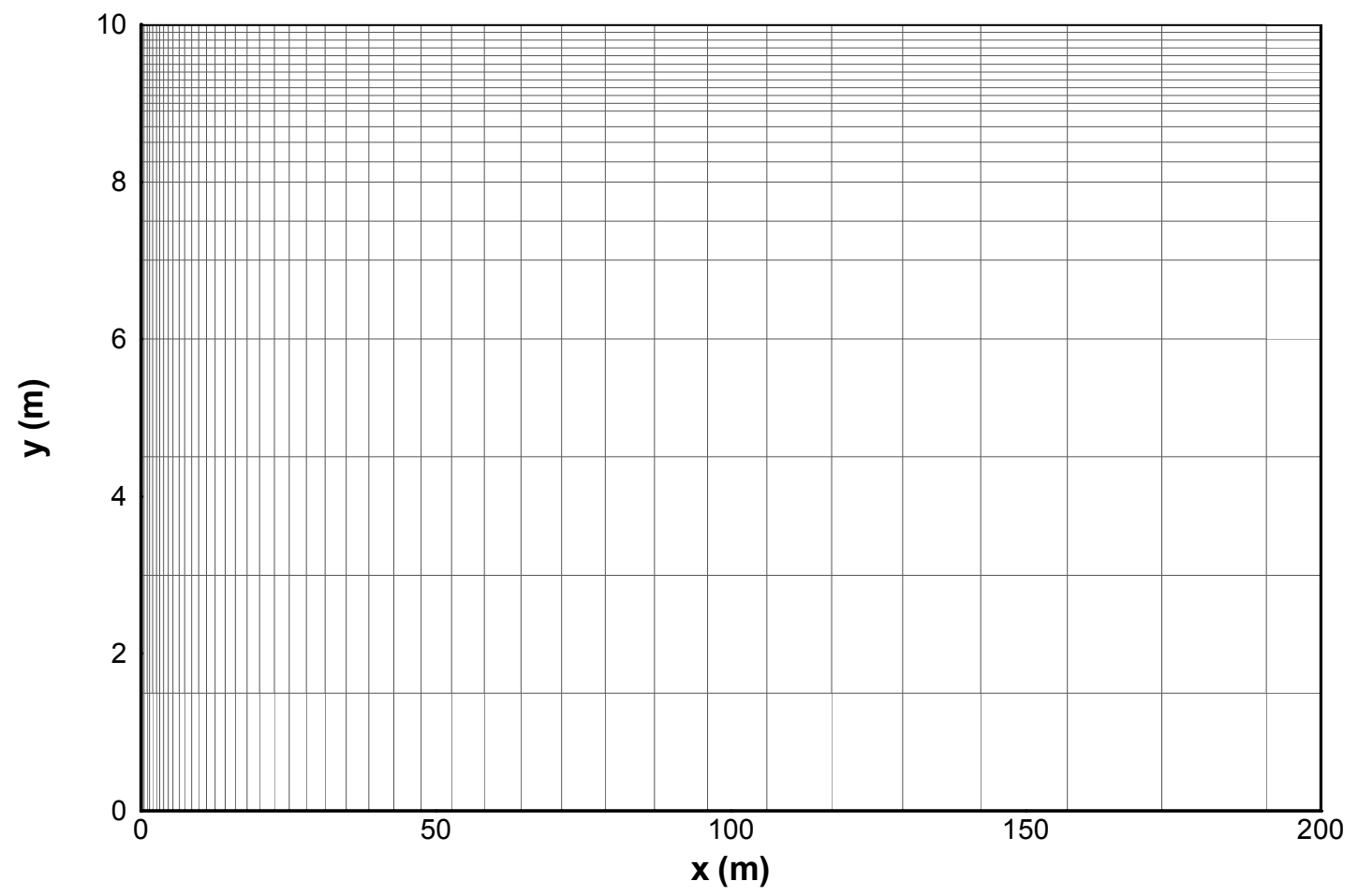

Figure 4.9.2. 2D PORFLOW Cartesian Grid for Problem 4.9.

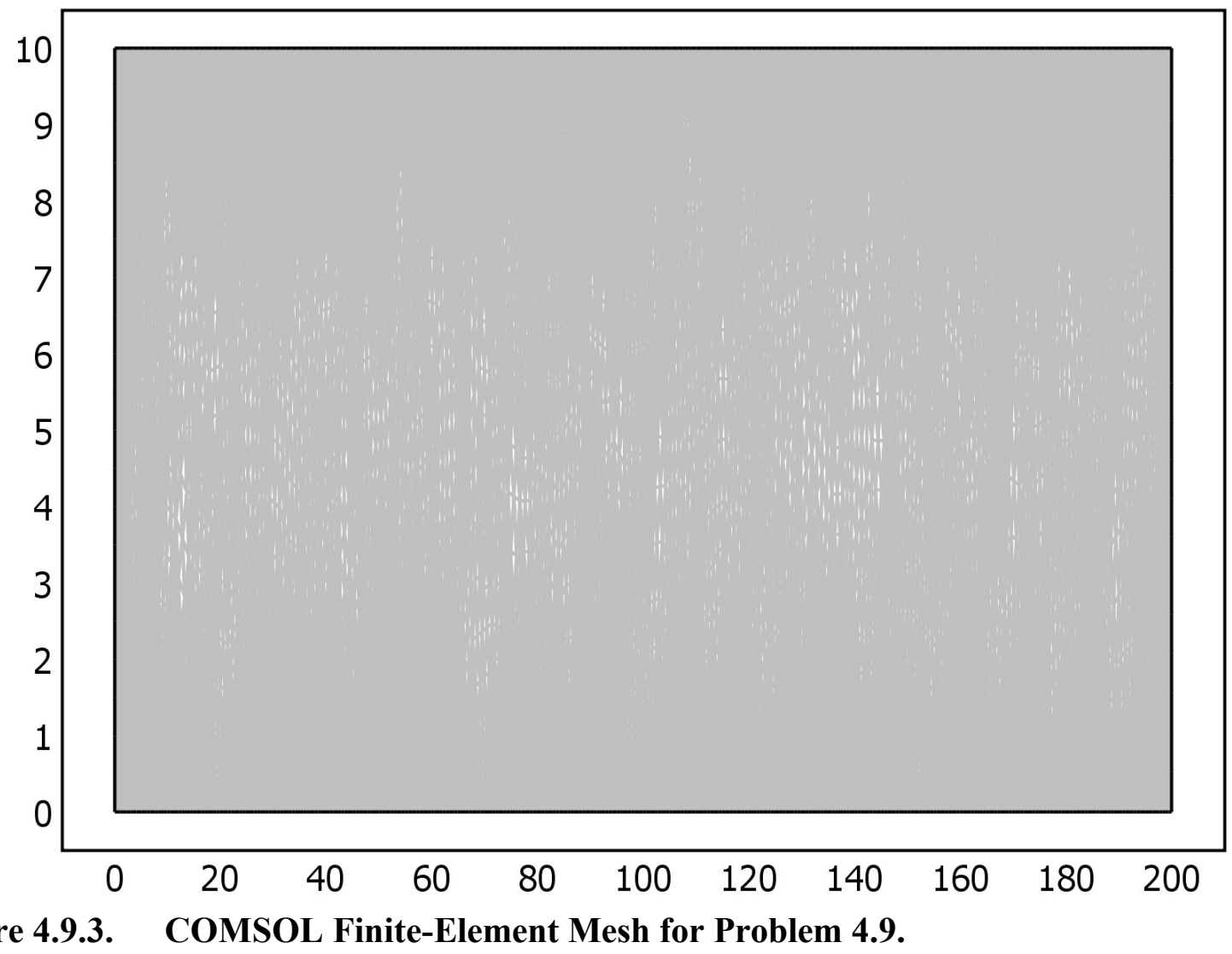

Figure 4.9.3. COMSOL Finite-Element Mesh for Problem 4.9. 


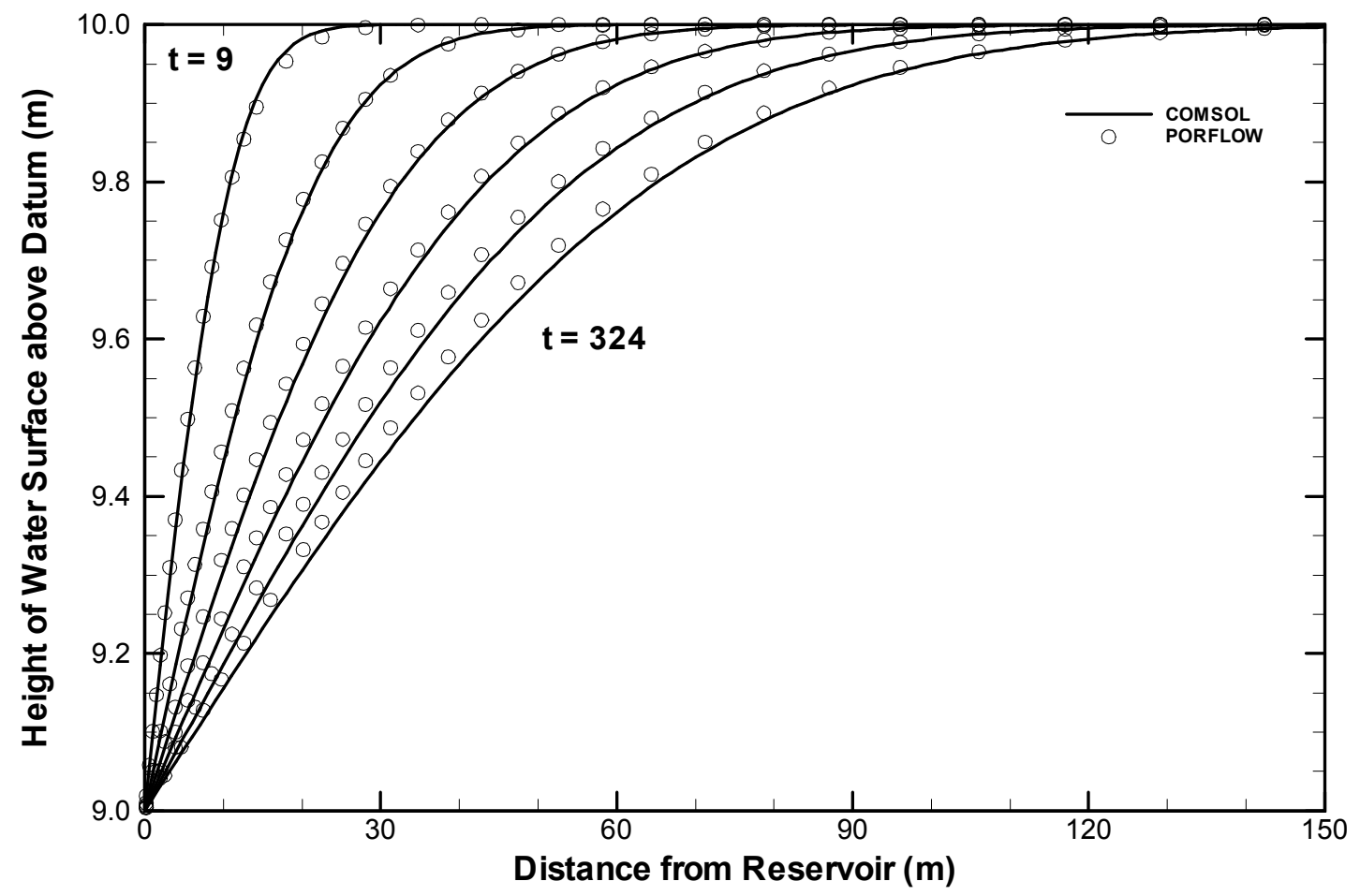

Figure 4.9.4. Time History of Phreatic Surface Due to Seepage.

Table 4.9.1. Input Commands for Problem 4.9.

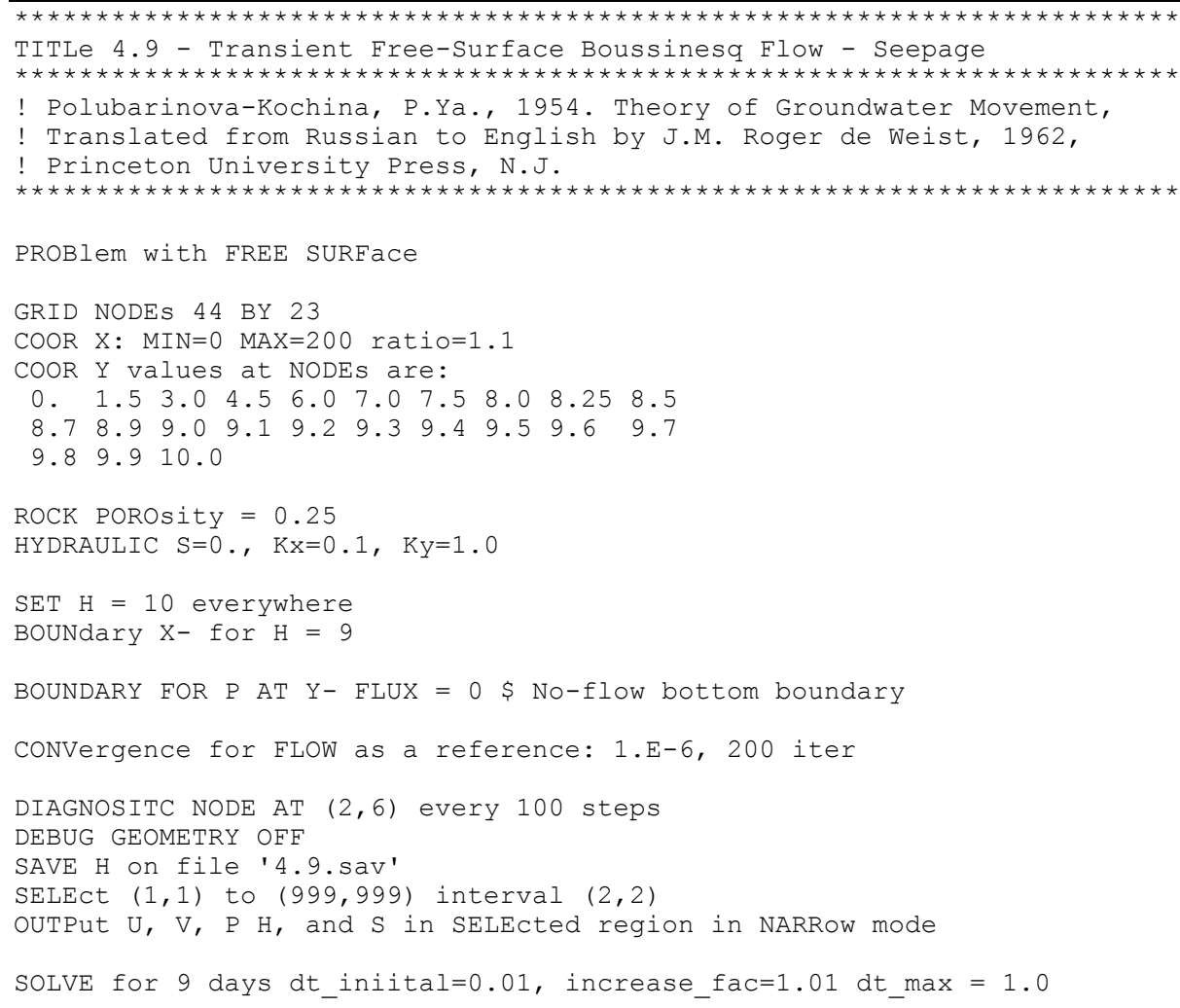


SAVE NOW

SOLVe for 27 days

SAVE NOW

SOLVe for 45 days

SAVE NOW

SOLVe for 63 days

SAVE NOW

SOLVe for 81 days

SAVE NOW

SOLVe for 99 days

END

\subsection{Unsaturated Vertical Soil Column}

Two test cases were designed to confirm correct implementation of soil characteristic curves and Richard's equation. The first test case essentially reproduces the water retention curve under no flow conditions. The second test case involves steady-state unsaturated flow at constant saturation that involves relative permeability. The soil characteristic curves chosen are for "Silt Loam G.E. 3" and are taken from van Genuchten (1980). The van Genuchten models for capillary suction-water retention and relative permeability are

$$
\begin{aligned}
& \mathrm{S}_{\mathrm{e}}=\left\{\begin{array}{cc}
\frac{1}{\left[1+(\alpha \psi)^{\mathrm{n}}\right]^{\mathrm{m}}} & \psi>0 \\
1 & \psi \leq 0
\end{array}\right. \\
& \mathrm{k}_{\mathrm{rw}}=\mathrm{S}_{\mathrm{e}}^{1 / 2}\left[1-\left(1-\mathrm{S}_{\mathrm{e}}^{1 / \mathrm{m}}\right)^{\mathrm{m}}\right]^{2}
\end{aligned}
$$

where $S_{e}$ is the "effective saturation" defined by

$$
\mathrm{S}_{\mathrm{e}}=\frac{\mathrm{S}_{\mathrm{w}}-\mathrm{S}_{\mathrm{wr}}}{1-\mathrm{S}_{\mathrm{wr}}}
$$

with empirical parameters $\alpha$ and $\mathrm{m}$.

For Silt Loam G.E. 3 the empirical parameters take on the values

$$
\begin{gathered}
\mathrm{S}_{\mathrm{wr}}=0.331 \\
\alpha=0.129 \mathrm{ft}^{-1} \\
\mathrm{~m}=0.515 \Rightarrow \mathrm{n}=\frac{1}{1-\mathrm{m}}=2.0619
\end{gathered}
$$

The saturated hydraulic conductivity is $\mathrm{K}=0.163 \mathrm{ft} /$ day (van Genuchten, 1980). 
Figures 4.10.1 and 4.10.2 show the PORFLOW and COMSOL meshes used in the numerical simulation of the test cases, respectively. The 1-D PORFLOW mesh consists of 251 nodes in the $\mathrm{x}$ direction and 3 nodes in the y direction. The COMSOL finite-element mesh consists of 3232 triangular elements and 1689 mesh points.

Water retention profile case: The input commands for the PORFLOW simulation are given in Table 4.10.1. Because the steady-state head is zero along the column, there is no flow in the column. Figure 4.10.3 shows the predicted column saturation profile for the COMSOL and PORFLOW numerical simulations. The agreement is excellent.

Steady-state unsaturated flow at constant saturation case: The input commands for the PORFLOW simulation are given in Table 4.10.2. For a saturation of $75 \%$, the capillary pressure is $0.005125952 \mathrm{ft}$ and the relative permeability is 0.043098523 . For boundary conditions of $9.377711175 \mathrm{ft}$ applied to both ends of the column, the Darcy velocity throughout the column is constant and equal to:

$$
\mathrm{U}=\mathrm{k}_{\mathrm{rw}} \mathrm{K} \frac{\Delta \mathrm{h}}{\Delta \mathrm{x}}=(0.043098523)\left(0.163 \frac{\mathrm{ft}}{\text { day }}\right) \frac{50 \mathrm{ft}}{50 \mathrm{ft}}=0.007025 \frac{\mathrm{ft}}{\text { day }}
$$

The PORFLOW code reproduces the constant saturation of $75 \%$ and Darcy velocity in Equation 4.10.5 as shown in Figure 4.10.4.

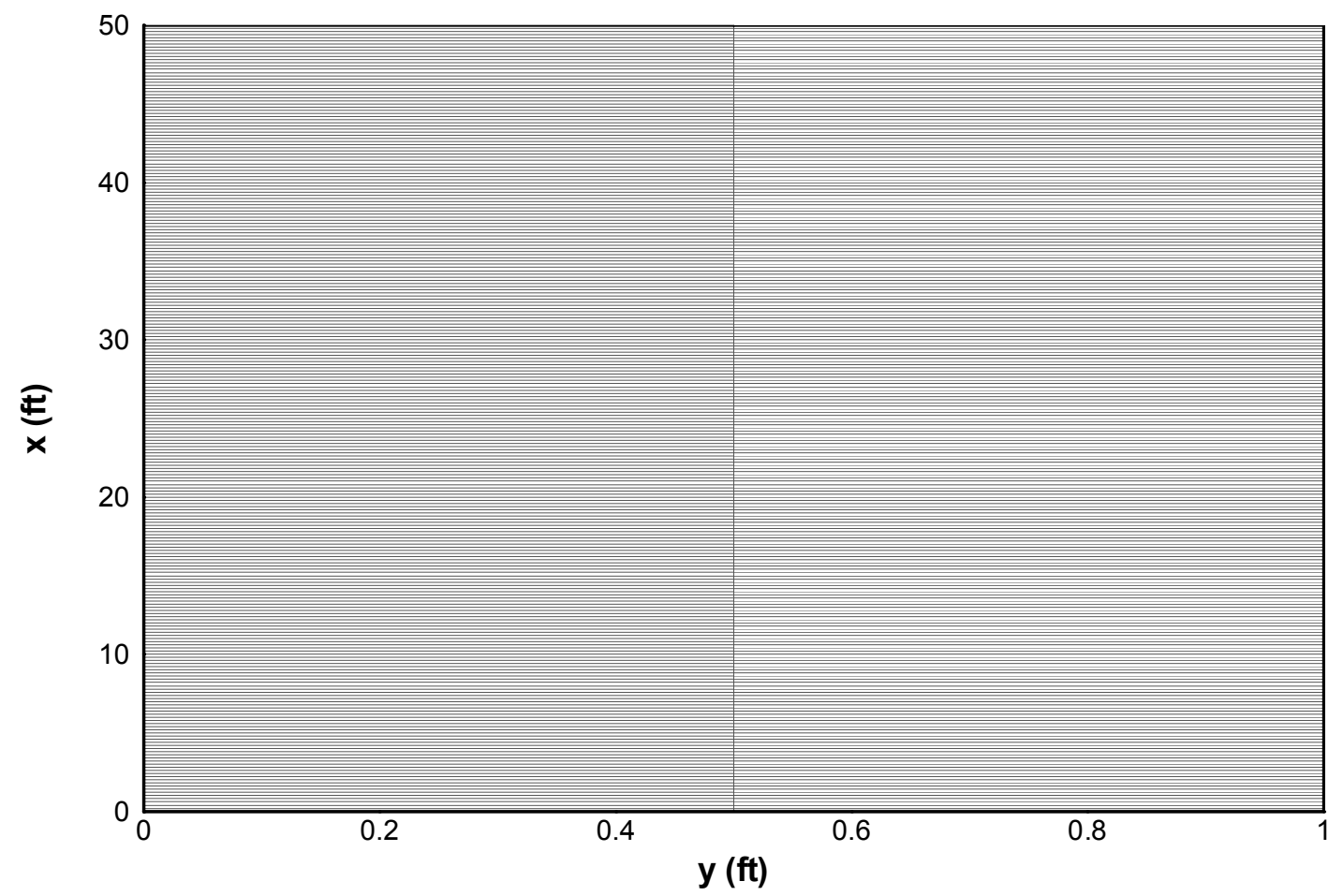

Figure 4.10.1. 1D PORFLOW Grid for Problem 4.10. 


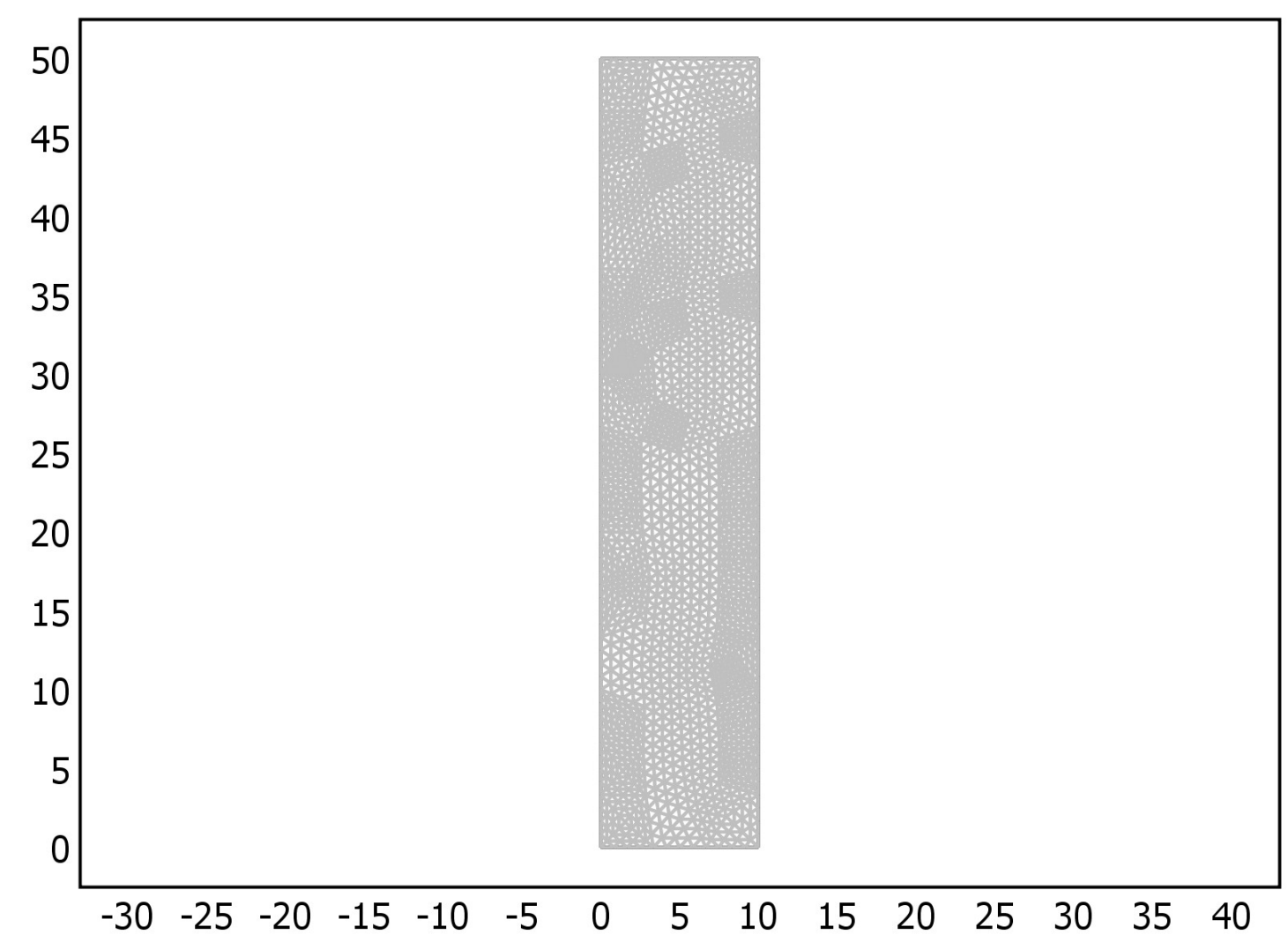

Figure 4.10.2. COMSOL Finite-Element Mesh for Problem 4.10. 


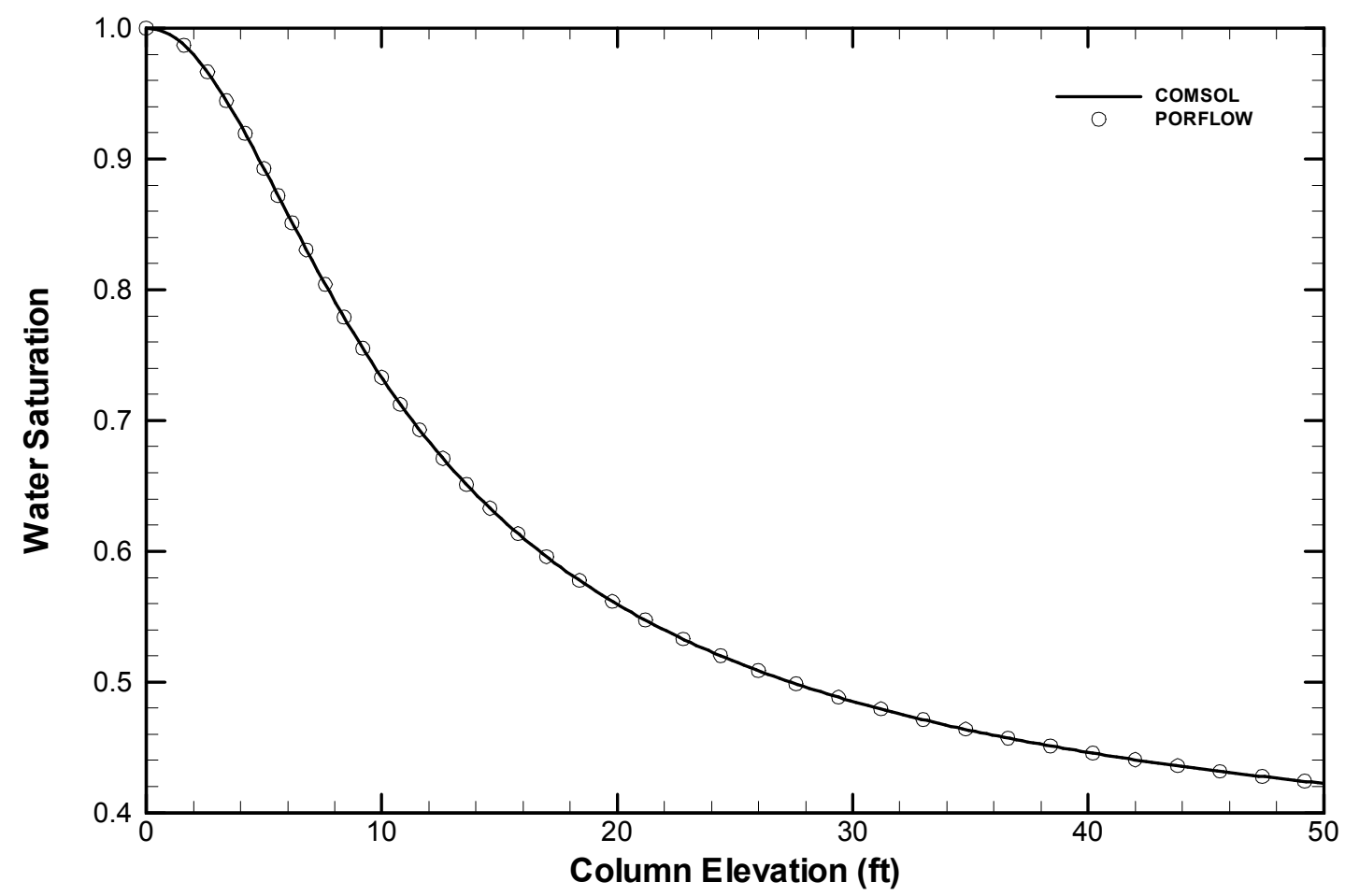

Figure 4.10.3. Predicted Column Saturation Profile for COMSOL and PORFLOW Simulations.

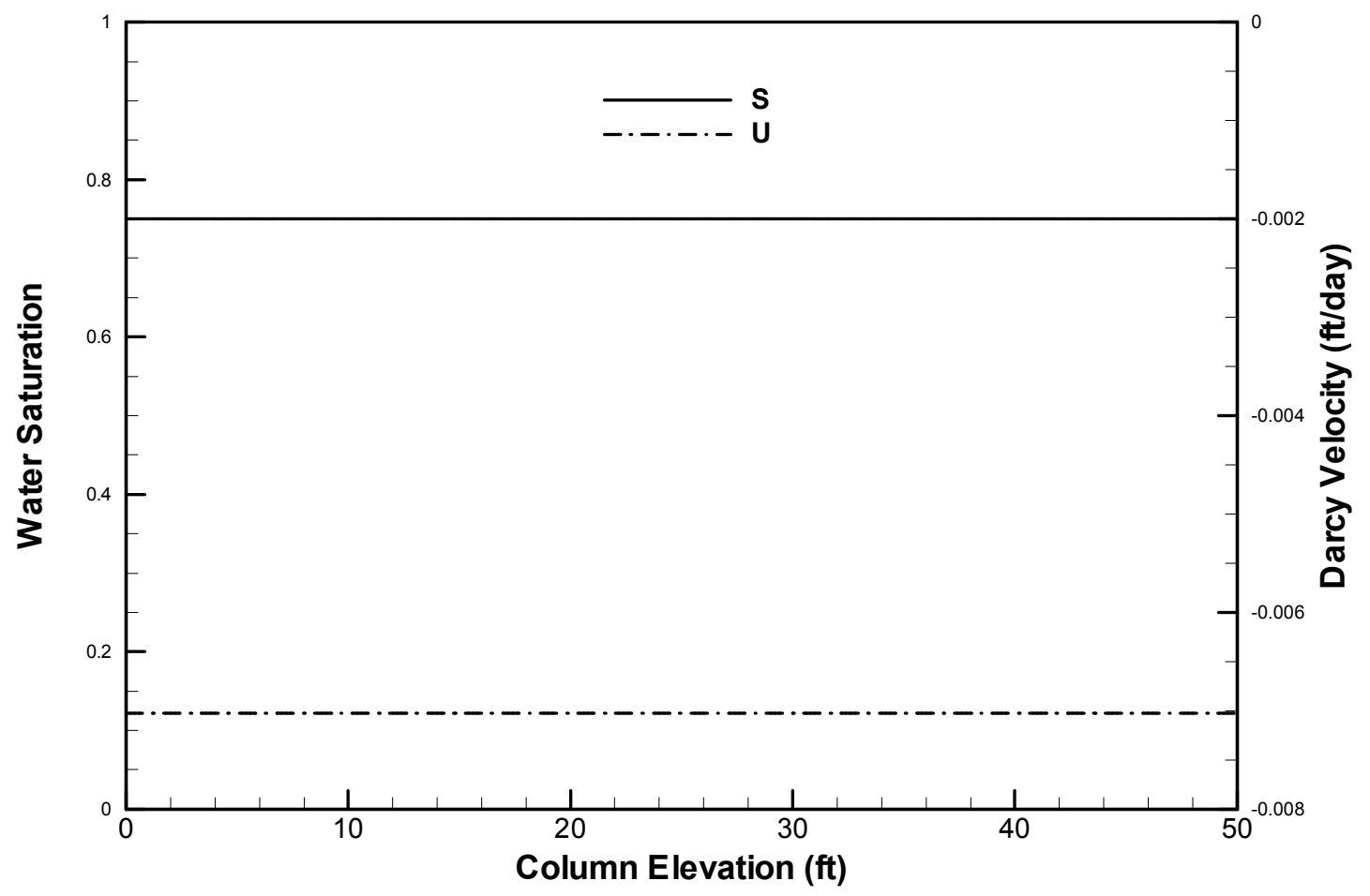

Figure 4.10.4. PORFLOW Simulation of Steady-state Unsaturated Flow at Constant Saturation (75\%). 
Table 4.10.1. Input Commands for Problem 4.10 (Water Retention Profile).

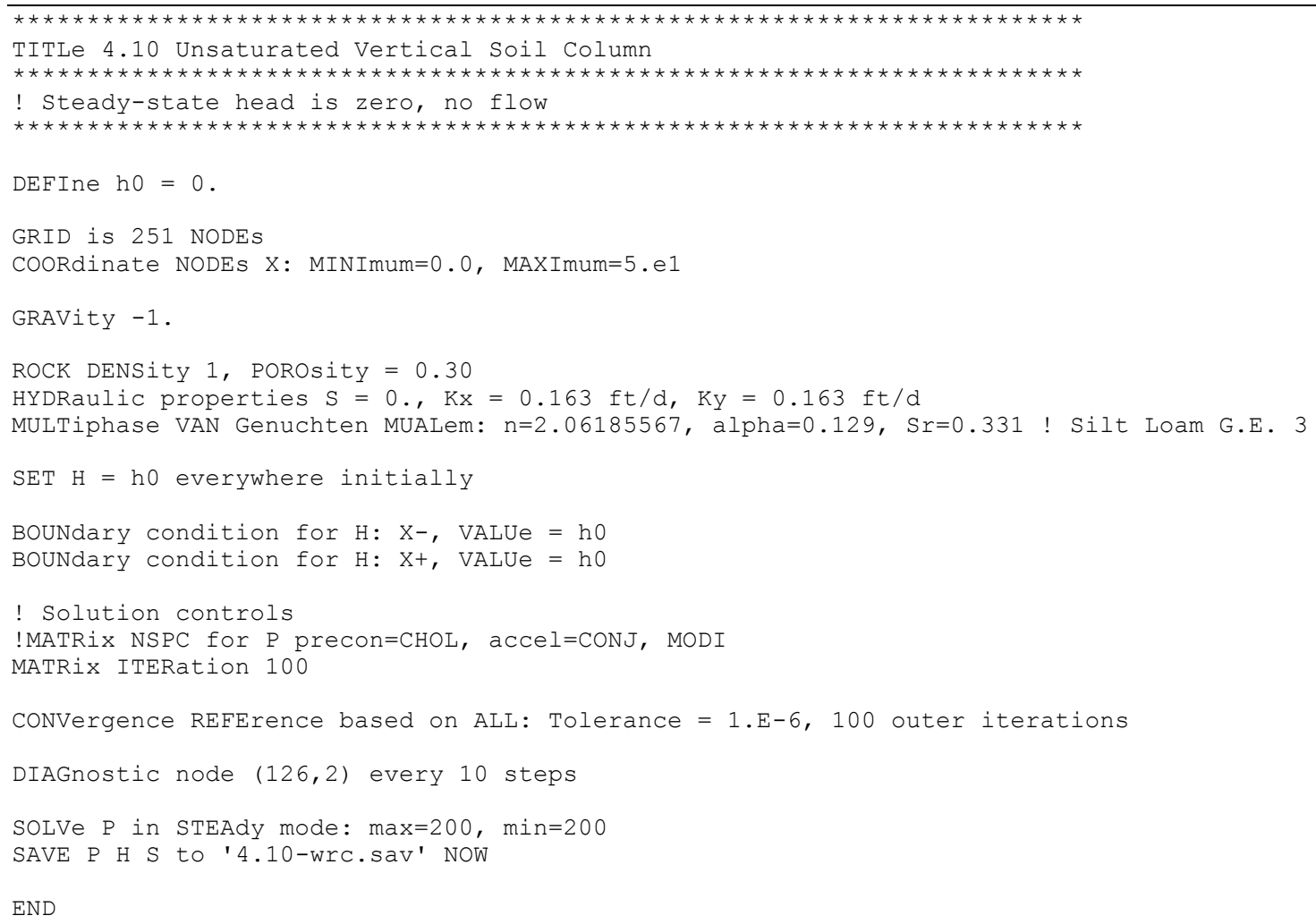

Table 4.10.2. Input Commands for Problem 4.10 (Constant Saturation).

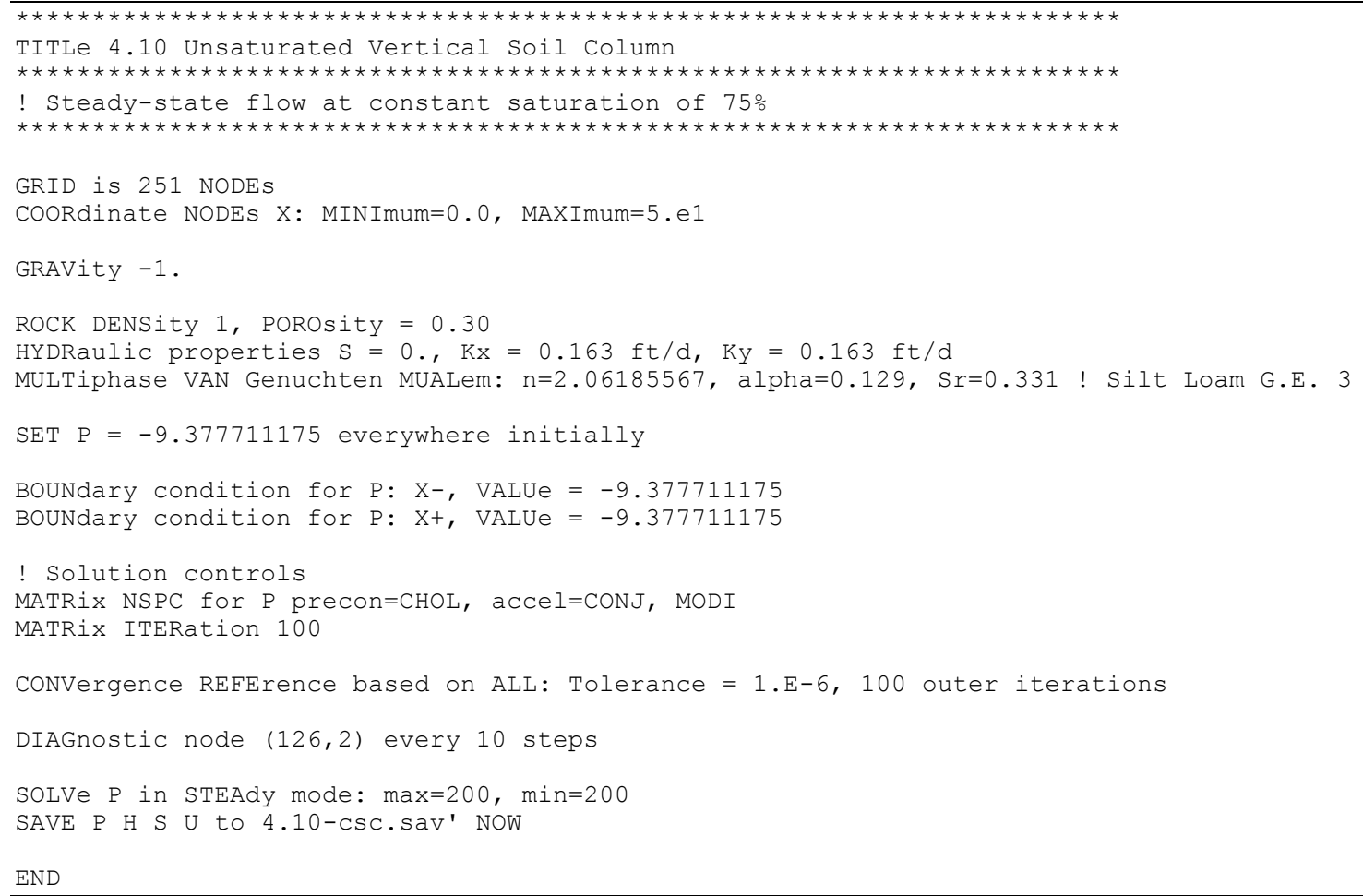




\section{Group 2: Contaminant Transport Problems}

In the following three sections, we present one, two, and three dimensional solute transport examples, respectively. They are classical cases ideal for studying the basic behavior of an advection-dispersion equation solver. In the one-dimensional case, we shall test the equation solver in various ways by varying its control parameters over a wide range of values. This enables modelers to see the inherent weaknesses of this solver and help to minimize such weaknesses in their own problems. The majority of behaviors presented below are observed in all advectiondispersion solvers. Ultimately, the users must rely on their own experience and it is highly recommended that several transport simulations of the same problem be performed. Comparisons to these simulations will provide excellent insight into how adequate and optimal their solution is. The test cases selected all have analytic solutions for a clear picture as to how well PORFLOW handles these transport conditions.

\subsection{One-Dimensional Saturated Solute Transport in a Uniform Flow Field}

This problem deals with one-dimensional advection-dispersion of a non-conservative solute species through a semi-infinite porous medium and is used to demonstrate the impact that various PORFLOW options (i.e., numerical approximations) have on its solution. The 1D advectiondispersion equation is ideal for testing an algorithm's behavior over a wide range of conditions. A physical schematic of this problem is shown in Figure 5.1.1. As illustrated, a non-conservative contaminant is continuously released from a fully penetrating channel into a shallow confined aquifer unit whose groundwater flow is assumed uniform. Both hydrodynamic dispersion and molecular diffusion are allowed, as well as, the possibility of radioactive decay and/or adsorption of the species. It is assumed that the contaminant concentration level in the neighboring reservoir remains constant, the aquifer's flow rate is uniform and constant, and the homogeneous aquifer's properties (such as porosity, soil type, water saturation) are uniform and constant.

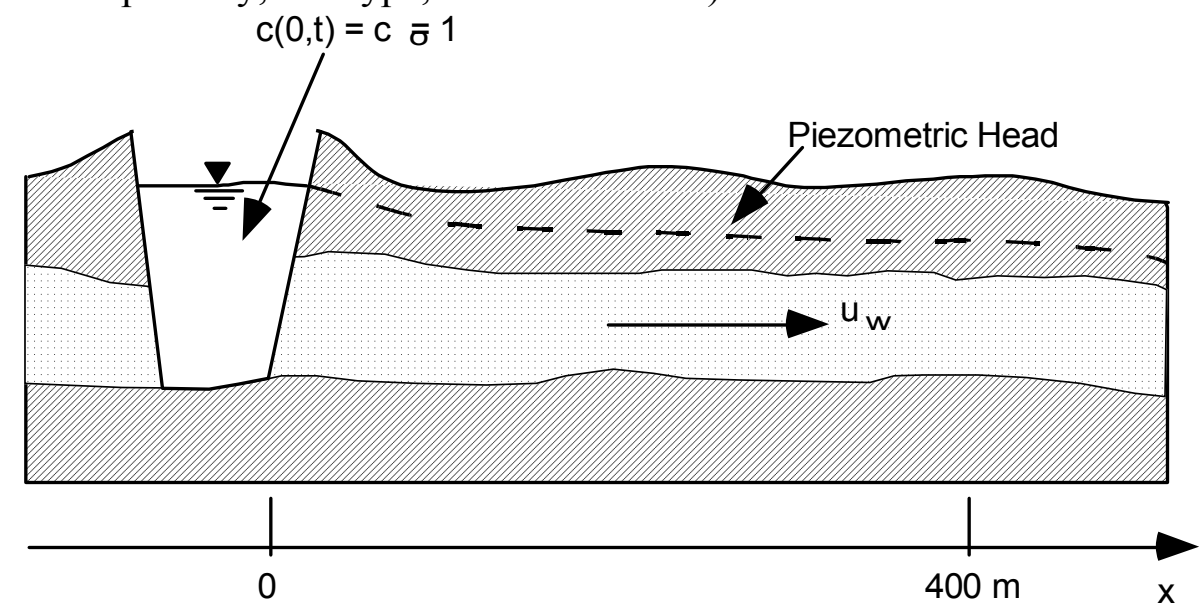

Figure 5.1.1. Schematic Diagram for $1 D$ Solute Transport in a Confined Aquifer.

Analytical solution: The conservative form of the multi-dimensional advection-dispersion equation for solute transport through a variably saturated porous media with radioactive decay and point sources is given as 


$$
\frac{\partial}{\partial \mathrm{t}}\left(\theta_{\mathrm{e}} \mathrm{Rc}\right)+\nabla \cdot(\mathrm{Uc})=\nabla \cdot(\Gamma \nabla \mathrm{c})-\theta_{\mathrm{e}} \mathrm{R} \lambda \mathrm{c}+\mathrm{qc}{ }^{*}
$$

where

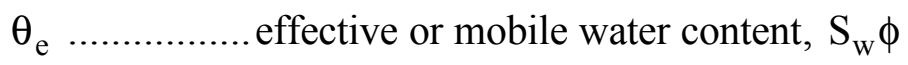

$$
\begin{aligned}
& R \text {............... retardation factor, } 1+\rho_{\mathrm{b}} \mathrm{k}_{\mathrm{d}} / \theta_{\mathrm{e}} \\
& \rho_{\mathrm{b}} \ldots \ldots \ldots \ldots \ldots \text {......... bulk soil density, } \rho_{\mathrm{s}}(1-\phi) \\
& \rho_{\mathrm{s}} \ldots \ldots \ldots \ldots \ldots . . . . . . \text { particle density } \\
& \mathrm{k}_{\mathrm{d}} \text {............... distribution coefficient } \\
& \text { U ................ Darcy velocity vector } \\
& \text { c .................. solute concentration } \\
& \Gamma \ldots \ldots \ldots \ldots \ldots . . . . . . \text { apparent hydrodynamic dispersion tensor, } \theta_{\mathrm{e}} \mathrm{D} \\
& \lambda \text {................ radioactive decay constant, } \ln (2) / \mathrm{t}_{1 / 2} \\
& \text { q ….............. volumetric flow source per unit volume of porous medium } \\
& c^{*} \text {................. solute concentration evaluated within source/sink flow term }
\end{aligned}
$$

Equation 5.1.1 is in conservative form and the non-conservative form is considered more amendable to spatial discretization. To accomplish the transformation, use is made of the flow (mixture mass balance) equation in its incompressible form

$$
\frac{\partial \theta_{\mathrm{e}}}{\partial \mathrm{t}}=-\nabla \cdot \mathrm{U}+\mathrm{q}
$$

Substituting Equation 5.1.2 into 5.1.1, with expanded advective and mass accumulation terms, yields

$$
\theta_{\mathrm{e}} \mathrm{R} \frac{\partial \mathrm{c}}{\partial \mathrm{t}}+\mathrm{c} \frac{\partial \theta_{\mathrm{e}}}{\partial \mathrm{t}}+\mathrm{c} \frac{\partial}{\partial \mathrm{t}}\left(\rho_{\mathrm{b}} \mathrm{k}_{\mathrm{d}}\right)+\mathrm{U} \cdot \nabla \mathrm{c}-\mathrm{c} \frac{\partial \theta_{\mathrm{e}}}{\partial \mathrm{t}}+\mathrm{qc}=\nabla \cdot\left(\theta_{\mathrm{e}} \mathrm{D}\right)-\theta_{\mathrm{e}} \mathrm{R} \lambda \mathrm{c}+\mathrm{qc}{ }^{*}
$$

Assuming that the time derivative of $\rho_{\mathrm{b}} \mathrm{k}_{\mathrm{d}}$ is negligible, Equation 5.1.3 reduces to

$$
\theta_{\mathrm{e}} \mathrm{R} \frac{\partial \mathrm{c}}{\partial \mathrm{t}}+\mathrm{U} \cdot \nabla \mathrm{c}=\nabla \cdot\left(\theta_{\mathrm{e}} \mathrm{D}\right)-\theta_{\mathrm{e}} \mathrm{R} \lambda \mathrm{c}-\mathrm{q}\left(\mathrm{c}-\mathrm{c}^{*}\right)
$$

Taking the 1D form of Equation 5.1.4 and assuming that no point sources/sinks exist within the domain, constant water saturation level, and that material coefficients are constants, results in

$$
\frac{\partial \mathrm{c}}{\partial \mathrm{t}}=\mathrm{D}_{\mathrm{xx}}^{\prime} \frac{\partial^{2} \mathrm{c}}{\partial \mathrm{x}^{2}}-\mathrm{u}_{\mathrm{x}}^{\prime} \frac{\partial \mathrm{c}}{\partial \mathrm{x}}-\lambda \mathrm{c}
$$




$$
\begin{gathered}
\mathrm{u}_{\mathrm{x}}=\frac{\mathrm{U}_{\mathrm{x}}}{\theta_{\mathrm{e}}} \\
\mathrm{u}_{\mathrm{x}}^{\prime}=\frac{\mathrm{u}_{\mathrm{x}}}{\mathrm{R}} \\
\mathrm{D}_{\mathrm{xx}}=\alpha_{\mathrm{L}} \mathrm{u}_{\mathrm{x}}+\theta_{\mathrm{e}} \tau \mathrm{D}_{\mathrm{m}} \\
\mathrm{D}_{\mathrm{xx}}^{\prime}=\frac{\mathrm{D}_{\mathrm{xx}}}{\mathrm{R}}
\end{gathered}
$$

where

$$
\begin{aligned}
& \alpha_{L} \ldots \ldots \ldots \ldots \ldots \text {......... longitudinal dispersivity } \\
& \tau \ldots \ldots \ldots \ldots \ldots . . . \text { tortuosity of the porous medium } \\
& D_{m} \ldots \ldots \ldots \ldots \ldots \text { bulk molecular diffusion coefficient }
\end{aligned}
$$

For our semi-infinite confined aquifer the initial conditions are:

$$
\mathrm{c}(\mathrm{x}, 0)=0
$$

and the boundary conditions are:

$$
\begin{aligned}
& c(0, t)=c_{0} \\
& c(\infty, t)=0
\end{aligned}
$$

For the first boundary condition we are assuming that at $\mathrm{x}=0$ the contaminant concentration reaches its ultimate value $c_{0}$ immediately upon commencement of flow and remains at that value throughout all positive times ( $\mathrm{x}=0$ boundary represents an inflow boundary). The second boundary condition is equivalent to assuming that the dispersive flux of solute is zero at plus infinity or

$$
\lim _{x \rightarrow \infty}\left(\frac{\partial c}{\partial x}\right)=0
$$

Equation 5.1.5, a linear partial differential equation subject to the initial and boundary conditions given by Eqs. 5.1.6a to 5.1.6d can be solved by applying Laplace and Fourier transforms. The general solution has been derived by Grobner and Hofreiter (1950) and takes the form:

$$
\left.c(x, t)=\frac{1}{2} c_{0} \exp \left(\frac{u_{x}^{\prime} x}{2 D_{x x}^{\prime}}\right) \exp (-x \beta) \operatorname{erfc}\left(\frac{x-\sigma t}{2 \sqrt{D_{x x}^{\prime} t}}\right)+\exp (x \beta) \operatorname{erfc}\left(\frac{x+\sigma t}{2 \sqrt{D_{x x}^{\prime} t}}\right)\right]
$$


where

$$
\beta^{2}=\left(\frac{u_{x}^{\prime}}{2 D_{x x}^{\prime}}\right)^{2}+\frac{\lambda}{D_{x x}^{\prime}} \text { and } \sigma^{2}=\left(u_{x}^{\prime}\right)^{2}+4 \lambda D_{x x}^{\prime}
$$

When there is no radioactive decay $(\lambda=0)$, Equation 5.1.7 reduces to

$$
c(x, t)=\frac{1}{2} c_{0}\left[\operatorname{erfc}\left(\frac{x-u_{x}^{\prime} t}{2 \sqrt{D_{x x}^{\prime} t}}\right)+\exp \left(\frac{u_{x}^{\prime} x}{D_{x x}^{\prime}}\right) \operatorname{erfc}\left(\frac{x+u_{x}^{\prime} t}{2 \sqrt{D_{x x}^{\prime} t}}\right)\right]
$$

derived also by Ogata and Banks (1961). The absence of adsorption is achieved by setting the retardation factor to unity $\left(\mathrm{R}=1, \mathrm{k}_{\mathrm{d}}=0\right)$. Note that the original derivation by Grobner and Hofreiter was performed on the limited case of a saturated media in the absence of adsorption. The more general case presented here results in the same solution but is based upon retarded material coefficients. The evaluation of the analytical expression, Equation 5.1.7, for a specific problem is performed numerically using the FORTRAN code GH in Section B.

PORFLOW numerical simulation and comparison: Values of the physical parameters used in the verification simulations are presented in Table 5.1.1. The base case grid chosen for this problem consists of 201 nodes uniformly spaced ( 2 meters) along the x-axis and 3 nodes in the $\mathrm{y}$ direction. Figure 5.1.2 illustrates the PORFLOW mesh chosen. At the channel inlet boundary (left face), the concentration of solute in the incoming water is set to $1.0 \mathrm{~kg} / \mathrm{m}^{3}$. Due to the finite overall length of our mesh, at the outflow boundary (right face) the dispersive flux is set to zero, while the advective flux is calculated as part of the solution. Since this is a 1D problem, solute concentration gradients do not exist in the transverse directions ( $\mathrm{y}$ and $\mathrm{z}$ directions). The aquifer is assumed to be completely saturated. 
Table 5.1.1. Values of the Physical Parameters, Mesh Spacing, Time Steps, and Key Computed Parameters Used in the One-Dimensional Transport Simulations.

\begin{tabular}{|c|c|c|}
\hline Physical parameters & Base Case & Range tested \\
\hline Darcy velocity, $\mathrm{U}_{\mathrm{x}}$ & $1.0 \mathrm{~m} / \mathrm{d}$ & - \\
\hline Porosity , $\phi$ & 0.25 & - \\
\hline Longitudinal dispersivity, $\alpha$ & $5.0 \mathrm{~m}$ & $0.01,5.0$ \\
\hline $\begin{array}{l}\text { Apparent molecular dispersion coefficient, } \\
\qquad \theta_{\mathrm{e}} \tau \mathrm{D}^{*}\end{array}$ & $0.0 \mathrm{~m}^{2} / \mathrm{d}$ & - \\
\hline Water saturation, $S_{\mathrm{w}}$ & 1.0 & - \\
\hline Radioactive decay constant., $\lambda$ & $0.0 \mathrm{~d}^{-1}$ & $0.0,0.01$ \\
\hline Soil density, $\rho_{\mathrm{s}}$ & $1.0 \mathrm{~kg} / \mathrm{m}^{3}$ & - \\
\hline Solute distribution coefficient, $\mathrm{k}_{\mathrm{d}}$ & $0.0 \mathrm{~m}^{3} / \mathrm{kg}$ & $0.0,0.3333$ \\
\hline Boundary solute concentration, $\mathrm{c}_{0}$ & $1.0 \mathrm{~kg} / \mathrm{m}^{3}$ & - \\
\hline \multicolumn{3}{|l|}{ Grid specifics } \\
\hline $\mathrm{x}$ grid spacing, $\Delta \mathrm{x}$ & $2 \mathrm{~m}$ & $2,20,40,80$ \\
\hline $\mathrm{y}$ grid spacing, $\Delta \mathrm{y}$ & $0.5 \mathrm{~m}$ & - \\
\hline Number nodes in $\mathrm{x}$-direction & 201 & $201,21,11,6$ \\
\hline Number nodes in y-direction & 3 & - \\
\hline Longitudinal length & $400 \mathrm{~m}$ & - \\
\hline \multicolumn{3}{|l|}{ Time steps } \\
\hline Time duration & $50 \mathrm{~d}$ & - \\
\hline number time-steps & 500 & $20,10,5,2$ \\
\hline time-step size, $\Delta \mathrm{t}$ & $0.1 \mathrm{~d}$ & $2.5,5,10,25$ \\
\hline \multicolumn{3}{|l|}{ Key computed parameters } \\
\hline Retardation factor, $\mathrm{R}$ & 1.0 & $1.0,2.0$ \\
\hline Bulk soil density, $\rho_{b}$ & $0.75 \mathrm{~kg} / \mathrm{m}^{3}$ & - \\
\hline Phasic velocity, $u_{x}$ & $4.0 \mathrm{~m} / \mathrm{d}$ & - \\
\hline Retarded phasic velocity, $\mathrm{u}_{\mathrm{x}}^{\prime}$ & $4.0 \mathrm{~m} / \mathrm{d}$ & $4.0,2.0$ \\
\hline $\begin{array}{l}\text { Retarded longitudinal dispersion } \\
\text { coefficient, } \mathrm{D}_{\mathrm{xx}}^{\prime}\end{array}$ & $20.0 \mathrm{~m}^{2} / \mathrm{d}$ & $20.0,10.0$ \\
\hline Cell Fourier number, $\mathrm{Fo}_{\mathrm{x}}$ & 0.5 & $\begin{array}{c}0.5,0.0078125 \\
0.03125,0.125,0.5,1.0,2.0,5.0\end{array}$ \\
\hline Cell Courant number, $\mathrm{Co}_{\mathrm{x}}$ & 0.2 & $\begin{array}{c}0.2,0.125,0.25,0.5 \\
10,20,50\end{array}$ \\
\hline Cell Peclet number, $P_{\mathrm{x}}$ & 0.4 & $0.4,4.0,8.0,16.0,1000.0$ \\
\hline
\end{tabular}

For this problem several simulations were performed. As summarized in Table 5.1.2, simulations were performed for a base case and then twelve runs were made varying certain key physical parameters and PORFLOW options to demonstrate their impact on the results. For each simulation, a transient calculation was performed for a 50-day duration and the results from PORFLOW at two points in time (25 and 50 days) are compared to the analytical solution given 
by Equation 5.1.7. As shown in Table 5.1.1, a broad range of cell Fourier, cell material Courant and cell Peclet numbers were tested. For understanding behavior, stability and accuracy issues, these are very important quantities to consider.

Table 5.1.2. Summary of Simulations Performed (Base Case and Variations) on the OneDimensional Transport Problem.

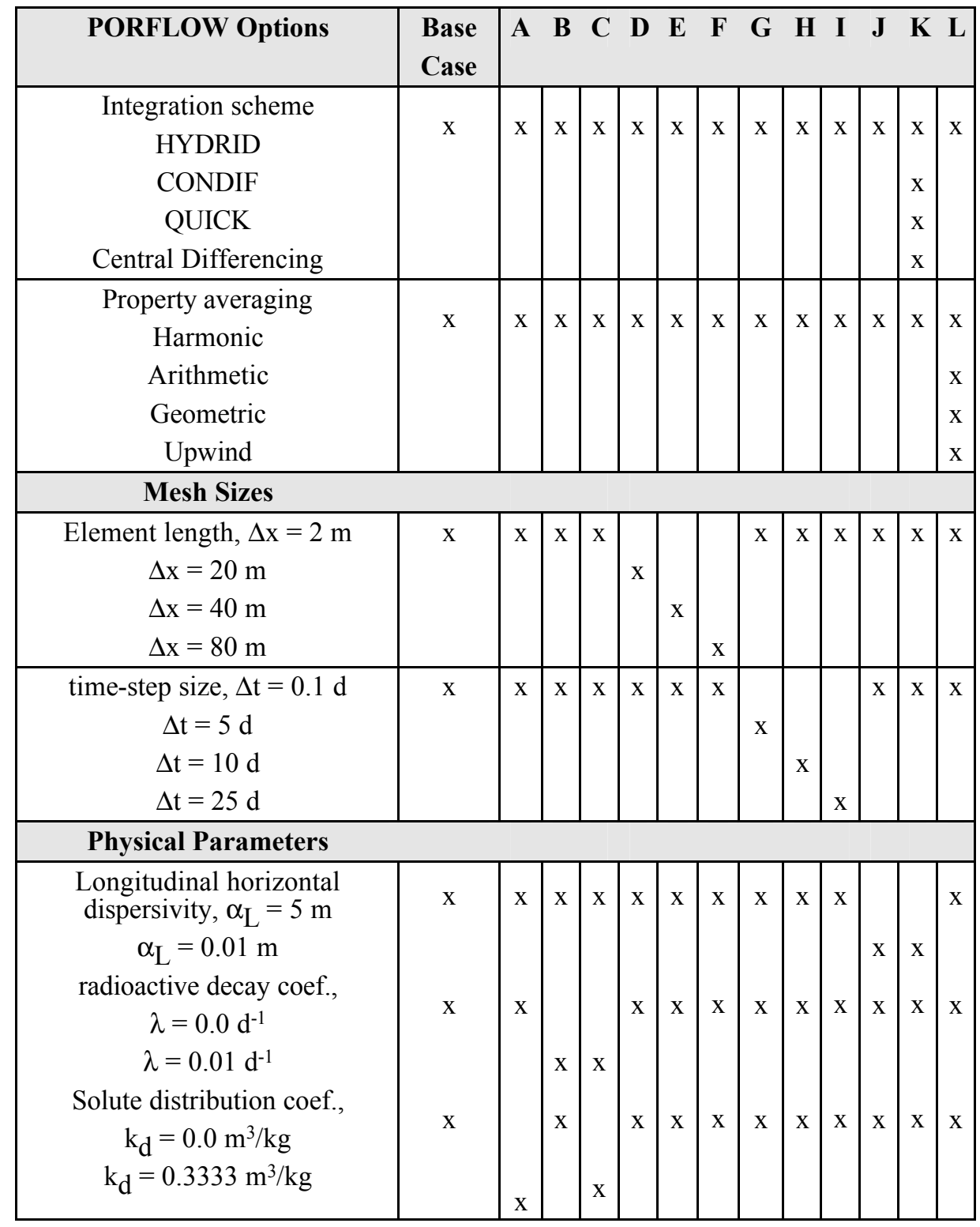

The results of all the simulations (both numerical and analytical) are shown in Figures 5.1.3 through 5.1.15. Each figure corresponds to a different combination of parameters (e.g., spatial and temporal approximations). These results are also presented in tabular form for comparison in Tables 5.1.3 through 5.1.17. The analytical results were computed from the computer code GH. The PORFLOW input commands for the 1D transport simulations are given in Tables 5.1.18 through 5.1.30b. 
The results presented in Figure 5.1.3 represent our base case. The concentration profiles at both time shots compare very close to the analytical profiles. Once the simulation time has reached 50 days, sufficient time has elapsed such that the concentration profile shape is unaffected by the inlet boundary condition and the mean transport distance (location of $50 \%$ of the solute) corresponds to the retarded velocity times elapsed time. For the base case at 50 days, the mean distance is 200 $\mathrm{m}$ (i.e., $4.0 \mathrm{~m} / \mathrm{d} \times 50 \mathrm{~d}$ ). Characteristic oscillatory overshoot or undershoot, exhibited by secondorder accurate centered spatial differencing, is not observed for the base case because the local cell Peclet number does not exceed 2.

By adjusting the solute distribution coefficient such that the retardation factor becomes 2 (Case A), the retarded dispersion and velocity are halved. These results can be seen in Figure 5.1.4 where (a) the mean transport distance at 50 days is now $100 \mathrm{~m}$ and (b) the spread of the plume has been greatly reduced. Compare the results in Figure 5.1.4 to 5.1.3.

By employing a non-zero radioactive decay coefficient such that the solute now becomes a nonconservative transport species (Case B), the solute concentration profiles are reduced especially at the higher concentration levels. These results can be seen in Figure 5.1.5 as compared to Figure 5.1.3.

By applying both retardation and radioactive decay coefficients simultaneously (Case C), we observe the composite effect as shown in Figure 5.1.6. These results show (a) the mean transport distance at 50 days is now less than $100 \mathrm{~m}$ and (b) the concentration profile has been reduced at the higher concentration levels.

The effect of varying the grid size spacing (Cases D, E, and F) can be seen in Figures 5.1.7 to 5.1.9. As shown, the "effective", numerical plus mechanical, dispersion coefficient continues to increase as the grid spacing increases. Thus, resolution of the concentration front diminishes. The cell Peclet numbers are 4, 8 and 16 for mesh sizes of $\Delta x=20 \mathrm{~m}, 40 \mathrm{~m}$ and $80 \mathrm{~m}$, respectively and we can begin to see significant undershoot and overshoot occurring.

The effect of varying the time-step size (Cases G, H, and I) can be seen in Figures 5.1.10 to 5.1.12. As shown, the "effective", numerical plus mechanical, dispersion coefficient continues to increase as the time-step size increases. Thus, resolution of the concentration front diminishes. At the time-step sizes $\Delta t=5,10$ and 25 days, the cell material Courant and Fourier numbers are exceeding unity and we can begin to see significant undershoot and overshoot occurring.

To see a strong effect of oscillation near the concentration front two case runs (Cases $\mathrm{J}$ and $\mathrm{K}$ ) were performed at a cell Peclet number of a thousand. This high of a cell Peclet number results in the transport of nearly square wave (i.e., plug flow) over the time and distance ranges of interest here. Using the central differencing scheme for integration of the transport equation (Case J) we see oscillatory behavior as illustrated in Figure 5.1.13. The central spatial differencing scheme attempt to capture the very steep concentration resulted in upstream oscillations. These oscillations can be minimized or eliminated by refining the grid spacing. We will restrict ourselves to the grid spacing use in the base case. Applying the default HYBRID, CONDIF or Modified 
QUICK nodal integration schemes (Case K) eliminates the oscillations but results in a significant increase in artificial dispersion.

PORFLOW offers several options for averaging of material properties from node to node. Figure 5.1.15 (Case L) illustrates the impact of arithmetic, harmonic (default option), geometric and upwind averaging of the dispersion coefficient in the transport equation. Since the dispersion coefficient does not vary spatially in this example, you would expect these various averaging schemes to produce identical results as shown in the figure. A better test would be to have the dispersion coefficient varying spatially and then compare the various averaging options.

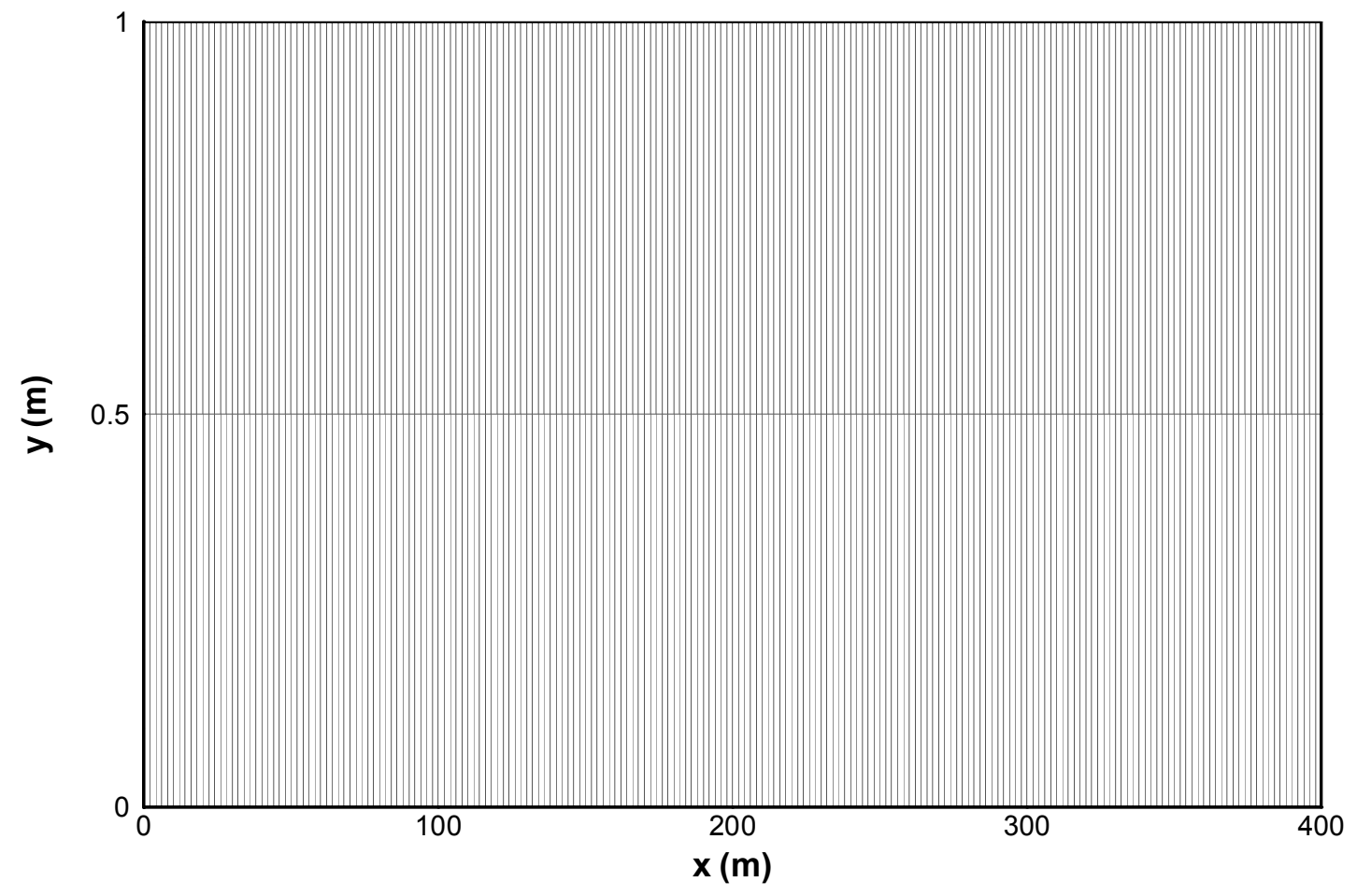

Figure 5.1.2. PORFLOW Grid for 1-D Base Case Transport Simulation. 


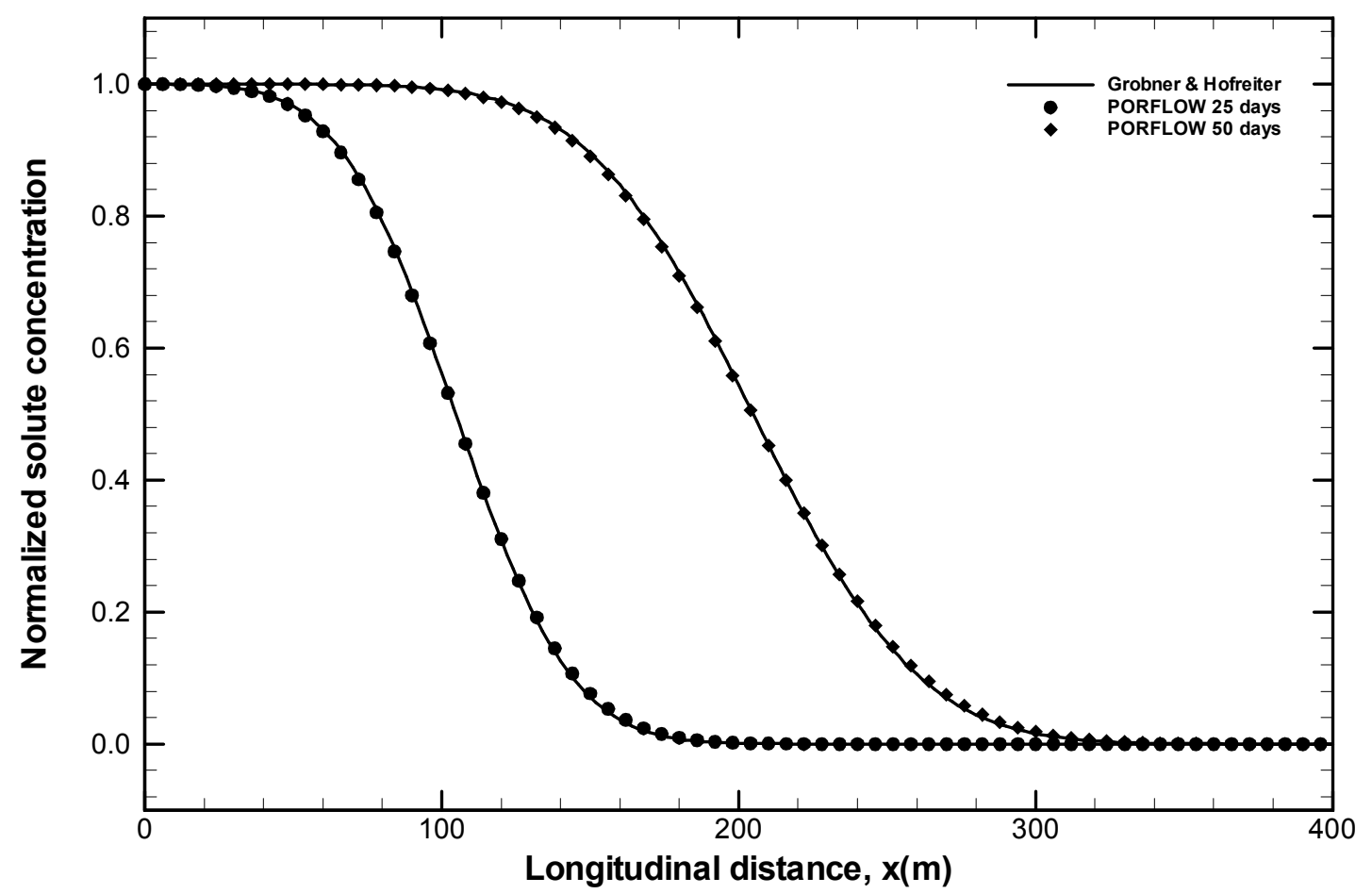

Figure 5.1.3. Concentration Profiles for the 1D Transport of the Base Case.

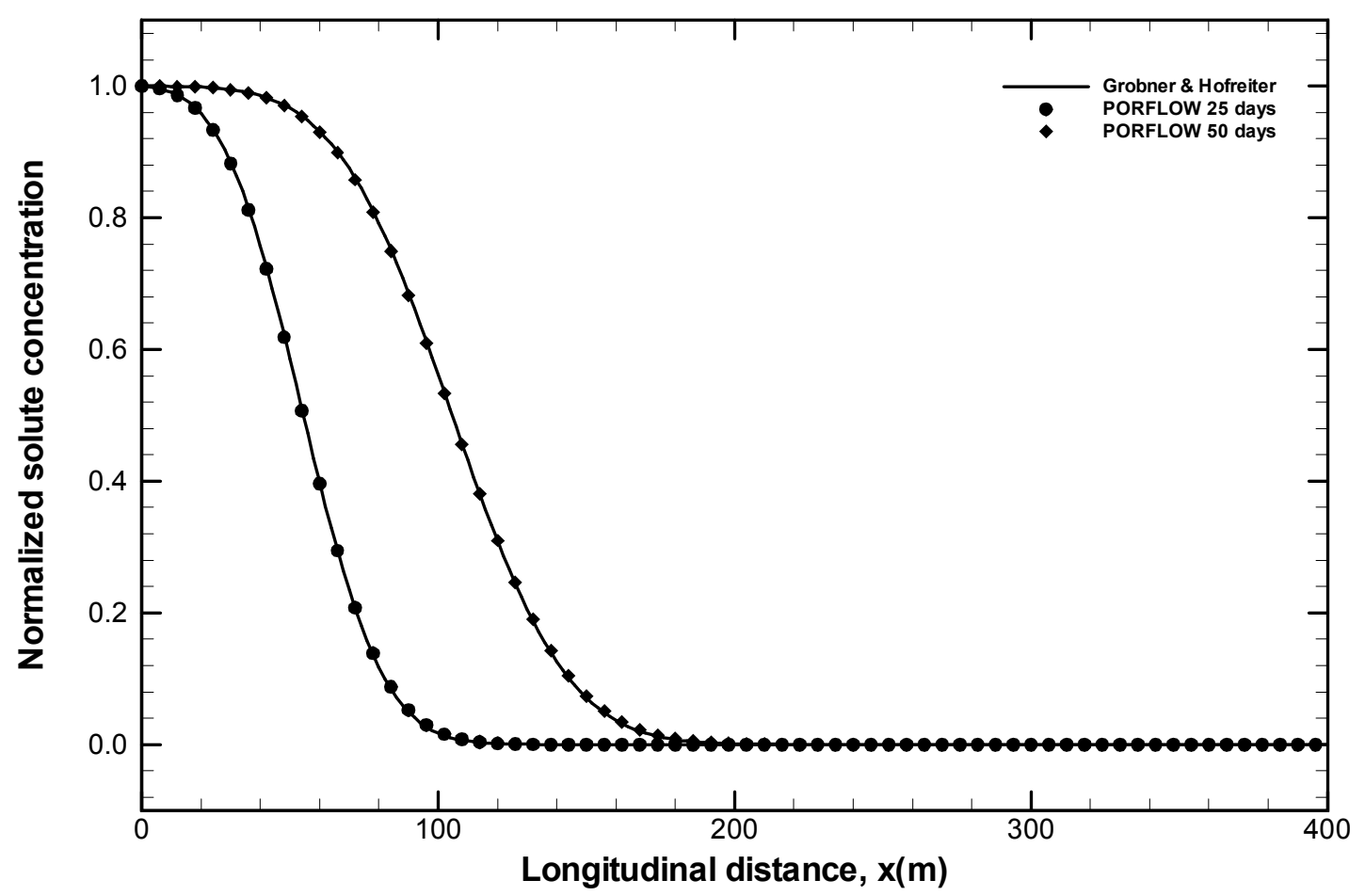

Figure 5.1.4 Concentration Profiles for the 1D Transport Showing Effect of Retardation (Case A). 


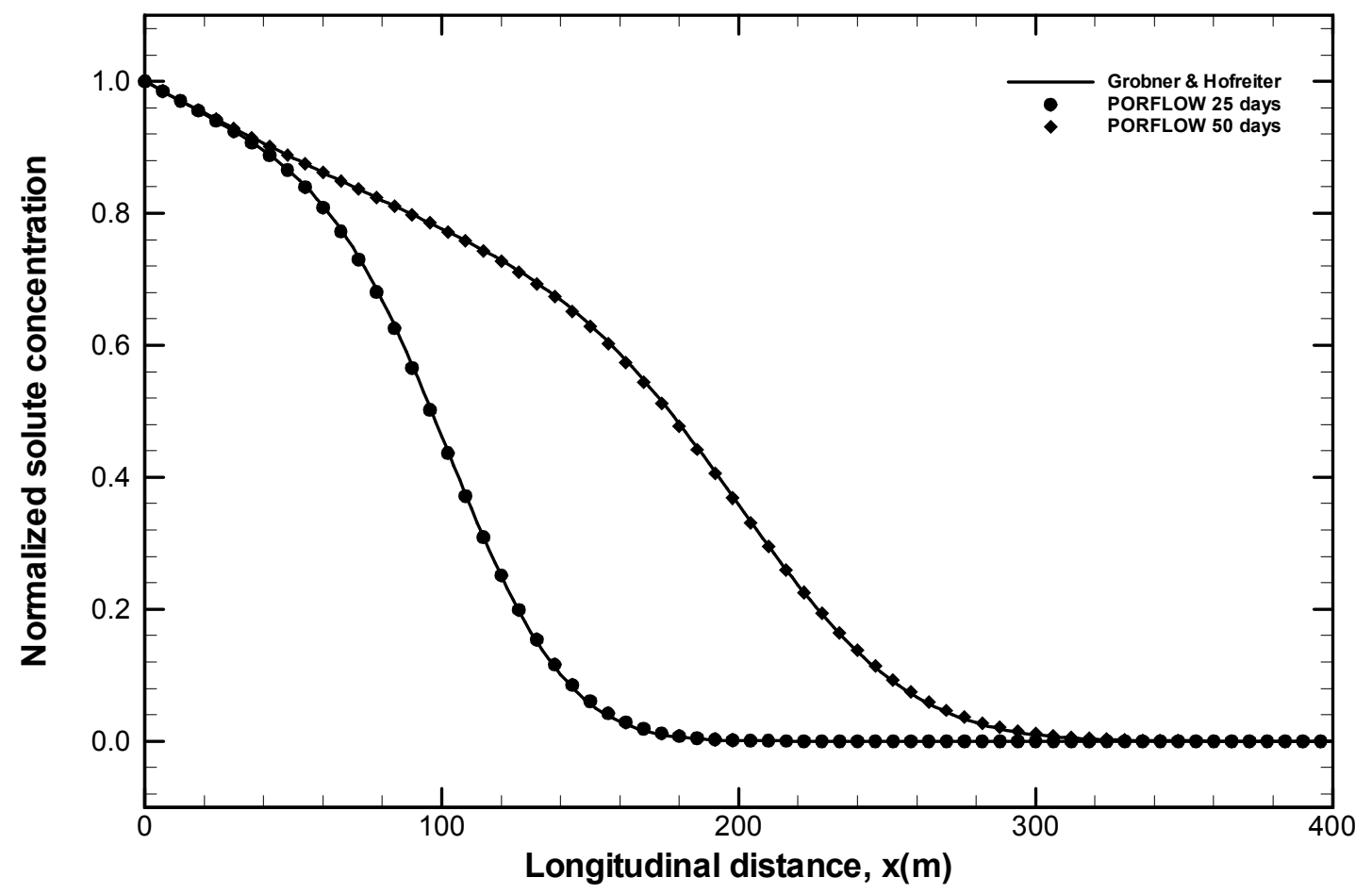

Figure 5.1.5 Concentration Profiles for the 1D Transport Showing Effect of Radioactive Decay (Case B).

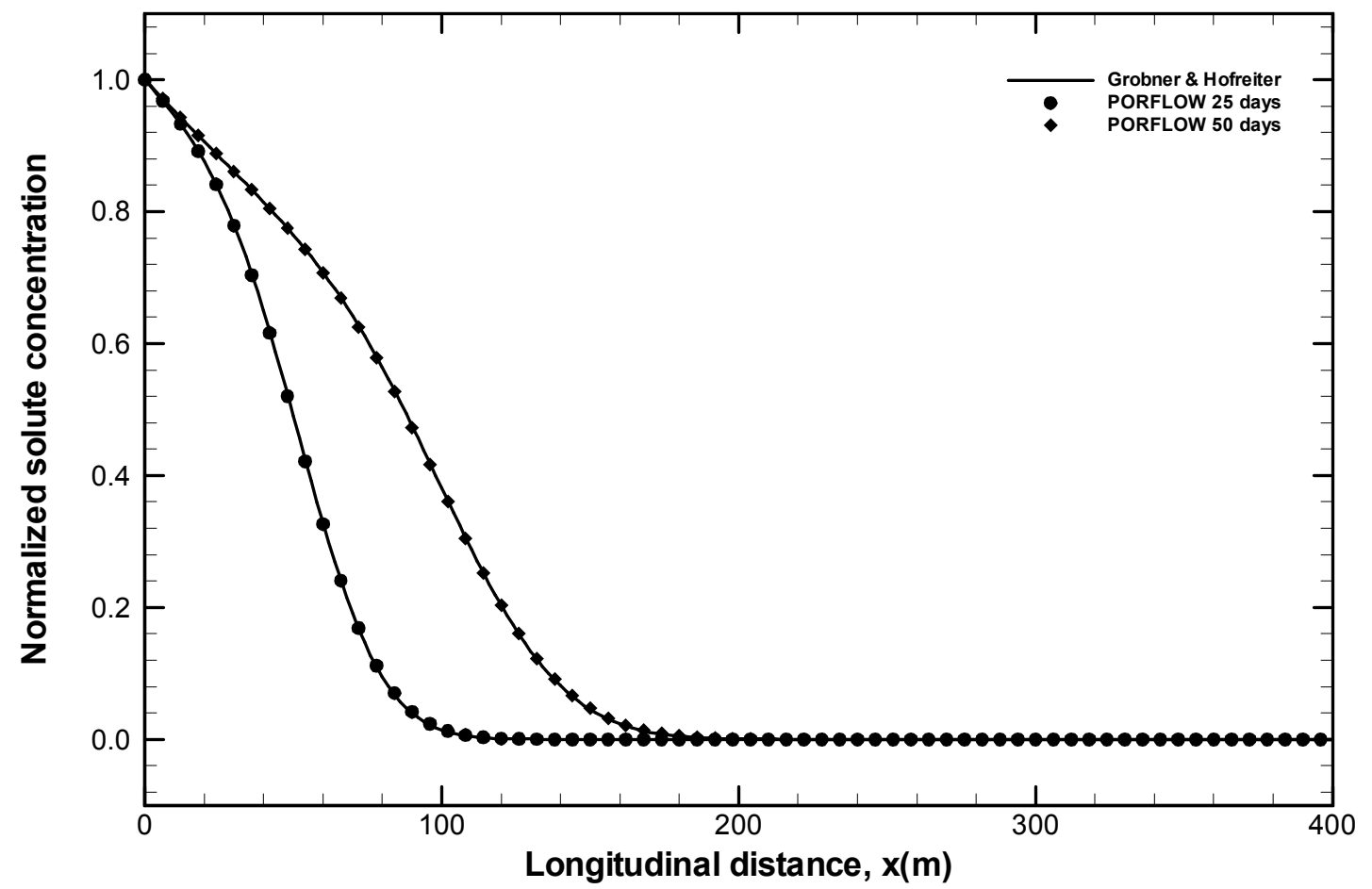

Figure 5.1.6 Concentration Profiles for the 1D Transport Showing Combined Effect of Retardation and Radioactive Decay (Case C). 


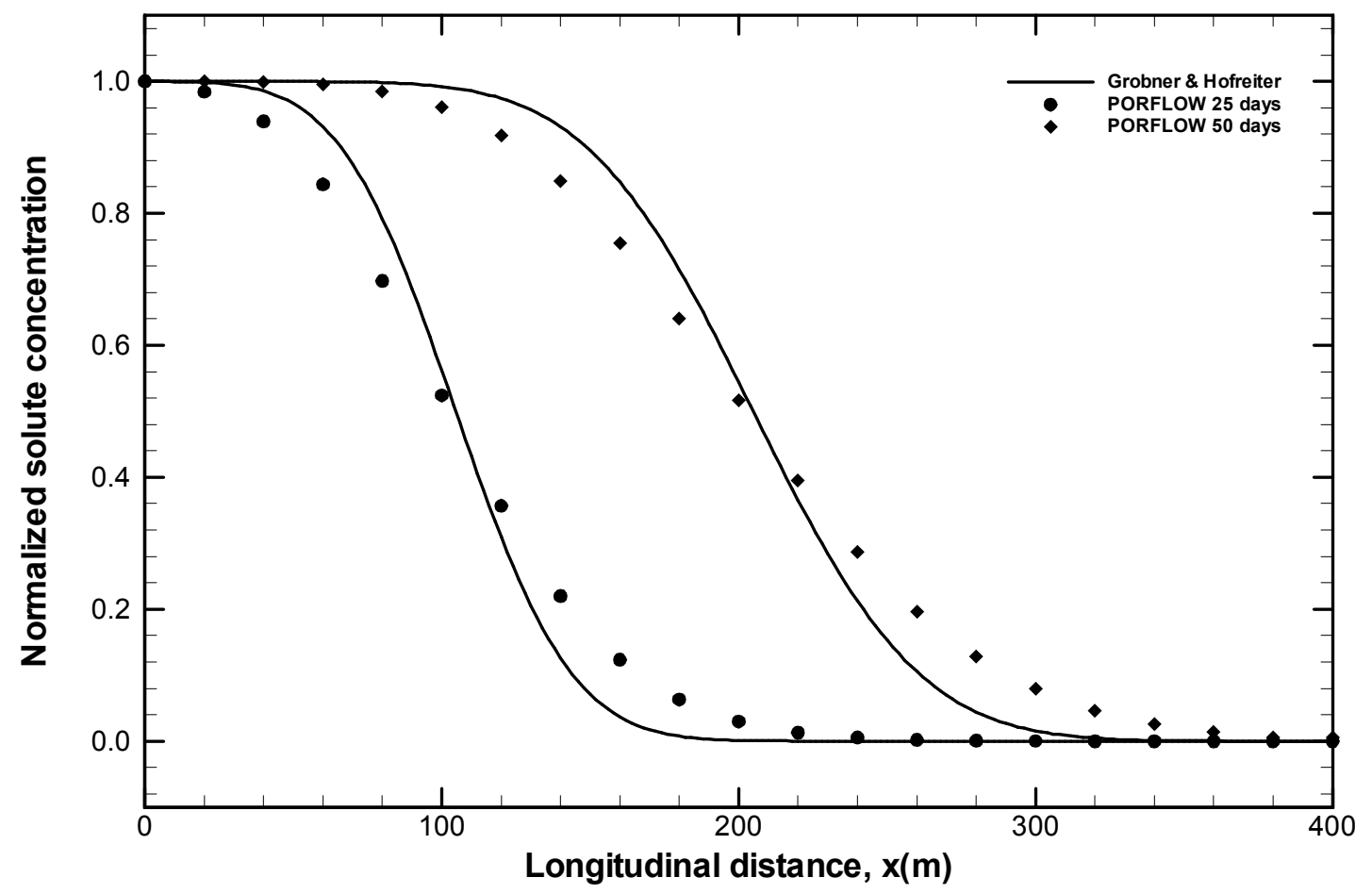

Figure 5.1.7 Concentration Profiles for the 1D Transport Showing Effect of Grid Size, $\Delta \mathbf{x}=20 \mathrm{~m}$ (Case D).

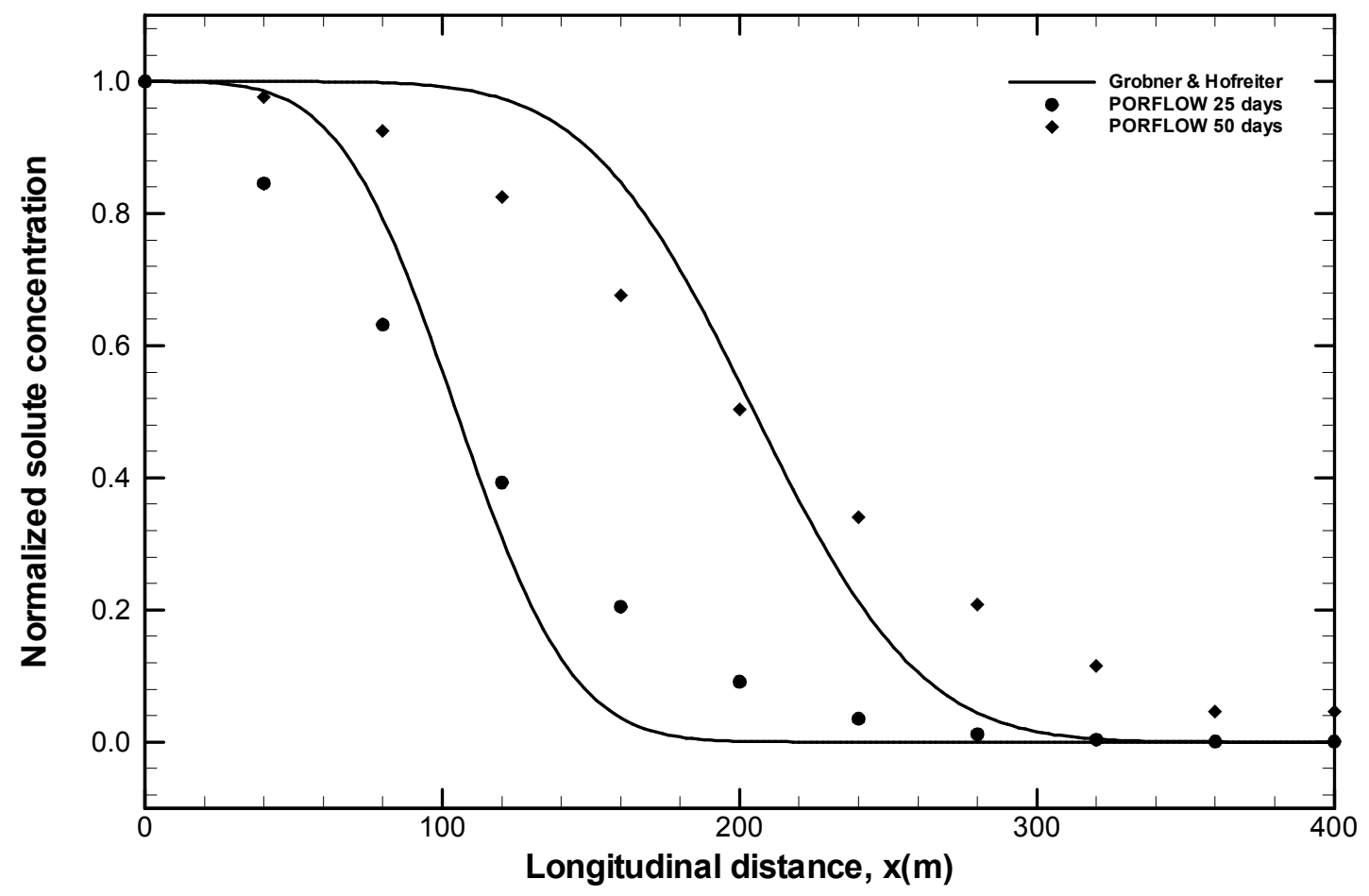

Figure 5.1.8 Concentration Profiles for the 1D Transport Showing Effect of Grid Size, $\Delta x=40$ m (Case E). 


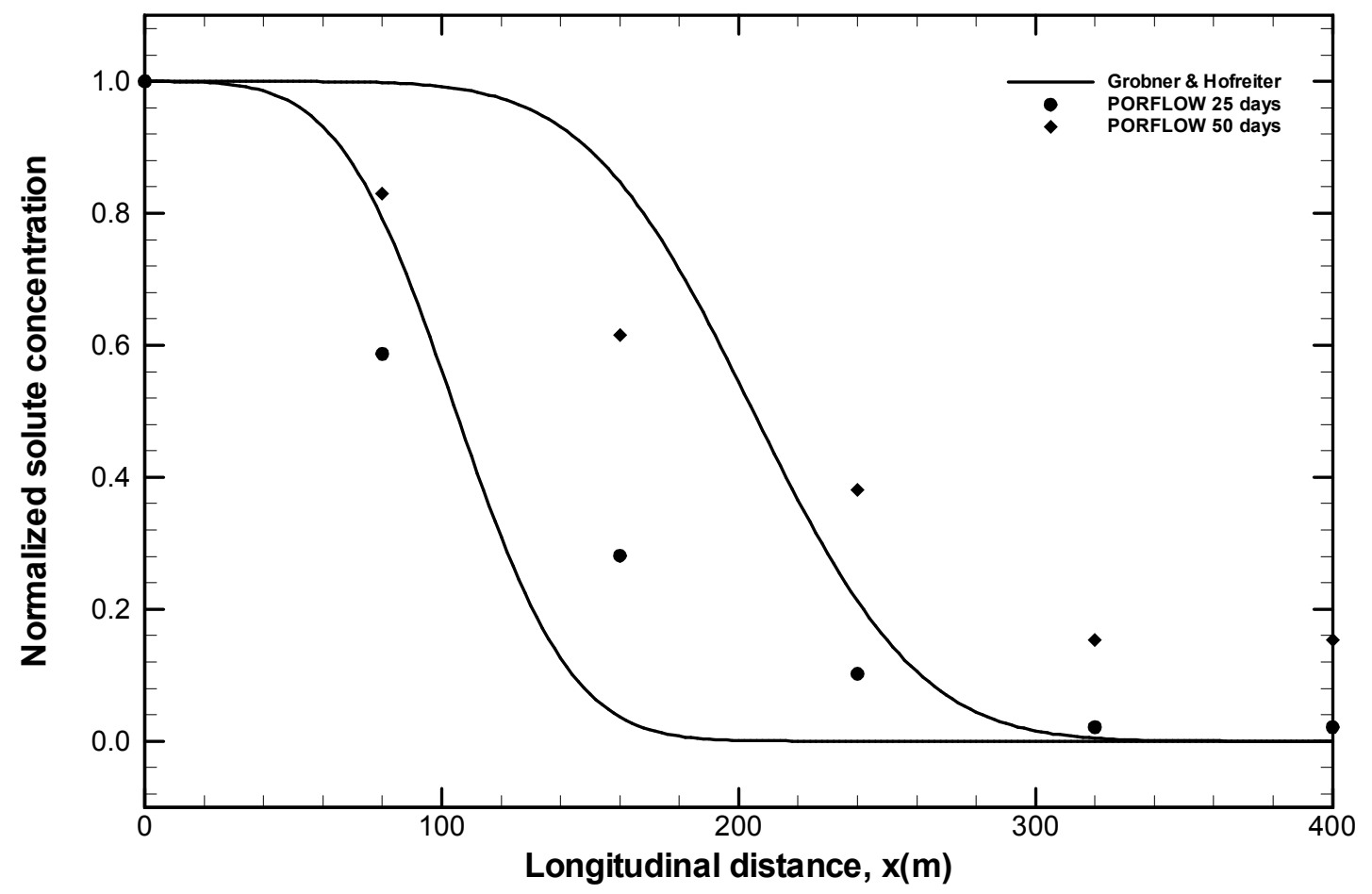

Figure 5.1.9 Concentration Profiles for the 1D Transport Showing Effect of Grid Size, $\Delta x=80$ m (Case F).

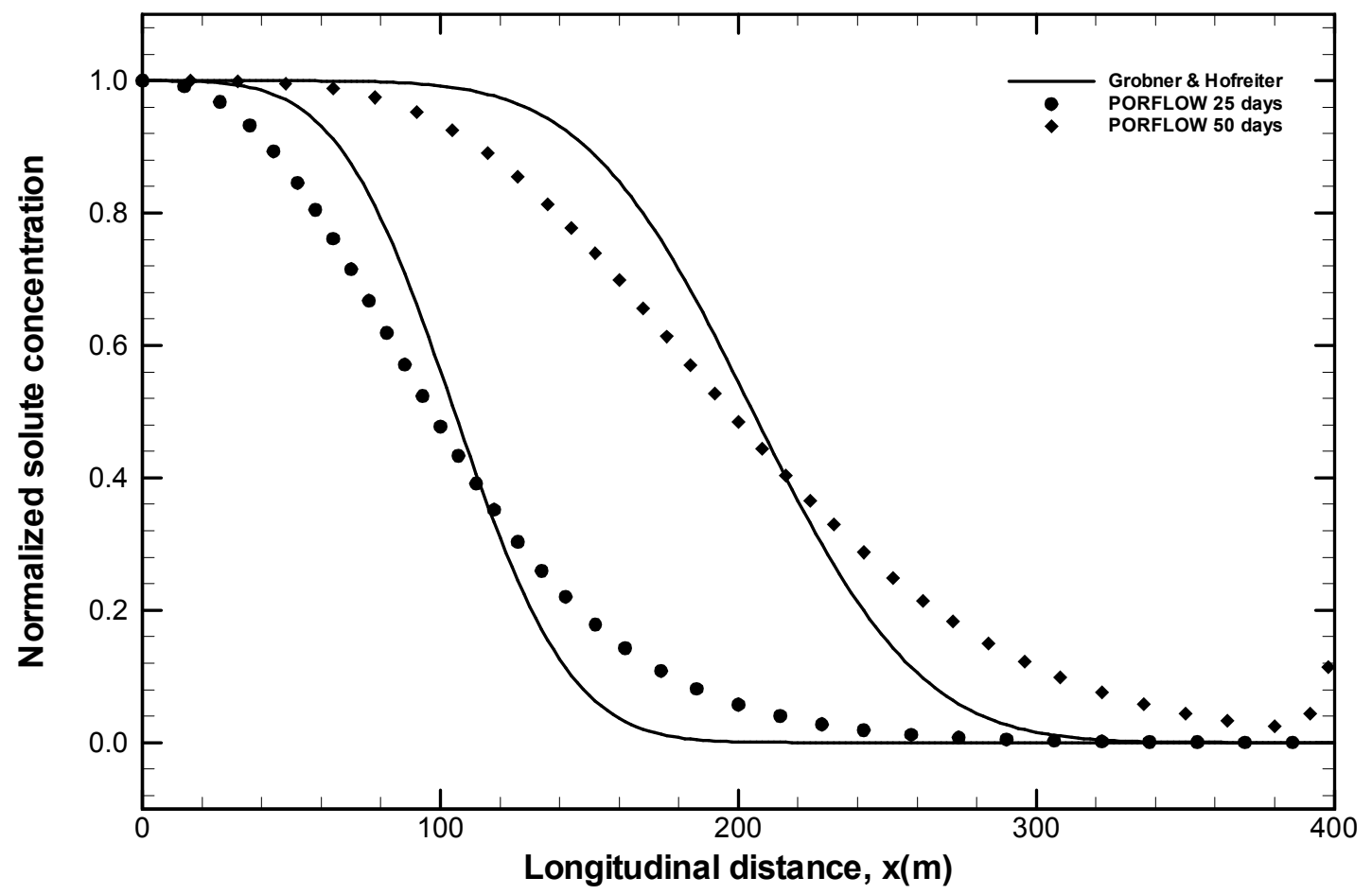

Figure 5.1.10 Concentration Profiles for the 1D Transport Showing Effect of Time Step Size, $\Delta \mathbf{t}=5 \mathrm{~d}$ (Case G). 


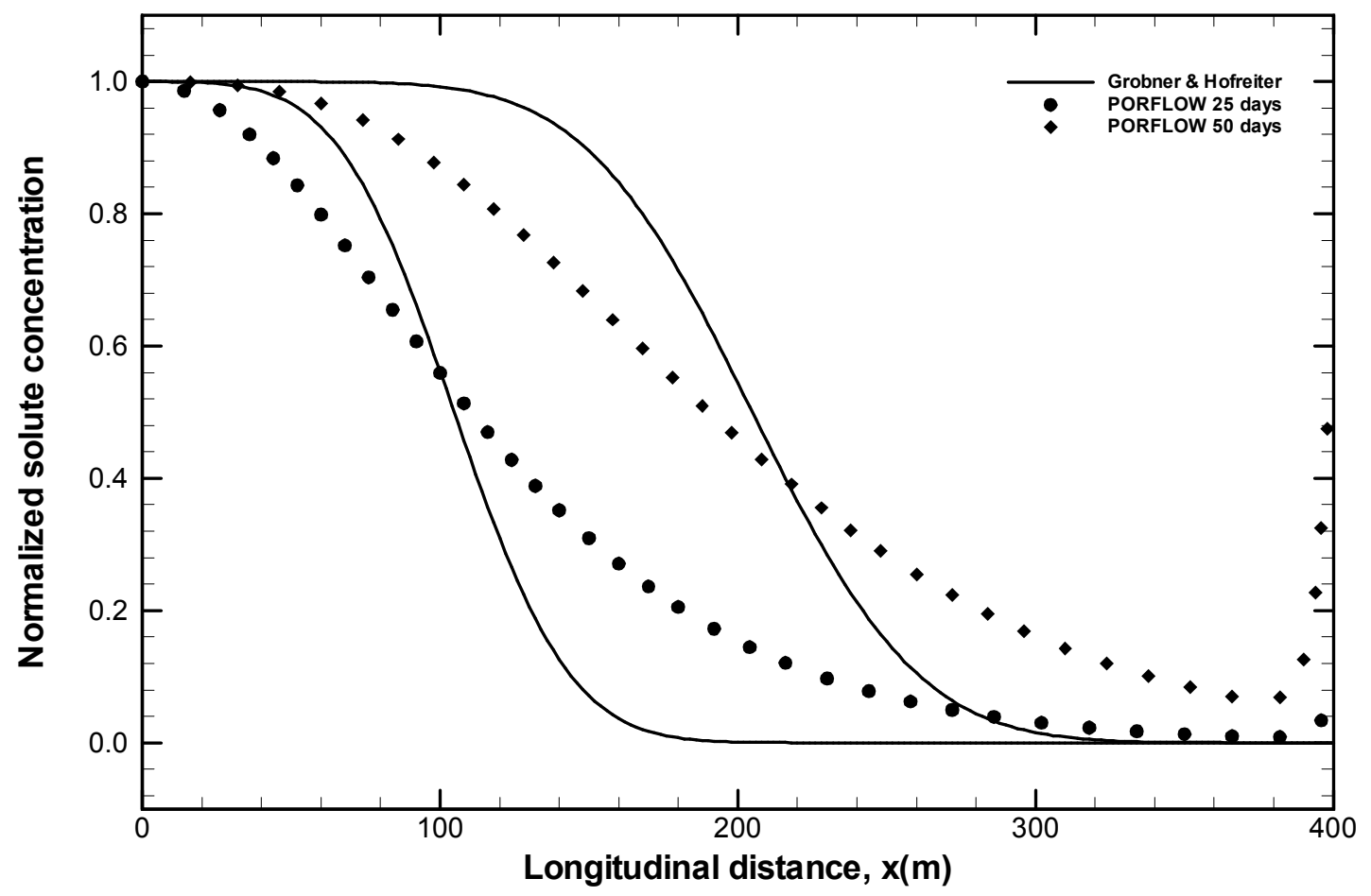

Figure 5.1.11 Concentration Profiles for the 1D Transport Showing Effect of Time Step Size, $\Delta \mathrm{t}=10 \mathrm{~d}$ (Case H).

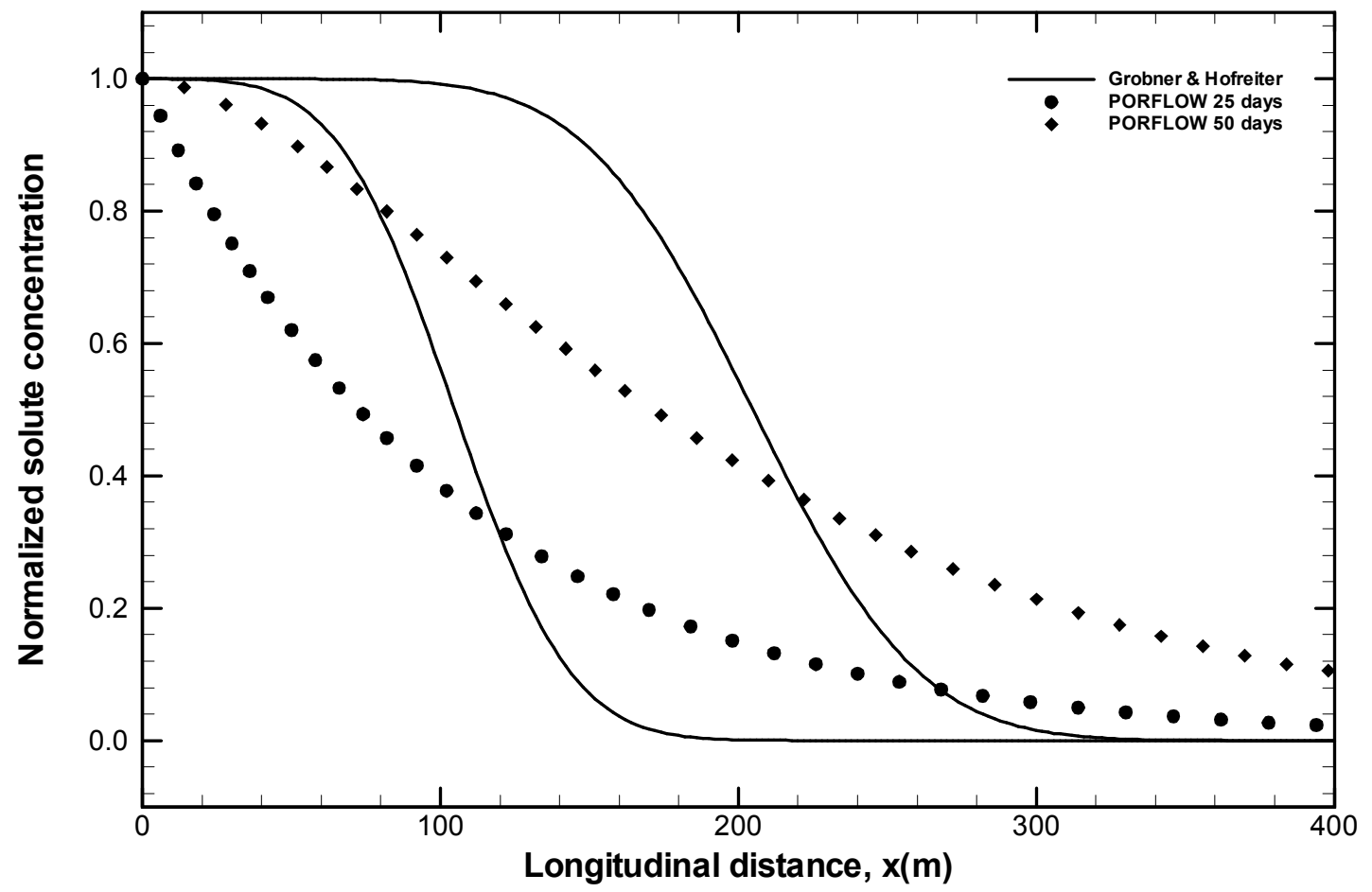

Figure 5.1.12 Concentration Profiles for the 1D Transport Showing Effect of Time Step Size, $\Delta \mathrm{t}=25 \mathrm{~d}$ (Case I). 


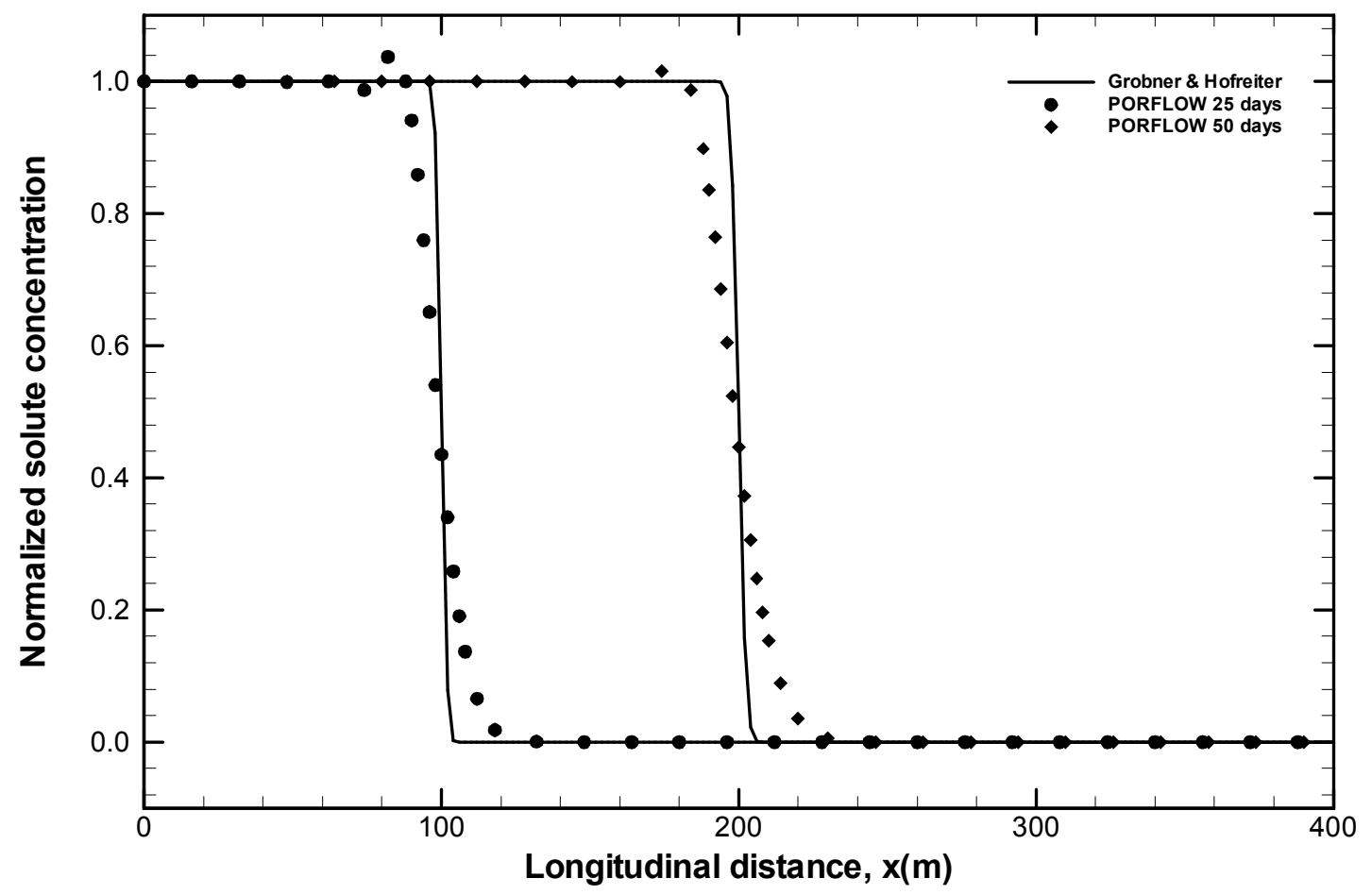

Figure 5.1.13 Concentration Profiles for the 1D Transport at High Peclet Number Showing Effect of Dispersion (Case J).

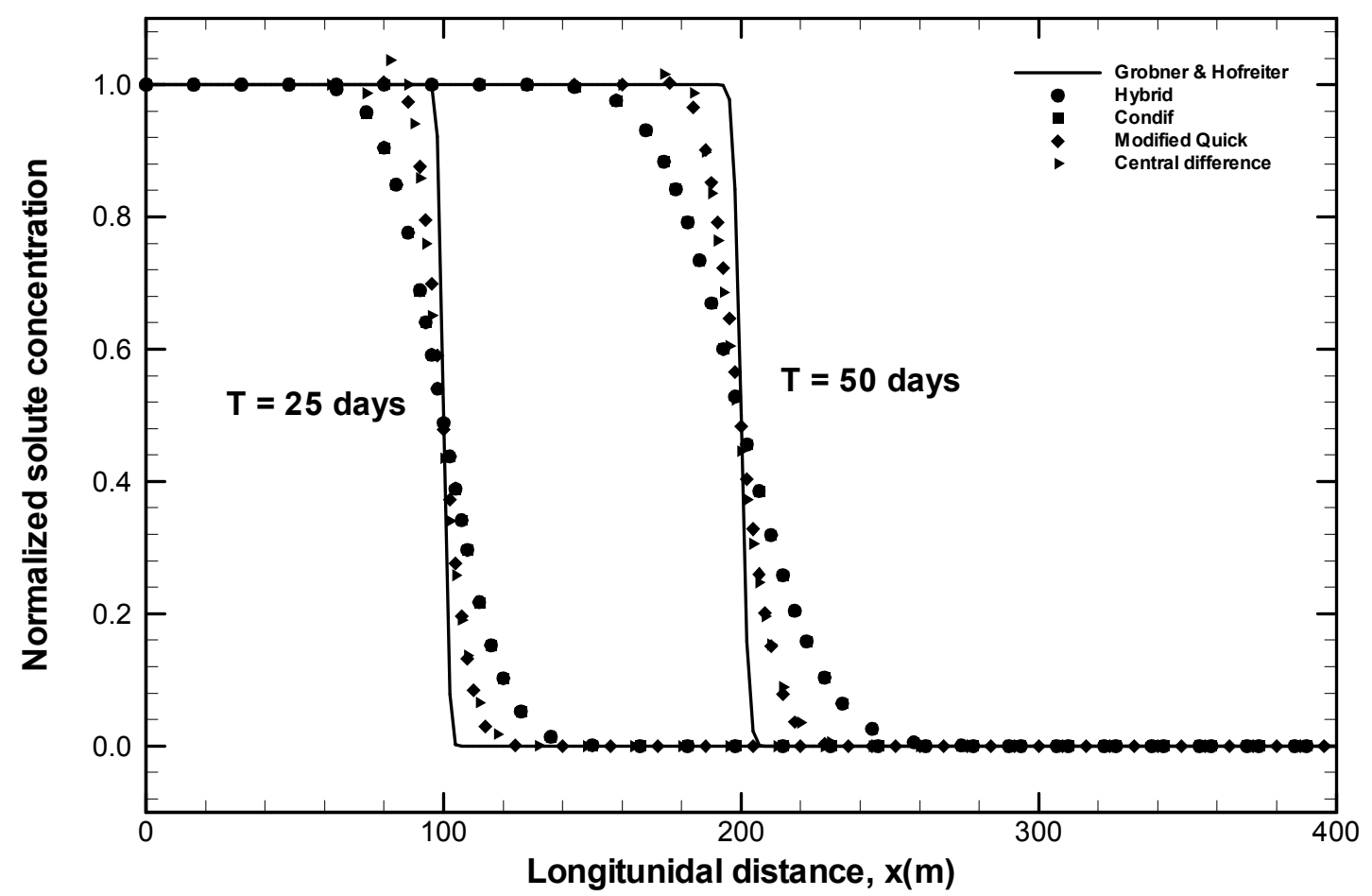

Figure 5.1.14 Concentration Profiles for the 1D Transport at High Peclet Number Showing Effect of Nodal Integration Schemes (Case K). 


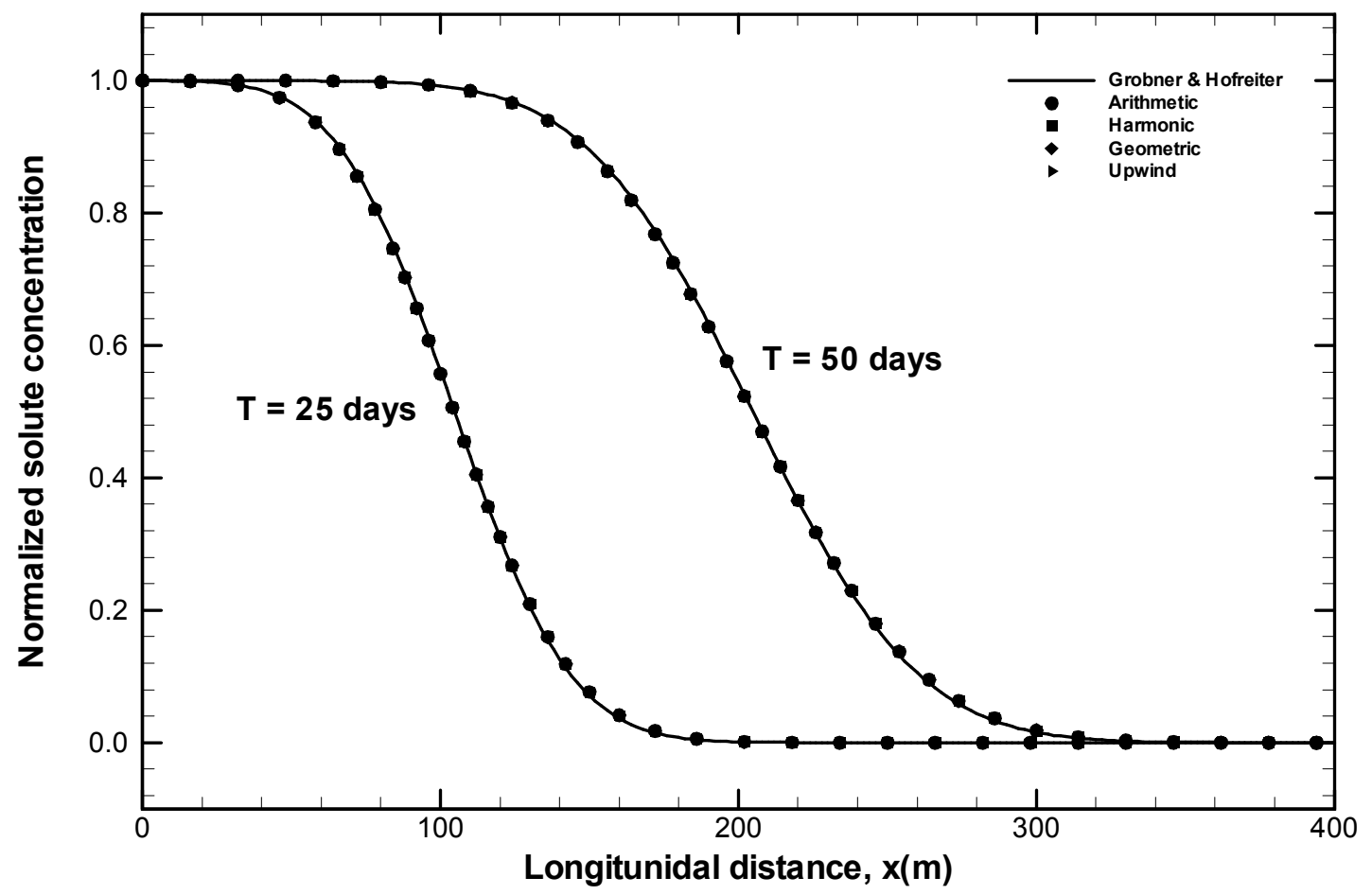

Figure 5.1.15 Concentration Profiles for the 1D Transport Showing Effect of Property Averaging Schemes (Case L).

Table 5.1.3. Comparison of Analytical and PORFLOW Numerical Results for the Transient 1D Transport Problem (Base Case)

\begin{tabular}{|c|c|c|c|c|}
\hline $\begin{array}{l}\text { Distance downstream } \mathrm{x} \\
(\mathrm{m})\end{array}$ & $\begin{array}{c}\text { Analytical } \\
(\mathrm{G \& H}) \\
(\mathrm{t}=25 \text { days })\end{array}$ & $\begin{array}{l}\text { Numerical } \\
\text { (PORFLOW) } \\
(\mathrm{t}=25 \text { days })\end{array}$ & $\begin{array}{c}\text { Analytical } \\
(\mathrm{G} \& \mathrm{H}) \\
(\mathrm{t}=50 \text { days })\end{array}$ & $\begin{array}{c}\text { Numerical } \\
\text { (PORFLOW) } \\
(\mathrm{t}=50 \text { days })\end{array}$ \\
\hline 0 & 1.0000 & 1.0000 & 1.0000 & 1.0000 \\
\hline 10 & 0.9996 & 0.9996 & 1.0000 & 1.0000 \\
\hline 20 & 0.9983 & 0.9983 & 1.0000 & 1.0000 \\
\hline 30 & 0.9945 & 0.9943 & 1.0000 & 1.0000 \\
\hline 40 & 0.9853 & 0.9846 & 0.9999 & 0.9999 \\
\hline 50 & 0.9662 & 0.9647 & 0.9999 & 0.9998 \\
\hline 60 & 0.9312 & 0.9284 & 0.9996 & 0.9996 \\
\hline 70 & 0.8744 & 0.8701 & 0.9991 & 0.9991 \\
\hline 80 & 0.7922 & 0.7867 & 0.9981 & 0.9979 \\
\hline 90 & 0.6856 & 0.6799 & 0.9960 & 0.9957 \\
\hline 100 & 0.5616 & 0.5572 & 0.9921 & 0.9916 \\
\hline 120 & 0.3096 & 0.3107 & 0.9742 & 0.9727 \\
\hline 130 & 0.2057 & 0.2094 & 0.9568 & 0.9546 \\
\hline 140 & 0.1262 & 0.1313 & 0.9311 & 0.9280 \\
\hline 150 & 0.0712 & 0.0765 & 0.8951 & 0.8910 \\
\hline 160 & 0.0368 & 0.0413 & 0.8472 & 0.8424 \\
\hline 170 & 0.0174 & 0.0207 & 0.7868 & 0.7816 \\
\hline
\end{tabular}




\begin{tabular}{|c|c|c|c|c|}
\hline $\begin{array}{l}\text { Distance downstream } \mathrm{x} \\
(\mathrm{m})\end{array}$ & $\begin{array}{c}\text { Analytical } \\
(\mathrm{G} \& H) \\
(\mathrm{t}=25 \text { days })\end{array}$ & $\begin{array}{c}\text { Numerical } \\
\text { (PORFLOW) } \\
(\mathrm{t}=25 \text { days })\end{array}$ & $\begin{array}{c}\text { Analytical } \\
(\mathrm{G} \& H) \\
(\mathrm{t}=50 \text { days })\end{array}$ & $\begin{array}{c}\text { Numerical } \\
\text { (PORFLOW) } \\
(\mathrm{t}=50 \text { days })\end{array}$ \\
\hline 180 & 0.0075 & 0.0096 & 0.7146 & 0.7094 \\
\hline 190 & 0.0030 & 0.0041 & 0.6325 & 0.6281 \\
\hline 200 & 0.0011 & 0.0017 & 0.5441 & 0.5409 \\
\hline 210 & 0.0003 & 0.0006 & 0.4535 & 0.4521 \\
\hline 220 & 0.0001 & 0.0002 & 0.3654 & 0.3659 \\
\hline 230 & 0.0000 & 0.0001 & 0.2840 & 0.2863 \\
\hline 240 & 0.0000 & 0.0000 & 0.2125 & 0.2163 \\
\hline 250 & 0.0000 & 0.0000 & 0.1528 & 0.1576 \\
\hline 260 & 0.0000 & 0.0000 & 0.1055 & 0.1106 \\
\hline 270 & 0.0000 & 0.0000 & 0.0698 & 0.0747 \\
\hline 280 & 0.0000 & 0.0000 & 0.0443 & 0.0485 \\
\hline 290 & 0.0000 & 0.0000 & 0.0269 & 0.0303 \\
\hline 300 & 0.0000 & 0.0000 & 0.0156 & 0.0182 \\
\hline 310 & 0.0000 & 0.0000 & 0.0086 & 0.0105 \\
\hline 320 & 0.0000 & 0.0000 & 0.0046 & 0.0059 \\
\hline 330 & 0.0000 & 0.0000 & 0.0023 & 0.0031 \\
\hline 340 & 0.0000 & 0.0000 & 0.0011 & 0.0016 \\
\hline 350 & 0.0000 & 0.0000 & 0.0005 & 0.0008 \\
\hline 360 & 0.0000 & 0.0000 & 0.0002 & 0.0004 \\
\hline 370 & 0.0000 & 0.0000 & 0.0001 & 0.0002 \\
\hline 380 & 0.0000 & 0.0000 & 0.0000 & 0.0001 \\
\hline 390 & 0.0000 & 0.0000 & 0.0000 & 0.0000 \\
\hline 400 & 0.0000 & 0.0000 & 0.0000 & 0.0000 \\
\hline
\end{tabular}

Table 5.1.4. Comparison of Analytical and PORFLOW Numerical Results for the Transient 1D Transport Problem (Case A)

\begin{tabular}{|c|c|c|c|c|}
\hline $\begin{array}{l}\text { Distance downstream } \mathrm{x} \\
(\mathrm{m})\end{array}$ & $\begin{array}{c}\text { Analytical } \\
(\mathrm{G} \& \mathrm{H}) \\
(\mathrm{t}=25 \text { days })\end{array}$ & $\begin{array}{c}\text { Numerical } \\
\text { (PORFLOW) } \\
(\mathrm{t}=25 \text { days })\end{array}$ & $\begin{array}{c}\text { Analytical } \\
(\mathrm{G} \& \mathrm{H}) \\
(\mathrm{t}=50 \text { days })\end{array}$ & $\begin{array}{c}\text { Numerical } \\
\text { (PORFLOW) } \\
(\mathrm{t}=50 \text { days })\end{array}$ \\
\hline 0 & 1.0000 & 1.0000 & 1.0000 & 1.0000 \\
\hline 10 & 0.9901 & 0.9902 & 0.9996 & 0.9997 \\
\hline 20 & 0.9578 & 0.9574 & 0.9983 & 0.9983 \\
\hline 30 & 0.8844 & 0.8826 & 0.9945 & 0.9945 \\
\hline 40 & 0.7576 & 0.7542 & 0.9853 & 0.9851 \\
\hline 50 & 0.5853 & 0.5817 & 0.9662 & 0.9656 \\
\hline 60 & 0.3981 & 0.3962 & 0.9312 & 0.9300 \\
\hline 70 & 0.2338 & 0.2347 & 0.8745 & 0.8724 \\
\hline 80 & 0.1170 & 0.1199 & 0.7923 & 0.7894 \\
\hline 90 & 0.0494 & 0.0525 & 0.6857 & 0.6826 \\
\hline 100 & 0.0175 & 0.0197 & 0.5617 & 0.5590 \\
\hline 120 & 0.0013 & 0.0017 & 0.3096 & 0.3099 \\
\hline 130 & 0.0003 & 0.0004 & 0.2058 & 0.2075 \\
\hline 140 & 0.0000 & 0.0001 & 0.1262 & 0.1289 \\
\hline 150 & 0.0000 & 0.0000 & 0.0712 & 0.0740 \\
\hline
\end{tabular}




\begin{tabular}{|c|c|c|c|c|}
\hline $\begin{array}{l}\text { Distance downstream } \mathrm{x} \\
(\mathrm{m})\end{array}$ & $\begin{array}{c}\text { Analytical } \\
(\mathrm{G} \& \mathrm{H}) \\
(\mathrm{t}=25 \text { days })\end{array}$ & $\begin{array}{l}\text { Numerical } \\
\text { (PORFLOW) } \\
(\mathrm{t}=25 \text { days })\end{array}$ & $\begin{array}{c}\text { Analytical } \\
(\mathrm{G} \& \mathrm{H}) \\
(\mathrm{t}=50 \text { days })\end{array}$ & $\begin{array}{c}\text { Numerical } \\
\text { (PORFLOW) } \\
(\mathrm{t}=50 \text { days })\end{array}$ \\
\hline 160 & 0.0000 & 0.0000 & 0.0368 & 0.0393 \\
\hline 170 & 0.0000 & 0.0000 & 0.0174 & 0.0192 \\
\hline 180 & 0.0000 & 0.0000 & 0.0075 & 0.0087 \\
\hline 190 & 0.0000 & 0.0000 & 0.0030 & 0.0036 \\
\hline 200 & 0.0000 & 0.0000 & 0.0011 & 0.0014 \\
\hline 210 & 0.0000 & 0.0000 & 0.0003 & 0.0005 \\
\hline 220 & 0.0000 & 0.0000 & 0.0001 & 0.0002 \\
\hline 230 & 0.0000 & 0.0000 & 0.0000 & 0.0000 \\
\hline 240 & 0.0000 & 0.0000 & 0.0000 & 0.0000 \\
\hline 250 & 0.0000 & 0.0000 & 0.0000 & 0.0000 \\
\hline 260 & 0.0000 & 0.0000 & 0.0000 & 0.0000 \\
\hline 270 & 0.0000 & 0.0000 & 0.0000 & 0.0000 \\
\hline 280 & 0.0000 & 0.0000 & 0.0000 & 0.0000 \\
\hline 290 & 0.0000 & 0.0000 & 0.0000 & 0.0000 \\
\hline 300 & 0.0000 & 0.0000 & 0.0000 & 0.0000 \\
\hline 310 & 0.0000 & 0.0000 & 0.0000 & 0.0000 \\
\hline 320 & 0.0000 & 0.0000 & 0.0000 & 0.0000 \\
\hline 330 & 0.0000 & 0.0000 & 0.0000 & 0.0000 \\
\hline 340 & 0.0000 & 0.0000 & 0.0000 & 0.0000 \\
\hline 350 & 0.0000 & 0.0000 & 0.0000 & 0.0000 \\
\hline 360 & 0.0000 & 0.0000 & 0.0000 & 0.0000 \\
\hline 370 & 0.0000 & 0.0000 & 0.0000 & 0.0000 \\
\hline 380 & 0.0000 & 0.0000 & 0.0000 & 0.0000 \\
\hline 390 & 0.0000 & 0.0000 & 0.0000 & 0.0000 \\
\hline 400 & 0.0000 & 0.0000 & 0.0000 & 0.0000 \\
\hline
\end{tabular}

Table 5.1.5. Comparison of Analytical and PORFLOW Numerical Results for the Transient 1D Transport Problem (Case B)

\begin{tabular}{|c|cc|cc|}
\hline $\begin{array}{c}\text { Distance downstream x } \\
(\mathrm{m})\end{array}$ & $\begin{array}{c}\text { Analytical } \\
(\mathrm{G} \& \mathrm{H}) \\
(\mathrm{t}=25 \text { days })\end{array}$ & $\begin{array}{c}\text { Numerical } \\
(\text { PORFLOW })\end{array}$ & $\begin{array}{c}\text { Analytical } \\
(\mathrm{G} \& \mathrm{t}=25 \text { days })\end{array}$ & $\begin{array}{c}\text { Numerical } \\
(\mathrm{PORFLOW})\end{array}$ \\
\hline 0 & 1.0000 & 1.0000 & 1.0000 & 1.0000 \\
\hline 10 & 0.9753 & 0.9753 & 0.9756 & 0.9756 \\
\hline 20 & 0.9506 & 0.9505 & 0.9518 & 0.9518 \\
\hline 30 & 0.9245 & 0.9243 & 0.9286 & 0.9286 \\
\hline 40 & 0.8950 & 0.8944 & 0.9059 & 0.9059 \\
\hline 50 & 0.8587 & 0.8574 & 0.8838 & 0.8837 \\
\hline 60 & 0.8112 & 0.8088 & 0.8621 & 0.8620 \\
\hline 70 & 0.7484 & 0.7447 & 0.8407 & 0.8407 \\
\hline 80 & 0.6677 & 0.6630 & 0.8196 & 0.8195 \\
\hline 90 & 0.5705 & 0.5656 & 0.7984 & 0.7982 \\
\hline 100 & 0.4623 & 0.4584 & 0.7766 & 0.7763 \\
\hline 120 & 0.3524 & 0.3504 & 0.7537 & 0.7531 \\
\hline 130 & 0.2508 & 0.2513 & 0.7288 & 0.7278 \\
\hline
\end{tabular}




\begin{tabular}{|c|c|c|c|c|}
\hline $\begin{array}{l}\text { Distance downstream } \mathrm{x} \\
(\mathrm{m})\end{array}$ & $\begin{array}{c}\text { Analytical } \\
(\mathrm{G} \& \mathrm{H}) \\
(\mathrm{t}=25 \text { days })\end{array}$ & $\begin{array}{l}\text { Numerical } \\
\text { (PORFLOW) } \\
(\mathrm{t}=25 \text { days })\end{array}$ & $\begin{array}{c}\text { Analytical } \\
(\mathrm{G} \& \mathrm{H}) \\
(\mathrm{t}=50 \text { days })\end{array}$ & $\begin{array}{c}\text { Numerical } \\
\text { (PORFLOW) } \\
(\mathrm{t}=50 \text { days })\end{array}$ \\
\hline 140 & 0.1657 & 0.1683 & 0.7007 & 0.6992 \\
\hline 150 & 0.1012 & 0.1049 & 0.6684 & 0.6663 \\
\hline 160 & 0.0568 & 0.0608 & 0.6308 & 0.6281 \\
\hline 170 & 0.0293 & 0.0327 & 0.5871 & 0.5839 \\
\hline 180 & 0.0138 & 0.0163 & 0.5371 & 0.5335 \\
\hline 190 & 0.0060 & 0.0075 & 0.4812 & 0.4777 \\
\hline 200 & 0.0023 & 0.0032 & 0.4210 & 0.4178 \\
\hline 210 & 0.0008 & 0.0013 & 0.3584 & 0.3559 \\
\hline 220 & 0.0003 & 0.0005 & 0.2960 & 0.2946 \\
\hline 230 & 0.0001 & 0.0002 & 0.2366 & 0.2365 \\
\hline 240 & 0.0000 & 0.0001 & 0.1826 & 0.1836 \\
\hline 250 & 0.0000 & 0.0000 & 0.1358 & 0.1378 \\
\hline 260 & 0.0000 & 0.0000 & 0.0972 & 0.0998 \\
\hline 270 & 0.0000 & 0.0000 & 0.0668 & 0.0696 \\
\hline 280 & 0.0000 & 0.0000 & 0.0440 & 0.0468 \\
\hline 290 & 0.0000 & 0.0000 & 0.0278 & 0.0303 \\
\hline 300 & 0.0000 & 0.0000 & 0.0168 & 0.0189 \\
\hline 310 & 0.0000 & 0.0000 & 0.0097 & 0.0113 \\
\hline 320 & 0.0000 & 0.0000 & 0.0054 & 0.0065 \\
\hline 330 & 0.0000 & 0.0000 & 0.0028 & 0.0036 \\
\hline 340 & 0.0000 & 0.0000 & 0.0014 & 0.0019 \\
\hline 350 & 0.0000 & 0.0000 & 0.0007 & 0.0010 \\
\hline 360 & 0.0000 & 0.0000 & 0.0003 & 0.0005 \\
\hline 370 & 0.0000 & 0.0000 & 0.0001 & 0.0002 \\
\hline 380 & 0.0000 & 0.0000 & 0.0001 & 0.0001 \\
\hline 390 & 0.0000 & 0.0000 & 0.0000 & 0.0000 \\
\hline 400 & 0.0000 & 0.0000 & 0.0000 & 0.0000 \\
\hline
\end{tabular}

Table 5.1.6. Comparison of Analytical and PORFLOW Numerical Results for the Transient 1D Transport Problem (Case C)

\begin{tabular}{|c|cc|cc|}
\hline $\begin{array}{c}\text { Distance downstream } x \\
(\mathrm{~m})\end{array}$ & $\begin{array}{c}\text { Analytical } \\
(\mathrm{G} \& \mathrm{H}) \\
(\mathrm{t}=25 \text { days })\end{array}$ & $\begin{array}{c}\text { Numerical } \\
(\text { PORFLOW) } \\
(\mathrm{t}=25 \text { days })\end{array}$ & $\begin{array}{c}\text { Analytical } \\
(\mathrm{G} \& \mathrm{H}) \\
(\mathrm{t}=50 \text { days })\end{array}$ & $\begin{array}{c}\text { Numerical } \\
(\text { PORFLOW }) \\
(\mathrm{t}=50 \text { days })\end{array}$ \\
\hline 0 & 1.0000 & 1.0000 & 1.0000 & 1.0000 \\
\hline 10 & 0.9452 & 0.9452 & 0.9522 & 0.9521 \\
\hline 20 & 0.8764 & 0.8760 & 0.9061 & 0.9060 \\
\hline 30 & 0.7805 & 0.7789 & 0.8607 & 0.8607 \\
\hline 40 & 0.6495 & 0.6465 & 0.8145 & 0.8143 \\
\hline 50 & 0.4908 & 0.4875 & 0.7647 & 0.7642 \\
\hline 60 & 0.3283 & 0.3264 & 0.7082 & 0.7073 \\
\hline 70 & 0.1905 & 0.1909 & 0.6417 & 0.6402 \\
\hline 80 & 0.0945 & 0.0965 & 0.5638 & 0.5617 \\
\hline 90 & 0.0396 & 0.0419 & 0.4754 & 0.4731 \\
\hline 100 & 0.0139 & 0.0156 & 0.3811 & 0.3791 \\
\hline
\end{tabular}




\begin{tabular}{|c|c|c|c|c|}
\hline $\begin{array}{l}\text { Distance downstream } \mathrm{x} \\
(\mathrm{m})\end{array}$ & $\begin{array}{c}\text { Analytical } \\
(\mathrm{G} \& \mathrm{H}) \\
(\mathrm{t}=25 \text { days })\end{array}$ & $\begin{array}{l}\text { Numerical } \\
\text { (PORFLOW) } \\
(\mathrm{t}=25 \text { days })\end{array}$ & $\begin{array}{c}\text { Analytical } \\
(\mathrm{G \& H}) \\
(\mathrm{t}=50 \text { days })\end{array}$ & $\begin{array}{c}\text { Numerical } \\
\text { (PORFLOW) } \\
(\mathrm{t}=50 \text { days })\end{array}$ \\
\hline 120 & 0.0041 & 0.0050 & 0.2879 & 0.2867 \\
\hline 130 & 0.0010 & 0.0014 & 0.2035 & 0.2033 \\
\hline 140 & 0.0002 & 0.0003 & 0.1336 & 0.1344 \\
\hline 150 & 0.0000 & 0.0001 & 0.0812 & 0.0826 \\
\hline 160 & 0.0000 & 0.0000 & 0.0454 & 0.0470 \\
\hline 170 & 0.0000 & 0.0000 & 0.0233 & 0.0248 \\
\hline 180 & 0.0000 & 0.0000 & 0.0110 & 0.0120 \\
\hline 190 & 0.0000 & 0.0000 & 0.0047 & 0.0054 \\
\hline 200 & 0.0000 & 0.0000 & 0.0019 & 0.0022 \\
\hline 210 & 0.0000 & 0.0000 & 0.0007 & 0.0009 \\
\hline 220 & 0.0000 & 0.0000 & 0.0002 & 0.0003 \\
\hline 230 & 0.0000 & 0.0000 & 0.0001 & 0.0001 \\
\hline 240 & 0.0000 & 0.0000 & 0.0000 & 0.0000 \\
\hline 250 & 0.0000 & 0.0000 & 0.0000 & 0.0000 \\
\hline 260 & 0.0000 & 0.0000 & 0.0000 & 0.0000 \\
\hline 270 & 0.0000 & 0.0000 & 0.0000 & 0.0000 \\
\hline 280 & 0.0000 & 0.0000 & 0.0000 & 0.0000 \\
\hline 290 & 0.0000 & 0.0000 & 0.0000 & 0.0000 \\
\hline 300 & 0.0000 & 0.0000 & 0.0000 & 0.0000 \\
\hline 310 & 0.0000 & 0.0000 & 0.0000 & 0.0000 \\
\hline 320 & 0.0000 & 0.0000 & 0.0000 & 0.0000 \\
\hline 330 & 0.0000 & 0.0000 & 0.0000 & 0.0000 \\
\hline 340 & 0.0000 & 0.0000 & 0.0000 & 0.0000 \\
\hline 350 & 0.0000 & 0.0000 & 0.0000 & 0.0000 \\
\hline 360 & 0.0000 & 0.0000 & 0.0000 & 0.0000 \\
\hline 370 & 0.0000 & 0.0000 & 0.0000 & 0.0000 \\
\hline 380 & 0.0000 & 0.0000 & 0.0000 & 0.0000 \\
\hline 390 & 0.0000 & 0.0000 & 0.0000 & 0.0000 \\
\hline 400 & 0.0000 & 0.0000 & 0.0000 & 0.0000 \\
\hline
\end{tabular}

Table 5.1.7. Comparison of Analytical and PORFLOW Numerical Results for the Transient 1D Transport Problem (Case D)

\begin{tabular}{|c|c|c|c|c|}
\hline $\begin{array}{l}\text { Distance downstream x } \\
\text { (m) }\end{array}$ & $\begin{array}{l}\text { Analytical } \\
(\mathrm{G} \& \mathrm{H}) \\
(\mathrm{t}=25 \text { days })\end{array}$ & $\begin{array}{c}\text { Numerical } \\
\text { (PORFLOW) } \\
(\mathrm{t}=25 \text { days })\end{array}$ & $\begin{array}{c}\text { Analytical } \\
(\mathrm{G \& H}) \\
(\mathrm{t}=50 \text { days })\end{array}$ & $\begin{array}{c}\text { Numerical } \\
\text { (PORFLOW) } \\
(\mathrm{t}=50 \text { days })\end{array}$ \\
\hline 0 & 1.0000 & 1.0000 & 1.0000 & 1.0000 \\
\hline 20 & 0.9983 & 0.9840 & 1.0000 & 0.9997 \\
\hline 40 & 0.9853 & 0.9391 & 0.9999 & 0.9987 \\
\hline 60 & 0.9312 & 0.8436 & 0.9996 & 0.9949 \\
\hline 80 & 0.7922 & 0.6975 & 0.9981 & 0.9844 \\
\hline 100 & 0.5616 & 0.5243 & 0.9921 & 0.9609 \\
\hline 120 & 0.3096 & 0.3567 & 0.9742 & 0.9173 \\
\hline 140 & 0.1262 & 0.2199 & 0.9311 & 0.8485 \\
\hline 160 & 0.0368 & 0.1234 & 0.8472 & 0.7543 \\
\hline
\end{tabular}




\begin{tabular}{|c|cc|cc|}
\hline $\begin{array}{c}\text { Distance downstream } x \\
(\mathrm{~m})\end{array}$ & $\begin{array}{c}\text { Analytical } \\
(\mathrm{G} \& \mathrm{H}) \\
(\mathrm{t}=25 \text { days })\end{array}$ & $\begin{array}{c}\text { Numerical } \\
(\text { PORFLOW })\end{array}$ & $\begin{array}{c}\text { Analytical } \\
(\mathrm{G} \& \mathrm{t}=25 \text { days })\end{array}$ & $\begin{array}{c}\text { Numerical } \\
(\mathrm{PORFLOW})\end{array}$ \\
\hline 180 & 0.0075 & 0.0634 & 0.7146 & 0.6402 \\
\hline 200 & 0.0011 & 0.0299 & 0.5441 & 0.5166 \\
\hline 220 & 0.0001 & 0.0131 & 0.3654 & 0.3952 \\
\hline 240 & 0.0000 & 0.0053 & 0.2125 & 0.2864 \\
\hline 260 & 0.0000 & 0.0020 & 0.1055 & 0.1966 \\
\hline 280 & 0.0000 & 0.0007 & 0.0443 & 0.1279 \\
\hline 300 & 0.0000 & 0.0002 & 0.0156 & 0.0790 \\
\hline 320 & 0.0000 & 0.0001 & 0.0046 & 0.0464 \\
\hline 340 & 0.0000 & 0.0000 & 0.0011 & 0.0259 \\
\hline 360 & 0.0000 & 0.0000 & 0.0002 & 0.0138 \\
\hline 380 & 0.0000 & 0.0000 & 0.0000 & 0.0089 \\
\hline 400 & 0.0000 & 0.0000 & 0.0000 & 0.0089 \\
\hline
\end{tabular}

Table 5.1.8. Comparison of Analytical and PORFLOW Numerical Results for the Transient 1D Transport Problem (Case E)

\begin{tabular}{|c|c|c|c|c|}
\hline $\begin{array}{l}\text { Distance downstream } \mathrm{x} \\
(\mathrm{m})\end{array}$ & $\begin{array}{c}\text { Analytical } \\
(\mathrm{G \& H}) \\
(\mathrm{t}=25 \text { days })\end{array}$ & $\begin{array}{l}\text { Numerical } \\
\text { (PORFLOW) } \\
(\mathrm{t}=25 \text { days })\end{array}$ & $\begin{array}{l}\text { Analytical } \\
(\mathrm{G} \& \mathrm{H}) \\
(\mathrm{t}=50 \text { days })\end{array}$ & $\begin{array}{c}\text { Numerical } \\
\text { (PORFLOW) } \\
(\mathrm{t}=50 \text { days })\end{array}$ \\
\hline 0 & 1.0000 & 1.0000 & 1.0000 & 1.0000 \\
\hline 40 & 0.9853 & 0.8456 & 0.9999 & 0.9762 \\
\hline 80 & 0.7922 & 0.6316 & 0.9981 & 0.9253 \\
\hline 120 & 0.3096 & 0.3929 & 0.9742 & 0.8246 \\
\hline 160 & 0.0368 & 0.2050 & 0.8472 & 0.6762 \\
\hline 200 & 0.0011 & 0.0911 & 0.5441 & 0.5040 \\
\hline 240 & 0.0000 & 0.0351 & 0.2125 & 0.3400 \\
\hline 280 & 0.0000 & 0.0119 & 0.0443 & 0.2078 \\
\hline 320 & 0.0000 & 0.0036 & 0.0046 & 0.1155 \\
\hline 360 & 0.0000 & 0.0007 & 0.0002 & 0.0462 \\
\hline 400 & 0.0000 & 0.0007 & 0.0000 & 0.0462 \\
\hline
\end{tabular}

Table 5.1.9. Comparison of Analytical and PORFLOW Numerical Results for the Transient 1D Transport Problem (Case F)

\begin{tabular}{|c|cc|cc|}
\hline $\begin{array}{c}\text { Distance downstream } \mathrm{x} \\
(\mathrm{m})\end{array}$ & $\begin{array}{c}\text { Analytical } \\
(\mathrm{G} \& \mathrm{H}) \\
(\mathrm{t}=25 \text { days })\end{array}$ & $\begin{array}{c}\text { Numerical } \\
(\text { PORFLOW }) \\
(\mathrm{t}=25 \text { days })\end{array}$ & $\begin{array}{c}\text { Analytical } \\
(\mathrm{G} \& \mathrm{H}) \\
(\mathrm{t}=50 \text { days })\end{array}$ & $\begin{array}{c}\text { Numerical } \\
(\text { PORFLOW })\end{array}$ \\
$(\mathrm{t}=50$ days $)$
\end{tabular}


Table 5.1.10. Comparison of Analytical and PORFLOW Numerical Results for the Transient 1D Transport Problem (Case G)

\begin{tabular}{|c|c|c|c|c|}
\hline $\begin{array}{l}\text { Distance downstream } \mathrm{x} \\
(\mathrm{m})\end{array}$ & $\begin{array}{c}\text { Analytical } \\
(\mathrm{G} \& \mathrm{H}) \\
(\mathrm{t}=25 \text { days })\end{array}$ & $\begin{array}{c}\text { Numerical } \\
\text { (PORFLOW) } \\
(\mathrm{t}=25 \text { days })\end{array}$ & $\begin{array}{c}\text { Analytical } \\
(\mathrm{G \& H}) \\
(\mathrm{t}=50 \text { days })\end{array}$ & $\begin{array}{c}\text { Numerical } \\
\text { (PORFLOW) } \\
(\mathrm{t}=50 \text { days })\end{array}$ \\
\hline 0 & 1.0000 & 1.0000 & 1.0000 & 1.0000 \\
\hline 10 & 0.9996 & 0.9954 & 1.0000 & 0.9999 \\
\hline 20 & 0.9983 & 0.9819 & 1.0000 & 0.9997 \\
\hline 30 & 0.9945 & 0.9555 & 1.0000 & 0.9992 \\
\hline 40 & 0.9853 & 0.9141 & 0.9999 & 0.9979 \\
\hline 50 & 0.9662 & 0.8583 & 0.9999 & 0.9953 \\
\hline 60 & 0.9312 & 0.7907 & 0.9996 & 0.9907 \\
\hline 70 & 0.8744 & 0.7150 & 0.9991 & 0.9832 \\
\hline 80 & 0.7922 & 0.6351 & 0.9981 & 0.9720 \\
\hline 90 & 0.6856 & 0.5549 & 0.9960 & 0.9563 \\
\hline 100 & 0.5616 & 0.4775 & 0.9921 & 0.9353 \\
\hline 120 & 0.4318 & 0.4051 & 0.9854 & 0.9087 \\
\hline 130 & 0.3096 & 0.3392 & 0.9742 & 0.8764 \\
\hline 140 & 0.2057 & 0.2807 & 0.9568 & 0.8386 \\
\hline 150 & 0.1262 & 0.2298 & 0.9311 & 0.7958 \\
\hline 160 & 0.0712 & 0.1863 & 0.8951 & 0.7487 \\
\hline 170 & 0.0368 & 0.1496 & 0.8472 & 0.6984 \\
\hline 180 & 0.0174 & 0.1191 & 0.7868 & 0.6457 \\
\hline 190 & 0.0075 & 0.0941 & 0.7146 & 0.5919 \\
\hline 200 & 0.0030 & 0.0738 & 0.6325 & 0.5379 \\
\hline 210 & 0.0011 & 0.0575 & 0.5441 & 0.4848 \\
\hline 220 & 0.0003 & 0.0445 & 0.4535 & 0.4334 \\
\hline 230 & 0.0001 & 0.0343 & 0.3654 & 0.3843 \\
\hline 240 & 0.0000 & 0.0263 & 0.2840 & 0.3382 \\
\hline 250 & 0.0000 & 0.0200 & 0.2125 & 0.2955 \\
\hline 260 & 0.0000 & 0.0152 & 0.1528 & 0.2562 \\
\hline 270 & 0.0000 & 0.0115 & 0.1055 & 0.2207 \\
\hline 280 & 0.0000 & 0.0086 & 0.0698 & 0.1888 \\
\hline 290 & 0.0000 & 0.0064 & 0.0443 & 0.1606 \\
\hline 300 & 0.0000 & 0.0048 & 0.0269 & 0.1357 \\
\hline 310 & 0.0000 & 0.0036 & 0.0156 & 0.1140 \\
\hline 320 & 0.0000 & 0.0027 & 0.0086 & 0.0952 \\
\hline 330 & 0.0000 & 0.0020 & 0.0046 & 0.0791 \\
\hline 340 & 0.0000 & 0.0014 & 0.0023 & 0.0654 \\
\hline 350 & 0.0000 & 0.0011 & 0.0011 & 0.0538 \\
\hline 360 & 0.0000 & 0.0008 & 0.0005 & 0.0440 \\
\hline 370 & 0.0000 & 0.0006 & 0.0002 & 0.0359 \\
\hline 380 & 0.0000 & 0.0004 & 0.0001 & 0.0292 \\
\hline 390 & 0.0000 & 0.0003 & 0.0000 & 0.0251 \\
\hline 400 & 0.0000 & 0.0003 & 0.0000 & 0.0349 \\
\hline
\end{tabular}


Table 5.1.11. Comparison of Analytical and PORFLOW Numerical Results for the Transient 1D Transport Problem (Case H)

\begin{tabular}{|c|c|c|c|c|}
\hline $\begin{array}{l}\text { Distance downstream } x \\
(\mathrm{~m})\end{array}$ & $\begin{array}{c}\text { Analytical } \\
(\mathrm{G} \& \mathrm{H}) \\
(\mathrm{t}=25 \text { days })\end{array}$ & $\begin{array}{l}\text { Numerical } \\
\text { (PORFLOW) } \\
(\mathrm{t}=25 \text { days })\end{array}$ & $\begin{array}{c}\text { Analytical } \\
(\mathrm{G \& H}) \\
(\mathrm{t}=50 \text { days })\end{array}$ & $\begin{array}{c}\text { Numerical } \\
\text { (PORFLOW) } \\
(\mathrm{t}=50 \text { days })\end{array}$ \\
\hline 0 & 1.0000 & 1.0000 & 1.0000 & 1.0000 \\
\hline 10 & 0.9996 & 0.9921 & 1.0000 & 0.9996 \\
\hline 20 & 0.9983 & 0.9734 & 1.0000 & 0.9982 \\
\hline 30 & 0.9945 & 0.9432 & 1.0000 & 0.9950 \\
\hline 40 & 0.9853 & 0.9027 & 0.9999 & 0.9893 \\
\hline 50 & 0.9662 & 0.8538 & 0.9999 & 0.9802 \\
\hline 60 & 0.9312 & 0.7988 & 0.9996 & 0.9672 \\
\hline 70 & 0.8744 & 0.7400 & 0.9991 & 0.9498 \\
\hline 80 & 0.7922 & 0.6794 & 0.9981 & 0.9279 \\
\hline 90 & 0.6856 & 0.6188 & 0.9960 & 0.9016 \\
\hline 100 & 0.5616 & 0.5594 & 0.9921 & 0.8711 \\
\hline 120 & 0.4318 & 0.5024 & 0.9854 & 0.8369 \\
\hline 130 & 0.3096 & 0.4485 & 0.9742 & 0.7995 \\
\hline 140 & 0.2057 & 0.3981 & 0.9568 & 0.7596 \\
\hline 150 & 0.1262 & 0.3517 & 0.9311 & 0.7177 \\
\hline 160 & 0.0712 & 0.3093 & 0.8951 & 0.6746 \\
\hline 170 & 0.0368 & 0.2708 & 0.8472 & 0.6309 \\
\hline 180 & 0.0174 & 0.2362 & 0.7868 & 0.5872 \\
\hline 190 & 0.0075 & 0.2053 & 0.7146 & 0.5439 \\
\hline 200 & 0.0030 & 0.1779 & 0.6325 & 0.5016 \\
\hline 210 & 0.0011 & 0.1536 & 0.5441 & 0.4606 \\
\hline 220 & 0.0003 & 0.1323 & 0.4535 & 0.4212 \\
\hline 230 & 0.0001 & 0.1136 & 0.3654 & 0.3837 \\
\hline 240 & 0.0000 & 0.0973 & 0.2840 & 0.3482 \\
\hline 250 & 0.0000 & 0.0832 & 0.2125 & 0.3149 \\
\hline 260 & 0.0000 & 0.0710 & 0.1528 & 0.2838 \\
\hline 270 & 0.0000 & 0.0604 & 0.1055 & 0.2550 \\
\hline 280 & 0.0000 & 0.0513 & 0.0698 & 0.2284 \\
\hline 290 & 0.0000 & 0.0435 & 0.0443 & 0.2039 \\
\hline 300 & 0.0000 & 0.0369 & 0.0269 & 0.1816 \\
\hline 310 & 0.0000 & 0.0311 & 0.0156 & 0.1613 \\
\hline 320 & 0.0000 & 0.0263 & 0.0086 & 0.1429 \\
\hline 330 & 0.0000 & 0.0222 & 0.0046 & 0.1263 \\
\hline 340 & 0.0000 & 0.0186 & 0.0023 & 0.1113 \\
\hline 350 & 0.0000 & 0.0157 & 0.0011 & 0.0979 \\
\hline 360 & 0.0000 & 0.0132 & 0.0005 & 0.0860 \\
\hline 370 & 0.0000 & 0.0110 & 0.0002 & 0.0754 \\
\hline 380 & 0.0000 & 0.0093 & 0.0001 & 0.0668 \\
\hline 390 & 0.0000 & 0.0085 & 0.0000 & 0.0660 \\
\hline 400 & 0.0000 & 0.0139 & 0.0000 & 0.1255 \\
\hline
\end{tabular}


Table 5.1.12. Comparison of Analytical and PORFLOW Numerical Results for the Transient 1D Transport Problem (Case I)

\begin{tabular}{|c|c|c|c|c|}
\hline $\begin{array}{l}\text { Distance downstream } \mathrm{x} \\
(\mathrm{m})\end{array}$ & $\begin{array}{c}\text { Analytical } \\
(\mathrm{G} \& \mathrm{H}) \\
(\mathrm{t}=25 \text { days })\end{array}$ & $\begin{array}{l}\text { Numerical } \\
\text { (PORFLOW) } \\
(\mathrm{t}=25 \text { days })\end{array}$ & $\begin{array}{c}\text { Analytical } \\
(\mathrm{G \& H}) \\
(\mathrm{t}=50 \text { days })\end{array}$ & $\begin{array}{c}\text { Numerical } \\
\text { (PORFLOW) } \\
(\mathrm{t}=50 \text { days })\end{array}$ \\
\hline 0 & 1.0000 & 1.0000 & 1.0000 & 1.0000 \\
\hline 10 & 1.0000 & 0.9810 & 1.0000 & 0.9991 \\
\hline 20 & 0.9999 & 0.9624 & 1.0000 & 0.9978 \\
\hline 30 & 0.9999 & 0.9442 & 1.0000 & 0.9961 \\
\hline 40 & 0.9998 & 0.9264 & 1.0000 & 0.9942 \\
\hline 50 & 0.9996 & 0.9089 & 1.0000 & 0.9920 \\
\hline 60 & 0.9995 & 0.8917 & 1.0000 & 0.9895 \\
\hline 70 & 0.9993 & 0.8748 & 1.0000 & 0.9868 \\
\hline 80 & 0.9990 & 0.8583 & 1.0000 & 0.9838 \\
\hline 90 & 0.9987 & 0.8421 & 1.0000 & 0.9806 \\
\hline 100 & 0.9983 & 0.8261 & 1.0000 & 0.9771 \\
\hline 120 & 0.9978 & 0.8105 & 1.0000 & 0.9734 \\
\hline 130 & 0.9972 & 0.7952 & 1.0000 & 0.9695 \\
\hline 140 & 0.9965 & 0.7802 & 1.0000 & 0.9655 \\
\hline 150 & 0.9956 & 0.7654 & 1.0000 & 0.9612 \\
\hline 160 & 0.9945 & 0.7509 & 1.0000 & 0.9567 \\
\hline 170 & 0.9932 & 0.7367 & 1.0000 & 0.9521 \\
\hline 180 & 0.9917 & 0.7228 & 1.0000 & 0.9473 \\
\hline 190 & 0.9899 & 0.7092 & 1.0000 & 0.9423 \\
\hline 200 & 0.9878 & 0.6957 & 1.0000 & 0.9372 \\
\hline 210 & 0.9853 & 0.6826 & 0.9999 & 0.9319 \\
\hline 220 & 0.9825 & 0.6697 & 0.9999 & 0.9265 \\
\hline 230 & 0.9792 & 0.6570 & 0.9999 & 0.9210 \\
\hline 240 & 0.9754 & 0.6446 & 0.9999 & 0.9154 \\
\hline 250 & 0.9711 & 0.6324 & 0.9999 & 0.9096 \\
\hline 260 & 0.9662 & 0.6205 & 0.9999 & 0.9037 \\
\hline 270 & 0.9607 & 0.6087 & 0.9998 & 0.8978 \\
\hline 280 & 0.9545 & 0.5972 & 0.9998 & 0.8917 \\
\hline 290 & 0.9475 & 0.5859 & 0.9997 & 0.8855 \\
\hline 300 & 0.9398 & 0.5748 & 0.9997 & 0.8793 \\
\hline 310 & 0.9312 & 0.5640 & 0.9996 & 0.8729 \\
\hline 320 & 0.9218 & 0.5533 & 0.9996 & 0.8665 \\
\hline 330 & 0.9114 & 0.5429 & 0.9995 & 0.8601 \\
\hline 340 & 0.9001 & 0.5326 & 0.9994 & 0.8535 \\
\hline 350 & 0.8878 & 0.5225 & 0.9993 & 0.8469 \\
\hline 360 & 0.8744 & 0.5126 & 0.9991 & 0.8403 \\
\hline 370 & 0.8601 & 0.5029 & 0.9990 & 0.8336 \\
\hline 380 & 0.8447 & 0.4934 & 0.9988 & 0.8268 \\
\hline 390 & 0.8282 & 0.4841 & 0.9986 & 0.8200 \\
\hline 400 & 0.8107 & 0.4750 & 0.9983 & 0.8132 \\
\hline
\end{tabular}


Table 5.1.13. Comparison of Analytical and PORFLOW Numerical Results for the Transient 1D Transport Problem (Case J)

\begin{tabular}{|c|c|c|c|c|}
\hline $\begin{array}{l}\text { Distance downstream } x \\
(\mathrm{~m})\end{array}$ & $\begin{array}{c}\text { Analytical } \\
(\mathrm{G} \& \mathrm{H}) \\
(\mathrm{t}=25 \text { days })\end{array}$ & $\begin{array}{l}\text { Numerical } \\
\text { (PORFLOW) } \\
(\mathrm{t}=25 \text { days })\end{array}$ & $\begin{array}{c}\text { Analytical } \\
(\mathrm{G \& H}) \\
(\mathrm{t}=50 \text { days })\end{array}$ & $\begin{array}{c}\text { Numerical } \\
\text { (PORFLOW) } \\
(\mathrm{t}=50 \text { days })\end{array}$ \\
\hline 0 & 1.0000 & 1.0000 & 1.0000 & 1.0000 \\
\hline 10 & 1.0000 & 1.0000 & 1.0000 & 1.0000 \\
\hline 20 & 1.0000 & 1.0000 & 1.0000 & 1.0000 \\
\hline 30 & 1.0000 & 1.0000 & 1.0000 & 1.0000 \\
\hline 40 & 1.0000 & 1.0000 & 1.0000 & 1.0000 \\
\hline 50 & 1.0000 & 1.0000 & 1.0000 & 1.0000 \\
\hline 60 & 1.0000 & 0.9975 & 1.0000 & 1.0000 \\
\hline 70 & 1.0000 & 0.9979 & 1.0000 & 1.0000 \\
\hline 80 & 1.0000 & 1.0200 & 1.0000 & 1.0000 \\
\hline 90 & 1.0000 & 0.9410 & 1.0000 & 1.0000 \\
\hline 100 & 0.5000 & 0.4350 & 1.0000 & 1.0000 \\
\hline 120 & 0.0000 & 0.0959 & 1.0000 & 1.0000 \\
\hline 130 & 0.0000 & 0.0113 & 1.0000 & 1.0000 \\
\hline 140 & 0.0000 & 0.0008 & 1.0000 & 0.9998 \\
\hline 150 & 0.0000 & 0.0000 & 1.0000 & 1.0000 \\
\hline 160 & 0.0000 & 0.0000 & 1.0000 & 0.9999 \\
\hline 170 & 0.0000 & 0.0000 & 1.0000 & 0.9994 \\
\hline 180 & 0.0000 & 0.0000 & 1.0000 & 0.9998 \\
\hline 190 & 0.0000 & 0.0000 & 1.0000 & 1.0250 \\
\hline 200 & 0.0000 & 0.0000 & 1.0000 & 0.8358 \\
\hline 210 & 0.0000 & 0.0000 & 0.5000 & 0.4457 \\
\hline 220 & 0.0000 & 0.0000 & 0.0000 & 0.1531 \\
\hline 230 & 0.0000 & 0.0000 & 0.0000 & 0.0353 \\
\hline 240 & 0.0000 & 0.0000 & 0.0000 & 0.0057 \\
\hline 250 & 0.0000 & 0.0000 & 0.0000 & 0.0007 \\
\hline 260 & 0.0000 & 0.0000 & 0.0000 & 0.0001 \\
\hline 270 & 0.0000 & 0.0000 & 0.0000 & 0.0000 \\
\hline 280 & 0.0000 & 0.0000 & 0.0000 & 0.0000 \\
\hline 290 & 0.0000 & 0.0000 & 0.0000 & 0.0000 \\
\hline 300 & 0.0000 & 0.0000 & 0.0000 & 0.0000 \\
\hline 310 & 0.0000 & 0.0000 & 0.0000 & 0.0000 \\
\hline 320 & 0.0000 & 0.0000 & 0.0000 & 0.0000 \\
\hline 330 & 0.0000 & 0.0000 & 0.0000 & 0.0000 \\
\hline 340 & 0.0000 & 0.0000 & 0.0000 & 0.0000 \\
\hline 350 & 0.0000 & 0.0000 & 0.0000 & 0.0000 \\
\hline 360 & 0.0000 & 0.0000 & 0.0000 & 0.0000 \\
\hline 370 & 0.0000 & 0.0000 & 0.0000 & 0.0000 \\
\hline 380 & 0.0000 & 0.0000 & 0.0000 & 0.0000 \\
\hline 390 & 0.0000 & 0.0000 & 0.0000 & 0.0000 \\
\hline 400 & 0.0000 & 0.0000 & 0.0000 & 0.0000 \\
\hline
\end{tabular}


Table 5.1.14. Comparison of Analytical and PORFLOW Numerical Results for the Transient 1D Transport Problem (Case K, Time $=25$ days)

\begin{tabular}{|c|c|c|c|c|c|}
\hline $\begin{array}{c}\text { Distance } \\
\text { downstream x } \\
(\mathrm{m})\end{array}$ & $\begin{array}{c}\text { Analytical } \\
(\mathrm{G} \& H)\end{array}$ & $\begin{array}{l}\text { Numerical } \\
\text { (PORFLOW) } \\
\text { (HYBRID) }\end{array}$ & $\begin{array}{c}\text { Numerical } \\
\text { (PORFLOW) } \\
\text { (CONDIF) }\end{array}$ & $\begin{array}{c}\text { Numerical } \\
\text { (PORFLOW) } \\
\text { (QUICK) }\end{array}$ & $\begin{array}{c}\text { Numerical } \\
\text { (PORFLOW) } \\
\text { (CENTRAL) }\end{array}$ \\
\hline 0.0 & 1.0000 & 1.0000 & 1.0000 & 1.0000 & 1.0000 \\
\hline 10.0 & 1.0000 & 1.0000 & 1.0000 & 1.0000 & 1.0000 \\
\hline 20.0 & 1.0000 & 1.0000 & 1.0000 & 1.0000 & 1.0000 \\
\hline 30.0 & 1.0000 & 1.0000 & 1.0000 & 1.0000 & 1.0000 \\
\hline 40.0 & 1.0000 & 1.0000 & 1.0000 & 1.0000 & 1.0000 \\
\hline 50.0 & 1.0000 & 0.9998 & 0.9998 & 1.0000 & 1.0000 \\
\hline 60.0 & 1.0000 & 0.9972 & 0.9972 & 1.0000 & 0.9975 \\
\hline 70.0 & 1.0000 & 0.9782 & 0.9782 & 0.9999 & 0.9979 \\
\hline 80.0 & 1.0000 & 0.9044 & 0.9044 & 1.0040 & 1.0200 \\
\hline 90.0 & 1.0000 & 0.7343 & 0.7343 & 0.9353 & 0.9410 \\
\hline 100.0 & 0.5000 & 0.4887 & 0.4887 & 0.4789 & 0.4350 \\
\hline 110.0 & 0.0000 & 0.2552 & 0.2552 & 0.0843 & 0.0959 \\
\hline 120.0 & 0.0000 & 0.1027 & 0.1027 & 0.0034 & 0.0113 \\
\hline 130.0 & 0.0000 & 0.0318 & 0.0318 & 0.0000 & 0.0008 \\
\hline 140.0 & 0.0000 & 0.0076 & 0.0076 & 0.0000 & 0.0000 \\
\hline 150.0 & 0.0000 & 0.0014 & 0.0014 & 0.0000 & 0.0000 \\
\hline 160.0 & 0.0000 & 0.0002 & 0.0002 & 0.0000 & 0.0000 \\
\hline 170.0 & 0.0000 & 0.0000 & 0.0000 & 0.0000 & 0.0000 \\
\hline 180.0 & 0.0000 & 0.0000 & 0.0000 & 0.0000 & 0.0000 \\
\hline 190.0 & 0.0000 & 0.0000 & 0.0000 & 0.0000 & 0.0000 \\
\hline 200.0 & 0.0000 & 0.0000 & 0.0000 & 0.0000 & 0.0000 \\
\hline 210.0 & 0.0000 & 0.0000 & 0.0000 & 0.0000 & 0.0000 \\
\hline 220.0 & 0.0000 & 0.0000 & 0.0000 & 0.0000 & 0.0000 \\
\hline 230.0 & 0.0000 & 0.0000 & 0.0000 & 0.0000 & 0.0000 \\
\hline 240.0 & 0.0000 & 0.0000 & 0.0000 & 0.0000 & 0.0000 \\
\hline 250.0 & 0.0000 & 0.0000 & 0.0000 & 0.0000 & 0.0000 \\
\hline 260.0 & 0.0000 & 0.0000 & 0.0000 & 0.0000 & 0.0000 \\
\hline 270.0 & 0.0000 & 0.0000 & 0.0000 & 0.0000 & 0.0000 \\
\hline 280.0 & 0.0000 & 0.0000 & 0.0000 & 0.0000 & 0.0000 \\
\hline 290.0 & 0.0000 & 0.0000 & 0.0000 & 0.0000 & 0.0000 \\
\hline 300.0 & 0.0000 & 0.0000 & 0.0000 & 0.0000 & 0.0000 \\
\hline 310.0 & 0.0000 & 0.0000 & 0.0000 & 0.0000 & 0.0000 \\
\hline 320.0 & 0.0000 & 0.0000 & 0.0000 & 0.0000 & 0.0000 \\
\hline 330.0 & 0.0000 & 0.0000 & 0.0000 & 0.0000 & 0.0000 \\
\hline 340.0 & 0.0000 & 0.0000 & 0.0000 & 0.0000 & 0.0000 \\
\hline 350.0 & 0.0000 & 0.0000 & 0.0000 & 0.0000 & 0.0000 \\
\hline 360.0 & 0.0000 & 0.0000 & 0.0000 & 0.0000 & 0.0000 \\
\hline 370.0 & 0.0000 & 0.0000 & 0.0000 & 0.0000 & 0.0000 \\
\hline 380.0 & 0.0000 & 0.0000 & 0.0000 & 0.0000 & 0.0000 \\
\hline 390.0 & 0.0000 & 0.0000 & 0.0000 & 0.0000 & 0.0000 \\
\hline 400.0 & 0.0000 & 0.0000 & 0.0000 & 0.0000 & 0.0000 \\
\hline
\end{tabular}


Table 5.1.15. Comparison of Analytical and PORFLOW Numerical Results for the Transient 1D Transport Problem (Case K, Time $=\mathbf{5 0}$ days)

\begin{tabular}{|c|c|c|c|c|c|}
\hline $\begin{array}{c}\text { Distance } \\
\text { downstream } \mathrm{x} \\
(\mathrm{m})\end{array}$ & $\begin{array}{c}\text { Analytical } \\
(\mathrm{G} \& H)\end{array}$ & $\begin{array}{l}\text { Numerical } \\
\text { (PORFLOW) } \\
\text { (HYBRID) }\end{array}$ & $\begin{array}{l}\text { Numerical } \\
\text { (PORFLOW) } \\
\text { (CONDIF) }\end{array}$ & $\begin{array}{c}\text { Numerical } \\
\text { (PORFLOW) } \\
\text { (QUICK) }\end{array}$ & $\begin{array}{c}\text { Numerical } \\
\text { (PORFLOW) } \\
\text { (CENTRAL) }\end{array}$ \\
\hline 0.0 & 1.0000 & 1.0000 & 1.0000 & 1.0000 & 1.0000 \\
\hline 10.0 & 1.0000 & 1.0000 & 1.0000 & 1.0000 & 1.0000 \\
\hline 20.0 & 1.0000 & 1.0000 & 1.0000 & 1.0000 & 1.0000 \\
\hline 30.0 & 1.0000 & 1.0000 & 1.0000 & 1.0000 & 1.0000 \\
\hline 40.0 & 1.0000 & 1.0000 & 1.0000 & 1.0000 & 1.0000 \\
\hline 50.0 & 1.0000 & 1.0000 & 1.0000 & 1.0000 & 1.0000 \\
\hline 60.0 & 1.0000 & 1.0000 & 1.0000 & 1.0000 & 1.0000 \\
\hline 70.0 & 1.0000 & 1.0000 & 1.0000 & 1.0000 & 1.0000 \\
\hline 80.0 & 1.0000 & 1.0000 & 1.0000 & 1.0000 & 1.0000 \\
\hline 90.0 & 1.0000 & 1.0000 & 1.0000 & 1.0000 & 1.0000 \\
\hline 100.0 & 1.0000 & 1.0000 & 1.0000 & 1.0000 & 1.0000 \\
\hline 110.0 & 1.0000 & 1.0000 & 1.0000 & 1.0000 & 1.0000 \\
\hline 120.0 & 1.0000 & 1.0000 & 1.0000 & 1.0000 & 1.0000 \\
\hline 130.0 & 1.0000 & 0.9997 & 0.9997 & 1.0000 & 0.9998 \\
\hline 140.0 & 1.0000 & 0.9981 & 0.9981 & 1.0000 & 1.0000 \\
\hline 150.0 & 1.0000 & 0.9912 & 0.9912 & 1.0000 & 0.9999 \\
\hline 160.0 & 1.0000 & 0.9696 & 0.9696 & 0.9999 & 0.9994 \\
\hline 170.0 & 1.0000 & 0.9172 & 0.9172 & 1.0010 & 0.9998 \\
\hline 180.0 & 1.0000 & 0.8180 & 0.8180 & 0.9946 & 1.0250 \\
\hline 190.0 & 1.0000 & 0.6697 & 0.6697 & 0.8517 & 0.8358 \\
\hline 200.0 & 0.5000 & 0.4919 & 0.4919 & 0.4828 & 0.4457 \\
\hline 210.0 & 0.0000 & 0.3188 & 0.3188 & 0.1508 & 0.1531 \\
\hline 220.0 & 0.0000 & 0.1805 & 0.1805 & 0.0232 & 0.0353 \\
\hline 230.0 & 0.0000 & 0.0888 & 0.0888 & 0.0015 & 0.0057 \\
\hline 240.0 & 0.0000 & 0.0379 & 0.0379 & 0.0000 & 0.0007 \\
\hline 250.0 & 0.0000 & 0.0140 & 0.0140 & 0.0000 & 0.0001 \\
\hline 260.0 & 0.0000 & 0.0045 & 0.0045 & 0.0000 & 0.0000 \\
\hline 270.0 & 0.0000 & 0.0013 & 0.0013 & 0.0000 & 0.0000 \\
\hline 280.0 & 0.0000 & 0.0003 & 0.0003 & 0.0000 & 0.0000 \\
\hline 290.0 & 0.0000 & 0.0001 & 0.0001 & 0.0000 & 0.0000 \\
\hline 300.0 & 0.0000 & 0.0000 & 0.0000 & 0.0000 & 0.0000 \\
\hline 310.0 & 0.0000 & 0.0000 & 0.0000 & 0.0000 & 0.0000 \\
\hline 320.0 & 0.0000 & 0.0000 & 0.0000 & 0.0000 & 0.0000 \\
\hline 330.0 & 0.0000 & 0.0000 & 0.0000 & 0.0000 & 0.0000 \\
\hline 340.0 & 0.0000 & 0.0000 & 0.0000 & 0.0000 & 0.0000 \\
\hline 350.0 & 0.0000 & 0.0000 & 0.0000 & 0.0000 & 0.0000 \\
\hline 360.0 & 0.0000 & 0.0000 & 0.0000 & 0.0000 & 0.0000 \\
\hline 370.0 & 0.0000 & 0.0000 & 0.0000 & 0.0000 & 0.0000 \\
\hline 380.0 & 0.0000 & 0.0000 & 0.0000 & 0.0000 & 0.0000 \\
\hline 390.0 & 0.0000 & 0.0000 & 0.0000 & 0.0000 & 0.0000 \\
\hline 400.0 & 0.0000 & 0.0000 & 0.0000 & 0.0000 & 0.0000 \\
\hline
\end{tabular}


Table 5.1.16. Comparison of Analytical and PORFLOW Numerical Results for the Transient 1D Transport Problem (Case $\mathrm{L}$, Time $=25$ days)

\begin{tabular}{|c|c|c|c|c|c|}
\hline $\begin{array}{c}\text { Distance } \\
\text { downstream } \mathrm{x} \\
(\mathrm{m})\end{array}$ & $\begin{array}{l}\text { Analytical } \\
\text { (G\&H) }\end{array}$ & $\begin{array}{l}\text { Numerical } \\
\text { (PORFLOW) } \\
\text { (Arithmetic) }\end{array}$ & $\begin{array}{l}\text { Numerical } \\
\text { (PORFLOW) } \\
\text { (Harmonic) }\end{array}$ & $\begin{array}{l}\text { Numerical } \\
\text { (PORFLOW) } \\
\text { (Geometric) }\end{array}$ & $\begin{array}{c}\text { Numerical } \\
\text { (PORFLOW) } \\
\text { (Upwind) }\end{array}$ \\
\hline 0.0 & 1.0000 & 1.0000 & 1.0000 & 1.0000 & 1.0000 \\
\hline 10.0 & 0.9996 & 0.9996 & 0.9996 & 0.9996 & 0.9996 \\
\hline 20.0 & 0.9983 & 0.9983 & 0.9983 & 0.9983 & 0.9983 \\
\hline 30.0 & 0.9945 & 0.9943 & 0.9943 & 0.9943 & 0.9943 \\
\hline 40.0 & 0.9853 & 0.9846 & 0.9846 & 0.9846 & 0.9846 \\
\hline 50.0 & 0.9662 & 0.9647 & 0.9647 & 0.9647 & 0.9647 \\
\hline 60.0 & 0.9312 & 0.9284 & 0.9284 & 0.9284 & 0.9284 \\
\hline 70.0 & 0.8744 & 0.8701 & 0.8701 & 0.8701 & 0.8701 \\
\hline 80.0 & 0.7922 & 0.7867 & 0.7867 & 0.7867 & 0.7867 \\
\hline 90.0 & 0.6856 & 0.6799 & 0.6799 & 0.6799 & 0.6799 \\
\hline 100.0 & 0.5616 & 0.5572 & 0.5572 & 0.5572 & 0.5572 \\
\hline 110.0 & 0.4318 & 0.4299 & 0.4299 & 0.4299 & 0.4299 \\
\hline 120.0 & 0.3096 & 0.3107 & 0.3107 & 0.3107 & 0.3107 \\
\hline 130.0 & 0.2057 & 0.2094 & 0.2094 & 0.2094 & 0.2094 \\
\hline 140.0 & 0.1262 & 0.1313 & 0.1313 & 0.1313 & 0.1313 \\
\hline 150.0 & 0.0712 & 0.0765 & 0.0765 & 0.0765 & 0.0765 \\
\hline 160.0 & 0.0368 & 0.0413 & 0.0413 & 0.0413 & 0.0413 \\
\hline 170.0 & 0.0174 & 0.0207 & 0.0207 & 0.0207 & 0.0207 \\
\hline 180.0 & 0.0075 & 0.0096 & 0.0096 & 0.0096 & 0.0096 \\
\hline 190.0 & 0.0030 & 0.0041 & 0.0041 & 0.0041 & 0.0041 \\
\hline 200.0 & 0.0011 & 0.0017 & 0.0017 & 0.0017 & 0.0017 \\
\hline 210.0 & 0.0003 & 0.0006 & 0.0006 & 0.0006 & 0.0006 \\
\hline 220.0 & 0.0001 & 0.0002 & 0.0002 & 0.0002 & 0.0002 \\
\hline 230.0 & 0.0000 & 0.0001 & 0.0001 & 0.0001 & 0.0001 \\
\hline 240.0 & 0.0000 & 0.0000 & 0.0000 & 0.0000 & 0.0000 \\
\hline 250.0 & 0.0000 & 0.0000 & 0.0000 & 0.0000 & 0.0000 \\
\hline 260.0 & 0.0000 & 0.0000 & 0.0000 & 0.0000 & 0.0000 \\
\hline 270.0 & 0.0000 & 0.0000 & 0.0000 & 0.0000 & 0.0000 \\
\hline 280.0 & 0.0000 & 0.0000 & 0.0000 & 0.0000 & 0.0000 \\
\hline 290.0 & 0.0000 & 0.0000 & 0.0000 & 0.0000 & 0.0000 \\
\hline 300.0 & 0.0000 & 0.0000 & 0.0000 & 0.0000 & 0.0000 \\
\hline 310.0 & 0.0000 & 0.0000 & 0.0000 & 0.0000 & 0.0000 \\
\hline 320.0 & 0.0000 & 0.0000 & 0.0000 & 0.0000 & 0.0000 \\
\hline 330.0 & 0.0000 & 0.0000 & 0.0000 & 0.0000 & 0.0000 \\
\hline 340.0 & 0.0000 & 0.0000 & 0.0000 & 0.0000 & 0.0000 \\
\hline 350.0 & 0.0000 & 0.0000 & 0.0000 & 0.0000 & 0.0000 \\
\hline 360.0 & 0.0000 & 0.0000 & 0.0000 & 0.0000 & 0.0000 \\
\hline 370.0 & 0.0000 & 0.0000 & 0.0000 & 0.0000 & 0.0000 \\
\hline 380.0 & 0.0000 & 0.0000 & 0.0000 & 0.0000 & 0.0000 \\
\hline 390.0 & 0.0000 & 0.0000 & 0.0000 & 0.0000 & 0.0000 \\
\hline 400.0 & 0.0000 & 0.0000 & 0.0000 & 0.0000 & 0.0000 \\
\hline
\end{tabular}


Table 5.1.17. Comparison of Analytical and PORFLOW Numerical Results for the Transient 1D Transport Problem (Case L, Time $=\mathbf{5 0}$ days)

\begin{tabular}{|c|c|c|c|c|c|}
\hline $\begin{array}{c}\text { Distance } \\
\text { downstream x } \\
(\mathrm{m})\end{array}$ & $\begin{array}{c}\text { Analytical } \\
(\mathrm{G} \& H)\end{array}$ & $\begin{array}{l}\text { Numerical } \\
\text { (PORFLOW) } \\
\text { (Arithmetic) }\end{array}$ & $\begin{array}{l}\text { Numerical } \\
\text { (PORFLOW) } \\
\text { (Harmonic) }\end{array}$ & $\begin{array}{l}\text { Numerical } \\
\text { (PORFLOW) } \\
\text { (Geometric) }\end{array}$ & $\begin{array}{c}\text { Numerical } \\
\text { (PORFLOW) } \\
\text { (Upwind) }\end{array}$ \\
\hline 0.0 & 1.0000 & 1.0000 & 1.0000 & 1.0000 & 1.0000 \\
\hline 10.0 & 1.0000 & 1.0000 & 1.0000 & 1.0000 & 1.0000 \\
\hline 20.0 & 1.0000 & 1.0000 & 1.0000 & 1.0000 & 1.0000 \\
\hline 30.0 & 1.0000 & 1.0000 & 1.0000 & 1.0000 & 1.0000 \\
\hline 40.0 & 0.9999 & 0.9999 & 0.9999 & 0.9999 & 0.9999 \\
\hline 50.0 & 0.9999 & 0.9998 & 0.9998 & 0.9998 & 0.9998 \\
\hline 60.0 & 0.9996 & 0.9996 & 0.9996 & 0.9996 & 0.9996 \\
\hline 70.0 & 0.9991 & 0.9991 & 0.9991 & 0.9991 & 0.9991 \\
\hline 80.0 & 0.9981 & 0.9979 & 0.9979 & 0.9979 & 0.9979 \\
\hline 90.0 & 0.9960 & 0.9957 & 0.9957 & 0.9957 & 0.9957 \\
\hline 100.0 & 0.9921 & 0.9916 & 0.9916 & 0.9916 & 0.9916 \\
\hline 110.0 & 0.9854 & 0.9844 & 0.9844 & 0.9844 & 0.9844 \\
\hline 120.0 & 0.9742 & 0.9727 & 0.9727 & 0.9727 & 0.9727 \\
\hline 130.0 & 0.9568 & 0.9546 & 0.9546 & 0.9546 & 0.9546 \\
\hline 140.0 & 0.9311 & 0.9280 & 0.9280 & 0.9280 & 0.9280 \\
\hline 150.0 & 0.8951 & 0.8910 & 0.8910 & 0.8910 & 0.8910 \\
\hline 160.0 & 0.8472 & 0.8424 & 0.8424 & 0.8424 & 0.8424 \\
\hline 170.0 & 0.7868 & 0.7816 & 0.7816 & 0.7816 & 0.7816 \\
\hline 180.0 & 0.7146 & 0.7094 & 0.7094 & 0.7094 & 0.7094 \\
\hline 190.0 & 0.6325 & 0.6281 & 0.6281 & 0.6281 & 0.6281 \\
\hline 200.0 & 0.5441 & 0.5409 & 0.5409 & 0.5409 & 0.5409 \\
\hline 210.0 & 0.4535 & 0.4521 & 0.4521 & 0.4521 & 0.4521 \\
\hline 220.0 & 0.3654 & 0.3659 & 0.3659 & 0.3659 & 0.3659 \\
\hline 230.0 & 0.2840 & 0.2863 & 0.2863 & 0.2863 & 0.2863 \\
\hline 240.0 & 0.2125 & 0.2163 & 0.2163 & 0.2163 & 0.2163 \\
\hline 250.0 & 0.1528 & 0.1576 & 0.1576 & 0.1576 & 0.1576 \\
\hline 260.0 & 0.1055 & 0.1106 & 0.1106 & 0.1106 & 0.1106 \\
\hline 270.0 & 0.0698 & 0.0747 & 0.0747 & 0.0747 & 0.0747 \\
\hline 280.0 & 0.0443 & 0.0485 & 0.0485 & 0.0485 & 0.0485 \\
\hline 290.0 & 0.0269 & 0.0303 & 0.0303 & 0.0303 & 0.0303 \\
\hline 300.0 & 0.0156 & 0.0182 & 0.0182 & 0.0182 & 0.0182 \\
\hline 310.0 & 0.0086 & 0.0105 & 0.0105 & 0.0105 & 0.0105 \\
\hline 320.0 & 0.0046 & 0.0059 & 0.0059 & 0.0059 & 0.0059 \\
\hline 330.0 & 0.0023 & 0.0031 & 0.0031 & 0.0031 & 0.0031 \\
\hline 340.0 & 0.0011 & 0.0016 & 0.0016 & 0.0016 & 0.0016 \\
\hline 350.0 & 0.0005 & 0.0008 & 0.0008 & 0.0008 & 0.0008 \\
\hline 360.0 & 0.0002 & 0.0004 & 0.0004 & 0.0004 & 0.0004 \\
\hline 370.0 & 0.0001 & 0.0002 & 0.0002 & 0.0002 & 0.0002 \\
\hline 380.0 & 0.0000 & 0.0001 & 0.0001 & 0.0001 & 0.0001 \\
\hline 390.0 & 0.0000 & 0.0000 & 0.0000 & 0.0000 & 0.0000 \\
\hline 400.0 & 0.0000 & 0.0000 & 0.0000 & 0.0000 & 0.0000 \\
\hline
\end{tabular}


Table 5.1.18. Input Commands for 1D Transport (Base Case)

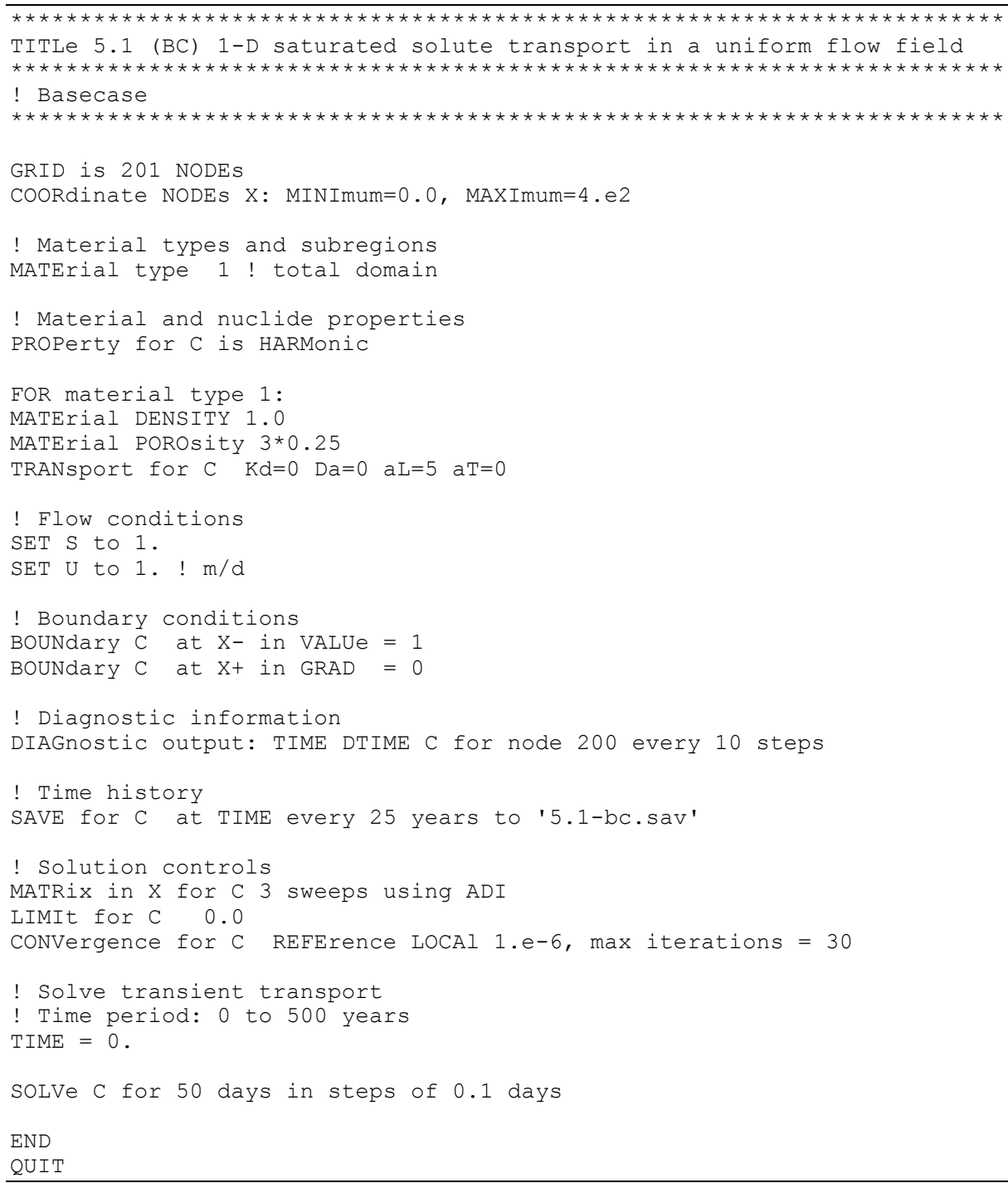

Table 5.1.19. Input Commands for 1D Transport (Case A)

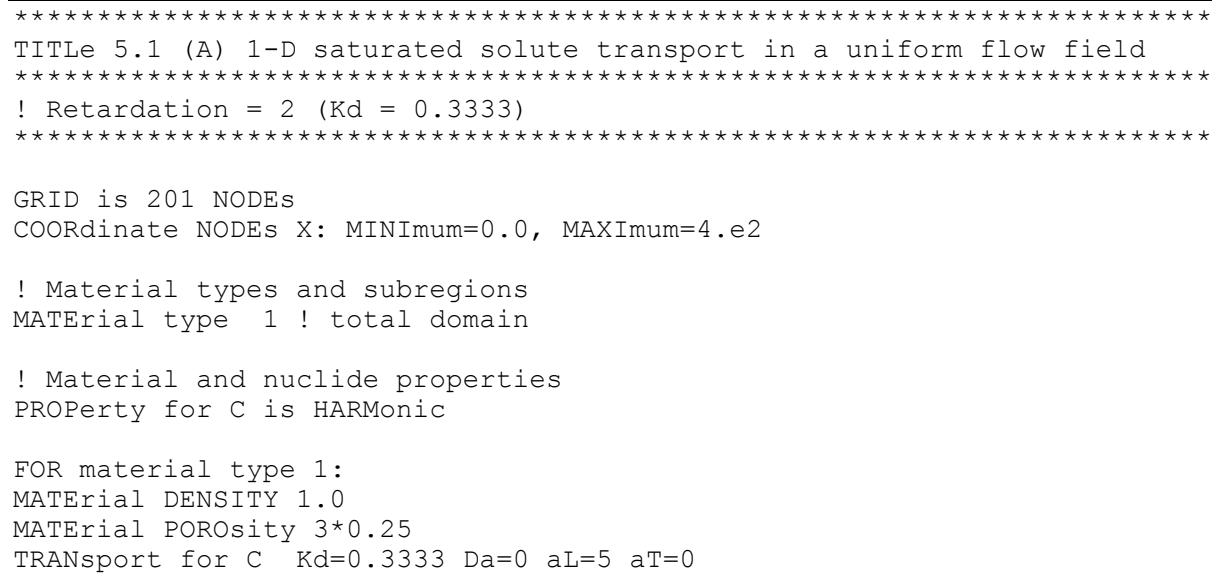




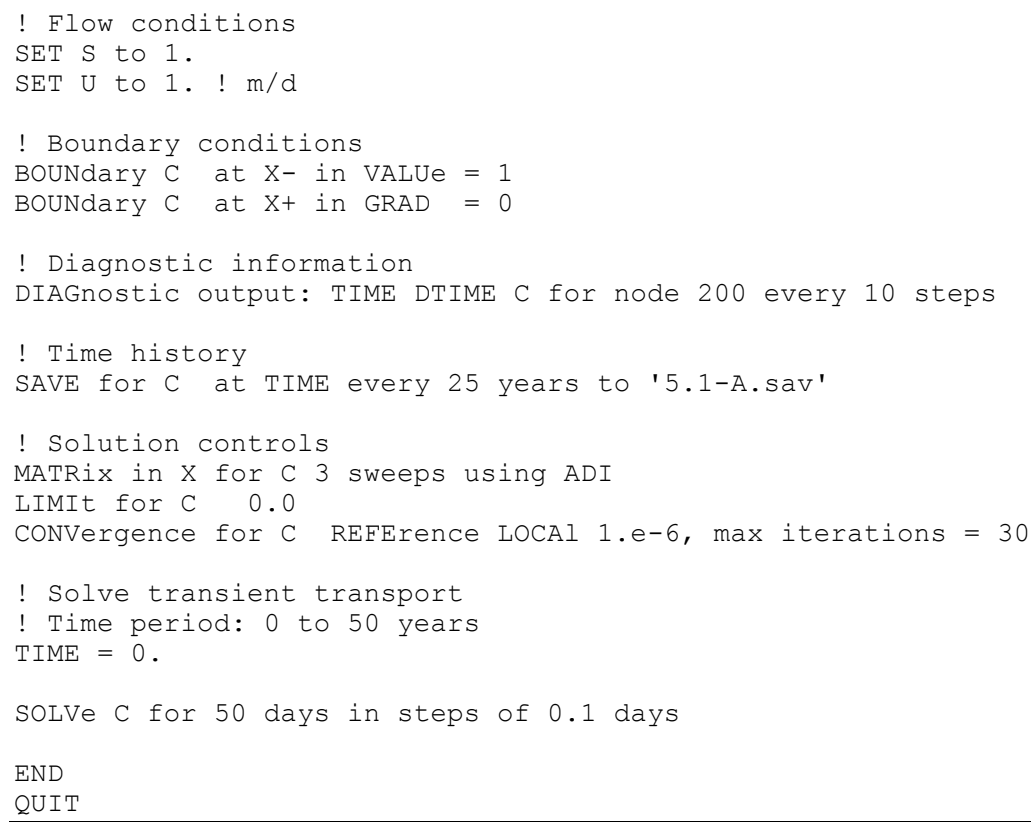

\section{Table 5.1.20. Input Commands for 1D Transport (Case B)}

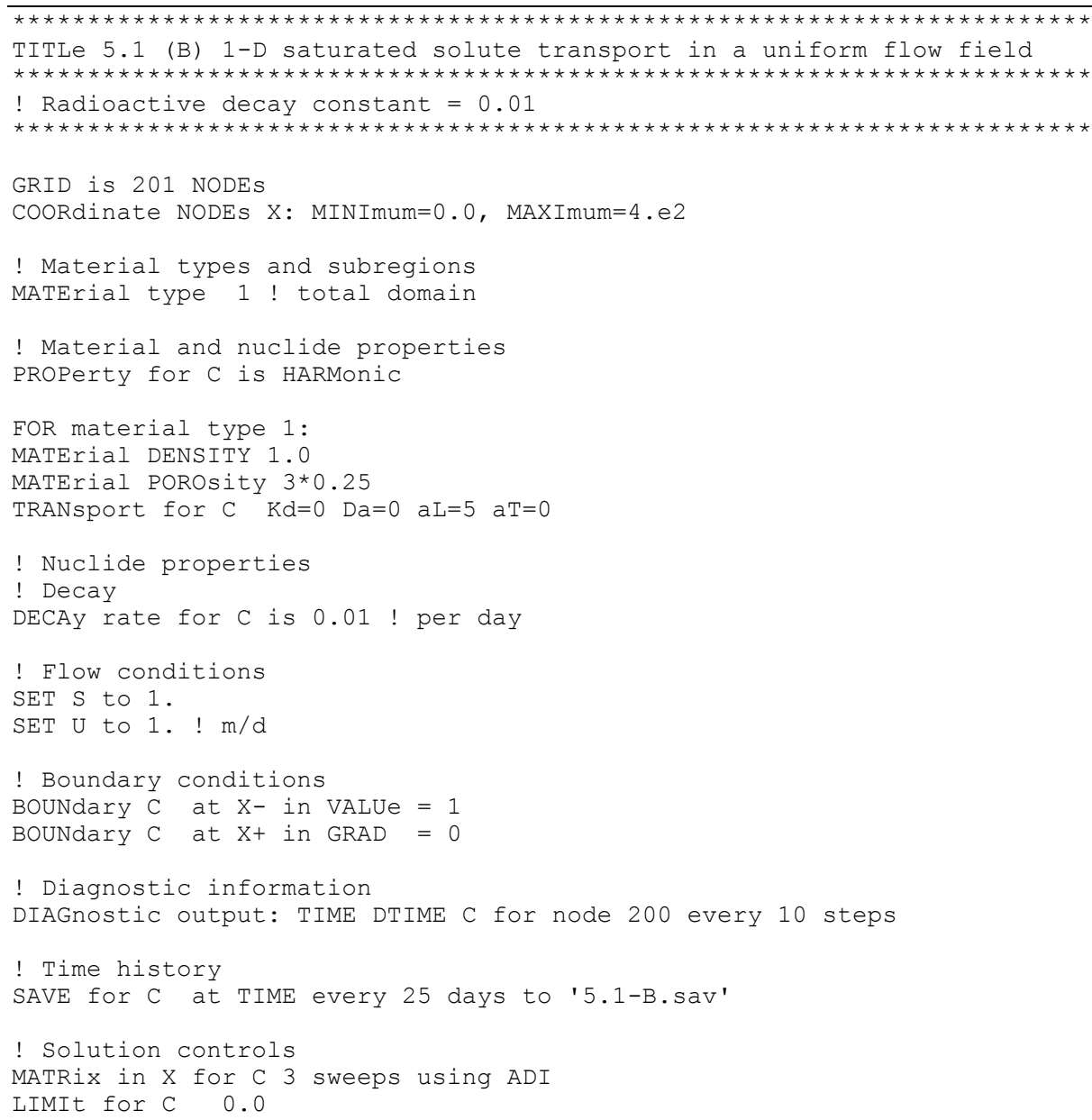


! Solve transient transport

! Time period: 0 to 50 years

$\mathrm{TIME}=0$.

Solve C for 50 days in steps of 0.1 days

END

QUIT

\section{Table 5.1.21. Input Commands for 1D Transport (Case C)}

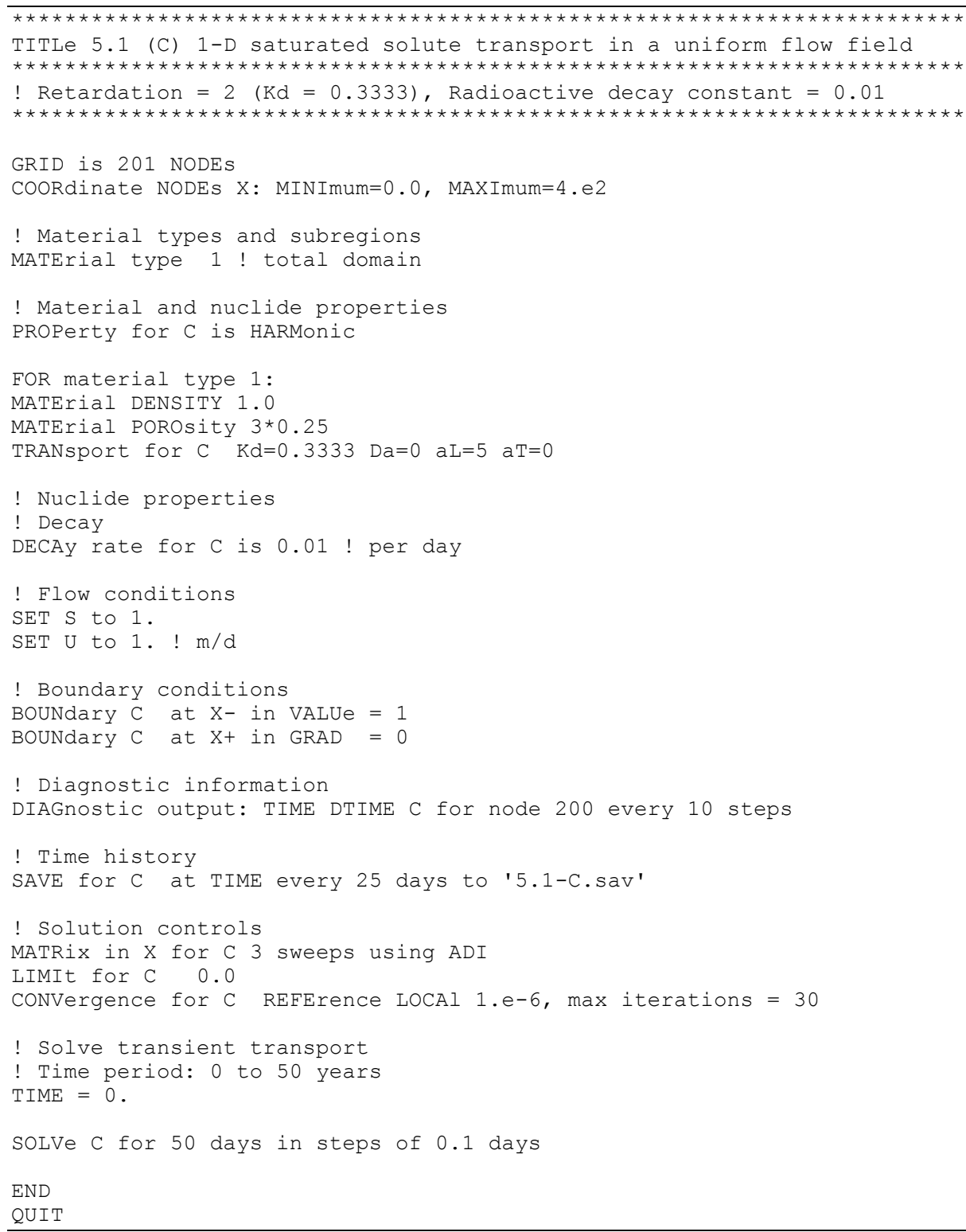

Table 5.1.22. Input Commands for 1D Transport (Case D)

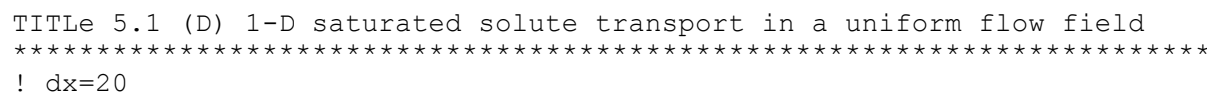




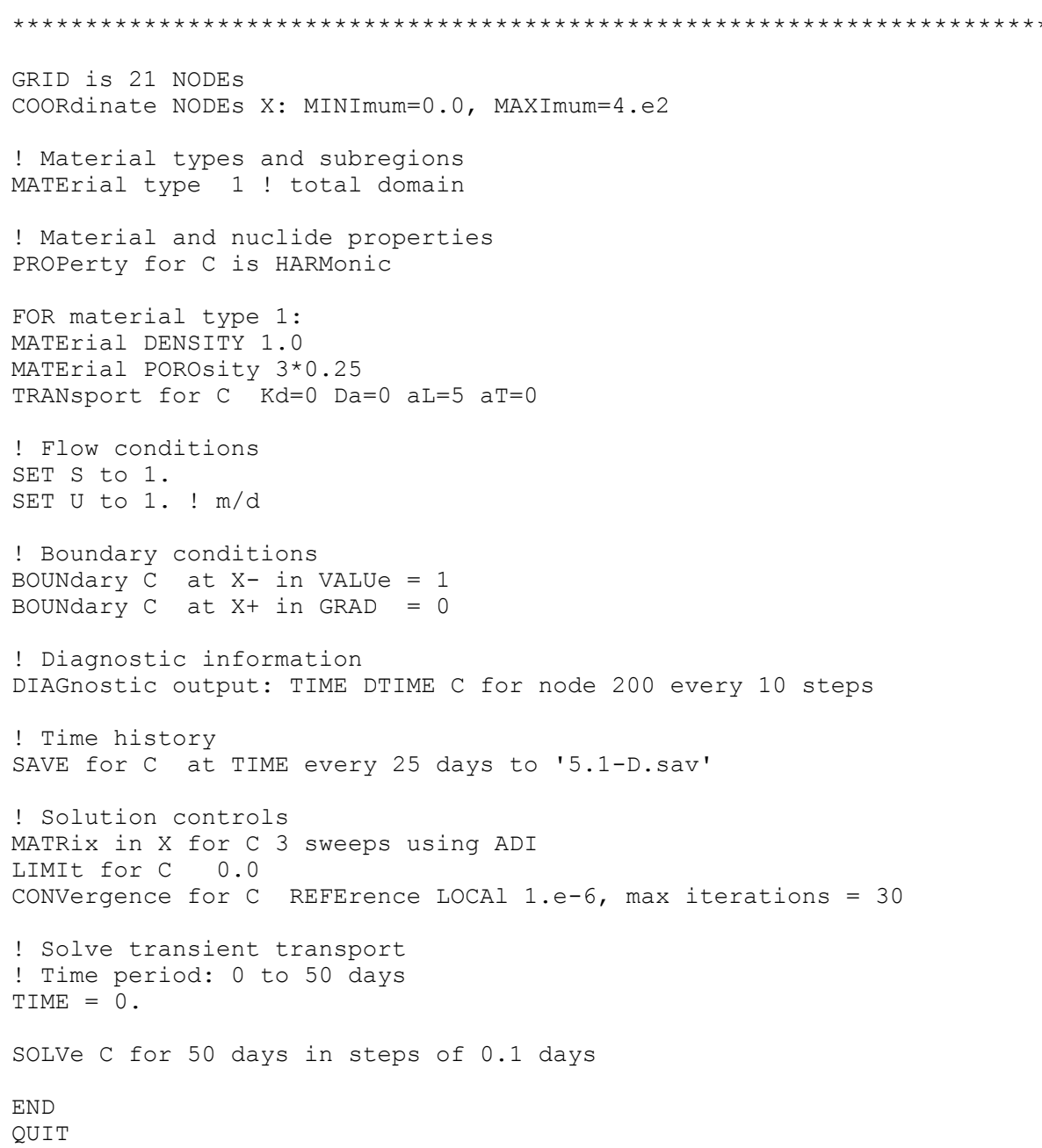

Table 5.1.23. Input Commands for 1D Transport (Case E)

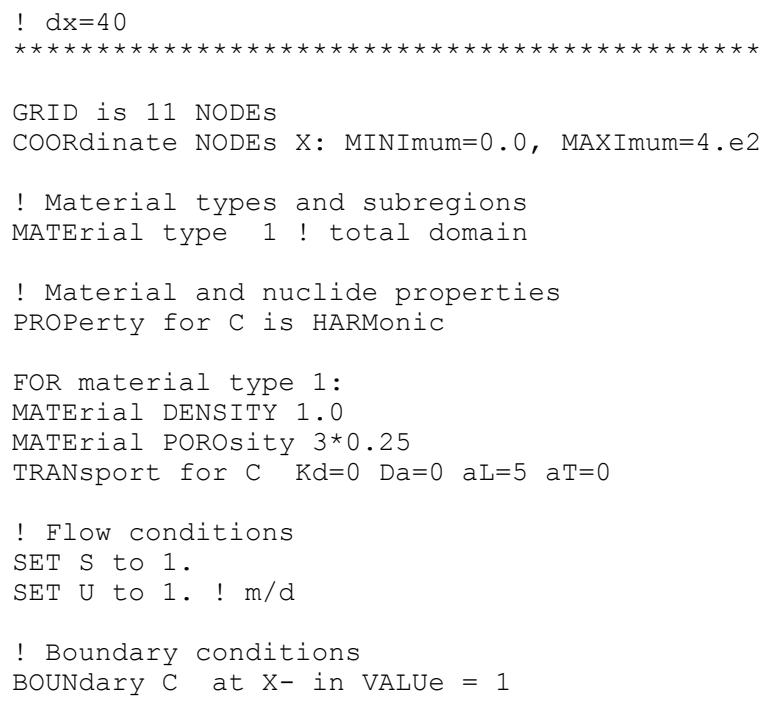




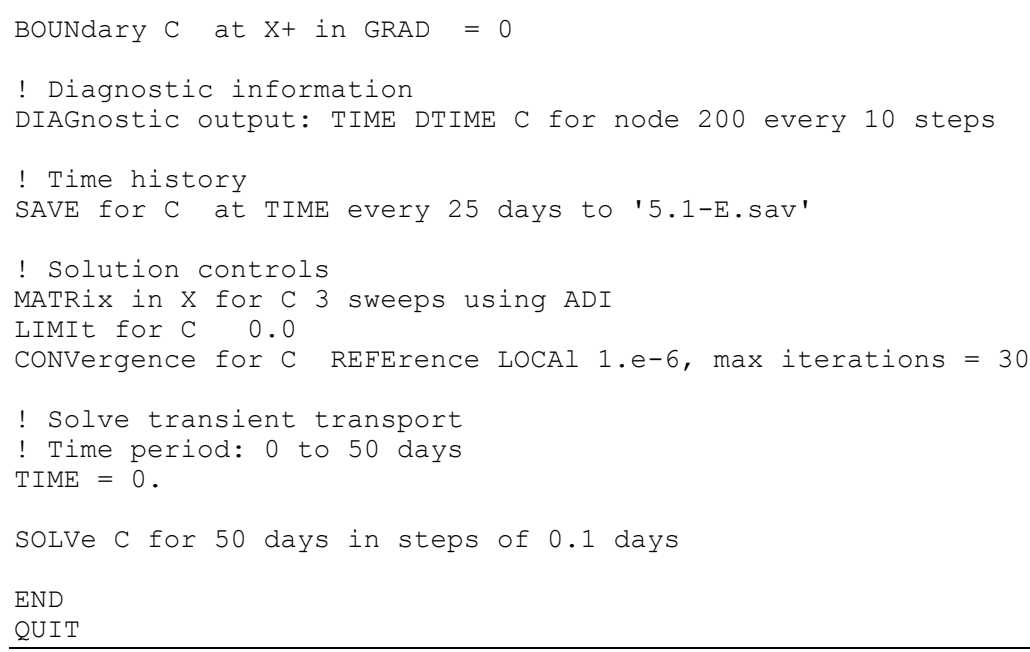

Table 5.1.24. Input Commands for 1D Transport (Case F)

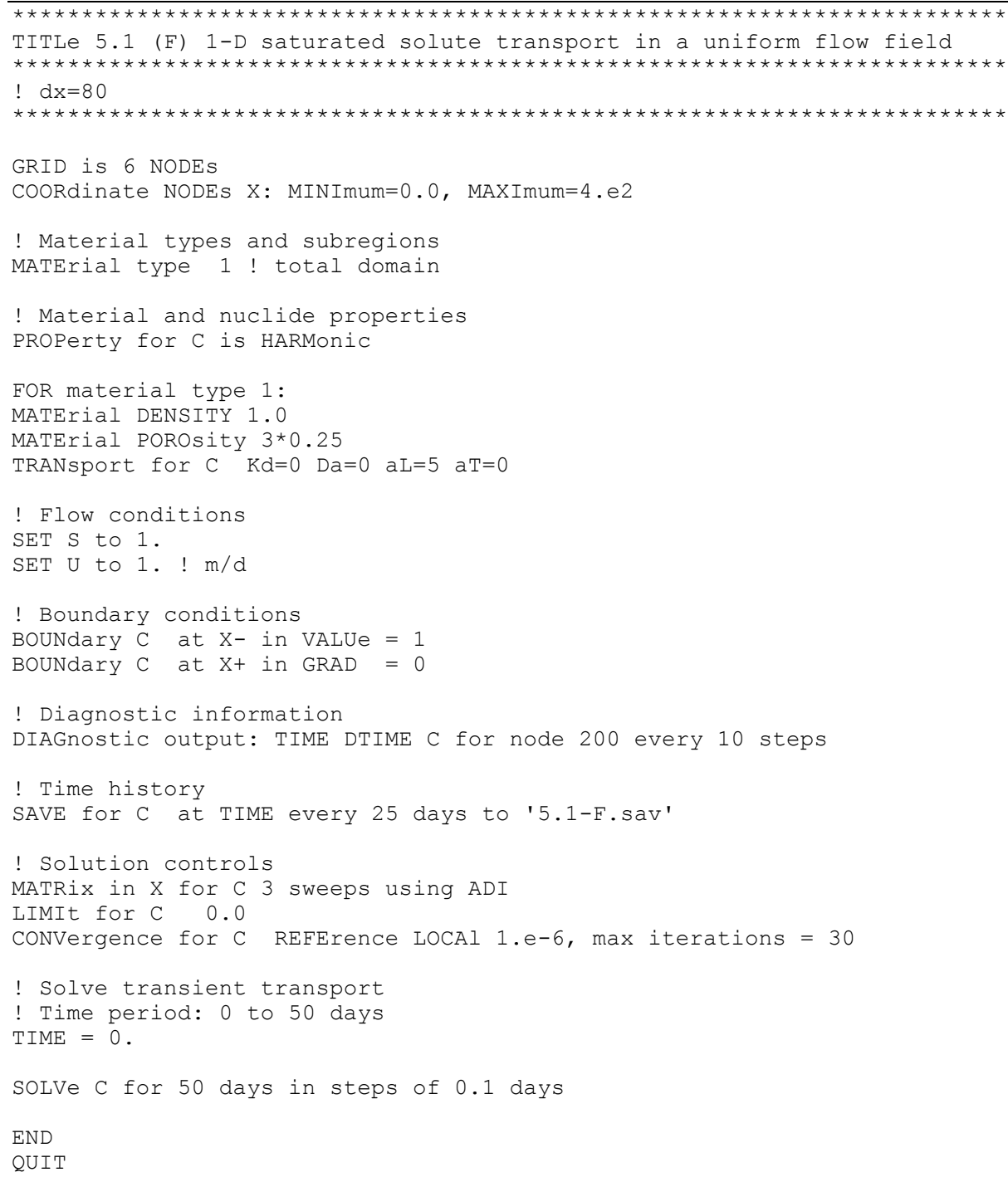


Table 5.1.25. Input Commands for 1D Transport (Case G)

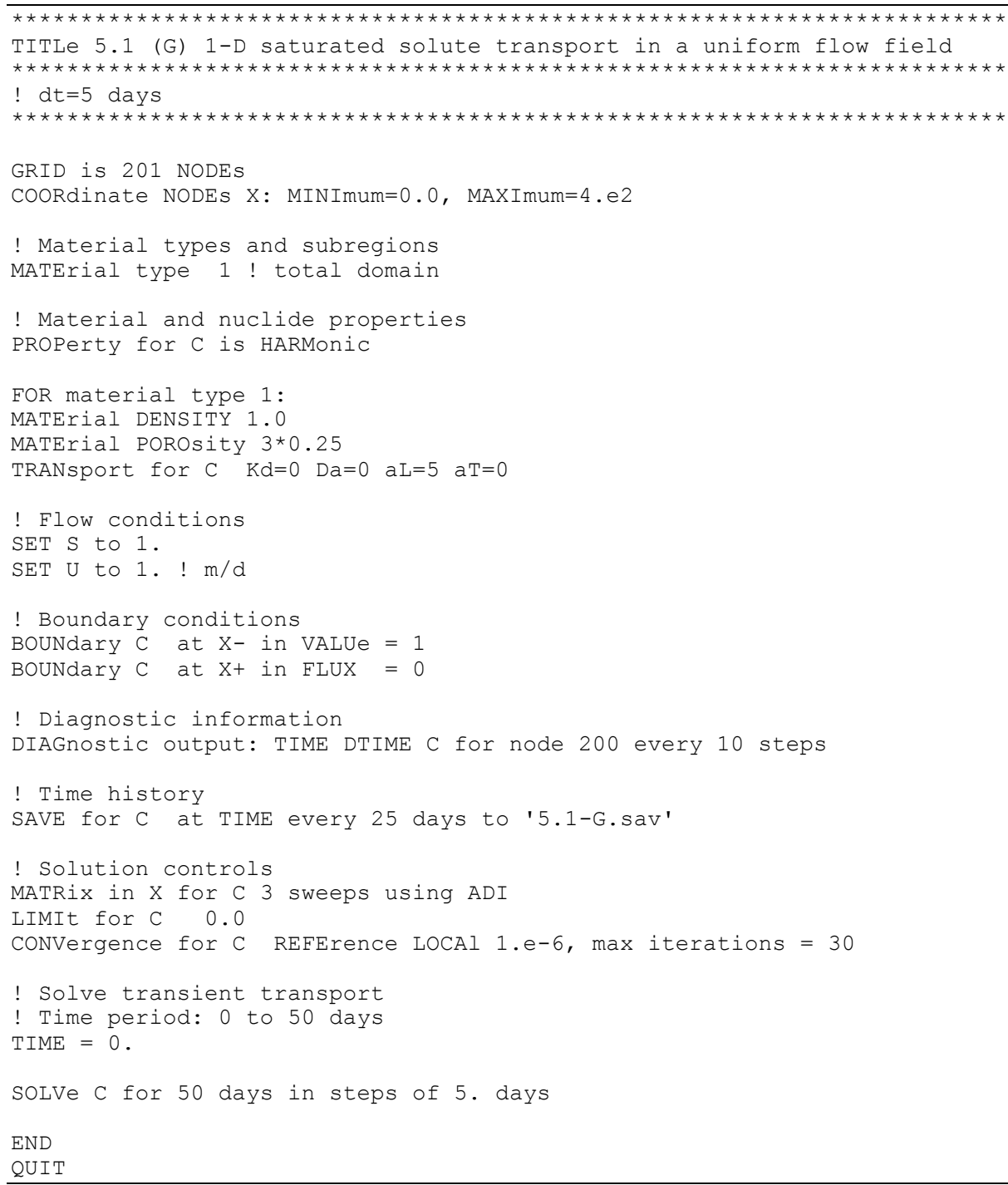

Table 5.1.26. Input Commands for 1D Transport (Case H)

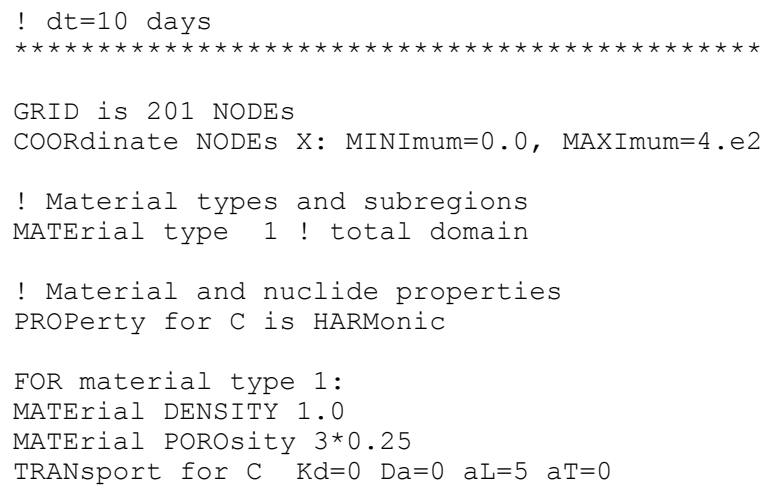




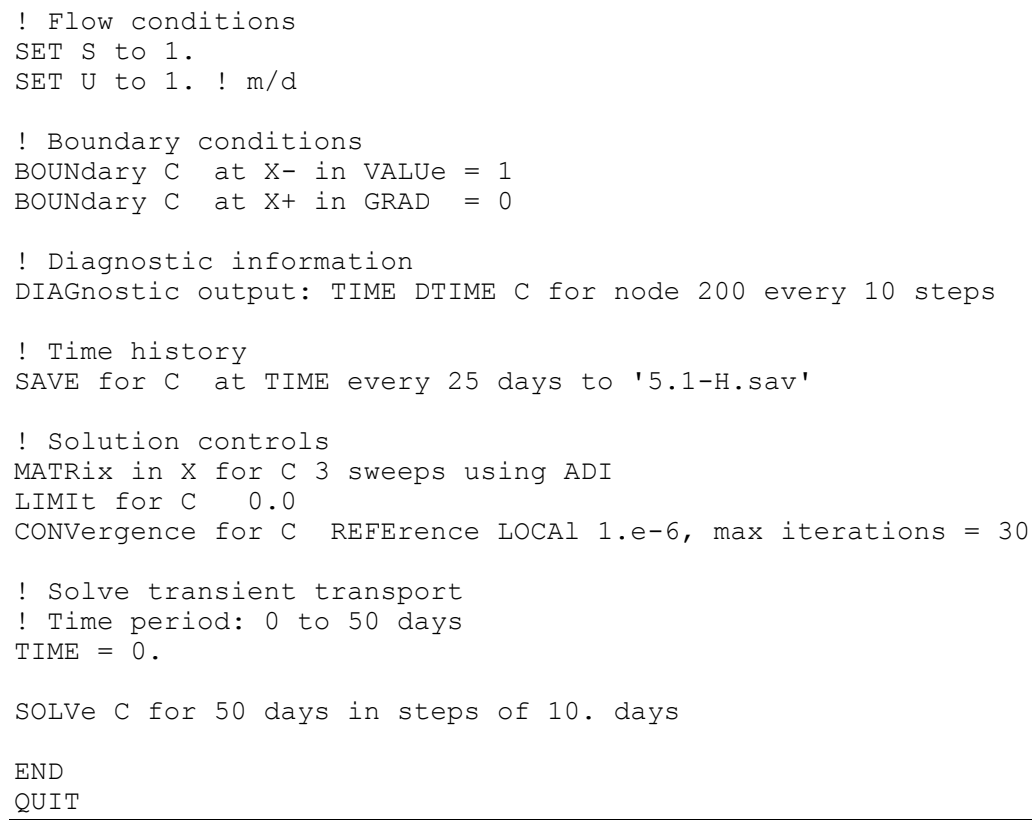

Table 5.1.27. Input Commands for 1D Transport (Case I)

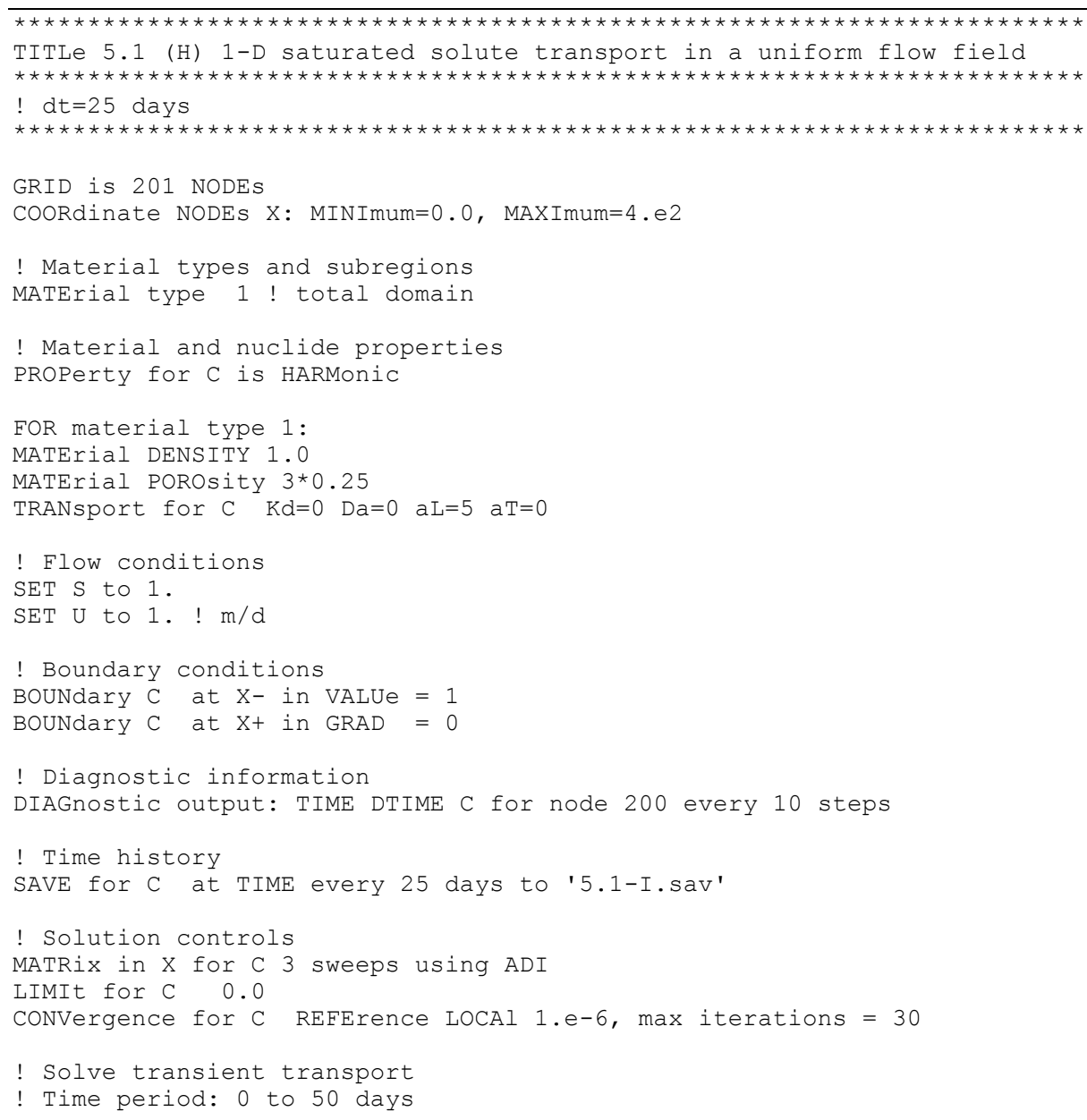


TIME $=0$.

\section{Table 5.1.28. Input Commands for 1D Transport (Case J)}

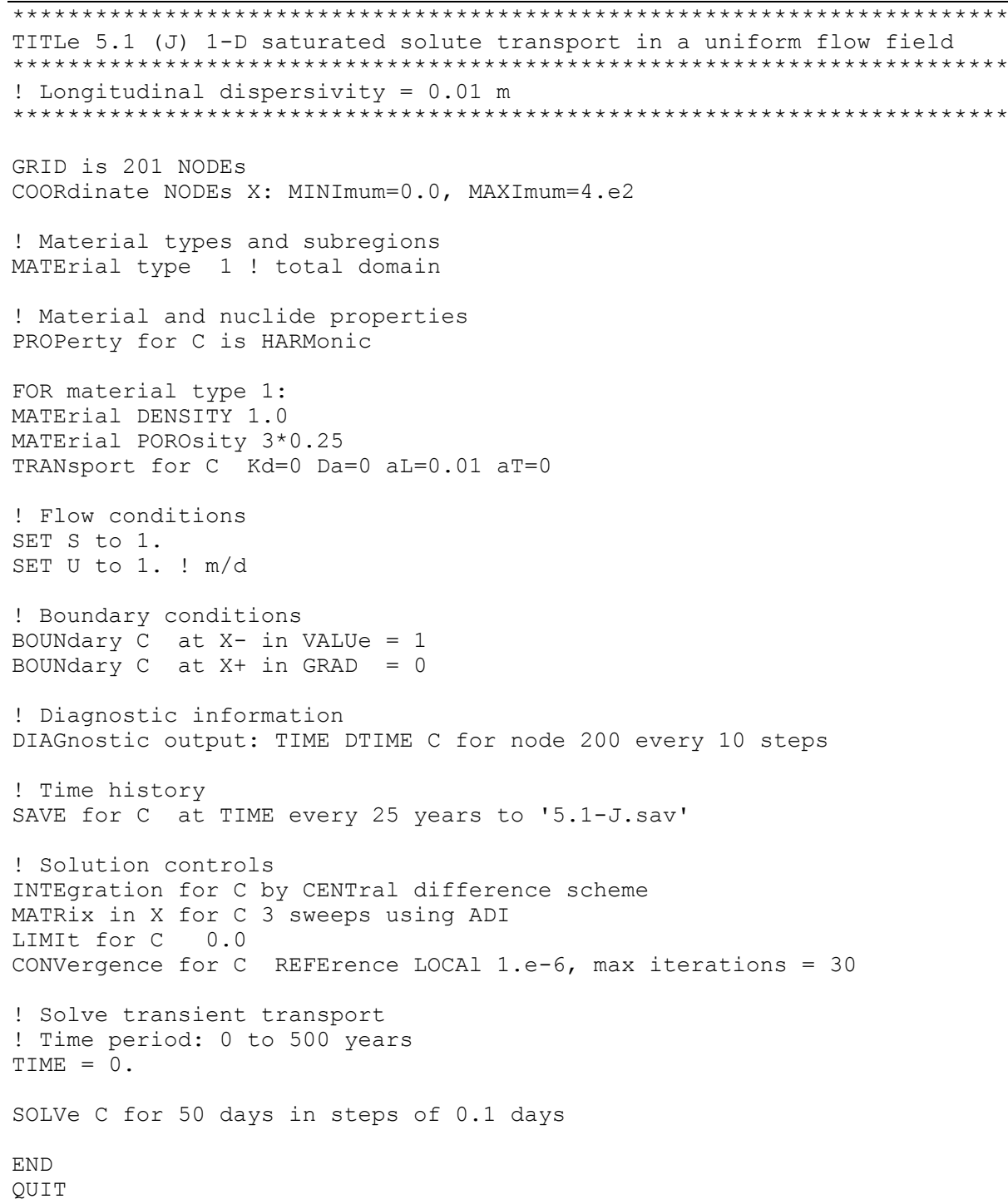

Table 5.1.29a. Input Commands for 1D Transport (Case $K, T=25$ days)

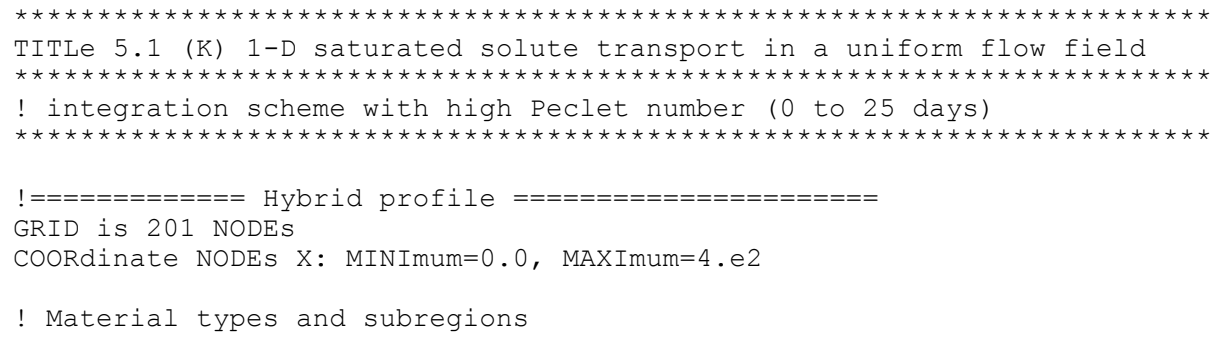


PROPerty for $\mathrm{C}$ is HARMonic

FOR material type 1:

MATErial DENSITY 1.0

MATErial POROsity $3 * 0.25$

TRANsport for $\mathrm{C} \quad \mathrm{Kd}=0 \quad \mathrm{Da}=0 \mathrm{aL}=0.01 \mathrm{aT}=0$

! Flow conditions

SET S to 1.

SET U to 1. ! $\mathrm{m} / \mathrm{d}$

! Boundary conditions

Boundary $\mathrm{C}$ at $\mathrm{X}$ - in $\mathrm{VALUe}=1$

BOUNdary $\mathrm{C}$ at $\mathrm{X}+$ in GRAD $=0$

! Diagnostic information

DIAGnostic output: TIME DTIME C for node 200 every 10 steps

! Solution controls

INTEgration for C by HYBRid profile

MATRix in $X$ for $C 3$ sweeps using ADI

LIMIt for $\mathrm{C} 0.0$

CONVergence for C REFErence LOCAl $1 . e-6$, max iterations $=30$

! Solve transient transport

! Time period: 0 to 25 days

TIME $=0$.

SOLVe $C$ for 25 days in steps of 0.1 days

SAVE for C NOW to '5.1-K1-25.sav'

END

! $============$ = Hybrid profile $======================$

! $=============$ CONDif Scheme $======================$

GRID is 201 NODES

COORdinate NODES X: MINImum $=0.0$, MAXImum $=4 . e 2$

! Material types and subregions

MATErial type 1 ! total domain

PROPerty for $\mathrm{C}$ is HARMonic

FOR material type 1:

MATErial DENSITY 1.0

MATErial POROsity $3 * 0.25$

TRANsport for C $\mathrm{Kd}=0$ Da=0 aL=0.01 aT=0

! Flow conditions

SET S to 1 .

SET U to 1. ! $\mathrm{m} / \mathrm{d}$

! Boundary conditions

Boundary $\mathrm{C}$ at $\mathrm{X}$ - in VALUe $=1$

BOUNdary $\mathrm{C}$ at $\mathrm{X}+$ in GRAD $=0$

! Diagnostic information

DIAGnostic output: TIME DTIME C for node 200 every 10 steps

! Solution controls

INTEgration for $C$ by CONDif scheme

MATRix in $X$ for $C 3$ sweeps using ADI

LIMIt for C 0.0

CONVergence for C REFErence LOCAl 1.e-6, $\max$ iterations $=30$

! Solve transient transport

! Time period: 0 to 25 days

TIME $=0$

SOLVe $C$ for 25 days in steps of 0.1 days

SAVE for C NOW to '5.1-K2-25.sav'

END 


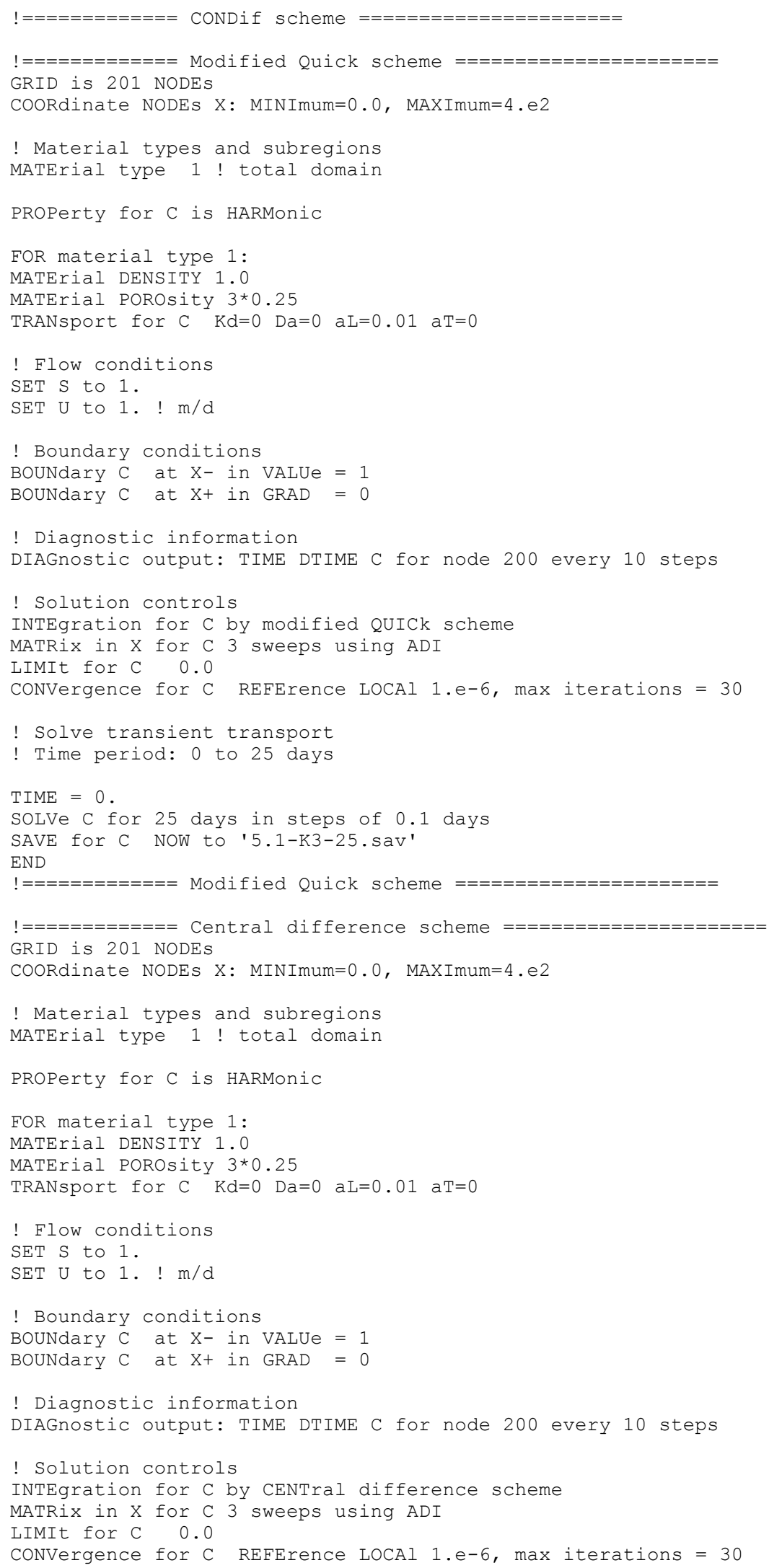


! Solve transient transport

! Time period: 0 to 25 days

$\mathrm{TIME}=0$.

SOLVe $\mathrm{C}$ for 25 days in steps of 0.1 days

SAVE for C Now to '5.1-K4-25.sav'

END

$\mathrm{I}============$ Central difference scheme $====================$

QUIT

Table 5.1.29b. Input Commands for 1D Transport (Case $\mathrm{K}, \mathrm{T}=\mathbf{5 0}$ days)

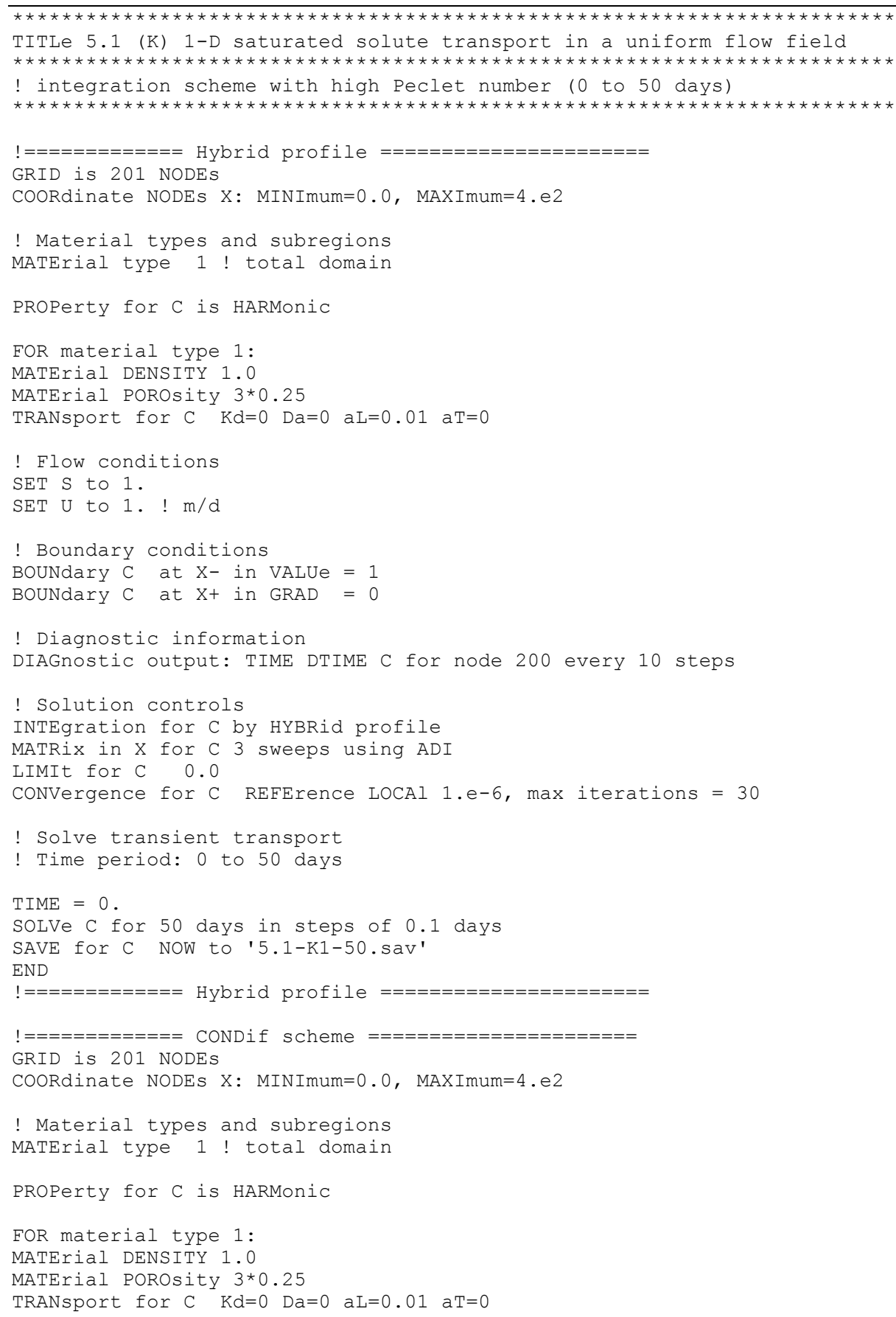




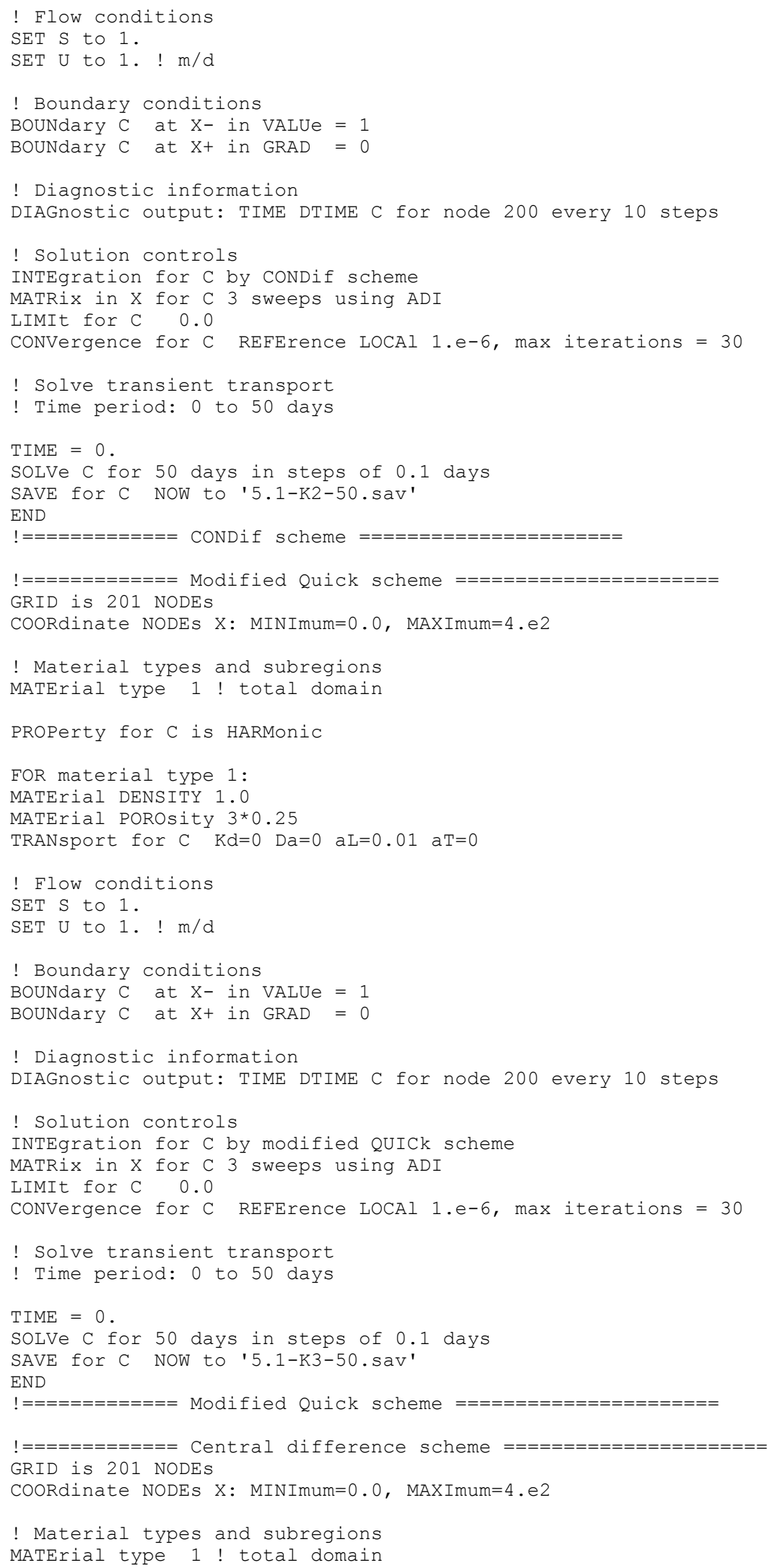




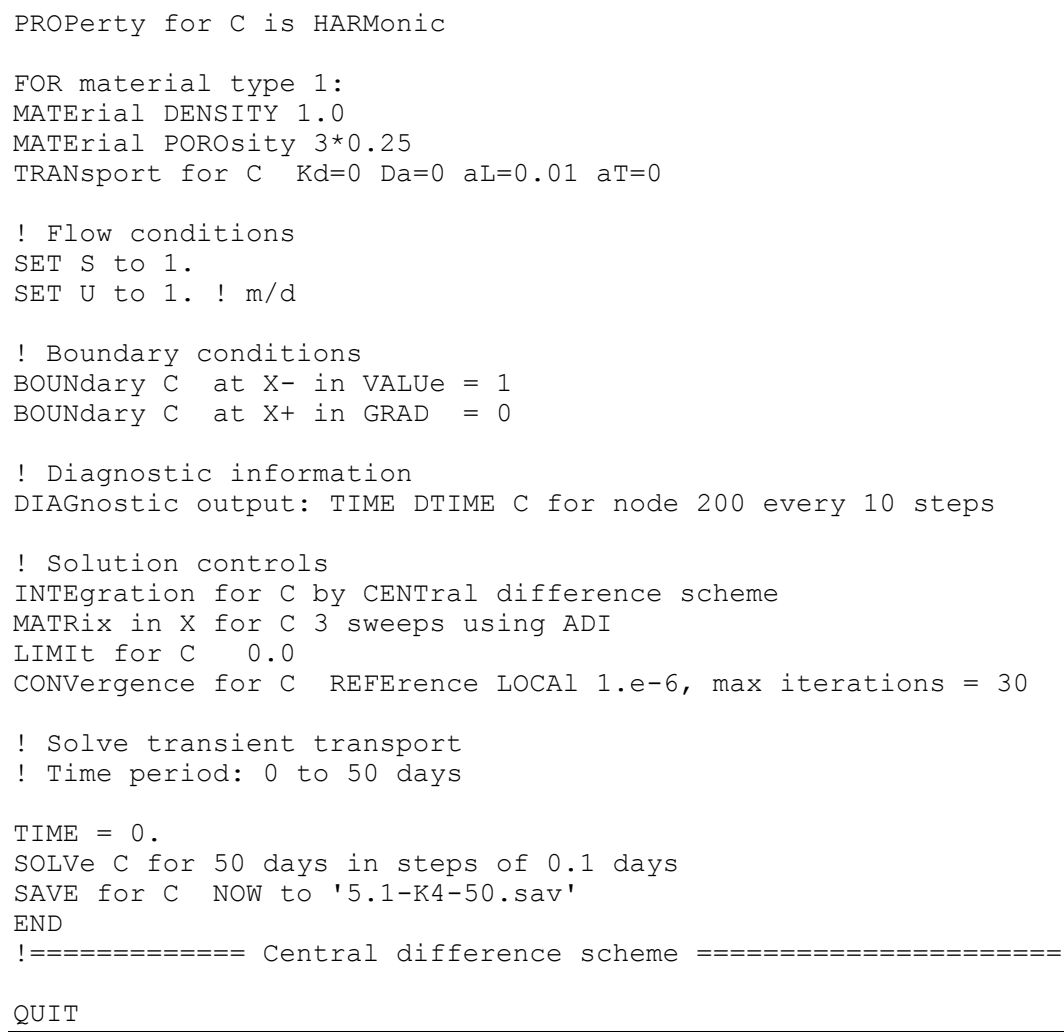

Table 5.1.30a. Input Commands for 1D Transport (Case $\mathrm{L}, \mathrm{T}=\mathbf{2 5}$ days)

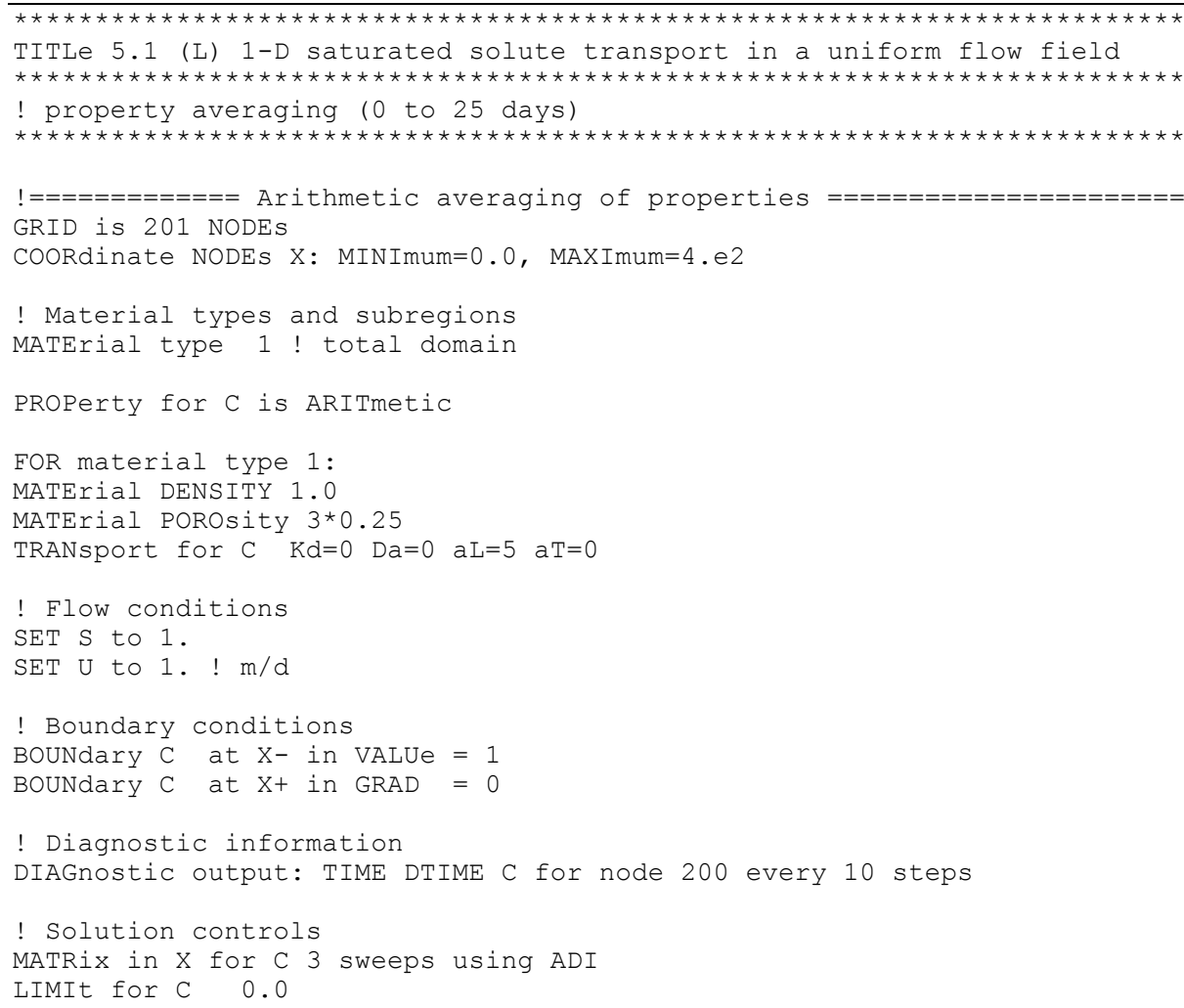




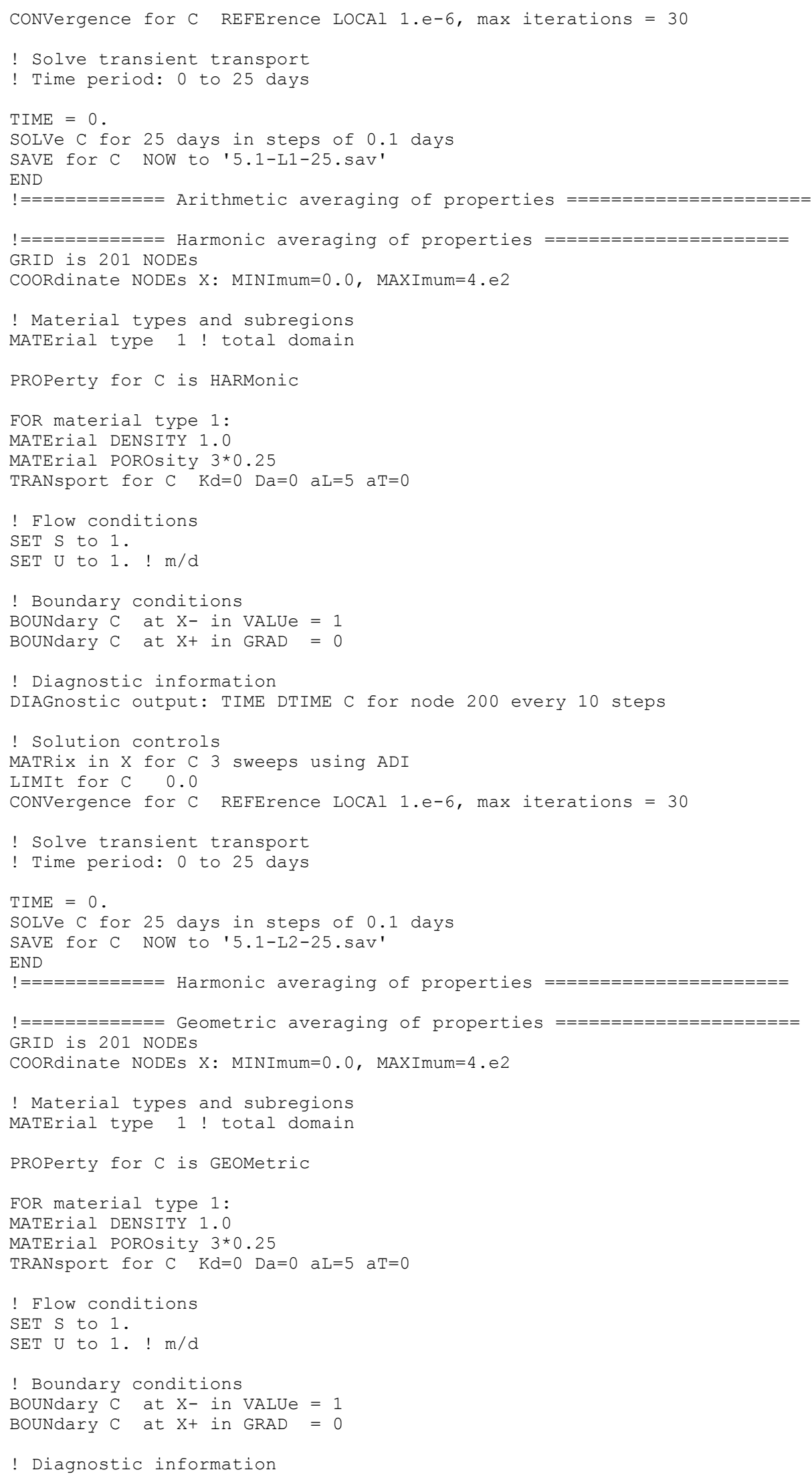


DIAGnostic output: TIME DTIME C for node 200 every 10 steps

! Solution controls

MATRix in $X$ for C 3 sweeps using ADI

LIMIt for $\mathrm{C} 0.0$

CONVergence for C REFErence LOCAl $1 . e-6$, $\max$ iterations $=30$

! Solve transient transport

! Time period: 0 to 25 days

TIME $=0$.

SOLVe $\mathrm{C}$ for 25 days in steps of 0.1 days

SAVE for C NOW to '5.1-L3-25.sav'

END

! $=============$ GeometriC averaging of properties $======================$

! $=============$ Upwind averaging of properties $======================$

GRID is 201 NODES

COORdinate NODES $\mathrm{X}$ : MINImum $=0.0$, MAXImum $=4 . e 2$

! Material types and subregions

MATErial type 1 ! total domain

PROPerty for $\mathrm{C}$ is UPWInd

FOR material type 1:

MATErial DENSITY 1.0

MATErial POROsity $3 * 0.25$

TRANsport for $C \quad K d=0 \quad \mathrm{Da}=0 \quad \mathrm{aL}=5 \quad \mathrm{aT}=0$

! Flow conditions

SET S to 1 .

SET U to 1. ! $\mathrm{m} / \mathrm{d}$

! Boundary conditions

Boundary $\mathrm{C}$ at $\mathrm{X}$ - in VALUe $=1$

BOUNdary $\mathrm{C}$ at $\mathrm{X}+$ in GRAD $=0$

! Diagnostic information

DIAGnostic output: TIME DTIME C for node 200 every 10 steps

! Solution controls

MATRix in $X$ for C 3 sweeps using ADI

LIMIt for $\mathrm{C} 0.0$

CONVergence for C REFErence LOCAl $1 . e-6$, max iterations $=30$

! Solve transient transport

! Time period: 0 to 25 days

TIME $=0$.

SOLVe $\mathrm{C}$ for 25 days in steps of 0.1 days

SAVE for C NOW to '5.1-L4-25.sav'

END

! $=============$ Upwind averaging of properties $======================$

QUIT

Table 5.1.30b. Input Commands for 1D Transport (Case $L, T=50$ days)

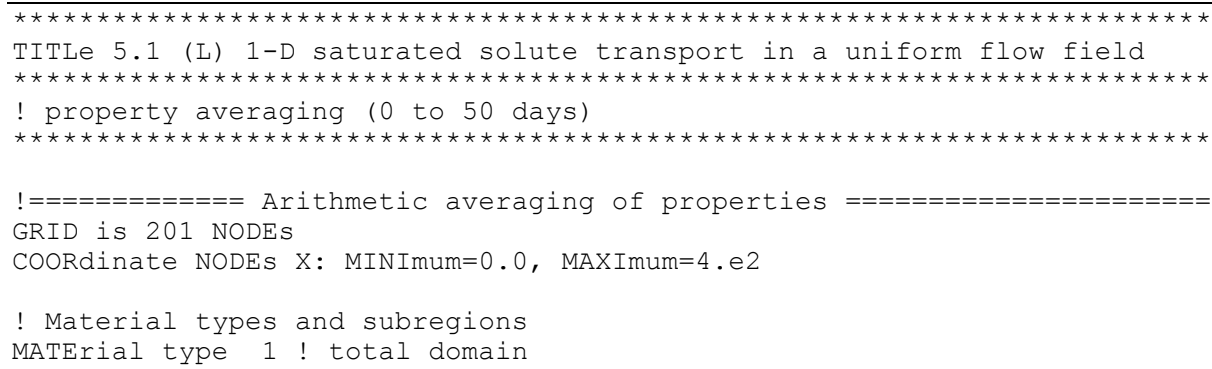


PROPerty for $\mathrm{C}$ is ARITmetic

FOR material type 1 :

MATErial DENSITY 1.0

MATErial POROsity $3 * 0.25$

TRANsport for $C \mathrm{Kd}=0$ Da=0 aL=5 $\mathrm{aT}=0$

! Flow conditions

SET S to 1.

SET U to 1. ! $\mathrm{m} / \mathrm{d}$

! Boundary conditions

Boundary $\mathrm{C}$ at $\mathrm{X}$ - in VALUe $=1$

BOUNdary $C$ at $\mathrm{X}+$ in GRAD $=0$

! Diagnostic information

DIAGnostic output: TIME DTIME C for node 200 every 10 steps

! Solution controls

MATRix in $\mathrm{X}$ for $\mathrm{C} 3$ sweeps using ADI

LIMIt for $\mathrm{C} 0.0$

CONVergence for $C$ REFErence LOCAl $1 . e-6, \max$ iterations $=30$

! Solve transient transport

! Time period: 0 to 50 days

$\operatorname{TIME}=0$.

SOLVe $C$ for 50 days in steps of 0.1 days

SAVE for C NOW to '5.1-L1-50.sav'

END

! $=============$ Arithmetic averaging of properties

! $=============$ HarmoniC averaging of properties $======================$

GRID is 201 NODES

COORdinate NODES X: MINImum=0.0, MAXImum $=4 . e 2$

! Material types and subregions

MATErial type 1 ! total domain

PROPerty for $\mathrm{C}$ is HARMonic

FOR material type 1:

MATErial DENSITY 1.0

MATErial POROsity $3 * 0.25$

TRANsport for $\mathrm{C} \quad \mathrm{Kd}=0 \quad \mathrm{Da}=0$ aL=5 $\mathrm{aT}=0$

! Flow conditions

SET S to 1 .

SET U to 1. ! $\mathrm{m} / \mathrm{d}$

! Boundary conditions

Boundary $\mathrm{C}$ at $\mathrm{X}$ - in VALUe $=1$

BOUNdary $\mathrm{C}$ at $\mathrm{X}+$ in GRAD $=0$

! Diagnostic information

DIAGnostic output: TIME DTIME C for node 200 every 10 steps

! Solution controls

MATRix in $X$ for $C 3$ sweeps using ADI

LIMIt for $\mathrm{C} 0.0$

CONVergence for C REFErence LOCAl 1.e-6, max iterations = 30

! Solve transient transport

! Time period: 0 to 50 days

TIME $=0$.

SOLVe $C$ for 50 days in steps of 0.1 days

SAVE for C NOW to '5.1-L2-50.sav'

END

! $=============$ HarmoniC averaging of properties $======================$

! $=============$ GeometriC averaging of properties $======================$

GRID is 201 NODES 
COORdinate NODES $\mathrm{X}$ : MINImum=0.0, MAXImum $=4 . e 2$

! Material types and subregions

MATErial type 1 ! total domain

PROPerty for $\mathrm{C}$ is GEOMetric

FOR material type 1 :

MATErial DENSITY 1.0

MATErial POROsity $3 * 0.25$

TRANsport for $C \mathrm{Kd}=0 \quad \mathrm{Da}=0$ aL=5 $\mathrm{aT}=0$

! Flow conditions

SET S to 1 .

SET U to 1. ! $\mathrm{m} / \mathrm{d}$

! Boundary conditions

Boundary $\mathrm{C}$ at $\mathrm{X}$ - in VALUe $=1$

BOUNdary $C$ at $\mathrm{X}+$ in GRAD $=0$

! Diagnostic information

DIAGnostic output: TIME DTIME C for node 200 every 10 steps

! Solution controls

MATRix in $\mathrm{X}$ for C 3 sweeps using ADI

LIMIt for $\mathrm{C} 0.0$

CONVergence for C REFErence LOCAl $1 . e-6, \max$ iterations $=30$

! Solve transient transport

! Time period: 0 to 50 days

TIME $=0$.

SOLVe $C$ for 50 days in steps of 0.1 days

SAVE for C NOW to '5.1-L3-50.sav'

END

! $=============$ Geometric averaging of properties $======================$

! $=============$ Upwind averaging of properties $======================$

GRID is 201 NODES

COORdinate NODES $\mathrm{X}$ : MINImum=0.0, MAXImum $=4 . e 2$

! Material types and subregions

MATErial type 1 ! total domain

PROPerty for $\mathrm{C}$ is UPWInd

FOR material type 1:

MATErial DENSITY 1.0

MATErial POROsity $3 * 0.25$

TRANsport for $C \mathrm{Kd}=0 \mathrm{Da}=0$ aL=5 $\mathrm{aT}=0$

! Flow conditions

SET S to 1 .

SET U to 1. ! $\mathrm{m} / \mathrm{d}$

! Boundary conditions

Boundary $\mathrm{C}$ at $\mathrm{X}$ - in VALUe $=1$

BOUNdary $\mathrm{C}$ at $\mathrm{X}+$ in $\mathrm{GRAD}=0$

! Diagnostic information

DIAGnostic output: TIME DTIME C for node 200 every 10 steps

! Solution controls

MATRix in $\mathrm{X}$ for $\mathrm{C} 3$ sweeps using ADI

LIMIt for $\mathrm{C} 0.0$

CONVergence for C REFErence LOCAl 1.e-6, $\max$ iterations $=30$

! Solve transient transport

! Time period: 0 to 50 days

TIME $=0$.

Solve $\mathrm{C}$ for 50 days in steps of 0.1 days

SAVE for C NOW to '5.1-L4-50.sav' 
END

! $=============$ Upwind averaging of properties

QUIT

\subsection{Two-Dimensional Saturated Solute Transport in a Uniform Flow Field}

This problem deals with two-dimensional advection-dispersion of a non-conservative solute species from a point source through an infinite porous medium. It is used to demonstrate the impact that grid orientation with transverse dispersion has on the solution from PORFLOW. A physical schematic of this problem is shown in Figure 5.2.1(a). In practice, the idealized conditions are analogous to continual leakage or injection of a contaminant into a shallow confined aquifer from a small leaking landfill or an improperly sealed fully penetrating injection well (gradients in the vertical direction are assumed to be negligible). It is assumed that the total rate of fluid leakage or injection into the aquifer is negligible and does not disturb the ambient groundwater flow regime. Analytically the problem is treated as a point source in the 2-D areal plane.

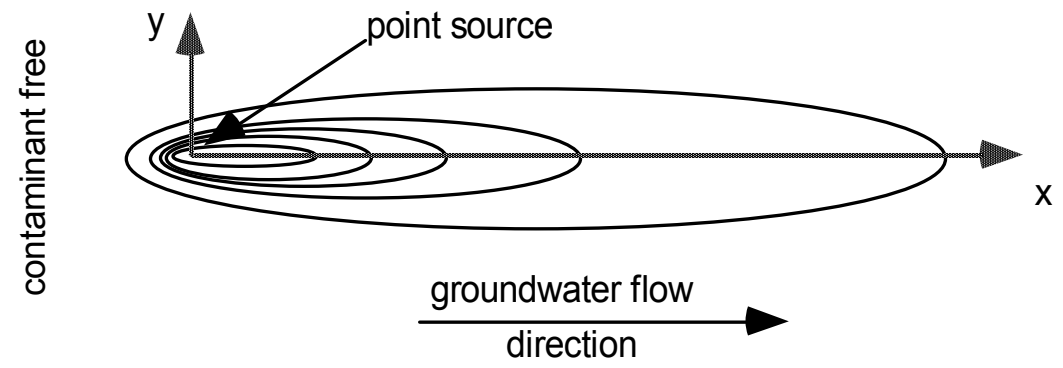

(a)

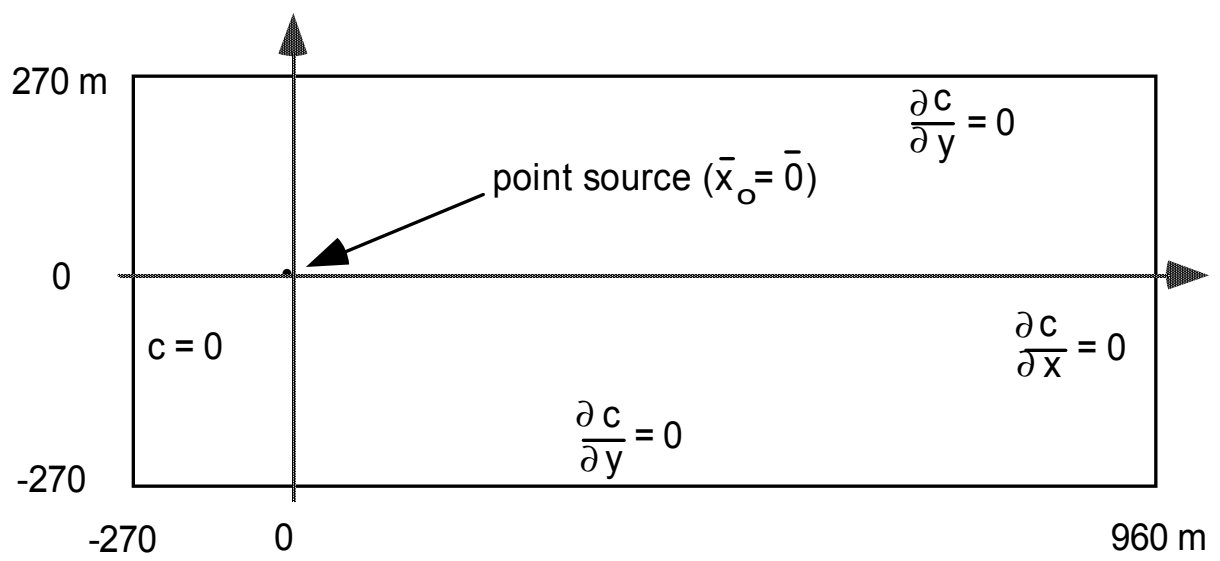

(b)

\section{Figure 5.2.1. Schematic Diagram for 2D Solute Transport in a Confined Aquifer}

As illustrated in Figure 5.2.1(b), a non-conservative contaminant is continuously released from a point source downstream of an inflow boundary (containing zero contaminant) into a shallow confined aquifer unit whose groundwater flow is assumed to be uniform. Both hydrodynamic dispersion and molecular diffusion are allowed, as well as, the possibility of radioactive decay and/or adsorption of the transported species. It is assumed that the contaminant mass flow rate at 
the point source remains constant, the aquifer's flow rate is uniform and constant, and the homogeneous aquifer's properties (such as porosity, soil type, water saturation) are uniform and constant.

Analytical solution: Equation 5.1.4 represents the non-conservative form of the multidimensional advection-dispersion equation for solute transport through a variably saturated porous media. Taking the 2-D form of Equation 5.1.4 and assuming that one point exists at the areal location $\mathrm{x}=\mathrm{y}=0$, constant water saturation level, and that material coefficients are constants, results in

$$
\frac{\partial c}{\partial t}=D_{x x}^{\prime} \frac{\partial^{2} c}{\partial x^{2}}+D_{y y}^{\prime} \frac{\partial^{2} c}{\partial y^{2}}+D_{x y}^{\prime} \frac{\partial^{2} c}{\partial x \partial y}+D_{y x}^{\prime} \frac{\partial^{2} c}{\partial y \partial x}-u_{x}^{\prime} \frac{\partial c}{\partial x}-u_{y}^{\prime} \frac{\partial c}{\partial y}-\lambda c-\frac{q\left(c-c^{*}\right)}{\theta_{e} R}
$$

where

$$
\begin{aligned}
& \mathrm{D}_{\mathrm{xx}}^{\prime}=\frac{\alpha_{\mathrm{L}} \frac{\mathrm{U}_{\mathrm{x}}^{2}}{|\mathrm{U}|}+\alpha_{\mathrm{T}} \frac{\mathrm{U}_{\mathrm{y}}^{2}}{|\mathrm{U}|}+\theta_{\mathrm{e}} \tau \mathrm{D}_{\mathrm{m}}}{\theta_{\mathrm{e}} \mathrm{R}} \\
& \mathrm{D}_{\mathrm{yy}}^{\prime}=\frac{\alpha_{\mathrm{L}} \frac{\mathrm{U}_{\mathrm{y}}^{2}}{|\mathrm{U}|}+\alpha_{\mathrm{T}} \frac{\mathrm{U}_{\mathrm{x}}^{2}}{|\mathrm{U}|}+\theta_{\mathrm{e}} \tau \mathrm{D}_{\mathrm{m}}}{\theta_{\mathrm{e}} \mathrm{R}} \\
& \mathrm{D}_{\mathrm{xy}}^{\prime}=\mathrm{D}_{\mathrm{yx}}^{\prime}=\frac{\left(\alpha_{\mathrm{L}}-\alpha_{\mathrm{T}}\right) \frac{\mathrm{U}_{\mathrm{x}} \mathrm{U}_{\mathrm{y}}}{|\mathrm{U}|}}{\theta_{\mathrm{e}} \mathrm{R}}
\end{aligned}
$$

and $\alpha_{\mathrm{T}}$ is the transverse dispersivity.

If the flow field is aligned with the x-axis Equation 5.2.1 reduces to

$$
\frac{\partial c}{\partial t}=D_{x x}^{\prime} \frac{\partial^{2} c}{\partial x^{2}}+D_{y y}^{\prime} \frac{\partial^{2} c}{\partial y^{2}}-u_{x}^{\prime} \frac{\partial c}{\partial x}-\lambda c-\frac{q\left(c-c^{*}\right)}{\theta_{e} R}
$$

For our infinite confined aquifer the initial conditions are:

$$
c(x, y, 0)=0
$$

and the boundary conditions are:

$$
c( \pm \infty, y, t)=0
$$




$$
c(x, \pm \infty, t)=0
$$

These boundary conditions are equivalent to assuming that the dispersive flux of solute is zero at plus or minus infinity or

$$
\begin{aligned}
& \lim _{x \rightarrow \pm \infty}\left(\frac{\partial c}{\partial x}\right)=0 \\
& \lim _{y \rightarrow \pm \infty}\left(\frac{\partial c}{\partial y}\right)=0
\end{aligned}
$$

For finite times there exists finite values of $\mathrm{x}$ and $\mathrm{y}$ where Equations $5.2 .4 \mathrm{~d}$ and $5.2 .4 \mathrm{e}$ remain valid.

As mentioned above, it is assumed that the total rate of fluid flow, q, into the aquifer due to the source is negligible and does not disturb the ambient groundwater flow regime. In order to have a finite mass flow rate of contaminant requires

$$
\lim _{\mathrm{q} \rightarrow 0}\left(\mathrm{q} \mathrm{c}^{*}\right)<\infty \Rightarrow \lim _{\mathrm{q} \rightarrow 0}\left(\mathrm{c}^{*}\right)= \pm \infty
$$

Equation 5.2.3, a linear partial differential equation subject to the initial and boundary conditions given by Equations 5.2.4a to 5.2.4c, can be solved by applying Laplace and Fourier transforms to derive at the appropriate Green's functions. For details, see Yeh (1981). The general solution for a continuous point source takes the form:

$$
\mathrm{c}(\mathrm{x}, \mathrm{y}, \mathrm{t})=\frac{\mathrm{qc}}{\theta_{\mathrm{e}} \mathrm{R}} \int_{0}^{\mathrm{t}} \mathrm{G}(\mathrm{x}|\xi ; \mathrm{y}| \eta ; \mathrm{t} \mid \tau) \mathrm{d} \tau
$$

where $\mathrm{G}(\mathrm{x}|\xi ; \mathrm{y}| \eta ; \mathrm{t} \mid \tau)$ is the Green's function over the domain space. For our analytical problem, we shall limit our flow field to flow parallel to the $x$-axis only. This results in a dispersion tensor that is diagonal and a separable Green's function. It can be shown that for a simple geometry such as a separable coordinate system, Green's function can be expressed as:

$$
\mathrm{G}(\mathrm{x}|\xi ; \mathrm{y}| \eta ; \mathrm{t} \mid \tau)=\mathrm{G}_{1}(\mathrm{x}|\xi ; \mathrm{t}| \tau) \mathrm{G}_{2}(\mathrm{y}|\eta ; \mathrm{t}| \tau)
$$

where for a point source in the x-direction (infinite domain, parallel to flow):

$$
\mathrm{G}_{1}(\mathrm{x}|\xi ; \mathrm{t}| \tau)=\frac{\mathrm{S}(\mathrm{t}-\tau)}{\sqrt{4 \pi \mathrm{D}_{\mathrm{xx}}^{\prime}(\mathrm{t}-\tau)}} \exp \left[-\frac{\left\{(\mathrm{x}-\xi)-\mathrm{u}_{\mathrm{x}}^{\prime}(\mathrm{t}-\tau)\right\}^{2}}{4 \mathrm{D}_{\mathrm{xx}}^{\prime}(\mathrm{t}-\tau)}-\lambda(\mathrm{t}-\tau)\right]
$$

and for a point source in the y-direction (infinite domain, transverse to flow): 


$$
G_{2}(y|\eta ; t| \tau)=\frac{S(t-\tau)}{\sqrt{4 \pi D_{y y}^{\prime}(t-\tau)}} \exp \left[-\frac{(y-\eta)^{2}}{4 D_{y y}^{\prime}(t-\tau)}\right]
$$

where $S(t-\tau)$ is the step function.

The evaluation of the analytical expressions, Equations 5.2.5a to $5.2 .5 \mathrm{~d}$, for a specific problem is performed numerically using the FORTRAN program AT123D in Section C.

PORFLOW numerical simulation and comparison: The PORFLOW 2-D Cartesian models for this consist of two grids. One grid is a rectangular domain in which the aquifer groundwater flow is in the x-direction only. The other grid is a square domain in which the aquifer groundwater flow is diagonal to the mesh orientation. The point source in both grids is located at $\mathrm{x}=\mathrm{y}=0$. Zero diffusive flux boundary conditions are applied at the $+X,-Y$ and $+Y$ faces of the mesh.

Even though we are considering an aquifer unit with infinite extent in the areal directions, our numerical model has finite size. At our inflow boundary $(-X)$ we shall assume that the incoming fluid remains contaminant free (i.e., the contaminant concentration immediately upstream of the source does not extend back up to the inflow boundary). For the parameters chosen (i.e., longitudinal dispersivity, Darcy velocity, and source location), the above assumption remains valid over the time of interest.

In addition, PORFLOW requires input about the total mass flowrate per unit thickness entering the aquifer at the point source. An arbitrarily small but finite value for $\mathrm{qc}^{*}$ was chosen to maintain computed solute concentration values near the source to acceptable values. Steep concentration gradients near a source can result in oscillatory behavior unless the local grid is sufficiently refined. For demonstration purposes, we have chosen uniform coarse grids and are primarily interested in results away from the source location. The simulations are done by specifying a velocity field and running the solute transport option only. In this way, the flow rate entering the aquifer due to the point source does not alter the aquifer flow field.

Values of the physical parameters used in the verification simulations are presented in Table 5.2.1. For the conservative solute transport cases, the parameters were selected based on data from a field investigation on hexavalent chromium contamination reported by Perlumutter and Lieber (1970) and Wilson and Miller (1978). For the non-conservative solute transport cases, the values of retardation and decay constants were chosen arbitrarily to test the performance of PORFLOW transport modules. 
Table 5.2.1. Values of the Physical Parameters, Mesh Spacing, Time Steps, and Key Computed Parameters Used in the Two-Dimensional Transport Simulations.

\begin{tabular}{|c|c|c|}
\hline Physical parameters & Base Case & Range tested \\
\hline Darcy velocity, $\mathrm{U}_{\mathrm{X}}$ & $0.161 \mathrm{~m} / \mathrm{d}$ & - \\
\hline Porosity,$\phi$ & 0.35 & - \\
\hline Longitudinal dispersivity, $\alpha_{\mathrm{L}}$ & $21.3 \mathrm{~m}$ & - \\
\hline Transverse dispersivity, $\alpha_{\mathrm{T}}$ & $4.3 \mathrm{~m}$ & - \\
\hline $\begin{array}{l}\text { Apparent molecular dispersion coefficient, } \\
\qquad \theta_{\mathrm{e}} \tau \mathrm{D}_{\mathrm{m}}\end{array}$ & $0.0 \mathrm{~m}^{2} / \mathrm{d}$ & - \\
\hline Water saturation, $\mathrm{S}_{\mathrm{W}}$ & 1.0 & - \\
\hline Radioactive decay constant., $\lambda$ & $0.0 \mathrm{~d}^{-1}$ & $0.0,0.005$ \\
\hline Soil density, $\rho_{\mathrm{S}}$ & $1.23077 \mathrm{~kg} / \mathrm{m}^{3}$ & - \\
\hline Solute distribution coefficient, $\mathrm{k}_{\mathrm{d}}$ & $0.0 \mathrm{~m}^{3} / \mathrm{kg}$ & $0.0,0.4375$ \\
\hline Boundary solute concentration, $\mathrm{c}_{\mathrm{O}}$ & $0.0 \mathrm{~kg} / \mathrm{m}^{3}$ & - \\
\hline $\begin{array}{l}\text { Contaminant total mass flowrate per unit } \\
\text { aquifer thickness (point source), qc* }\end{array}$ & $7.040119 \mathrm{E}-3 \mathrm{~kg} / \mathrm{d} / \mathrm{m}$ & - \\
\hline \multicolumn{3}{|l|}{ Grid specifics } \\
\hline Grid spacing, $\Delta x=\Delta y$ & $15 \mathrm{~m}$ & - \\
\hline Number nodes in $\mathrm{x}$-dir & 83 & - \\
\hline \multirow{2}{*}{ Number nodes in y-dir } & 37 (parallel grid) & - \\
\hline & 83 (diagonal grid) & \\
\hline \multicolumn{3}{|l|}{ Time steps } \\
\hline Time duration & $1400 \mathrm{~d}$ & - \\
\hline number time-steps & 1400 & - \\
\hline time-step size, $\Delta \mathrm{t}$ & $1 \mathrm{~d}$ & - \\
\hline
\end{tabular}




\begin{tabular}{|c|c|c|}
\hline \multicolumn{3}{|l|}{ Key computed parameters } \\
\hline Retardation factor, $\mathrm{R}$ & 1.0 & 2.0 \\
\hline Bulk soil density, $\rho_{b}$ & $0.8 \mathrm{~kg} / \mathrm{m}^{3}$ & - \\
\hline Phasic velocity, $\mathrm{u}_{\mathrm{x}}$ & $\begin{array}{c}0.46 \mathrm{~m} / \mathrm{d}(\mathrm{P}) \\
0.3253 \mathrm{~m} / \mathrm{d}(\mathrm{D})\end{array}$ & - \\
\hline Retarded phasic velocity, $\mathrm{u}_{\mathrm{x}}^{\prime}$ & $\begin{array}{c}0.46 \mathrm{~m} / \mathrm{d}(\mathrm{P}) \\
0.3253 \mathrm{~m} / \mathrm{d}(\mathrm{D})\end{array}$ & $\begin{array}{c}0.23 \mathrm{~m} / \mathrm{d}(\mathrm{P}) \\
0.1626 \mathrm{~m} / \mathrm{d}(\mathrm{D})\end{array}$ \\
\hline Phasic velocity, $\mathrm{u}_{\mathrm{y}}$ & $\begin{array}{c}0.0 \mathrm{~m} / \mathrm{d}(\mathrm{P}) \\
0.3253 \mathrm{~m} / \mathrm{d}(\mathrm{D})\end{array}$ & - \\
\hline Retarded phasic velocity, $\mathrm{u}_{\mathrm{y}}^{\prime}$ & $\begin{array}{c}0.0 \mathrm{~m} / \mathrm{d}(\mathrm{P}) \\
0.3253 \mathrm{~m} / \mathrm{d}(\mathrm{D})\end{array}$ & $\begin{array}{c}0.0 \mathrm{~m} / \mathrm{d}(\mathrm{P}) \\
0.1626 \mathrm{~m} / \mathrm{d}(\mathrm{D})\end{array}$ \\
\hline $\begin{array}{l}\text { Retarded longitudinal dispersion } \\
\text { coefficient, } \mathrm{D}_{\mathrm{xx}}^{\prime}\end{array}$ & $\begin{array}{l}9.798 \mathrm{~m}^{2} / \mathrm{d}(\mathrm{P}) \\
5.888 \mathrm{~m}^{2} / \mathrm{d}(\mathrm{D})\end{array}$ & $\begin{array}{l}4.899 \mathrm{~m}^{2} / \mathrm{d}(\mathrm{P}) \\
2.944 \mathrm{~m}^{2} / \mathrm{d}(\mathrm{D})\end{array}$ \\
\hline $\begin{array}{l}\text { Retarded transverse dispersion coefficient, } \\
\qquad \mathrm{D}_{\mathrm{yy}}^{\prime}\end{array}$ & $\begin{array}{l}1.978 \mathrm{~m}^{2} / \mathrm{d}(\mathrm{P}) \\
5.888 \mathrm{~m}^{2} / \mathrm{d}(\mathrm{D})\end{array}$ & $\begin{array}{l}0.989 \mathrm{~m}^{2} / \mathrm{d}(\mathrm{P}) \\
2.944 \mathrm{~m}^{2} / \mathrm{d}(\mathrm{D})\end{array}$ \\
\hline Retarded cross dispersion coefficient, $\mathrm{D}_{\mathrm{xy}}^{\prime}$ & $\begin{array}{c}0.0 \mathrm{~m}^{2} / \mathrm{d}(\mathrm{P}) \\
3.91 \mathrm{~m}^{2} / \mathrm{d}(\mathrm{D})\end{array}$ & $\begin{array}{c}0.0 \mathrm{~m}^{2} / \mathrm{d}(\mathrm{P}) \\
1.955 \mathrm{~m}^{2} / \mathrm{d}(\mathrm{D})\end{array}$ \\
\hline Cell Fourier number, $\mathrm{Fo}_{\mathrm{x}} ; \mathrm{Fo}_{\mathrm{y}}$ & $0.0435 ; 0.0088(\mathrm{P})$ & $0.0218 ; 0.0044(\mathrm{P})$ \\
\hline Cell Courant number, $\mathrm{Co}_{\mathrm{x}} ; \mathrm{Co}_{\mathrm{y}}$ & $0.0307 ; 0.0(\mathrm{P})$ & $0.0153 ; 0.0(\mathrm{P})$ \\
\hline Cell Peclet number, $\mathrm{Pe}_{\mathrm{x}} ; \mathrm{Pe}_{\mathrm{y}}$ & $0.7042 ; 3.4883(\mathrm{P})$ & $0.7042,3.4883(\mathrm{P})$ \\
\hline
\end{tabular}

In 1D transport, only longitudinal dispersion is active and in a 2D transport problem, both longitudinal and transverse dispersion can occur. In a general 2D transport problem, the offdiagonal terms of the dispersion coefficient tensor are typically non-zero. In PORFLOW the resulting dispersive flux cross-term products such as

$$
\frac{\partial}{\partial x}\left[D_{x y}\left(\frac{\partial c}{\partial y}\right)\right]
$$

are handled consistently and are not "lumped". The lumping approximation is more commonly used in finite difference algorithms (Faust, et. al., 1993) to accommodate their matrix solution requirements. Typically, lumping greatly over estimates transverse dispersion. Also for many algorithms, grid orientation effects occur even though these cross-product dispersion terms are handled in a consistent formulation.

To examine these grid orientation effects in PORFLOW, two base case grids were chosen for this problem: (1) a grid aligned parallel to the aquifer flow direction consisting of 3071 nodes uniformly spaced (15 $\mathrm{m}$ in length) with 83 nodes along the $\mathrm{x}$-axis and 37 along the $\mathrm{y}$-axis and (2) a grid aligned at a $45^{\circ}$ diagonal to the aquifer flow direction consisting of 6889 nodes uniformly spaced (15 m in length) with 83 nodes along the $\mathrm{x}$-axis and y-axis. Figures 5.2.2 and 5.2.3 illustrate PORFLOW grids chosen. At the channel inlet boundary(s) (-X face; $-\mathrm{X}$ face and $-\mathrm{Y}$ face), the concentration of solute in the incoming water is set to $0.0 \mathrm{~kg} / \mathrm{m}^{3}$. Due to the finite 
overall length of our mesh, at the outflow boundary(s) $(+\mathrm{X}$ face; $+\mathrm{X}$ face and $+\mathrm{Y}$ face) the dispersive flux (GRAD) is set to zero, while the advective flux is calculated as part of the solution. In addition, for the parallel grid the dispersive flux is set to zero at the transverse faces ( $-Y$ face and $+Y$ face). The nodal spacing for both grid orientations is the same and the results shown should represent grid orientation effects only.

For this problem several simulations were performed. As summarized in Table 5.2.2, simulations were performed for both base cases (parallel and diagonal grids) and then two additional runs were made varying certain key physical parameters and PORFLOW options to demonstrate their impact on the results. For each simulation, a transient calculation was performed for a 1400-day duration and the results from PORFLOW at this end time are compared to the analytical solution given by Equation 5.2.5a. As shown in Table 5.2.1., a range of cell Peclet, cell material Courant, and cell Fourier numbers were tested.

The results of all the simulations (both numerical and analytical) are shown in Figures 5.2.4 through 5.2.15. These results are also presented in tabular form for comparison in Tables 5.2.3 through 5.2.7. The input commands for the 2D transport simulations are given in Tables 5.2.8 through 5.2.11 for Case A through D, respectively. The analytical results were computed from the numerical code AT123D shown in Section C.

\section{Table 5.2.2. Summary of Simulations Performed (Base Case and Variations) on the Two- Dimensional Transport Problem}

\begin{tabular}{|c|c|c|c|c|c|c|}
\hline FACT Options & $\begin{array}{l}\text { Base case } \\
\text { (parallel) }\end{array}$ & $\begin{array}{r}\text { Base case } \\
\text { (diagonal) }\end{array}$ & $\overline{\mathbf{A}}$ & $\bar{B}$ & $\bar{C}$ & $\mathbf{D}$ \\
\hline \multicolumn{7}{|l|}{ Mesh Options } \\
\hline $\begin{array}{l}\text { parallel grid } \\
\text { diagonal grid }\end{array}$ & $\mathrm{x}$ & $\mathrm{x}$ & $\mathrm{x}$ & $\mathrm{x}$ & $\bar{x}$ & $\mathrm{x}$ \\
\hline \multicolumn{7}{|l|}{ Physical Parameters } \\
\hline $\begin{array}{l}\text { radioactive decay coef., } \lambda= \\
\qquad 0.0 \mathrm{~d}^{-1}\end{array}$ & $\mathrm{x}$ & $\mathrm{x}$ & $\mathrm{x}$ & $\mathrm{x}$ & $\mathrm{x}$ & \\
\hline$\lambda=0.005 \mathrm{~d}^{-1}$ & & & & & & $\mathrm{x}$ \\
\hline $\begin{array}{l}\text { Solute distribution coef., } \mathrm{k}_{\mathrm{d}}= \\
\qquad 0.0 \mathrm{~m}^{3} / \mathrm{kg}\end{array}$ & $\mathrm{x}$ & $\mathrm{x}$ & $\mathrm{x}$ & $\mathrm{x}$ & & $\mathrm{x}$ \\
\hline $\mathrm{k}_{\mathrm{d}}=0.4375 \mathrm{~m}^{3} / \mathrm{kg}$ & & & & & $\mathrm{x}$ & \\
\hline
\end{tabular}

The concentration plume presented in Figure 5.2.4 represents our base case parallel grid simulation (Case A). The aquifer flow direction is aligned with the grid and the concentration plume does not exhibit any oscillatory behavior near the continuous point source using the default HYBRID nodal integration scheme. The cell Peclet number, $\mathrm{Pe}_{\mathrm{y}}$, transverse to the plume centerline is greater than 2. Therefore, you would expect to see slight oscillatory behavior in the concentrations transverse to the plume centerline. The HYBRID nodal integration scheme eliminated upstream oscillations in the high Peclet number 1D transport simulation by increasing the artificial dispersion. Similarly, oscillatory behavior transverse to the plume centerline is being suppressed by increased artificial dispersion in that direction. 
Figures 5.2.5 and 5.2.6 present concentration profiles for 2D transport of the base case (Case A) along and transverse to the plume centerline at 1400 days, respectively. There is excellent agreement between the PORFLOW numerical results and the results from AT123D. Tables 5.2.3 and 5.2.4 complement the data shown in the figures above. The PORFLOW input commands for Case A are given in Table 5.2.8.

The concentration plume presented in Figure 5.2.7 presents the base case diagonal grid simulation (Case B). The aquifer flow direction is aligned at a 45-degree angle with respect to the grid. The alternating stripes of concentration contours transverse to the plume centerline exhibit oscillatory behavior perhaps due to less diagonal dominance of the dispersion tensor with off diagonal terms. The HYBRID nodal integration scheme was used for this case as well. A point of interest or warning is that PORFLOW by default only computes the diagonal terms of the dispersion tensor. The user must issue the "CONDuction with TENSor diffusivity" command to invoke computation of the off-diagonal dispersion terms.

Figures 5.2.8 and 5.2.9 present concentration profiles for 2D transport of the base case (Case B) along and transverse to the plume centerline at 1400 days, respectively. As shown in Figure 5.2.8, PORFLOW under estimates the concentration upstream of the point source due to increased artificial dispersion in the transverse direction. There is excellent agreement between the PORFLOW numerical results and AT123D away from the source. Tables 5.2.3 and 5.2.4 complement the data shown in the figures above. The PORFLOW input commands for Case B are given in Table 5.2.9.

The concentration plume presented in Figure 5.2.10 shows the effect of retardation on the base case parallel grid simulation (Case $\mathrm{C}$ ). By adjusting the solute distribution coefficient such that the retardation factor becomes 2, the retarded dispersion coefficient and velocity are halved. These results can be compared to the no retardation base case (Case A) in Figures 5.2.11 and 5.2.12 where the spread of the plume has been greatly reduced. The agreement between the PORFLOW simulation results and AT123D is excellent. Tables 5.2.6 and 5.2.7 summarize the analytical and PORFLOW concentrations along and transverse to the plume centerline at 1400 days, respectively. The PORFLOW input commands for Case $\mathrm{C}$ are shown in Table 5.2.10.

The concentration plume presented in Figure 5.2.13 shows the effect of radioactive decay on the base case parallel grid simulation (Case D). By employing a non-zero radioactive decay coefficient, the solute now becomes a non-conservative transport species. These results can be compared to the non-decaying base case (Case A) in Figures 5.2.14 and 5.2.15 where the solute concentration profiles are reduced especially at the higher concentration levels. The agreement between the PORFLOW simulation results and AT123D is excellent. Tables 5.2.6 and 5.2.7 summarize the analytical and PORFLOW concentrations along and transverse to the plume centerline at 1400 days, respectively. The PORFLOW input commands for Case D are shown in Table 5.2.11.

By employing a non-zero radioactive decay coefficient such that the solute now becomes a nonconservative transport species (Cases D), the solute concentration profiles are reduced especially 
at the higher concentration levels. These results can be compared to the base case (Case A) in Figure 5.2.14 and 5.2.15.

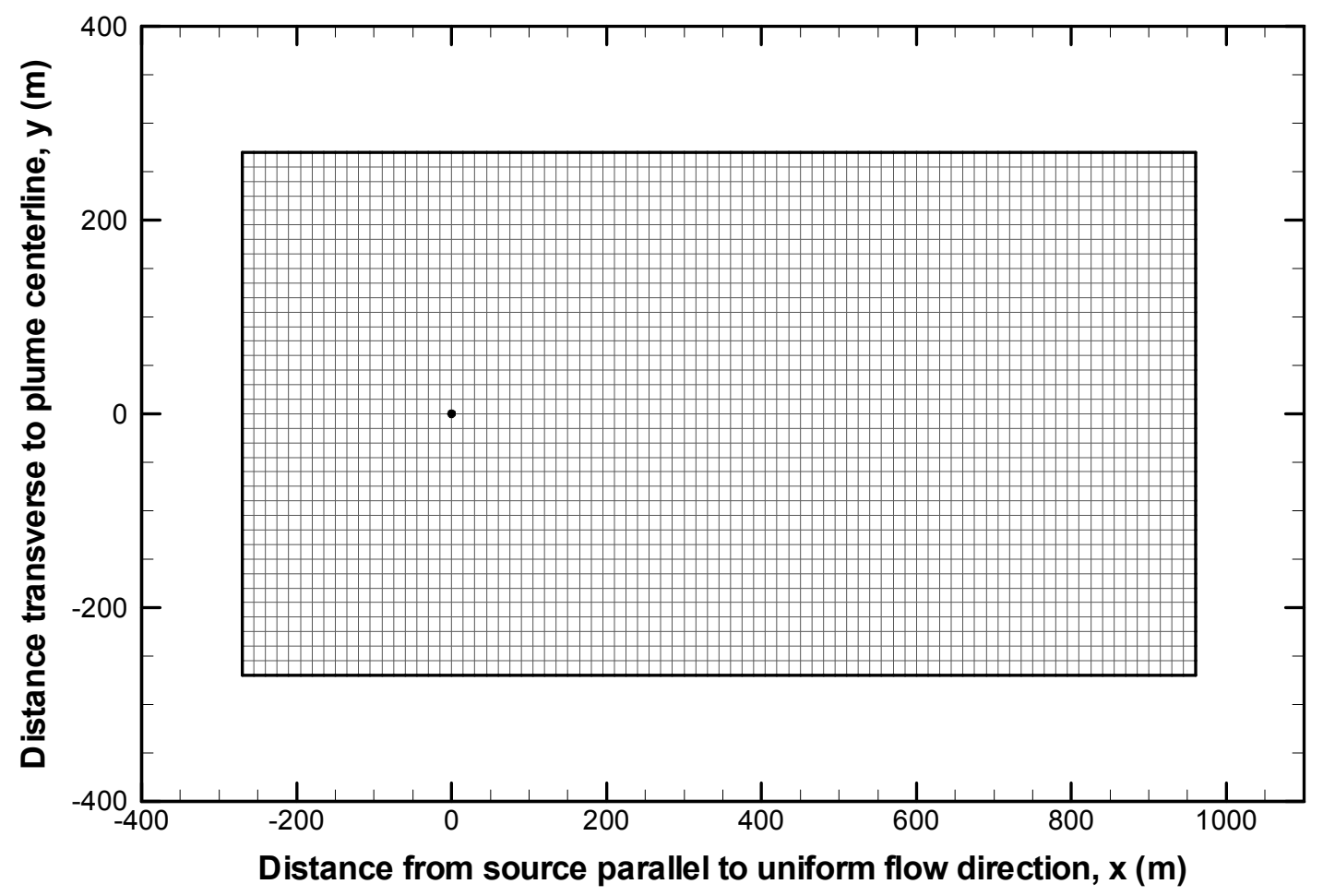

Figure 5.2.2. PORFLOW Parallel Grid for 2D Transport Simulations.

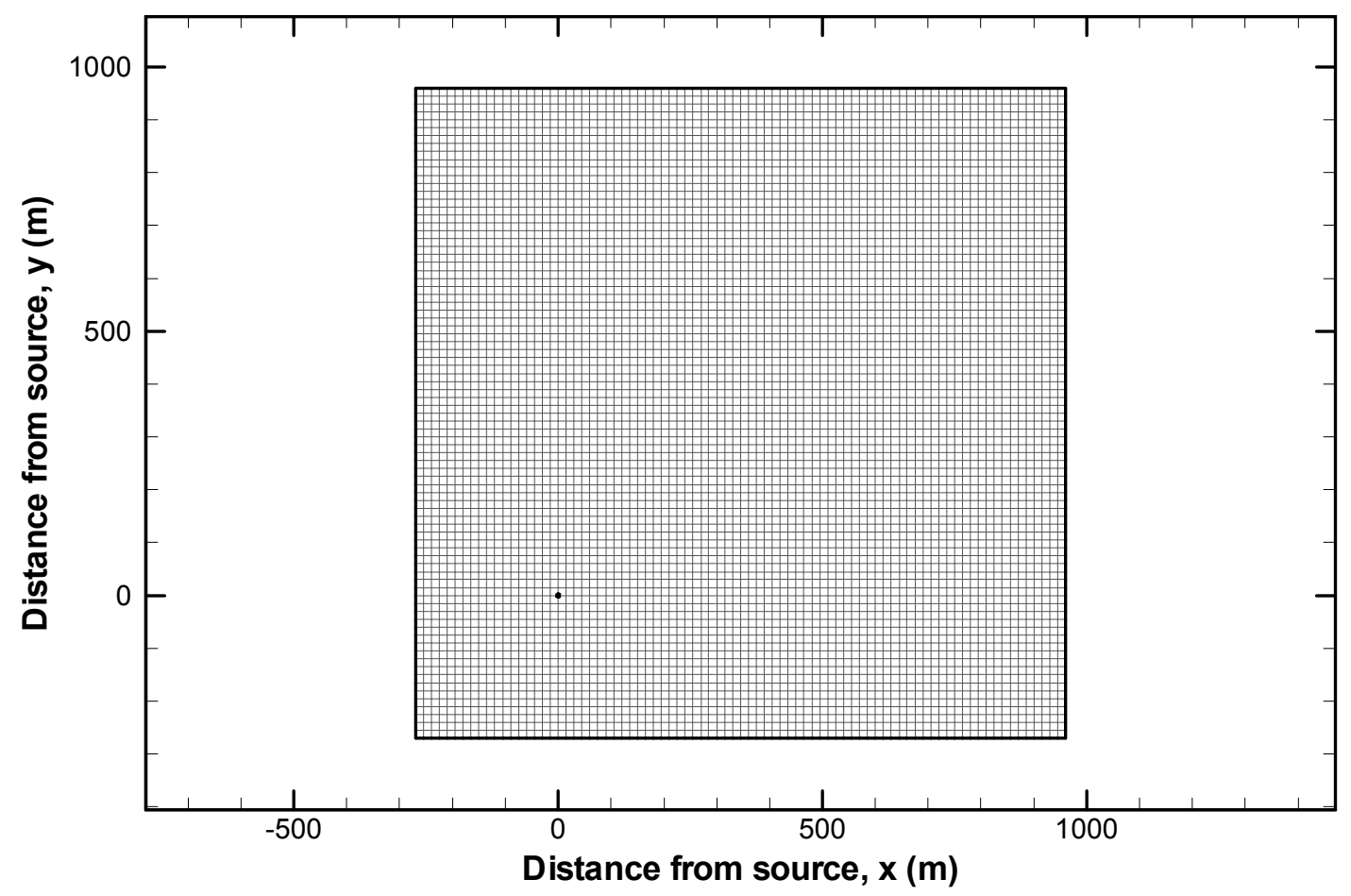

Figure 5.2.3. PORFLOW Diagonal Grid for 2D Transport Simulation. 


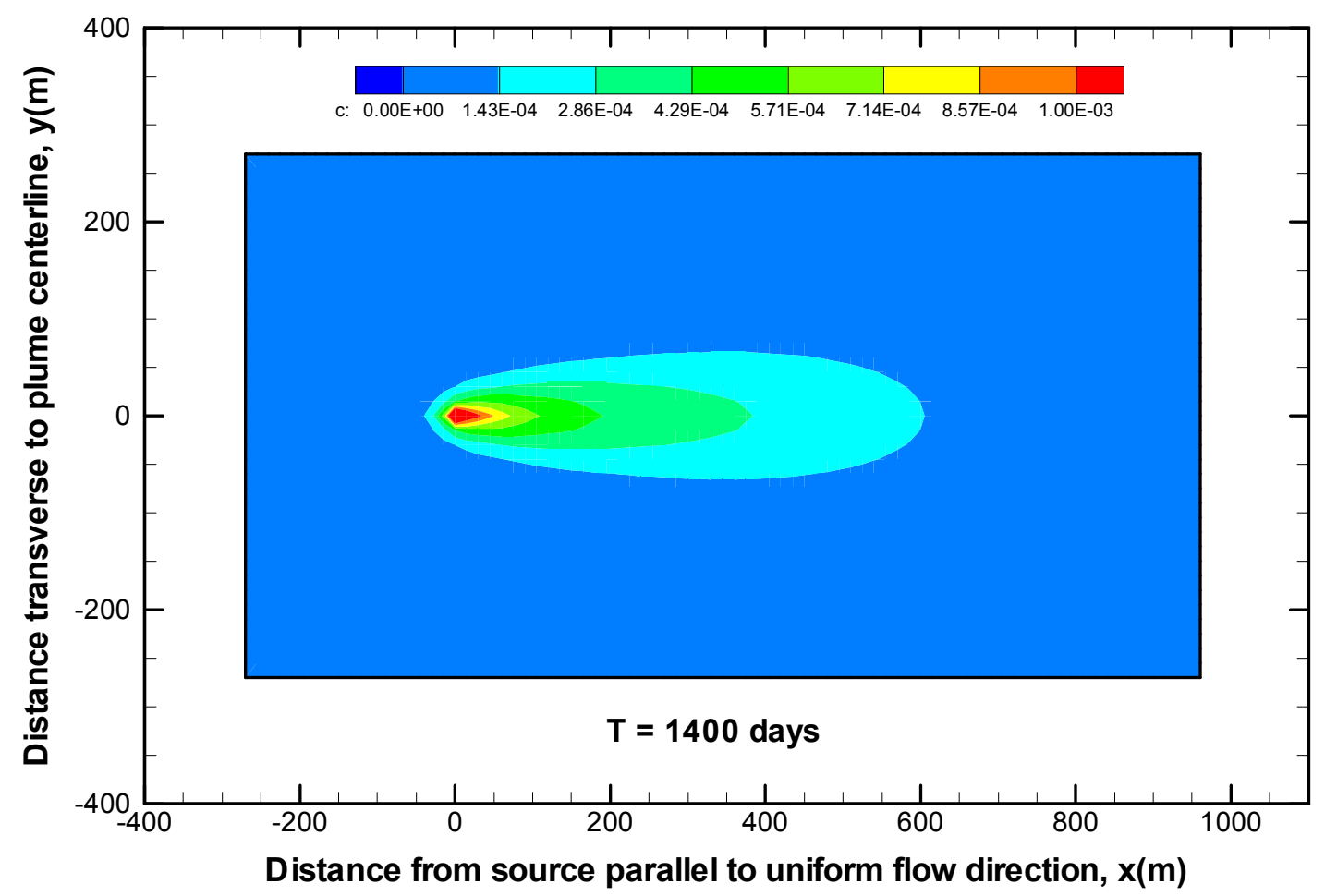

Figure 5.2.4. PORFLOW Concentration Plume for 2D Transport of the Base Case on the Parallel Grid (Case A).

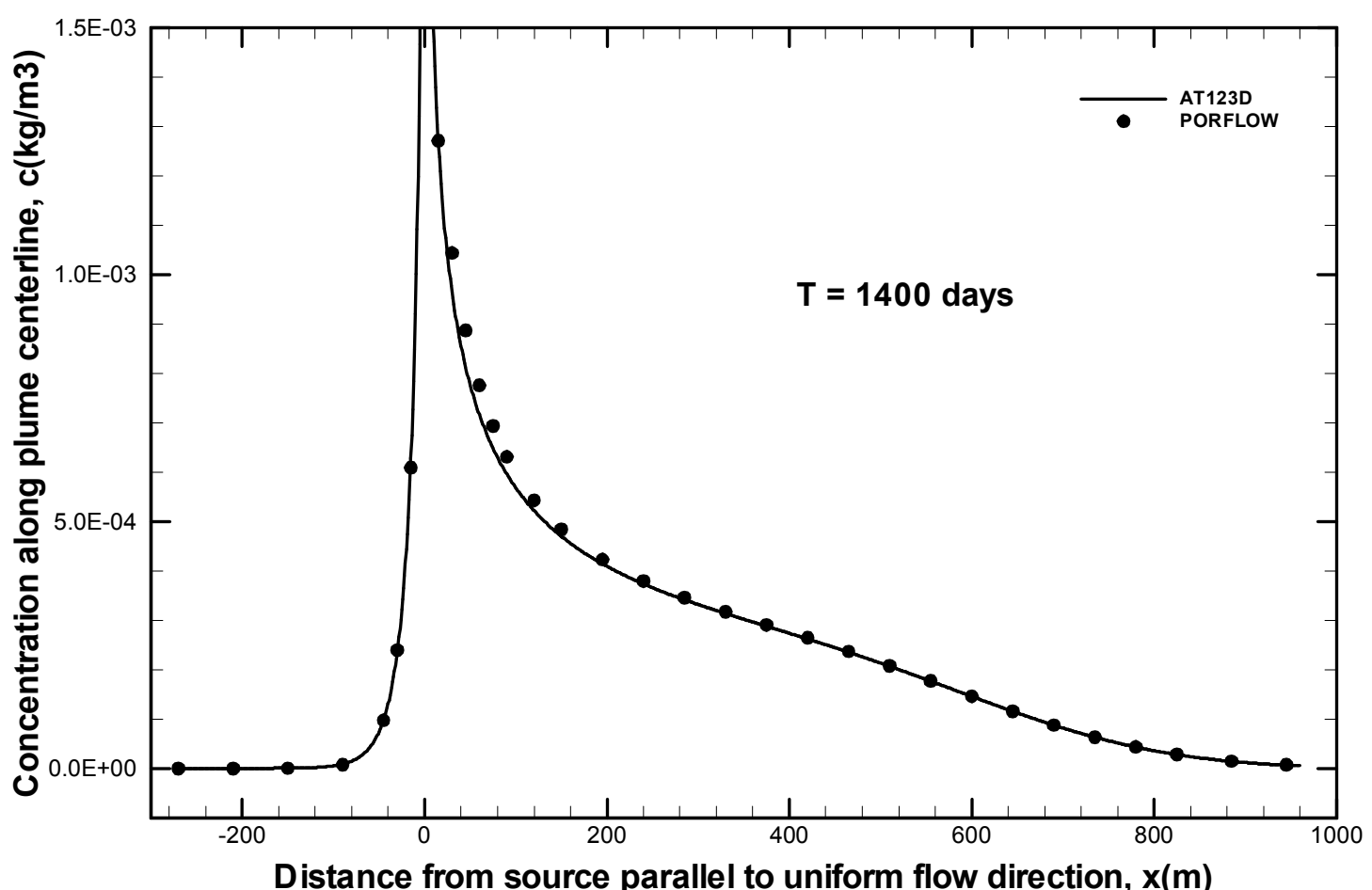

Figure 5.2.5. Concentration Profiles for 2D Transport of the Base Case along the Plume Centerline (Case A). 


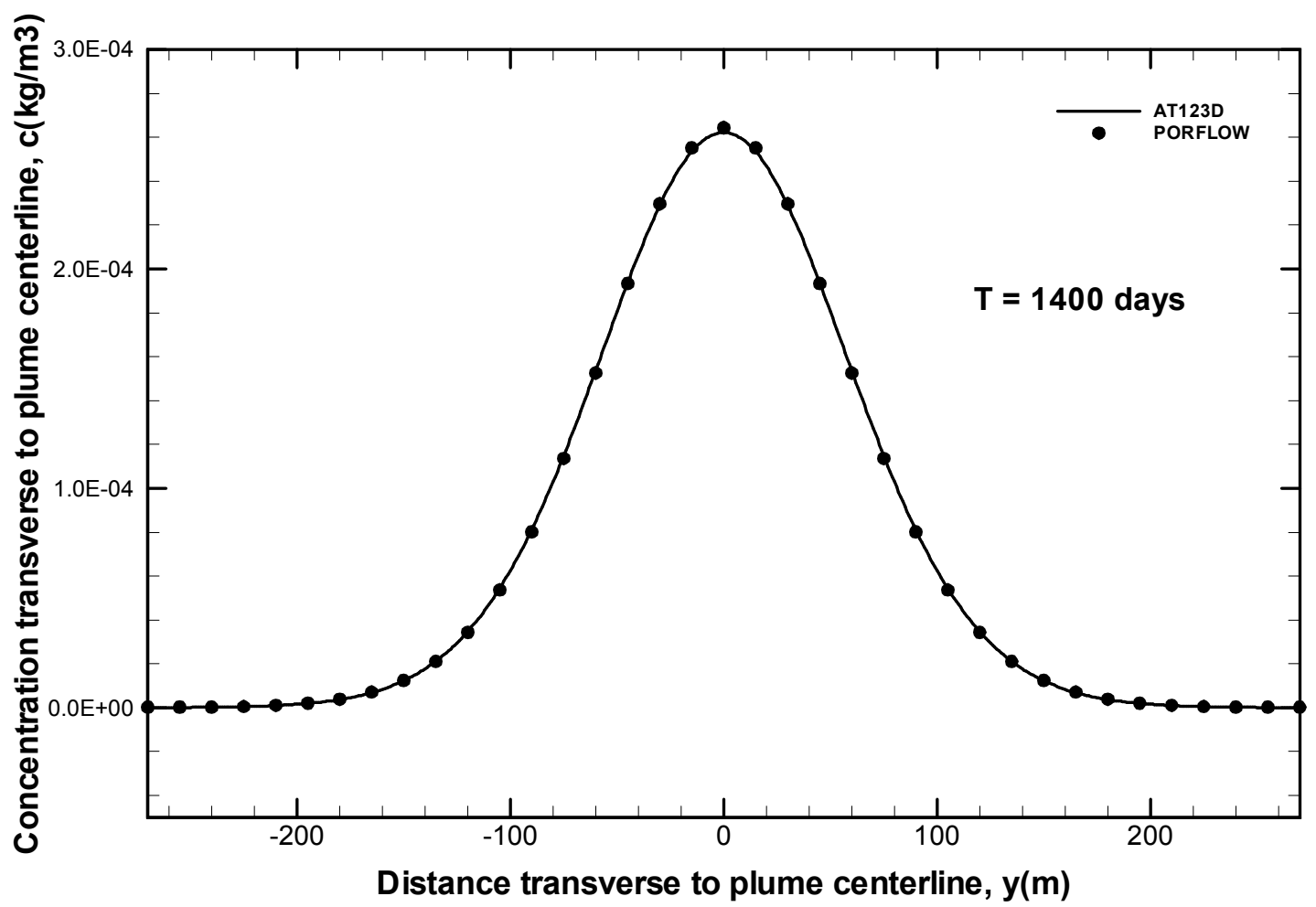

Figure 5.2.6. Concentration Profiles for 2D Transport of the Base Case Transverse to the Plume Centerline at $\mathrm{x}=\mathbf{4 2 0}$ meters (Case A).

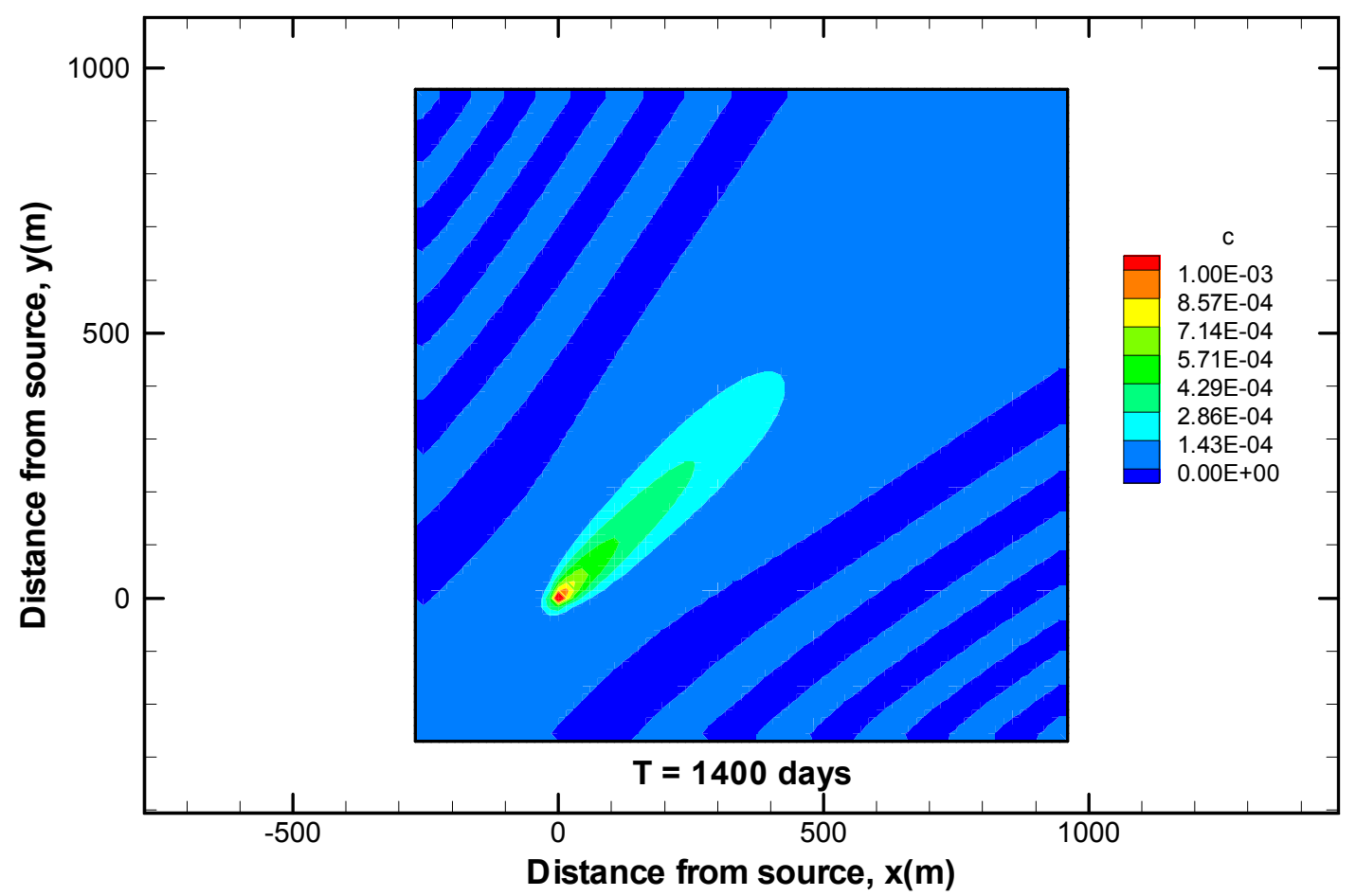

Figure 5.2.7. PORFLOW Concentration Plume for 2D Transport of the Base Case on the Diagonal Grid (Case B). 


\section{PORFLOW Testing and Verification Document}

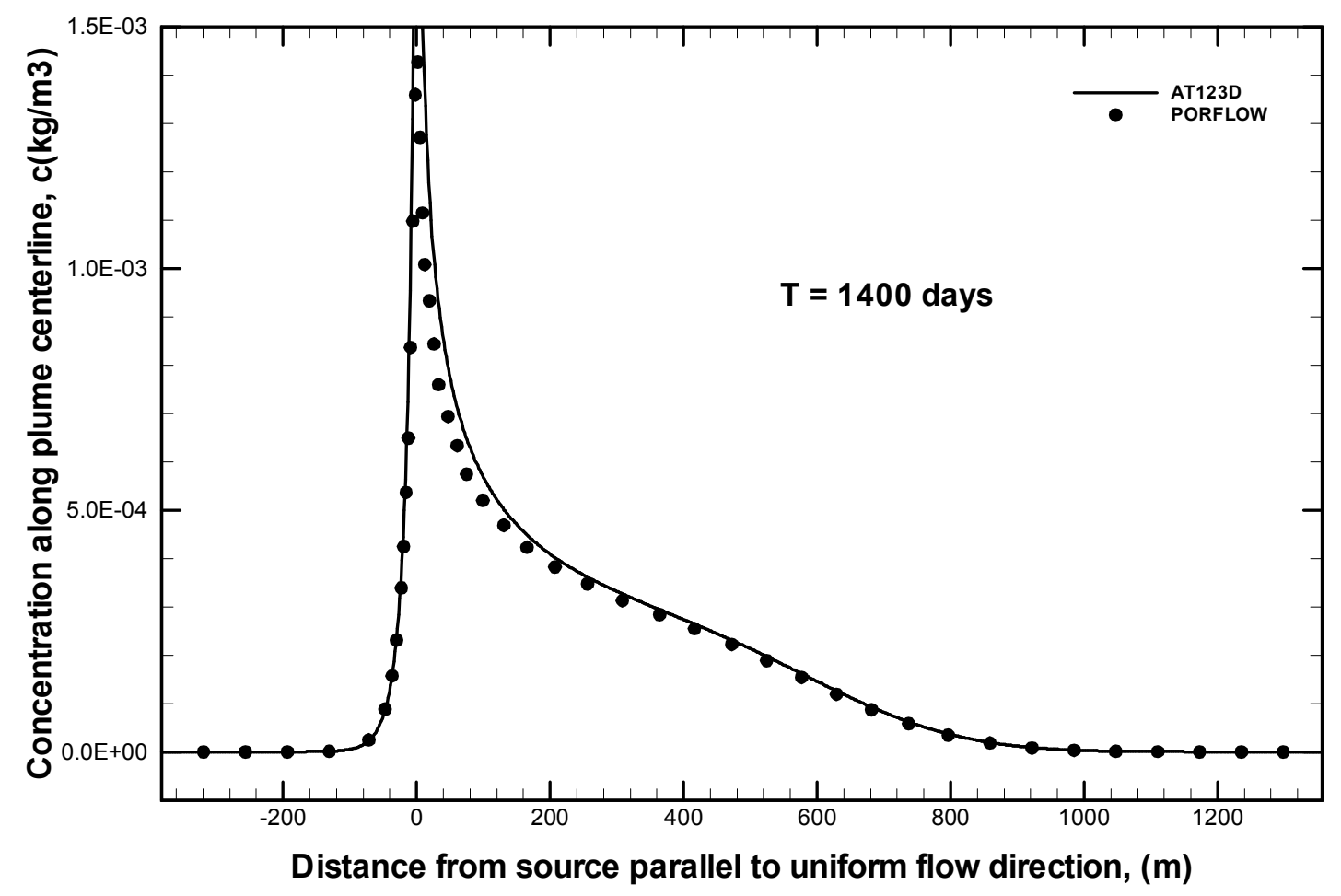

Figure 5.2.8. Concentration Profiles for 2D Transport of the Base Case along the Plume Centerline (Case B).

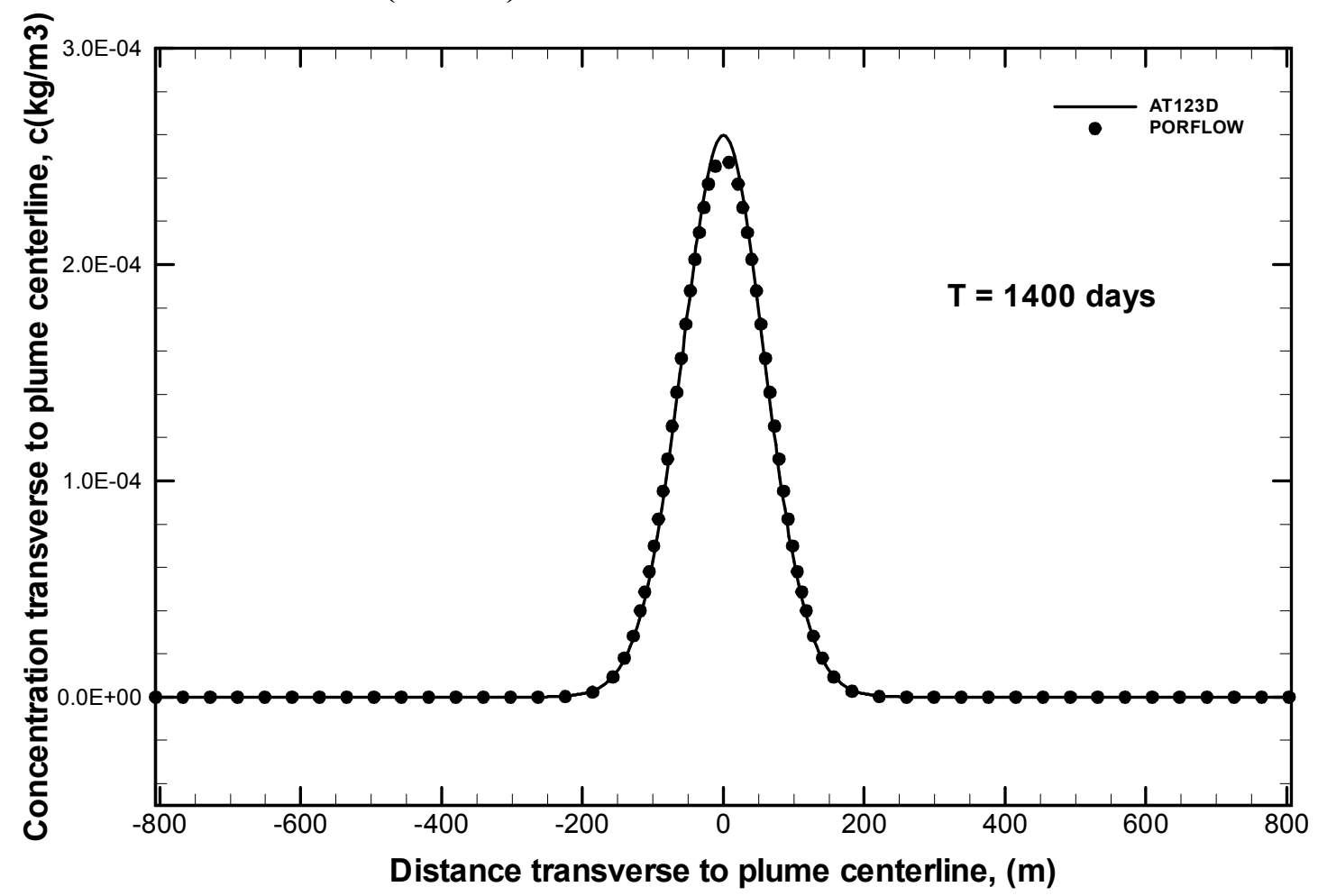

Figure 5.2.9. Concentration Profiles for 2D Transport of the Base Case Transverse to the Plume Centerline at $\mathrm{x}=\mathbf{4 2 4}$ meters (Case B). 


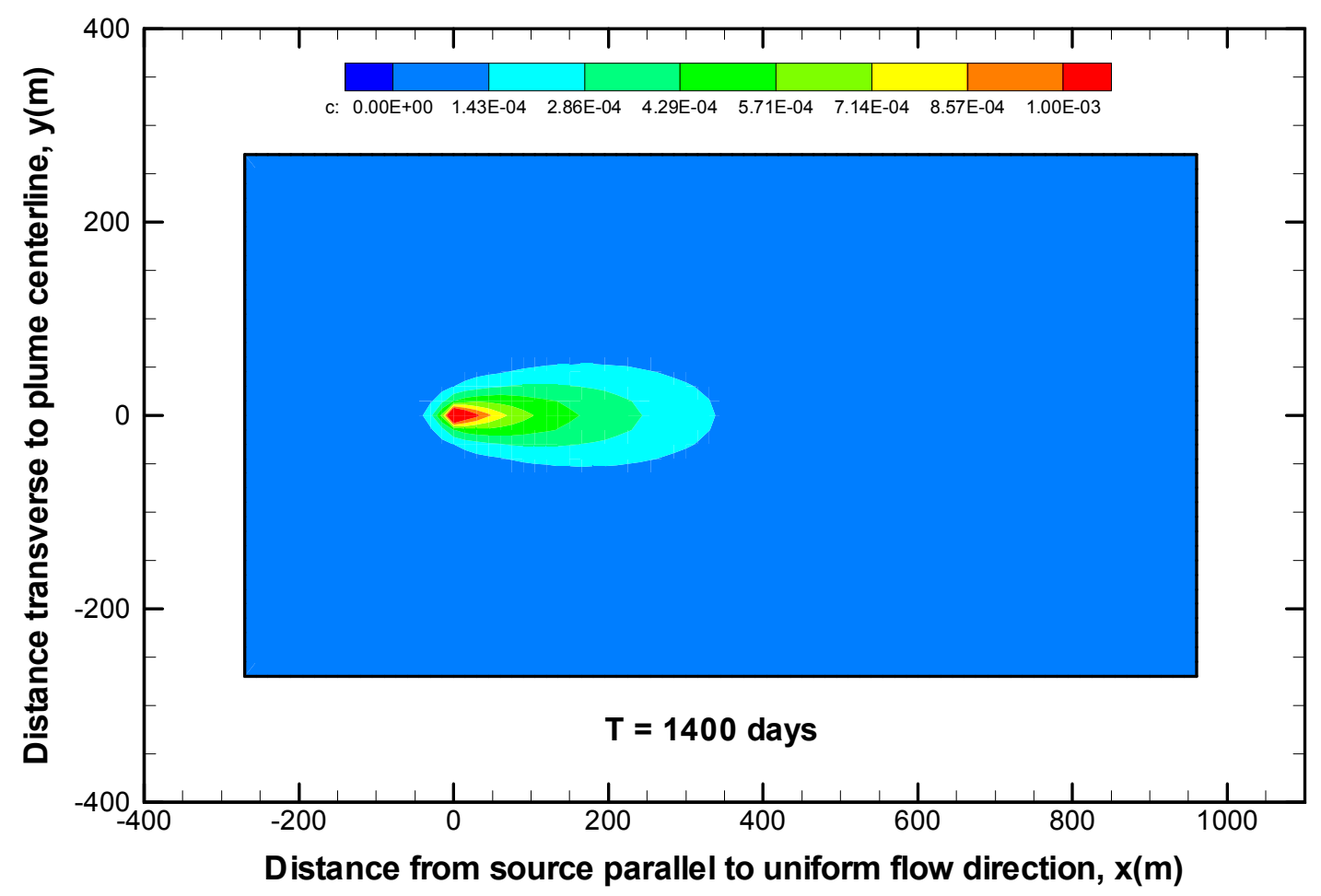

Figure 5.2.10 PORFLOW Concentration Plume for 2D Transport Showing the Effect of Retardation (Case C).

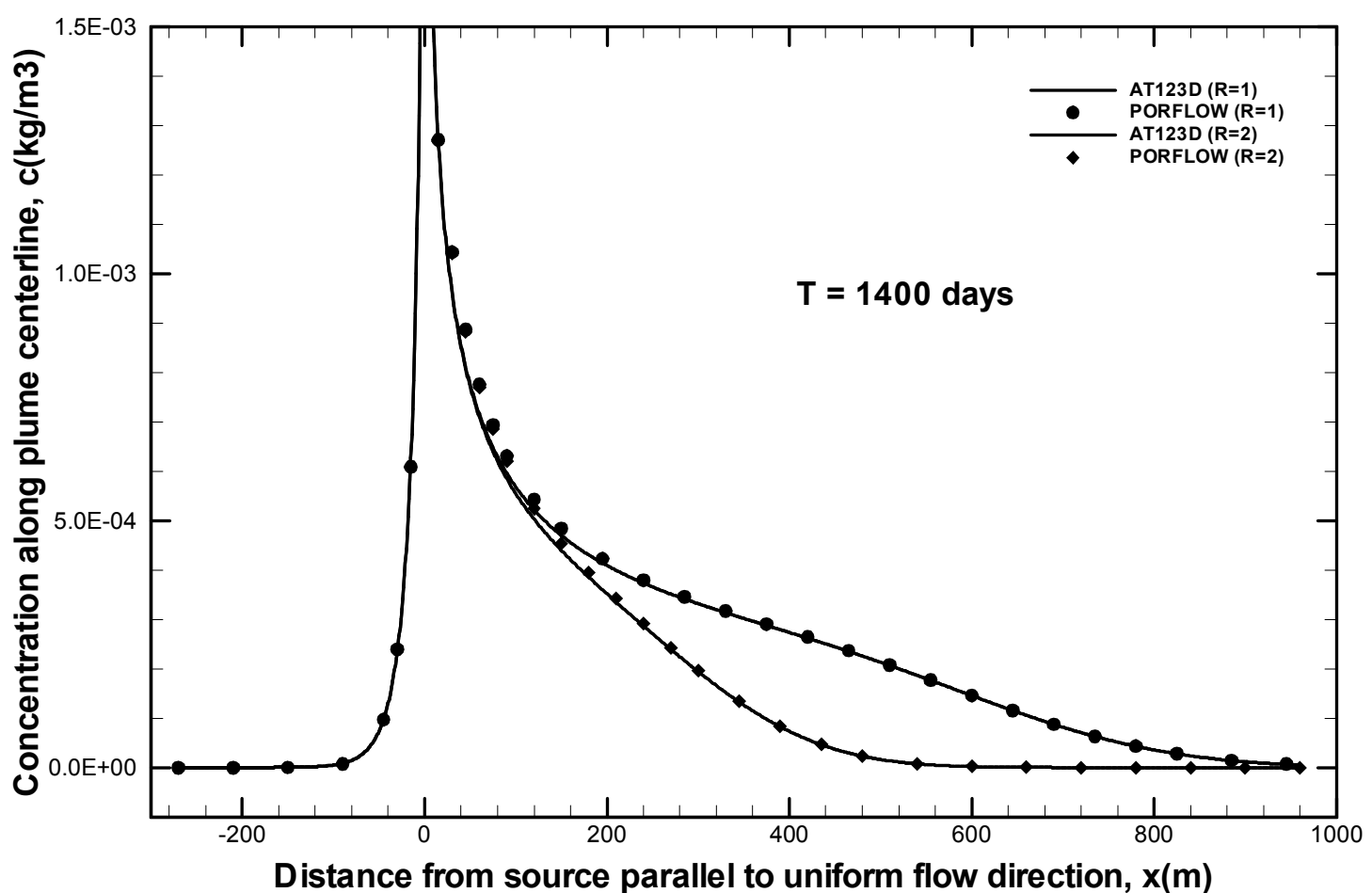

Figure 5.2.11 Concentration Profiles for 2D Transport Showing the Effect of Retardation along the Plume Centerline (Case C). 


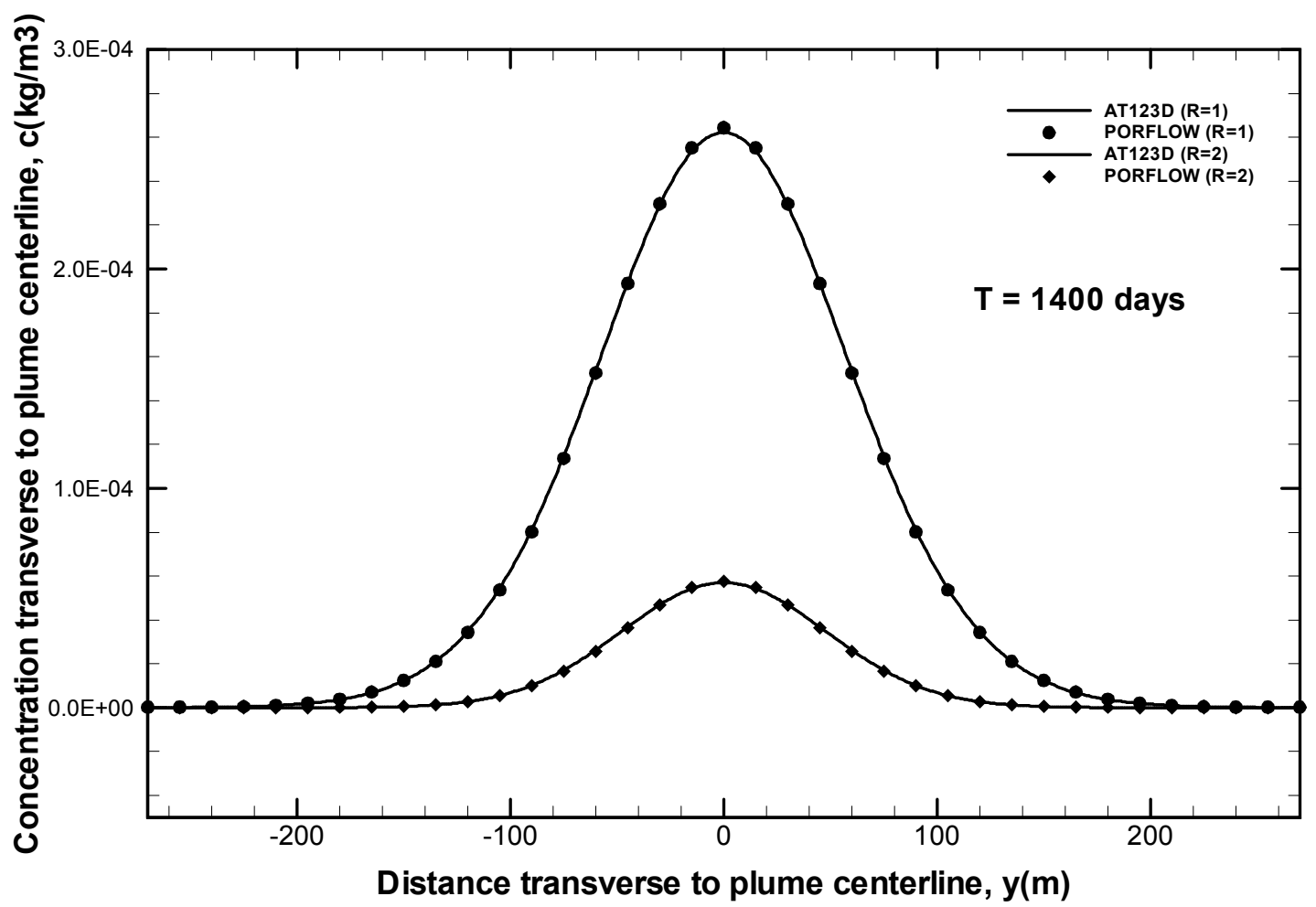

Figure 5.2.12 Concentration Profiles for 2D Transport Showing the Effect of Retardation Transverse to the Plume Centerline at $x=420$ meters (Case $C$ ).

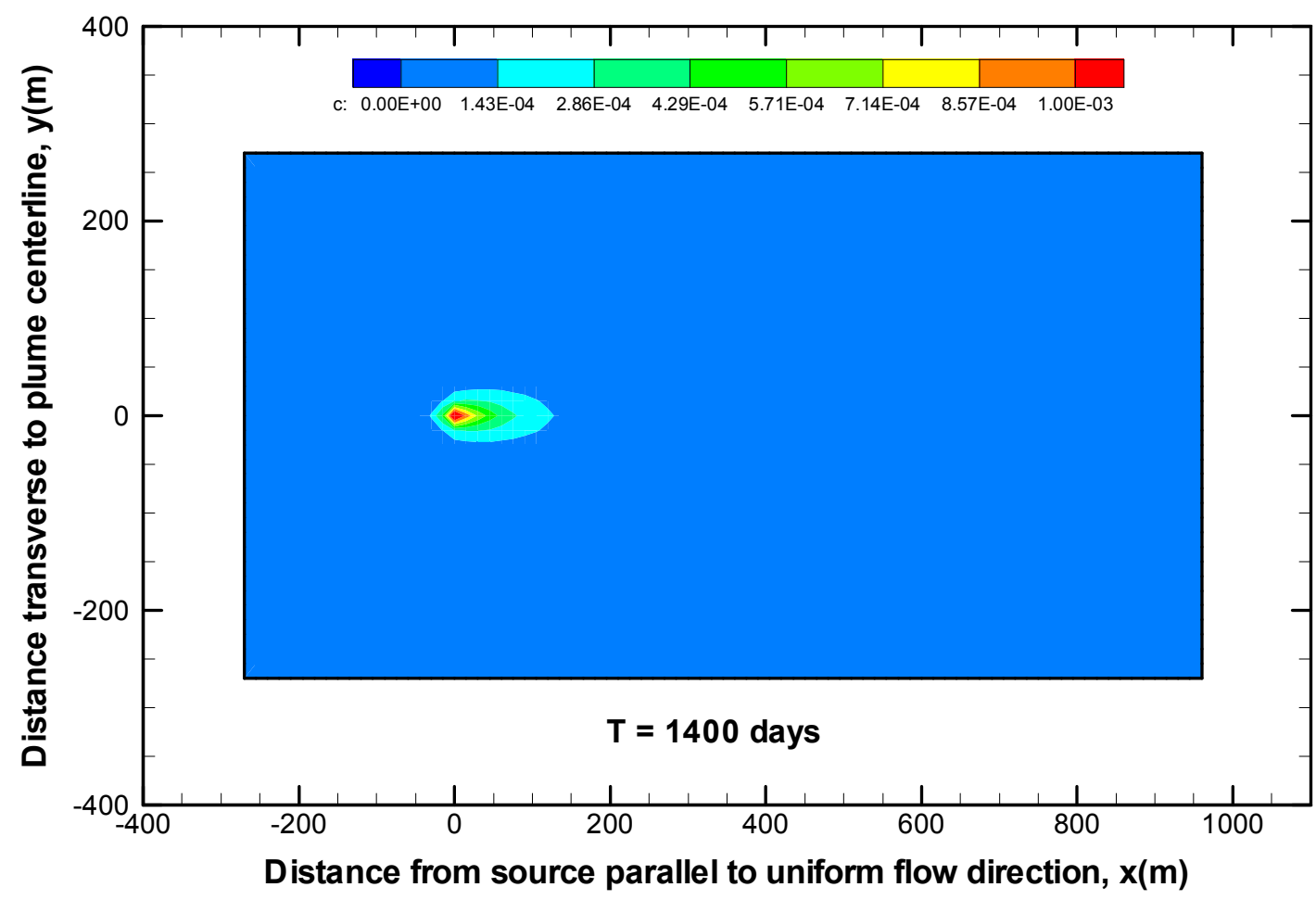

Figure 5.2.13 PORFLOW Concentration Plume for 2D Transport Showing the Effect of Radioactive Decay (Case D). 


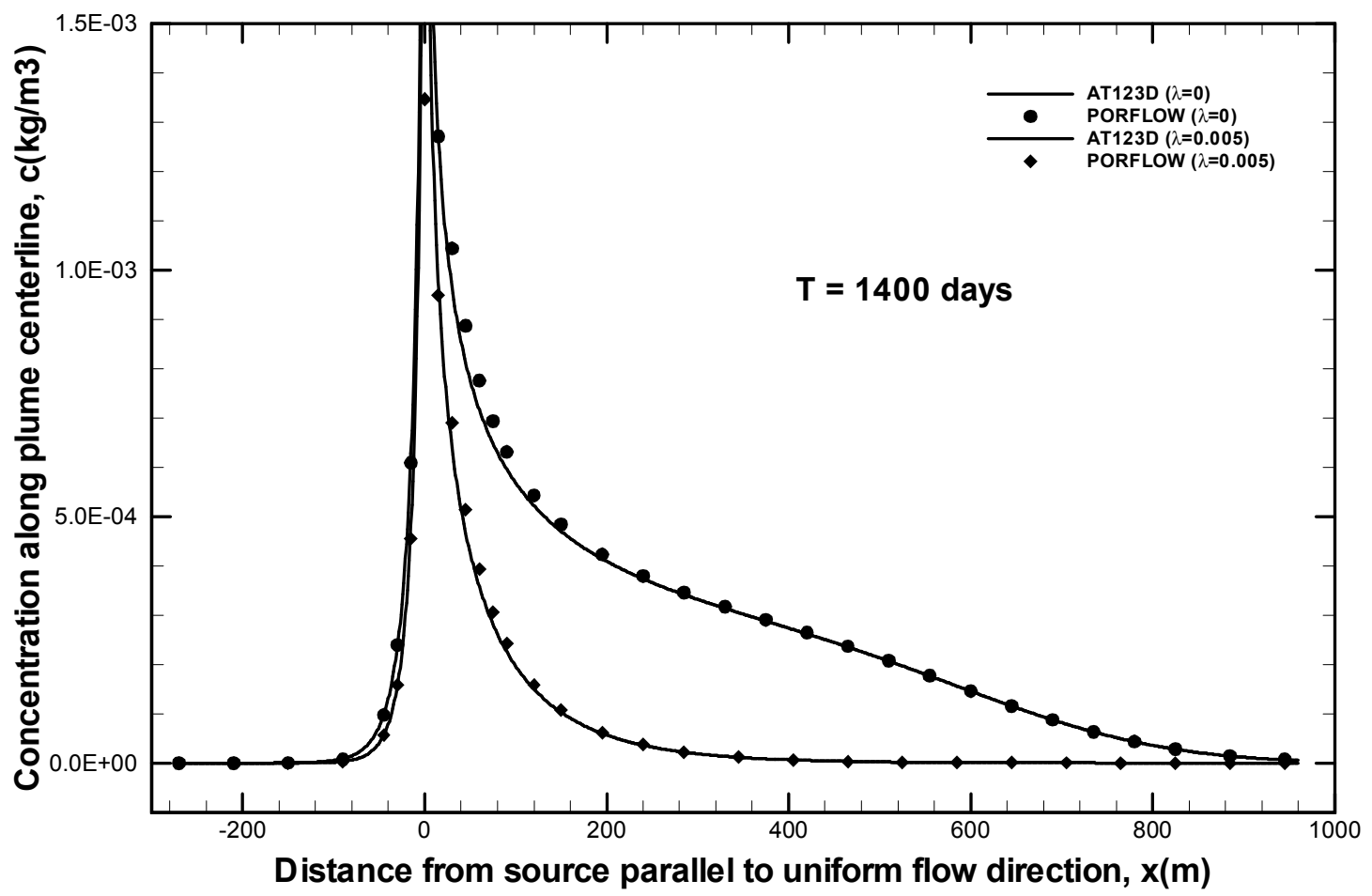

Figure 5.2.14 Concentration Profiles for 2D Transport Showing the Effect of Radioactive Decay along the Plume Centerline (Case D).

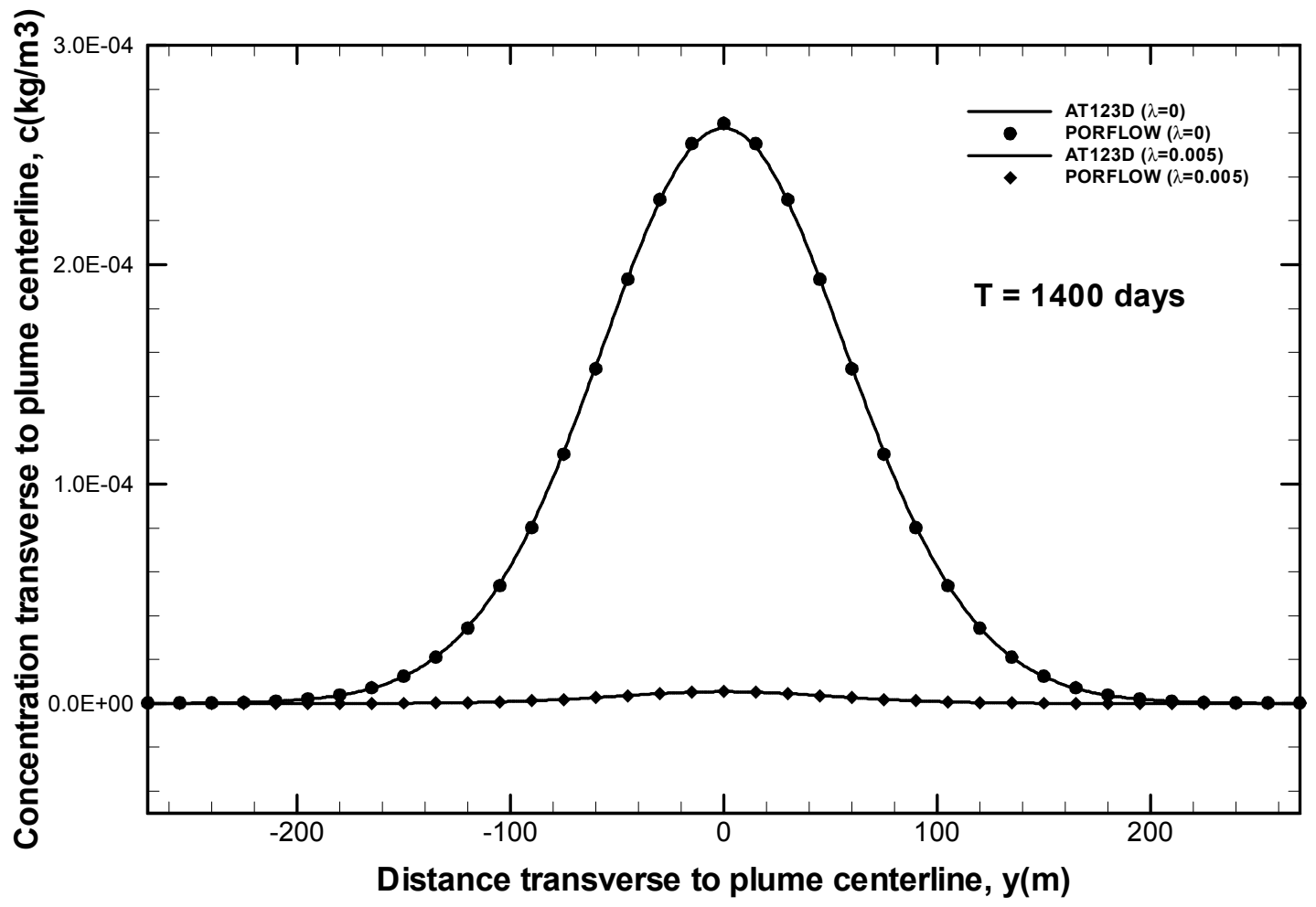

Figure 5.2.15 Concentration Profiles for 2D Transport Showing the Effect of Radioactive Decay Transverse to the Plume Centerline at $x=420$ meters (Case D). 
Table 5.2.3. Effect Grid Orientation has on the Numerical Concentration Results along the Plume Centerline for the Transient 2D Transport Simulation (Cases A and B, T = 1400 days).

\begin{tabular}{|c|cc|c|cc|}
\hline $\begin{array}{c}\text { Distance } \\
\text { down- } \\
\text { stream, } \\
\text { x (m) }\end{array}$ & $\begin{array}{c}\text { Analytical } \\
\text { (AT123D) }\end{array}$ & $\begin{array}{c}\text { Numerical } \\
\text { (PORFLOW) }\end{array}$ & $\begin{array}{c}\text { Distance } \\
\text { down- } \\
\text { stream, }(\mathrm{m})\end{array}$ & $\begin{array}{c}\text { Analytical } \\
\text { (AT123D) }\end{array}$ & $\begin{array}{c}\text { Numerical } \\
\text { (PORFLOW) }\end{array}$ \\
\hline-270.0 & $1.0987 \mathrm{E}-09$ & $0.0000 \mathrm{E}+00$ & -381.8 & $4.6641 \mathrm{E}-12$ & $3.5420 \mathrm{E}-12$ \\
\hline-240.0 & $4.7766 \mathrm{E}-09$ & $2.1880 \mathrm{E}-09$ & -339.4 & $3.7091 \mathrm{E}-11$ & $2.8480 \mathrm{E}-11$ \\
\hline-210.0 & $2.0884 \mathrm{E}-08$ & $1.3000 \mathrm{E}-08$ & -297.0 & $2.9432 \mathrm{E}-10$ & $3.1960 \mathrm{E}-10$ \\
\hline-180.0 & $9.2081 \mathrm{E}-08$ & $6.3920 \mathrm{E}-08$ & -254.6 & $2.3433 \mathrm{E}-09$ & $2.6350 \mathrm{E}-09$ \\
\hline-150.0 & $4.1095 \mathrm{E}-07$ & $3.0830 \mathrm{E}-07$ & -212.1 & $1.8828 \mathrm{E}-08$ & $2.0960 \mathrm{E}-08$ \\
\hline-120.0 & $1.8670 \mathrm{E}-06$ & $1.5090 \mathrm{E}-06$ & -169.7 & $1.5381 \mathrm{E}-07$ & $1.6790 \mathrm{E}-07$ \\
\hline-90.0 & $8.7236 \mathrm{E}-06$ & $7.6390 \mathrm{E}-06$ & -127.3 & $1.2921 \mathrm{E}-06$ & $1.3740 \mathrm{E}-06$ \\
\hline-60.0 & $4.2841 \mathrm{E}-05$ & $4.0890 \mathrm{E}-05$ & -84.9 & $1.1426 \mathrm{E}-05$ & $1.1690 \mathrm{E}-05$ \\
\hline-30.0 & $2.3592 \mathrm{E}-04$ & $2.3970 \mathrm{E}-04$ & -42.4 & $1.1389 \mathrm{E}-04$ & $1.0870 \mathrm{E}-04$ \\
\hline 0.0 & $5.8747 \mathrm{E}-03$ & $1.6090 \mathrm{E}-03$ & 0.0 & $5.5942 \mathrm{E}-03$ & $1.5000 \mathrm{E}-03$ \\
\hline 30.0 & $9.6481 \mathrm{E}-04$ & $1.0440 \mathrm{E}-03$ & 42.4 & $8.3350 \mathrm{E}-04$ & $7.2830 \mathrm{E}-04$ \\
\hline 60.0 & $7.1652 \mathrm{E}-04$ & $7.7560 \mathrm{E}-04$ & 84.9 & $6.1293 \mathrm{E}-04$ & $5.6240 \mathrm{E}-04$ \\
\hline 90.0 & $5.9668 \mathrm{E}-04$ & $6.3120 \mathrm{E}-04$ & 127.3 & $5.0801 \mathrm{E}-04$ & $4.7650 \mathrm{E}-04$ \\
\hline 120.0 & $5.2226 \mathrm{E}-04$ & $5.4330 \mathrm{E}-04$ & 169.7 & $4.4317 \mathrm{E}-04$ & $4.2090 \mathrm{E}-04$ \\
\hline 150.0 & $4.7011 \mathrm{E}-04$ & $4.8410 \mathrm{E}-04$ & 212.1 & $3.9757 \mathrm{E}-04$ & $3.8070 \mathrm{E}-04$ \\
\hline 180.0 & $4.3079 \mathrm{E}-04$ & $4.4090 \mathrm{E}-04$ & 254.6 & $3.6261 \mathrm{E}-04$ & $3.4920 \mathrm{E}-04$ \\
\hline
\end{tabular}




\begin{tabular}{|c|cc|c|cc|}
\hline $\begin{array}{c}\text { Distance } \\
\text { down- } \\
\text { stream, } \\
\text { x }(m)\end{array}$ & $\begin{array}{c}\text { Analytical } \\
\text { (AT123D) }\end{array}$ & $\begin{array}{c}\text { Numerical } \\
\text { (PORFLOW) }\end{array}$ & $\begin{array}{c}\text { Distance } \\
\text { down- } \\
\text { stream, }(m)\end{array}$ & $\begin{array}{c}\text { Analytical } \\
\text { (AT123D) }\end{array}$ & $\begin{array}{c}\text { Numerical } \\
\text { (PORFLOW) }\end{array}$ \\
\hline 210.0 & $3.9957 \mathrm{E}-04$ & $4.0730 \mathrm{E}-04$ & 297.0 & $3.3377 \mathrm{E}-04$ & $3.2260 \mathrm{E}-04$ \\
\hline 240.0 & $3.7375 \mathrm{E}-04$ & $3.7990 \mathrm{E}-04$ & 339.4 & $3.0825 \mathrm{E}-04$ & $2.9850 \mathrm{E}-04$ \\
\hline 270.0 & $3.5158 \mathrm{E}-04$ & $3.5660 \mathrm{E}-04$ & 381.8 & $2.8406 \mathrm{E}-04$ & $2.7520 \mathrm{E}-04$ \\
\hline 300.0 & $3.3188 \mathrm{E}-04$ & $3.3610 \mathrm{E}-04$ & 424.3 & $2.5975 \mathrm{E}-04$ & $2.5150 \mathrm{E}-04$ \\
\hline 330.0 & $3.1374 \mathrm{E}-04$ & $3.1740 \mathrm{E}-04$ & 466.7 & $2.3435 \mathrm{E}-04$ & $2.2640 \mathrm{E}-04$ \\
\hline 360.0 & $2.9645 \mathrm{E}-04$ & $2.9950 \mathrm{E}-04$ & 509.1 & $2.0740 \mathrm{E}-04$ & $1.9970 \mathrm{E}-04$ \\
\hline 390.0 & $2.7943 \mathrm{E}-04$ & $2.8210 \mathrm{E}-04$ & 551.5 & $1.7904 \mathrm{E}-04$ & $1.7160 \mathrm{E}-04$ \\
\hline 420.0 & $2.6223 \mathrm{E}-04$ & $2.6440 \mathrm{E}-04$ & 594.0 & $1.4996 \mathrm{E}-04$ & $1.4300 \mathrm{E}-04$ \\
\hline 450.0 & $2.4451 \mathrm{E}-04$ & $2.4630 \mathrm{E}-04$ & 636.4 & $1.2127 \mathrm{E}-04$ & $1.1500 \mathrm{E}-04$ \\
\hline 480.0 & $2.2606 \mathrm{E}-04$ & $2.2750 \mathrm{E}-04$ & 678.8 & $9.4293 \mathrm{E}-05$ & $8.8870 \mathrm{E}-05$ \\
\hline 510.0 & $2.0682 \mathrm{E}-04$ & $2.0790 \mathrm{E}-04$ & 721.2 & $7.0220 \mathrm{E}-05$ & $6.5810 \mathrm{E}-05$ \\
\hline 540.0 & $1.8686 \mathrm{E}-04$ & $1.8760 \mathrm{E}-04$ & 763.7 & $4.9926 \mathrm{E}-05$ & $4.6570 \mathrm{E}-05$ \\
\hline 570.0 & $1.6641 \mathrm{E}-04$ & $1.6690 \mathrm{E}-04$ & 806.1 & $3.3797 \mathrm{E}-05$ & $3.1410 \mathrm{E}-05$ \\
\hline 600.0 & $1.4582 \mathrm{E}-04$ & $1.4610 \mathrm{E}-04$ & 848.5 & $2.1734 \mathrm{E}-05$ & $2.0170 \mathrm{E}-05$ \\
\hline 630.0 & $1.2552 \mathrm{E}-04$ & $1.2570 \mathrm{E}-04$ & 891.0 & $1.3251 \mathrm{E}-05$ & $1.2300 \mathrm{E}-05$ \\
\hline 660.0 & $1.0596 \mathrm{E}-04$ & $1.0610 \mathrm{E}-04$ & 933.4 & $7.6472 \mathrm{E}-06$ & $7.1260 \mathrm{E}-06$ \\
\hline 690.0 & $8.7618 \mathrm{E}-05$ & $8.7750 \mathrm{E}-05$ & 975.8 & $4.1720 \mathrm{E}-06$ & $3.9150 \mathrm{E}-06$ \\
\hline 720.0 & $7.0870 \mathrm{E}-05$ & $7.1070 \mathrm{E}-05$ & 1018.2 & $2.1491 \mathrm{E}-06$ & $2.0400 \mathrm{E}-06$ \\
\hline 750.0 & $5.6013 \mathrm{E}-05$ & $5.6300 \mathrm{E}-05$ & 1060.7 & $1.0443 \mathrm{E}-06$ & $1.0070 \mathrm{E}-06$ \\
\hline 780.0 & $4.3215 \mathrm{E}-05$ & $4.3590 \mathrm{E}-05$ & 1103.1 & $4.7826 \mathrm{E}-07$ & $4.7110 \mathrm{E}-07$ \\
\hline 810.0 & $3.2518 \mathrm{E}-05$ & $3.2970 \mathrm{E}-05$ & 1145.5 & $2.0631 \mathrm{E}-07$ & $2.0890 \mathrm{E}-07$ \\
\hline 840.0 & $2.3846 \mathrm{E}-05$ & $2.4350 \mathrm{E}-05$ & 1187.9 & $8.3775 \mathrm{E}-08$ & $8.7750 \mathrm{E}-08$ \\
\hline 870.0 & $1.7030 \mathrm{E}-05$ & $1.7540 \mathrm{E}-05$ & 1230.4 & $3.2006 \mathrm{E}-08$ & $3.4940 \mathrm{E}-08$ \\
\hline 900.0 & $1.1837 \mathrm{E}-05$ & $1.2340 \mathrm{E}-05$ & 1272.8 & $1.1499 \mathrm{E}-08$ & $1.3190 \mathrm{E}-08$ \\
\hline 930.0 & $8.0034 \mathrm{E}-06$ & $8.5240 \mathrm{E}-06$ & 1315.2 & $3.8838 \mathrm{E}-09$ & $4.7450 \mathrm{E}-09$ \\
\hline 960.0 & $5.2616 \mathrm{E}-06$ & $7.1230 \mathrm{E}-06$ & 1357.6 & $1.2327 \mathrm{E}-09$ & $2.8200 \mathrm{E}-09$ \\
\hline
\end{tabular}


Table 5.2.4. Effect Grid Orientation has on the Numerical Concentration Results

Transverse to the Plume Centerline (424 m downstream of the source) for the Transient 2D Transport Simulation (Cases $A$ and $B, T=1400$ days).

\begin{tabular}{|ccc|ccc|}
\hline $\begin{array}{c}\text { Distance } \\
\text { transverse, } \\
\mathrm{y}(\mathrm{m})\end{array}$ & $\begin{array}{c}\text { Analytical } \\
(\mathrm{AT} 123 \mathrm{D})\end{array}$ & $\begin{array}{c}\text { Numerical } \\
\text { (PORFLOW) }\end{array}$ & $\begin{array}{c}\text { Distance } \\
\text { transverse, } \\
(\mathrm{m})\end{array}$ & $\begin{array}{c}\text { Analytical } \\
(\mathrm{AT} 123 \mathrm{D})\end{array}$ & $\begin{array}{c}\text { Numerical } \\
\text { (PORFLOW) }\end{array}$ \\
\hline 0.0 & $2.5975 \mathrm{E}-04$ & $2.6184 \mathrm{E}-04$ & 0.0 & $2.5975 \mathrm{E}-04$ & $2.5150 \mathrm{E}-04$ \\
\hline 30.0 & $2.2708 \mathrm{E}-04$ & $2.2777 \mathrm{E}-04$ & 42.4 & $1.9880 \mathrm{E}-04$ & $1.9840 \mathrm{E}-04$ \\
\hline 60.0 & $1.5298 \mathrm{E}-04$ & $1.5180 \mathrm{E}-04$ & 84.9 & $9.1901 \mathrm{E}-05$ & $9.6740 \mathrm{E}-05$ \\
\hline 90.0 & $8.1128 \mathrm{E}-05$ & $8.0016 \mathrm{E}-05$ & 127.3 & $2.7535 \mathrm{E}-05$ & $2.8500 \mathrm{E}-05$ \\
\hline 120.0 & $3.4804 \mathrm{E}-05$ & $3.4501 \mathrm{E}-05$ & 169.7 & $5.7418 \mathrm{E}-06$ & $4.7750 \mathrm{E}-06$ \\
\hline 150.0 & $1.2394 \mathrm{E}-05$ & $1.2535 \mathrm{E}-05$ & 212.1 & $8.7465 \mathrm{E}-07$ & $3.6950 \mathrm{E}-07$ \\
\hline 180.0 & $3.7386 \mathrm{E}-06$ & $3.9229 \mathrm{E}-06$ & 254.6 & $9.9669 \mathrm{E}-08$ & $-3.1660 \mathrm{E}-09$ \\
\hline 210.0 & $9.6810 \mathrm{E}-07$ & $1.0753 \mathrm{E}-06$ & 297.0 & $8.5549 \mathrm{E}-09$ & $-2.0810 \mathrm{E}-09$ \\
\hline 240.0 & $2.1682 \mathrm{E}-07$ & $2.6725 \mathrm{E}-07$ & 339.4 & $5.5217 \mathrm{E}-10$ & $-7.7560 \mathrm{E}-12$ \\
\hline 270.0 & $4.2133 \mathrm{E}-08$ & $1.4013 \mathrm{E}-07$ & 381.8 & $2.6670 \mathrm{E}-11$ & $8.9890 \mathrm{E}-12$ \\
\hline
\end{tabular}

Table 5.2.5. Effect Grid Orientation has on the Numerical Concentration Results Transverse to the Plume Centerline ( $0 \mathrm{~m}$ downstream of the source) for the Transient $2 D$ Transport Problem (Cases A and B, T = 1400 days).

\begin{tabular}{|ccc|ccc|}
\hline $\begin{array}{c}\text { Distance } \\
\text { transverse, } \\
\mathrm{y}(\mathrm{m})\end{array}$ & $\begin{array}{c}\text { Analytical } \\
(\mathrm{AT123D})\end{array}$ & $\begin{array}{c}\text { Numerical } \\
\text { (PORFLOW) } \\
\text { (parallel grid) }\end{array}$ & $\begin{array}{c}\text { Distance } \\
\text { transverse, } \\
(\mathrm{m})\end{array}$ & $\begin{array}{c}\text { Analytical } \\
\text { (AT123D) }\end{array}$ & $\begin{array}{c}\text { Numerical } \\
\text { (PORFLOW) }\end{array}$ \\
\hline 0.0 & $5.8747 \mathrm{E}-03$ & $1.6090 \mathrm{E}-03$ & 0.0 & $5.8747 \mathrm{E}-03$ & $1.5000 \mathrm{E}-03$ \\
\hline 30.0 & $1.4251 \mathrm{E}-04$ & $1.4270 \mathrm{E}-04$ & 42.4 & $6.3611 \mathrm{E}-05$ & $4.7470 \mathrm{E}-05$ \\
\hline 60.0 & $2.1612 \mathrm{E}-05$ & $2.1910 \mathrm{E}-05$ & 84.9 & $4.9991 \mathrm{E}-06$ & $1.4400 \mathrm{E}-06$ \\
\hline 90.0 & $3.7120 \mathrm{E}-06$ & $3.8190 \mathrm{E}-06$ & 127.3 & $4.4351 \mathrm{E}-07$ & $3.8140 \mathrm{E}-08$ \\
\hline 120.0 & $6.6951 \mathrm{E}-07$ & $6.9970 \mathrm{E}-07$ & 169.7 & $4.0405 \mathrm{E}-08$ & $3.6090 \mathrm{E}-11$ \\
\hline 150.0 & $1.2302 \mathrm{E}-07$ & $1.3100 \mathrm{E}-07$ & 212.1 & $3.5513 \mathrm{E}-09$ & $-1.4170 \mathrm{E}-10$ \\
\hline 180.0 & $2.2522 \mathrm{E}-08$ & $2.4600 \mathrm{E}-08$ & 254.6 & $2.8281 \mathrm{E}-10$ & $-1.0280 \mathrm{E}-11$ \\
\hline 210.0 & $4.0198 \mathrm{E}-09$ & $4.5590 \mathrm{E}-09$ & 297.0 & $1.9238 \mathrm{E}-11$ & $2.7250 \mathrm{E}-13$ \\
\hline 240.0 & $6.8394 \mathrm{E}-10$ & $8.3160 \mathrm{E}-10$ & 339.4 & $1.0659 \mathrm{E}-12$ & $3.4380 \mathrm{E}-14$ \\
\hline 270.0 & $1.0857 \mathrm{E}-10$ & $3.7320 \mathrm{E}-10$ & 381.8 & $4.6459 \mathrm{E}-14$ & $0.0000 \mathrm{E}+00$ \\
\hline
\end{tabular}

Table 5.2.6. Effect Retardation or Radioactive Decay has on the Numerical Concentration along the Plume Centerline for the Transient 2D Transport Problem (Cases $C$ and $D, T=1400$ days).

\begin{tabular}{|c|c|c|c|c|}
\hline $\begin{array}{c}\text { Distance } \\
\text { downstream, } \\
\text { x (m) }\end{array}$ & $\begin{array}{l}\text { Analytical } \\
\text { (AT123D) }\end{array}$ & $\begin{array}{c}\text { Numerical } \\
\text { (PORFLOW) } \\
(\mathrm{R}=2.0) \\
\text { (parallel grid) }\end{array}$ & $\begin{array}{l}\text { Analytical } \\
\text { (AT123D) }\end{array}$ & $\begin{array}{c}\text { Numerical } \\
\text { (PORFLOW) } \\
(\lambda=0.005) \\
\text { (parallel grid) }\end{array}$ \\
\hline-270.0 & $7.4912 \mathrm{E}-10$ & $0.0000 \mathrm{E}+00$ & $8.1095 \mathrm{E}-11$ & $0.0000 \mathrm{E}+00$ \\
\hline
\end{tabular}




\begin{tabular}{|c|c|c|c|c|}
\hline $\begin{array}{c}\text { Distance } \\
\text { downstream, } \\
\text { x (m) }\end{array}$ & $\begin{array}{l}\text { Analytical } \\
\text { (AT123D) }\end{array}$ & $\begin{array}{c}\text { Numerical } \\
\text { (PORFLOW) } \\
(\mathrm{R}=2.0) \\
\text { (parallel grid) }\end{array}$ & $\begin{array}{l}\text { Analytical } \\
\text { (AT123D) }\end{array}$ & $\begin{array}{c}\text { Numerical } \\
\text { (PORFLOW) } \\
(\lambda=0.005) \\
\text { (parallel grid) }\end{array}$ \\
\hline-240.0 & $3.6661 \mathrm{E}-09$ & 1.7890E-09 & $4.6152 \mathrm{E}-10$ & $2.3820 \mathrm{E}-10$ \\
\hline-210.0 & $1.7526 \mathrm{E}-08$ & $1.1140 \mathrm{E}-08$ & $2.6462 \mathrm{E}-09$ & $1.7160 \mathrm{E}-09$ \\
\hline-180.0 & $8.2424 \mathrm{E}-08$ & $5.7620 \mathrm{E}-08$ & $1.5319 \mathrm{E}-08$ & $1.0820 \mathrm{E}-08$ \\
\hline-150.0 & $3.8459 \mathrm{E}-07$ & $2.8940 \mathrm{E}-07$ & $8.9856 \mathrm{E}-08$ & $6.8310 \mathrm{E}-08$ \\
\hline-120.0 & $1.7988 \mathrm{E}-06$ & $1.4570 \mathrm{E}-06$ & $5.3704 \mathrm{E}-07$ & $4.4050 \mathrm{E}-07$ \\
\hline-90.0 & $8.5563 \mathrm{E}-06$ & $7.5040 \mathrm{E}-06$ & $3.3046 \mathrm{E}-06$ & $2.9420 \mathrm{E}-06$ \\
\hline-60.0 & $4.2453 \mathrm{E}-05$ & $4.0560 \mathrm{E}-05$ & $2.1413 \mathrm{E}-05$ & $2.0740 \mathrm{E}-05$ \\
\hline-30.0 & $2.3506 \mathrm{E}-04$ & $2.3900 \mathrm{E}-04$ & $1.5640 \mathrm{E}-04$ & $1.5840 \mathrm{E}-04$ \\
\hline 0.0 & $6.1250 \mathrm{E}-03$ & $1.6080 \mathrm{E}-03$ & $5.6364 \mathrm{E}-03$ & $1.3470 \mathrm{E}-03$ \\
\hline 30.0 & $9.6132 \mathrm{E}-04$ & $1.0410 \mathrm{E}-03$ & $6.3960 \mathrm{E}-04$ & $6.8970 \mathrm{E}-04$ \\
\hline 60.0 & $7.1002 \mathrm{E}-04$ & 7.6940E-04 & $3.5813 \mathrm{E}-04$ & $3.9350 \mathrm{E}-04$ \\
\hline 90.0 & $5.8524 \mathrm{E}-04$ & $6.2000 \mathrm{E}-04$ & $2.2603 \mathrm{E}-04$ & $2.4310 \mathrm{E}-04$ \\
\hline 120.0 & $5.0317 \mathrm{E}-04$ & $5.2440 \mathrm{E}-04$ & $1.5022 \mathrm{E}-04$ & $1.5850 \mathrm{E}-04$ \\
\hline 150.0 & $4.3995 \mathrm{E}-04$ & $4.5390 \mathrm{E}-04$ & $1.0279 \mathrm{E}-04$ & $1.0710 \mathrm{E}-04$ \\
\hline 180.0 & $3.8561 \mathrm{E}-04$ & $3.9520 \mathrm{E}-04$ & 7.1669E-05 & $7.4050 \mathrm{E}-05$ \\
\hline 210.0 & $3.3531 \mathrm{E}-04$ & $3.4200 \mathrm{E}-04$ & $5.0628 \mathrm{E}-05$ & $5.2030 \mathrm{E}-05$ \\
\hline 240.0 & $2.8686 \mathrm{E}-04$ & $2.9140 \mathrm{E}-04$ & $3.6112 \mathrm{E}-05$ & $3.6990 \mathrm{E}-05$ \\
\hline 270.0 & $2.3971 \mathrm{E}-04$ & $2.4260 \mathrm{E}-04$ & $2.5950 \mathrm{E}-05$ & $2.6520 \mathrm{E}-05$ \\
\hline 300.0 & $1.9447 \mathrm{E}-04$ & $1.9630 \mathrm{E}-04$ & $1.8757 \mathrm{E}-05$ & $1.9140 \mathrm{E}-05$ \\
\hline 330.0 & $1.5234 \mathrm{E}-04$ & $1.5340 \mathrm{E}-04$ & $1.3622 \mathrm{E}-05$ & $1.3880 \mathrm{E}-05$ \\
\hline 360.0 & $1.1472 \mathrm{E}-04$ & $1.1550 \mathrm{E}-04$ & $9.9313 \mathrm{E}-06$ & $1.0110 \mathrm{E}-05$ \\
\hline 390.0 & $8.2723 \mathrm{E}-05$ & $8.3370 \mathrm{E}-05$ & $7.2631 \mathrm{E}-06$ & $7.3920 \mathrm{E}-06$ \\
\hline 420.0 & $5.6936 \mathrm{E}-05$ & $5.7620 \mathrm{E}-05$ & $5.3249 \mathrm{E}-06$ & $5.4180 \mathrm{E}-06$ \\
\hline 450.0 & $3.7304 \mathrm{E}-05$ & $3.8040 \mathrm{E}-05$ & $3.9112 \mathrm{E}-06$ & $3.9780 \mathrm{E}-06$ \\
\hline 480.0 & $2.3213 \mathrm{E}-05$ & $2.3960 \mathrm{E}-05$ & $2.8762 \mathrm{E}-06$ & $2.9250 \mathrm{E}-06$ \\
\hline 510.0 & $1.3694 \mathrm{E}-05$ & $1.4390 \mathrm{E}-05$ & $2.1161 \mathrm{E}-06$ & $2.1520 \mathrm{E}-06$ \\
\hline 540.0 & $7.6460 \mathrm{E}-06$ & $8.2300 \mathrm{E}-06$ & $1.5562 \mathrm{E}-06$ & $1.5820 \mathrm{E}-06$ \\
\hline 570.0 & $4.0354 \mathrm{E}-06$ & $4.4830 \mathrm{E}-06$ & $1.1427 \mathrm{E}-06$ & $1.1620 \mathrm{E}-06$ \\
\hline 600.0 & $2.0110 \mathrm{E}-06$ & $2.3250 \mathrm{E}-06$ & $8.3693 \mathrm{E}-07$ & $8.5100 \mathrm{E}-07$ \\
\hline 630.0 & $9.4535 \mathrm{E}-07$ & $1.1480 \mathrm{E}-06$ & $6.1048 \mathrm{E}-07$ & $6.2080 \mathrm{E}-07$ \\
\hline 660.0 & $4.1888 \mathrm{E}-07$ & $5.4030 \mathrm{E}-07$ & $4.4282 \mathrm{E}-07$ & $4.5050 \mathrm{E}-07$ \\
\hline 690.0 & $1.7483 \mathrm{E}-07$ & $2.4220 \mathrm{E}-07$ & $3.1888 \mathrm{E}-07$ & $3.2460 \mathrm{E}-07$ \\
\hline 720.0 & $6.8689 \mathrm{E}-08$ & $1.0350 \mathrm{E}-07$ & $2.2755 \mathrm{E}-07$ & $2.3180 \mathrm{E}-07$ \\
\hline 750.0 & $2.5393 \mathrm{E}-08$ & $4.2170 \mathrm{E}-08$ & $1.6062 \mathrm{E}-07$ & $1.6390 \mathrm{E}-07$ \\
\hline 780.0 & $8.8287 \mathrm{E}-09$ & $1.6400 \mathrm{E}-08$ & $1.1195 \mathrm{E}-07$ & $1.1450 \mathrm{E}-07$ \\
\hline 810.0 & $2.8859 \mathrm{E}-09$ & $6.0890 \mathrm{E}-09$ & $7.6895 \mathrm{E}-08$ & $7.8940 \mathrm{E}-08$ \\
\hline 840.0 & $8.8655 \mathrm{E}-10$ & $2.1600 \mathrm{E}-09$ & $5.1968 \mathrm{E}-08$ & $5.3610 \mathrm{E}-08$ \\
\hline 870.0 & $2.5589 \mathrm{E}-10$ & $7.3230 \mathrm{E}-10$ & $3.4500 \mathrm{E}-08$ & $3.5820 \mathrm{E}-08$ \\
\hline 900.0 & $6.9375 \mathrm{E}-11$ & $2.3750 \mathrm{E}-10$ & $2.2465 \mathrm{E}-08$ & $2.3530 \mathrm{E}-08$ \\
\hline 930.0 & $1.7663 \mathrm{E}-11$ & $7.3150 \mathrm{E}-11$ & $1.4328 \mathrm{E}-08$ & $1.5260 \mathrm{E}-08$ \\
\hline 960.0 & $4.2224 \mathrm{E}-12$ & $3.8240 \mathrm{E}-11$ & 8.9392E-09 & $1.2370 \mathrm{E}-08$ \\
\hline
\end{tabular}


Table 5.2.7. Effect Retardation or Radioactive Decay has on the Numerical Concentration Transverse to the Plume Centerline for the Transient 2D Transport Problem at $x=420$ meters (Cases $C$ and $D, T=1400$ days).

\begin{tabular}{|c|cc|cc|}
\hline $\begin{array}{c}\text { Distance } \\
\text { transverse, } \\
\mathrm{y}(\mathrm{m})\end{array}$ & $\begin{array}{c}\text { Analytical } \\
\text { (AT123D) }\end{array}$ & $\begin{array}{c}\text { Numerical } \\
(\text { PORFLOW) } \\
(\mathrm{R}=2.0)\end{array}$ & $\begin{array}{c}\text { Analytical } \\
(\mathrm{AT} 123 \mathrm{D})\end{array}$ & $\begin{array}{c}\text { Numerical } \\
(\text { PORFLOW) } \\
\left(\begin{array}{c}\lambda=0.005) \\
\text { (parallel grid) }\end{array}\right.\end{array}$ \\
\hline 0.0 & $5.6936 \mathrm{E}-05$ & $5.7620 \mathrm{E}-05$ & $5.3249 \mathrm{E}-06$ & $5.4180 \mathrm{E}-06$ \\
\hline 30.0 & $4.6872 \mathrm{E}-05$ & $4.6930 \mathrm{E}-05$ & $4.4559 \mathrm{E}-06$ & $4.4860 \mathrm{E}-06$ \\
\hline 60.0 & $2.6285 \mathrm{E}-05$ & $2.5780 \mathrm{E}-05$ & $2.6453 \mathrm{E}-06$ & $2.6110 \mathrm{E}-06$ \\
\hline 90.0 & $1.0171 \mathrm{E}-05$ & $9.9310 \mathrm{E}-06$ & $1.1536 \mathrm{E}-06$ & $1.1310 \mathrm{E}-06$ \\
\hline 120.0 & $2.7592 \mathrm{E}-06$ & $2.7950 \mathrm{E}-06$ & $3.8698 \mathrm{E}-07$ & $3.8590 \mathrm{E}-07$ \\
\hline 150.0 & $5.3229 \mathrm{E}-07$ & $5.9630 \mathrm{E}-07$ & $1.0464 \mathrm{E}-07$ & $1.0910 \mathrm{E}-07$ \\
\hline 180.0 & $7.3815 \mathrm{E}-08$ & $9.9360 \mathrm{E}-08$ & $2.3748 \mathrm{E}-08$ & $2.6490 \mathrm{E}-08$ \\
\hline 210.0 & $7.4096 \mathrm{E}-09$ & $1.3260 \mathrm{E}-08$ & $4.6656 \mathrm{E}-09$ & $5.6860 \mathrm{E}-09$ \\
\hline 240.0 & $5.4058 \mathrm{E}-10$ & $1.4380 \mathrm{E}-09$ & $8.0986 \mathrm{E}-10$ & $1.1130 \mathrm{E}-09$ \\
\hline 270.0 & $2.8724 \mathrm{E}-11$ & $4.2490 \mathrm{E}-10$ & $1.2558 \mathrm{E}-10$ & $5.0830 \mathrm{E}-10$ \\
\hline
\end{tabular}

Table 5.2.8. Input Commands for 2D Transport (Case A)

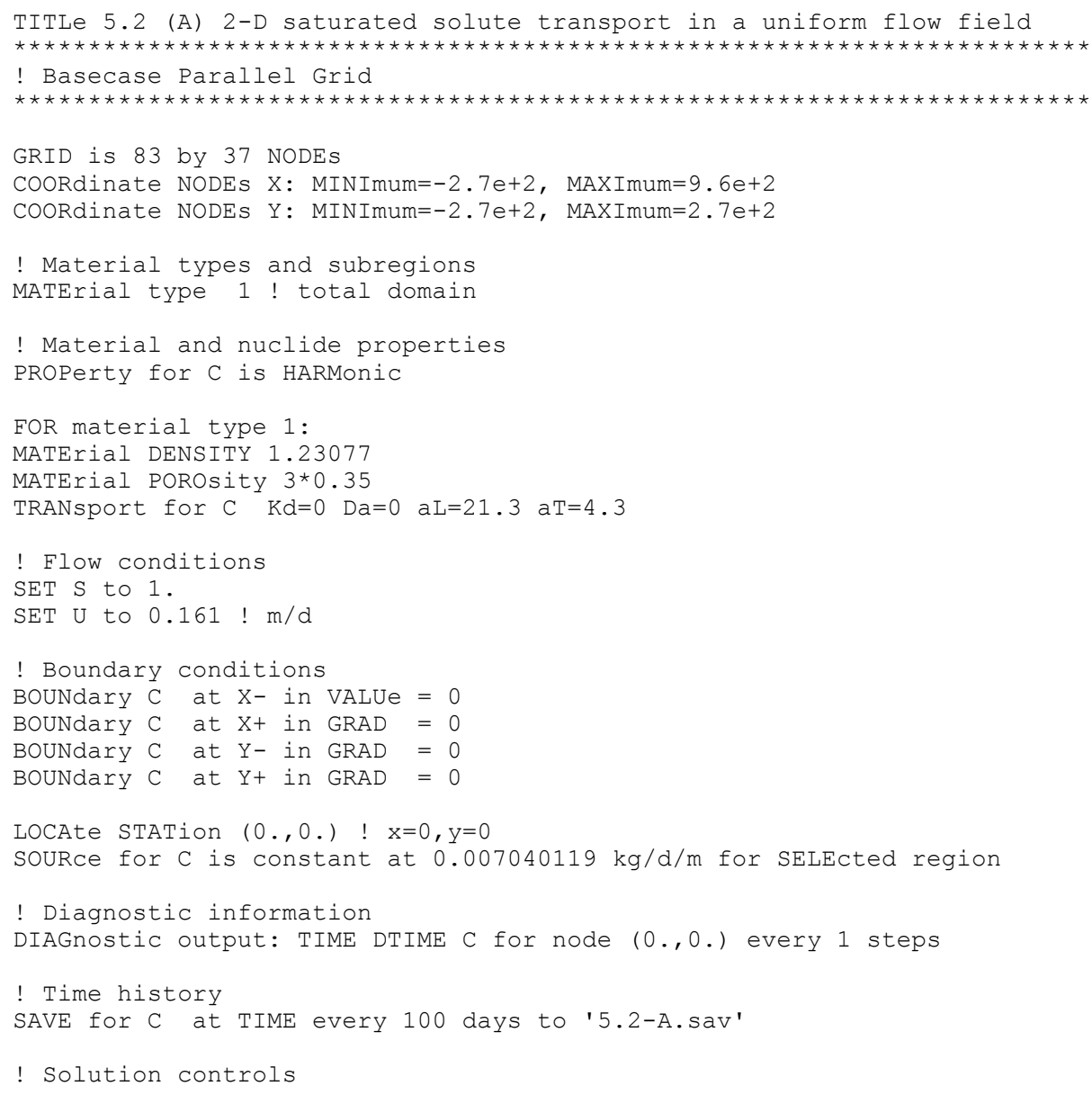


MATRix in $X$ for $C 3$ sweeps using ADI

! LIMIt for $\mathrm{C} 0.0$

CONVergence for C REFErence LOCAl 1.e-6, max iterations = 30

! Solve transient transport

! Time period: 0 to 1400 years

TIME $=0$

Solve C for 1400 days in steps of 1 days

END

QUIT

Table 5.2.9. Input Commands for 2D Transport (Case B)

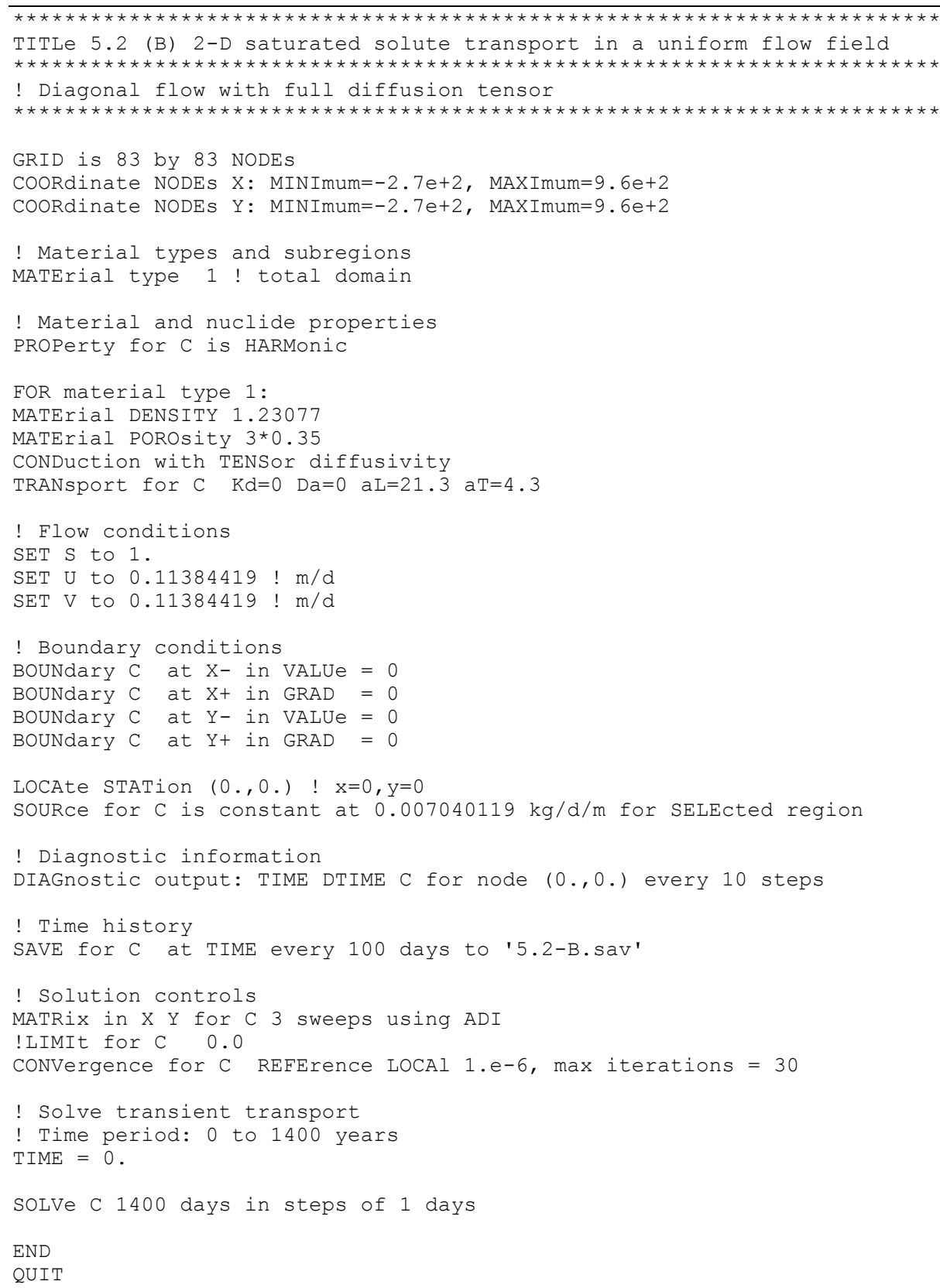

Table 5.2.10. Input Commands for 2D Transport (Case C) 
Table 5.2.11. Input Commands for 2D Transport (Case D)

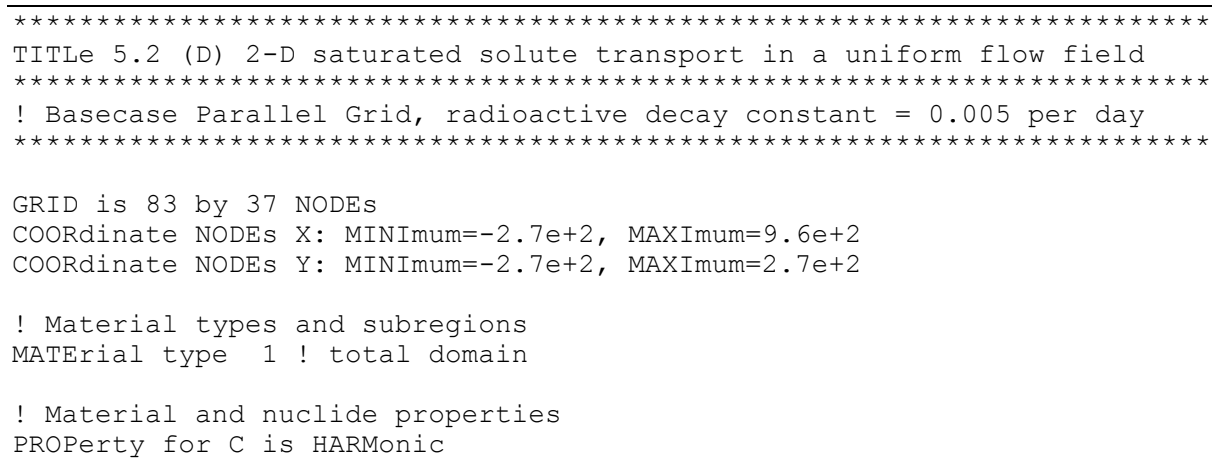




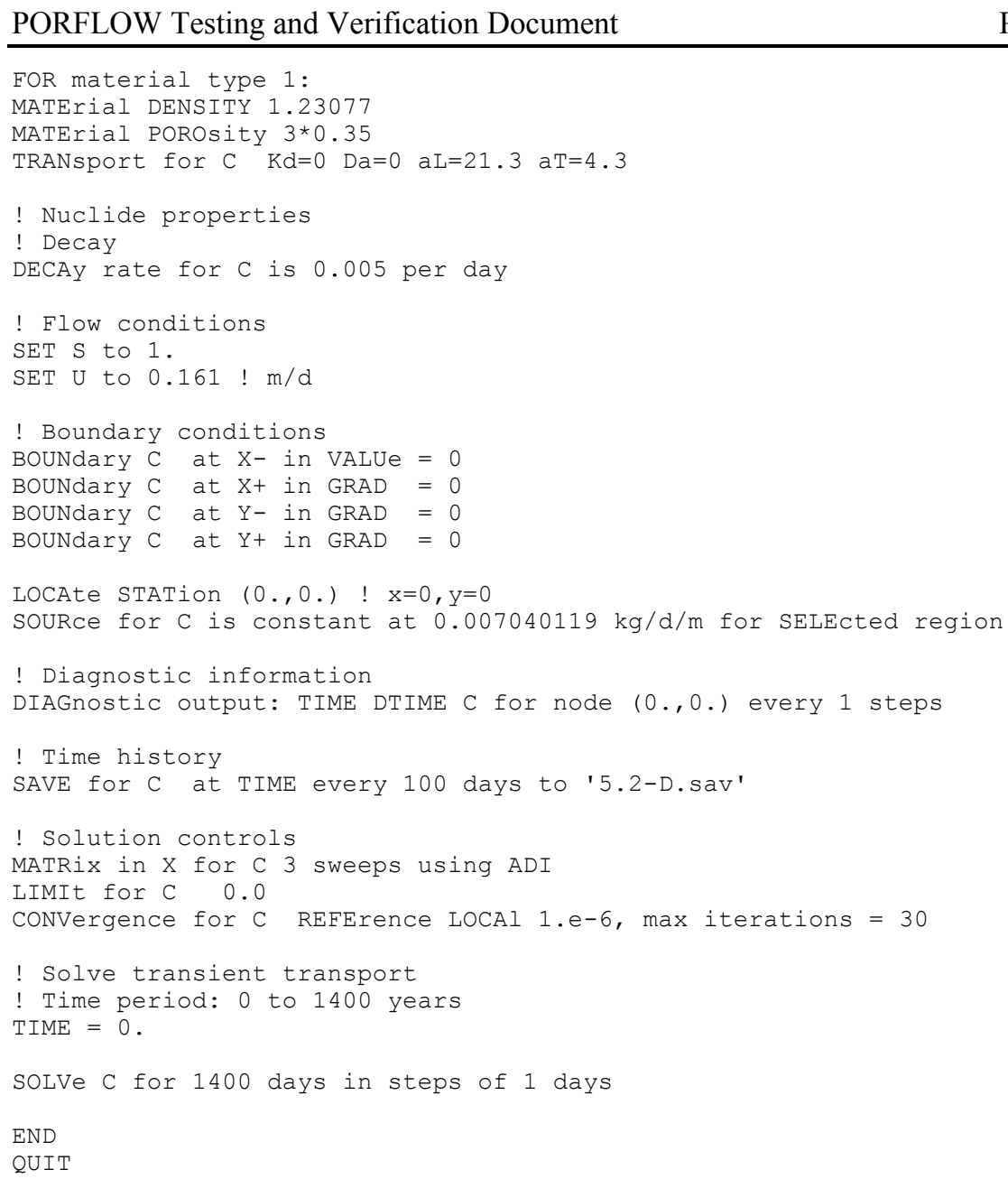

\subsection{Three-Dimensional Saturated Solute Transport in a Uniform Flow Field}

This problem deals with three-dimensional advection-dispersion of a conservative solute species from a point source through an infinite porous medium. Based upon the problem definition, this problem results in an analytic solution that is axisymmetric in solute concentrations. However, the problem will be solved analytically and numerically in 3D Cartesian coordinates. It is used to demonstrate PORFLOW's capability to solve 3D transport problems and to yield 3D results that are indeed axisymmetric. This problem also tests PORFLOW's formulation of transverse dispersion in more than one dimension. The physical schematic of this problem is essentially the same as for the 2D transport problem discussed in Section 5.2 and shown in Figure 5.2.1(a). In practice, the idealized conditions are analogous to continual leakage, leaching, or injection of a contaminant into a large confined aquifer from buried waste in a landfill or an improperly sealed partially penetrating injection well. It is assumed that the total rate of fluid leakage, leaching, or injection into the aquifer is negligible and does not disturb the ambient groundwater flow regime. Analytically and numerically, the problem is treated as a point source in 3D Cartesian coordinates.

As illustrated in Figure 5.2.1(b), a conservative contaminant is continuously released from a point source downstream of an inflow boundary (containing zero contaminant) into a large aquifer unit whose groundwater flow is assumed to be uniform. The point source is located at a depth such 
that end effects at the top and bottom of the aquifer unit are negligible. Both hydrodynamic dispersion and molecular diffusion are allowed for the transported species. It is assumed that the contaminant mass flow rate at the point source remains constant, the aquifer's flow rate is uniform and constant, the aquifer is sufficiently large to neglect end effects, and the homogeneous aquifer's properties (such as porosity, soil type, water saturation) are uniform and constant.

Analytical solution: Equation 5.1.4 represents the non-conservative form of the multidimensional advection-dispersion equation for solute transport through a variably saturated porous media. Taking the 3D form of Equation 5.1.4 and assuming that one point exists at the areal location $\mathrm{x}=\mathrm{y}=\mathrm{z}=0$, constant water saturation level, and that material coefficients are constants, results in

$$
\begin{aligned}
\frac{\partial c}{\partial t} & =D_{x x}^{\prime} \frac{\partial^{2} c}{\partial x^{2}}+D_{x y}^{\prime} \frac{\partial^{2} c}{\partial x \partial y}+D_{x z}^{\prime} \frac{\partial^{2} c}{\partial x \partial z}+D_{y x}^{\prime} \frac{\partial^{2} c}{\partial y \partial x}+D_{y y}^{\prime} \frac{\partial^{2} c}{\partial y^{2}}+D_{y z}^{\prime} \frac{\partial^{2} c}{\partial y \partial z} \\
& +D_{z x}^{\prime} \frac{\partial^{2} c}{\partial z \partial x}+D_{z y}^{\prime} \frac{\partial^{2} c}{\partial z \partial y}+D_{z z}^{\prime} \frac{\partial^{2} c}{\partial z^{2}}-u_{x}^{\prime} \frac{\partial c}{\partial x}-u_{y}^{\prime} \frac{\partial c}{\partial y}-u_{z}^{\prime} \frac{\partial c}{\partial z}-\lambda c-\frac{q\left(c-c^{*}\right)}{\theta_{e} R}
\end{aligned}
$$

where

$$
\begin{gathered}
\mathrm{D}_{\mathrm{xx}}^{\prime}=\frac{\alpha_{\mathrm{L}} \frac{\mathrm{U}_{\mathrm{x}}^{2}}{|\mathrm{U}|}+\alpha_{\mathrm{T}} \frac{\mathrm{U}_{\mathrm{y}}^{2}+\mathrm{U}_{\mathrm{z}}^{2}}{|\mathrm{U}|}+\theta_{\mathrm{e}} \tau \mathrm{D}_{\mathrm{m}}}{\theta_{\mathrm{e}} \mathrm{R}} \\
\mathrm{D}_{\mathrm{yy}}^{\prime}=\frac{\alpha_{\mathrm{L}} \frac{\mathrm{U}_{\mathrm{y}}^{2}}{|\mathrm{U}|}+\alpha_{\mathrm{T}} \frac{\mathrm{U}_{\mathrm{x}}^{2}+\mathrm{U}_{\mathrm{z}}^{2}}{|\mathrm{U}|}+\theta_{\mathrm{e}} \tau \mathrm{D}_{\mathrm{m}}}{\theta_{\mathrm{e}} \mathrm{R}} \\
\mathrm{D}_{\mathrm{zz}}^{\prime}=\frac{\alpha_{\mathrm{L}} \frac{\mathrm{U}_{\mathrm{z}}^{2}}{|\mathrm{U}|}+\alpha_{\mathrm{T}} \frac{\mathrm{U}_{\mathrm{x}}^{2}+\mathrm{U}_{\mathrm{y}}^{2}}{|\mathrm{U}|}+\theta_{\mathrm{e}} \tau \mathrm{D}_{\mathrm{m}}}{\theta_{\mathrm{e}} \mathrm{R}} \\
\mathrm{D}_{\mathrm{xy}}^{\prime}=\mathrm{D}_{\mathrm{yx}}^{\prime}=\frac{\left(\alpha_{\mathrm{L}}-\alpha_{\mathrm{T}}\right) \frac{\mathrm{U}_{\mathrm{x}} \mathrm{U}_{\mathrm{y}}}{|\mathrm{U}|}}{\theta_{\mathrm{e}} \mathrm{R}} \\
\mathrm{D}_{\mathrm{xz}}^{\prime}=\mathrm{D}_{\mathrm{zx}}^{\prime}=\frac{\left(\alpha_{\mathrm{L}}-\alpha_{\mathrm{T}}\right) \frac{\mathrm{U}_{\mathrm{x}} \mathrm{U}_{\mathrm{z}}}{|\mathrm{U}|}}{\theta_{\mathrm{e}} \mathrm{R}}
\end{gathered}
$$




$$
D_{y z}^{\prime}=D_{z y}^{\prime}=\frac{\left(\alpha_{L}-\alpha_{T}\right) \frac{U_{y} U_{z}}{|U|}}{\theta_{\mathrm{e}} R}
$$

If the flow field is aligned with the x-axis, Equation 5.3.1 reduces to

$$
\frac{\partial c}{\partial t}=D_{x x}^{\prime} \frac{\partial^{2} c}{\partial x^{2}}+D_{y y}^{\prime} \frac{\partial^{2} c}{\partial y^{2}}+D_{z z}^{\prime} \frac{\partial^{2} c}{\partial z^{2}}-u_{x}^{\prime} \frac{\partial c}{\partial x}-\lambda c-\frac{q\left(c-c^{*}\right)}{\theta_{e} R}
$$

For our infinite confined aquifer the initial conditions are:

$$
\mathrm{c}(\mathrm{x}, \mathrm{y}, \mathrm{z}, 0)=0
$$

and the boundary conditions are:

$$
\begin{aligned}
& c( \pm \infty, y, z, t)=0 \\
& c(x, \pm \infty, z, t)=0 \\
& c(x, y, \pm \infty, t)=0
\end{aligned}
$$

These boundary conditions are equivalent to assuming that the dispersive flux of solute is zero at plus or minus infinity or

$$
\begin{aligned}
& \lim _{x \rightarrow \pm \infty}\left(\frac{\partial c}{\partial x}\right)=0 \\
& \lim _{y \rightarrow \pm \infty}\left(\frac{\partial c}{\partial y}\right)=0 \\
& \lim _{z \rightarrow \pm \infty}\left(\frac{\partial c}{\partial z}\right)=0
\end{aligned}
$$

For finite times there exists finite values of $\mathrm{x}, \mathrm{y}$ and $\mathrm{z}$ where Equations $5.3 .4 \mathrm{e}, 5.3 .4 \mathrm{f}$ and $5.3 .4 \mathrm{~g}$ remain valid.

As mentioned above, it is assumed that the total rate of fluid flow, q, into the aquifer due to the source is negligible and does not disturb the ambient groundwater flow regime. In order to have a finite mass flow rate of contaminant requires

$$
\lim _{\mathrm{q} \rightarrow 0}\left(\mathrm{qc}^{*}\right)<\infty \Rightarrow \lim _{\mathrm{q} \rightarrow 0}\left(\mathrm{c}^{*}\right)= \pm \infty
$$


Equation 5.3.3, a linear partial differential equation subject to the initial and boundary conditions given by Equations 5.3.4a to 5.3.4d, can be solved by applying Laplace and Fourier transforms to derive at the appropriate Green's functions. For details, see Yeh (1981). The general solution for a continuous point source takes the form:

$$
c(x, y, z, t)=\frac{\mathrm{qc}^{*}}{\theta_{\mathrm{e}} \mathrm{R}} \int_{0}^{\mathrm{t}} \mathrm{G}(\mathrm{x}|\xi ; \mathrm{y}| \eta ; \mathrm{z}|\zeta ; \mathrm{t}| \tau) \mathrm{d} \tau
$$

where $\mathrm{G}(\mathrm{x}|\xi ; \mathrm{y}| \eta ; \mathrm{z}|\zeta ; \mathrm{t}| \tau)$ is the Green's function over the domain space. For our analytical problem, we shall limit our flow field to flow parallel to the $\mathrm{x}$-axis only. This results in a dispersion tensor that is diagonal and a separable Green's function. It can be shown that for a simple geometry such as a separable coordinate system, Green's function can be expressed as:

$$
\mathrm{G}(\mathrm{x}|\xi ; \mathrm{y}| \eta ; \mathrm{z}|\zeta ; \mathrm{t}| \tau)=\mathrm{G}_{1}(\mathrm{x}|\xi ; \mathrm{t}| \tau) \mathrm{G}_{2}(\mathrm{y}|\eta ; \mathrm{t}| \tau) \mathrm{G}_{3}(\mathrm{z}|\zeta ; \mathrm{t}| \tau)
$$

where for a point source in the x-direction (infinite domain, parallel to flow):

$$
\mathrm{G}_{1}(\mathrm{x}|\xi ; \mathrm{t}| \tau)=\frac{\mathrm{S}(\mathrm{t}-\tau)}{\sqrt{4 \pi \mathrm{D}_{\mathrm{xx}}^{\prime}(\mathrm{t}-\tau)}} \exp \left[-\frac{\left\{(\mathrm{x}-\xi)-\mathrm{u}_{\mathrm{x}}^{\prime}(\mathrm{t}-\tau)\right\}^{2}}{4 \mathrm{D}_{\mathrm{xx}}^{\prime}(\mathrm{t}-\tau)}-\lambda(\mathrm{t}-\tau)\right]
$$

and for a point source in the y-direction (infinite domain, transverse to flow):

$$
\mathrm{G}_{2}(\mathrm{y}|\eta ; \mathrm{t}| \tau)=\frac{\mathrm{S}(\mathrm{t}-\tau)}{\sqrt{4 \pi \mathrm{D}_{\mathrm{yy}}^{\prime}(\mathrm{t}-\tau)}} \exp \left[-\frac{(\mathrm{y}-\eta)^{2}}{4 \mathrm{D}_{\mathrm{yy}}^{\prime}(\mathrm{t}-\tau)}\right]
$$

and for a point source in the z-direction (infinite domain, transverse to flow):

$$
\mathrm{G}_{3}(\mathrm{z}|\zeta ; \mathrm{t}| \tau)=\frac{\mathrm{S}(\mathrm{t}-\tau)}{\sqrt{4 \pi \mathrm{D}_{\mathrm{zz}}^{\prime}(\mathrm{t}-\tau)}} \exp \left[-\frac{(\mathrm{z}-\zeta)^{2}}{4 \mathrm{D}_{\mathrm{zz}}^{\prime}(\mathrm{t}-\tau)}\right]
$$

where $S(t-\tau)$ is the step function.

The evaluation of the analytical expressions, Equations 5.3.5a to 5.2.5e, for a specific problem is performed numerically using the FORTRAN program AT123D in Section C.

PORFLOW numerical simulation and comparison: In PORFLOW, we will model this axisymmetric problem using a 3D mesh containing equally spaced nodes in all three directions. The point source given above will be located at the center of the yz plane, 270 meters from the inflow boundary in the $\mathrm{x}$-direction $(\mathrm{x}=\mathrm{y}=\mathrm{z}=0)$.

Even though we are considering an aquifer unit with infinite extent in the areal and vertical directions, our numerical model has finite size. At our inflow boundary we shall assume that the incoming fluid remains contaminant free (i.e., the contaminant concentration immediately 
upstream of the source does not extend back up to the inflow boundary). We also assume that the vertical extent of the top and bottom faces of our mesh from the point source is sufficient distance that negligible amounts of contaminant reaches these boundary faces. For the parameters chosen (i.e., longitudinal dispersivity, Darcy velocity, and source location), the above assumption remains valid over the time of interest.

In addition, PORFLOW requires input about the total mass flowrate entering the aquifer at the point source. An arbitrarily small but finite value for $\mathrm{qc}^{*}$ was chosen to maintain computed solute concentration values near the source to acceptable values. Steep concentration gradients near a source can result in oscillatory behavior unless the local grid is sufficiently refined. For demonstration purposes, we have chosen uniform coarse grids and are primarily interested in results away from the source location. The simulations are done by specifying a velocity field and running the solute transport option only. In this way, the flow rate entering the aquifer due to the point source does not alter the aquifer flow field.

Values of the physical parameters used in the verification simulations are presented in Table 5.3.1. For the conservative solute transport case the parameters were selected based on data from a field investigation on hexavalent chromium contamination reported by Perlumutter and Lieber (1970) and Wilson and Miller (1978)

In 1D transport, only longitudinal dispersion is active and in a 2D transport problem, both longitudinal and transverse dispersion can occur. In 3D transport, transverse dispersion occurs throughout the plane perpendicular to the flow direction. In a general 3D transport problem, the off-diagonal terms of the dispersion coefficient tensor are typically non-zero. If the grid is aligned parallel to the groundwater flow direction, then only the diagonal terms of the hydrodynamic dispersion tensor are non-zero. 
Table 5.3.1. Values of the Physical Parameters, Mesh Spacing, Time Steps, and Key Computed Parameters Used in the Three-Dimensional Transport Simulations

\begin{tabular}{|c|c|}
\hline Physical parameters & Base Case \\
\hline Darcy velocity, $\mathrm{U}_{\mathrm{x}}$ & $0.161 \mathrm{~m} / \mathrm{d}$ \\
\hline Porosity, $\phi$ & 0.35 \\
\hline Longitudinal dispersivity, $\alpha_{L}$ & $21.3 \mathrm{~m}$ \\
\hline Transverse dispersivity, $\alpha_{\mathrm{T}}$ & $4.3 \mathrm{~m}$ \\
\hline $\begin{array}{l}\text { Apparent molecular dispersion coefficient, } \\
\theta_{\mathrm{e}} \tau \mathrm{D}^{*}\end{array}$ & $0.0 \mathrm{~m}^{2} / \mathrm{d}$ \\
\hline Water saturation, $\mathrm{S}_{\mathrm{W}}$ & 1.0 \\
\hline radioactive decay coef., $\lambda$ & $0.0 \mathrm{~d}^{-1}$ \\
\hline Soil density, $\rho_{\mathrm{S}}$ & $1.23077 \mathrm{~kg} / \mathrm{m}^{3}$ \\
\hline Solute distribution coefficient, $\mathrm{k}_{\mathrm{d}}$ & $0.0 \mathrm{~m}^{3} / \mathrm{kg}$ \\
\hline Boundary solute concentration, $c_{o}$ & $0.0 \mathrm{~kg} / \mathrm{m}^{3}$ \\
\hline $\begin{array}{c}\text { Contaminant total mass flowrate (point } \\
\text { source), } \mathrm{qc}^{*}\end{array}$ & $0.117922 \mathrm{~kg} / \mathrm{d}$ \\
\hline \multicolumn{2}{|l|}{ Grid specifics } \\
\hline Mesh spacing, $\Delta x=\Delta y=\Delta z$ & $15 \mathrm{~m}$ \\
\hline Number nodes in $\mathrm{x}$-dir & 83 \\
\hline Number nodes in y-dir & 37 \\
\hline Number nodes in z-dir & 37 \\
\hline \multicolumn{2}{|l|}{ Time steps } \\
\hline $\begin{array}{c}\text { Time duration } \\
\text { number time-steps } \\
\text { time-step size, } \Delta \mathrm{t}\end{array}$ & $\begin{array}{c}1400 \mathrm{~d} \\
140 \\
10 \mathrm{~d}\end{array}$ \\
\hline \multicolumn{2}{|l|}{ Key computed parameters } \\
\hline Retardation factor, $\mathrm{R}$ & 1.0 \\
\hline Bulk soil density, $\rho_{b}$ & $0.8 \mathrm{~kg} / \mathrm{m}^{3}$ \\
\hline Phasic velocity, $\mathrm{u}_{\mathrm{x}}$ & $0.46 \mathrm{~m} / \mathrm{d}$ \\
\hline Retarded phasic velocity, $\mathrm{u}_{\mathrm{x}}^{\prime}$ & $0.46 \mathrm{~m} / \mathrm{d}$ \\
\hline $\begin{array}{l}\text { Retarded longitudinal dispersion } \\
\text { coefficient, } \mathrm{D}_{\mathrm{xx}}^{\prime}\end{array}$ & $9.798 \mathrm{~m}^{2} / \mathrm{d}$ \\
\hline $\begin{array}{l}\text { Retarded transverse dispersion coefficients, } \\
\qquad \mathrm{D}_{\mathrm{yy}}^{\prime}=\mathrm{D}_{\mathrm{zz}}^{\prime}\end{array}$ & $1.978 \mathrm{~m}^{2} / \mathrm{d}$ \\
\hline $\begin{array}{l}\text { Retarded cross dispersion coefficients, } \\
\qquad \mathrm{D}_{\mathrm{xy}}^{\prime}=\mathrm{D}_{\mathrm{xz}}^{\prime}=\mathrm{D}_{\mathrm{yz}}^{\prime}\end{array}$ & $0.0 \mathrm{~m}^{2} / \mathrm{d}$ \\
\hline Cell Fourier number, $\mathrm{Fo}_{\mathrm{x}} ; \mathrm{Fo}_{\mathrm{y}}=\mathrm{Fo}_{\mathrm{z}}$ & $0.4355 ; 0.0879$ \\
\hline Cell Courant number, $\mathrm{Co}_{\mathrm{x}} ; \mathrm{Co}_{\mathrm{y}}=\mathrm{Co}_{\mathrm{z}}$ & $0.3067 ; 0.0$ \\
\hline Cell Peclet number, $\mathrm{Pe}_{\mathrm{x}} ; \mathrm{Pe}_{\mathrm{y}}=\mathrm{Pe}_{\mathrm{z}}$ & $0.7042 ; 3.4884$ \\
\hline
\end{tabular}

To examine in PORFLOW transverse dispersion into a plane perpendicular to groundwater flow without the added complication of cross-term products resulting from the dispersion tensor, a base case grid was chosen for a problem that is aligned parallel to the aquifer flow direction. This 
grid consists of 113,627 nodes uniformly sized (15 m in length) with 83 nodes along the $\mathrm{x}$-axis and 37 along the $y$ - and z-axes, respectively. This number of nodes is close to the current license limit of 128,000 nodes.

Figure 5.3.1 illustrates the PORFLOW grid chosen with a chair cut showing the point source location (vertex of chair cut). At the channel inlet boundary (-X face), the concentration of solute in the incoming water is set to $0.0 \mathrm{~kg} / \mathrm{m} 3$. Due to the finite overall length of our mesh, at the outflow boundary ( $+\mathrm{X}$ face) the dispersive flux (GRAD) is set to zero, while the advective flux is computed as part of the solution. In addition, the dispersive flux is set to zero at the transverse faces ( $-Y$ face, $+Y$ face, $-Z$ face and $+Z$ face). The aquifer is assumed to be completely saturated.

For this problem only the base case simulation was performed. For this simulation, a transient calculation was performed for a 1400-day duration and the results from PORFLOW at this end time were compared to the analytical solution given by Equation 5.3.5.

The results from the simulations, analytical and numerical, are shown in Figures 5.3.2 through 5.3.4. These results are also presented in tabular form in Tables 5.3.2 and 5.3.3. The PORFLOW input commands for the 3D transport simulation are shown in Table 5.3.4. The analytical results were computed using the FORTRAN code AT123D in Section C.

The PORFLOW concentration plume at 1400 days is presented in Figure 5.3.2. A section of the domain has been cut out to highlight the plume centerline and its basic axisymmetric development in the transverse direction.

The concentration profiles for the 3D transport simulation along and transverse to the plume centerline are shown in Figures 5.3.3 and 5.3.4, respectively. Figure 5.3.4 shows computed concentration profiles transverse to the groundwater flow direction and plume centerline (y-axis and z-axis) 120 meters downstream of the point source. The results away from the source show good comparison between the analytical solution and the numerical results for both transverse directions. These results indicate that the concentration plume predicted by PORFLOW is axisymmetric as well. 


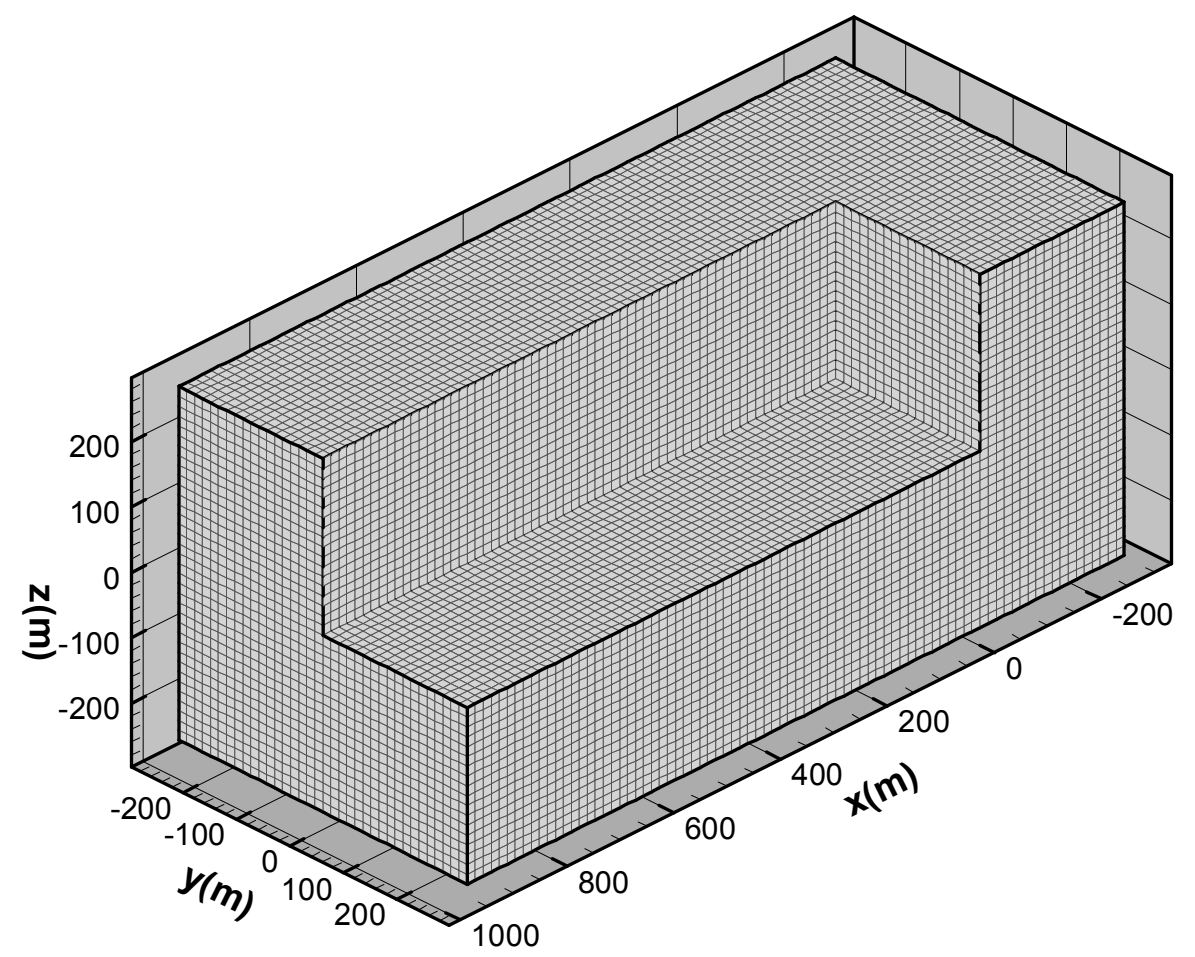

Figure 5.3.1. PORFLOW Grid for 3D Transport Simulation.

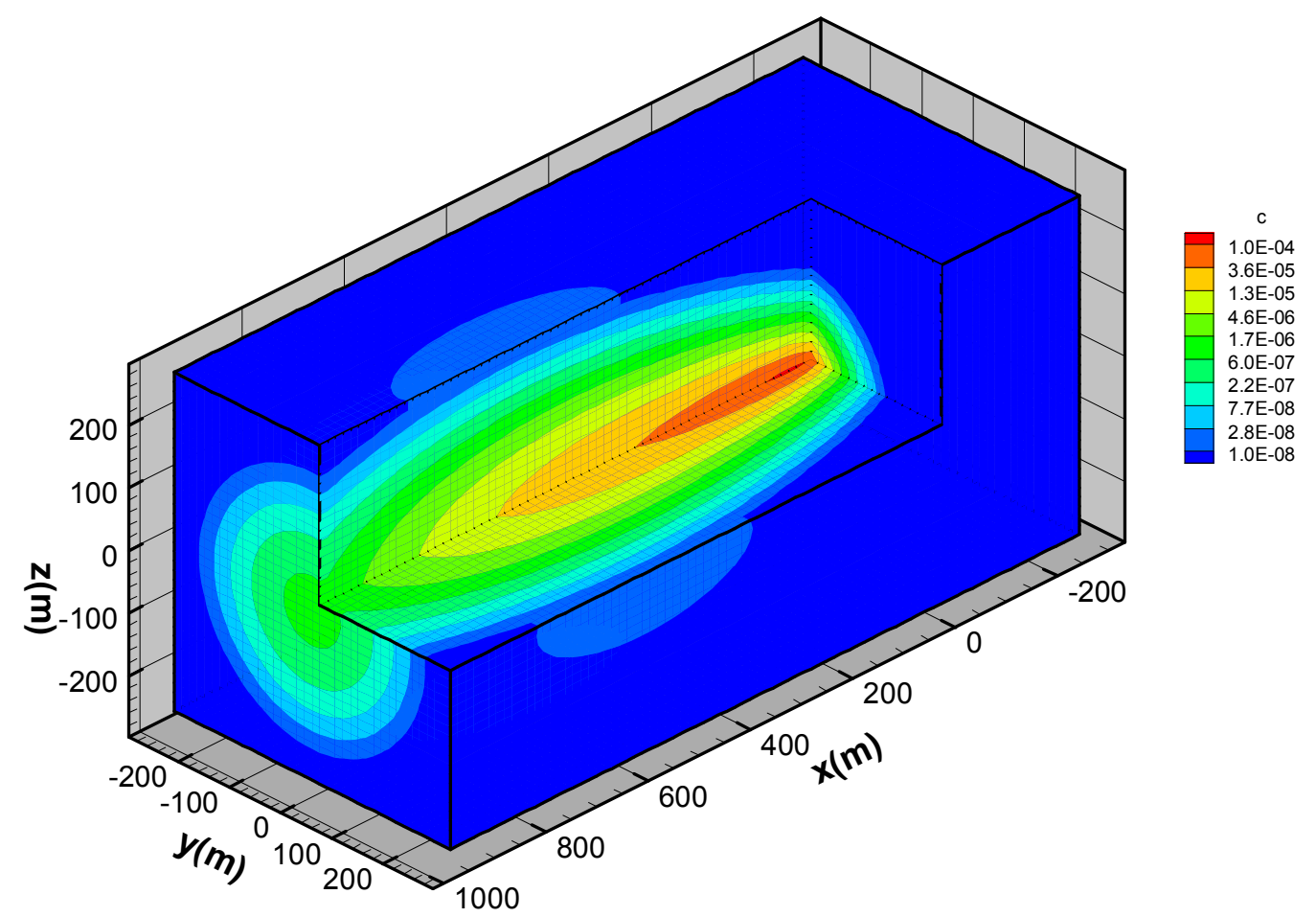

Figure 5.3.2 PORFLOW Concentration Plume for 3D Transport of the Base Case on a Parallel Grid. 


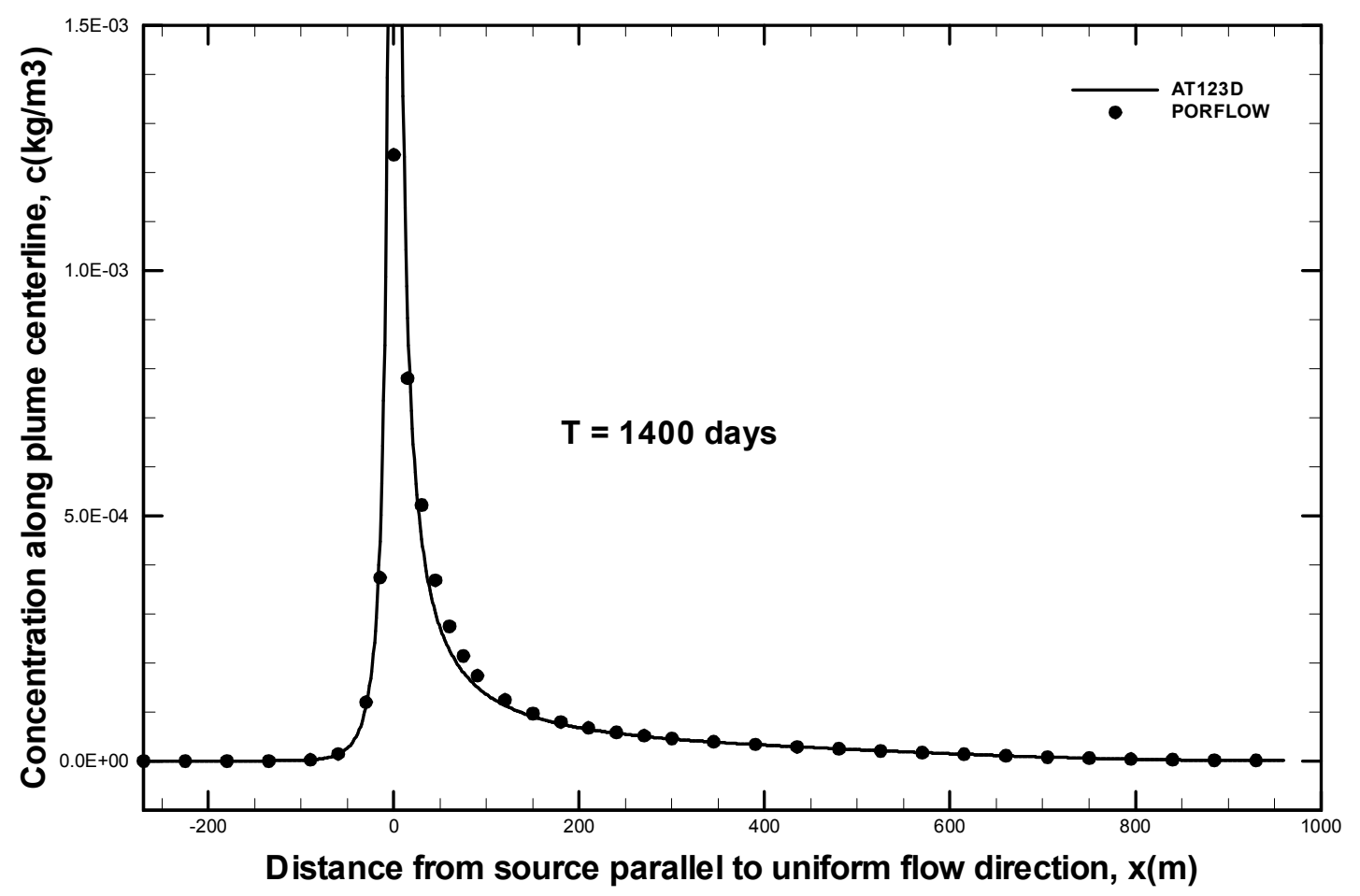

Figure 5.3.3 Concentration Profile for 3D Transport of the Base Case along the Plume Centerline.

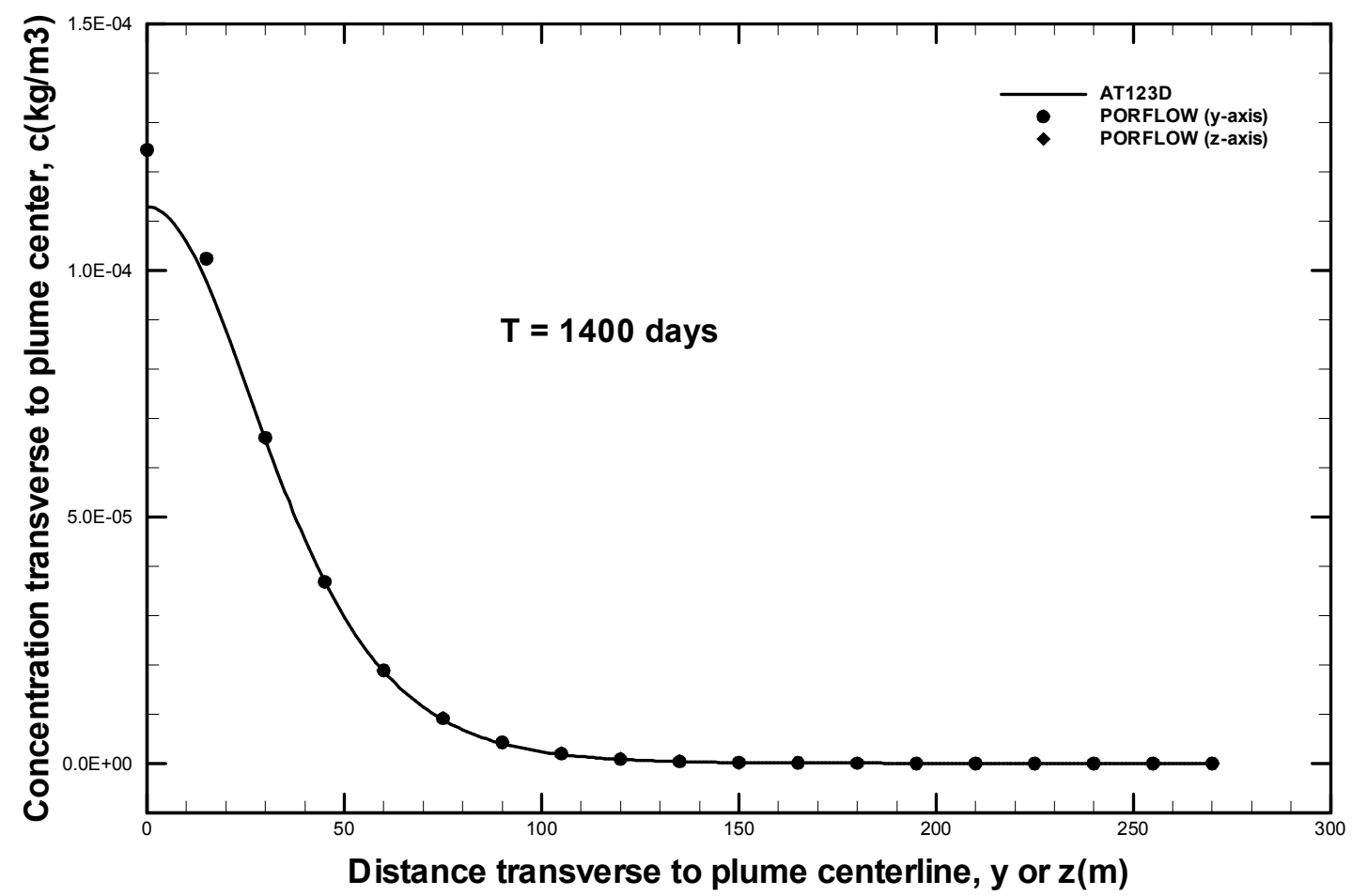

Figure 5.3.4 Concentration Profiles for 3D Transport of the Base Case Transverse to the Plume Centerline (in both $\mathrm{y}$ and $\mathrm{z}$ directions) located at $\mathrm{x}=120$ meters. 
Table 5.3.2. Concentration Profile for 3D Transport of the Base Case along the Plume Centerline at 1400 days.

\begin{tabular}{|c|c|c|}
\hline $\begin{array}{c}\text { Distance } \\
\text { downstream, } \\
\text { x (m) }\end{array}$ & $\begin{array}{l}\text { Analytical } \\
\text { (AT123D) }\end{array}$ & $\begin{array}{c}\text { Numerical } \\
\text { (PORFLOW) }\end{array}$ \\
\hline-270.0 & $1.5587 \mathrm{E}-10$ & $0.0000 \mathrm{E}+00$ \\
\hline-240.0 & $7.1915 \mathrm{E}-10$ & $3.4620 \mathrm{E}-10$ \\
\hline-210.0 & $3.3669 \mathrm{E}-09$ & $2.1680 \mathrm{E}-09$ \\
\hline-180.0 & $1.6080 \mathrm{E}-08$ & $1.1540 \mathrm{E}-08$ \\
\hline-150.0 & $7.8953 \mathrm{E}-08$ & $6.1729 \mathrm{E}-08$ \\
\hline-120.0 & $4.0372 \mathrm{E}-07$ & $3.4599 \mathrm{E}-07$ \\
\hline-90.0 & $2.2017 \mathrm{E}-06$ & $2.0999 \mathrm{E}-06$ \\
\hline-60.0 & $1.3507 \mathrm{E}-05$ & $1.4469 \mathrm{E}-05$ \\
\hline-30.0 & $1.1048 \mathrm{E}-04$ & $1.1969 \mathrm{E}-04$ \\
\hline 0.0 & $7.7226 \mathrm{E}-01$ & $1.2359 \mathrm{E}-03$ \\
\hline 30.0 & $4.5181 \mathrm{E}-04$ & $5.2137 \mathrm{E}-04$ \\
\hline 60.0 & $2.2590 \mathrm{E}-04$ & $2.7449 \mathrm{E}-04$ \\
\hline 90.0 & $1.5059 \mathrm{E}-04$ & $1.7350 \mathrm{E}-04$ \\
\hline 120.0 & $1.1293 \mathrm{E}-04$ & $1.2450 \mathrm{E}-04$ \\
\hline 150.0 & $9.0319 \mathrm{E}-05$ & $9.6869 \mathrm{E}-05$ \\
\hline 180.0 & $7.5226 \mathrm{E}-05$ & 7.9329E-05 \\
\hline 210.0 & $6.4417 \mathrm{E}-05$ & $6.7209 \mathrm{E}-05$ \\
\hline 240.0 & $5.6270 \mathrm{E}-05$ & $5.8270 \mathrm{E}-05$ \\
\hline 270.0 & $4.9876 \mathrm{E}-05$ & $5.1370 \mathrm{E}-05$ \\
\hline 300.0 & $4.4683 \mathrm{E}-05$ & $4.5810 \mathrm{E}-05$ \\
\hline 330.0 & $4.0333 \mathrm{E}-05$ & $4.1190 \mathrm{E}-05$ \\
\hline 360.0 & $3.6580 \mathrm{E}-05$ & $3.7220 \mathrm{E}-05$ \\
\hline 390.0 & $3.3249 \mathrm{E}-05$ & $3.3700 \mathrm{E}-05$ \\
\hline 420.0 & $3.0214 \mathrm{E}-05$ & $3.0510 \mathrm{E}-05$ \\
\hline 450.0 & $2.7381 \mathrm{E}-05$ & $2.7540 \mathrm{E}-05$ \\
\hline 480.0 & $2.4687 \mathrm{E}-05$ & $2.4740 \mathrm{E}-05$ \\
\hline 510.0 & $2.2092 \mathrm{E}-05$ & $2.2060 \mathrm{E}-05$ \\
\hline 540.0 & $1.9577 \mathrm{E}-05$ & $1.9490 \mathrm{E}-05$ \\
\hline 570.0 & $1.7142 \mathrm{E}-05$ & $1.7040 \mathrm{E}-05$ \\
\hline 600.0 & $1.4801 \mathrm{E}-05$ & $1.4700 \mathrm{E}-05$ \\
\hline 630.0 & $1.2576 \mathrm{E}-05$ & $1.2510 \mathrm{E}-05$ \\
\hline 660.0 & $1.0498 \mathrm{E}-05$ & $1.0480 \mathrm{E}-05$ \\
\hline 690.0 & $8.5957 \mathrm{E}-06$ & $8.6350 \mathrm{E}-06$ \\
\hline 720.0 & $6.8932 \mathrm{E}-06$ & $6.9950 \mathrm{E}-06$ \\
\hline 750.0 & 5.4073E-06 & $5.5650 \mathrm{E}-06$ \\
\hline 780.0 & $4.1444 \mathrm{E}-06$ & $4.3460 \mathrm{E}-06$ \\
\hline 810.0 & $3.1005 \mathrm{E}-06$ & $3.3300 \mathrm{E}-06$ \\
\hline 840.0 & $2.2620 \mathrm{E}-06$ & $2.5020 \mathrm{E}-06$ \\
\hline 870.0 & $1.6081 \mathrm{E}-06$ & $1.8450 \mathrm{E}-06$ \\
\hline 900.0 & $1.1133 \mathrm{E}-06$ & $1.3340 \mathrm{E}-06$ \\
\hline 930.0 & $7.5006 \mathrm{E}-07$ & $9.5320 \mathrm{E}-07$ \\
\hline 960.0 & $4.9154 \mathrm{E}-07$ & $8.1150 \mathrm{E}-07$ \\
\hline
\end{tabular}


Table 5.3.3. Concentration Profiles for 3D Transport of the Base Case Transverse to the Plume Centerline located at $x=120$ meters (1400 days).

\begin{tabular}{|c|ccc|}
\hline $\begin{array}{c}\text { Distance } \\
\text { transverse, } \\
\text { y or z }(m)\end{array}$ & $\begin{array}{c}\text { Analytical } \\
\text { (AT123D) }\end{array}$ & $\begin{array}{c}\text { Numerical } \\
\text { (PORFLOW) } \\
\text { (y-axis) }\end{array}$ & $\begin{array}{c}\text { Numerical } \\
\text { (PORFLOW) } \\
\text { (z-axis) }\end{array}$ \\
\hline 0.0 & $1.1293 \mathrm{E}-04$ & $1.2450 \mathrm{E}-04$ & $1.2450 \mathrm{E}-04$ \\
\hline 30.0 & $6.5699 \mathrm{E}-05$ & $6.6050 \mathrm{E}-05$ & $6.6050 \mathrm{E}-05$ \\
\hline 60.0 & $1.8645 \mathrm{E}-05$ & $1.8830 \mathrm{E}-05$ & $1.8830 \mathrm{E}-05$ \\
\hline 90.0 & $4.0297 \mathrm{E}-06$ & $4.2600 \mathrm{E}-06$ & $4.2600 \mathrm{E}-06$ \\
\hline 120.0 & $7.9368 \mathrm{E}-07$ & $8.7710 \mathrm{E}-07$ & $8.7710 \mathrm{E}-07$ \\
\hline 150.0 & $1.5041 \mathrm{E}-07$ & $1.7270 \mathrm{E}-07$ & $1.7270 \mathrm{E}-07$ \\
\hline 180.0 & $2.7710 \mathrm{E}-08$ & $3.3030 \mathrm{E}-08$ & $3.3030 \mathrm{E}-08$ \\
\hline 210.0 & $4.9259 \mathrm{E}-09$ & $6.1340 \mathrm{E}-09$ & $6.1340 \mathrm{E}-09$ \\
\hline 240.0 & $8.3177 \mathrm{E}-10$ & $1.1130 \mathrm{E}-09$ & $1.1130 \mathrm{E}-09$ \\
\hline 270.0 & $1.3098 \mathrm{E}-10$ & $4.9760 \mathrm{E}-10$ & $4.9760 \mathrm{E}-10$ \\
\hline
\end{tabular}

Table 5.3.4. Input Commands for Problem 5.3.

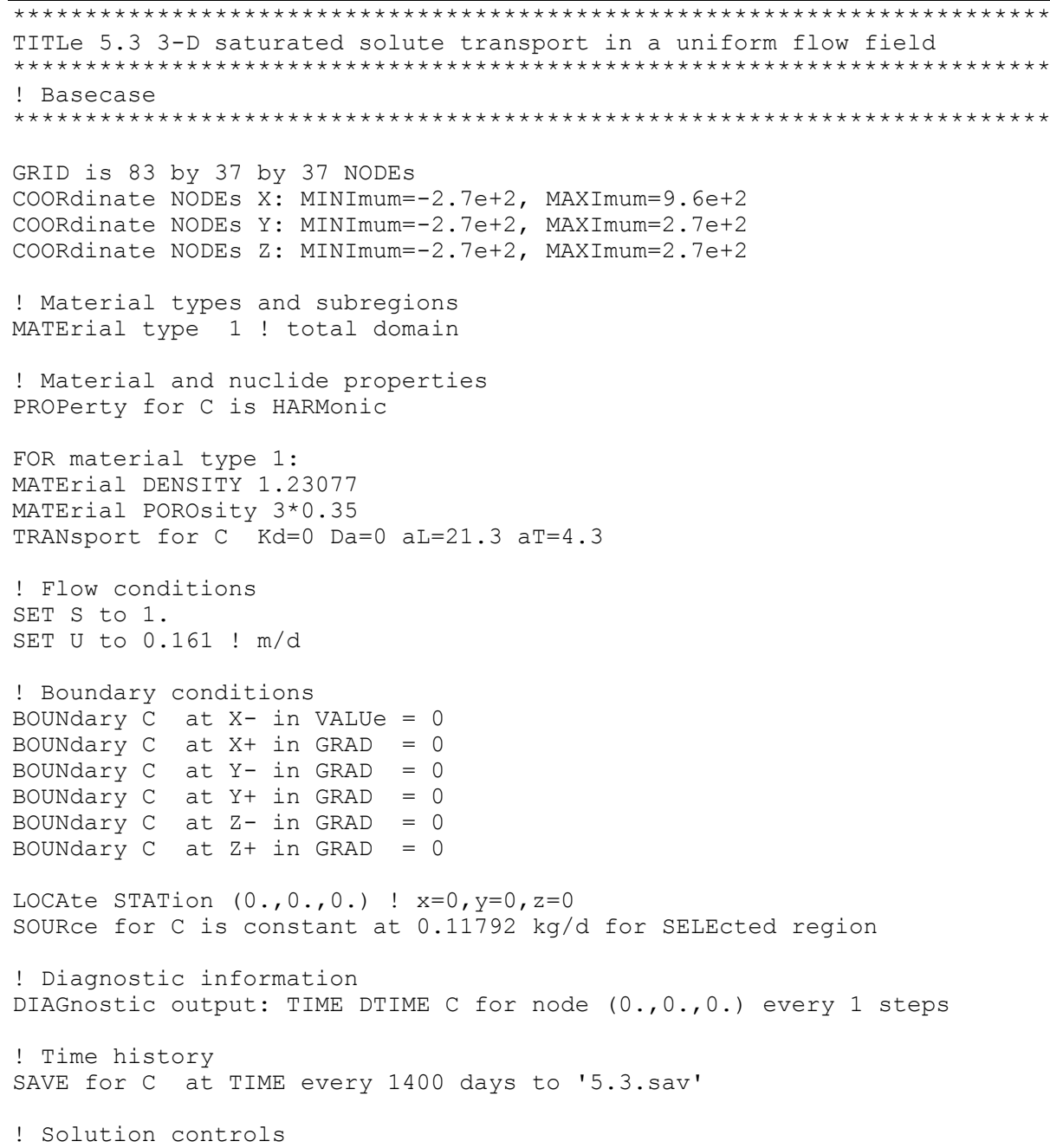


MATRix in $X$ Y $Z$ for $C 3$ sweeps using ADI

!LIMIt for $\mathrm{C} \quad 0.0$

CONVergence for $C$ REFErence LOCAl 1.e-6, $\max$ iterations $=30$

! Solve transient transport

! Time period: 0 to 1400 years

$\mathrm{TIME}=0$.

SOLVe $C$ for 1400 days in steps of 10 days

END

QUIT

\subsection{Slit and Engineered Trench Radon Air Pathway Transport Simulation}

One-dimensional radon transport models were developed using COMSOL and PORFLOW numerical simulators to compute Rn-222 concentration profiles and fluxes from the Silt and Engineered Trenches over a 1000-year period. The Slit and Engineered Trenches are below grade earthen disposal units, are very similar in design, and foot print. The first time period evaluated, which covers the operational and institutional control periods, was extended from 125 to 1000 years for this verification study. The second time period, which covers the post-closure compliance period (125 to 1125 years), was not modeled.

The potential parent radionuclides that can contribute to the formation of $\mathrm{Rn}-222$ are shown in Figure 5.4.1. The diagram illustrates the decay chains that lead to the creation of Rn-222 and the half-lives for each member of the chain. Since the half-life of U-238 is extremely long (4.5 billion years), members of the decay chain above U-238 can be ignored as potential contributors to the formation of Rn-222 during the time of interest. The Th-230 decay chain (Th-230 $\rightarrow$ Ra$226 \rightarrow \mathrm{Rn}-222$ ), which includes three radionuclides, was chosen as the verification candidate.

In the current formulation of the radon air pathway transport model, the following assumptions are utilized:

- Advection for all species is set to zero

- No absorption of species

- No partitioning of radon between gas and liquid phases. Radon is always in the gas phase.

- Molecular diffusion of radon gas only

- Material porosity specified to represent air-filled porosity

- No flux boundary condition specified below the lower waste zone for all species

- Zero concentration boundary condition for radon at the ground surface to maximize flux of radon at the land surface. No flux boundary condition for the remaining species at the land surface. 
- The initial activity of Th-230 is set to $0.125 \mathrm{Ci}$ in each of the lower and upper waste zones.

Incorporating the assumptions above and transforming from concentration to activity, the onedimensional advection-diffusion equation for each species becomes:

$$
\begin{gathered}
\frac{\partial \alpha_{\mathrm{a}}}{\partial \mathrm{t}}=-\lambda_{\mathrm{a}} \alpha_{\mathrm{a}} \\
\frac{\partial \alpha_{\mathrm{b}}}{\partial \mathrm{t}}=\lambda_{\mathrm{b}} \alpha_{\mathrm{a}}-\lambda_{\mathrm{b}} \alpha_{\mathrm{b}} \\
\frac{\partial \alpha_{\mathrm{c}}}{\partial \mathrm{t}}=\mathrm{D}_{\mathrm{xx}}^{\prime} \frac{\partial^{2} \alpha_{\mathrm{c}}}{\partial \mathrm{x}^{2}}+\lambda_{\mathrm{c}} \alpha_{\mathrm{b}}-\lambda_{\mathrm{c}} \alpha_{\mathrm{c}} \\
\mathrm{D}_{\mathrm{xx}}^{\prime}=\theta_{\mathrm{e}} \tau \mathrm{D}_{\mathrm{m}}
\end{gathered}
$$

where

$$
\begin{aligned}
& \text { a, b, c ........... Th-230, Ra-226 and } \mathrm{Rn}-222 \text {, respectively } \\
& \alpha \ldots \ldots \ldots \ldots \ldots . . . . . \text { activity of radionuclide, } \lambda \mathrm{c}
\end{aligned}
$$

PORFLOW numerical simulation and comparison: The COMSOL and PORFLOW material regions are defined as follows: (1) Lower Waste Zone from 0 to $4.16 \mathrm{~m}$; (2) Upper Waste Zone from 4.16 to $4.92 \mathrm{~m}$; (3) Clean Soil from 4.92 to $6.13 \mathrm{~m}$; and (4) Closure Cap from 6.13 to 8.03 $\mathrm{m}$. The PORFLOW 1D mesh consists of 3 nodes in the $\mathrm{x}$-direction and 55 nodes in the $\mathrm{y}$ direction. The material zones and PORFLOW mesh are shown in Figure 5.4.2. The COMSOL 1D finite-element mesh contains 273 nodes and 272 elements. The PORFLOW input commands; geometry specification, zone identification and material specification are shown in Tables 5.4.1 to 5.4.4, respectively.

Figures 5.4.3 and 5.4.4 show a comparison of COMSOL and PORFLOW concentration profiles of Th-230 and Ra-226 at 1000 years, respectively. The agreement is excellent. At 1000 years, the activity of Th-230 is at $99.1 \%$ of the original activity. As seen in Figure 5.4.4, the activity of Ra-226 is increasing from 100 to 500 to 1000 years. The two species are immobile within each waste zone as expected.

The radon concentration profile at 1000 years is shown in Figure 5.4.5 as computed by COMSOL and PORFLOW. The short-lived radon is in secular equilibrium with its long-lived parent radium. The radon peak is slightly lower due to molecular diffusion to the land surface over the past 1000 years. The agreement between COMSOL and PORFLOW is excellent.

Figure 5.4.6 represents the radon dispersive flux at the land surface in $\mathrm{pCi} / \mathrm{m}^{2} / \mathrm{sec}$ over a 1000 year period. The radon flux continues to monotonically grow throughout time because of the 
rising activity of the radium parent. The agreement is excellent between the COMSOL and PORFLOW numerical results. 


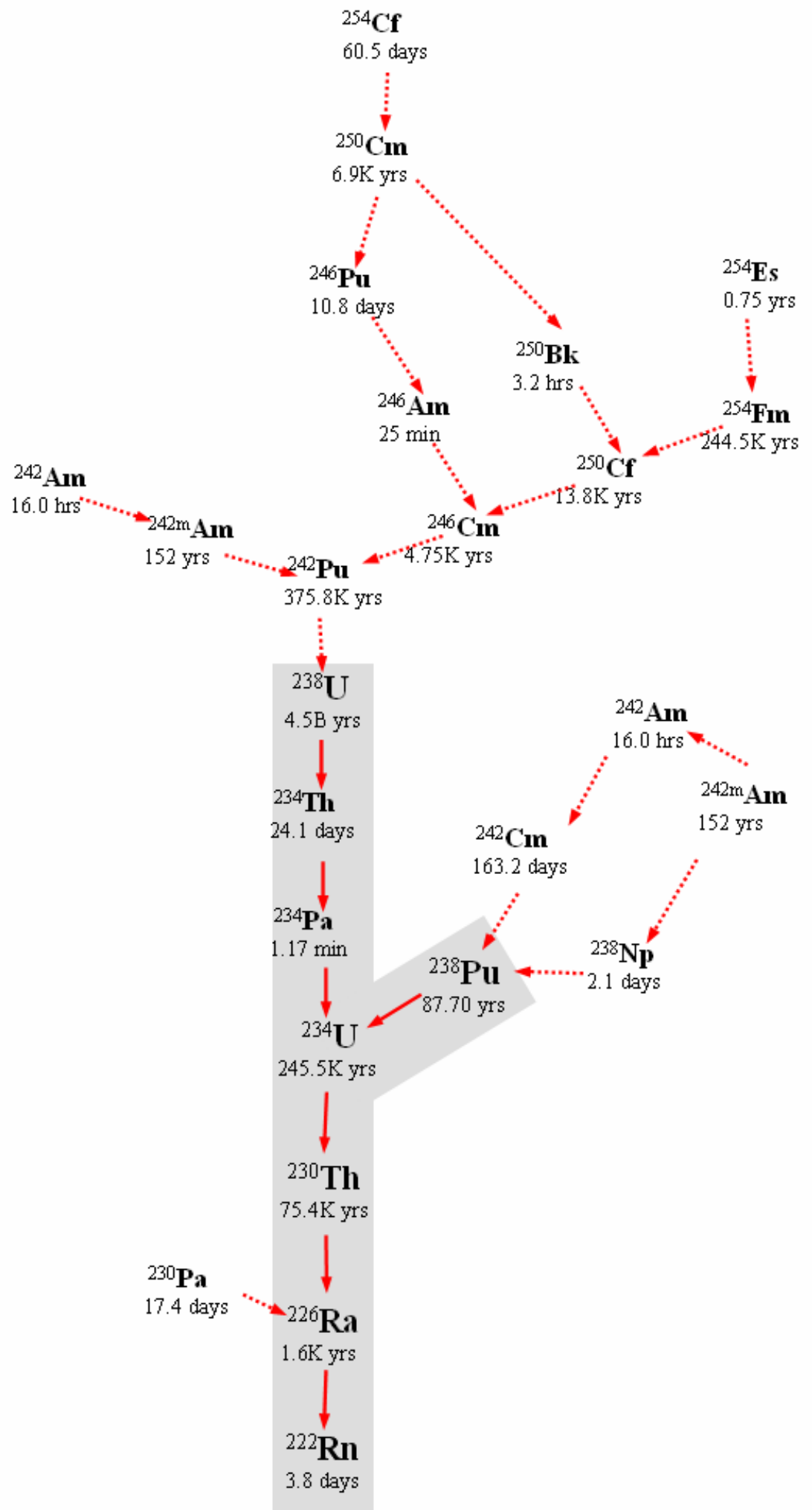

Figure 5.4.1. Radioactive Decay Chains Leading to Rn-222. 


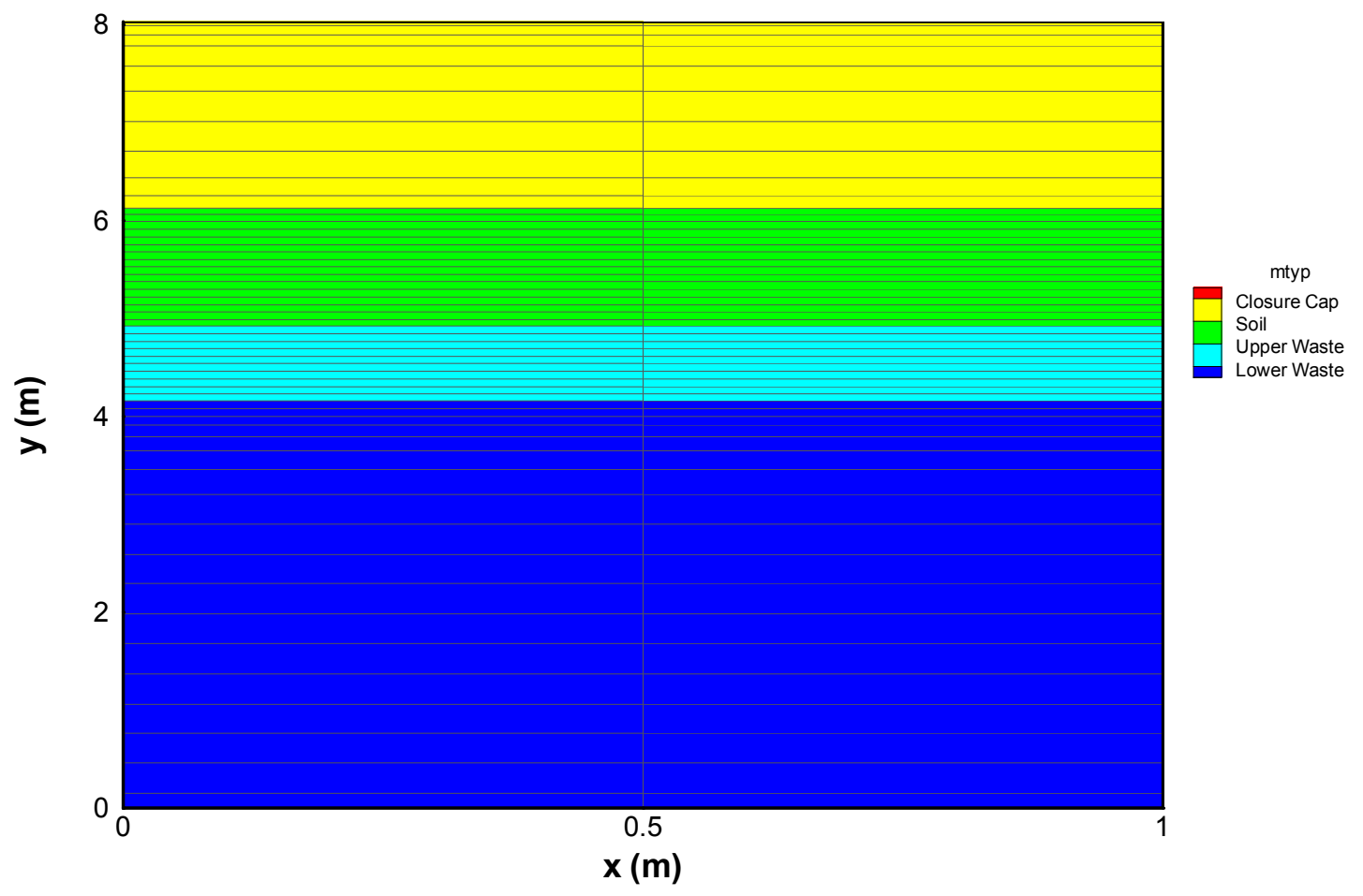

Figure 5.4.2. PORFLOW 1D Conceptual Model and Mesh for Radon Transport in the Slit and Engineered Trenches.

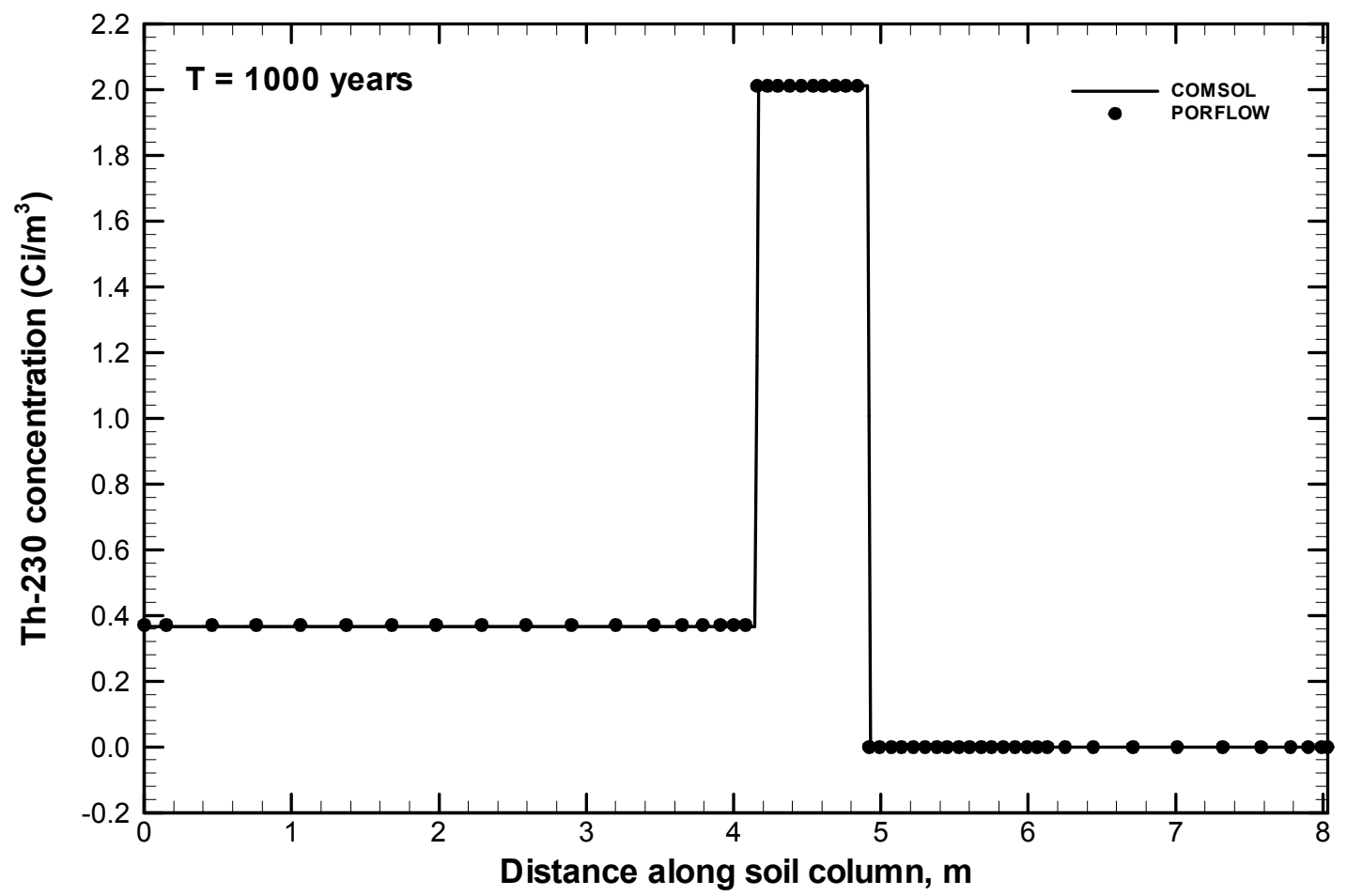

Figure 5.4.3. Comparison of COMSOL and PORFLOW Th-230 Concentration Profiles at 1000 years. 


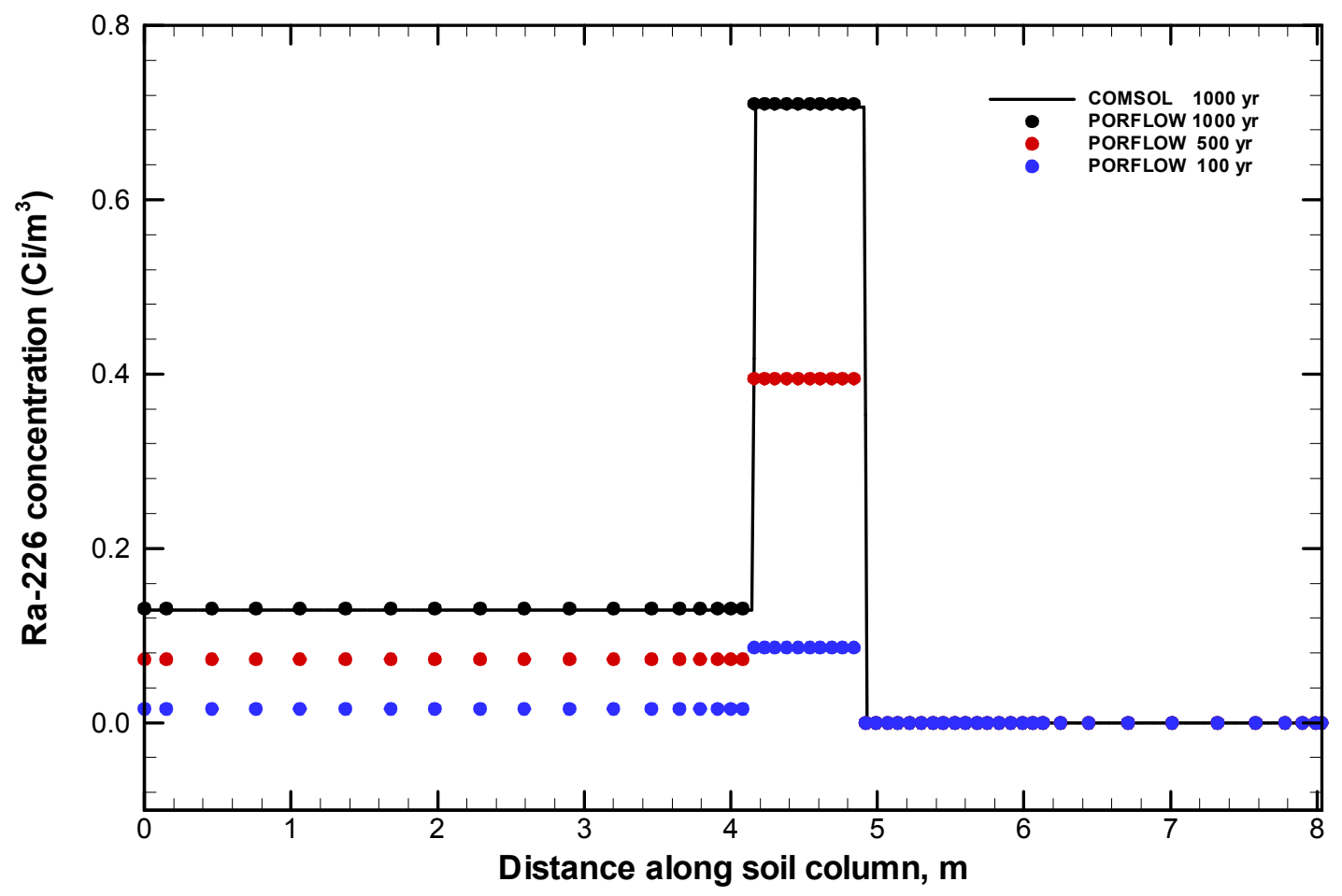

Figure 5.4.4. Comparison of COMSOL and PORFLOW Ra-226 Concentration Profiles.

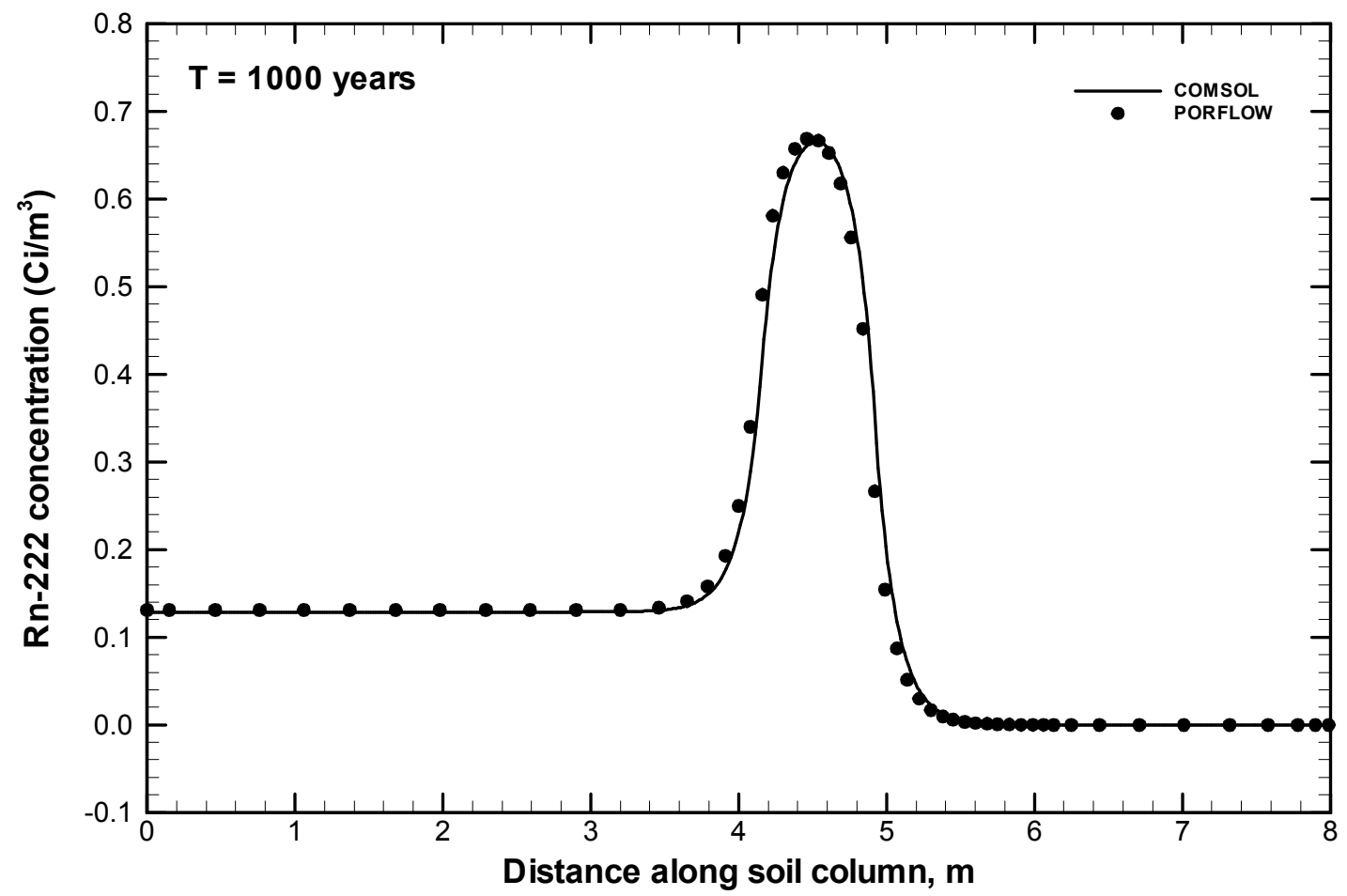

Figure 5.4.5. Comparison of COMSOL and PORFLOW Rn-222 Concentration Profiles at 1000 years. 


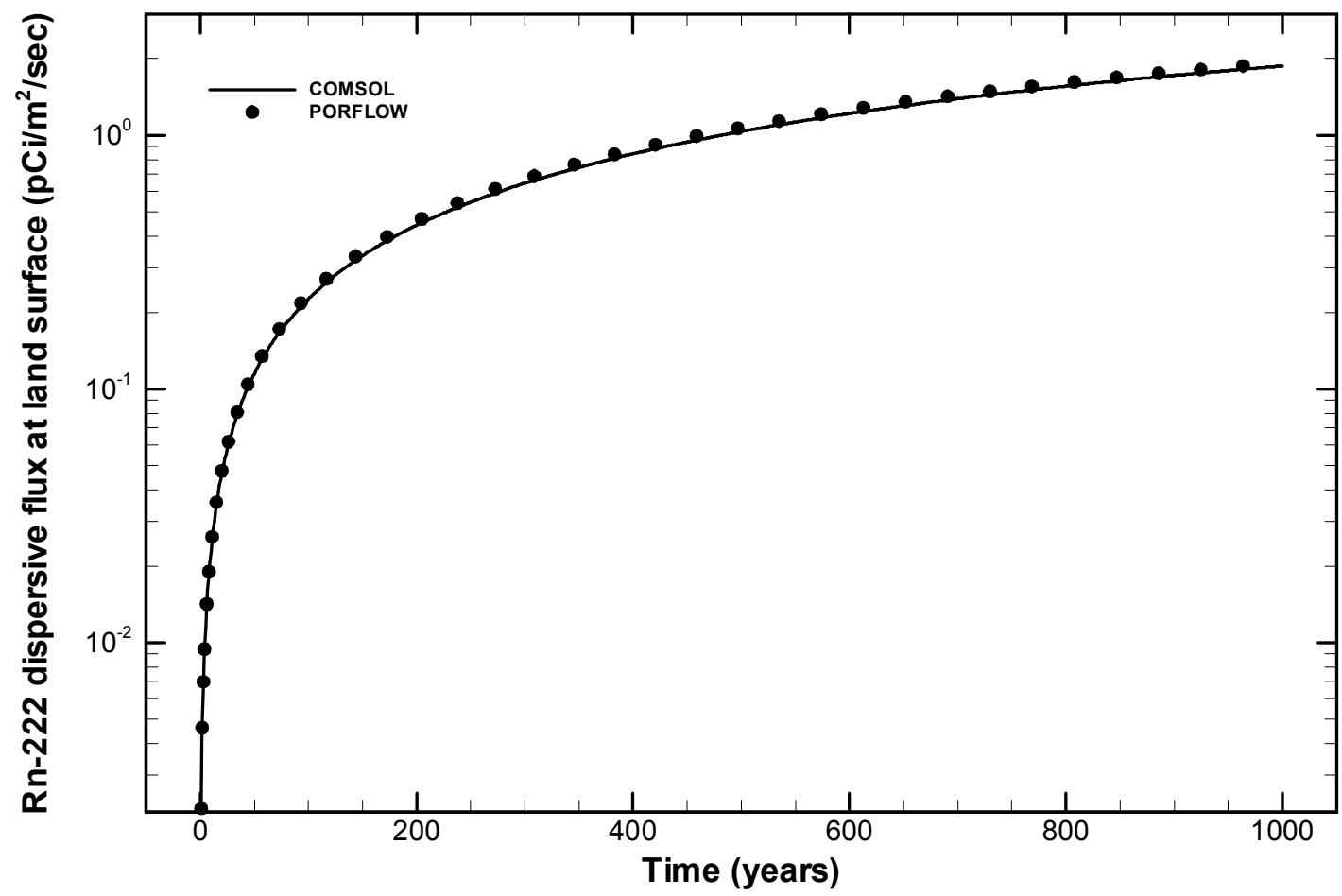

Figure 5.4.6. Comparison of COMSOL and PORFLOW Rn-222 Dispersive Flux at the Land Surface.

Table 5.4.1. Input Commands for Problem 5.4.

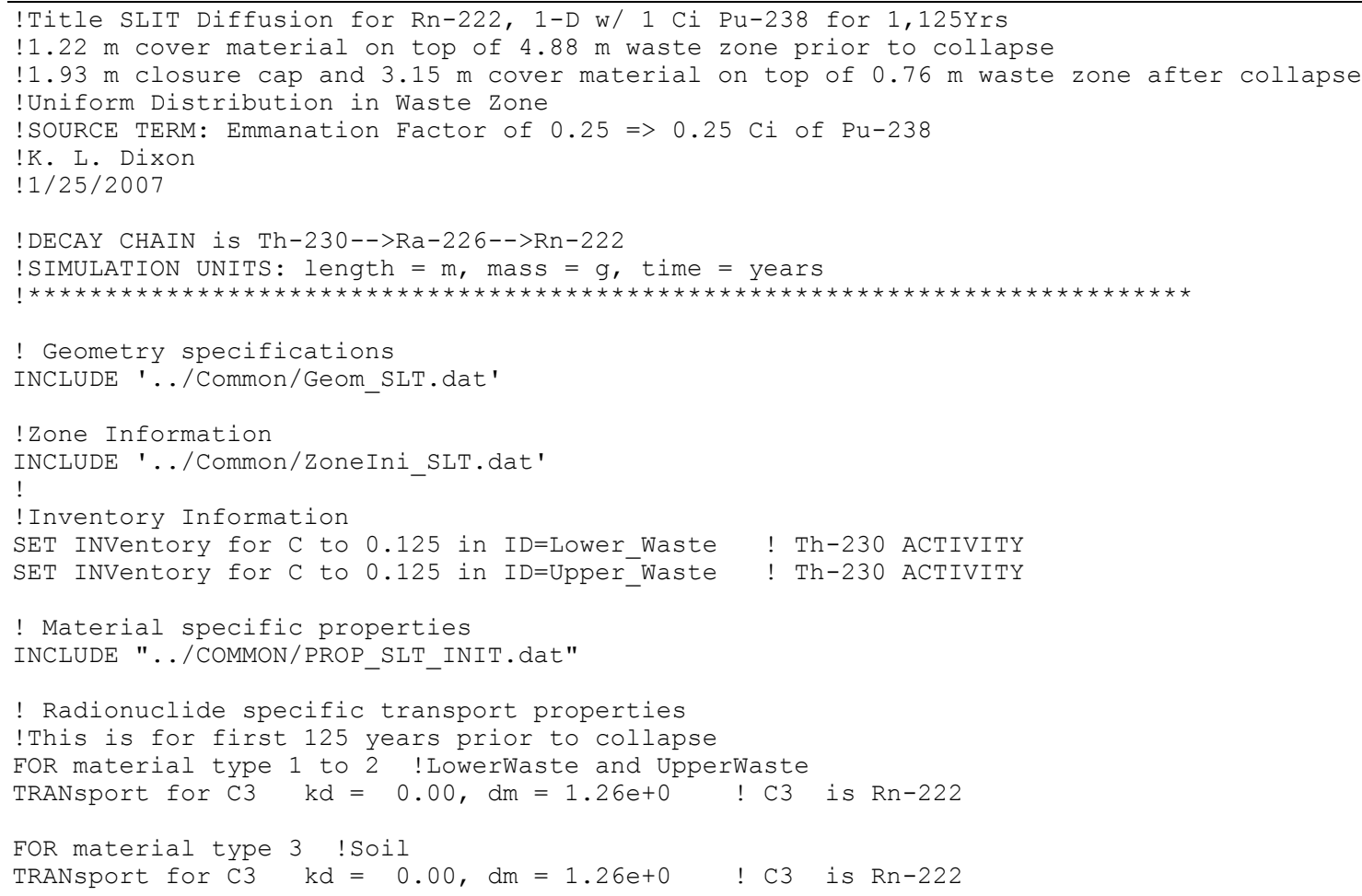


WASHINGTON SAVANNAH RIVER COMPANY

Report: WSRC-STI-2007-00150

Revision (Date): $\quad \operatorname{Rev} 0$ (6/15/2007)

PORFLOW Testing and Verification Document

Page:

146 of 193

FOR material type 4 !Closure Cap

TRANsport for C3 $\mathrm{kd}=0.00, \mathrm{dm}=3.47 \mathrm{e}+2$ ! C3 is Rn-222

BOUN C X- FLUX $=0$.

! Th-230

BOUN C $\quad \mathrm{X}+\quad$ FLUX $=0$

BOUN C Y- $\mathrm{FLUX}=0$.

BOUN C Y + FLUX $=0$

BOUN C2 X- FLUX $=0$.

! $\mathrm{Ra}-226$

BOUN C2 X+ FLUX $=0$

BOUN C2 Y- FLUX $=0$

BOUN C2 $\mathrm{Y}+\mathrm{FLUX}=0$

BOUN C3 $\mathrm{X}-\quad \mathrm{FLUX}=0$.

BOUN C3 X+ FLUX $=0$.

BOUN C3 Y- $\mathrm{FLUX}=0$

BOUN C3 $\mathrm{Y}+\mathrm{VALU}=0$.

! Decay Info

DECAy half LIFE for $C$ is $7.538 e+04$ years

DECAy half LIFE for C2 is $1.600 e+03$ years

! Th-230 from Nuclear Wallet Cards, BNL

DECAy half LIFE for C3 is $1.047 \mathrm{e}-02$ years

Ra-226 from Nuclear Wallet Cards, BNI

REGEnerate C2 from C 4.711E+01 ! C/C2

REGEnerate C3 from C2 1.528E+05! $\mathrm{C} 2 / \mathrm{C} 3$

PROPerty for C C2 C3 is HARMonic ! GEOM mean

MATRIX in $Y$ direction in 3 sweeps using ADI

DIAGnostic node for TIME C C2 C3 at $(2,26)$ every 100 steps

FLUX C for ID=DOMAIN by TIME every 1 years to 'FLUX.out'

FLUX C2 for ID=DOMAIN by TIME every 1 years to 'FLUX.out'

FLUX C3 for ID=DOMAIN by TIME every 1 years to 'FLUX.out'

SAVE C C2 C3 for ID=DOMAIN to 'Th-230.sav' at TIME interval of 100 years

TIME $=0$

CONV C REFE GLOBal resid $=1.0 e-06$, max_iter 10, min_iter 2, F_threshold $=1 . e-5$

CONV C2 REFE GLOBal resid $=1.0 e-06$, max iter 10, min iter 2, F threshold $=1 . e-5$

CONV C3 REFE GLOBal resid $=1.0 e-06, m_{-}$iter 10, min_iter 2, F_threshold $=1 . e-5$

DISAble FLOW

SoLVe 1000 yrs, init 1.e-5, inc 1.005, max 1.

!Pre-collapse

END

Table 5.4.2. Geometry Specification for Problem 5.4.

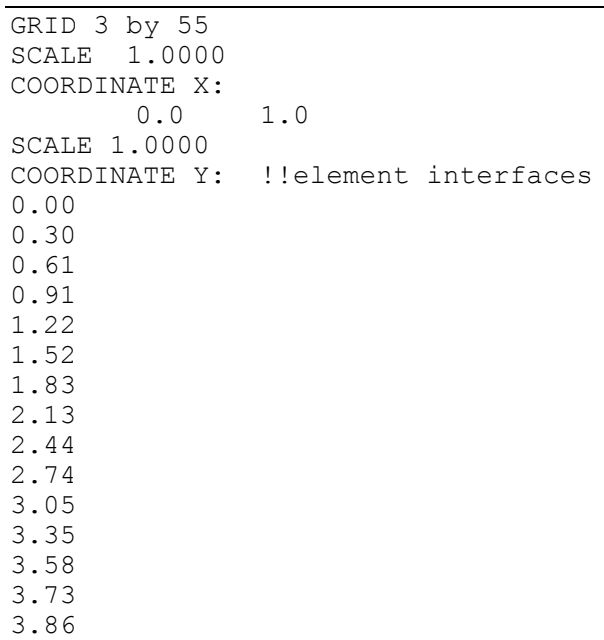


Table 5.4.3. Zone Identification for Problem 5.4.

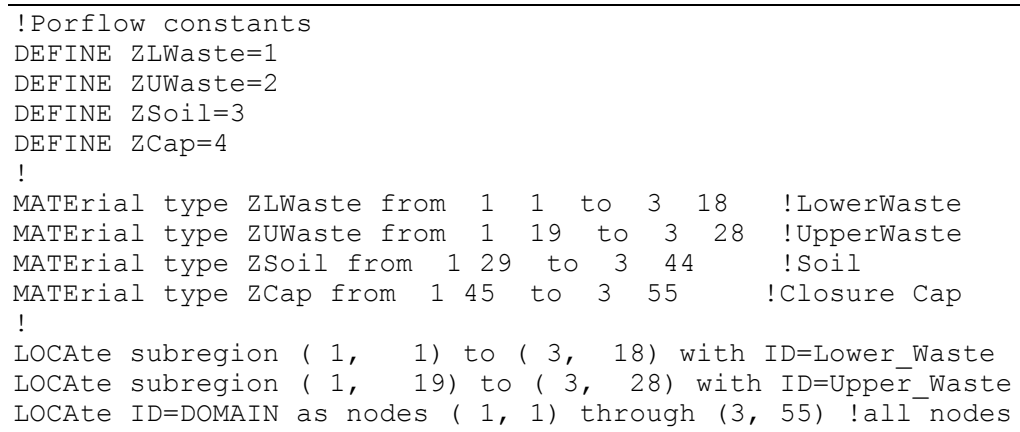

Table 5.4.4. Material Specification for Problem 5.4.

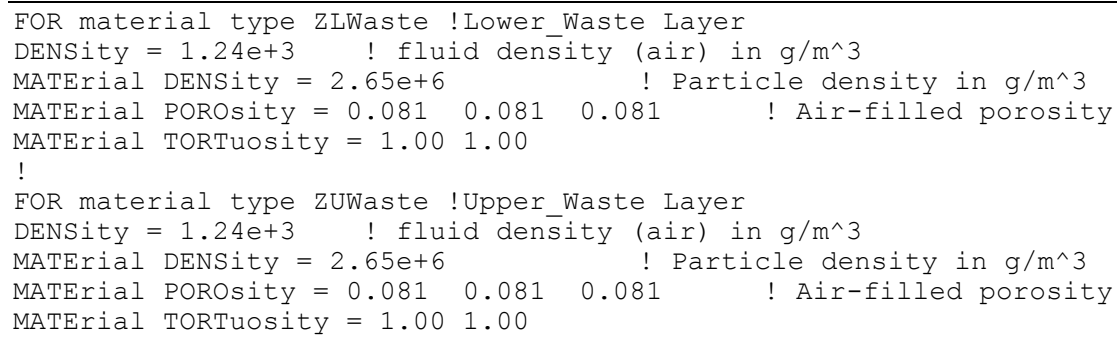


FOR material type ZSoil !Soil Layer

DENSity $=1.24 \mathrm{e}+3 \quad$ ! fluid density $\left(\right.$ air) in $\mathrm{g} / \mathrm{m}^{\wedge} 3$

MATErial DENSity $=2.65 \mathrm{e}+6 \quad$ ! Particle density in $\mathrm{g} / \mathrm{m}^{\wedge} 3$

MATErial POROsity $=0.081 \quad 0.081 \quad 0.081 \quad$ ! Air-filled porosity

MATErial TORTuosity $=1.001 .00$

FOR material type zCap !Closure Cap

DENSity $=1.24 \mathrm{e}+3 \quad$ ! fluid density (air) in $\mathrm{g} / \mathrm{m}^{\wedge} 3$

MATErial DENSity $=2.65 \mathrm{e}+6 \quad$ ! Particle density in $\mathrm{g} / \mathrm{m} \wedge 3$

MATErial POROsity $=1.001 .001 .00 \quad$ ! Air-filled porosity

MATErial TORTuosity $=1.001 .00$

\subsection{Impact of PORFLOW Retardation Model on Variably Saturated Solute Transport.}

The conventional definition of the retardation factor as used by the majority of the groundwater modeling community for a linear adsorption isotherm is shown in Equation 5.1.1 as

$$
\mathrm{R}=1+\rho_{\mathrm{s}}(1-\phi) \mathrm{k}_{\mathrm{d}} / \theta_{\mathrm{e}}
$$

The current conceptual model in PORFLOW for linear sorption assumes that the diffusion in the solid is negligible and that the contact between the fluid and solid is only through the wetted surface (advective or mobile phase fluid). The nonwetted surface in contact with air does not participate in sorption. Therefore, the retardation factor in PORFLOW is computed as

$$
\mathrm{R}=1+\rho_{\mathrm{s}}\left(\mathrm{S}_{\mathrm{w}}-\theta_{\mathrm{e}}\right) \mathrm{k}_{\mathrm{d}} / \theta_{\mathrm{e}}=1+\rho_{\mathrm{s}}(1-\phi) \mathrm{k}_{\mathrm{d}} / \phi
$$

Equations 5.5.1 and 5.5.2 are identical under saturated conditions. For variably saturated transport, Equation 5.5.2 always computes a smaller retardation factor than Equation 5.5.1. The impact is less loading to the solid, higher solute concentrations and higher retarded phasic velocities. Is this a conservative approach? Most likely in every case. Obviously, the further conditions are away from saturation, the greater the impact on solute concentrations and transport travel times. This approach is non-standard and should be replaced by the conventional and accepted form of the retardation factor.

PORFLOW numerical simulation and comparison: A one-dimensional solute transport simulation at a constant saturation and Darcy velocity of 50\% and 4 meters per year, respectively, was executed in COMSOL and PORFLOW. The COMSOL finite-element grid consists of 240 elements and 241 nodes. The PORFLOW grid is shown in Figure 5.5.1 and contains 201 nodes in the $\mathrm{x}$-direction and 3 nodes in the y-direction. The extent of the mesh is 400 meters. The simulation time was 50 years using time steps of 0.01 year. The solute concentration profiles were written every 25 years.

Two species are tracked in the simulation. A decaying parent with a half-live of 25 years and a non-decaying stable daughter. Each species has a distribution coefficient of 0.5 and a longitudinal dispersivity of 4 meters. The material particle density is 2 with a porosity of 0.5 . The bulk density is therefore equal to 1 (water like soil). The retardation factors are computed as 3 and 2 using Equations 5.5.1 and 5.5.2, respectively. The corresponding retarded pore or phasic velocities are 5.333 and 8 meters per year, respectively. 
The dispersive flux for each species is set to zero at the outflow boundary $(+\mathrm{X})$. The inflow boundary condition at $-\mathrm{X}$ is a unit concentration and zero concentration for the parent and daughter, respectively.

Figure 5.5.2 shows a comparison between the COMSOL and PORFLOW parent and daughter solute concentration profiles at 25 and 50 years. The COMSOL variably saturated transport equation uses the standard form of the retardation factor. As the results show, PORFLOW produces higher solute concentrations than COMSOL. The peak in solute concentration for the daughter species has moved further down the soil column due to a higher retarded pore velocity.

The imbedded construct for computing the retardation factor in PORFLOW can be overridden by use of the RETArdation keyword command with appropriate modifiers. The retardation factor was computed as a function of moisture content using the POWEr function

$$
\begin{aligned}
& R=A(B+C)^{D}+E \\
& A=\rho_{s}(1-\phi) k_{d}, B=\theta_{e}, C=0, D=-1, E=1
\end{aligned}
$$

The RETAdation command is issued for each species and subregion. If no subregion is specified, the command is applied to the whole domain.

Figure 5.5.3 shows the same comparison as in Figure 5.5.2 but where PORFLOW now uses the conventional form of the retardation factor. The comparison is excellent. PORFLOW should be modified to use the conventional form as an option. The input commands for this problem is given in Table 5.5.1. 


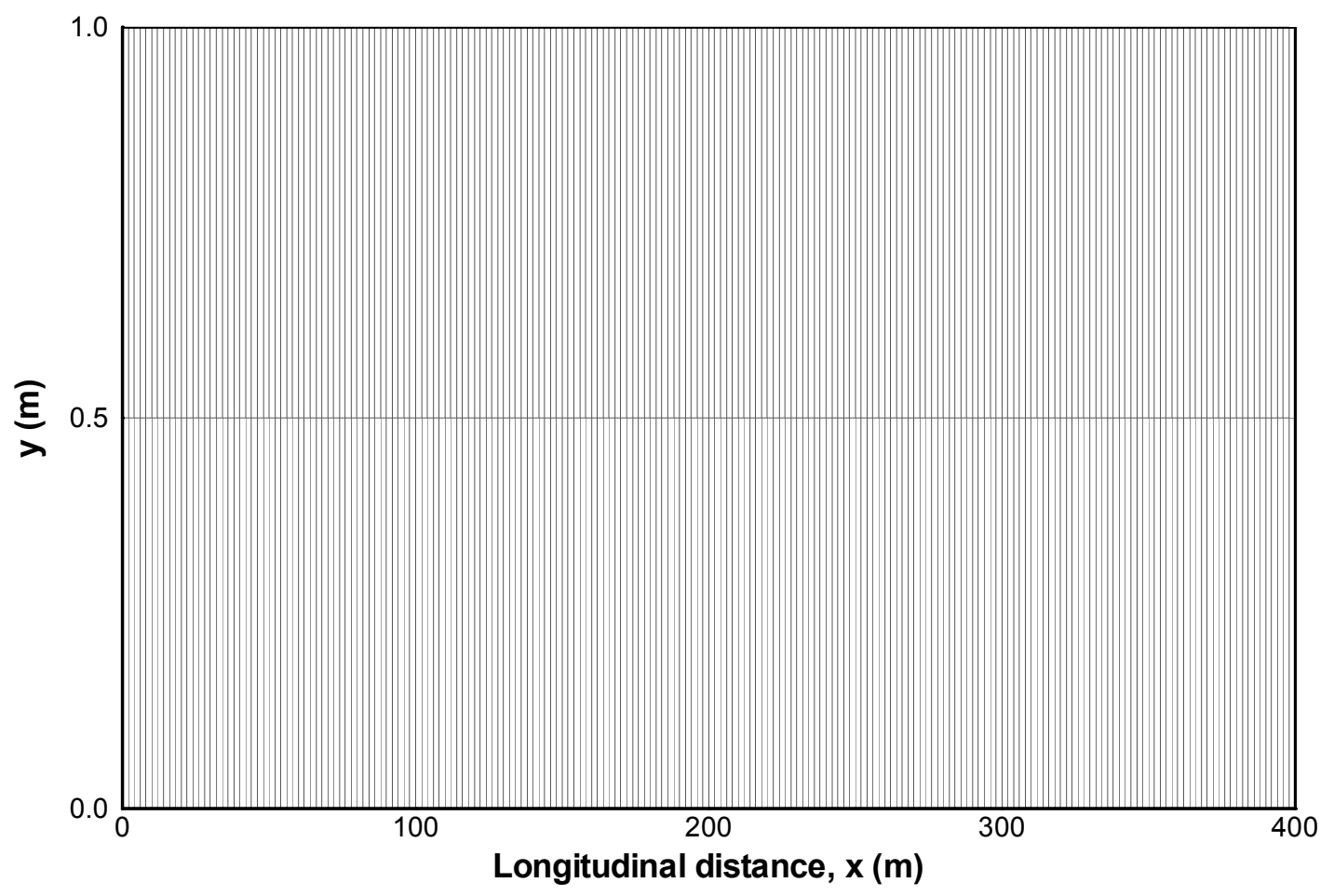

Figure 5.5.1. PORFLOW Grid for 1D Variably Saturated Transport.

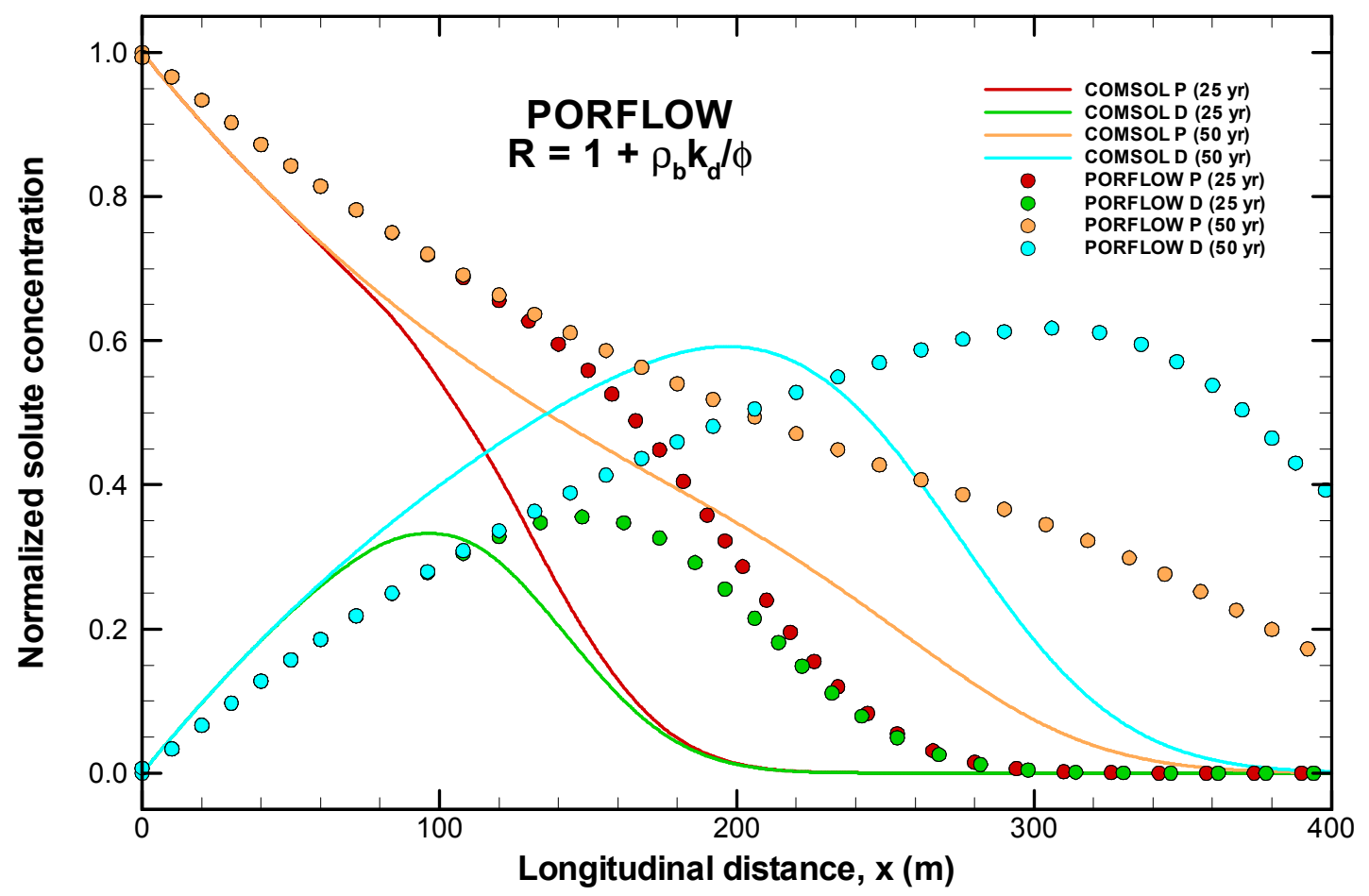

Figure 5.5.2. Comparison of COMSOL and PORFLOW Parent and Daughter Solute Concentration Profiles at 25 and 50 years (PORFLOW using Eq. 5.5.2). 


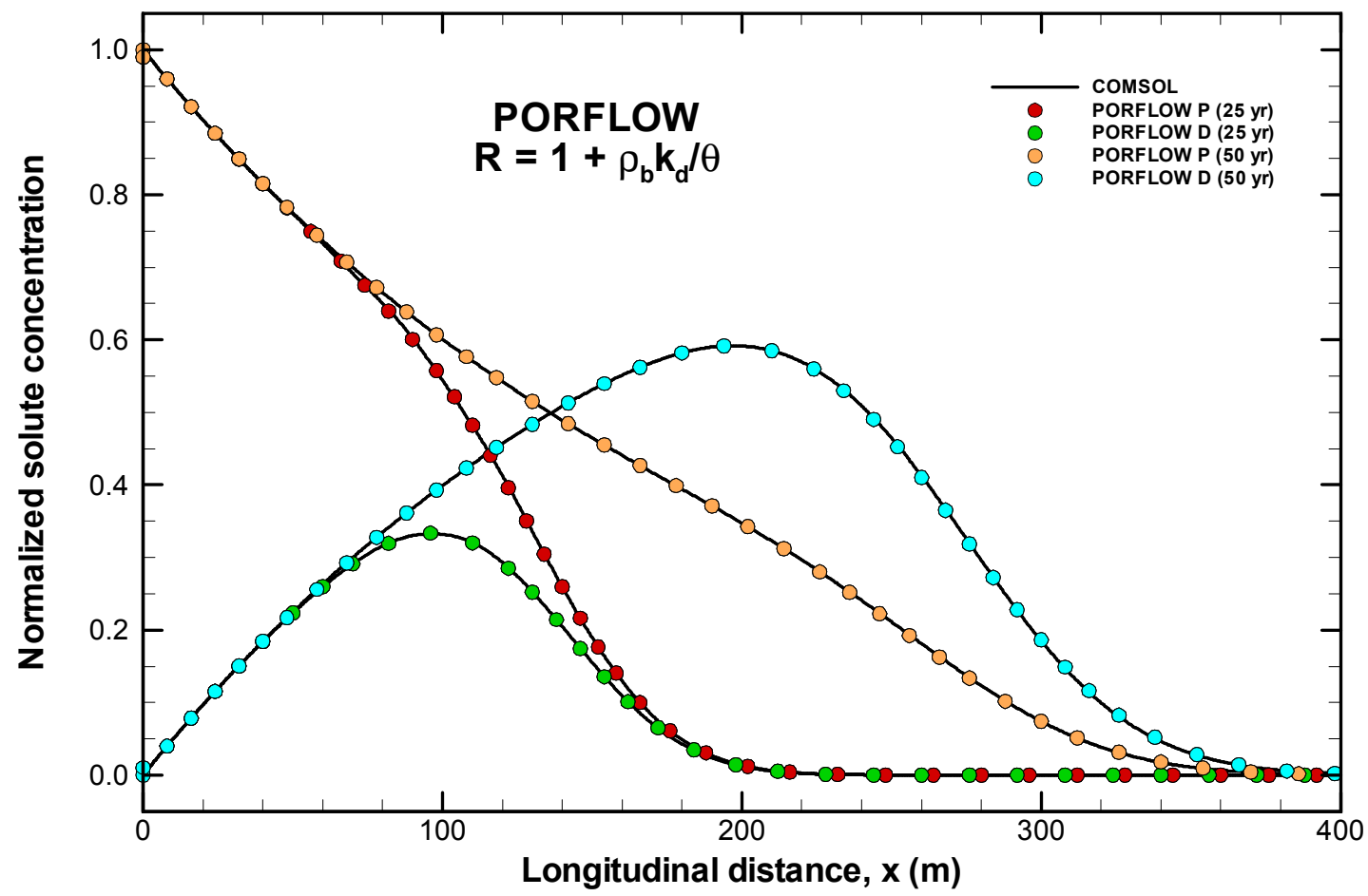

Figure 5.5.3. Comparison of COMSOL and PORFLOW Parent and Daughter Solute Concentration Profiles at 25 and 50 years (PORFLOW using Eq. 5.5.1)

Table 5.5.1. Input Commands for Problem 5.5.

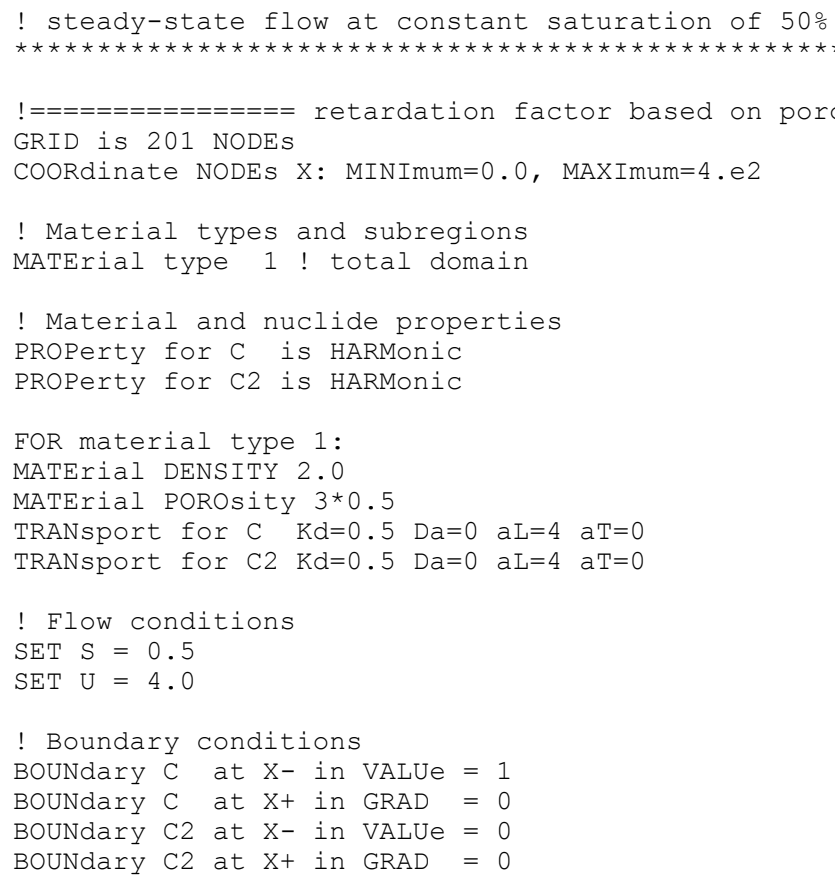


! Decaying species

DECAy half LIFE for $C$ is 25 years

REGEneration of $\mathrm{C} 2$ from $\mathrm{C}$ is 1 .

SET C2 0 .

! Diagnostic information

DIAGnostic output: TIME DTIME C for node 200 every 10 steps

! Time history

SAVE for C C2 at TIME every 25 years to '5.5-phi.sav'

! Solution controls

MATRix in $\mathrm{X}$ for $\mathrm{C}$ C2 33 sweeps using ADI

CONVergence for $C$ REFErence LOCAl 1.e-6, max iterations = 30

CoNVergence for C2 REFErence LOCAl 1.e-6, max iterations = 30

! Solve transient transport

! Time period: 0 to 500 years

$\mathrm{TIME}=0$.

SOLVe C C2 for 50 days in steps of 0.01 days

END

! $==============$ retardation factor based on portity $==============$

! $=============$ retardation factor based on water content $============$

GRID is 201 NODES

COORdinate NODEs $\mathrm{X}$ : MINImum $=0.0$, MAXImum $=4 . e 2$

! Material types and subregions

MATErial type 1 ! total domain

! Material and nuclide properties

PROPerty for $\mathrm{C}$ is HARMonic

PROPerty for C2 is HARMonic

FOR material type 1:

MATErial DENSITY 2.0

MATErial POROsity $3 * 0.5$

TRANsport for $\mathrm{C} \mathrm{Kd}=0.5 \mathrm{Da}=0 \quad \mathrm{aL}=4 \quad \mathrm{aT}=0$

TRANsport for $\mathrm{C} 2 \mathrm{Kd}=0.5 \mathrm{Da}=0 \quad \mathrm{aL}=4 \quad \mathrm{aT}=0$

! Flow conditions

SET $S=0.5$

SET U $=4.0$

SET MOIS as LINEar function: $0 .+(0.5)(\mathrm{S})$

! Boundary conditions

Boundary $\mathrm{C}$ at $\mathrm{X}-$ in VALUe $=1$

BOUNdary $\mathrm{C}$ at $\mathrm{X}+$ in GRAD $=0$

Boundary $\mathrm{C} 2$ at $\mathrm{X}$ - in VALUe $=0$

BOUNdary $\mathrm{C} 2$ at $\mathrm{X}+$ in GRAD $=0$

! Decaying species

DECAy half LIFE for $C$ is 25 years

REGEneration of $\mathrm{C} 2$ from $\mathrm{C}$ is 1 .

SET C2 0 .

!Compute retardation factor based on water content

RETArdation for $C$ as a POWEr function: $(0.5) *(\operatorname{MOIS}+0)^{\wedge}(-1)+(1) ! \mathrm{Kd}=0.5$

RETArdation for C2 as a POWEr function: $(0.5) *(\operatorname{MOIS}+0)^{\wedge}(-1)+(1) ! \mathrm{Kd}=0.5$

! Diagnostic information

DIAGnostic output: TIME DTIME C for node 200 every 10 steps

! Time history

SAVE for C C2 at TIME every 25 years to '5.5-wC.sav'

! Solution controls 
MATRix in $\mathrm{X}$ for $\mathrm{C} C 2 \quad 33$ sweeps using ADI

CONVergence for $C$ REFErence LOCAl 1.e-6, $\max$ iterations $=30$

CONVergence for C2 REFErence LOCAl 1.e-6, max iterations = 30

! Solve transient transport

! Time period: 0 to 500 years

TIME $=0$.

SOLVe C C2 for 50 days in steps of 0.01 days

END

$!=============$ retardation factor based on water content

QUIT

\section{Group 3: Numerical Dispersion}

The impact of numerical and mechanical dispersion in PORFLOW on peak solute concentrations is addressed in this section with regard to mesh spacing in a one-dimensional pulsed soil column. Numerical dispersion in two and three dimensions is not addressed here but will be addressed in a subsequent study. The node limitation of 128,000 in PORFLOW prohibits the investigation of numerical dispersion in a three-dimensional aquifer model.

Numerical dispersion in one dimension can be minimized by not allowing the solute concentration to advect more than a cell length within a time step. The Courant condition for advective transport is

$$
(\delta \mathrm{t})_{\mathrm{A}} \leq \frac{\delta \mathrm{x}}{\mathrm{u}_{\mathrm{x}}^{\prime}}
$$

where $(\delta \mathrm{t})_{\mathrm{A}}$ is the time step for advective transport, $\delta \mathrm{x}$ is the cell length and $\mathrm{u}_{\mathrm{x}}^{\prime}$ is the retarded phasic velocity in the $\mathrm{x}$-direction. Numerical oscillations in the solute concentration due to diffusion and dispersion can be eliminated by invoking the Von Neumann criterion for dispersive transport as

$$
(\delta \mathrm{t})_{\mathrm{D}} \leq \frac{(\delta \mathrm{x})^{2}}{3 \mathrm{D}_{\mathrm{xx}}^{\prime}}
$$

where $(\delta t)_{D}$ is the time step for dispersive and diffusive transport and $D_{x x}^{\prime}$ is the retarded longitudinal dispersion coefficient. Numerical dispersion can be negligible when $\delta x \leq \alpha_{L}$, because mechanical dispersion is equally or more important than advective transport. For a refined mesh, the time step for dispersive transport may become more restrictive than the time step for advective transport, due to the quadratic dependence on mesh size in Equation 6.2.

The current engineering judgment for modeling groundwater transport at SRS is not to invoke mechanical dispersion in PORFLOW, only advective and molecular transport. For coarser grids in the aquifer, the idea is that numerical dispersion will be on par with mechanical dispersion based on reasonable values for the longitudinal and transverse dispersivities. This is all conjecture. In 
the next section, we will demonstrate the impact of numerical and mechanical dispersion on the peak concentrations of a pulsed one-dimensional soil column.

\subsection{Numerical and Mechanical Dispersion in a One-Dimensional Saturated Soil Column.}

PORFLOW numerical simulation and comparison: A series of one-dimensional solute transport simulations under saturated conditions and 10 meters per year Darcy velocity were executed in PORFLOW. Ten PORFLOW grids were generated with equally sized mesh spacing ranging from 0.1 to 60 meters. The extent of the PORFLOW grid was from -200 meters to 800 meters. There were adjustments made to the extent of the grid to accommodate a source node at $\mathrm{x}=0$. The number of nodes range from 10001 to 19 for mesh spacing of 0.1 and 60 meters, respectively.

An inflow boundary condition of zero concentration was specified at $-X$ and a dispersive flux of zero was specified at $+X$.

A conservative species was injected at $\mathrm{x}=0$ with a source strength of $10 \mathrm{~kg} / \mathrm{yr} / \mathrm{m}$ for a duration of 1 year and a time step of 0.001 year. This results in a pulse of unit concentration traveling at a phasic velocity of 40 meters per year. The width of the pulse is 40 meters. The transport simulations were executed for an additional 4.5 years at a time step of 0.001 year. The centerline of the pulse arrives at $x=200$ meters in 5.5 years.

Figure 6.1.1 shows the normalized solute concentration profiles at 5.5 years for mesh spacing ranging for 0.1 to 60 meters. During these simulations, mechanical dispersion and molecular diffusion are set to zero. At a mesh spacing of 0.1 meters, PORFLOW matches the peak concentration of the analytical pulse profile. As the mesh gets coarser, the resulting spread of the pulse is due to numerical dispersion. With increasing mesh spacing, PORFLOW continues to deviate more from the analytical pulse as shown in Figure 6.1.2. Next, we turn on mechanical dispersion to see the impact on the solute concentration profiles.

The centerline of the pulse travels 200 meters in 5.5 years. A rule-of-thumb longitudinal dispersivity equal to one-tenth the travel distance was chosen as 20 meters. Figure 6.1.3 represents the same conditions shown in Figure 6.1.1 except with mechanical dispersion. The peak concentration has dropped from 1 to 0.175 . Below a mesh spacing of 20 meters, PORFLOW has excellent agreement with the analytical solution. Numerical dispersion appears to become significant above a mesh spacing of 40 meters.

The input commands for PORFLOW are given in Tables 6.1.1 and 6.1.2.

Fine mesh resolution is needed to capture the peak solute concentration in PORFLOW if no mechanical dispersion is present. If mechanical dispersion is used in PORFLOW, the modeler needs to determine the appropriate mesh spacing and time stepping to mitigate the impact of numerical dispersion especially in multi-dimensional transport. 


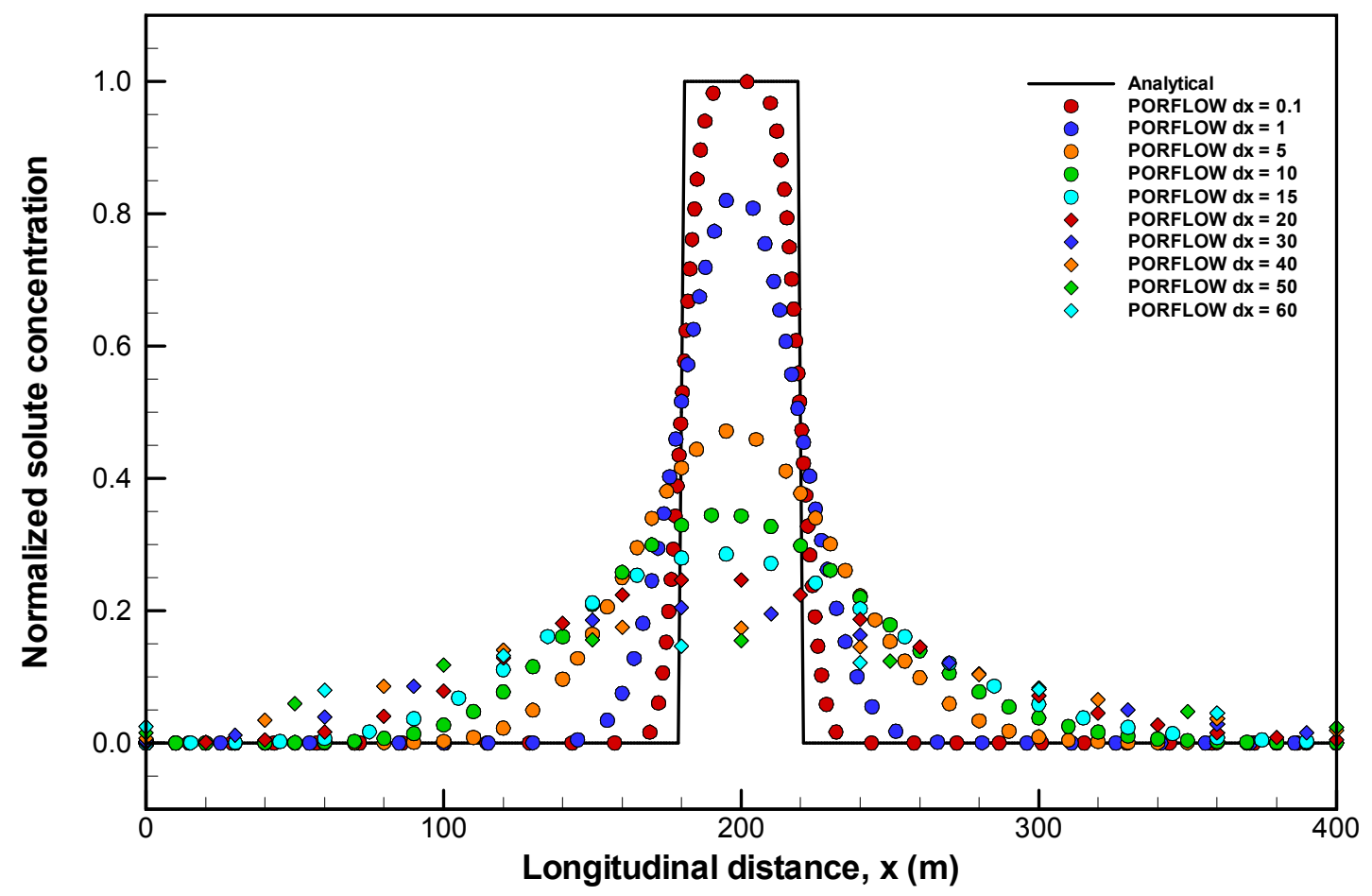

Figure 6.1.1. Normalized Solute Concentration Profiles at 5.5 Years for Variable Mesh Spacing in PORFLOW with Numerical Dispersion.

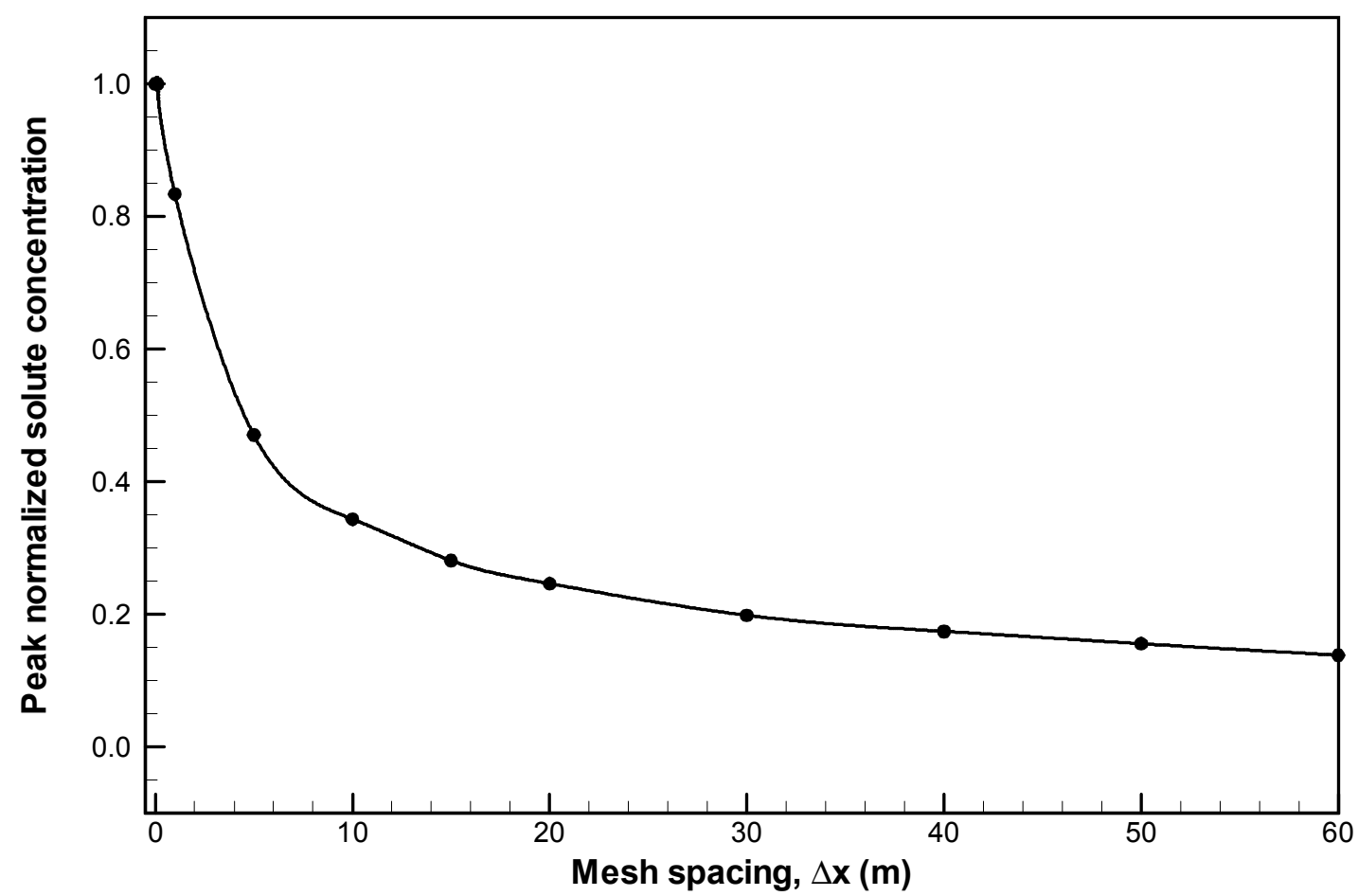

Figure 6.1.2. Peak Normalized Solute Concentration as a Function of Mesh Spacing in PORFLOW at 5.5 Years with Numerical Dispersion. 


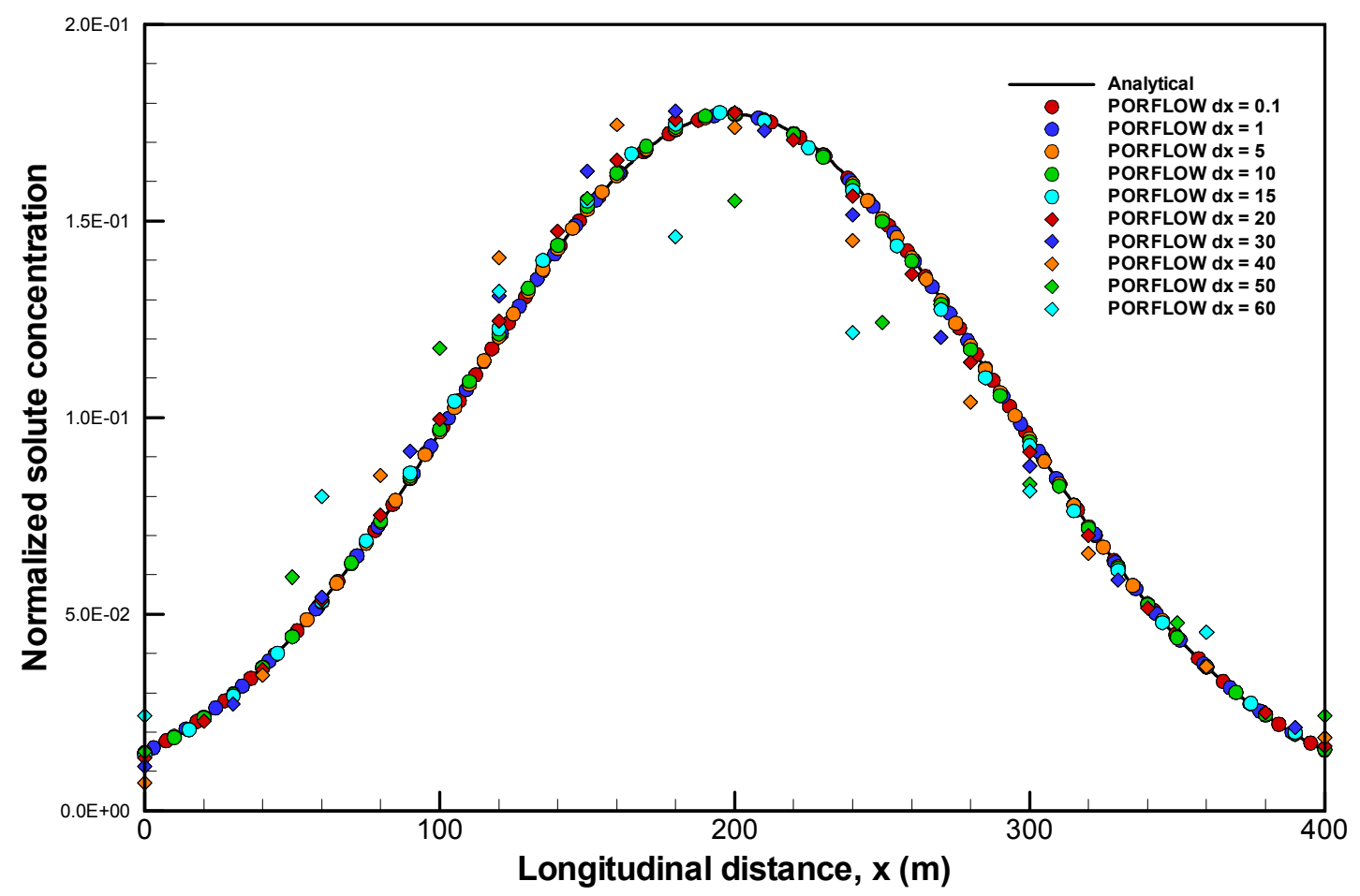

Figure 6.1.3. Normalized Solute Concentration Profiles at 5.5 Years for Variable Mesh Spacing in PORFLOW with Mechanical Dispersion.

Table 6.1.1. Input Commands for Problem 6.1 (Numerical Dispersion).

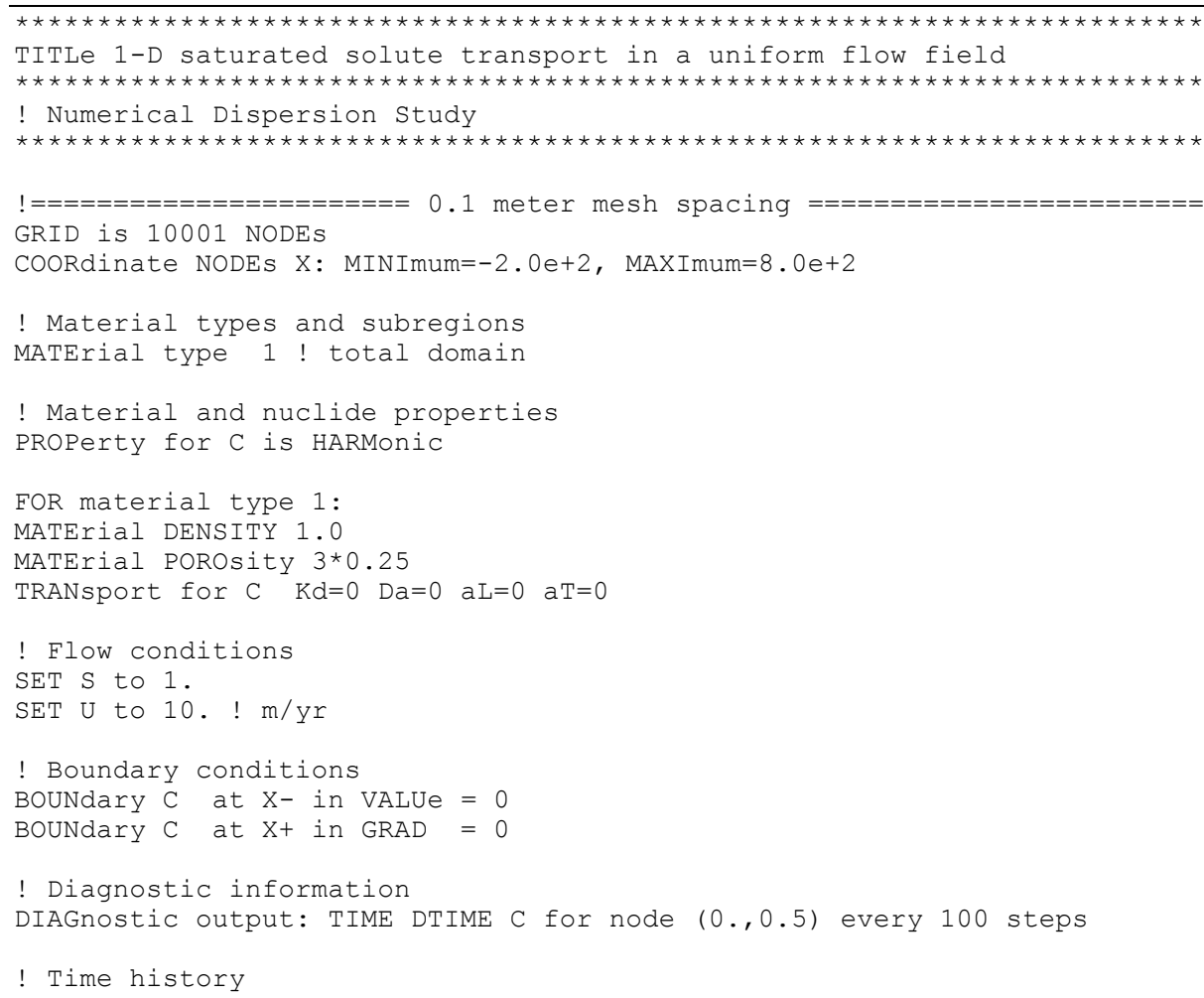


SAVE for $C$ at TIME every 5.5 years to ' $6.1-\mathrm{A}-\mathrm{L} 01 . \mathrm{Sav}$ '

! Solution controls

MATRix in $X$ for $C 3$ sweeps using ADI

LIMIt for $\mathrm{C} 0.0$

CONVergence for C REFErence LOCAl $1 . e-6$, $\max$ iterations $=30$

! Solve transient transport

! Time period: 0 to 5.5 years

TIME $=0$

LOCAte STATion $(0,0.5) \quad ! \quad \mathrm{x}=0, \mathrm{y}=0$

SOURce for $\mathrm{C}$ is constant at $10 \mathrm{~kg} / \mathrm{y} / \mathrm{m}$ for SELEcted region

SOLVe $C$ for 1 years in steps of 0.001 years

SOURce OFF for $\mathrm{C}$ for most recently SELEcted region

SOLVe $\mathrm{C}$ for 4.5 years in steps of 0.001 years

END

! $=======================0.1$ meter Mesh spacing $========================$

! $=======================1.0$ meter mesh $\operatorname{spaCing}========================$

GRID is 1001 NODES

COORdinate NODES $\mathrm{X}$ : MINImum $=-2.0 e+2$, MAXImum $=8.0 e+2$

! Material types and subregions

MATErial type 1 ! total domain

! Material and nuclide properties

PROPerty for $\mathrm{C}$ is HARMonic

FOR material type 1 :

MATErial DENSITY 1.0

MATErial POROsity $3 * 0.25$

TRANsport for $\mathrm{C} \quad \mathrm{Kd}=0$ Da=0 aL=0 aT=0

! Flow conditions

SET S to 1 .

SET U to 10. ! m/yr

! Boundary conditions

Boundary $\mathrm{C}$ at $\mathrm{X}-$ in $\mathrm{VALUe}=0$

BOUNdary $\mathrm{C}$ at $\mathrm{X}+$ in GRAD $=0$

! Diagnostic information

DIAGnostic output: TIME DTIME C for node $(0 ., 0.5)$ every 100 steps

! Time history

SAVE for C at TIME every 5.5 years to ' $6.1-A-L 02 . s a v '$

! Solution controls

MATRix in $X$ for $C 3$ sweeps using ADI

LIMIt for C 0.0

CONVergence for C REFErence LOCAl 1.e-6, max iterations = 30

! Solve transient transport

! Time period: 0 to 5.5 years

TIME $=0$.

LOCAte STATion $(0,0.5) \quad ! \quad \mathrm{x}=0, \mathrm{y}=0$

SOURCe for $\mathrm{C}$ is constant at $10 . \mathrm{kg} / \mathrm{y} / \mathrm{m}$ for SELEcted region

SOLVe $C$ for 1 years in steps of 0.001 years

SOURce OFF for $C$ for most recently SELEcted region

SOLVe C for 4.5 years in steps of 0.001 years

END

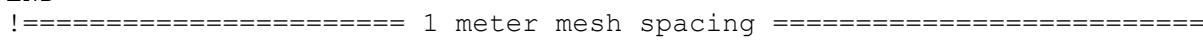

$!======================05$ meter mesh spacing

GRID is 201 NODES

COORdinate NODEs $\mathrm{X}$ : MINImum $=-2.0 e+2, \operatorname{MAXImum}=8.0 e+2$ 
! Material types and subregions MATErial type 1 ! total domain

! Material and nuclide properties

PROPerty for $\mathrm{C}$ is HARMonic

FOR material type 1 :

MATErial DENSITY 1.0

MATErial POROsity $3 * 0.25$

TRANsport for $\mathrm{C} \quad \mathrm{Kd}=0 \quad \mathrm{Da}=0 \quad \mathrm{aL}=0 \quad \mathrm{aT}=0$

! Flow conditions

SET $S$ to 1

SET U to 10. ! $\mathrm{m} / \mathrm{yr}$

! Boundary conditions

Boundary $\mathrm{C}$ at $\mathrm{X}$ - in VALUe $=0$

BOUNdary $\mathrm{C}$ at $\mathrm{X}+$ in GRAD $=0$

! Diagnostic information

DIAGnostic output: TIME DTIME C for node $(0,0.5)$ every 100 steps

! Time history

SAVE for C at TIME every 5.5 years to '6.1-A-L03.sav'

! Solution controls

MATRix in $X$ for $C 3$ sweeps using ADI

LIMIt for $\mathrm{C} 0.0$

CONVergence for C REFErence LOCAl 1.e-6, max iterations $=30$

! Solve transient transport

! Time period: 0 to 5.5 years

$\mathrm{TIME}=0$.

LOCAte STATion $(0,0.5) \quad ! \mathrm{x}=0, \mathrm{y}=0$

SOURce for $\mathrm{C}$ is constant at $10 \mathrm{~kg} / \mathrm{y} / \mathrm{m}$ for SELEcted region

SOLVe C for 1 years in steps of 0.001 years

SOURCe OFF for $C$ for most recently SELEcted region

Solve $\mathrm{C}$ for 4.5 years in steps of 0.001 years

END

! $=====================5$ meter mesh $\operatorname{spacing}=======================$

! $====================10$ meter mesh $\operatorname{spacing}======================$

GRID is 101 NODES

COORdinate NODEs X: MINImum $=-2.0 e+2$, MAXImum $=8.0 e+2$

! Material types and subregions

MATErial type 1 ! total domain

! Material and nuclide properties

PROPerty for $\mathrm{C}$ is HARMonic

FOR material type 1:

MATErial DENSITY 1.0

MATErial POROsity $3 * 0.25$

TRANsport for $\mathrm{C} K d=0$ Da=0 $\mathrm{aL}=0 \quad \mathrm{TT}=0$

! Flow conditions

SET S to 1.

SET U to 10. ! m/yr

! Boundary conditions

Boundary $\mathrm{C}$ at $\mathrm{X}$ - in VALUe $=0$

BOUNdary $\mathrm{C}$ at $\mathrm{X}+$ in GRAD $=0$

! Diagnostic information

DIAGnostic output: TIME DTIME C for node $(0 ., 0.5)$ every 100 steps

! Time history

SAVE for C at TIME every 5.5 years to '6.1-A-L04.sav' 
! Solution controls

MATRix in $X$ for $C 3$ sweeps using ADI

LIMIt for $\mathrm{C} 0.0$

CONVergence for $C$ REFErence LOCAl 1.e-6, max iterations = 30

! Solve transient transport

! Time period: 0 to 5.5 years

$\mathrm{TIME}=0$.

LoCAte STATion $(0,0.5) \quad ! \mathrm{x}=0, \mathrm{y}=0$

SOURce for $\mathrm{C}$ is constant at $10 \mathrm{~kg} / \mathrm{y} / \mathrm{m}$ for sELEcted region

Solve C for 1 years in steps of 0.001 years

SOURce OFF for $C$ for most recently SELEcted region

SOLVe $\mathrm{C}$ for 4.5 years in steps of 0.001 years

END

$!====================10$ meter mesh spacing

! $====================15$ meter mesh $\operatorname{spacing}=====================$

GRID is 69 NODES

COORdinate NODEs X: MINImum $=-2.1 e+2$, MAXImum=8.1e+2

! Material types and subregions

MATErial type 1 ! total domain

! Material and nuclide properties

PROPerty for $\mathrm{C}$ is HARMonic

FOR material type 1 :

MATErial DENSITY 1.0

MATErial POROsity $3 * 0.25$

TRANsport for $\mathrm{C} \mathrm{Kd}=0$ Da=0 $\mathrm{aL}=0 \quad \mathrm{aT}=0$

! Flow conditions

SET S to 1 .

SET U to 10. ! $\mathrm{m} / \mathrm{yr}$

! Boundary conditions

Boundary $\mathrm{C}$ at $\mathrm{X}$ - in VALUe $=0$

BOUNdary $\mathrm{C}$ at $\mathrm{X}+$ in $\mathrm{GRAD}=0$

! Diagnostic information

DIAGnostic output: TIME DTIME C for node (0.,0.5) every 100 steps

! Time history

SAVE for $C$ at TIME every 5.5 years to ' $6.1-A-L 05$. sav' $^{\prime}$

! Solution controls

MATRix in $X$ for $C 3$ sweeps using ADI

LIMIt for $\mathrm{C} 0.0$

CONVergence for $C$ REFErence LOCAl 1.e-6, max iterations $=30$

! Solve transient transport

! Time period: 0 to 5.5 years

$\mathrm{TIME}=0$.

LOCAte STATion $(0,0.5) \quad ! \mathrm{x}=0, \mathrm{y}=0$

SOURce for $\mathrm{C}$ is constant at $10 \mathrm{~kg} / \mathrm{y} / \mathrm{m}$ for SELEcted region

SOLVe $\mathrm{C}$ for 1 years in steps of 0.001 years

SOURCe OFF for $C$ for most recently SELEcted region

SoLVe C for 4.5 years in steps of 0.001 years

END

! $=====================15$ meter $\operatorname{mesh}$ spacing $======================$

! $======================20$ meter mesh $\mathrm{spaCing}=======================$

GRID is 51 NODES

COORdinate NODEs X: MINImum $=-2 . e+2$, MAXImum $=8 . e+2$

! Material types and subregions

MATErial type 1 ! total domain 


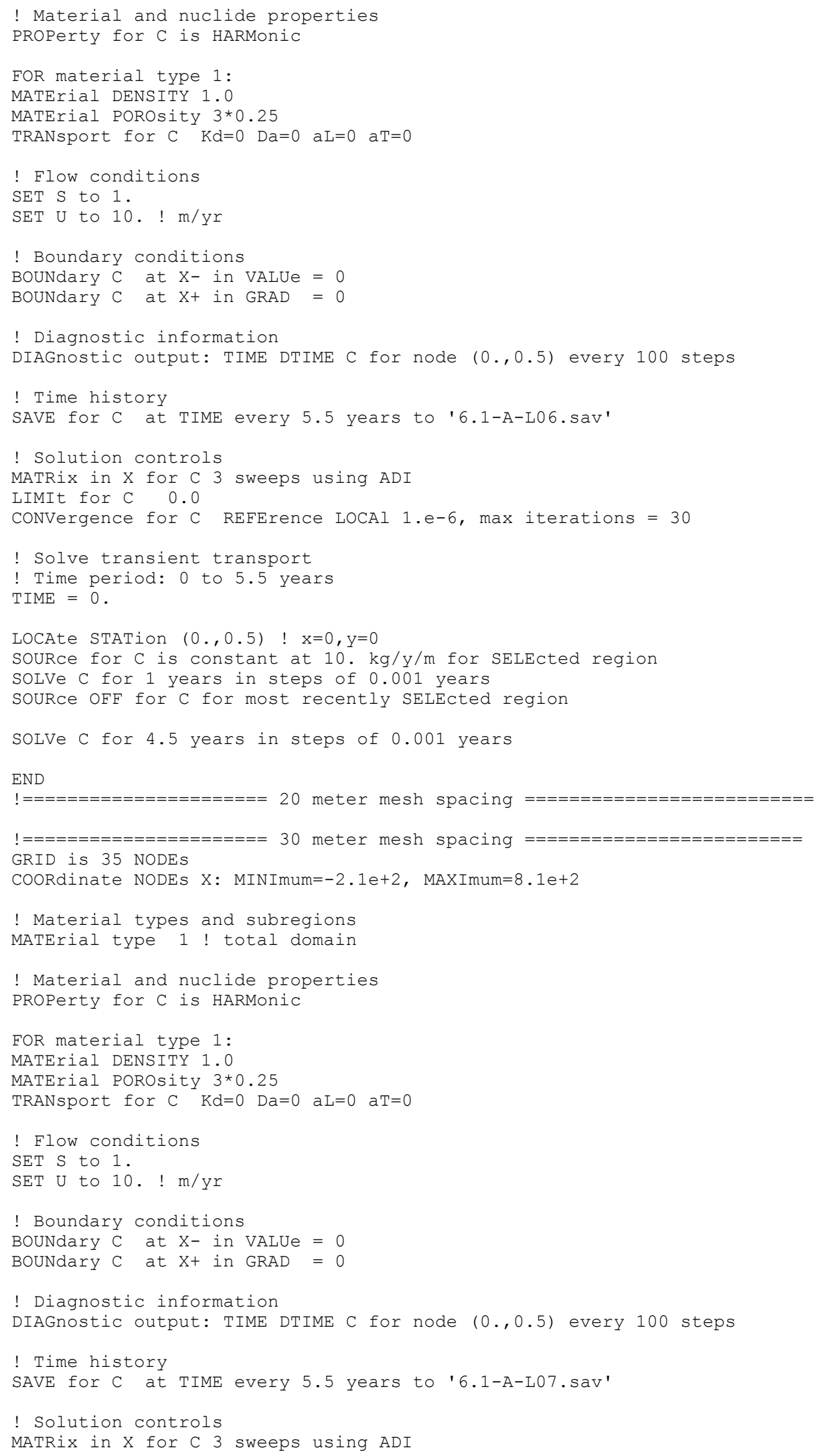


LIMIt for $\mathrm{C} 0.0$

CONVergence for C REFErence LOCAl 1.e-6, $\max$ iterations $=30$

! Solve transient transport

! Time period: 0 to 5.5 years

TIME $=0$.

LOCAte STATion $(0,0.5) \quad ! \quad \mathrm{x}=0, \mathrm{y}=0$

SOURce for $\mathrm{C}$ is constant at $10 . \mathrm{kg} / \mathrm{y} / \mathrm{m}$ for sELEcted region

SOLVe $\mathrm{C}$ for 1 years in steps of 0.001 years

SOURce OFF for $C$ for most recently SELEcted region

SOLVe C for 4.5 years in steps of 0.001 years

END

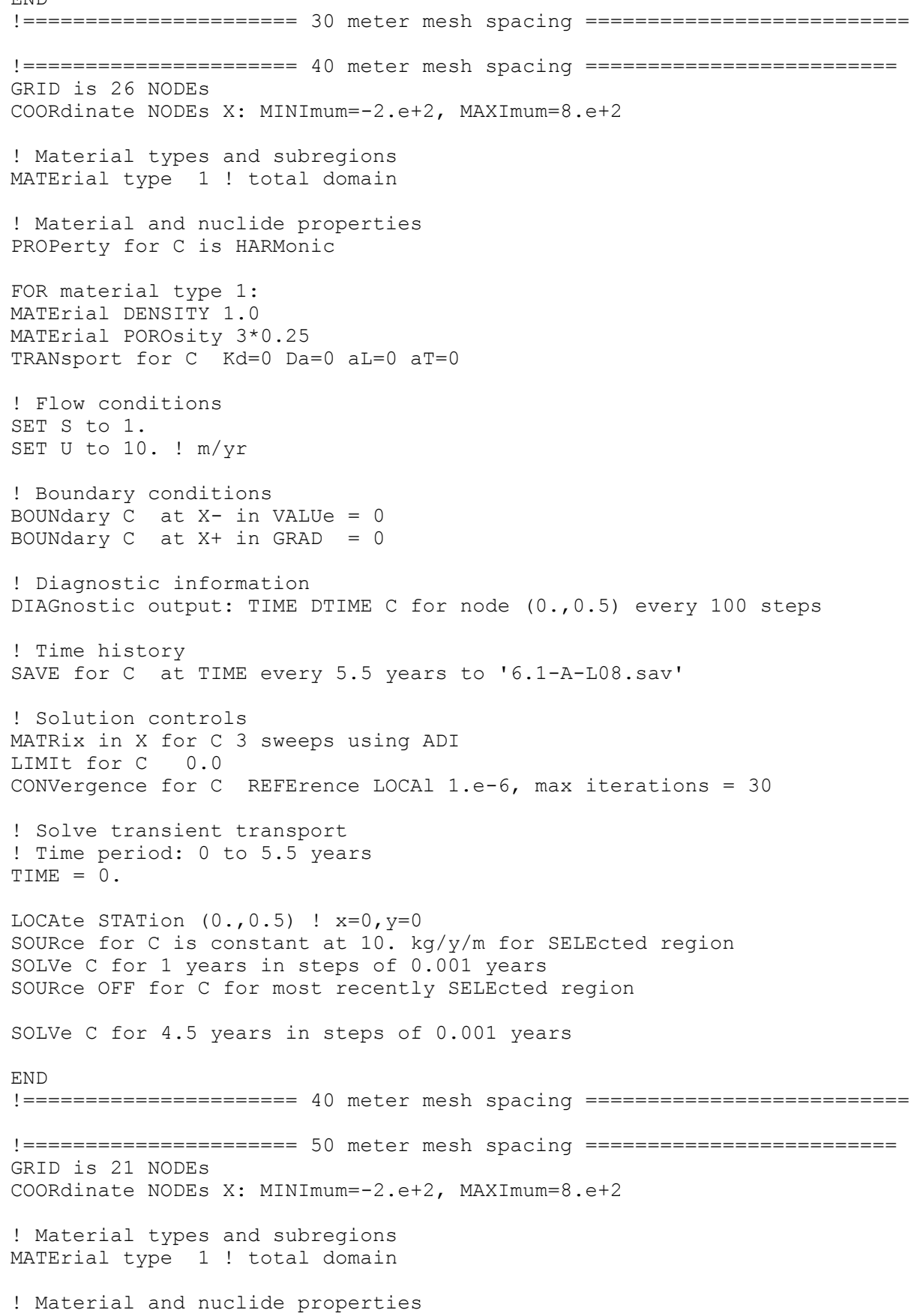


PROPerty for $\mathrm{C}$ is HARMonic

FOR material type 1 :

MATErial DENSITY 1.0

MATErial POROsity $3 * 0.25$

TRANsport for $\mathrm{C} \quad \mathrm{Kd}=0$ Da=0 aL=0 $\mathrm{aT}=0$

! Flow conditions

SET S to 1 .

SET U to 10. ! m/yr

! Boundary conditions

Boundary $\mathrm{C}$ at $\mathrm{X}$ - in $\mathrm{VALUe}=0$

BOUNdary $C$ at $\mathrm{X}+$ in GRAD $=0$

! Diagnostic information

DIAGnostic output: TIME DTIME C for node $(0 ., 0.5)$ every 100 steps

! Time history

SAVE for C at TIME every 5.5 years to ' $6.1-\mathrm{A}-\mathrm{L} 09$. .sav'

! Solution controls

MATRix in $X$ for $C 3$ sweeps using ADI

LIMIt for $\mathrm{C} 0.0$

CONVergence for C REFErence LOCAl $1 . e-6, \max$ iterations $=30$

! Solve transient transport

! Time period: 0 to 5.5 years

TIME $=0$

LOCAte STATion $(0,0.5) \quad ! \mathrm{x}=0, \mathrm{y}=0$

SOURce for $C$ is constant at $10 . \mathrm{kg} / \mathrm{y} / \mathrm{m}$ for SELEcted region

SOLVe $C$ for 1 years in steps of 0.001 years

SOURce OFF for $\mathrm{C}$ for most recently SELEcted region

SOLVe C for 4.5 years in steps of 0.001 years

END

! $======================4$ meter mesh spacing $==========================$

! $=====================060$ meter mesh spacing

GRID is 19 NODES

COORdinate NODES X: MINImum $=-2.4 \mathrm{e}+2, \operatorname{MAXImum}=8.4 \mathrm{e}+2$

! Material types and subregions

MATErial type 1 ! total domain

! Material and nuclide properties

PROPerty for $\mathrm{C}$ is HARMonic

FOR material type 1 :

MATErial DENSITY 1.0

MATErial POROsity $3 * 0.25$

TRANsport for $\mathrm{C} \quad \mathrm{Kd}=0$ Da=0 aL=0 aT=0

! Flow conditions

SET $S$ to 1.

SET U to 10.! m/yr

! Boundary conditions

BOUNdary $C$ at $X-$ in VALUe $=0$

BOUNdary $\mathrm{C}$ at $\mathrm{X}+$ in GRAD $=0$

! Diagnostic information

DIAGnostic output: TIME DTIME C for node $(0 ., 0.5)$ every 100 steps

! Time history

SAVE for C at TIME every 5.5 years to ' $6.1-\mathrm{A}-\mathrm{L} 10 . \mathrm{Sav}^{\prime}$

! Solution controls

MATRix in $X$ for $C 3$ sweeps using ADI

LIMIt for $\mathrm{C} 0.0$

CONVergence for C REFErence LOCAl 1.e-6, max iterations $=30$ 
! Solve transient transport

! Time period: 0 to 5.5 years

$\mathrm{TIME}=0$.

LOCAte STATion $(0 ., 0.5) \quad ! \mathrm{x}=0, \mathrm{y}=0$

SOURce for $\mathrm{C}$ is constant at $10 \mathrm{~kg} / \mathrm{y} / \mathrm{m}$ for SELEcted region

SOLVe $\mathrm{C}$ for 1 years in steps of 0.001 years

SOURce OFF for $C$ for most recently SELEcted region

SOLVe C for 4.5 years in steps of 0.001 years

END

! $=====================60$ meter mesh spacing

QUIT

Table 6.1.2. Input Commands for Problem 6.1 (Mechanical Dispersion).

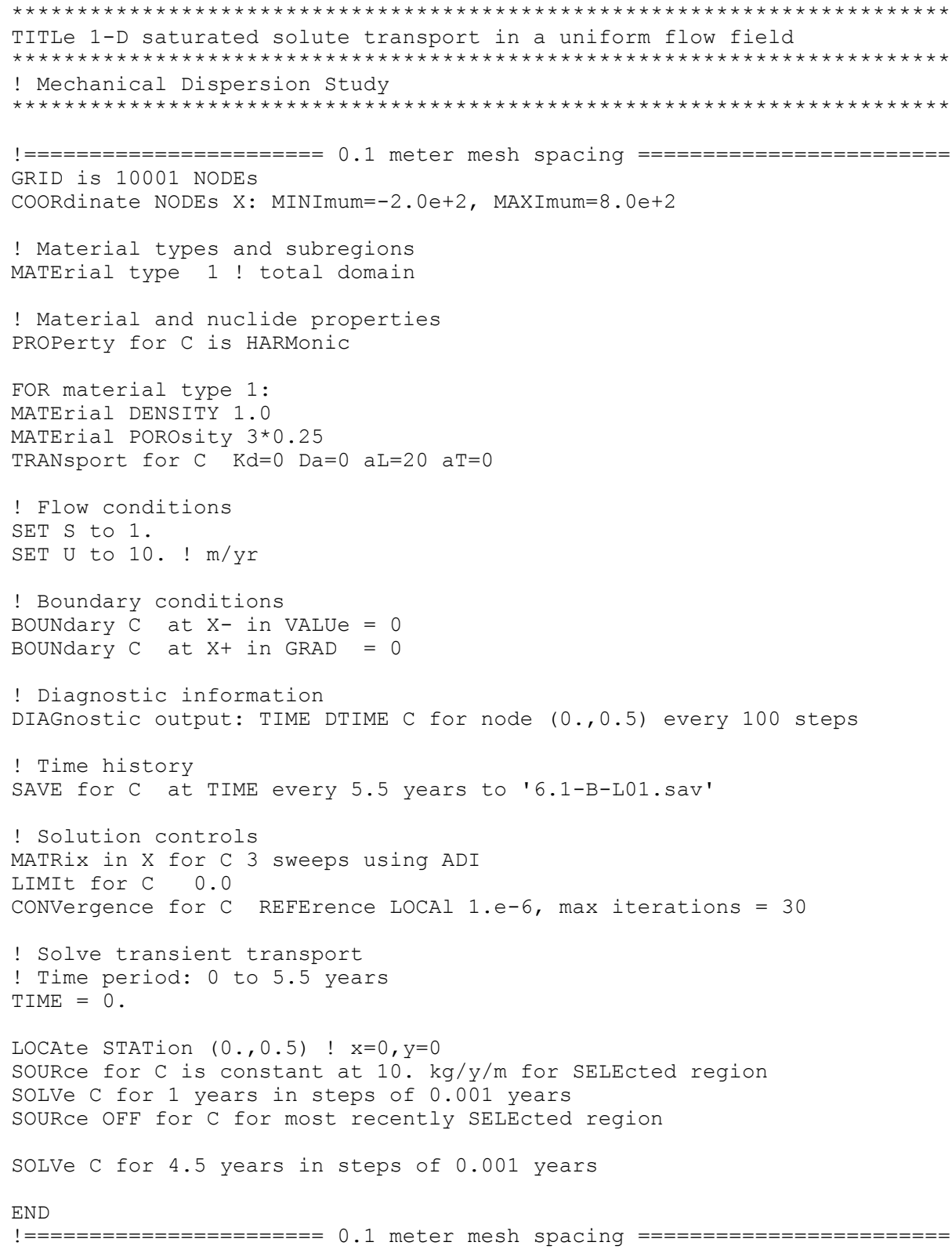




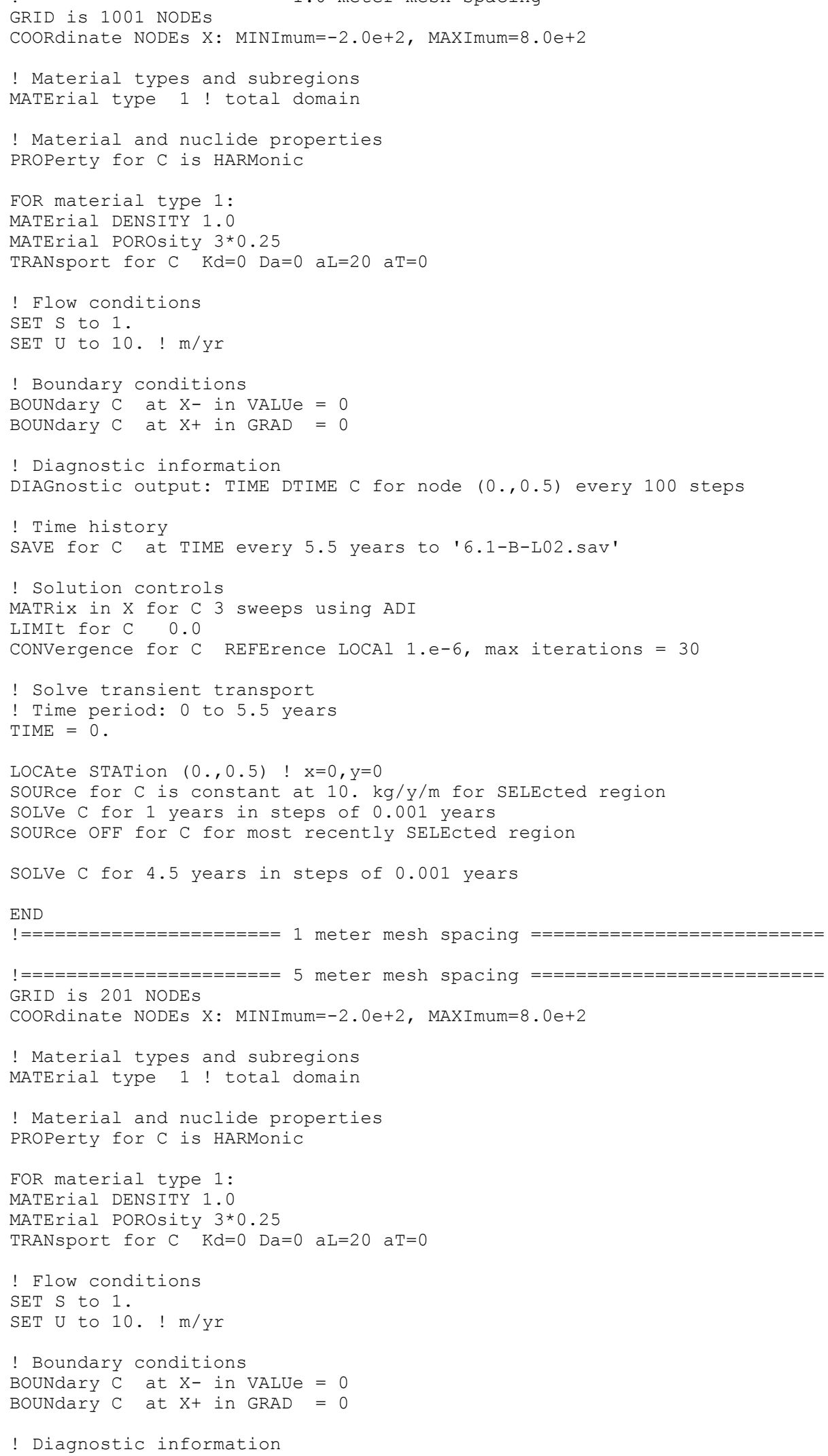


DIAGnostic output: TIME DTIME C for node $(0 ., 0.5)$ every 100 steps

! Time history

SAVE for C at TIME every 5.5 years to ' $6.1-$ B-L03.sav'

! Solution controls

MATRix in $X$ for $C 3$ sweeps using ADI

LIMIt for $\mathrm{C} 0.0$

CONVergence for C REFErence LOCAl $1 . e-6, \max$ iterations $=30$

! Solve transient transport

! Time period: 0 to 5.5 years

TIME $=0$.

LOCAte STATion $(0,0.5) \quad ! \quad x=0, y=0$

SOURce for $\mathrm{C}$ is constant at $10 \mathrm{~kg} / \mathrm{y} / \mathrm{m}$ for SELEcted region

SOLVe $C$ for 1 years in steps of 0.001 years

SOURce OFF for $C$ for most recently SELEcted region

SOLVe C for 4.5 years in steps of 0.001 years

END

! $=======================4$ meter mesh spacing $==========================$

! $=======================10$ meter Mesh spacing $==========================$

GRID is 101 NODES

COORdinate NODES X: MINImum $=-2.0 e+2$, MAXImum $=8.0 e+2$

! Material types and subregions

MATErial type 1 ! total domain

! Material and nuclide properties

PROPerty for $\mathrm{C}$ is HARMonic

FOR material type 1 :

MATErial DENSITY 1.0

MATErial POROsity $3 * 0.25$

TRANsport for $\mathrm{C} \mathrm{Kd}=0 \mathrm{Da}=0$ aL=20 aT=0

! Flow conditions

SET S to 1 .

SET U to 10. ! m/yr

! Boundary conditions

BOUNdary $\mathrm{C}$ at $\mathrm{X}-$ in VALUe $=0$

BOUNdary $C$ at $\mathrm{X}+$ in GRAD $=0$

! Diagnostic information

DIAGnostic output: TIME DTIME C for node $(0 ., 0.5)$ every 100 steps

! Time history

SAVE for C at TIME every 5.5 years to ' 6.1 -B-L04.sav'

! Solution controls

MATRix in $X$ for $C 3$ sweeps using ADI

LIMIt for $\mathrm{C} 0.0$

CONVergence for C REFErence LOCAl 1.e-6, max iterations $=30$

! Solve transient transport

! Time period: 0 to 5.5 years

TIME $=0$.

LOCAte STATion $(0,0.5) \quad ! \quad \mathrm{x}=0, \mathrm{y}=0$

SOURce for $\mathrm{C}$ is constant at $10 . \mathrm{kg} / \mathrm{y} / \mathrm{m}$ for SELEcted region

SOLVe $\mathrm{C}$ for 1 years in steps of 0.001 years

SOURce OFF for $\mathrm{C}$ for most recently SELEcted region

SOLVe C for 4.5 years in steps of 0.001 years

END

! $=======================10$ meter mesh spacing $==========================$

! $=======================15$ meter mesh spacing 
GRID is 69 NODES

COORdinate NODES X: MINImum $=-2.1 \mathrm{e}+2$, MAXImum $=8.1 \mathrm{e}+2$

! Material types and subregions

MATErial type 1 ! total domain

! Material and nuclide properties

PROPerty for $\mathrm{C}$ is HARMonic

FOR material type 1 :

MATErial DENSITY 1.0

MATErial POROsity $3 * 0.25$

TRANsport for $C \quad K d=0 \quad \mathrm{Da}=0 \quad \mathrm{aL}=20 \quad \mathrm{aT}=0$

! Flow conditions

SET S to 1.

SET U to 10 . ! m/yr

! Boundary conditions

BOUNdary $\mathrm{C}$ at $\mathrm{X}$ - in VALUe $=0$

BOUNdary $\mathrm{C}$ at $\mathrm{X}+$ in GRAD $=0$

! Diagnostic information

DIAGnostic output: TIME DTIME C for node $(0,0.5)$ every 100 steps

! Time history

SAVE for C at TIME every 5.5 years to ' $6.1-$ B-L05.sav'

! Solution controls

MATRix in $X$ for $C 3$ sweeps using ADI

LIMIt for $\mathrm{C} 0.0$

CONVergence for C REFErence LOCAl 1.e-6, max iterations $=30$

! Solve transient transport

! Time period: 0 to 5.5 years

TIME $=0$

LOCAte STATion $(0,0.5) ! x=0, y=0$

SOURce for $\mathrm{C}$ is constant at $10 \mathrm{~kg} / \mathrm{y} / \mathrm{m}$ for SELEcted region

SOLVe $\mathrm{C}$ for 1 years in steps of 0.001 years

SOURCe OFF for $\mathrm{C}$ for most recently SELEcted region

SOLVe $\mathrm{C}$ for 4.5 years in steps of 0.001 years

END

! $=======================15$ meter mesh spacing $=========================$

! $=======================20$ meter mesh spacing $=========================$

GRID is 51 NODES

COORdinate NODEs X: MINImum $=-2 . e+2$, MAXImum $=8 \cdot e+2$

! Material types and subregions

MATErial type 1 ! total domain

! Material and nuclide properties

PROPerty for $\mathrm{C}$ is HARMonic

FOR material type 1 :

MATErial DENSITY 1.0

MATErial POROsity $3 * 0.25$

TRANsport for $\mathrm{C} \mathrm{Kd}=0$ Da=0 $\mathrm{aL}=20$ aT=0

! Flow conditions

SET $S$ to 1 .

SET U to 10. ! m/yr

! Boundary conditions

Boundary $\mathrm{C}$ at $\mathrm{X}$ - in VALUe $=0$

BOUNdary $\mathrm{C}$ at $\mathrm{X}+$ in GRAD $=0$

! Diagnostic information

DIAGnostic output: TIME DTIME C for node $(0 ., 0.5)$ every 100 steps 
! Time history

SAVE for $C$ at TIME every 5.5 years to ' $6.1-B-L 06$. sav' $^{\prime}$

! Solution controls

MATRix in $X$ for $C 3$ sweeps using ADI

LIMIt for $\mathrm{C} 0.0$

CONVergence for C REFErence LOCAl 1.e-6, max iterations = 30

! Solve transient transport

! Time period: 0 to 5.5 years

$\mathrm{TIME}=0$

LOCAte STATion $(0,0.5) \quad ! \mathrm{x}=0, \mathrm{y}=0$

SOURce for $C$ is constant at $10 . \mathrm{kg} / \mathrm{y} / \mathrm{m}$ for SELEcted region

SOLVe $\mathrm{C}$ for 1 years in steps of 0.001 years

SOURCe OFF for $\mathrm{C}$ for most recently sELEcted region

Solve C for 4.5 years in steps of 0.001 years

END

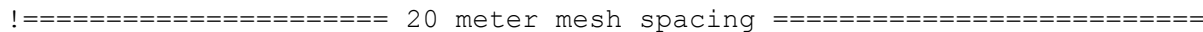

! $===================30$ meter mesh spacing $=====================$

GRID is 35 NODES

COORdinate NODEs $\mathrm{X}$ : MINImum $=-2.1 e+2$, MAXImum $=8.1 e+2$

! Material types and subregions

MATErial type 1 ! total domain

! Material and nuclide properties

PROPerty for $\mathrm{C}$ is HARMonic

FOR material type 1:

MATErial DENSITY 1.0

MATErial POROsity $3 * 0.25$

TRANsport for $\mathrm{C} \quad \mathrm{Kd}=0 \quad \mathrm{Da}=0 \quad \mathrm{aL}=20$ aT=0

! Flow conditions

SET S to 1

SET U to 10 . ! $\mathrm{m} / \mathrm{yr}$

! Boundary conditions

Boundary $\mathrm{C}$ at $\mathrm{X}-$ in VALUe $=0$

BOUNdary $\mathrm{C}$ at $\mathrm{X}+$ in GRAD $=0$

! Diagnostic information

DIAGnostic output: TIME DTIME C for node $(0 ., 0.5)$ every 100 steps

! Time history

SAVE for C at TIME every 5.5 years to '6.1-B-L07.sav'

! Solution controls

MATRix in $X$ for $C 3$ sweeps using ADI

LIMIt for $\mathrm{C} 0.0$

CONVergence for C REFErence LOCAl 1.e-6, max iterations = 30

! Solve transient transport

! Time period: 0 to 5.5 years

$\mathrm{TIME}=0$

LOCAte STATion $(0,0.5) \quad ! \mathrm{x}=0, \mathrm{y}=0$

SOURce for $\mathrm{C}$ is constant at $10 \mathrm{~kg} / \mathrm{y} / \mathrm{m}$ for SELEcted region

SOLVe $\mathrm{C}$ for 1 years in steps of 0.001 years

SOURCe OFF for $C$ for most recently SELEcted region

Solve $\mathrm{C}$ for 4.5 years in steps of 0.001 years

END

! $=====================30$ meter mesh $\operatorname{mparing}=======================$

! $====================40$ meter mesh spacing

GRID is 26 NODES

COORdinate NODEs $\mathrm{X}$ : MINImum $=-2 . e+2$, MAXImum $=8 . e+2$ 


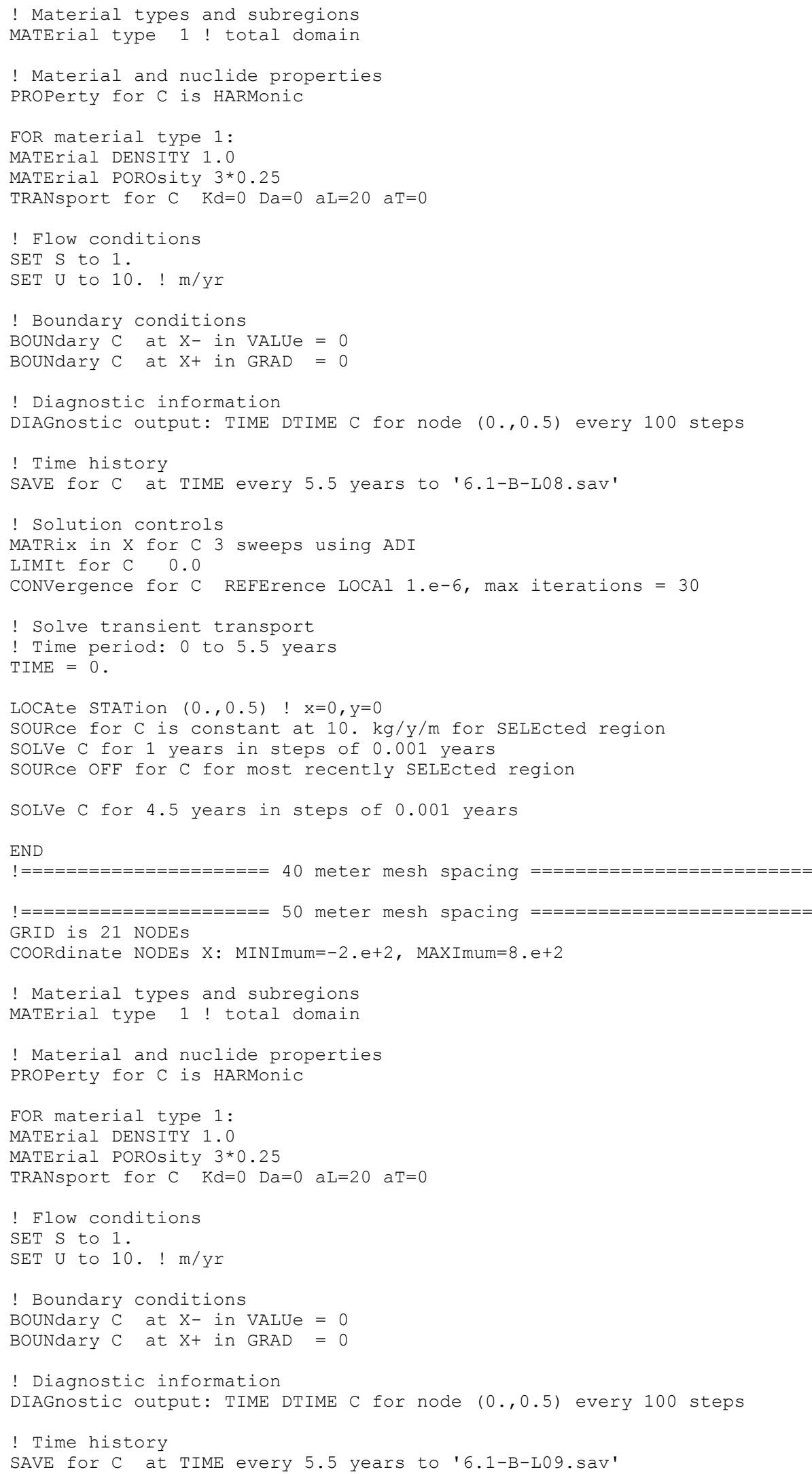




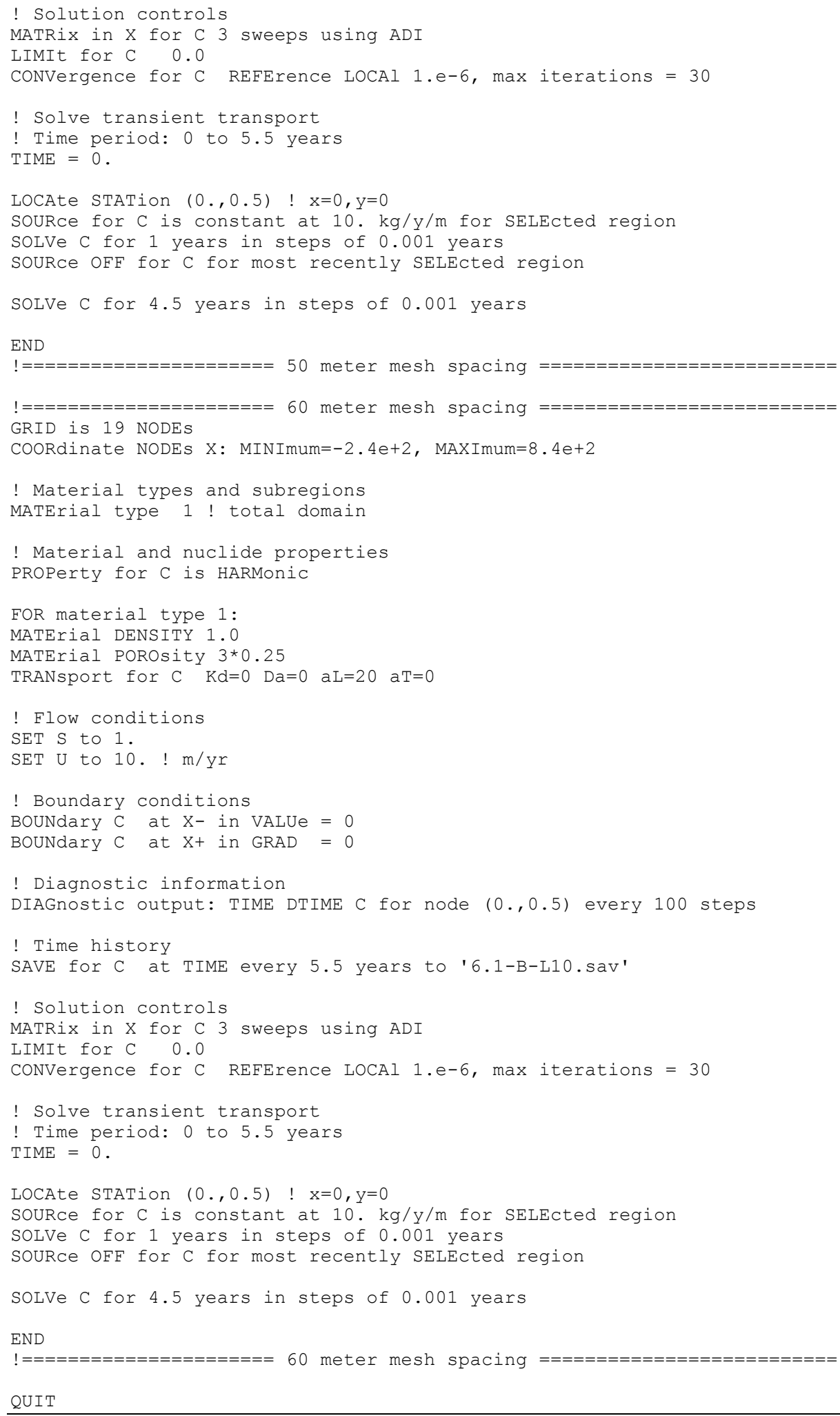




\section{Group 4: Keyword Commands}

This section deals with the testing of several PORFLOW keyword commands that are of interest to the Performance Assessment modelers at SRNL. The list of keywords tested is not exhaustive by any means and will continue to grow as needed by the modeling community.

\subsection{DECAy and REGEneration}

Mode 1 (Direct Linear Decay) of the DECAy command is used to specify rate constants and mode of decay of a dependent variable due to physical, chemical or radioactive decay. The REGEneration command specifies the regeneration rate of one species from another in the decay chain. The U-230 decay chain, U-230 $\rightarrow$ Th-226 $\rightarrow$ Ra-222 $\rightarrow$ Rn-218 $\rightarrow$ Po-214 $\rightarrow$ Pb-210 $\rightarrow$ Bi$210 \rightarrow$ Po-210, was used to verify that PORFLOW correctly computes transient concentrations of the parent and progeny species undergoing radioactive decay only (no transport). The PORFLOW results were compared to the classical Bateman equation.

Analytical solution: Bateman (1910) developed a general equation for serial decay chains, such as the heavy decay chain of U-238. Assuming that the concentration of all the daughters are initially zero (i.e. $\mathrm{n}_{\mathrm{i}}(0)=0$ for $\mathrm{i}>1$ ), the concentration of the $\mathrm{i}$-th radionuclide can be determined from

$$
\mathrm{n}_{\mathrm{i}}(\mathrm{t})=\lambda_{1} \lambda_{2} \cdots \lambda_{\mathrm{i}-1} \mathrm{n}_{1}(0) \sum_{\mathrm{j}=1}^{\mathrm{i}} \frac{\mathrm{e}^{-\lambda_{\mathrm{j}} \mathrm{t}}}{\prod_{\substack{\mathrm{k}=1 \\ \mathrm{k} \neq \mathrm{j}}}^{\mathrm{i}}\left(\lambda_{\mathrm{k}}-\lambda_{\mathrm{j}}\right)}
$$

PORFLOW numerical simulation and comparison: A one-element PORFLOW model of unit size was constructed. The initial inventory of U-230 was set equal to one mole. No transport properties were specified for all the species. Zero flux boundary conditions were imposed for all species. A 200-year simulation with a time step of 0.01 years was executed. The history of U230 and its daughters was recorded every 0.1 years. The PORFLOW input commands are shown in Table 7.1.1.

Figure 7.1.1 shows a comparison of the transient concentrations of the last three daughters in the U-230 decay chain to the Bateman equation. The Bateman and PORFLOW results are shown as lines and circles, respectively. The agreement is excellent. The parent and earlier daughters decay so quickly and are not visible within the scale shown in the figure. 


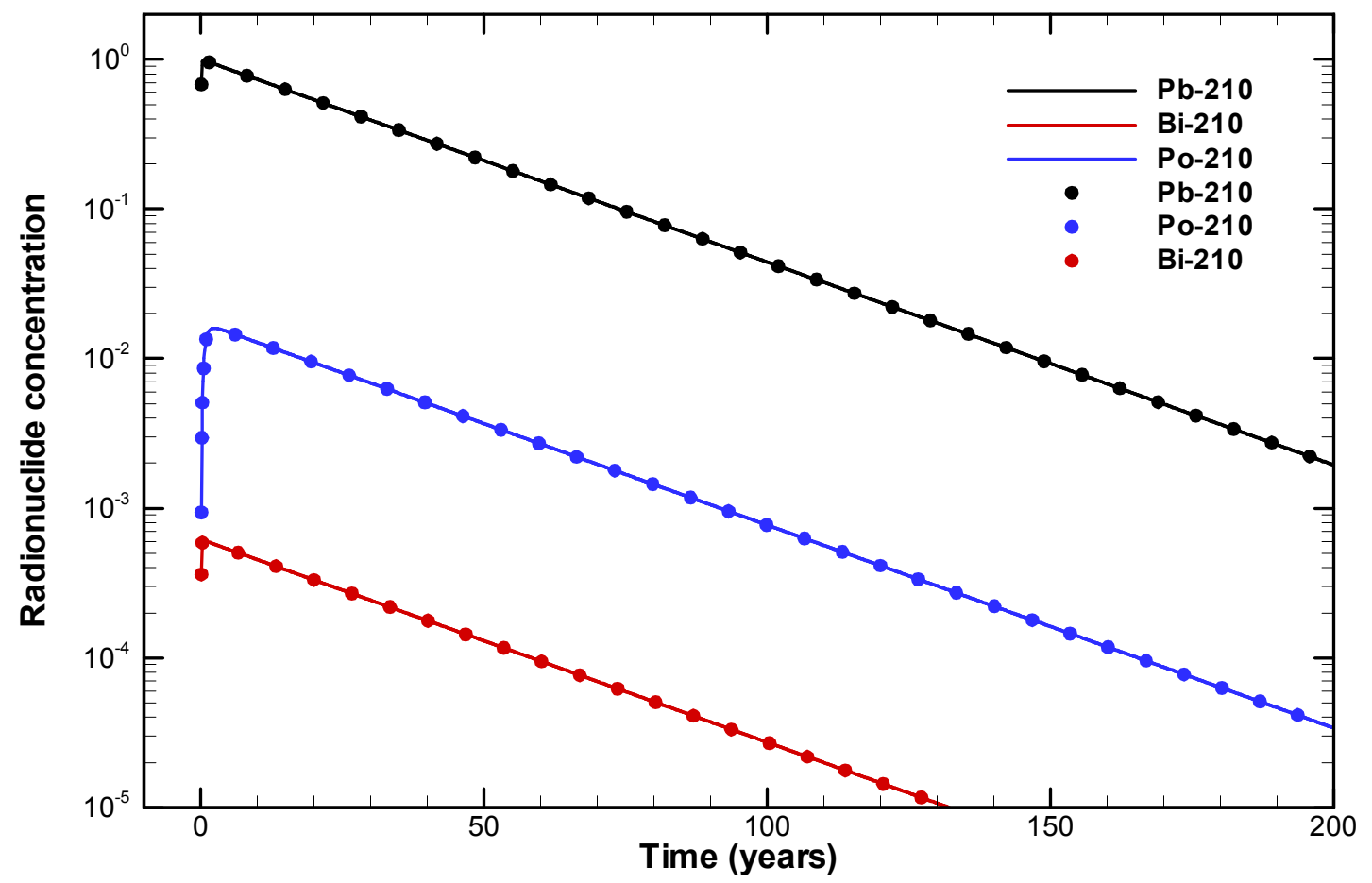

Figure 7.1.1. Comparison of Radionuclide Concentrations in the U-230 Decay Chain to the Bateman Equation.

Table 7.1.1. Input Commands for Problem 7.1.

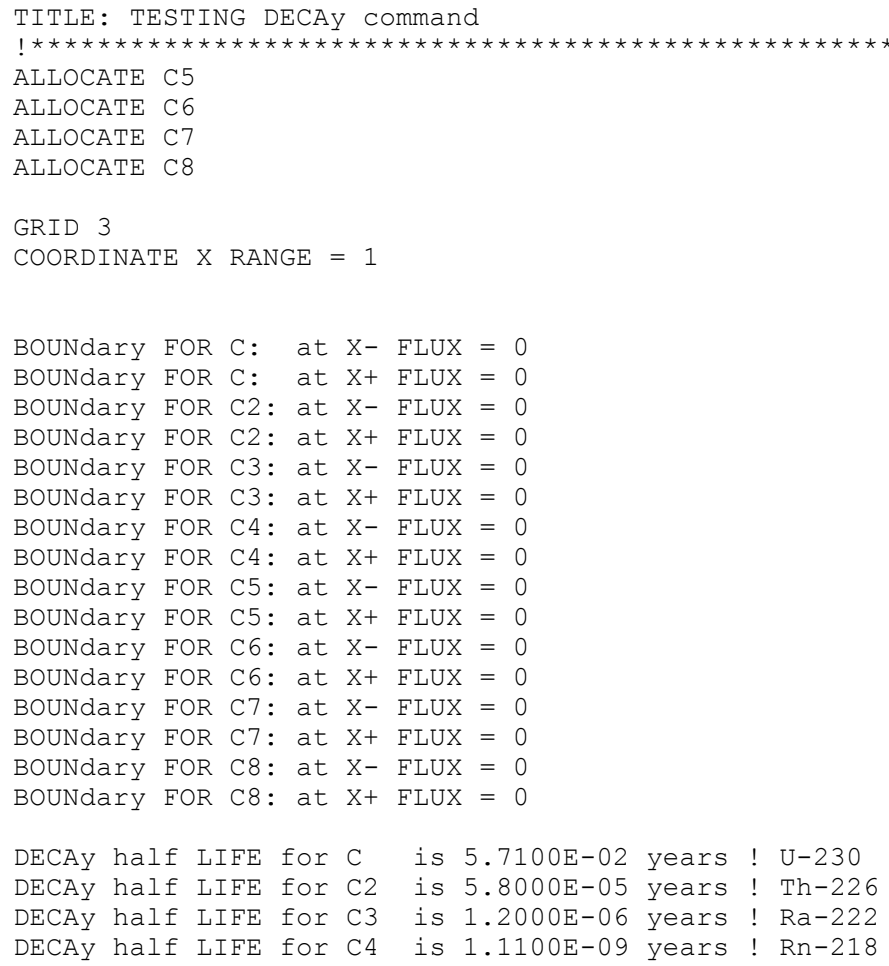


DECAy half LIFE for C5 is $5.2000 \mathrm{E}-12$ years ! Po-214

DECAy half LIFE for $\mathrm{C} 6$ is $2.2200 \mathrm{E}+01$ years ! $\mathrm{Pb}-210$

DECAy half LIFE for C7 is $1.3700 \mathrm{E}-02$ years! Bi-210

DECAy half LIFE for C8 is $3.8100 \mathrm{E}-01$ years ! Po-210

REGEneration of $\mathrm{C} 2$ from $\mathrm{C}$ is $1.00000 \mathrm{E}+00$ ! Th-226 from U-230

REGEneration of C3 from C2 is $1.00000 \mathrm{E}+00$ ! Ra-222 from Th-226

REGEneration of $\mathrm{C} 4$ from $\mathrm{C} 3$ is $1.00000 \mathrm{E}+00$ ! Rn-218 from Ra-222

REGEneration of C5 from C4 is $1.00000 \mathrm{E}+00$ ! Po-214 from Rn-218

REGEneration of $\mathrm{C} 6$ from $\mathrm{C} 5$ is $1.00000 \mathrm{E}+00$ ! Pb-210 from Po-214

REGEneration of C7 from C6 is $1.00000 \mathrm{E}+00$ ! Bi-210 from $\mathrm{Pb}-210$

REGEneration of $\mathrm{C} 8$ from $\mathrm{C} 7$ is $1.00000 \mathrm{E}+00$ ! Po-210 from Bi-210

SET C2 0

SET C3 0

SET C4 0

SET C5 0

SET C6 0

SET C7 0

SET C8 0

! Initial condition

SET C INVEntory to 1 mole with UNIForm concentration

DIAgnostics for TIME C at: $(2,2)$ every 10 steps

! Solution controls

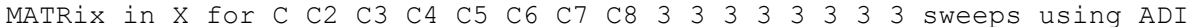

Convergence for C C2 C3 C4 C5 C6 C7 C8 REFErence LOCAl 1.e-6, max iterations = 30

SELEct $(2,2)$

HISTory for C C2 C3 C4 at TIME interval of 0.1 year to file 'DECAy-1.his' for SELEcted

HISTory for C5 C6 C7 C8 at TIME interval of 0.1 year to file 'DECAy-2.his' for SELEcted

SOLVE C C2 C3 C4 C5 C6 C7 C8 200 years in steps of 0.01

SAVE OFF

END

\subsection{DISTribution and RETArdation}

Mode 1 (Distribution Coefficient as a General Function) of the DISTribution command with the TABLe modifier was used to specify the distribution coefficient, $k d$, as a function of fluid concentration in tabular form. The complementary RETArdation command with the TABLe modifier was used to specify retardation as a function of fluid concentration in tabular form. The table of values was formulated to demonstrate that the DIST or RETA command could be used to limit the solubility of a species. If the fluid concentration is below the solubility limit, the $\mathrm{kd}$ or $\mathrm{R}$ remains constant at the nominal value. If the inventory or effective concentration (fluid and solid) of the species exceeds the solubility limit of the species, the $\mathrm{kd}$ or $\mathrm{R}$ is adjusted to maintain the fluid concentration at the solubility limit. The fluid concentration is computed in PORFLOW as

$$
\mathrm{C}=\frac{\mathrm{C}_{\mathrm{e}}}{\mathrm{R} \theta}
$$

where

C ..................concentration of the species in the fluid phase

$\mathrm{C}_{\mathrm{e}} \ldots \ldots \ldots \ldots \ldots . . . . .$. inventory or effective concentration of the species in the porous medium 
PORFLOW numerical simulation and comparison: A one-element PORFLOW model of unit size was constructed. The dry solid density and the porosity were set to 2 and 0.5 , respectively. The fluid phase is saturated. Zero flux boundary conditions were imposed. A 600-year simulation with a time step of 0.1 years was executed. A point source of strength 0.01 per year was turned on for 200 years. A subsequent point sink of strength 0.01 per year was then turned on for the next 100 years. The point source/sink cycle was then repeated.

A table of $(\mathrm{C}, \mathrm{kd})$ values with three sets was specified for the DIST command. The kd is set to a constant value of $0.4(\mathrm{R}=2)$ for fluid concentrations ranging from 0 to 1 . Above a fluid concentration of 1 the $\mathrm{kd}$ is adjusted so that the product of the retardation factor and water content offsets any increase in the inventory or effective concentration to maintain the solubility limit. This results in a species solubility limit of 1 . Once the effective concentration drops below the solubility limit, the kd is constant at 0.4 . Similarly, for the RETA command a table of $(C, R)$ values are specified instead.

Figure 7.2.1. shows the implementation of a solubility-limited source based on the DIST and RETA command. During the first 100 years the liquid and solid concentrations are equal $\left(\rho_{\mathrm{s}} \mathrm{kd}=\right.$ 1) and track the injection rate of 0.01 per year. At 100 years, the liquid and solid concentrations equal the solubility limit of 1 . Between 100 and 200 years, the effective concentration of the species attempts to increase from 1 to 2 . Any delta increase in the effective concentration above the solubility limit of 1 is offset by an increase in the product of the retardation factor and water content to keep the fluid concentration at 1 . Any mass injected above the solubility limit of the species is discarded by PORFLOW and is reflected by an increase in the flux balance discrepancy in the diagnostic file. Between the time of 200 to 300 years, the solute is extracted at a rate of 0.01 per year. The actual inventory at 200 years is 1 instead of 2 since PORFLOW has discarded the additional mass. This drives the fluid concentration from 1 to 0 . The rest of the transient is a repeat of the previous point source/sink cycle. The DIST and RETA commands (Mode 1) function as designed by the developer is nonconservative, due to loss inventory, for implementation as a means to limit the solubility of the species. The fluid concentration should ideally track the solid line in Figure 7.2.1.

The PORFLOW input commands utilizing the DIST and RETA commands are shown in Table 7.2.1 and 7.2.2, respectively. 


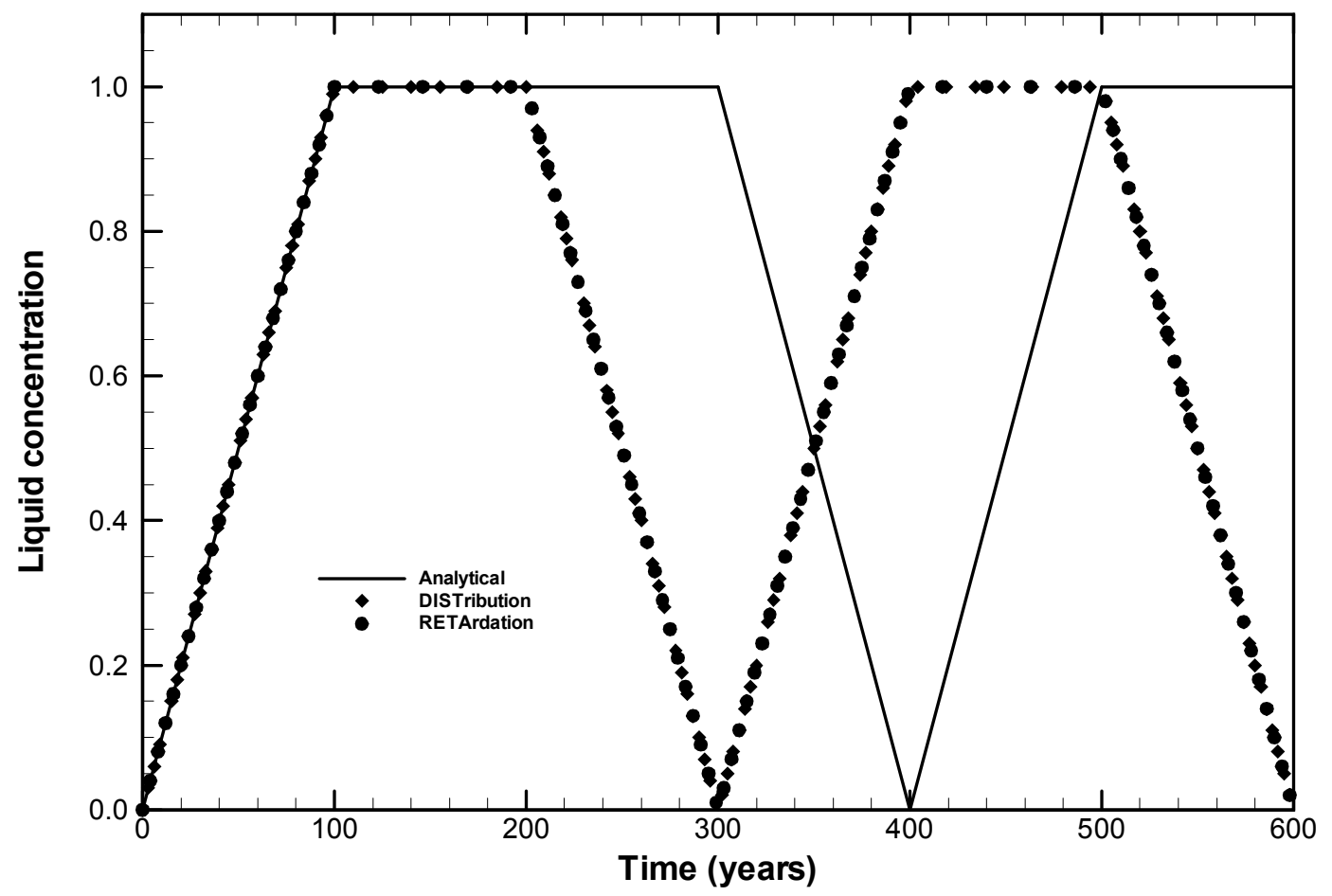

Figure 7.2.1. Implementation of Solubility Limited Source based on the DIST and RETA Command.

Table 7.2.1. Input Commands for Problem 7.2 (DIST command).

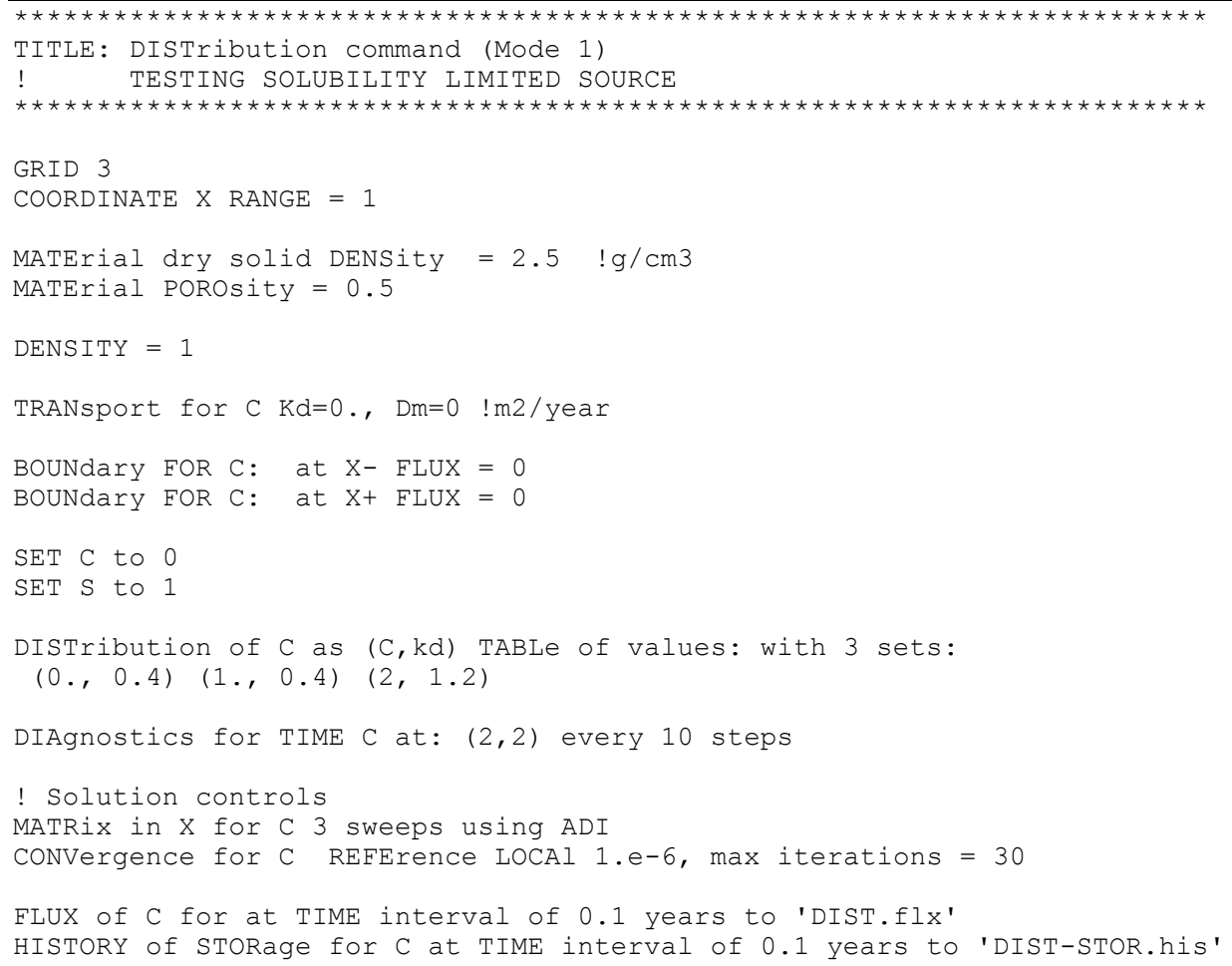


SELEct $(2,2)$

HISTORY for $C$ at TIME interval of 0.1 years to 'DIST-C.his' for SELEcted

SELECt $(2,2)$

SOURce for C by VOLUme: 0.01 per year

SOLVE C 200 years in steps of 0.1

SOURce is OFF for C

SOURce for C by VoLUme: -0.01 per year

SOLVE C 100 years in steps of 0.1

SOURce is OFF for $C$

SOURce for C by VOLUme: 0.01 per year

SOLVE C 200 years in steps of 0.1

SOURce is OFF for $C$

SOURce for C by VoLUme: -0.01 per year

SOLVE C 100 years in steps of 0.1

END

Table 7.2.2. Input Commands for Problem 7.2 (RETA command).

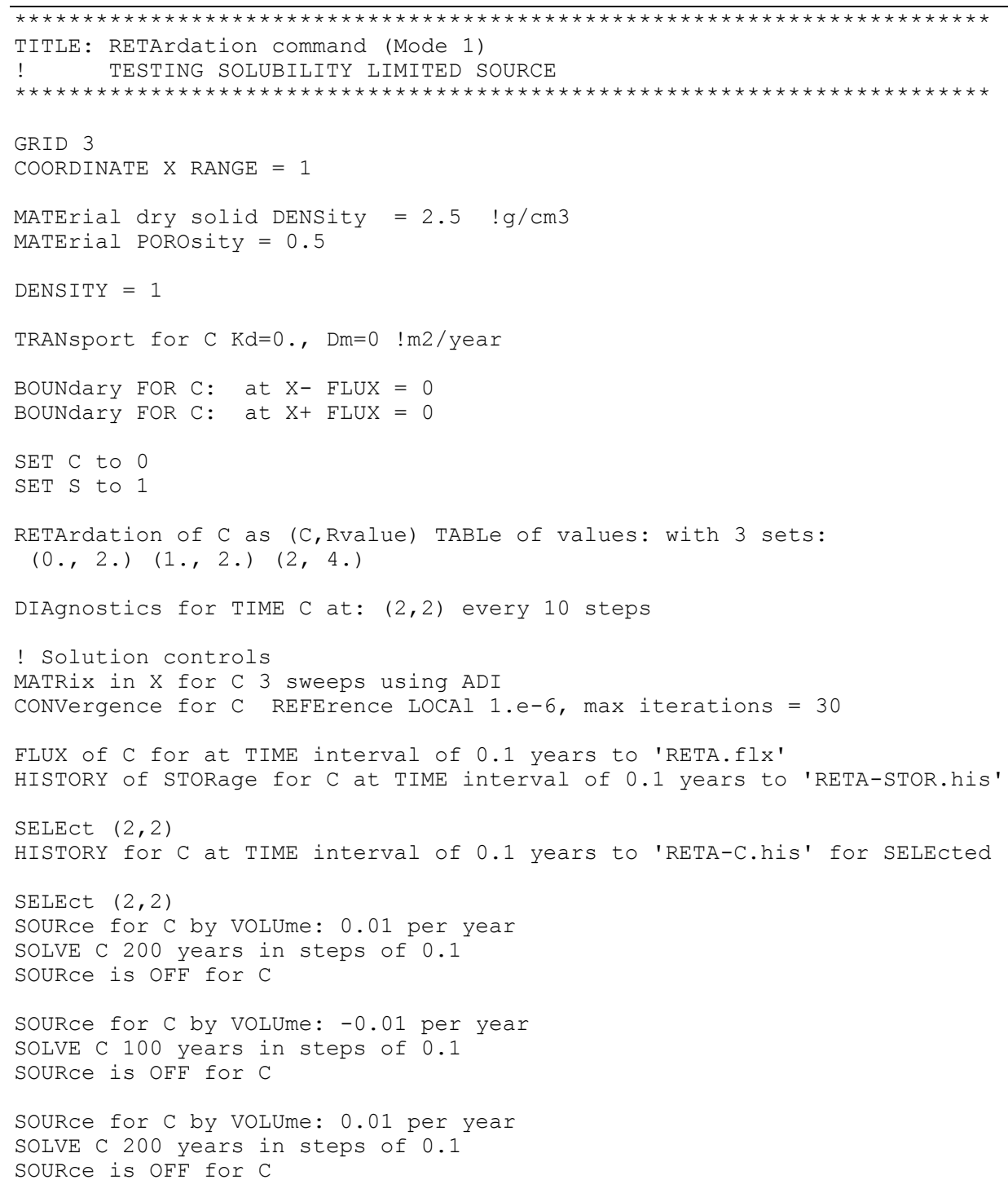


SOURce for C by VoLUme: -0.01 per year

SOLVE C 100 years in steps of 0.1

END

\subsection{STATistics}

The STATistics command provides a means to compute and obtain output of the statistics for an independent variable for a selected subregion. The computed statistics consist of the minimum, maximum, mean and standard deviation, and the location of the minimum and the maximum. The statistic of interest for PA modeling at SRNL is the maximum concentration within a subregion or in a subregion beyond a facility or compliance boundary (100 meter well). The STATistics command was tested on the 3D saturated solute transport problem in Section 5.3.

The PORFLOW input commands for Problem 5.3 were augmented with two STATistics commands as shown in Table 7.3.1. The first command determines the location and maximum concentration within the computational domain every 100 years of the simulation. The second command determines the same information for a subregion beyond $\mathrm{x}=700$ meters.

The maximum concentration for the entire domain exist at the point source located at $\mathrm{x}=\mathrm{y}=\mathrm{z}=$ 0. The element number for the point source is 49590 as shown in Table 7.3.2 using the LOCAte command. The STATistics command, with no subregion specified, locates the maximum concentration for the entire domain at element 49590 as shown in Table 7.3.3. The command has generated the correct result.

A subregion was selected 700 meters downstream of the point source. The LOCAte EXCLude POLYgon command was used to exclude any elements below $x=700$ meters. The highest concentrations downstream of the point source exist along the plume centerline ( $I \geq 20, \mathrm{~J}=19, \mathrm{~K}$ $=19)$. According to Table 7.3.4, 20825 elements were selected in the subregion. The table was edited to show only the elements along the plume centerline. The STATistics command for subregion ID=RECT locates the maximum concentration at element 49637 as shown in Table 7.3.5. This element is along the plume centerline (see Table 7.3.4); therefore, the command has generated the correct result.

\section{Table 7.3.1. Input Commands for Problem 7.3.}

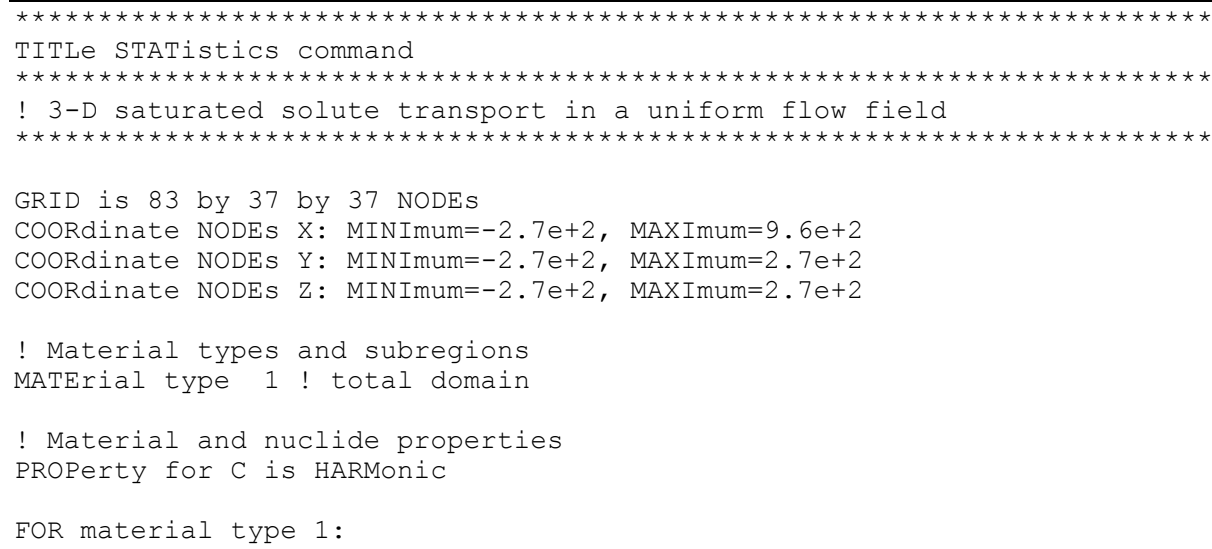




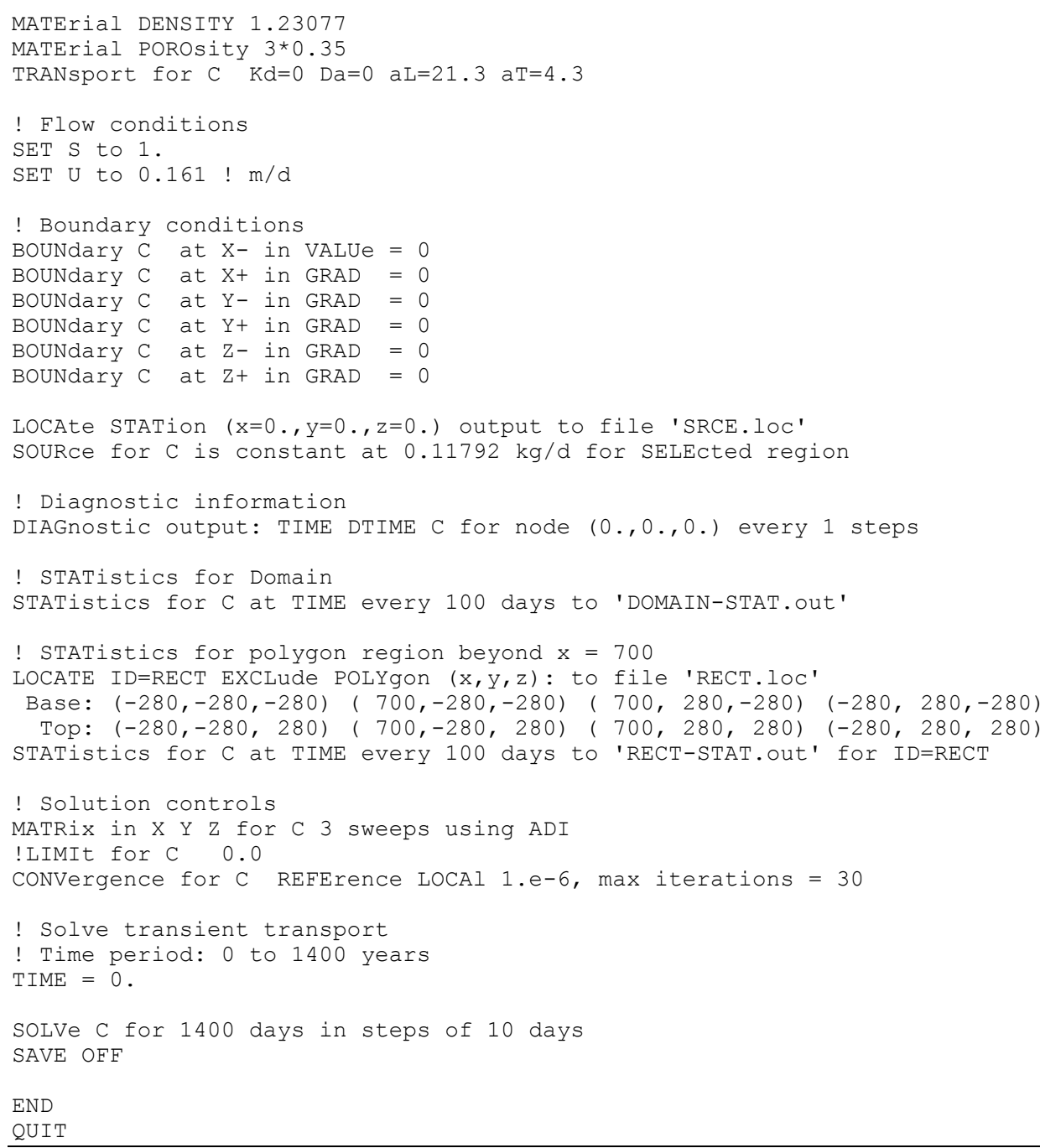

Table 7.3.2. Location of Point Source for Problem 7.3.

\begin{tabular}{|c|c|c|c|c|c|}
\hline $\begin{array}{l}\text { LOCATE LI } \\
\text { END HEADE } \\
49590\end{array}$ & T of Elen & s & $=\mathrm{SR}$ & $.10 \mathrm{C}$ & ! \\
\hline Serial\# & Element\# & I & $\mathrm{J}$ & K & \\
\hline 1 & 49590 & 19 & 19 & 19 & \\
\hline
\end{tabular}

Table 7.3.3. Statistics File Showing Maximum Value and Element for Domain.

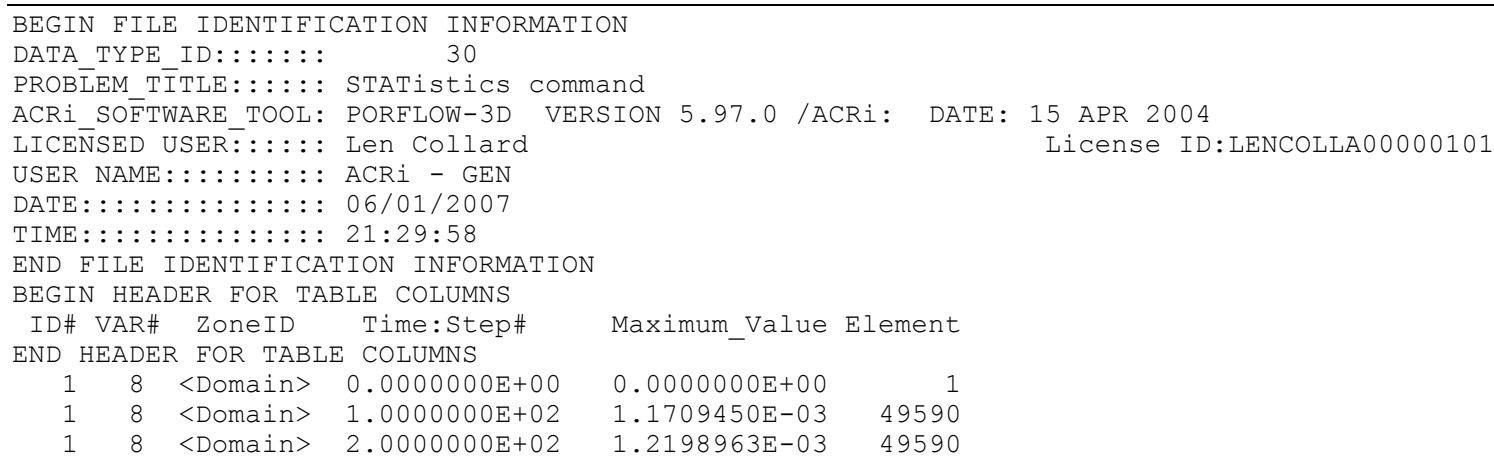




\begin{tabular}{|c|c|c|c|c|c|}
\hline 1 & 8 & $<$ Domain $>$ & $3.0000000 \mathrm{E}+02$ & $1.2305669 \mathrm{E}-03$ & 49590 \\
\hline 1 & 8 & $<$ Domain $>$ & $4.0000000 \mathrm{E}+02$ & $1.2340566 \mathrm{E}-03$ & 49590 \\
\hline 1 & 8 & $<$ Domain $>$ & $5.0000000 \mathrm{E}+02$ & $1.2354029 \mathrm{E}-03$ & 49590 \\
\hline 1 & 8 & $<$ Domain $>$ & $6.0000000 \mathrm{E}+02$ & 1.2359710E-03 & 49590 \\
\hline 1 & 8 & $<$ Domain $>$ & $7.0000000 \mathrm{E}+02$ & 1.2362248E-03 & 49590 \\
\hline 1 & 8 & $<$ Domain $>$ & $8.0000000 \mathrm{E}+02$ & $1.2363427 \mathrm{E}-03$ & 49590 \\
\hline 1 & 8 & $<$ Domain $>$ & $9.0000000 \mathrm{E}+02$ & 1.2363992E-03 & 49590 \\
\hline 1 & 8 & $<$ Domain $>$ & $1.0000000 \mathrm{E}+03$ & $1.2364268 E-03$ & 49590 \\
\hline 1 & 8 & $<$ Domain $>$ & $1.1000000 \mathrm{E}+03$ & $1.2364406 \mathrm{E}-03$ & 49590 \\
\hline 1 & 8 & $<$ Domain $>$ & 1. $2000000 \mathrm{E}+03$ & $1.2364475 E-03$ & 49590 \\
\hline 1 & 8 & $<$ Domain $>$ & $1.3000000 \mathrm{E}+03$ & 1.2364511E-03 & 49590 \\
\hline 1 & 8 & $<$ Domain $>$ & $1.4000000 \mathrm{E}+03$ & $1.2364529 \mathrm{E}-03$ & 49590 \\
\hline
\end{tabular}

Table 7.3.4. Location of Plume Centerline Nodes Beyond $x=700$ meters.

\begin{tabular}{|c|c|c|c|c|c|c|c|c|c|c|}
\hline $\begin{array}{l}\text { LOCATE LI } \\
\text { END HEADEI }\end{array}$ & $\begin{array}{l}\text { ST of Elem } \\
\text { R }^{2}\end{array}$ & nents I & $=\mathrm{RECT}$ & .100 & & 20825 & & & & \\
\hline & & & & & 49637 & 49638 & 49639 & 49640 & 49641 & 49642 \\
\hline $\begin{array}{l}49643 \\
49653\end{array}$ & 49644 & 49645 & 49646 & & 49647 & 49648 & 49649 & 49650 & 49651 & 49652 \\
\hline Serial\# & Element\# & I & $\mathrm{J}$ & $\mathrm{K}$ & & & & & & \\
\hline 10405 & 49637 & 66 & 19 & 19 & & & & & & \\
\hline 10406 & 49638 & 67 & 19 & 19 & & & & & & \\
\hline 10407 & 49639 & 68 & 19 & 19 & & & & & & \\
\hline 10408 & 49640 & 69 & 19 & 19 & & & & & & \\
\hline 10409 & 49641 & 70 & 19 & 19 & & & & & & \\
\hline 10410 & 49642 & 71 & 19 & 19 & & & & & & \\
\hline 10411 & 49643 & 72 & 19 & 19 & & & & & & \\
\hline 10412 & 49644 & 73 & 19 & 19 & & & & & & \\
\hline 10413 & 49645 & 74 & 19 & 19 & & & & & & \\
\hline 10414 & 49646 & 75 & 19 & 19 & & & & & & \\
\hline 10415 & 49647 & 76 & 19 & 19 & & & & & & \\
\hline 10416 & 49648 & 77 & 19 & 19 & & & & & & \\
\hline 10417 & 49649 & 78 & 19 & 19 & & & & & & \\
\hline 10418 & 49650 & 79 & 19 & 19 & & & & & & \\
\hline 10419 & 49651 & 80 & 19 & 19 & & & & & & \\
\hline 10420 & 49652 & 81 & 19 & 19 & & & & & & \\
\hline 10421 & 49653 & 82 & 19 & 19 & & & & & & \\
\hline
\end{tabular}

Table 7.3.5. Statistics File Showing Maximum Value and Element Beyond $x=700$ m.

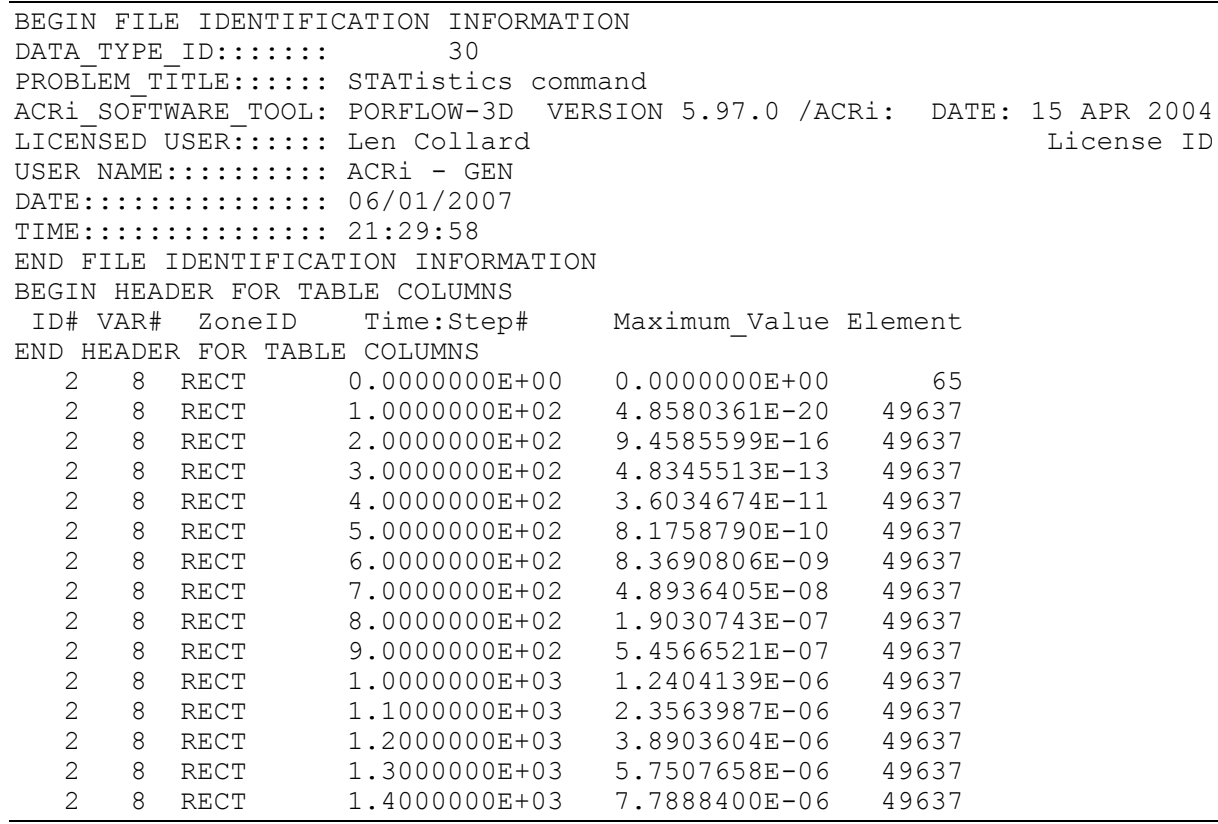




\section{References}

Abramowitz, M. and Stegun, I., 1970. Handbook of Mathematical Functions (Hardcover), National Bureau of Standards (1970).

ACRI, Inc., 1994. "Validation Version 2.50," Analytical \& Computational Research, Inc., 19851994.

ACRI, Inc., 2004. “User's Manual Version 5.0 Rev:5,”, Analytical \& Computational Research, Inc., 1985-2002.

Anderson, P. F., 1993, "A manual of instructional problems for the U.S.G.S. MODFLOW model", EPA/600/R-93/010, Environmental Protection Agency.

Bateman, H., 1910. "The solution of a system of differential equations occurring in the theory of radio-active transformations," Proc. Cambridge Phil. Soc., 16, p. 423 (1910).

COMSOL AB, 2006. "COMSOL Multiphysics: Earth Science Module”, Version 3.3, August 2006.

de Marsily, G., 1986. Quantitative Hydrogeology, Academic Press, Inc.

Faust, C. R., P. N. Sims, C. P. Spalding, P. F. Anderson, B. H. Lester, M. G. Shupe, and A. Harrover, 1993 (GeoTrans, Inc.). FTWORK: Groundwater Flow and Solute Transport in Three Dimensions, Documentation, Version 12.9, prepared for GeoTrans, Inc., Sterling, Virginia 20166

Freeze, R. A., and J. A. Cherry, 1979, Groundwater, Prentice-Hall, Englewood Cliffs, New Jersey.

GeoTrans, 1993, "FTWORK: Groundwater flow and solute transport in three dimensions; Documentation version 2.8".

Grobner, W., and N. Hofreiter, 1950. Integraltafel, Springer, Vienna, Vol. 2 (Vol. 1, 1949).

Hamm, L., L. and Aleman, S. E., 2000. "FACT (Version 2.0) Subsurface Flow and Contaminant Transport Documentation and User's Guide (U)," Westinghouse Savannah River Company, March 2000.

Hantush, M. S. and C. E. Jacob, 1955, "Non-steady radial flow in an infinite leaky aquifer", Am. Geophys. Union Trans., v36, 95-100.

Hantush, M. S., and R. G. Thomas, 1966, "A method for analyzing a drawdown test in anisotropic aquifers", Water Resources Res. v2 n2, 281-285.

Ogata, A. and R. B. Banks, 1961. A solution of the differential equation of longitudinal dispersion in a porous media, U.S. Geol. Survey, Professional Paper 411-A.

Permlmutter, N.M. and M. Lieber, 1970. Dispersion of plating wastes and sewage contaminants in groundwater and surface water, South Farmingdale-Massapequa Area, Nassau County, New York, U.S. Geol. Surv. Water Supply Paper 1879-G.

Polubarinova-Kochina, 1962. "Theory of Ground Water Movement," Translated from the Russian by J. M. Roger De Wiest, Princeton University Press, Princeton, New Jersey, 1962.

Theis, C. V., 1935, "The relation between the lowering of the piezometric surface and the rate and duration of discharge of a well using groundwater storage", Trans. Am. Geophys. Union, Ann. Meet., 16th, 519-524.

van Genuchten, M. T., 1980, "A Closed-form Equation for Predicting the Hydraulic Conductivity of Unsaturated Soils", Soil Sci. Am. J., v44, pp. 892-898. 
Wilson, J.L. and P.J. Miller, 1978. Two-dimensional plume in uniform ground-water flow, J. of the Hydraulics Div., Amer. Soc. Civ. Engrs., 104, HY4.

Yeh, G.T. 1981. AT123D: Analytical transient one-, two-, and three-dimensional simulation of transport in the aquifer system, Oak Ridge National Lab., Tennessee, Report No. ORNL5602.

\section{A Hantush and Jacob Numerical Code}

\section{A.1 FORTRAN Listing for HantushJacob.f90}

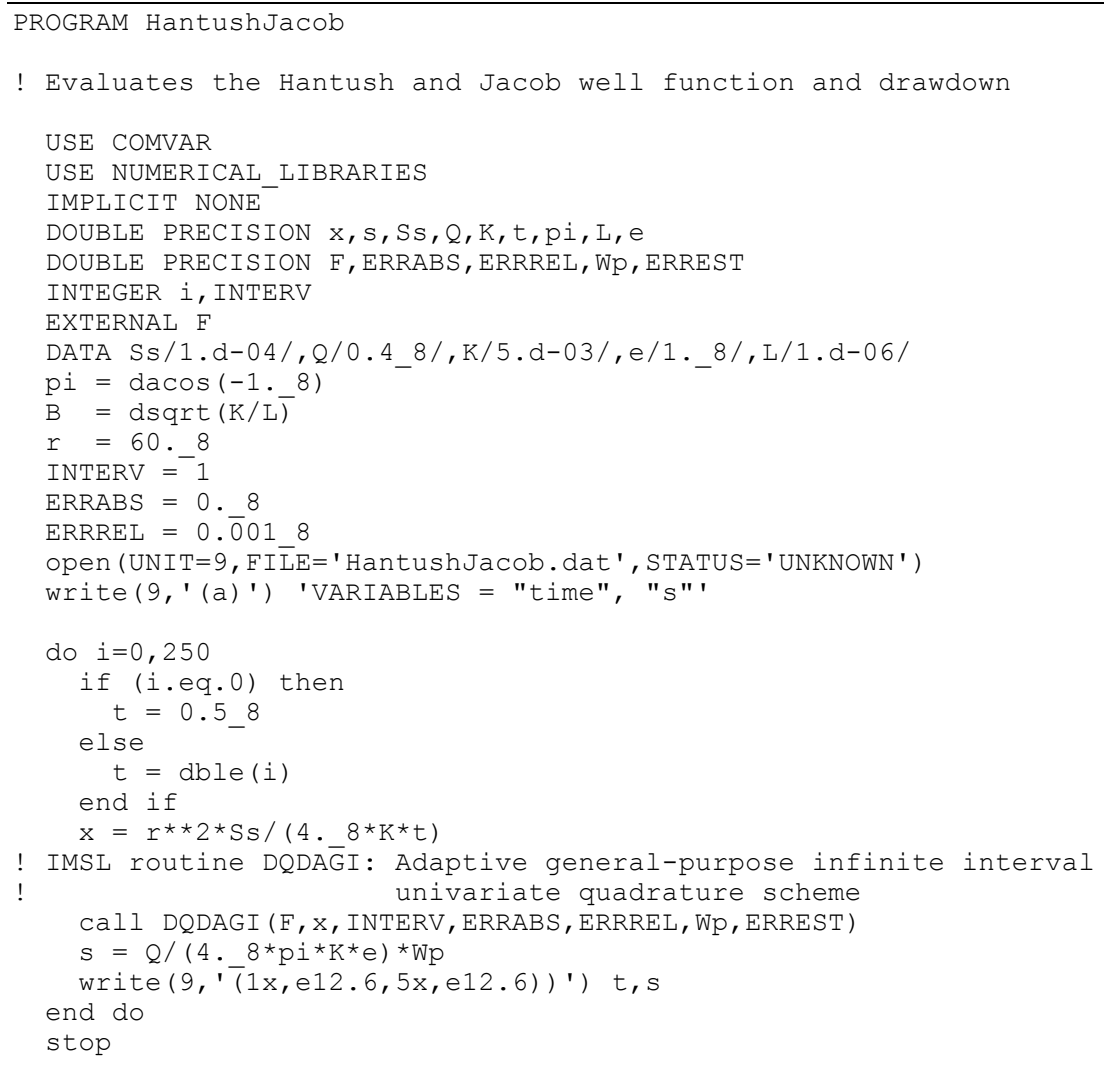

END PROGRAM HantushJacob

\section{A.2 FORTRAN Listing for F.f90}

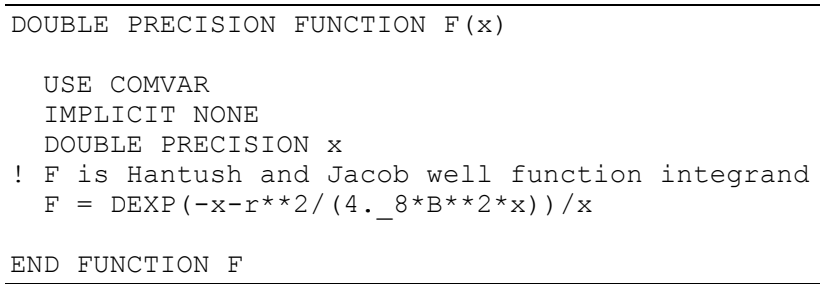

\section{A.3 FORTRAN Listing for Comvar.f90}

MODULE COMVAR 


\section{B Grobner and Hofreiter Numerical Code}

\section{B.1 FORTRAN Listing for GH.f90}

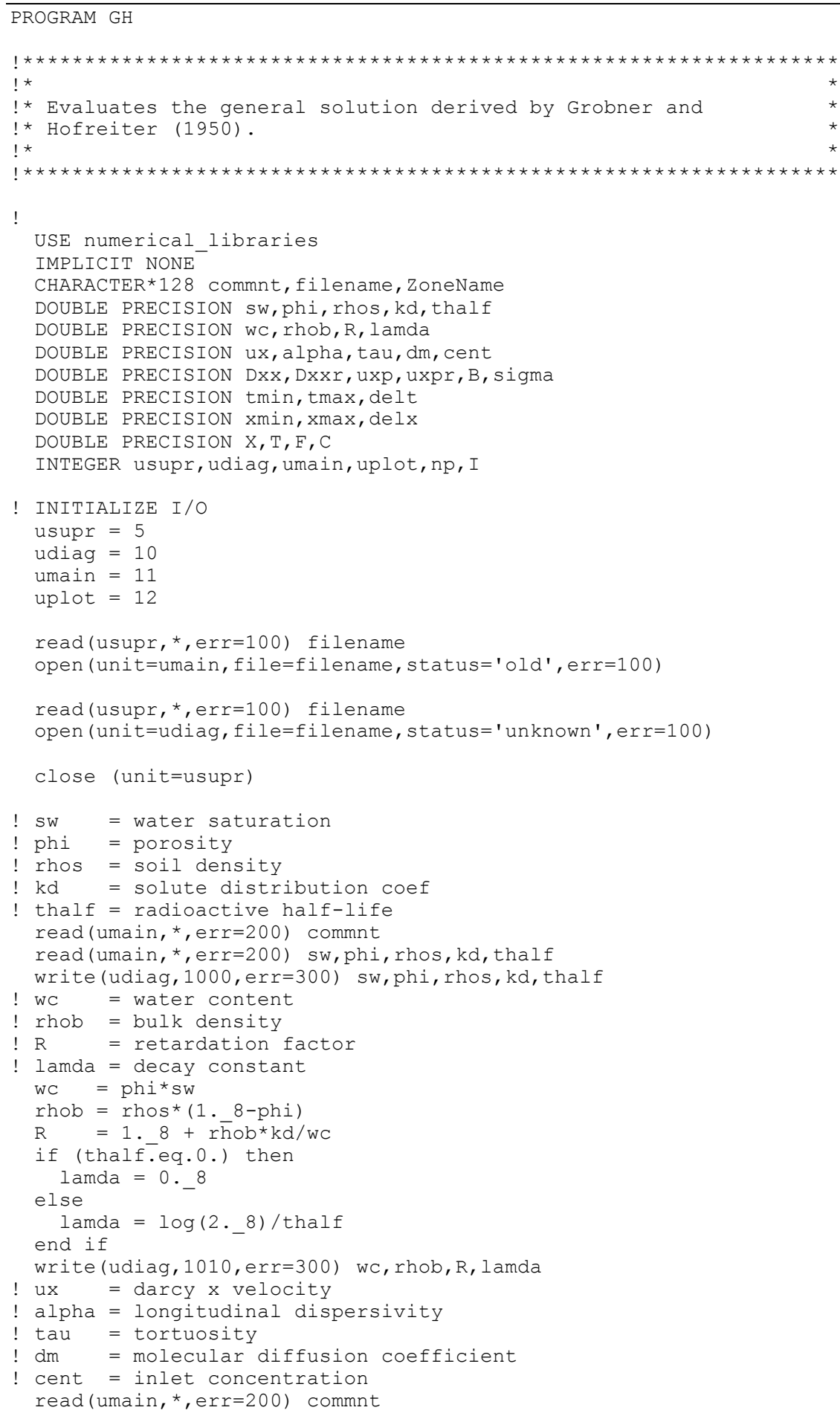


read (umain, *, err $=200$ ) ux, alpha, tau, dm, cent

write (udiag, 1020, err=300) ux, alpha, tau, dm, cent

! $\operatorname{uxp}=$ phasic velocity

! uxpr $=$ retarded phasic velocity

! $\mathrm{Dxx}=$ dispersion coefficient

! Dxxr $=$ retarded dispersion coefficient

$\mathrm{uxp}=\mathrm{ux} / \mathrm{wC}$

uxpr $=u x p / R$

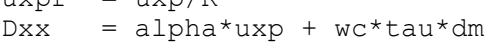

$\mathrm{Dxxr}=\mathrm{Dxx} / \mathrm{R}$

$\mathrm{B}=\operatorname{dsqrt}\left(\left(0.5{ }^{8 * u x p r} / \mathrm{Dxxr}\right) * * 2+1\right.$ amda/Dxxr $)$

sigma $=$ dsqrt (uxpr $\overline{ }$ *2 $+4.8 * 1$ amda*Dxxr)

write (udiag, 1030, err=300) uxp, uxpr, Dxx, Dxxr, B, sigma

! tmin = minimum time value

! $t \max =$ maximum time value

! delt = delta time

read (umain, *, err $=200$ ) commnt

read (umain, *, err $=200$ ) tmin, tmax, delt

! $x$ min $=$ minimum $\mathrm{x}$ value

! $x \max =$ maximum $\mathrm{x}$ value

! $\operatorname{del} x=x$ grid spacing

read (umain, *, err $=200$ ) commnt

read (umain, *, err $=200$ ) xmin, xmax, delx

$\mathrm{np}=\max (\operatorname{int}((\operatorname{xmax}-x \min ) / \operatorname{del} \mathrm{x})+1,1)$

$\mathrm{T}=\operatorname{tmin}$

read (umain, *, err $=200$ ) commnt

! Loop over time

do while $(T<=t \max )$

read (umain, *, err $=200$ ) filename

open (unit=uplot, file=filename, status='unknown', err=100)

read (umain, *, err $=200$ ) ZoneName

write (uplot,' (a)') 'VARIABLES = "x" "y" "c" "mtyp" '

write (uplot, 1500) trim(ZoneName)

$\mathrm{X}=\mathrm{xmin}$

! Loop over $x$

do $I=1, n p$

$\mathrm{F}=\operatorname{derfC}(0.58 *(\mathrm{X}+\operatorname{sigma} * \mathrm{~T}) / \operatorname{dsqrt}(\operatorname{Dxxr} * \mathrm{~T}))$

if (F.eq.0.8) then

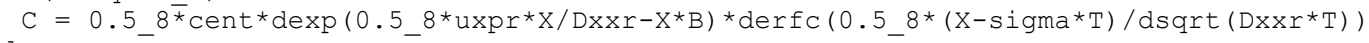
else

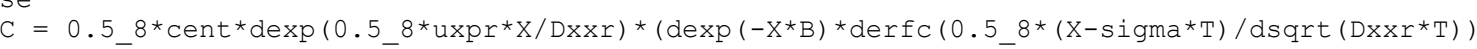

$\&$ end if

$+\operatorname{dexp}(X * B) * \operatorname{derfC}(0.58 *(X+\operatorname{sigma} * T) / \operatorname{dsqrt}(D x \times r * T)))$

write (uplot,' (2(f10.2), es12.3e3,i7)') $\mathrm{x}, 0 ., \mathrm{C}, 1$

$\mathrm{X}=\mathrm{x}+\operatorname{del} \mathrm{x}$

end do

close (unit=uplot)

$\mathrm{T}=\mathrm{T}+\operatorname{del} \mathrm{t}$

end do

close (unit=umain)

stop 'Normal termination'

pause

! bail out during open error

100 stop 'OPEN error'

pause

! bail out during read error

200 stop 'READ error' pause

bail out during write error

300 stop 'WRITE error'

pause

1000 format (/,'water saturation is ',f5.3, \&

/, 'porosity is ', f5.3, \&

/,'soil density is ',f5.3, \&

/,' solute distribution coefficient is ',f5.3, \&

/,' 'half life is ', es9.3)

1010 format (/,' water content is ',f5.3, \&

/,' 'bulk density is ', f5.3, \&

/, 'retardation factor is ', f5.3, \&

/,' decay constant is ', es9.3) 


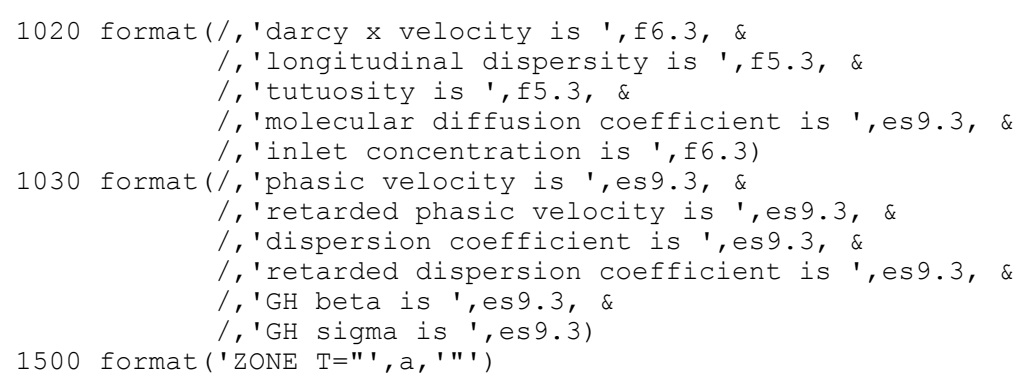

\section{B.2 Superfile Input for 1D Transport Base Case}

\section{B.3 Main Input for 1D Transport Base Case}

\begin{tabular}{|c|c|c|c|c|}
\hline $\begin{array}{l}\text { /sw, } \\
1 . \\
\text { /ux, } \\
1 . \\
\text { /tmin, } \\
25 . \\
\text { /xmin, } \\
0 . \\
\text { /Tecplot } \\
5.1-\mathrm{bc}-25 \\
\text { "5.1-bc } \\
5.1-\mathrm{bc}-50 \\
\text { "5.1-bc }\end{array}$ & $\begin{array}{l}\text { phi, } \\
0.25 \\
\text { alpha, } \\
5 . \\
\text { tmax, } \\
50 . \\
\text { xmax, } \\
400 . \\
\text { output/ } \\
5 . p l t \\
(25) " \\
\text {.plt } \\
(50) "\end{array}$ & $\begin{array}{l}\text { rhos, } \\
1 . \\
\text { tau, } \\
1 . \\
\text { delt/ } \\
25 . \\
\text { delx/ } \\
2 .\end{array}$ & $\begin{array}{l}\mathrm{kd}, \\
0 . \\
\mathrm{dm}, \\
0 .\end{array}$ & $\begin{array}{l}\text { thalf/ } \\
0 . \\
\text { cent/ } \\
1 .\end{array}$ \\
\hline
\end{tabular}

\section{AT123D Numerical Code}

\section{C.1 FORTRAN Listing for AT123D.f90}

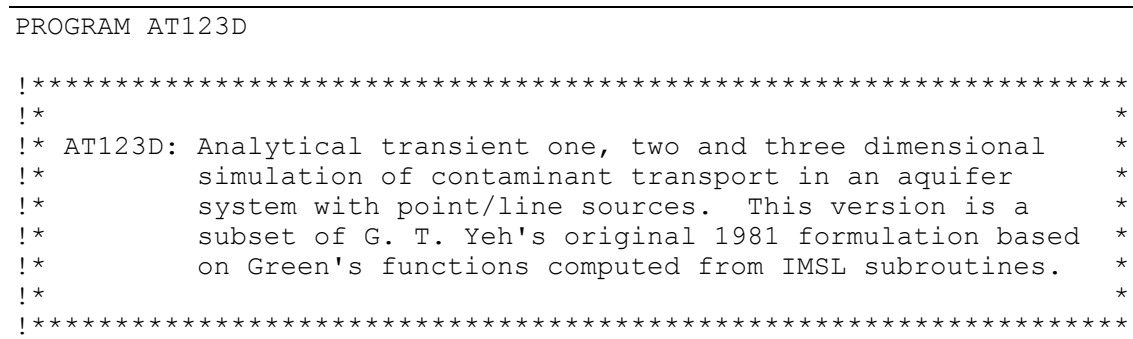

USE comvar

USE numerical_libraries

IMPLICIT NONE

DOUBLE PRECISION, ALLOCATABLE : : alist(:), blist(:), rlist(:), \&

DOUBLE PRECISION sw, phi, rhos, kd, thalf

DOUBLE PRECISION wC, rhob, $R$

DOUBLE PRECISION ux, uy, theta

DOUBLE PRECISION uxp, uyp, up

DOUBLE PRECISION DL, DT, tau, Dm

DOUBLE PRECISION Dxx, Dyy, Dzz

DOUBLE PRECISION QCS, dz, QCl, src beg, src end

DOUBLE PRECISION errabs, errrel, êrrest

DOUBLE PRECISION cost, sint

DOUBLE PRECISION tmin, tmax, delt 
DOUBLE PRECISION xmin, xmax, delx

DOUBLE PRECISION ymin, ymax, dely

DOUBLE PRECISION zmin, zmax, delz

DOUBLE PRECISION XT,YT, C, INTG

CHARACTER *128 commnt, filename, Zone

CHARACTER $* 15$ timestr

CHARACTER * 4 option

INTEGER, ALLOCATABLE : : iord(:)

INTEGER usupr, umain, udiag, uplot, ierr

INTEGER irule, maxsub, neval, nsubin

INTEGER I, J, K, npts

EXTERNAL G

! INITIALIZE I/O

usupr $=5$

umain $=10$

udiag $=15$

uplot $=20$

read (usupr, *, err $=100$ ) filename

open (unit=umain, file=filename, status='old', err=100)

read(usupr, *, err $=100$ ) filename

open (unit=udiag, file=filename, status=' unknown', err=100)

read (usupr, *, err $=100$ ) filename

open (unit=uplot, file=filename, status=' unknown', err=100)

close (unit=usupr)

! ndim = '1D', '2D' or '3D' analytical simulation

! $\mathrm{sx}=$ type of source in $\mathrm{x}$-dir ('PS' or 'LS')

! sy = type of source in y-dir ('PS' or 'LS')

! sz = type of source in z-dir ('PS' or 'LS')

! option = 'GRID', 'LINE' or 'PNTS' specified

! Zone = Tecplot zone prefix name

read (umain, *, err $=200$ ) commnt

read (umain, *, err $=200$ ) ndim, sx, sy, sz, option, zone

write (udiag, 1000 , err=300) ndim, sx, sy, sz, option, zone

! sw = water saturation

! phi $=$ porosity

! rhos = soil density

! $\mathrm{kd}=$ solute distribution coef

! thalf = radioactive half-life

read (umain, *, err $=200$ ) commnt

read (umain, *, err $=200$ ) sw, phi, rhos, kd, thalf

write (udiag, 1010, err=300) sw, phi, rhos, kd, thalf

wC $=p h i * s w$

rhob $=(1.8-$ phi $) *$ rhos

$\mathrm{R}=1 \cdot \overline{8}+\mathrm{rhob} * \mathrm{kd} / \mathrm{wC}$

if (thalf.eq.0.) then

lamda $=0.8$

else

lamda $=\log \left(2 \cdot{ }_{-}\right) /$thalf

end if

write (udiag, 1020, err=300) wc, rhob, R, lamda

! ux = Darcy $\mathrm{x}$-dir velocity

! uy $=$ Darcy y-dir velocity

! theta $=$ Rotation angle (degrees counterclockwise from $x$-axis)

read (umain, *, err $=200$ ) commnt

read (umain, *, err $=200$ ) ux, uy, theta

write (udiag, 1030, err=300) ux, uy, theta

$\mathrm{uxp}=\mathrm{ux} / \mathrm{wC}$

uxpr $=\mathrm{uxp} / \mathrm{R}$

uyp $=u y / w c$

uypr $=\mathrm{uyp} / \mathrm{R}$

$\operatorname{up}=\operatorname{sqrt}($ uxp*uxp+uyp*uyp)

! DLh $=$ Longitudinal dispersivity

! DTh = Transverse dispersivity

! tau = tortuosity of porous medium

! $\mathrm{Dm}=$ molecular diffusion coefficient

read (umain, *, err $=200$ ) commnt

read (umain, *, err $=200$ ) DL, DT, tau, Dm

write (udiag, 1040, err=300) DL, DT, tau, Dm 


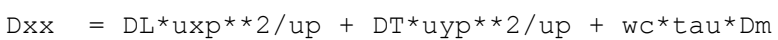

Dyy $=\mathrm{DL}^{\star}$ uyp $* * 2 / \mathrm{up}+\mathrm{DT}^{*}$ uxp**2/up $+\mathrm{wC}^{*}$ tau ${ }^{*} \mathrm{Dm}$

$\mathrm{Dzz}=\mathrm{DT} * \mathrm{uxp} * \star 2 / \mathrm{up}+\mathrm{DT}^{\star} \mathrm{Uyp}^{\star} * 2 / \mathrm{Up}+\mathrm{wc} * \mathrm{tau} * \mathrm{Dm}$

$\mathrm{Dxxr}=\mathrm{Dxx} / \mathrm{R}$

Dyyr $=$ Dyy/R

$\mathrm{Dzzr}=\mathrm{Dzz} / \mathrm{R}$

write (udiag, 1050, err=300) Dxx, Dyy, Dzz, Dxxr, Dyyr, Dzzr

! $\mathrm{Qcp}=$ point source mass flowrate

! $\mathrm{dz}=$ aquifer thickness

! Xs $=\mathrm{x}$ location of point source

! Ys = y location of point source

! ZS = z location of point source

! Ls = start of line source

! Le = end of line source

read (umain, *, err $=200$ ) commnt

read (umain, *, err $=200$ ) QCS, dz, Xs, Ys, Zs, Ls, Le

$\mathrm{Qcl}=\mathrm{Qcs} / \mathrm{dz}$

! src beg = start time for source

! src_end = end time for source

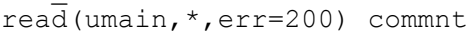

read (umain, *, err $=200$ ) src beg, src end

! irule = choice of quadrature rule (Gauss-Kronrod)

! $\quad=1(7-15$ points $)$

$=2(10-21$ points $)$

$=3(15-31$ points $)$

$=4(20-41$ points $)$

$=5(25-51$ points $)$

$=6(30-61$ points $)$

maxsub $=$ number of sub intervals allowed

errabs = absolute error

! errrel = relative error

read (umain, *, err $=200$ ) commnt

read (umain, *, err $=200$ ) irule, maxsub, errabs, errrel

allocate (alist (maxsub), stat=ierr)

allocate (blist (maxsub), stat=ierr)

allocate (rlist (maxsub), stat=ierr)

allocate (elist (maxsub), stat=ierr)

allocate (iord (maxsub), stat=ierr)

! tmin = minimum time value

! $\operatorname{tmax}=$ maximum time value

! delt = delta time

read (umain, *, err $=200$ ) commnt

read (umain, *, err $=200$ ) tmin, tmax, delt

pi $=\operatorname{dacos}(-1.8)$

theta $=$ theta $/ 1 \cdot 8 \overline{\mathrm{d}}+2 * \mathrm{p} i$

cost $=\cos ($ theta)

sint $=\sin ($ theta)

select case (option)

case ('GRID')

xmin = starting $\mathrm{x}$ value for grid

xmax $=$ ending $x$ value for grid

del $\mathrm{x}=\mathrm{x}$ grid spacing

read (umain, *, err $=200$ ) commnt

read (umain, *, err $=200$ ) xmin, xmax, delx

$I=\max (\operatorname{int}((\operatorname{xmax}-x \min ) / \operatorname{del} x)+1,1)$

ymin = starting $y$ value for grid

ymax = ending $y$ value for grid

dely $=y$ grid spacing

read (umain, *, err $=200$ ) commnt

read (umain, *, err $=200$ ) ymin, ymax, dely

$\mathrm{J}=\max (\operatorname{int}((\operatorname{ymax}-y \min ) / \operatorname{dely})+1,1)$

zmin = starting $\mathrm{z}$ value for grid

zmax $=$ ending $\mathrm{z}$ value for grid

delz $=z$ grid spacing

read (umain, *, err $=200$ ) commnt

read (umain, *, err $=200$ ) zmin, zmax, delz

$\mathrm{K}=\max (\operatorname{int}((\mathrm{zmax}-\mathrm{zmin}) / \operatorname{del} \mathrm{z})+1,1)$

$\mathrm{T}=\mathrm{tmin}$

write (uplot, ' (a) ', err=300) 'VARIABLES = "x" "y" "z" "c"'

! Loop over time

do while $(T<=$ tmax $)$

write (timestr, 2000 , err $=300$ ) T 
write (uplot, 2010, err=300) trim(Zone)//trim(timestr), I, J, K

! Loop over Z

$\mathrm{z}=\mathrm{zmin}$ 


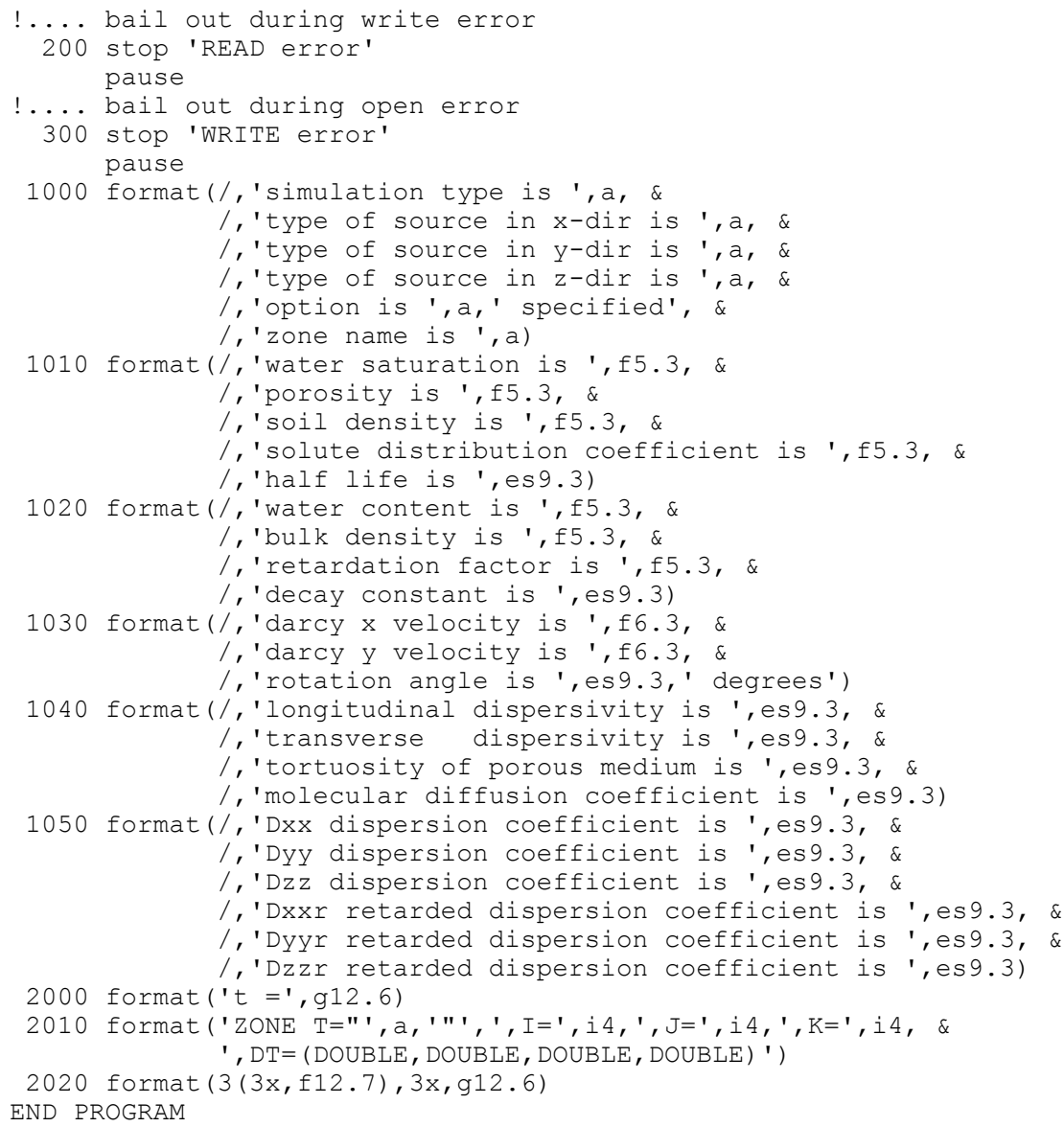

\section{C.2 FORTRAN Listing for COMVAR.f90}

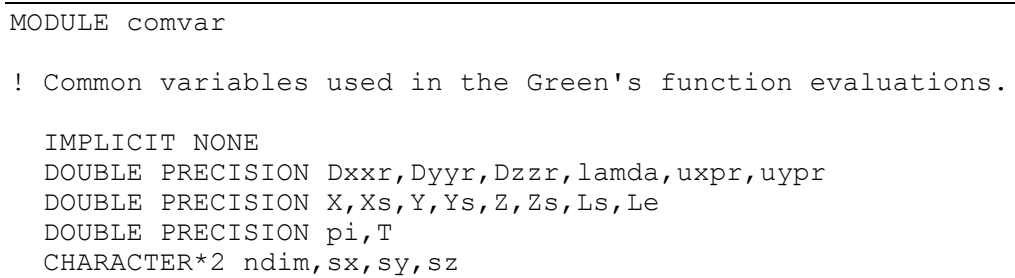

END MODULE comvar

\section{C.3 FORTRAN Listing for G.f90}

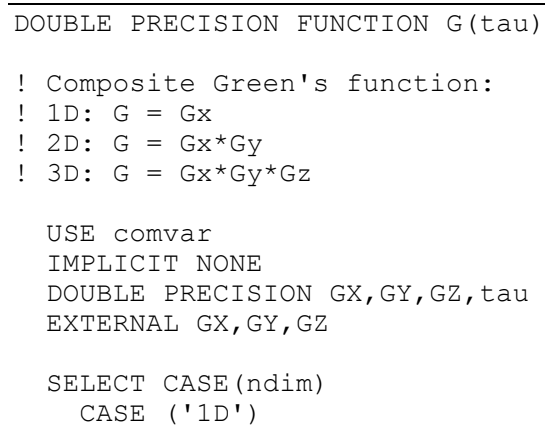




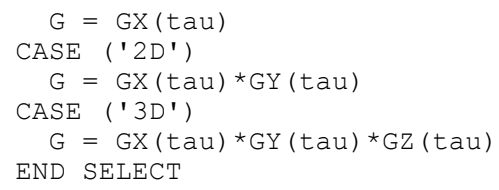

\section{C.4 FORTRAN Listing for Gx.f90}

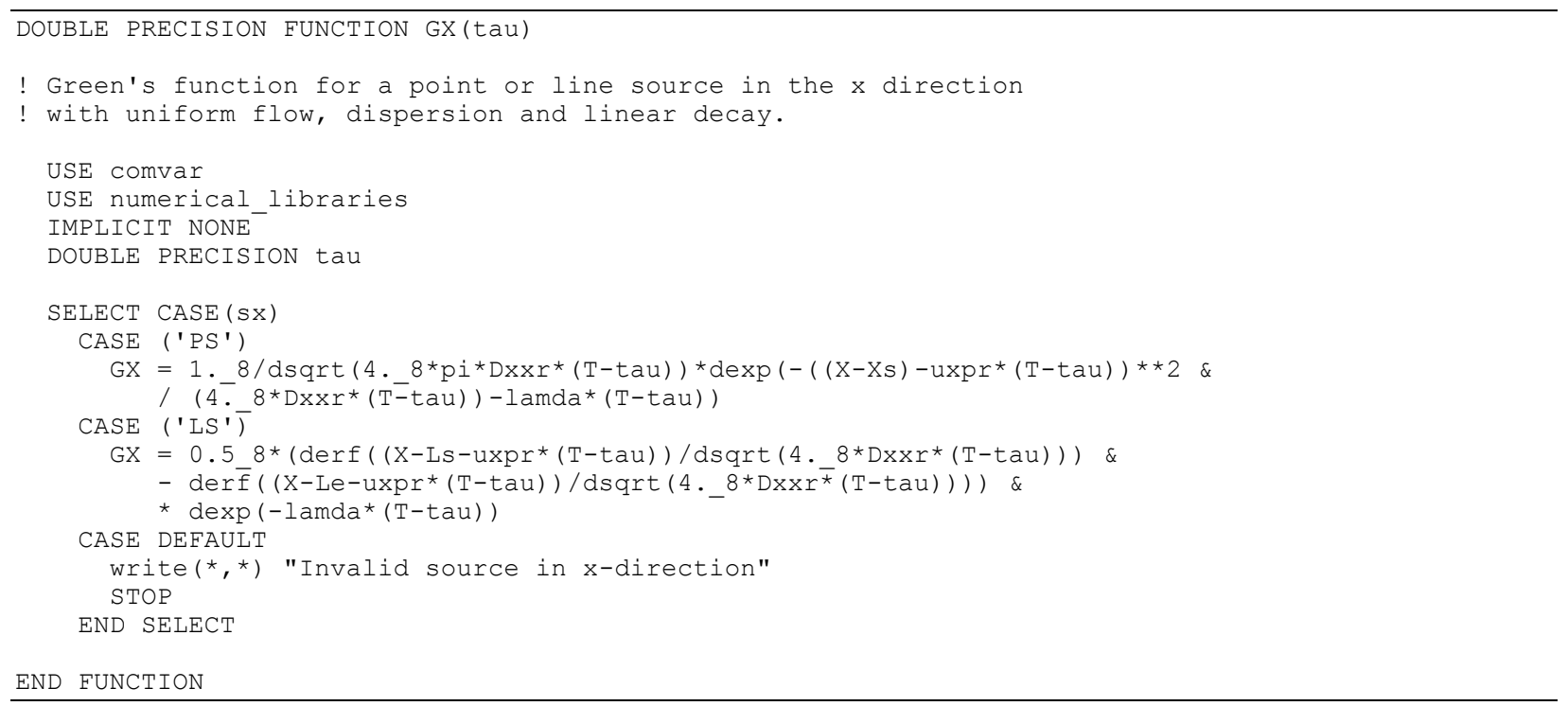

\section{C.5 FORTRAN Listing for Gy.f90}

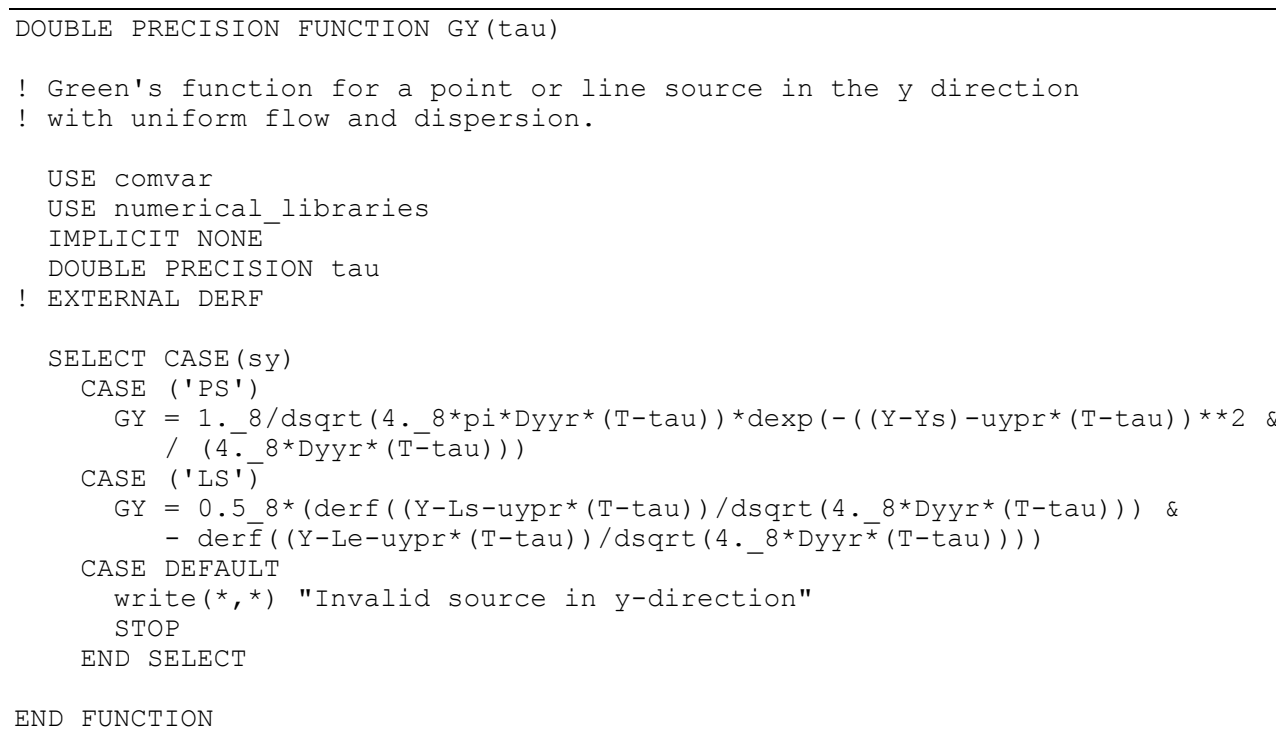

\section{C.6 FORTRAN Listing for Gz.f90}

DOUBLE PRECISION FUNCTION GZ(tau)

! Green's function for a point or line source in the $z$ direction

! with dispersion. 


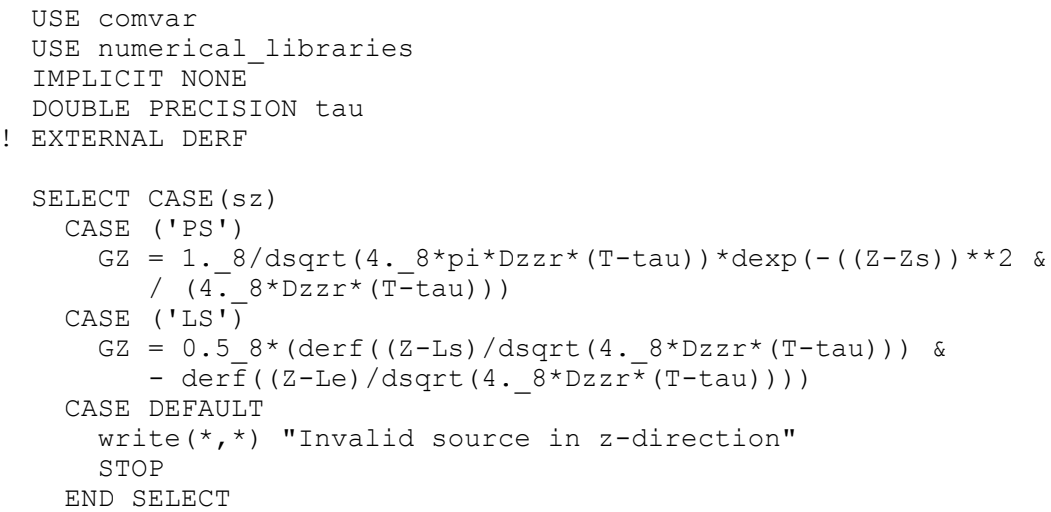

\section{C.7 Superfile Input for 2D Transport Base Case (Case A)}

AT123D-Y000.inp

AT123D-Y000.log

AT123D-Y000.plt

\section{C.8 Main Input for 2D Transport Base Case (Case A)}

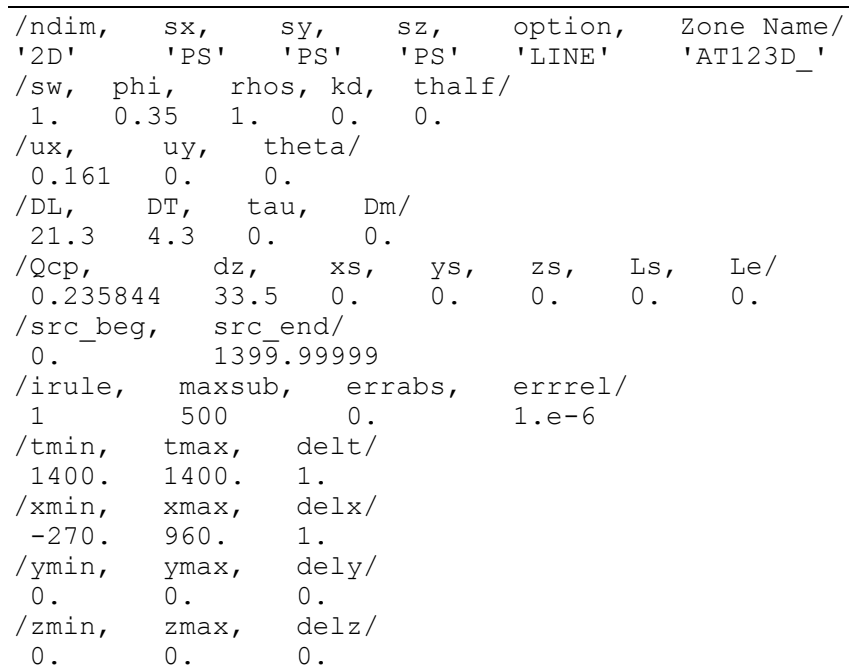

\section{Bateman Numerical Code}

\section{D.1 FORTRAN Listing for Bateman.f90}

PROGRAM Bateman

!... Computes the ith radionuclide concentration based on

!.... the Bateman Equation (n1-->n2-->n3-->...ni-->). The

!... concentrations of all the progeny are initially zero

!... (i.e. ni $(0)=0$ for $i>1)$

USE decay constants

IMPLICIT DOUBLE PRECISION $(\mathrm{a}-\mathrm{h}, \mathrm{o}-\mathrm{z})$

INTEGER, PARAMETER : : sor=8, soi=4

CHARACTER*128 commnt,filename 
CHARACTER*10, ALLOCATABLE : : $\operatorname{species(:)}$

$\operatorname{ALLOCATABLE}:: A(:,:), \operatorname{Aconv}(:)$, thalf $(:), t(:)$

ALLOCATABLE : : BTerm2 $(:,:), \operatorname{BTerm} 1(:)$

DOUBLE PRECISION, ALLOCATABLE : : $\mathrm{n}(:,:)$

DOUBLE PRECISION : : nO

DOUBLE PRECISION, EXTERNAL : : BTermi

!... INITIALIZE I/O

isup $=5$

iinp $=10$

$i \log =20$

icon $=21$

iact $=22$

iplt $=23$

read (isup, *, err $=100$ ) filename

open (unit=iinp, file=filename, status='old', err=300)

read (isup, *, err $=100$ ) filename

open (unit=ilog, file=filename, status='unknown', err=300)

read (isup, *, err $=100$ ) filename

open (unit=icon, file=filename, status=' unknown', err=300)

read (isup, *, err $=100$ ) filename

open (unit=iact, file=filename, status='unknown', err=300)

read (isup, *, err $=100$ ) filename

open (unit=iplt, file=filename, status='unknown', err=300)

!... PROCESS DECAY CHAIN INFO

!... nspecies $=$ number of radioactive species

read (iinp, *, err $=100$ ) commnt

read (iinp, *, err $=100$ ) nspecies

write (ilog, *, err=200) 'nspecies = ', nspecies

!... species = radioactive species name

!... thalf = radioactive species half-life

allocate (species (nspecies), stat=ierr)

call chkmem('Bateman', 'species', 10*nspecies, 0 , ierr)

allocate (thalf (nspecies), stat=ierr)

call chkmem('Bateman', 'thalf', sor*nspecies, 0, ierr)

read (iinp, *, err $=100$ ) commnt

do $i=1$, nspecies

read (iinp, *, err $=100)$ species(i), thalf(i)

write (ilog, *, err=200) $\operatorname{species(i),thalf(i)}$ end do

!... no = initial concentration of parent species

.... tend $=$ end time for transient decay

!... nint $=$ number of time intervals between 0 and tend

read (iinp, *, err $=100$ ) commnt

read (iinp, *, err $=100$ ) no, tend, nint

delt $=$ tend/dble (nint)

.... lamda = radioactive species decay constant $\ln (2) / t 1 / 2$

!... Aconv = activity conversion factor for each progeny

allocate (lamda (nspecies), stat=ierr)

call chkmem('Bateman', 'lamda', sor*nspecies, 0 , ierr)

allocate (Aconv (nspecies), stat=ierr)

call chkmem ('Bateman', 'Aconv', sor*nspecies, 0 , ierr)

do $i=1$, nspecies

lamda $(i)=\log (2.8) /$ thalf $(i)$

$\operatorname{Aconv}(i)=\operatorname{lamda}(\bar{i}) / \operatorname{lamda}(1)$

write (ilog, 111, err=200) i, lamda (i)

111 format ('lamda (',i1,') = ',e12.6) end do

!...t $=$ time to evaluate Bateman Eq.

allocate (t (0:nint), stat=ierr)

call chkmem('Bateman', 't', sor*nint, 0 , ierr)

$t=0.8$

!... $n(i, j)^{-}=$radioactive species concentrations $(i=$ species, $j=t i m e)$

!...A $(i, j)=$ radioactive species activities

allocate (n (nspecies, 0 :nint), stat=ierr)

call chkmem('Bateman', 'n', sor*nspecies*nint, 0 , ierr)

allocate (A (nspecies, 0 :nint), stat=ierr) 
call chkmem('Bateman', 'A', sor*nspecies*nint, 0, ierr)

!.... Initialize concentrations and activities

$\mathrm{n}=0.8$

$\mathrm{A}=0 .-8$

$\mathrm{n}(1,0)=\mathrm{n} 0$

$A(1,0)=\operatorname{Aconv}(1) * n(1,0)$

!... Compute Bateman Eq.time-independent terms for each species

allocate (BTerm1 (nspecies), stat=ierr)

call chkmem('Bateman', 'BTerm1', sor*nspecies, 0 , ierr)

allocate (BTerm2 (nspecies, nspecies), stat=ierr)

call chkmem('Bateman', 'BTerm2', sor*nspecies*nspecies, 0, ierr)

do $i=1$, nspecies

BTerm1 (i) = BTermi (i)*n0

write (ilog, 123, err $=200)$ i, BTerm1 (i)

123 format ('BTerm1 $\left(', i 1,{ }^{\prime}\right)={ }^{\prime}$, e12.6)

do $j=1, i$

$\operatorname{BTerm} 2(i, j)=1.8$

do $k=1$, $i$

if $(k==j)$ then

factor $=1$. 8

else

factor $=$ lamda $(k)-l$ amda $(j)$

end if

$\operatorname{BTerm} 2(i, j)=$ factor*BTerm2 $(i, j)$

end do

write (ilog, 124, err=200) i,j,BTerm2 $(i, j)$

124 format ('BTerm2(',i1, ', ', i1,' ) = ', e12.6) end do

end do

!... LOOP OVER TIME

do it $=1$, nint

$t(i t)=d b l e(i t) * d e l t$

do $i=1$, nspecies

do $j=1, i$

$n(i, i t)=n(i, i t)+\exp (-\operatorname{lamda}(j) * t(i t)) / \operatorname{BTerm} 2(i, j)$

end do

$\mathrm{n}(\mathrm{i}, \mathrm{it})=\operatorname{BTerm} 1(i) * \mathrm{n}(i, i t)$

$A(i, i t)=A \operatorname{conv}(i) *_{n}(i, i t)$

end do

end do

!... WRITE CSV FILE of CONCENTRATIONS

write (icon,*) 'Time', (', ', trim(species(i)), i=1, nspecies)

do it $=0$, nint

write (icon, ' (e12.6,12(a,e12.6))') t(it), (', ',n(i,it), i=1,nspecies)

end do

!... WRITE CSV FILE of ACTIVITIES

write (iact, *) 'Time', (', ', trim(species (i)), $i=1$, nspecies)

do it $=0$, nint

write (iact,' (e12.6,12(a,e12.6))') t(it), (', ', A (i, it), i=1, nspecies)

end do

!.... WRITE TECPLOT FILE OF CONCENTRATIONS AND ACTIVITIES

write (iplt, *, err=200) 'Title = "Bateman Eqn. for ', \&

species(1) (1:len_trim(species(1))),' decay chain"'

write (iplt, ${ }^{*}$, err $=200$ ) 'Variables $=$ '

write (iplt, *, err=200) ' "Time"'

do $i=1$, nspecies

write (iplt, *, err=200) ' "'//trim(species (i))//'"'

end do

write (iplt, *, err=200) ' ZONE $T=$ "Concentrations"'

write (iplt, *, err=200) 'DT=( ', ('DOUBLE ',i=1, nspecies+1), ')'

do it $=0$, nint

write (iplt, ' (7 (es13.6,1x))', err=200) t(it), (n (i,it), i=1, nspecies)

end do

write (iplt, *, err=200) ' ZONE T = "Activities"'

write (iplt, *, err=200) 'DT=( ', ('DOUBLE ',i=1, nspecies+1), ')'

do it $=0$, nint

write(iplt,' (7e13.6)', err=200) t(it), (A (i,it), i=1, nspecies)

end do

stop 'Normal termination'

pause

!... bail out during read error

100 stop 'READ error'

pause 


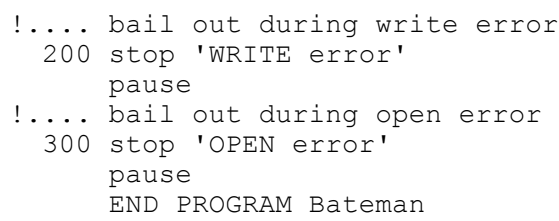

\section{D.2 FORTRAN Listing for BTermi.f90}

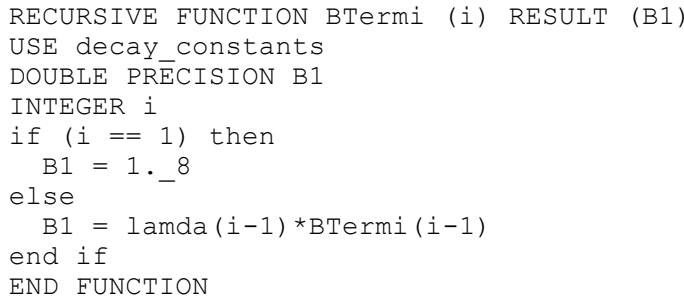

\section{D.3 FORTRAN Listing for BTermij.f90}

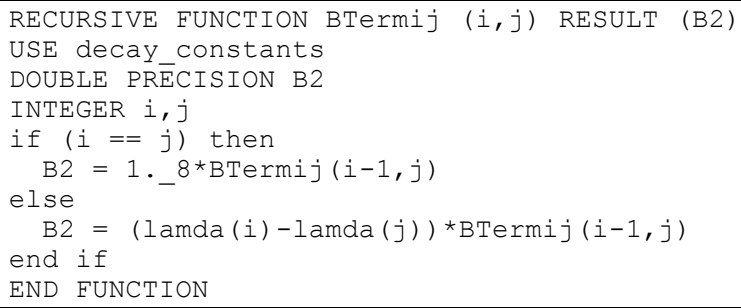

\section{D.4 FORTRAN Listing for chkmem.f90}

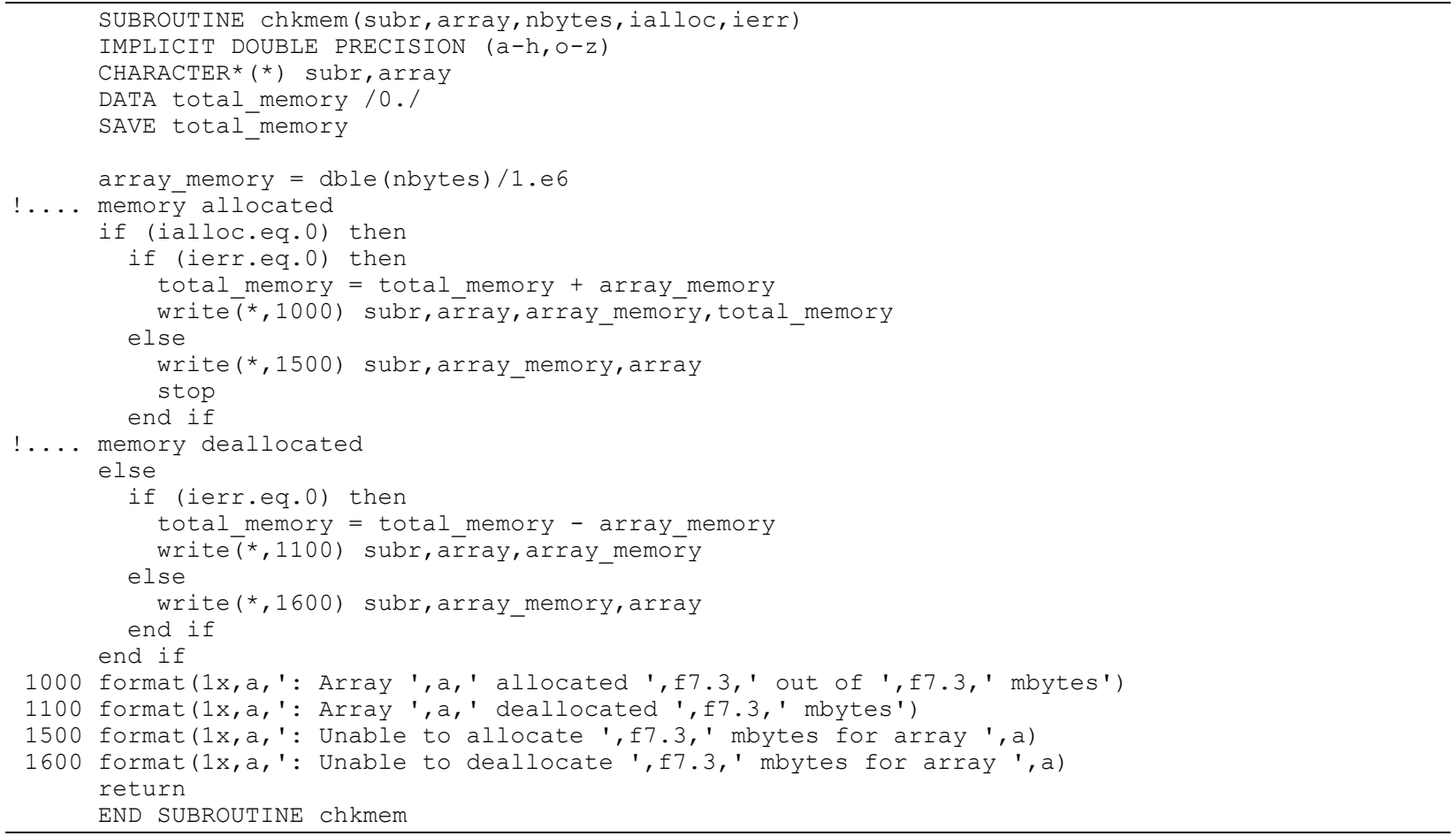


D.5 FORTRAN Listing for decay_constants.f90

MODULE decay_constants

DOUBLE PRECISION, ALLOCATABLE :: lamda(:) END MODULE decay_constants

\section{D.6 Superfile Input for U-230 Decay Chain}

$\mathrm{U}-230$. dat

$\mathrm{U}-230 . \log$

$\mathrm{U}-230-\mathrm{C} . \mathrm{CSV}$

$\mathrm{U}-230-\mathrm{A} \cdot \mathrm{CSV}$

$\mathrm{U}-230 \cdot \mathrm{plt}$

\section{D.7 Main Input for U-230 Decay Chain}

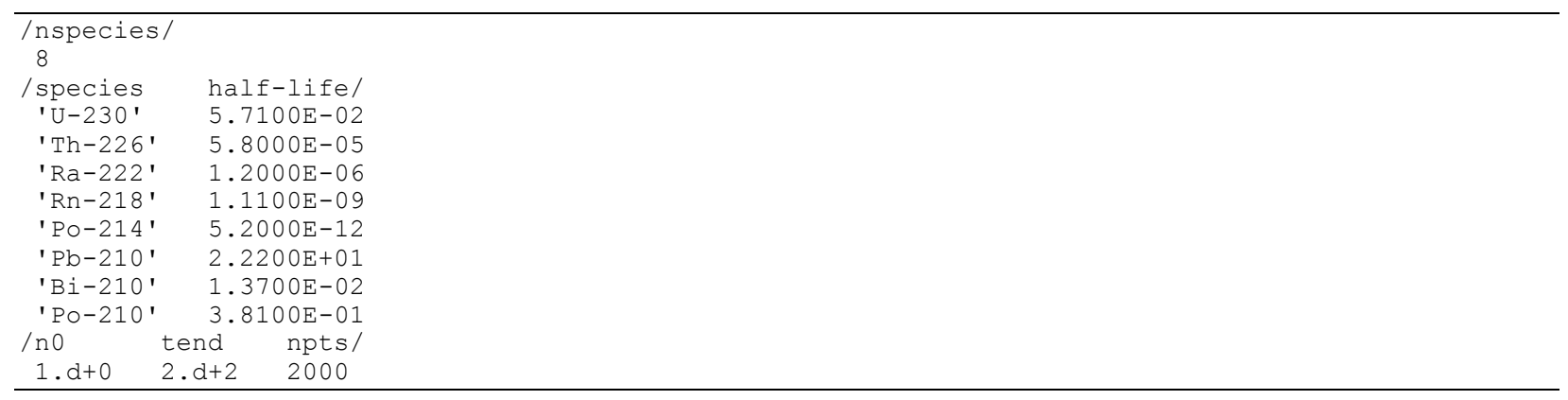

